\title{
GEOLOGY, MINERALOGY, AND GEOCHEMISTRY OF THE VAZANTE NORTHERN EXTENSION ZINC SILICATE DEPOSIT, MINAS GERAIS, BRAZIL
}

by

Paul R. Slezak

A thesis submitted to the Department of Geological Sciences and Geological Engineering In conformity with the requirements for

the degree of Master of Science

\author{
Queen's University \\ Kingston, Ontario, Canada
}

(February, 2012)

Copyright (CPaul R. Slezak, 2012 


\begin{abstract}
The Vazante Northern Extension is a continuation of the structurally controlled, hypogene nonsulfide zinc deposit from the Vazante Mine, Minas Gerais, Brazil. The deposit is hosted in Neoproterozoic carbonates of the Serra do Poço Verde Formation located in the Brasília Fold Belt along the western margin of the São Francisco Craton. The Northern Extension deposit is hosted within a shear zone that strikes 200 to 225 and dips $30^{\circ}-70^{\circ} \mathrm{NW}$ and is associated with substantial folding that occurred in the same deformation $\left(\mathrm{D}_{2}\right)$ event and later transcurrent faulting. The shear zone is composed of a tectonic-hydrothermal breccia that can be subdivided into four types: Type 1 Breccia- distally located from the willemite $\left(\mathrm{Zn}_{2} \mathrm{SiO}_{4}\right)$ ore, weakly brecciated dolostone with some Fe-carbonate alteration; Type 2 Breccia-Strong Fe-carbonate alteration, intense brecciation; Type 3 Breccia-hematite replacement breccia, usually proximal to the Type 4 Breccia; Type 4 Breccia-willemite ore breccia, containing three generations of hypogene willemite $\left(\mathrm{Zn}_{2} \mathrm{SiO}_{4}\right)$ as well as hematite $\left(\mathrm{Fe}_{2} \mathrm{O}_{3}\right)$, and franklinite $\left(\mathrm{ZnFe}_{2} \mathrm{O}_{4}\right)$. In the southern part of the deposit, late sulfide-rich veinlets are observed cutting the hypogene zinc silicate mineralization, and contain galena, sphalerite, native silver, covellite, and stromeyerite $\left(\mathrm{Cu}_{1-\mathrm{x}} \mathrm{Ag}_{1-}\right.$ $\left.{ }_{\mathrm{x}} \mathrm{S}\right)$. Geochemical studies conducted on the tectonic-hydrothermal breccia showed a strong regional signature enriched in $\mathrm{Ag}, \mathrm{Cd}, \mathrm{Pb}$, and $\mathrm{Zn}$. The presence of willemite as opposed to sphalerite and the ubiquity of hematite suggest that the zinc silicate mineralization has formed from the mixing between a low sulfur, acid, oxidizing metalliferous basinal brine similar to the fluids found in MVT deposits with a cooler, more oxidizing, meteoric fluid.
\end{abstract}




\section{Co-Authorship}

Chapter 3 of this thesis was prepared as a separate manuscript for a journal publication as a coauthored paper. This chapter is co-authored with Dr. Gema R. Olivo who contributed to field work; structural, mineralogical, and geochemical interpretations; and acted in as a supervisor and editor throughout the entirety of this thesis; Gustavo D. Oliveira, who contributed extensively to the organization and interpretation of field work, core logging, and sampling; and Dr. Marcel A. Dardenne*, who contributed to background research, field interpretations, and stratigraphic studies.

*Deceased 


\section{Acknowledgements}

Paul R. Slezak gratefully acknowledges Votorantim Metals for their continuous support and collaboration and also thanks Society of Economic Geologists (SEG) for his student research grant. This research was also generously supported by National Science and Engineering Research Council (NSERC) grants to Dr. G.R. Olivo.

I would like to extend my sincere gratitude towards my thesis advisor, Dr. Gema R. Olivo, for her continuous guidance and patience throughout this project. I would also like to thank her for her assistance in the field as well as her help for all the various lab related parts of this project, and in particular, her help in reviewing and editing this thesis. Most importantly, I want to thank Dr. Olivo for introducing me to Brazil and all of its opportunities.

I would like to thank Dr. Marcel A. Dardenne of the University of Brasília for his valuable input in understanding the Brasília Fold Belt as well as his jovial attitude while out in the field. He was a wonderful person who just simply fun to be around. He will be greatly missed.

A very special thanks goes out to Gustavo Diniz-Oliveira of Votorantim Metals for all his input and advice about the Vazante region, but most importantly for acting as my "handler" while I was in Brazil. Gustavo always made it possible so I could map and access the Votorantim's exploration drill core and data. Without this information I would not have been able to complete this project. 
This project was made possible by Votorantim Metals, who provided access to information relevant to the project. I would like to thank the Vazante Exploration Team, in particular, who came out into the field, bounced around ideas, helped me learn Brazilian Portuguese, and were excellent roommates. Thank you Basilio Botura Neto, Fernando Henrique Baia, Luiz Fernando de Mello Montano, João Henrique Boniatti, Joyce Teixeira, and Samual Bouças do Lago.

My field work and core logging was made possible by Celio Rodrigues Gomes, Jocelino Alves Da Fonseca, and Rondennell Procópio da Silva of Votorantim and by Jaison Pereira Costa, Jeovani Pererra Da Silva, João Paulo Rodrigues Frois, Fernando Rodrigues da Silva, Geraldo Vasconcelos Borges, Gilberto Maria Pereira, Leandro José Rodrigues, and Nilberto Jose Pereira of WÇA. I would also like to apologize to João Paulo Rodrigues Frois for making him hike through the streams and spider webs to find a few small outcrops.

Thank you to Dr. Nelia Dunbar of New Mexico Tech and the New Mexico Bureau of Mines and Geology for helping me with my EMP analysis. I really appreciate the time you put in helping me as well as the excellent quality of the analyses.

I would like to thank my colleagues at Queen's University, especially Liane Millington, for all the good times, being available to bounce around ideas, and helping me find my way to the labs.

Finally, I would like to thank my family for their love and support. 


\section{Table of Contents}

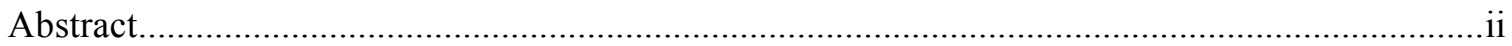

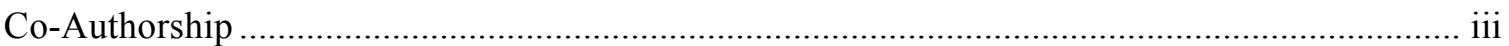

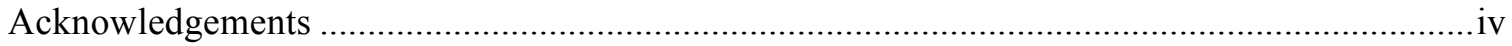

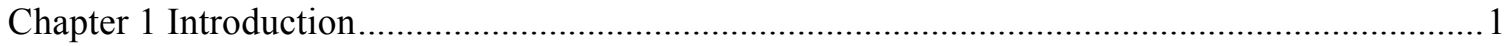

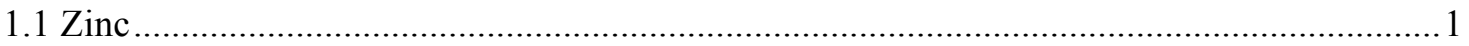

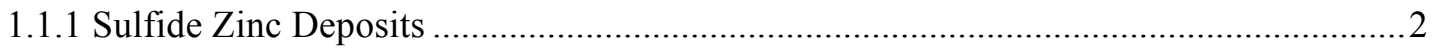

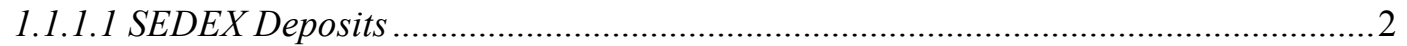

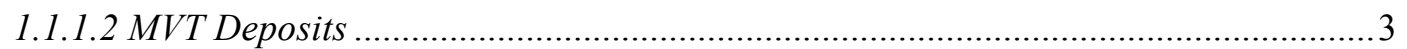

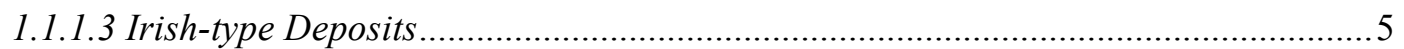

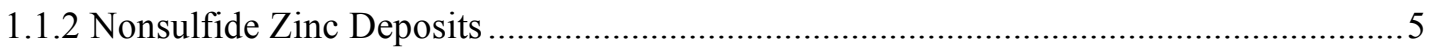

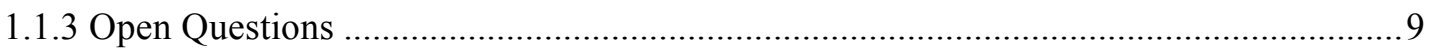

1.1.4 The Vazante Mine and Northern Extension Deposit..................................................... 9

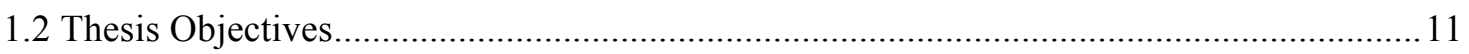

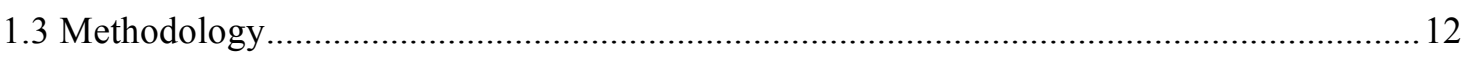

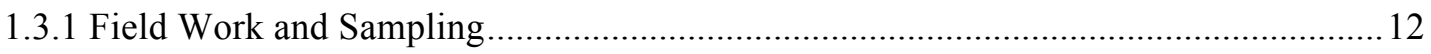

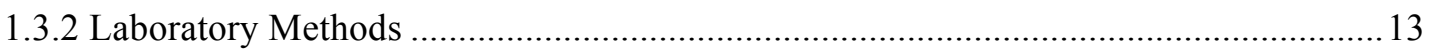

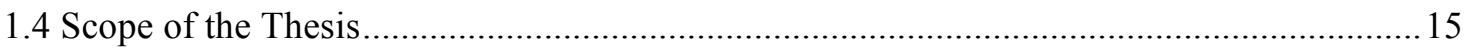

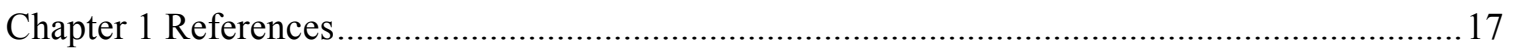

Chapter 2 Literature Review: Regional Geology, Tectonic Evolution, and Metallogeny of the

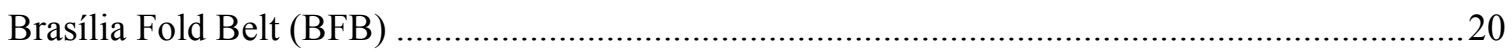

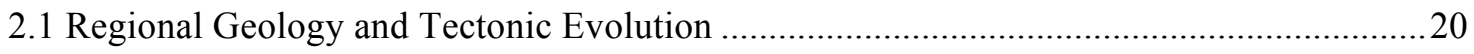

2.2 Mineral Deposits Hosted in the Vazante Group ............................................................ 30

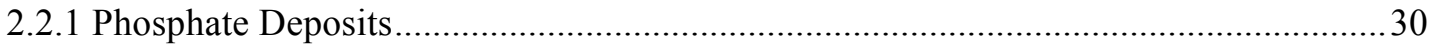

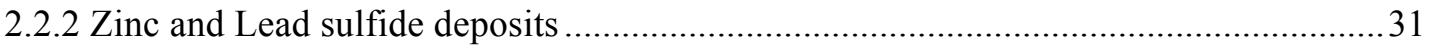

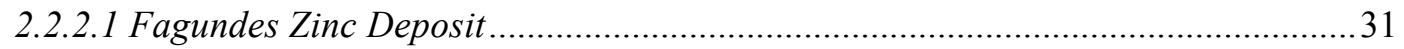

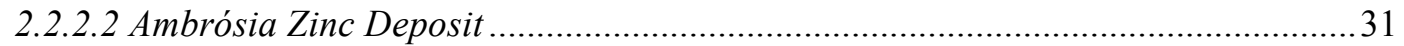

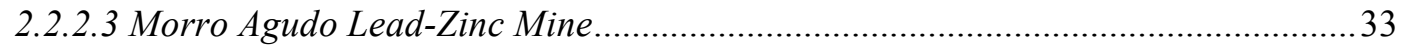

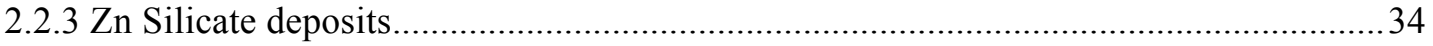

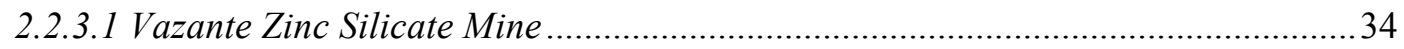

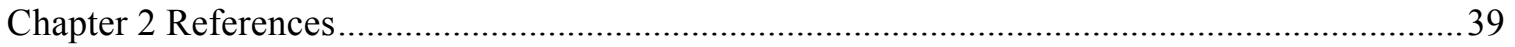


Chapter 3 Geology, Mineralogy, and Geochemistry of the Vazante Northern Extension Zinc

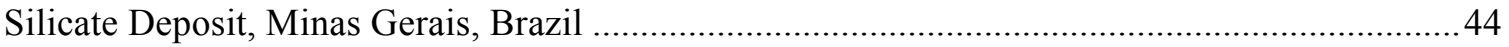

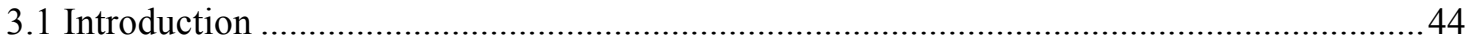

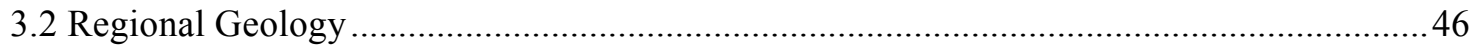

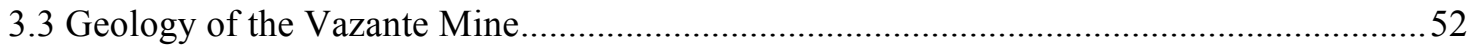

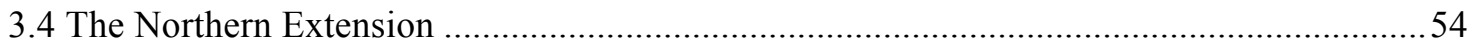

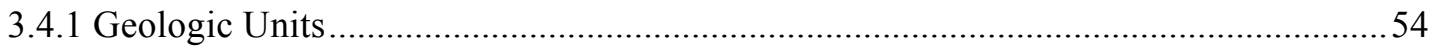

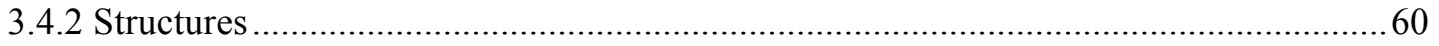

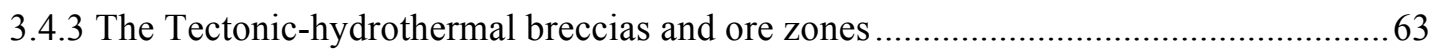

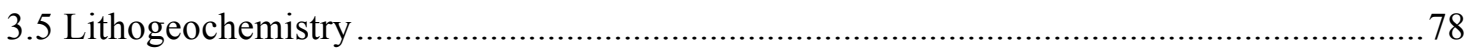

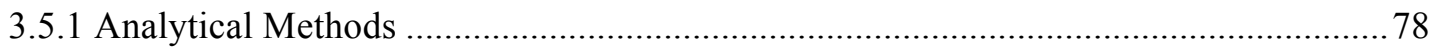

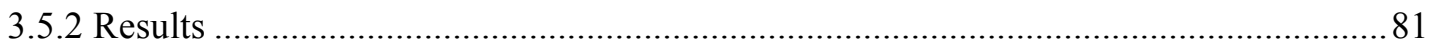

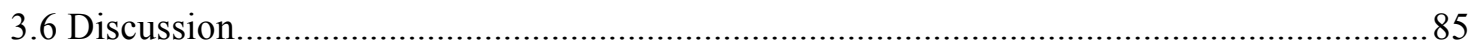

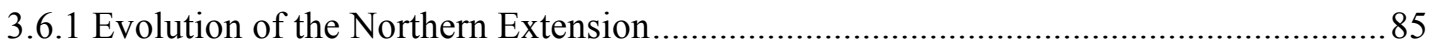

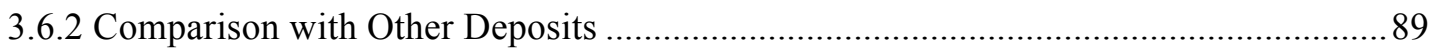

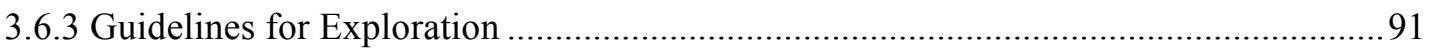

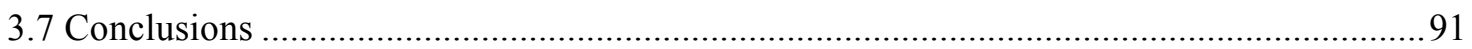

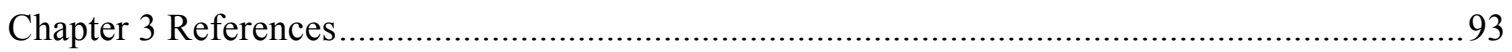

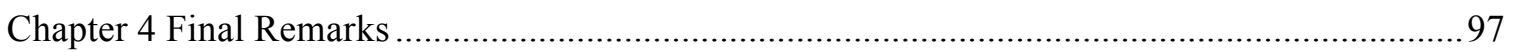

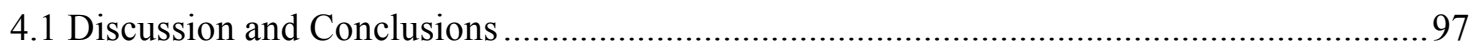

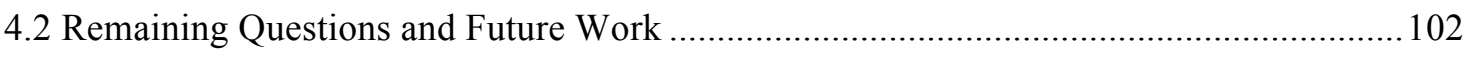

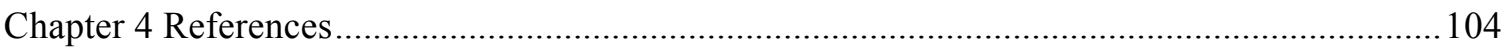

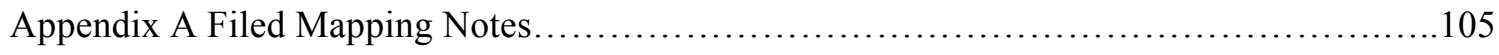

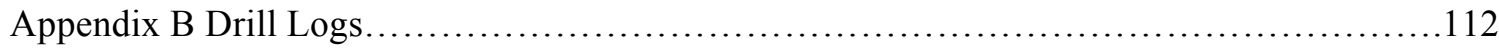

Appendix C Thin Section Descriptions............................................ 156

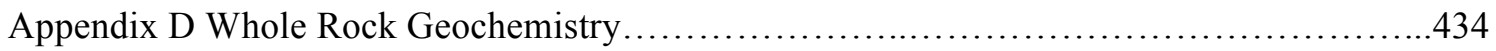




\section{List of Figures}

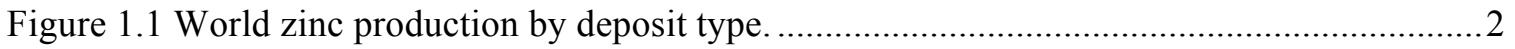

Figure 1.2 Comparison of Vazante Zn grade and tonnage versus world SEDEX deposits .............3

Figure 1.3 Vazante grade and tonnage compared to world MVT deposits.................................... 4

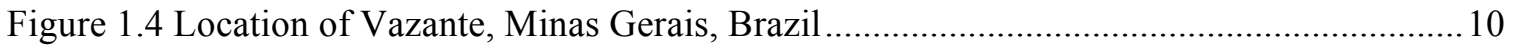

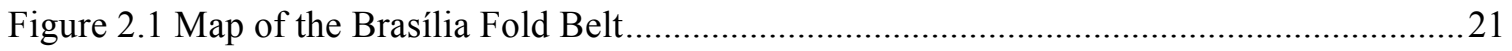

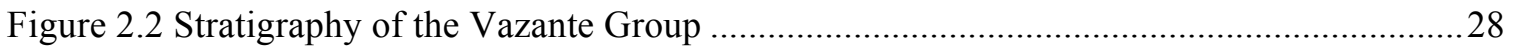

Figure 2.3 Map of the southeastern Brasília Fold Belt with the locations of the $\mathrm{Zn}-\mathrm{Pb}$ deposits .. 32

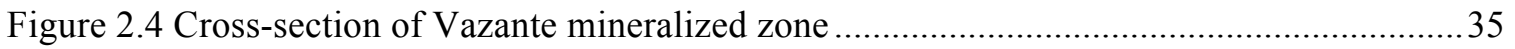

Figure 2.5 Paragenetic sequence for the mineralization in the Vazante Mine ...............................36

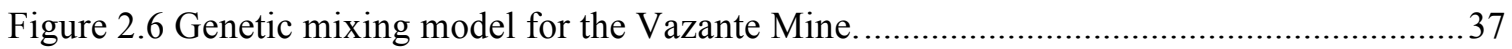

Figure 3.1 A) Geological map of Brazil with the Tocantins Province. B) Map of the southeastern

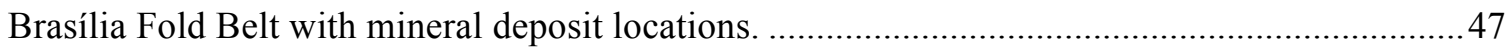

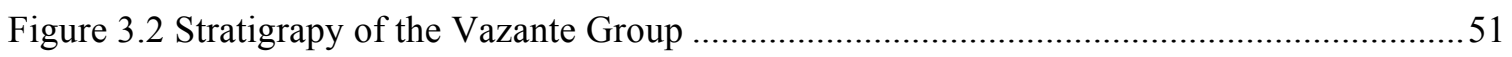

Figure 3.3 Aerial photographs of the Vazante Mine and Northern Extension deposit ..................53

Figure 3.4 Geological of the northernmost section of the Northern Extension..............................55

Figure 3.5 Northern Exenstion topographic map with drill hole sample locations........................56

Figure 3.63 -dimensional cross section of the Northern Extension..............................................57

Figure 3.7 Drill core photographs depicting rock types of the Vazante Region.................67

Figure 3.8 Photographs howing the plunging antiform-synform in the Serra do Garrote Fm.......60

Figure $3.9 \mathrm{~S}_{0}$ compositional layering, $\mathrm{S}_{1}$ cleavage, and $\mathrm{S}_{2}$ crenulations found in phyllitic units ...60

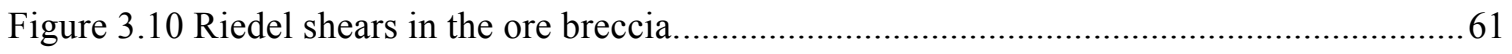

Figure 3.11 A) Stereonet plots of $S_{0} / S_{1}$ compositional layering/foliation, $S_{2}$ crenulation cleavage, trend and plunges of major fold axes, and the average fold axis B) Plots of the poles of the conjectural fault planes bounding the tectonic-hydrothermal breccia and the later E-W transverse faults

Figure 3.12 BSEI of A) Ferroan dolomite growths in carbonate alteration veinlets. B) Ferroan

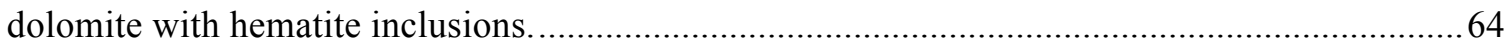

Figure 3.13 Photographs of drill cores illustrating the various types of breccias ..........................65

Figure 3.14 Photomicrographs showing the minerals and their textural relationships in the ore .. 76

Figure 3.15 Photomicrographs and BSEI showing the sulfide veins and veinlets in the ore zone.77 
Figure 3.16 Paragenetic sequence of the mineralized zone in the Northern Extension

Figure 3.17 Box and whisker plots of major element gains and losses.................................... 82

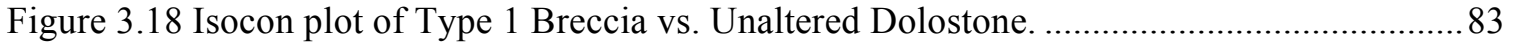

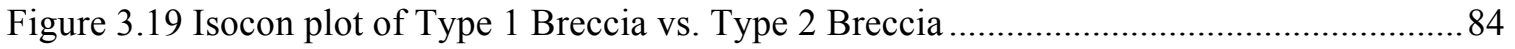

Figure 3.20 Isocon plot of Type 2 Breccia vs. Type 3 Breccia ................................................. 84

Figure 3.21 Isocon plot of Type 3 (hematite) Breccia vs. Type 4 (willemite) Breccia................. 85

Figure 4.1 Genetic model of the Northern Extension Deposit..............................97 


\section{List of Tables}

Table 1.1 Summary of the characteristics of major hypogene nonsulfide zinc deposits............7

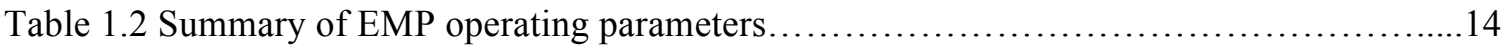

Table 2.1 Stratigraphic units of the Northern Brasília Fold Belt............................23

Table 2.2 Conditions for willemite and sphalerite precipitation..............................

Table 3.1 List of samples and mineral compositions analyzed by EMP A) Dolomite, B)

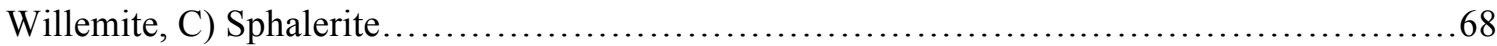

Table 3.2 Comparison of fluid conditions favorable for willemite and sphalerite...............87

Table 3.3 Summary of the major hypogene zinc silicate deposits............................90 


\section{List of Reactions}

Reaction 1 between an iron-bearing carbonate and an iron-bearing brine.......................86

Reaction 2 between an iron-bearing carbonate and a zinc and iron-bearing brine ......................87

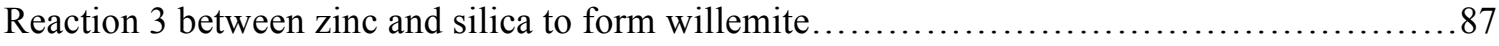

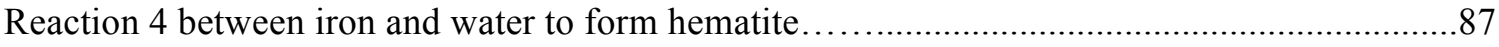




\section{List of Mineral Formulas}

\begin{tabular}{|c|c|}
\hline Mineral Name & Formula \\
\hline Anglesite & $\mathrm{PbSO}_{4}$ \\
\hline Barite & $\mathrm{BaSO}_{4}$ \\
\hline Chalcocite & $\mathrm{Cu}_{2} \mathrm{~S}$ \\
\hline Covellite & $\mathrm{CuS}$ \\
\hline Galena & $\mathrm{PbS}$ \\
\hline Franklinite & $\mathrm{ZnFe}_{2} \mathrm{O}_{4}$ \\
\hline Hematite & $\mathrm{Fe}_{2} \mathrm{O}_{3}$ \\
\hline Hemimorphite & $\mathrm{Zn}_{4} \mathrm{Si}_{2} \mathrm{O}_{7}(\mathrm{OH})_{2} \cdot 2 \mathrm{H}_{2} \mathrm{O}$ \\
\hline Hydrozincite & $\mathrm{Zn}_{5}(\mathrm{OH})_{6}\left(\mathrm{CO}_{3}\right)_{2}$ \\
\hline Phosphohedyphane & $\mathrm{Ca}_{2} \mathrm{~Pb}_{3}\left(\mathrm{PO}_{4}\right)_{3} \mathrm{Cl}$ \\
\hline Sauconite & $\mathrm{NaO} .3(\mathrm{Zn}, \mathrm{Mg})_{3}(\mathrm{Si}, \mathrm{Al})_{4} \cdot \mathrm{OH}_{2} \mathrm{nH}_{2} \mathrm{O}$ \\
\hline Smithsonite & $\mathrm{ZnCO}_{3}$ \\
\hline Sphalerite & $\mathrm{ZnS}$ \\
\hline Stromeyerite & $\mathrm{Cu}_{1-\mathrm{x}} \mathrm{Ag}_{1-\mathrm{x}} \mathrm{S}$ \\
\hline Willemite & $\mathrm{Zn}_{2} \mathrm{SiO}_{4}$ \\
\hline Zincite & $\mathrm{ZnO}$ \\
\hline
\end{tabular}




\section{Chapter 1}

\section{Introduction}

\subsection{Zinc}

Zinc is the fourth most widely produced metal in the world after iron, aluminum, and copper (USGS, 2011). Although its main use is for the galvanization of steel, it is also a major component in brass and zinc-based alloys. The top zinc producing countries in the world are: China, Peru, Australia, the United States, and Canada (USGS, 2011). Most of the world's zinc is currently produced from sedimentary exhalative (SEDEX), volcanogenic massive sulfide (VMS), and Mississippi Valley-type (MVT) sulfide deposits. Zinc oxide deposits account for approximately $12 \%$ of the world's production (Fig. 1.1; Hunt, 2006; Goodfellow and Lydon, 2007) and approximately $4.4 \%$ of the world's zinc resource (Penney et al, 2004).

Zinc oxide deposits, more commonly referred to as nonsulfide zinc deposits, were originally mined in Ancient Rome and Medieval Europe. Ancient Romans and Medieval Europeans used these deposits as a source of zinc to make brass, using "calamine" which is composed of zinc oxide and carbonate ore. In the $19^{\text {th }}$ century, the development of the Wälz kiln in Europe allowed for greater processing of the calamine ore (Boni and Large, 2003). In the early $20^{\text {th }}$ century, nonsulfide zinc orebodies were no longer viewed as economically significant due to the development of the differential flotation process and advancements in the smelting of sulfides. With the recent advent of hydrometallurgical acid-leaching, solvent-extraction, and electrowinning technologies, nonsulfide zinc ore deposits have regained some commercial interest as major sources of high-grade (15-20\%) zinc (Large, 2001; Hitzman et al., 2003).

Currently, the zinc sulfide deposits, (i.e. SEDEX, VMS, and MVT) provide lead, silver, and copper ( \pm gold) as co-products or by-products. Nonsulfide zinc deposits typically lack these 
additional metals; however, with the high ore grades in zinc nonsulfide deposits, the lack of additional metals found in sulfide deposits can be offset by cheaper processing and environmental costs typically associated with sulfide ores (Hitzman et al., 2003). The characteristics and genesis of the sulfide zinc deposits has been extensively investigated (e.g., Goodfellow et al., 1986; Guilbert and Park, 1986; Goodfellow et al., 1993; Leach and Sangster, 1993; Hitzman and Beaty, 1996; Nelson et al., 2002; Goodfellow and Lydon, 2007; and Paradis et al., 2007) and are summarized below. However, little is known about the characteristics and genesis of large nonsulfide zinc deposits. The Vazante deposit, which includes the Vazante Mine and Northern Extension, is an example of large nonsulfide zinc deposit and is the focus of this study.

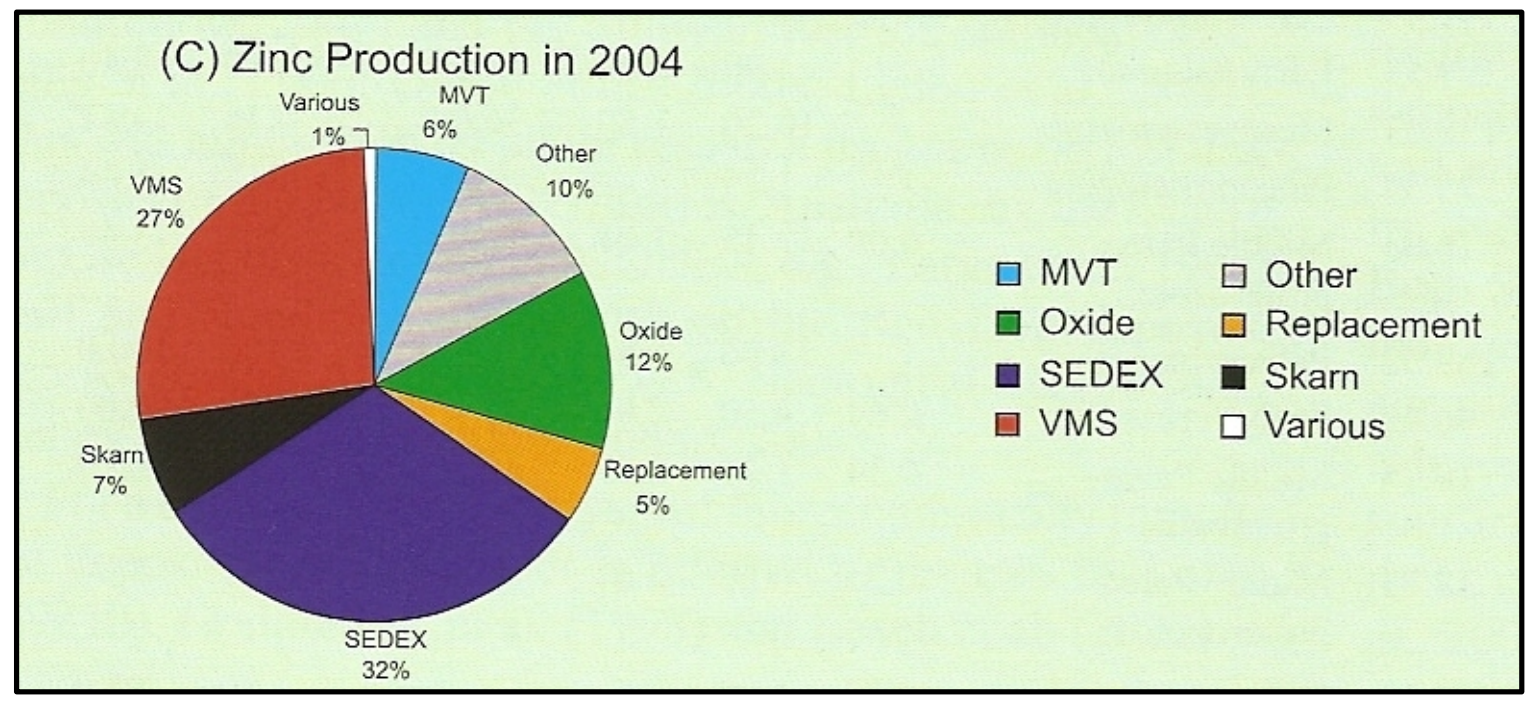

Fig. 1.1 World zinc production per deposit type (from Hunt (2006).

\subsubsection{Sulfide Zinc Deposits}

\subsubsection{SEDEX Deposits}

Sedimentary exhalative (SEDEX) deposits are mineral deposit types that consist of interbedded sulfide minerals with sedimentary rocks, both of which were deposited on the sea floor in association with a hydrothermal conduit. SEDEX deposits can be quite substantial in tonnage and account for a substantial amount of the world's lead and zinc reserves (32\%- Fig. 1.2, Goodfellow and Lydon, 2007). 
SEDEX deposit ages are bimodally distributed to the Middle Proterozoic and Paleozoic.

They are hosted in the intracratonic rift basins (Goodfellow and Lydon, 2007), reactivated rift margins (Goodfellow et al., 1986; Goodfellow and Lydon, 2007), and back-arc rifts with large structures acting as fluid conduits for metal precipitation (Nelson et al., 2002;

Goodfellow and Lydon, 2007). The rock types that host SEDEX deposits consist of marine basin sedimentary rocks such as chert and shale (Goodfellow and Lydon, 2007).

\section{Mineralization within SEDEX}

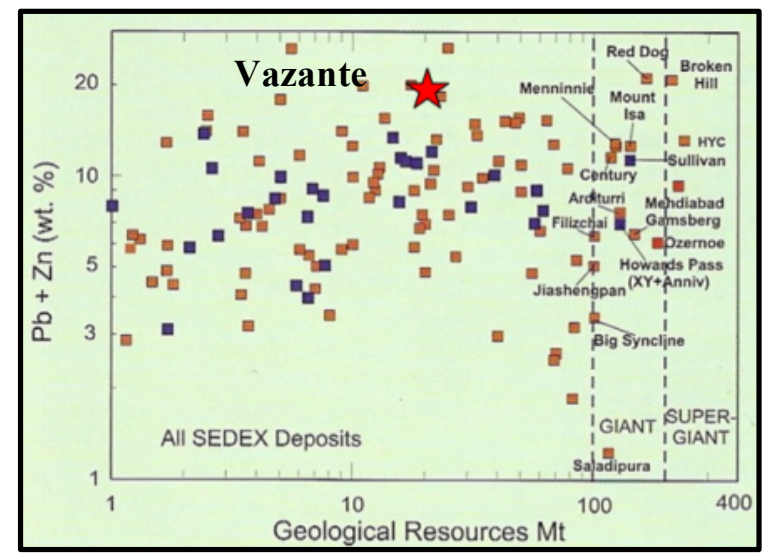

Fig. 1.2 Comparison of Vazante $\mathrm{Zn}$ grade and tonnage versus world SEDEX deposits (modified from Goodfellow and Lydon, 2007).

deposits consists of lead, zinc, and silver

sulfides. Copper and gold may also be of economic value in some SEDEX deposits. Alteration minerals are zoned with respect to distance from the hydrothermal source conduit. The alteration assemblages usually contain quartz, muscovite, chlorite, ankerite, siderite, tourmaline, and abundant Fe-sulfide minerals, such as pyrite and pyrrhotite (Goodfellow and Lydon, 2007).

SEDEX deposits form when hot $\left(\sim 300^{\circ} \mathrm{C}\right)$ sulfur-poor, oxidized basinal fluids circulate in a sedimentary rift basin, leaching metals until they find a fluid conduit, such as a fault or vent, in which to move upwards. There, the basinal fluids react with cooler, reduced, sulfur-bearing seawater, precipitating out lead-zinc sulfides (Goodfellow et al., 1993; Goodfellow and Lydon, 2007).

\subsubsection{MVT Deposits}

Mississippi Valley-type (MVT) deposits are a specific type of carbonate-hosted lead-zinc deposits that typically have little to no magmatic input (Guilbert and Park, 1986). Individual 
deposits are small (Fig. 1.3); however, deposits tend to occur in clusters, creating large mining

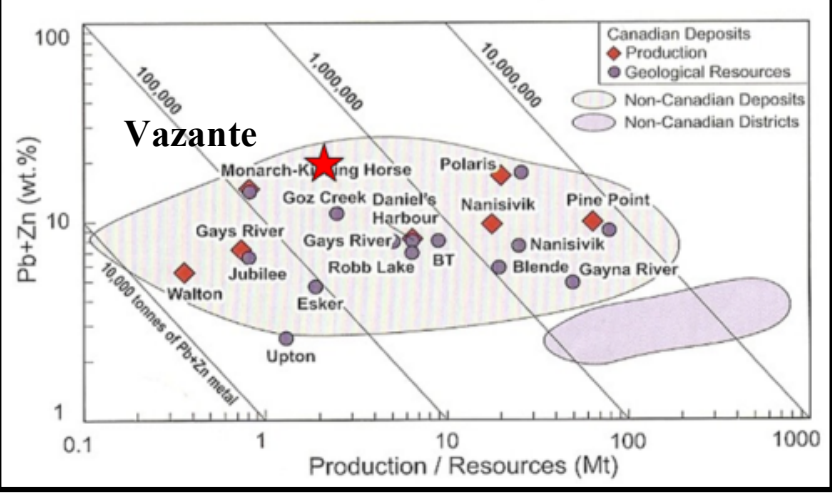

Fig. 1.3 Vazante grade and tonnage compared to world MVT deposits (modified from Paradis et al., 2007). districts, some of which are comparable in grade and tonnage to SEDEX deposits (Leach and Sangster, 1993). MVT deposits are hosted in dolostones that have replaced limestones through dolomitization. The mineralization age of these deposits ranges from the Proterozoic to the Jurassic, with the largest number of deposits being Cambrian in age. MVT deposits occur in carbonate platforms found in orogenic forelands, extensional basins, and intra-cratonic basins (Leach and Sangster, 1993).

Mineralization occurs as filling open space fractures in breccias, massive replacement, and disseminated crystals. Zinc, lead, and iron sulfide minerals are common; however, fluorite and barite may also be of economic significance. Alteration typically occurs as hydrothermal dolomite in dissolution and collapse breccias (Leach and Sangster, 1993; Paradis et al., 2007).

MVT deposits form when deep basinal brines migrate into carbonate sequences along basin margins. Presently, there are three possible models for metal precipitation. The first model involves mixing between a metal-rich brine and an $\mathrm{H}_{2} \mathrm{~S}$-bearing fluid in the open spaces of the carbonate rocks, precipitating out base metal sulfide minerals. The second model proposes that the brine transports both metals and a sulfate, which interacts with organic matter in the carbonate rocks to reduce the sulfates to sulfides, causing precipitation of the metals. The last model comprises a fluid containing both reduced sulfur and metals being carried in the basinal fluid. Precipitation is induced by cooling, dilution, or the loss of volatile compounds (Anderson, 1975; Guilbert and Park, 1986; Paradis et al., 2007). 


\subsubsection{Irish-type Deposits}

Irish-type deposits differ from SEDEX and MVT deposits in that they involve SEDEX style formation and mineralization but have carbonate host rock settings, much like an MVT deposit (Goodfellow and Lydon, 2007). Irish-type deposits are lead-zinc-silver deposits that are typified by the Navan, Lisheen, Galmoy, and Tynagh mineral deposits in Ireland (Hitzman and Beaty, 1996). Although Irish-type deposits vary in age, most of them are Carboniferous. They form in marine carbonate sequences proximal to normal faults that act as a conduit for metalliferous fluids (Hitzman and Beaty, 1996).

Mineralization in Irish-type deposits is stratabound or podiform in morphology and consists of lead, zinc, and silver-bearing minerals. Minerals containing barium and arsenic may also be present. Alteration minerals are usually hydrothermal dolomite, ferroan dolomite, and calcite (Hitzman and Beaty, 1996).

Irish-type deposits form as a result of metalliferous brines being focused upwards along structure and directly replacing carbonate rocks with sulfide mineralization, creating massive deposits that contain high amounts of copper, silver, and iron in addition to lead and zinc (Hitzman and Beaty, 1996).

\subsubsection{Nonsulfide Zinc Deposits}

According to Hitzman et al. (2003), there are two subtypes of nonsulfide zinc deposits: supergene deposits and hypogene deposits. Supergene deposits are formed by the oxidation of both zinc oxide and zinc sulfide deposits. They are typically hosted in carbonate rocks and the major zinc minerals are smithsonite $\left(\mathrm{ZnCO}_{3}\right)$, hemimorphite $\left(\mathrm{Zn}_{4} \mathrm{Si}_{2} \mathrm{O}_{7}(\mathrm{OH})_{2} \cdot 2 \mathrm{H}_{2} \mathrm{O}\right)$, hydrozincite $\left(\mathrm{Zn}_{5}(\mathrm{OH})_{6}\left(\mathrm{CO}_{3}\right)_{2}\right)$, and sauconite $\left(\mathrm{NaO} .3(\mathrm{Zn}, \mathrm{Mg})_{3}(\mathrm{Si}, \mathrm{Al})_{4} \cdot(\mathrm{OH})_{2} \mathrm{nH}_{2} \mathrm{O}\right)$ (Hitzman et al., 2003).

Supergene zinc deposits can be further divided into three categories: direct replacement, wall-rock replacement, and residual karst-fill. These deposits are thought to have been created by 
the oxidation of MVT deposits. Direct replacement deposits form when zinc sulfide minerals, such as sphalerite, are oxidized and replaced by smithsonite and hemimorphite. Other secondary lead, copper, and iron oxides may also occur associated with zinc minerals. Wall-rock replacement deposits form near the original sulfide body as a result of groundwater leaching zinc from the sulfide body and precipitating it into the surrounding carbonate host rocks. Residual karst-fill deposits are the result of zinc minerals accumulating in karst depressions and caves that were formed due to a weathering environment (Hitzman et al., 2003).

Hypogene nonsulfide zinc deposits, from now on referred to as hypogene zinc silicate deposits, are not derived from a pre-existing sulfide body. Hypogene zinc minerals include willemite $\left(\mathrm{Zn}_{2} \mathrm{SiO}_{4}\right)$, franklinite $\left(\mathrm{ZnFe}_{2} \mathrm{O}_{4}\right)$, and zincite $(\mathrm{ZnO})$ and may include the supergene zinc minerals smithsonite, hemimorphite, hydrozincite, and sauconite as well. Hypogene zinc silicate deposits are further divided into two categories based on their controls: structurally controlled and stratiform (Hitzman et al., 2003), though the "stratiform" category should be more aptly dubbed "metamorphic" hypogene nonsulfide zinc, as the type locality for these nonsulfide zinc deposits is the metamorphosed Franklin deposit. There are four major hypogene zinc silicate deposits: the Beltana-Aroona trend, Australia; the Franklin and Sterling deposit, New Jersey, U.S.A; the Kabwe-Lusaka district, Zambia; and the Vazante trend, Brazil (Table 1.1). 


\begin{tabular}{|c|c|c|c|c|c|c|c|c|c|c|c|}
\hline Deposits & Location & $\begin{array}{l}\text { Size } \\
\text { (Mt) }\end{array}$ & $\begin{array}{l}\text { Grade } \\
\text { (\% Zn) }\end{array}$ & Host rocks & Host rock Age & $\begin{array}{l}\text { Related } \\
\text { Structures }\end{array}$ & Ore minerals & Alteration & $\begin{array}{l}\mathrm{T}_{H} \\
\left({ }^{0} \mathrm{C}\right)\end{array}$ & $\begin{array}{l}\text { Salinity } \\
\text { (eq. wt } \\
\% \mathrm{NaCl} \text { ) }\end{array}$ & $\begin{array}{l}\text { Trace } \\
\text { Elements }\end{array}$ \\
\hline Beltana $^{1}$ & Australia & 0.86 & 37.9 & $\begin{array}{l}\text { limestone, } \\
\text { dolostone }\end{array}$ & Cambrian & $\begin{array}{l}\text { Beltana Fault, } \\
\text { district scale } \\
\text { folds, breccia }\end{array}$ & $\begin{array}{l}\text { Willemite, } \\
\text { coronadite, } \\
\text { hedyphane, } \\
\text { mimetite, } \\
\text { smithsonite }\end{array}$ & $\begin{array}{l}\text { Ferroan } \\
\text { dolomite, } \\
\text { hematite, } \\
\text { quartz }\end{array}$ & $\begin{array}{l}50- \\
170 \\
(w 1)\end{array}$ & $4-14$ & $\begin{array}{l}\mathrm{As}, \mathrm{Be}, \\
\mathrm{Cd}, \mathrm{Cu}, \\
\mathrm{Fe}, \mathrm{Ge}, \\
\mathrm{Mn}, \mathrm{Pb}, \\
\mathrm{Sb}, \mathrm{V}\end{array}$ \\
\hline Franklin² & U.S.A. & 24 & 19.6 & marble & Neoproterozoic & $\begin{array}{l}\text { Zero Fault, } \\
\text { district scale } \\
\text { folds }\end{array}$ & $\begin{array}{l}\text { franklinite, } \\
\text { willemite, } \\
\text { zincite, } \\
\text { tephroite, }\end{array}$ & $\begin{array}{l}\text { rhodonite, } \\
\text { magnetite, } \\
\text { calcsilicates }\end{array}$ & $\begin{array}{l}650- \\
700\end{array}$ & $\mathrm{n} / \mathrm{a}$ & $\begin{array}{l}\text { As, B, } \\
\text { Co, Cu, } \\
\text { Fe, Mn, } \\
\text { Ni, Pb, } \\
\text { V, W }\end{array}$ \\
\hline Kabwe $^{3 *}$ & Zambia & $13^{8}$ & $20-25^{8}$ & dolostone & Neoproterozoic & $\begin{array}{l}\text { Mine Club } \\
\text { fault zone, } \\
\text { district scale } \\
\text { folds, minor } \\
\text { breccia }\end{array}$ & $\begin{array}{l}\text { sphalerite, } \\
\text { willemite, } \\
\text { galena }\end{array}$ & $\begin{array}{l}\text { Quartz, } \\
\text { hematite, } \\
\text { smithsonite, } \\
\text { cerussite }\end{array}$ & $\begin{array}{l}98- \\
178 \\
(\mathrm{sp})\end{array}$ & 11.5 & $\begin{array}{l}\mathrm{Ag}, \mathrm{Be}^{8}, \\
\mathrm{Cd}, \mathrm{Cu}, \\
\mathrm{Ga}, \mathrm{Ge}, \\
\mathrm{In}, \mathrm{V}, \mathrm{Pb}\end{array}$ \\
\hline $\begin{array}{l}\text { Vazante } \\
\text { Trend }\end{array}$ & Brazil & $18^{4}$ & $19^{4}$ & dolostone & Neoproterozoic & $\begin{array}{l}\text { Vazante Shear } \\
\text { Zone, district } \\
\text { scale folds, } \\
\text { breccia }^{4}\end{array}$ & $\begin{array}{l}\text { willemite, } \\
\text { sphalerite, } \\
\text { franklinite, } \\
\text { sphalerite }\end{array}$ & $\begin{array}{l}\text { Quartz, } \\
\text { ferroan and } \\
\text { zincian } \\
\text { dolomite, } \\
\text { hematite }^{4}\end{array}$ & $\begin{array}{l}65- \\
180^{6} \\
(w 1)\end{array}$ & $3-15^{6}$ & $\begin{array}{l}\mathrm{Ag}, \mathrm{As}, \\
\mathrm{Be}, \mathrm{Cd}, \\
\mathrm{Co}, \mathrm{Cu}, \\
\mathrm{Ge}^{7}, \mathrm{Fe}, \\
\mathrm{Hg}, \mathrm{In}, \\
\mathrm{Mo}, \mathrm{Pb}, \\
\mathrm{S}, \mathrm{Sb}, \mathrm{Se}, \\
\mathrm{V}^{4}\end{array}$ \\
\hline
\end{tabular}

Table 1.1 Summary of the characteristics on zinc silicate deposits. Temperatures are taken from fluid inclusions within the cited mineral, except for Franklin where the temperatures were estimated based on the equilibrium assemblage of $\mathrm{Fe}_{3} \mathrm{O}_{4}-\mathrm{Mn}_{3} \mathrm{O}_{4}-\mathrm{ZnFe}_{2} \mathrm{O}_{4}$. $* \mathrm{Willemite}$ comes, in part, from oxidized sphalerite.

${ }^{1}$ (Groves et al., 2003); ${ }^{2}$ (Frondel and Baum, 1974); ${ }^{3}$ (Kamona and Friedrich, 2007); ${ }^{4}$ (This study); ${ }^{5}$ ( Monteiro et al., 1999); ${ }^{6}$ (Dardenne and Freitas-Silva, 1999); ${ }^{7}$ (Monteiro et al., 2007), ${ }^{8}$ (Boni et al., 2011). 
Hypogene zinc silicate deposits tend to occur in sedimentary basins, Neoproterozoic to Cambrian in age that have been metamorphosed to some degree, usually at least to the sub-greenschist facies (except Franklin, NJ, which is metamorphosed to amphibolites-granulite facies).

Hypogene deposits are found in carbonate rocks associated with major structures, such as district scale faults and folds (in the Beltana, Vazante, and Kabwe districts). Brecciation is commonly associated with mineralization; this is exemplified by the Vazante trend. The breccias at Beltana are associated with karst (Groves et al., 2003); whereas the breccias at Vazante and Kabwe are a result of tectonism and hydrothermal fluid flow (Dardenne, 2000; Kamona and Friedrich, 2007; this study).

All of the deposits are dominated by mineralogical associations containing the zinc silicate, willemite, as well as zinc oxides, such as zincite, franklinite, and the zinc carbonate smithsonite. Ferroan dolomite, hematite, and quartz are all common alteration minerals found in these types of deposits, with Franklin, NJ as the exception due to its high metamorphic grade. Additionally, all of the deposits show a moderate fluid inclusion homogenization temperature $\left(\mathrm{T}_{H}\right)$ of $50-180{ }^{\circ} \mathrm{C}$, which is interpreted as the minimum temperature of formation (Groves et al., 2003). Higher temperatures up to $300{ }^{\circ} \mathrm{C}$ have been determined through $\delta^{18} \mathrm{O}$ isotope analysis in Vazante (Monteiro et al., 1999; 2006; 2007; Appold and Monteiro, 2009) and Kabwe (Kamona and Friedrich, 2007). The extraordinarily high temperatures found in Franklin are a reflection of the amphibolite-granulite facies metamorphism.

The genetic model for hypogene nonsulfide deposits involves the migration of acidic, oxidizing brines $\left(50-180^{\circ} \mathrm{C}\right)$ with the metals transported as $\mathrm{Cl}^{-}$complexes through structural conduits where they interact with cooler, meteoric fluids. Through cooling, and an increase in $\mathrm{pH}$, the willemite precipitates preferentially over sphalerite (Brugger et al., 2003; Groves et al., 2003; Monteiro et al, 2006; Appold and Monteiro, 2009). 


\subsubsection{Open Questions}

The potential for producing zinc from high-grade deposits at lower costs has renewed interest in nonsulfide zinc deposits around the world. However, because zinc production has been dominated by sulfide deposits for the past century, little is known about nonsulfide zinc deposits, and especially with respect to hypogene nonsulfide zinc deposits. There are several questions relating to the formation of hypogene zinc deposits, in particular the structurally controlled type, which are dominated by zinc silicates. These questions include:

What are the most favorable environments for the formation for zinc silicate deposits? What role do structures play in the formation of zinc silicate deposits?

What is the ore and alteration mineral paragenesis?

What geochemical signatures do these deposits have?

What are the ore fluid characteristics of these deposits?

What are the processes related to its formation?

What geophysical and geochemical methods can be used to detect for zinc silicate deposits?

What other explorations methods can be applied to find these types of deposits?

\subsubsection{The Vazante Mine and Northern Extension Deposit}

The Vazante zinc silicate mine is located in the southern region of the Brasília Fold Belt near the town of Vazante, Minas Gerais, Brazil (Figure 2). It is the largest zinc producing mine in Brazil. Artisanal mining has taken place since the 1950's when the area was mined for the "calamine" (hemimorphite and smithsonite) zinc ore. It has been estimated that around $8 \mathrm{Mt}$ of calamine ore was mined in the Northern Extension of the Vazante Mine (Votorantim pers. commun., 2011). In 1969 and in 1983, open pit and underground mining began, respectively (Monteiro et al., 1999). 


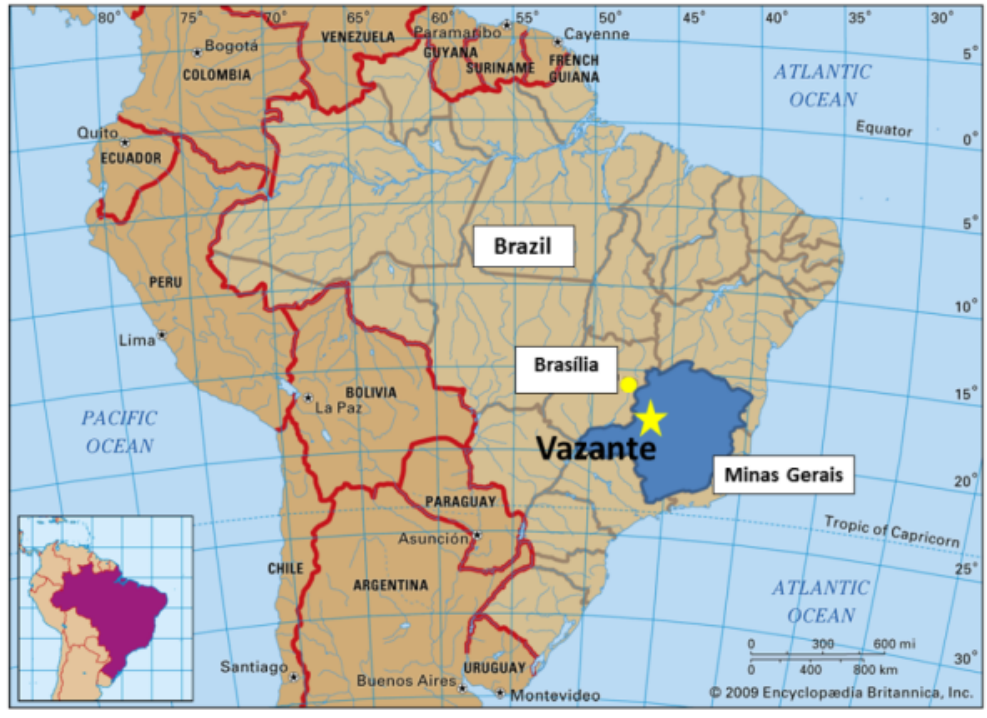

Fig. 1.4 Location of Vazante, Minas Gerais, Brazil (modified from Encyclopedia Britannica, 2011)
The Vazante Mine has estimated reserves of $18 \mathrm{Mt}$ at $19 \% \mathrm{Zn}$. The Northern Extension property contains additional reserves of $850 \mathrm{Kt}$ of ore at $16 \% \mathrm{Zn}$ (pers. commun., 2011). When compared to currently and historically producing SEDEX and MVT deposits, Vazante has average reserve zinc tonnages and high zinc grades (Figs. 3 \& 4).

The Vazante deposit has been studied by Rigobello et al. (1988); Dardenne and FreitasSilva (1999); Dardenne (2000); Monteiro et al. (1999, 2006, 2007); Misi et al. (2005); Cunha et al. (2007) and Rostirolla et al. (2007). Rigobello et al. (1988) and Dardenne (2000) first described the stratigraphy and host rocks as well as the structural setting of the Vazante Mine. Dardenne (2000) also described the tectonic history of the Brasília Fold Belt with respect to the Vazante region. Monteiro et al. (1999, 2006, 2007) conducted studies on the mineralization, alteration, and stable isotopes in the Vazante Mine and first proposed ideas for ore fluid models. Dardenne and Freitas-Silva (1999), Misi et al. (2005), and Cunha et al. (2007) all conducted Pb isotope dating studies in order to establish dates for the host rocks and mineralization events in the Brasília Fold Belt. They also undertook fluid inclusion studies to constrain the ore fluid salinities and temperatures of formation. Rostirolla et al. (2007) did regional studies to establish a metamorphic history of the Vazante region. A more detailed review of the previous work will be presented in Chapter 2 of this thesis. 
Votorantim has developed in the last years an extensive exploration program to evaluate the extension at depth and along the structure of the Northern Extension deposit, historically known as the Mineração Areiense S/A (MASA) mine and considered to be an extension of the main Vazante orebody. This includes wide-spread drilling in this region, providing a unique opportunity to characterize the various mineralized zones and integrate the data with the previous work conducted in the Vazante mine. This knowledge generated by the integration of the data from this study in the Northern Extension with those from the Vazante Mine will further our understanding of the processes related to the formation of hypogene zinc silicate deposits, leading to guidelines for exploration in this region and for this type of deposit globally.

\subsection{Thesis Objectives}

The main objectives of this investigation of the Vazante Northern Extension deposit include:

A) Determination of the major structural and lithological controls for the mineralized zones;

B) Characterization of the mineral paragenesis for the various mineralized and alteration zones in order to understand the evolution of fluid/rock interaction during the basin formation and deformation;

C) Identification of the critical processes responsible for the deposition of the main zinc silicate ore and the minor mineralized zones by integrating the data obtained in this study with thermodynamic data for this type of system and models proposed in previous work for similar deposits;

D) Recognition of the relevant geological and geochemical indicators to explore for this deposit type.

This study will provide a basic understanding of the genesis of these mineralized zones and it is expected that this will lead to the generation of an exploration model that will assist in finding new deposits in the Brasília Fold Belt. 


\subsection{Methodology}

\subsubsection{Field Work and Sampling}

To achieve the research objectives both field and laboratory work were undertaken. The objectives of the field work included mapping and logging the various units of the Vazante Group in the Vazante Northern Extension. Regional reconnaissance work was carried out to allow for integration of the local geology with the regional geology. Local mapping was conducted using a base topographic map provided by Votorantim, Brunton Pocket Transit, and Dakota 20 GPS at a 1:2000 scale to document the structures, rock types, mineralization, and hydrothermal alteration of the most northern exposures in the Northern Extension region. This area was chosen for detailed mapping because the open pits (referred to as Cavas $5 \mathrm{~A}, 6 \mathrm{~A}$, and $7 \mathrm{~A}$ ) from the old mine operated by Mineração Areiense S/A (MASA) allowed exposure of rock units that are normally covered by lateritic soils. The data acquired can be found in Appendix A and were integrated into the geological base map provide by Votorantim Metals.

Re-logging of drill core was conducted to examine mineralization and alteration of the host rocks in the subsurface, as well as to delineate the different stratigraphic units using less weathered samples than those found at surface (Appendix B). The re-logged drill holes were chosen based on transects perpendicular and along the major structural and mineralized trends in the Northern Extension region of the Vazante Mine.

To provide insights on the evolution of the deposit and on the critical factors related to the mineralizing event, 426 samples were selected from 30 drill holes to represent the various rock units, types of mineralization, and various degrees of alteration. These samples included representative samples of unaltered rock for comparison with the mineralized and altered zones. The sample intervals were recorded and the core was halved at the Vazante Mine. 


\subsubsection{Laboratory Methods}

Petrographic investigation, using an Olympus BX 60 transmitted/reflected light microscope with an Olympus TH3 reflected light power source, was completed in order to identify the minerals, textures, and paragenetic relationships for ninety-nine representative samples. The thin sections are $26 \times 46 \mathrm{~mm}$ in size, 30 microns thick, and polished to produce a reflected surface of microprobe quality. Petrographic reports were then generated to create an allencompassing mineral paragenesis from the microscope analyses of the thin section samples (Appendix C).

A FEI MLA 650 FEG-ESEM was used to verify the identification of some rare, unknown minerals, and evaluate the variation in major elements of carbonate and willemite. Backscattered electronic images (BSEI) were taken. The samples were not carbon coated and were analyzed under low vacuum pressure at $20.00 \mathrm{kV}$. Quantax energy dispersive X-ray spectrometer (EDS) readings were used to identify qualitative information about unknown minerals.

Electron microprobe (EMP) analysis was completed at the New Mexico Bureau of Mines and Mineral Resources (NMBBMR) at the New Mexico Institute of Mining and Technology (i.e. New Mexico Tech). The analyses were conducted using a Cameca SX-100 with three wavelength dispersive electrometers with secondary electron and high-speed backscattered electron detectors. All samples were carbon coated for the EMP analyses. A beam current of 20 $\mathrm{nA}$ was used for willemite and sphalerite, and a beam current of $10 \mathrm{nA}$ was used for carbonate analysis (Table 1.2). 


\begin{tabular}{|c|c|c|c|c|}
\hline Mineral & Element & $\underline{\text { Standard }}$ & Peak counting times (s) & Detection Limit (ppm) \\
\hline \multicolumn{5}{|l|}{ Willemite } \\
\hline & $\mathrm{Cr}$ & Chromite & 20 & 551 \\
\hline & $\mathrm{Fe}$ & Horneblend-Kak & 20 & 645 \\
\hline & $\mathrm{Mn}$ & Kaersuitite & 20 & 740 \\
\hline & $\mathrm{Zn}$ & $\mathrm{ZnSe}$ & 20 & 1000 \\
\hline \multicolumn{5}{|l|}{ Sphalerite } \\
\hline & $\mathrm{Cd}$ & $\mathrm{Cd}$ & 40 & 1021 \\
\hline & $\mathrm{Fe}$ & Horneblend-Kak & 20 & 512 \\
\hline & $\mathrm{Mn}$ & Kaersuitite & 40 & 576 \\
\hline & $\mathrm{S}$ & Pyrite & 20 & 438 \\
\hline & $\mathrm{Zn}$ & Sphalerite & 20 & 1203 \\
\hline \multicolumn{5}{|l|}{ Carbonate } \\
\hline & $\mathrm{Ba}$ & Siderite & 20 & 4397 \\
\hline & $\mathrm{Ca}$ & Calcite & 20 & 408 \\
\hline & $\mathrm{Fe}$ & Siderite & 10 & 1009 \\
\hline & $\mathrm{Mg}$ & Dolomite & 20 & 262 \\
\hline & $\mathrm{Mn}$ & Siderite & 20 & 778 \\
\hline & $\mathrm{Si}$ & Kaersuitite & 20 & 232 \\
\hline & $\mathrm{Sr}$ & Dolomite & 20 & 1138 \\
\hline & $\mathrm{Zn}$ & $\mathrm{ZnSe}$ & 20 & 1042 \\
\hline
\end{tabular}

Table 1.2 Table of elements analyzed by EMP with respect to each mineral, the standards, peak counting times, and detection limits.

The EMP was used to analyze the composition of each generation of willemite and to examine sphalerite and carbonate composition, allowing for a better understanding of the evolution of the hydrothermal system. The results of the petrographic and mineralogical investigation were integrated with field observations to determine the overall evolution of the deposit and can be found in Table 3.1 in Chapter 3.

Whole rock geochemical analyses of samples from each unit, alteration and mineralization zones were also completed. These analyses were done to determine the geochemical signatures related to the various styles of mineralization and alteration as well as the any possible mass changes involved in the alteration process. Of the 74 samples analyzed for 
trace elements, 39 of those samples were analyzed for whole rock geochemistry using X-ray fluorescence (XRF). The geochemical analysis was carried out by the ALS Laboratory Group in Canada. Detailed information of the analytical methods is provided in Chapter 3, and the results are provided in Appendix D.

Specific statistic and isocon methods were applied to evaluate the lithogeochemical data and are described in detail in Chapter 3.

\subsection{Scope of the Thesis}

The organization of the thesis follows the scope described below: Chapter 1 introduces the various zinc deposit types, both sulfide and nonsulfide. It elaborates on the different types of zinc nonsulfide deposits, with emphasis on what constitutes a hypogene zinc silicate deposit. The Vazante zinc silicate deposit is briefly introduced and the objectives of this study discussed, and the methods applied to achieve the objectives are briefly outlined.

Chapter 2 presents an overview of the Tocantins tectonic province and the Brasília Fold Belt (BFB). It then discusses the stratigraphy of the BFB with emphasis on the Vazante Group sediments, which host the Vazante Mine. The issues in dating the Vazante Group sediments are examined, and finally the prominent mineral deposits of the southern BFB are reviewed.

Chapter 3 is in the form of a manuscript reporting the results of this study and their interpretations, which will be submitted to a major journal in the field of Economic Geology. It presents a brief summary of the regional geology addressed in Chapter 2 and elaborates on the geologic units investigated in the Vazante Northern Extension. The structure and mineralogy of the Northern Extension deposit are described in detail, culminating in a mineral paragenesis of the deposit. The geochemical results are integrated with the structural and mineralogical data to provide the evolution of fluid-rock interaction for the deposit. The results and interpretations 
were integrated with previous work to further our understanding of nonsulfide zinc deposits and provide guidelines for their exploration.

Chapter 4 includes concluding remarks on this study and suggestions for future studies. 


\section{Chapter 1 References}

Anderson, G.M., 1975, Precipitation of Mississippi Valley-type ores: Economic Geology, v. 70, p. $937-942$.

Appold, M.S. and Monteiro, L.V.S., 2009, Numerical modeling of the hydrothermal zinc silicate and sulfide mineralization in the Vazante deposit, Brazil: Geofluids, v. 9, p. 96-115.

Boni, M. and Large, D., 2003, Nonsulfide Zinc mineralization in Europe: An overview: Economic Geology, v. 98, p. 715-729.

Boni, M., Rosario, T., Balassone, G., Gleeson, S.A., and Matthews, A., 2011, The carbonatehosted willemite prospects of the Zambezi Metamorphic Belt, Zambia: Minerlia Deposita, v. 46, p. 707-729.

Brugger, J., McPhail, D.C., Wallace, M., and Waters, J., 2003, Formation of willemite in hydrothermal environments: Economic Geology, v. 98, p. 819-835.

Cunha, I.A., Misi, A., Babinski, M., and Iyer, S.S.S., 2007, Lead isotope constraints on the genesis of $\mathrm{Pb}-\mathrm{Zn}$ deposits in the Neoproterozoic Vazante Group, Minas Gerais, Brazil: Gondwana Research, v. 11, p. 382-395.

Dardenne, M.A., 2000, The Brasília Fold Belt, in: The tectonic evolution of South America: Rio de Janeiro, 31st International Geological Congress, p. 231-263

Dardenne, M.A. and Freitas-Silva, F.H., 1999, Pb-Zn ore deposits of Bambuí and Vazante groups, in the São Francisco Craton and Brasília Fold Belt, Brazil, in: Base metal deposits of Brazil: Salvador, Ernesto von Sperling, p. 75-83.

Frondel, C. and Baum, J.L., 1974, Structure and mineralogy of the Franklin-zinc-iron-manganese deposit, New Jersey: Economic Geology, v. 69, p. 157-180.

Goodfellow, W.D., Jonasson, I.R., and Morin, J.A., 1986, Environment of formation of the Howards Pass (XY) Zn-Pb deposit, Selwyn, Basin, Yukon: Canadian Institute of Mining and Metallurgy, Special Volume, v. 37, p. 19-50.

Goodfellow, W.D. and Lydon, J.W., 2007, Sedimentary exhalative (SEDEX) deposits, in Goodfellow, W.D., ed., Mineral deposits of Canada: A synthesis of major deposit types, district metallogeny, the evolution of geological provinces and exploration methods: St. John's, Geological Association of Canada - Mineral Deposits Division, p. 163-183. 
Goodfellow, W.D., Lydon, J.W., and Turner, R.W., 1993, Geology and genesis of stratiform sediment-hosted (SEDEX) Zn-Pb-Ag sulphide deposits: Geological Association of Canada, Special Paper 40, p. 201-251.

Groves, I.M., Carman, C.E., and Dunlap, W.J., 2003, Geology of the Beltana Willemite Deposit, Flinders Range, South Australia: Economic Geology, v. 98, p. 797-818.

Guilbert, J. and Park, C.F., 1986, The geology of ore deposits: Long Grove, Waveland Press, Inc.

Hitzman, M.W. and Beaty, D.W., 1996, The Irish Zn-Pb-(Ba) orefield, in Sangster, D.F., ed., Carbonate-Hosted Lead-Zinc Deposits: Auburn Hills, Michigan, Socitey of Economic Geologists, $75^{\text {th }}$ Anniversary Volume, p. 112-143.

Hitzman, M.W., Reynolds, N.A., Sangster, D.F., Allen, C.R., and Carman, C.E., 2003, Classification, genesis, and exploration guides for nonsulfide zinc deposits: Economic Geology, v. 98, p. 685-714.

Hunt, B., 2006, Lead and zinc costs: Mines and projects: 2006 Edition, Surrey, England: Brook Hunt and Associates Ltd.

Kamona, A.F. and Friedrich, G.H., 2007, Geology, mineralogy, and stable isotopes of the Kabwe carbonate-hosted Pb-Zn deposit, Central Zambia: Ore Geology Reviews, v. 30, p. 217-243.

Large, D., 2001, The geology of nonsulfide zinc deposits: An overview: Erzmetall, v. 54, p. 264276.

Leach, D.L. and Sangster, D.F., 1993, Mississippi Valley-type lead-zinc deposits, in Sinclair, R.V., Thorpe, W.D., Duke, R.I., and Duke, J.M., eds., Mineral deposit modeling: St. John's, Newfoundland, Geological Association of Canada, p.289-314.

Minas Gerais, 2012, Encyclopedia Britannica Online: Retrieved 24 January, 2012, from (http://www.britannica.com/EBchecked/topic/383491/Minas-Gerais)

Misi, A., Iyer, S.S.S., Coelho, C.E.S., Tassinari, C.C.G., Franca-Rocha, W.J.S., Cunha, I.A., Gomes, A.S.R., Oliveira, T.F., Teixeira, J.B.G., and Filho, V.M.C., 2005, Sediment hosted lead-zinc deposits of the Neoproterozoic Bambuí Group and correlative sequences, São Francisco Craton, Brazil: A review and a possible metallogenic evolution model: Ore Geology Reviews, v. 26, p. 263-304.

Monteiro, J.V.S., Bettencourt, J.S., Spiro, B., Graca, R., and Oliveira, T.F., 1999, The Vazante zinc mine, Minas Gerais, Brazil: constraints on willemitic mineralization and fluid evolution: Exploration Mining Geology, v. 8, p. 21-42. 
Monteiro, L.V.S., Bettencourt, J.S., Juliani, C., and Oliveira, T.F., 2006, Geology, petrography, and mineral chemistry of the Vazante non-sulfide and Ambrosia and Fagundes sulfide-rich carbonate-hosted Zn-(Pb) deposits, Minas Gerais, Brazil: Ore Geology Reviews, v. 28, p. 201-234.

Monteiro, L.V.S., Bettencourt, J.S., Juliani, C., and Oliveira, T.F., 2007, Nonsulfide and sulfiderich zinc mineralizations in the Vazante, Ambrosia and Fagundes deposits, Minas Gerais, Brazil: Mass balance and stable isotope characteristics of the hydrothermal alterations: Gondwana Research, v. 11, p. 362-381.

Nelson, J., Paradis, S., Christensen, J., and Gabites, J., 2002, Canadian Cordilleran Mississippi Valley-type deposits: A case for Devonian-Mississippian back-arc hydrothermal origin: Economic Geology v. 97, p. 1013-1036.

Paradis, S., Hannigan, P., and Dewing, K, 2007, Mississippie Valley-type lead zinc deposits, in Goodfellow, W.D., ed., Mineral deposits of Canada: A synthesis of major deposit types, district metallogeny, the evolution of geological provinces and exploration methods: St. John's, Geological Association of Canada-Mineral Deposits Division, p.185-203.

Penney, S.R., Allen, M., Harrisson, S., Lees, T.C, Murphy, F.C., and Norman, A.R., 2004, The global distribution of zinc mineralisation, an analysis based on a new zinc deposit database: Applied Earth Science, v. 113, p. 171-182.

Rigobello, A.E., Branquinho, J.A., Silva Dantas, M.G., Oliveira, T.F., and Neves Filho, W., 1988, Mina de zinco de Vazante, Minas Gerais, in: Principais Depositos Minerais do Brasil: Brasília, DNPM, p. 101-110.

Rostirolla, S.P., Mancini, F., Reis Neto, J.M., Figueira, E.G., Araujo, E.C., 2002, Analise estrutural da mina de Vazante e adjacencias: Geometria, cinematica, e implicacoes para a hidrogeologia: Revista Brasileira de Geociências, v. 32, p. 59-68.

U.S. Geological Survey, Tolcin, A.C., 2011, Minerals Yearbook: Zinc (http://minerals.er.usgs.gov/minerals/pubs/commodity/zinc/myb1-2009-zinc.pdf) 


\section{Chapter 2}

\section{Literature Review: Regional Geology, Tectonic Evolution, and Metallogeny of the Brasília Fold Belt (BFB)}

\subsection{Regional Geology and Tectonic Evolution}

The Brasília Fold Belt is roughly $1000 \mathrm{~km}$ long and is part of the Tocantins Province, which is composed of three fold belts: the Araguaia Belt to the east of the Amazonia Craton, the Paraguay Belt on the southeastern margin of the Amazonia Craton, and the Brasília Fold Belt along the western edge of the São Francisco Craton (Fig. 2.1; Almeida et al., 1981; Pimentel et al., 2001; Valeriano et al., 2008; Rodrigues et al., 2010). The Paraguay and Araguaia fold belts are thrust towards the Amazonia Craton to the west; however, the central Brasília Fold Belt is thrust to the east onto the São Francisco Craton (Valeriano et al., 2008).

The Brasília Fold Belt was created through a series of tectonic events starting with continental rifting of the São Francisco Craton around $1700 \mathrm{Ma}$ and ending with the formation of the Brasília Fold Belt during the Brasiliano Orogeny (Misi et. al, 2005; Martins-Neto, 2009). The continental rift system evolved into a passive margin setting along the western edge of the São Francisco Craton, allowing for the deposition of intracratonic siliciclastic sediments and platform carbonates represented primarily by the Canastra-Paranoá-Vazante groups at around 1200-900 Ma (Valeriano et al., 2008; Martins-Neto, 2009). The latter is the host of the Vazante silicate zinc deposit. 


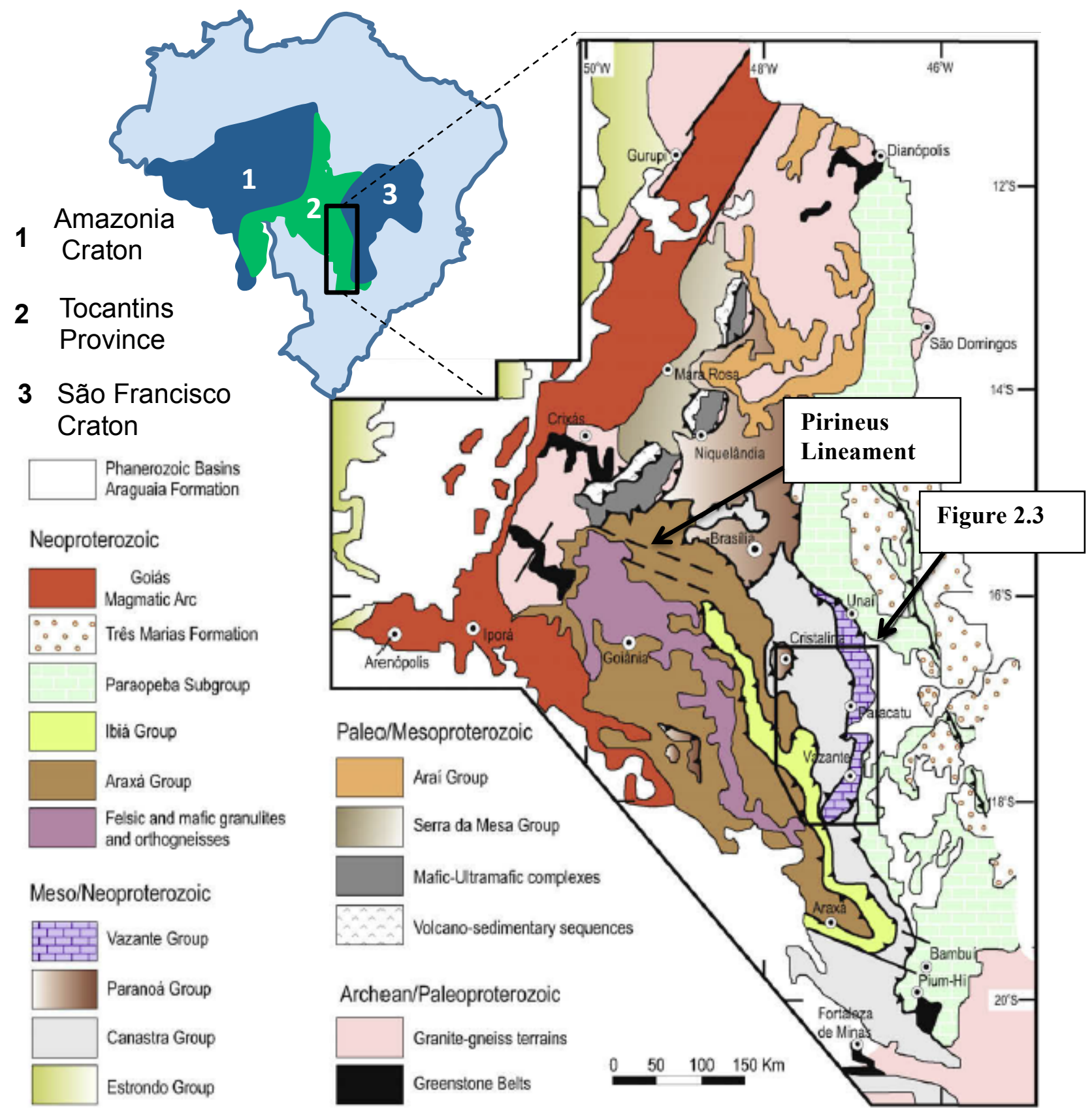

Fig. 2.1 Map of the Brasília Fold Belt (Modified from Rodrigues et al., 2010) 
Subduction of oceanic crust from the Brazilide Ocean beneath the São Francisco Craton also began around $900 \mathrm{Ma}$, causing magmatism that would lead to the formation of the Goiás magmatic arc (Dardenne, 2000; Valeriano et al., 2008; Martins-Neto, 2009). Continuing subduction caused the convergence of the Goiás magmatic arc and the São Francisco Craton around $850-750 \mathrm{Ma}$, the start of the Brasiliano Orogeny. This collision thrusted the passive margin sediments as thin-skinned nappes to the east onto the São Francisco Craton (Dardenne, 2000; Valeriano et al., 2008, Martins-Neto, 2009), and reached its metamorphic peak around 650 Ma (Martins-Neto, 2009). Dardenne (2000) also documented geochemical and isotopic evidence of a late Brasiliano collisional event around $630-610 \mathrm{Ma}$ and interpreted the data as related to the convergence of another magmatic arc on the eastern margin of the São Francisco Craton. Finally, the cratonic sediments of the Bambuí Group were deposited and construction of the Brasília Fold Belt was completed around 590 Ma (Martins-Neto, 2009).

The central region of the Brasília Fold Belt bulges to the west because of the Pirineus Lineament, which trends NW - SE (Fig. 2.1; Almeida et al., 1981; Marini et al., 1981) and further subdivides the Brasília Belt into northern and southern zones (Dardenne, 2000). The rocks in the northern zone of the Brasília Fold Belt either remain unmetamorphosed or are metamorphosed up to the greenschist facies. Their stratigraphic relationships remain well preserved and the units consist of the Araí, Serra da Mesa, and Paranoá groups as well as the Serra da Mesa, Serra do Encosto, and Serra Dourada granites and Palmeirópolis, Juscelâdia, and Indaianópolis volcanosedimentary sequences (summarized in Table 2.1). The former mentioned granites are mineralized with tin (Pimentel et al, 1991) and the latter volcanic-sedimentary sequences host the Palmeirópolis VMS deposit. Additionally, the Araí, Serra da Mesa, and Paranoá groups host various gold occurrences and artisanal mines (Guiliani et al., 1993; Araujo, 1999; Dardenne and Schobbenhaus, 2001). 


\begin{tabular}{|c|c|c|c|}
\hline Sedimentary units & Volcanic units & Description & Age (Ma) \\
\hline \multirow[t]{2}{*}{ Paranoá Group $^{1,2}$} & & $\begin{array}{l}\text { conglomerates overlain by } \\
\text { rhythmites with mudcracks } \\
\text { and evaporites, marine } \\
\text { rhythmites and quartzites, } \\
\text { limestones, and } \\
\text { stromatolitic dolomites }\end{array}$ & $1200-900$ \\
\hline & $\begin{array}{l}\text { Palmerópolis, Juscelâdia, } \\
\text { and Indaianópolis } \\
\text { Volcano-sedimentary } \\
\text { sequences }\end{array}$ & $\begin{array}{l}\text { fine-grained amphibolites } \\
\text { and gneiss, mica schists, } \\
\text { metachert, and } \\
\text { metavolcanics of varying } \\
\text { compositions }\end{array}$ & $1300-1170$ \\
\hline \multirow[t]{2}{*}{$\begin{array}{l}\text { Serra da Mesa } \\
\text { Group } \\
1,5,6,7\end{array}$} & & $\begin{array}{l}\text { quartzites, micaceous } \\
\text { schists, and marbles }\end{array}$ & $1600-1470$ \\
\hline & $\begin{array}{l}\text { Serra da Mesa, Serra do } \\
\text { Encosto, and Serra } \\
\text { Dourada granites }{ }^{1,8}\end{array}$ & anorogenic tin granites & $1658-1574$ \\
\hline Araí Group ${ }^{1,6,7,9}$ & & $\begin{array}{l}\text { intraformational quartz } \\
\text { conglomerates intercalated } \\
\text { with metasiltstone on top } \\
\text { of basal volcanics } \\
\text { including basalt, andesite, } \\
\text { dacite, an rhyolite }\end{array}$ & 1770 \\
\hline
\end{tabular}

Table 2.1 Summary of the characteristics of the northern Brasília Fold Belt geologic units. ${ }^{1}$ (Dardenne, 2000); ${ }^{2}$ (Dardenne, 1978); (Araujo, 1999); (Dardenne and Schobbenhaus, 2001); (Fuck and Marini, 1981); ${ }^{6,7}$ (Marini et al., 1984a, b); ${ }^{8}$ (Pimentel et al., 1991); ${ }^{9}$ (Pimentel et al., 2001)

The southern region of the Brasília Fold Belt, which comprises the Vazante, Canastra, Araxá, and Ibiá groups, was metamorphosed up to the greenschist facies and generally displays more intense tectonic deformation than the northern region. The Araxá, Vazante, Canastra, and Ibiá groups form a complex system of nappes that are thrust onto the Bambuí Group sediments to the east (Dardenne, 2000).

The Araxá Group is located in the very southern region of the Brasília Fold Belt, is highly deformed, and associated with numerous thrust events. It is composed of micaceous quartzites and a variety of schists including: chlorite-muscovite, biotite-garnet, staurolite, and feldspathic schists. Syntectonic meta-basalts, andesites, and rhyolites are associated with the schists in the Araxá Group (Dardenne, 2000; Pimentel et al., 2001). 
The Ibiá Group lies unconformably on top of the Canastra Group and is made up of two formations: the Cubatão and Rio Verde. The Cubatão Formation consists of thick diamictites (Dardenne, 1978). The Rio Verde Formation sits on top of the Cubatão Formation and consists of calciferous phyllites with some intercalations of quartzite (Dardenne, 2000).

The Araxá and Ibiá groups have overlapping ages (1100 - $800 \mathrm{Ma})$ based on U-Pb zircon ages obtained from the syntectonic intrusives and $\mathrm{Sm}-\mathrm{Nd}$ ages obtained from amphiboles (Pimentel et al., 2001). Dardenne (2000) suggests that these units represent laterally distinct sedimentary packages that were thrust onto the São Francisco cratonic margin; however, Pimentel et al. (2001) suggests that these sedimentary packages formed due to erosion from the same source.

The Canastra Group is considered to be the southern BFB equivalent sedimentary group to the Paranoá Group located in the northern BFB (Dardenne, 1978, 2000), and its age is estimated to be between 1200 - 900 Ma based on stromatolites (Dardenne, 2000) and around 1000 Ma based on Sm- Nd dating (Pimentel et al., 2001). It is composed primarily of phyllite and quartzite that has been metamorphosed up to the greenschist facies. There are 3 formations that make up the Canastra Group, which sits unconformably below the Ibiá Group and is tectonically thrust on top of the Vazante Group (Dardenne, 2000). They are from the bottom to the top: the Serra do Landim, Paracatú, and Chapada dos Pilões formations. The Serra do Landim Formation are interpreted to be the lowest section of the Canastra Group according to Freitas-Silva and Dardenne (1994); however, Madalosso and Valle (1978) have described the Serra do Landim as being the uppermost formation of the Vazante Group. The Serra do Landim comprises a pale green to gray-green calc-phyllite to calc-schist (Freitas-Silva and Dardenne, 1994; Dardenne, 2000). The Paracatú Formation is composed of gray carbonaceous phyllite with quartzite lenses (Almeida, 1969; Freitas-Silva and Dardenne, 1994; Dardenne, 2000) and hosts the Morro do Ouro gold mine located in Paracatú, Minas Gerais (Dardenne, 2000). The Chapada 
dos Pilões Formation lies on top of the Paracatú Formation and is made up of intercalated phyllites and quartzites, which have been interpreted as metamorphosed rhythmites and turbidites (Dardenne, 2000).

The Vazante Group is located in the northwest region of Minas Gerais where it stretches for nearly $250 \mathrm{~km} \mathrm{~N}$-S, lies tectonically below the Canastra Group; and is thrust on top of the younger Bambuí Group (Dardenne, 2000; Pimentel et al., 2001; Monteiro et al., 2007). It is primarily composed of intercalated phyllites and carbonates that have undergone metamorphism up to the greenschist facies. The age of the Vazante Group is controversial and poorly constrained due to the absence of igneous rocks and well constrained detrital zircon dating.

The Vazante Group (Fig. 2.2) is divided into 7 formations, which are from the bottom to the top: Retiro (also referred to as Santo Antônio do Bonito Formation; Cunha et al., 2007; Misi et al., 2005), Rocinha, Lagamar, Serra do Garrote, Serra do Poço Verde, Morro do Calcario, and Serra da Lapa (Dardenne, 2000). The Retiro formation consists of quartzite, conglomerate, diamictite (i.e., debris flows from glacio-marine environments), and slate (Dardenne, 2000; Cunha et al., 2007). It is host to the Coromandel phosphorite mineralization (Dardenne, 2000). The Retiro Formation grades upwards into the Rocinha Formation, which consists of pelite, siltstone rhythmites as well as phosphatic layers that host the Rocinha and Lagamar phosphorite deposits (Dardenne, 2000; Cunha et al., 2007). The top of the Rocinha Formation is a thick sequence of slate and metasiltstone that grades into carbonaceous and pyrite-bearing slate (Dardenne, 2000; Pimentel et al., 2001). These pyrite-bearing slates contain phosphorites and are host to the Rocinha phosphate deposit. The upper part of the formation contains quartzites and siltstones that host the Lagamar phosphate deposit (Dardenne, 2000). Above the Rocinha Formation is the Lagamar Formation which is a metapsamo-pelitic unit with a basal metaconglomerate. Overlaying these clastic units are dolomitic breccias, dark gray limestones, and light pink stromatolitic bioherms with columnar Conophyton and Jacutophyton stromatolites 
(Dardenne, 2000; Monteiro et al. 2006; Cunha et al., 2007). Above the Lagamar Formation is the Serra do Garrote Formation, which is made up of dark gray to green-gray phyllites with localized pyritic zones (Dardenne, 2000; Cunha et al. 2007; this study).

Sitting atop the Serra do Garrote Formation is the Serra do Poço Verde Formation, which hosts the Vazante zinc silicate mineralization. The Serra do Poço Verde Formation (Fig. 2.2) can further be subdivided into four members: Lower Morro do Pinheiro, Upper Morro do Pinheiro, Lower Pamplona, and Middle Pamplona (Dardenne, 2000). The Lower Morro do Pinheiro Member forms the base of the Serra do Poço Verde Formation. It consists of light gray and pink dolomite with stromatolites intercalated with oncolitic dolarenites and intraformational dolomite breccias. The Upper Morro do Pinheiro Member is a dark gray dolomite with bird's eyes and minor carbonaceous phyllites. The Lower Pamplona Member consists of gray to pink laminated dolomites intercalated with green and maroon phyllites. The Middle Pamplona Member consists of light gray to pink dolomite with algal laminations and dolarenites with stromatolites (Dardenne, 2000).

The Morro do Calcário Formation, also known as the Upper Pamplona Member according to Rigobello et al. (1988), rests on the top of the Serra do Poço Verde Formation, is comprised of carbonaceous shale, dolarenites, breccias, and stromatolitic bioherm facies (Dardenne, 2000; this study). It hosts of the Morro Agudo lead-zinc Mine (Dardenne, 2000), Fagundes zinc sulfide deposit, and Ambrósia zinc sulfide deposit (Monteiro et. al., 2006).

The upper most formation in the Vazante Group is the Lapa Formation, which sits unconformably on top of the Morro do Calcário formation. The Lapa Formation is composed of black, carbonaceous rhythmites with intercalated slates and phyllites. It also contains sericitechlorite phyllites, carbonate bearing metasiltstones with dolomite and quartz lenses (Dardenne, 2000; Monteiro et al., 2006). 
There is controversy surrounding the age of the Vazante Group. The first issues stems from the age of the stromatolites, Conophyton, which represents a large time interval of 1.35 $0.95 \mathrm{Ga}$. This broad age may indicate a possible stratigraphic correlation between the Vazante Group and the Paranoá Group (Cloud and Dardenne, 1973; Dardenne et al., 1979; Dardenne, 2000). Geochronological dating on sediments using ${ }^{207} \mathrm{~Pb} /{ }^{206} \mathrm{~Pb}, \mathrm{~K} / \mathrm{Ar}$, and $\mathrm{Rb} / \mathrm{Sr}$ methods indicate a metamorphic closing age of 750-570 Ma, giving the Vazante Group an age close to that of the 'younger' Bambuí Group sediments, which lie to the east (Amaral, 1968; Iyer et al, 1992;. Monteiro, 2002). In fact, Dardenne (2000) has suggested that the diamictites of the Retiro Formation may correspond to the diamictites in the Jequitaí Formation of the Bamuí Group. ${ }^{207} \mathrm{~Pb} /{ }^{206} \mathrm{~Pb}$ dating using galenas found in the Vazante and Morro Agudo mines by Freitas-Silva and Dardenne (1997) and Dardenne (2000) suggest an age range of $1200 \mathrm{Ma}$ to $650 \mathrm{Ma}$, the latter date being representative of the closing date of metamorphism in the region. Using $\mathrm{Sm}-\mathrm{Nd}$ isotope dating, Pimentel et al. (2001) places the $\mathrm{T}_{\mathrm{DM}}$ of the Vazante group around $2200-1800$ Ma., an age that is representative of the basement source rocks from the São Francisco Craton. Rodrigues (2008) and Rodrigues et al. (2010) conducted U-Pb and ${ }^{207} \mathrm{~Pb} /{ }^{206} \mathrm{~Pb}$ analyses that provided an age range of $1350-630 \mathrm{Ma}$, with the latter date representing the Brasiliano Orogeny. In addition, Rodrigues (2008) and Rodrigues et al. (2010) obtained Sm-Nd ages for the basal Retiro Formation of $935 \pm 14 \mathrm{Ma}$, which is younger than the rest of the overlying formations in the Vazante Group. However, it should be noted that no dates were obtained for the Serra do Poço Verde Formation, the host of the Vazante Northern Extension zinc silicate deposit. 


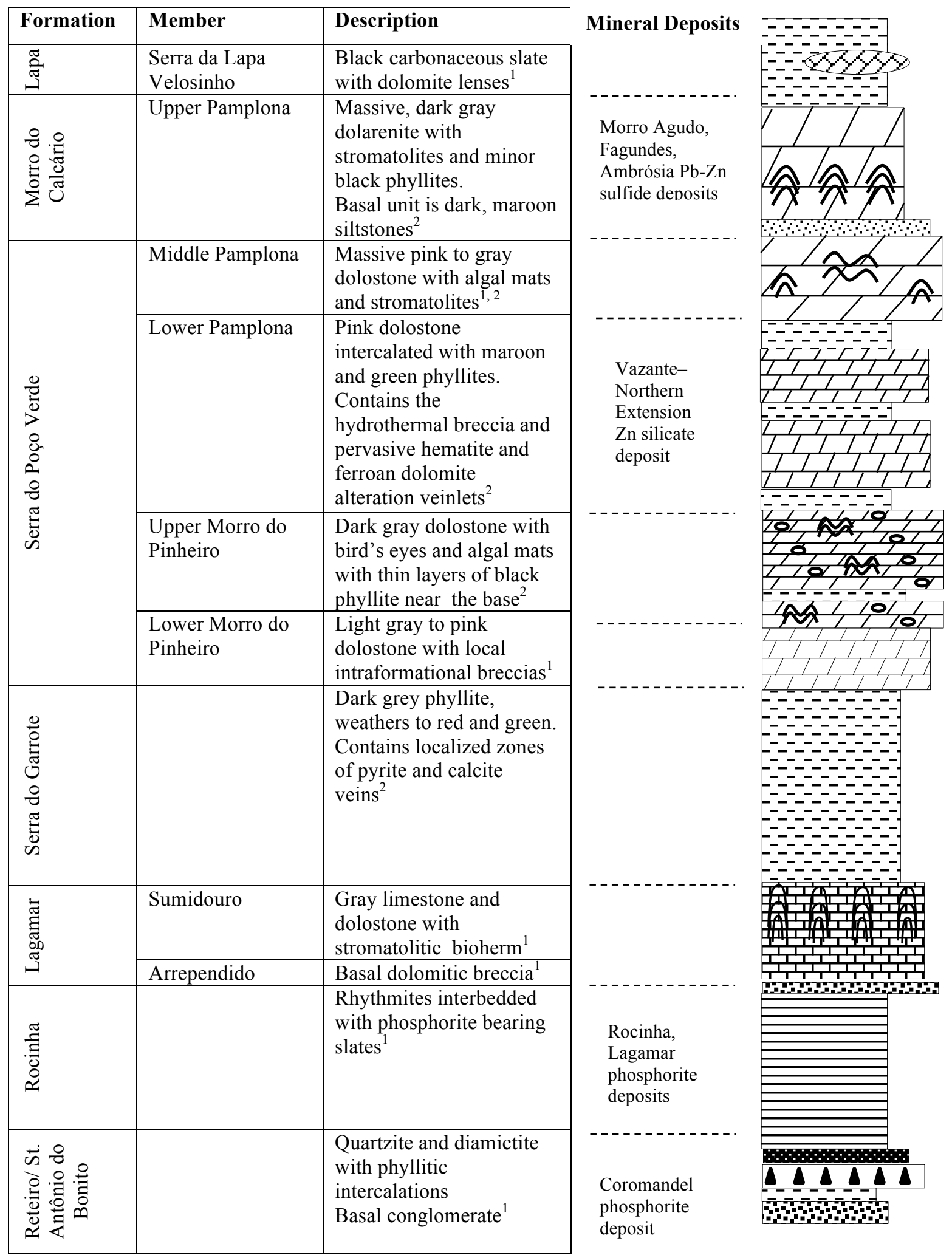

Fig. 2.2 Stratigraphy of the Vazante Group and the mineral deposits hosted in its units (modified from ${ }^{1}$ Dardenne, 2000; ${ }^{2}$ this study). 
The Bambuí Group consists of Neoproterozoic sediments on the eastern edge of the Brasília Fold Belt and in the São Francisco Basin. The Bambuí Group sits unconformably on the granite-gneiss basement rocks of the São Francisco Craton as well as on the metasedimentary rocks of the Araí and Paranoá Groups. It comprises 6 formations, which are from the bottom to the top: Jequitaí, Sete Lagoas, Serra da Santa Helena, Lagoa do Jacaré, Serra da Saudade, and Três Marias (Dardenne, 2000). The basal Jequitaí Formation is composed of diamictite beds containing limestone clasts, representing glacial episodes. The Sete Lagoas Formation consists of pelitic carbonates that grade into dolomites at the top of the formation. Above the Jequitaí Formation sits the carbonaceous pelites of the Serra da Santa Helena Formation. Sitting conformably on the Serra da Santa Helena Formation is the Lagoa do Jacaré Formation, which is composed of dark gray platform limestones. Next in the sequence are the sandy pelites that compose the Serra da Saudade Formation. The top of the Bambuí Group is made up of the Três Marias Formation, which is composed of arkoses and siltstones (Dardenne, 2000).

The age of detrital zircons found in the Bamuí Group sediments has a bimodal distribution, according to Rodrigues (2008) and Rodrigues et al. (2010). The Jequitaí Formation has detrital zircons that represent an age of about $900 \mathrm{Ma}$; however, the Sete Lagoas Formation contains zircons as young as $610 \mathrm{Ma}$. The latter age correlates to the $650-600 \mathrm{Ma}$ age range based on K/Ar and Rb/Sr isotope dating (Thomaz Filho et al., 1998).

The relationship between the Vazante and Bambuí Groups is not well constrained due to the overlap in detrital zircon, and other isotopic system data (1200 - 630 Ma). As previously mentioned, the passive margin sediments of the Vazante Group were thrust on top of the cratonic sediments of the Bambuí Group (Dardenne, 2000; Pimentel et al., 2001). It has been hypothesized that the Vazante sediments represent a slice of the Bambuí Group that has been thrust up atop itself and is therefore, not a separate stratigraphic group (Misi et al., 2007). Due to the tectonic complexity of the Brasília Fold Belt as well as the correlative issues between 
northern and southern sedimentary packages in the BFB, this theory is not unwarranted.

Chemostratigraphic investigation based on ${ }^{87} \mathrm{Sr} /{ }^{86} \mathrm{Sr}$ isotopes conducted by Misi et al. (2007) suggests a correlation between the carbonates of the Vazante and Bambuí groups. This interpretation differs from Azmy et al. (2001), who reported lower ${ }^{87} \mathrm{Sr} /{ }^{86} \mathrm{Sr}$ values indicating that the Vazante Group was, in fact, older than the Bambuí Group. Furthermore, Carbon isotope studies conducted by Misi et al. (2007) found that the $\delta^{13} \mathrm{C}$ values for Vazante carbonates were substantially lower $(+3.3 \%$ compared to $+9-16 \%$ ) than the Bambuí carbonates. However, without ages for the Serra do Poço Verde Formation, the primary carbonate unit within the Vazante Group, the relationship between the two groups will remain uncertain and under scrutiny.

\subsection{Mineral Deposits Hosted in the Vazante Group}

\subsubsection{Phosphate Deposits}

Two phosphate mines are hosted in the Vazante Group: the Rocinha and Lagamar deposits. The Rocinha phosphate mine is located near Patos do Minas, Minas Gerais, and is hosted in the carbonate and pyrite-bearing slates of the Rocinha Formation. The phosphate ore occurs as fine phosphatic laminations and phospharenites containing phosphate pellets and intraclasts (Dardenne, 2000). The phosphate is apatite according to Da Rocha Araujo et al. (1992), and the reserves are estimated to be $400 \mathrm{Mt}$ at $12 \% \mathrm{P}_{2} \mathrm{O}_{5}$ (Dardenne, 2000).

The Lagamar phosphate deposit is found in the rhythmic quartzite and siltstone sedimentary units of the upper Rocinha Formation. The deposit is almost entirely composed of phospharenite and the reserves are estimated at $5 \mathrm{Mt}$ at $25 \% \mathrm{P}_{2} \mathrm{O}_{5}$ (Dardenne, 2000).

A third phosphate mineralization exists in the Retiro Formation at the base of the Vazante group by the town Coromandel, from which its name is derived; however, this mineralization is currently uneconomic and poorly defined with respect to tonnage and grade (Dardenne, 2000). 


\subsubsection{Zinc and Lead sulfide deposits}

\subsubsection{Fagundes Zinc Deposit}

The Fagundes zinc sulfide deposit is a carbonate-hosted, epigenetic lead-zinc deposit. It is currently uneconomic and therefore, poorly delineated with respect to tonnage and grade. The Fagundes deposit is hosted in the Upper Pamplona Member of the Morro do Calcário Formation about $30 \mathrm{~km}$ North of Paracatú, Minas Gerais (Fig. 2.2 and Fig. 2.3). In this region, the Upper Pamplona comprises dolostone with bacterial mats and stromatolite columns with localized areas containing dolrudites composed of Upper Pamplona intraclasts. The depositional environment is interpreted as a fore and back reef facies (Monteiro, 2002 and Monteiro et al., 2006). The dolostones are silicified prior to mineralization. Mineralization is stratabound and composed of pyrite, sphalerite, and late galena. The pyrite and sphalerite are found in banded, colloform, and zoned crystal habits. The galena occurs in veins and veinlets associated with pyrite and, to a lesser extent, galena. Fluid sources are interpreted as hydrothermal in origin with salinities of 5 22 eq. wt $\% \mathrm{NaCl}$ and homogenization temperatures of $122-144{ }^{\circ} \mathrm{C}$ (Monteiro 2002, Monteiro et al., 2006, 2007; Cunha et al., 2007).

\subsubsection{Ambrósia Zinc Deposit}

The Ambrósia sulfide zinc deposit is also an uneconomic, carbonate-hosted, epigenetic lead-zinc deposit; however, unlike the Fagundes deposit, the Ambrósia deposit is controlled by a reverse fault that strikes $30^{\circ}$ and dips $60-80^{\circ} \mathrm{SE}$ and has been displaced by a N20E-trending transversenormal fault (Monteiro, 2002; Monteiro et al., 2007; Cunha et al., 2007). The mineralization is hosted in brecciated dolostones of the Upper Pamplona member of the Morro do Calcário Formation and is located $20 \mathrm{~km}$ Northeast of Paracatú, Minas Gerais. The deposit is uneconomic so the ore body morphology, tonnage, and grade are poorly defined. In the Ambrósia district, the 


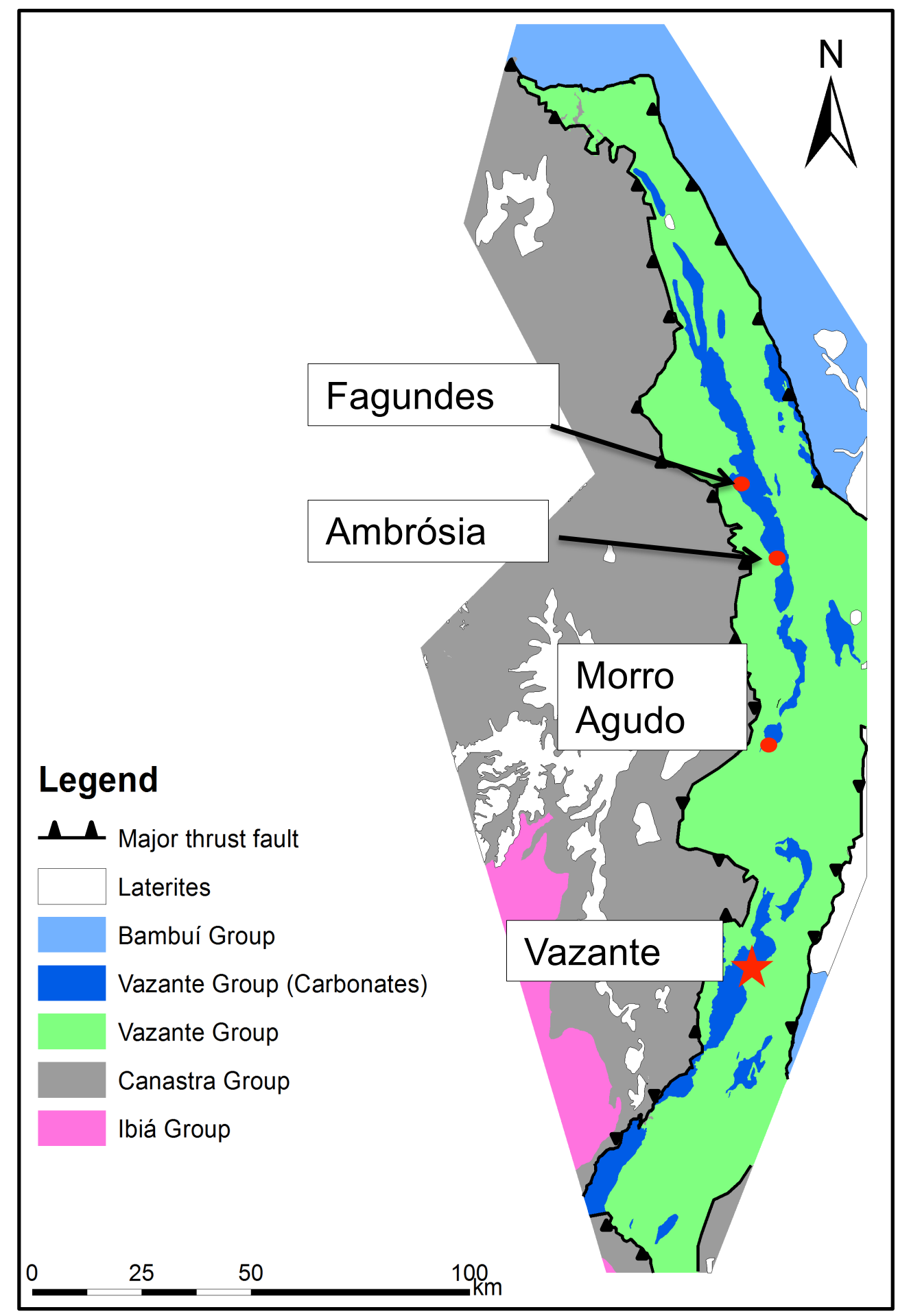

Fig. 2.3 Map of the southeastern Brasília Fold Belt with the locations of the $\mathrm{Zn}-\mathrm{Pb}$ deposits in the Vazante Group (modified from Votorantim, in prep.) 
Upper Pamplona is tectonically imbricated with the shales, phyllites, and dolostones of the Lower Pamplona Member of the Serra do Poço Verde Formation.

The host rocks are silicified and contain ankerite alteration (Monteiro, 2002; Monteiro et al., 2006, 2007). The mineralization is composed of pyrite, marcasite, sphalerite, galena, and course-grained dolomite. Veins, veinlets, and fracture fillings host the mineralization. The ore fluid salinities at Ambrósia range from $5-15$ eq. wt $\% \mathrm{NaCl}$, and the homogenization temperatures range from $120-265^{\circ} \mathrm{C}$ (Monteiro, 2002; Monteiro et al., 2006, 2007; Cunha et al., 2007).

\subsubsection{Morro Agudo Lead-Zinc Mine}

The Morro Agudo lead-zinc mine has reserves at $5 \mathrm{Mt}$ of $6.3 \% \mathrm{Zn}$ and $2.9 \% \mathrm{~Pb}$ (Cunha et al., 2007). This deposit has been categorized as a MVT deposit by Rigobello et al. (1988) and Iyer et al.(1992); a SEDEX by Misi et al. (1996) as well as Freitas-Silva and Dardenne (1997); and an Irish-type by Dardenne and Freitas-Silva (1999) Cunha et al. (2000), Dardenne (2000), Misi et al. (2000), and Monteiro (2002).

The Morro Agudo mine is hosted by dolarenitic breccias the Upper Pamplona member of the Morro do Calcário Formation. The mineralization is bounded by a normal fault, interpreted as a syn-sedimentary feeder zone and conduit for ore fluids that strikes N10-20W and dips $75^{\circ} \mathrm{S}$. The mineralization is both disseminated and massive along the fault zone and consists of sphalerite, galena, and pyrite associated with quartz and barite (Dardenne and Freitas-Silva, 1999; Misi et al., 2005; Cunha et al., 2007; Monteiro et al., 2007).

The Morro Agudo mineralization is divided into four ore zones: the N, M, JKL, and GHI, which are stratigraphically constrained to the Upper Pamplona Member of the Morro do Calcário Formation (Dardenne and Freitas-Silva, 1999; Misi et al., 2005; Cunha et al., 2007). The $\mathrm{N}$ orebody consists of fine-grained, alternating galena, sphalerite, pyrite, and chert beds. The 
$\mathrm{M}$ orebody is irregularly shaped and composed of coarse-grained galena and sphalerite (Misi et al., 2005; Cunha et al., 2007). The JKL orebody is made up of massive fine to coarse-grained sphalerite that cements oolitic dolostone beds (Misi et al, 2005). The GHI orebody consists mainly of coarse-grained sphalerite and galena that cements angular breccia clasts, some of which are derived from the $\mathrm{N}$ orebody (Cunha et al., 2007).

Fluid inclusion studies conducted by Cunha et al. (2007) in sphalerite crystals show ore fluid salinities ranging from $4-22$ eq. $\mathrm{Wt} \% \mathrm{NaCl}$, and homogenization temperatures ranging from $122-283{ }^{\circ} \mathrm{C}$.

\subsubsection{Zn Silicate deposits}

\subsubsection{Vazante Zinc Silicate Mine}

The Vazante Mine zinc silicate mineralization is hosted in a hydrothermal shear breccia found near the contact between the Lower Pamplona and Upper Morro do Pinheiro members of the Serra do Poço Verde Formation (Figs. 2.2, 2.3, and 2.4). Metamorphosed dikes are tectonically imbricated with the carbonates and hydrothermal breccias (Rigobello et al., 1988; Monteiro, et al., 1999, 2006, 2007; Cunha et al., 2007). Mineralization within the breccia is found along the Vazante fault zone that strikes N50E and dips $60-70^{\circ} \mathrm{NW}$ (Rigobello et al., 1988; Dardenne and Freitas-Silva, 1999; Monteiro et al., 1999; Dardenne, 2000; Monteiro et al., 2006, 2007). The fault is interpreted as a syn-sedimentary growth fault with multiple reactivations during the Brasiliano Orogeny. It began as a reverse, transcurrent fault during compression and later evolved into a normal, sinistral fault at the end of the orogenic event (Pinho, 1990; Monteiro, 1999; Dardenne, 2000). The shear zone in the Vazante Mine displays both brittle and ductile deformation, represented by brecciation and S-C foliation found within the shear zone, respectively (Monteiro et al., 1999). 


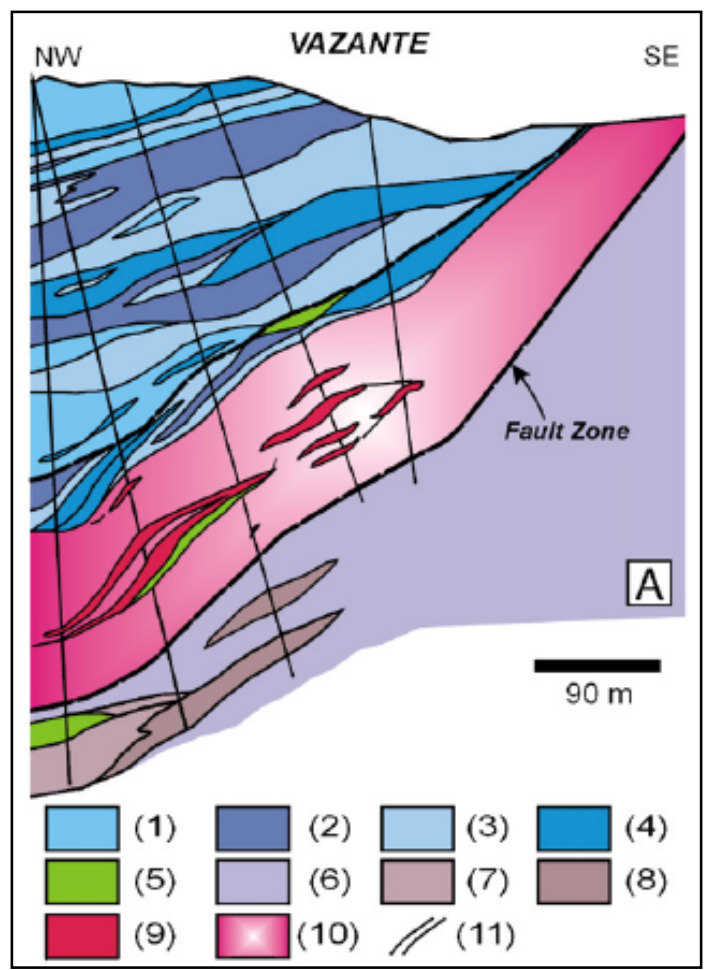

Fig. 2.4 Geological NW-SE cross-section of Vazante Mine showing the mineralized zone [(1) gray dolostone; (2) sericite phyllite; slate and metamarl; (3) pink

dolostone; (4) light gray dolostone, quartz sericite phyllite, (5) metabasite; (6-8) Upper Morro do Pinheiro Member: (6) dark-gray dolostone; (7) pyrite-bearing black phyllite; (8) pyrite-rich metamarl; (9) nonsulfide zinc ore; (10) hydraulic breccias; (11) limit of the Vazante Shear Zone] (from Monteiro et al., 2007).
Willemite is the main zinc ore in the

Vazante mine. It occurs as pods, veinlets, and meter-wide veins within the hydrothermal breccia and is locally controlled by antithetic faults in the deposit (Monteiro et al., 1999; Dardenne, 2000;

Hitzman et al., 2003; Monteiro et al., 2006, 2007).

The willemite ore extends along strike for nearly

$3 \mathrm{~km}$ and ranges in depth from the surface to over $400 \mathrm{~m}$ in the underground mine (Rigobello et al., 1988; (Votorantim, pers. commun., 2010).

According to Monteiro et al. (1999), small sulfide bodies consisting of Fe-poor sphalerite with minor galena are tectonically imbricated with the willemite orebodies, metabasites, and dolostones. In addition to the willemite and sulfides, franklinite, smithsonite, zincite, hematite, barite, and quartz are also found. The relationship between willemite and sphalerite is not well understood at Vazante. Monteiro et al. (1999) and Hitzman et al. (2003) suggested coeval precipitation of willemite and sphalerite.

However, Appold and Monteiro (2009) suggest that willemite formed after sphalerite. Willemite typically occurs as radiating or prismatic crystals and displays green cathode luminescence (CL) (Monteiro et al., 2006, 2007). The willemite composition, according to EMP studies conducted by Monteiro et al. (2006), contains minor amounts of $\mathrm{FeO}$ (up to $0.3 \mathrm{wt} \%$ ) and $\mathrm{CaO}$ (up to 0.13 $\%$ ), although the calcium could be related to contamination form the carbonate host rocks. The 
paragenetic sequence for the mineralization at the Vazante Mine was first described in detail by Monteiro et al. (1999) and is summarized in Figure 2.5.

The hydrothermal alteration within the Vazante district is largely controlled by the structures relating to brecciation. Siderite, ankerite, quartz and hematite are common alteration minerals found in veins and filling spaces between breccia clasts. Rare gahnite also occurs in some veinlets close to the ore mineralization (Monteiro et al., 1999). Alteration to the surrounding host rocks includes a color change from gray to pink dolostones caused by pervasive hematite alteration. Silicification of dolostone is also common (Monteiro et al., 1999; Dardenne, 2000; Monteiro et al., 2006, 2007).

\begin{tabular}{|c|c|c|c|c|}
\hline & \multirow{2}{*}{ Minerals } & \multirow[t]{2}{*}{ Premineralization stage } & Main mineralization stage & Late mineralization stage \\
\hline & & & $\begin{array}{l}\text { Brittle-ductile } \\
\text { structures }\end{array}$ & $\begin{array}{c}\text { Brittle-ductile and } \\
\text { brittle structures }\end{array}$ \\
\hline $\begin{array}{l}\text { Early } \\
\text { diagenetic } \\
\text { association }\end{array}$ & $\begin{array}{l}\text { Microspar to pseudospar } \\
\text { dolomite } \\
\text { Euhedral pyrite } \\
\text { Fibrous dolomite }\end{array}$ & $\infty$ & & \\
\hline $\begin{array}{l}\text { Late } \\
\text { diagenenetic } \\
\text { association }\end{array}$ & $\begin{array}{l}\text { Spar dolomite } \\
\text { Quartz } \\
\text { Pyrite (stylolites) } \\
\text { Saddle dolomite } \\
\text { Clay-minerals }\end{array}$ & $\sum_{2}^{\rightleftarrows}$ & & \\
\hline $\begin{array}{l}\text { Epigenetic } \\
\text { association }\end{array}$ & $\begin{array}{l}\text { Baroque dolomite } \\
\text { Siderite } \\
\text { Hematite } \\
\text { Jasper } \\
\text { Sphalerite } \\
\text { Galena } \\
\text { Willemite } \\
\text { Quartz } \\
\text { Magnetite } \\
\text { Franklinite } \\
\text { Zincite } \\
\text { Cloudy saddle dolomite } \\
\text { Barite } \\
\text { Apatite } \\
\text { Smithsonite } \\
\text { Talc } \\
\text { Chlorite }\end{array}$ & 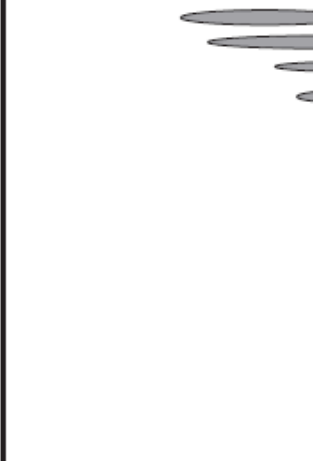 & 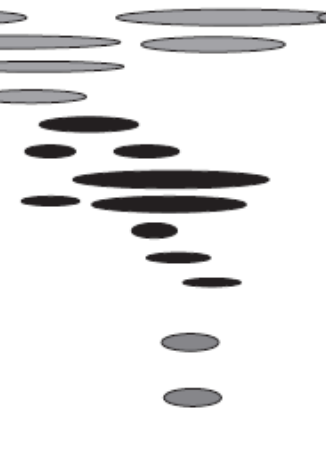 & $\stackrel{\sum}{\rightleftarrows}$ \\
\hline
\end{tabular}

Fig. 2.5 Paragenetic sequence for the mineralization in the Vazante Mine (from Monteiro et al., 2006)

Fluid inclusion studies conducted on the willemite at Vazante by Dardenne and FreitasSilva (1999) reveal salinities of 3 - 15 eq. Wt. \% NaCl and homogenization temperatures ranging 
from $65-180{ }^{\circ} \mathrm{C}$. Sphalerite fluid inclusion studies conducted by Cunha et al. (2007) show salinities around $15-23$ eq. Wt. $\% \mathrm{NaCl}$ and homogenization temperatures ranging from $201-$ $232{ }^{\circ} \mathrm{C}$.

Sulfur isotopes are limited in Vazante due to the lack of sulfur minerals within the deposit; however, Misi et al. (2005) were able to obtain sulfur isotope data from sphalerite and galena found at the Vazante mine. The isotopic ratios range from $+11.8 \%$ to $16.4 \%$ which indicated that the sulfur that was sourced from seawater.

The genetic model for Vazante, like many hypogene nonsulfide zinc deposits, is not well understood nor is it complete. Given the prevalence of hematite and willemite, the ore fluids would have to ultimately be

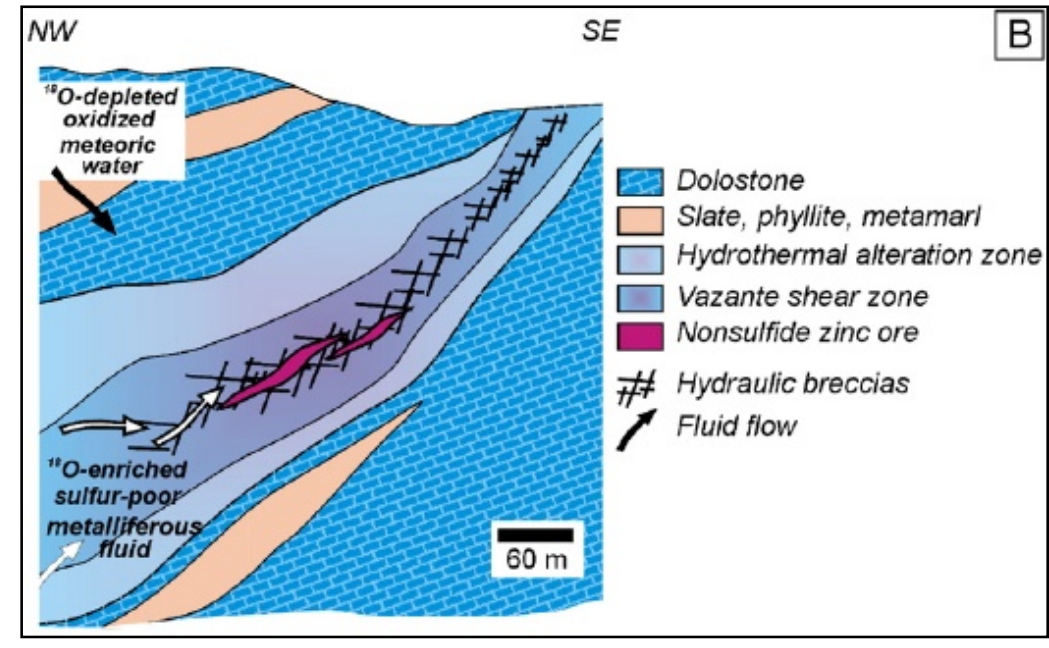

Fig. 2.6 Genetic mixing model for the Vazante Mine where a metalliferous fluid is focused through the Vazante Shear Zone and mixes with meteoric water from the surface (from Monteiro et al., 2007).

constrained to an oxidizing

environment. Monteiro et al. (2007) first proposed that an oxygen-enriched, hot, sulfur deficient metalliferous fluid would have mixed with oxygen-depleted, cool, meteoric waters within the Vazante shear zone (Figure 5). Appold and Monteiro (2009) explained the controls on willemite deposition by fluid mixing models. Table 2 below summarizes the mechanisms proposed by Appold and Monteiro (2009) to explain the precipitation of willemite and/or sphalerite under specific conditions. 


\begin{tabular}{l|ll}
\hline Factor & Willemite $(\mathrm{Wl})$ & Sphalerite $(\mathrm{Sp})$ \\
\hline Temperature $(T)$ & Precipitation not affected & As $T$ decreases, Sp precipitates \\
$\mathrm{pH}$ & $\begin{array}{l}\text { More sensitive to } \mathrm{pH} \text { than } \mathrm{Sp}, \text { as } \\
\mathrm{pH} \text { increases, Wl precipitates }\end{array}$ & $\begin{array}{l}\text { Less sensitive to } \mathrm{pH} \text { than Wl, as } \\
\mathrm{pH} \text { increases, Sp will precipitate } \\
\text { but to a lesser extent than W1 }\end{array}$ \\
Salinity & $\begin{array}{l}\text { A decrease in salinity precipitates } \\
\text { Wl and Sp (favors neither) }\end{array}$ & $\begin{array}{l}\text { A decrease in salinity precipitates } \\
\text { Wl and Sp (favors neither) }\end{array}$ \\
$\log f \mathrm{O}_{2}$ & No effect & $\begin{array}{l}\text { As Log } f \mathrm{O}_{2} \text { increases, sulfides } \\
\text { converted to sulfates }\end{array}$ \\
\hline
\end{tabular}

Table 2.2 Summary of the factors controlling willemite and sphalerite precipitation from a fluid

(Summarized from Brugger et al. (2003) and Appold and Monteiro (2009). Wl = willemite, $\mathrm{Sp}=$ sphalerite

Based on the factors outlined in Table 2, Appold and Monteiro (2009) interpreted the possible ore fluid as being a hot, saline, reducing, acidic, metalliferous fluid that mixed with cooler, oxidizing meteoric water. The ore would then have precipitated as a result of an increase in $\mathrm{pH}$ and possibly a small decrease in temperature (Brugger et al., 2003; Appold and Monteiro, 2009).

Recent work was conducted by Lemos (2011) in the Vazante Northern Extension zone. The main focus was to characterize willemite ore in order to assist in optimizing ore processing. Willemite samples from the field and drill core were crushed, categorized based on iron content, and analyzed for trace elements. Lemos (2011) found that the ore breccia containing high iron also contained higher chromium values and lower zinc values, indicating that iron and chromium were substituting for zinc. It was also found that there were no discernible differences in trace elements between iron-rich willemite breccia and iron-poor willemite breccia; however, the Northern Extension ore was determined to be depleted in the elements $\mathrm{Ag}, \mathrm{Cd}, \mathrm{Ge}$, and $\mathrm{Pb}$ when compared with the main Vazante orebody. This was interpreted to be due to the smaller content in sulfide in the North Extension ore (Lemos, 2011). 


\section{Chapter 2 References}

Almeida, F.F.M., 1969, Evolucao tectônica do Centro-Oeste Brasileiro: Anais da Academia Brasileira de Ciências, p. 280-296.

Almeida, F.F.M., Hasui, Y., Brito Neves, B.B., and Fuck, R.A., 1981, Brazilian structural provinces: an introduction: Earth-Science Reviews, v. 17, p. 1-29.

Amaral, G., 1968, Geologia e depositos de minerio na reigao de Vazante, estado de Minas Gerais: Ph.D. thesis, São Paulo, Brazil, Universidade de São Paulo.

Appold, M.S. and Monteiro, L.V.S., 2009, Numerical modeling of the hydrothermal zinc silicate and sulfide mineralization in the Vazante deposit, Brazil: Geofluids, v. 9, p. 96-115.

Araujo, S.M., 1999, The Palmeiroplolis volcanogenic massive sulfide deposit, Tocantins state, in: Base metal deposits of Brazil: Salvador, Ernesto von Sperling, p. 64-68

Azmy, K. Veizer, J., Misi, A., Oliveira, T.F., Sanches, A.L., and Dardenne, M.A., 2001, Dolomitization and isotope stratigraphy of the Vazante Formation, São Francisco Basin, Brazil: Precambrian Research, v. 112, p. 303-329.

Brugger, J., McPhail, D.C., Wallace, M., and Waters, J., 2003, Formation of willemite in hydrothermal environments: Economic Geology, v. 98, p. 819-835.

Cloud, P. and Dardenne, M.A., 1973, Proterozoic age of the Bambuí Group in Brazil: Geological Society of America Bulletin, v. 84(5), p. 1673-1676.

Cunha, I.A., Coelho, C.E.S., and Misi, A., 2000, Fluid inclusion study of the Morro Agudo Pb-Zn deposits, Minas Gerais, Brazil: Revista Brasileira de Geociências, v. 30, p. 318-321.

Cunha, I.A., Misi, A., Babinski, M., and Iyer, S.S.S., 2007, Lead isotope constraints on the genesis of $\mathrm{Pb}-\mathrm{Zn}$ deposits in the Neoproterozoic Vazante Group, Minas Gerais, Brazil: Gondwana Research, v. 11, p. 382-395.

Da Rocha Araujo, P.R., Flicoteaux, R., Parron, C., and Trompette, R., 1992, Phosphorites of Rocinha Mine - Patos de Minas (Minas Gerais, Brazil): Genesis and evolution of a middle Proterozoic deposit tectonized by the Brasiliano Orogeny: Economic Geology, v. 87, p. 332351.

Dardenne, M.A., 1978, Geologia da Região de Morro Agudo, Minas Gerais. s.l., Sociedade Brasilera de Geologia, p. 68-84. 
Dardenne, M.A., 1979, Le mineralisations plomb-zinc du Groupe Bambuí et leur contexte geologique: Ph.D. thesis, Paris, France, Universite Pierre et Marie Curie.

Dardenne, M.A., 2000, The Brasília Fold Belt, in: The tectonic evolution of South America: Rio de Janeiro, 31st International Geological Congress, p. 231-263.

Dardenne, M.A. and Freitas-Silva, F.H., 1999, Pb-Zn ore deposits of Bambuí and Vazante groups, in the São Francisco Craton and Brasília Fold Belt, Brazil, in: Base metal deposits of Brazil: Salvador, Ernesto von Sperling, p. 75-83.

Dardenne, M.A. and Schobbenhaus, C., 2001, Metalogenese do Brasil: Brasília, University of Brasília.

Freitas-Silva, F.H. and Dardenne, M.A., 1994, Proposta de subdiviSão estratigrafica formal para o Grupo Canastra no oeste de Minas Gerais e leste de Goias: Goiana, Sociedade Brasilera de Geologia, p. 161-163.

Freitas-Silva, F.H. and Dardenne, M.A., 1997, $\mathrm{Pb} / \mathrm{Pb}$ isotopic patterns of galenas from Morro do Ouro (Paracatu Formation), Morro Agudo/Vazante (Vazante Formation) and Bambuí Group deposits: Campos do Jordão, s.n., p. 118-120.

Fuck, R.A. and Marini, O.J., 1981, O Grupo Araxa e unidades homotaxiais: s.l., Sociedade Brasileira de Geociências, p. 118-130.

Giuliani, G., Olivo, G.R., Marini, O.J., and Michel, D., 1993, The Santa Rita gold deposit in the Proterozoic Paranoá Group, Goiás, Brazil: An example of fluid mixing during ore deposition: Ore Geology Reviews, v. 8, p. 503-523.

Hitzman, M.W., Reynolds, N.A., Sangster, D.F., Allen, C.R., and Carman, C.E., 2003, Classification, genesis, and exploration guides for nonsulfide zinc deposits: Economic Geology, v. 98, p. 685-714.

Iyer, S.S., Hoefs, J. and Krouse, H.R., 1992, Sulfur and lead isotope geochemistry of galenas from the Bamui Group, Minas Gerais, Brazil: implications for ore genesis: Economic Geology, v. 87, p. 437-443.

Lemos, M.G., 2011, Caracterização geológica e tecnológica do minério de zinco do Extremo Norte da Mina de Vazante, Minas Gerais: M.Sc. thesis, Campinas, Universidade de Campinas. 
Madalosso, A. and Valle, C.R.O., 1978, Consideraçoẽs sobre a estratigrafia e sedimentologia do Grupo Bambuí na região de Paracatú - Morro Agudo, MG: s.l., Sociedade Brasileira de Geociências, p. 622-634.

Marini, O.J., Fuck, R.A., Danni, J.C. and Dardenne, M.A., 1981, A evolução geotectônica da Faixa Brasília e de seu embasamento: Salvador, COM/SBG, p. 100-115.

Marini, O.J., Fuck, R.A., Danni, J.C.M., Dardenne, M.A., Loguercio, S.O. and Ramalho, R., 1984a, As faixas de dobramento Brasília, Uruacu, Paraguai-Araguaia e o Macico Mediano de Goiás, in: Geologia do Brasil: Brasília, DNPM, p. 251-303.

Marini, O.J., Fuck, R.A., Dardenne, M.A. and Danni, J.C.M., 1984b, Província Tocantins: setores central e sudeste, in: Almeida, F.F.M. and Hasui, Y. eds., O Precambriano do Brasil: São Paulo, Edgar Blucher, p. 205-264.

Martins-Neto, M.A., 2009, Sequence stratigraphic framework of Proterozoic successions in eastern Brazil: Marine and Petroleum Geology, V. 26, p. 163-176.

Misi, A., Iyer, S.S.S., and Tassinari, C.C.G., 1996, Lead-zinc deposits, Brazil: new SEDEX subtypes?: Salvador, s.n., p. 251-253.

Misi, A., Iyer, S.S.S., Kyle, J.R., Coelho, C.E.S, Tassinari,C.G.G., Franca-Rocha, W.J.S., Gomes, A.S.R., Cunha, I.A, Toulkeridis, T., and Sanches, A.L., 2000, A metallogenic evolution model for the lead-zinc deposits of the Meso and Neoproterozoic sedimentary basins of the São Francisco Craton, Bahia and Minas Gerais, Brazil: Revista Brasileira de Geociências, v. 30, p. 302-305.

Misi, A., Iyer, S.S.S., Coelho, C.E.S., Tassinari, C.C.G., Franca-Rocha, W.J.S., Cunha, I.A., Gomes, A.S.R., Oliveira, T.F., Teixeira, J.B.G., and Filho, V.M.C., 2005, Sediment hosted lead-zinc deposits of the Neoproterozoic Bambuí Group and correlative sequences, São Francisco Craton, Brazil: A review and a possible metallogenic evolution model: Ore Geology Reviews, v. 26, p. 263-304.

Misi, A., Kaufman, A.J., Veizer, J., Powis, K., Azmy, K., Boggiani, P.C., Gaucher, C., Teixeira, J.B.G., Sanches, A.L., and Iyer, S.S.S., 2007, Chemostratigraphic correlation of Neoproterozoic successions in South America: Chemical Geology v. 237, p. 143-167.

Monteiro, J.V.S., Bettencourt, J.S., Spiro, B., Graca, R., and Oliveira, T.F., 1999, The Vazante zinc mine, Minas Gerais, Brazil: constraints on willemitic mineralization and fluid evolution: Exploration Mining Geology, v. 8, p. 21-42. 
Monteiro, L.V.S., 2002, Modelamento metalogenetico do depositos de zinco de Vazante, Fagundes e Ambrosia, associados ao Grupo Vazante, Minas Gerais: Ph.D. thesis, São Paulo, Universidade de São Paulo.

Monteiro, L.V.S., Bettencourt, J.S., Juliani, C., and Oliveira, T.F., 2006, Geology, petrography, and mineral chemistry of the Vazante non-sulfide and Ambrosia and Fagundes sulfide-rich carbonate-hosted Zn-(Pb) deposits, Minas Gerais, Brazil: Ore Geology Reviews, v. 28, p. 201-234.

Monteiro, L.V.S., Bettencourt, J.S., Juliani, C., and Oliveira, T.F., 2007, Nonsulfide and sulfiderich zinc mineralizations in the Vazante, Ambrosia and Fagundes deposits, Minas Gerais, Brazil: Mass balance and stable isotope characteristics of the hydrothermal alterations: Gondwana Research, v. 11, p. 362-381.

Pimentel, M.M., Heaman, L., Fuck, R.A., and Marini, O.J., 1991, U-Pb zircon geochronology of Precambrian tin-bearing continental-type acid magmatism in Central Brazil: Precambrian Research, v. 52, p. 321-335.

Pimentel, M.M., Dardenne, M.A., Fuck, R.A., Viana, M.G., Junges, S.L., Fischel, D.P., Seer, H.J., and Dantas, E.L., 2001, Nd isotopes of the provenance of detrital sediments of the Neoproterozoic Brasília Belt, central Brazil: Journal of South American Earth Sciences, v. 14 , p. 571-585.

Pinho, J.M.M., 1990, Evolução tectônica da mineralizacao de zinco de Vazante, Brasília: Master's thesis, Brasília, Universidade de Brasília.

Rigobello, A.E., Branquinho, J.A., Silva Dantas, M.G., Oliveira, T.F., and Neves Filho, W., 1988, Mina de zinco de Vazante, Minas Gerais, in: Principais Depositos Minerais do Brasil: Brasília, DNPM, p. 101-110.

Rodrigues, J.B., 2008, Proveniencia de sedimentos dos grupos Canastra, Ibia, Vazante e Bambuí: Um estudo de zircoes detriticos e idades modelo Sm-Nd: Ph.D. theis, Brasília, University of Brasília.

Rodrigues, J.B., Pimentel, M.M., Dardenne, M.A., and Armstrong, R.A., 2010, Age, provenance and tectonic setting of the Canastra and Ibiá Groups (Brasília Belt, Brazil): Implications for the age of a Neoproterozoic glacial event in central Brazil: Journal of South American Earth Sciences, v. 29, p. 512-521.

Thomaz Filho, A., Kawashita, K., and Cordani, U.G., 1998, A origem do Grupo Bambuí no contexto da evolução geotectônica e de idades radiométricas: Anais da Academia Brasileira de Ciências, v. 70, p. 537-548. 
Valeriano, C.M., Pimentel, M.M., Heilbron, M., Almeida, J.C.H., and Trouw, R.A.J., 2008. Tectonic evolution of the Brasília Belt, Central Brazil, and early assembly of Gondwana: Geological Society, London, Special Publications, v. 294, p. 197-210. 


\section{Chapter 3}

\section{Geology, Mineralogy, and Geochemistry of the Vazante Northern Extension Zinc Silicate Deposit, Minas Gerais, Brazil}

\subsection{Introduction}

The increase in global population and in their standards of living is expected to foster the consumption of metals and in particular zinc, which has main applications ranging from galvanized alloys to fertilizer (USGS, 2011). Since the early $20^{\text {th }}$ century, zinc has been mainly produced from sulfide ores of sedimentary-exhalative (SEDEX), Mississippi Valley-type (MVT), and volcanogenic massive sulfide (VMS) deposits due to development of the flotation and smelting techniques (Boni and Large, 2003). However, the potential for producing zinc from high-grade nonsulfide deposits at lower costs using hydrometallurgical techniques has renewed interest in nonsulfide zinc deposits around the world. These nonsulfide zinc deposits comprise two major subtypes: supergene and hypogene (Hitzman et al., 2003). Supergene deposits are formed by the oxidation of both zinc oxide and zinc sulfide deposits and they are typically hosted in carbonate rocks. The most common zinc nonsulfide minerals in supergene deposits are: smithsonite $\left(\mathrm{ZnCO}_{3}\right)$, hemimorphite $\left(\mathrm{Zn}_{4} \mathrm{Si}_{2} \mathrm{O}_{7}(\mathrm{OH})_{2} \cdot 2 \mathrm{H}_{2} \mathrm{O}\right)$, hydrozincite $\left(\mathrm{Zn}_{5}(\mathrm{OH})_{6}\left(\mathrm{CO}_{3}\right)_{2}\right)$, and sauconite $\left(\mathrm{NaO} .3(\mathrm{Zn}, \mathrm{Mg})_{3}(\mathrm{Si}, \mathrm{Al})_{4} \cdot(\mathrm{OH})_{2} \mathrm{nH}_{2} \mathrm{O}\right)$ (Hitzman et al., 2003). Hypogene deposits, from now on referred to as hypogene zinc silicate deposits, are hydrothermal and not necessary derived from a pre-existing sulfide body. They comprise two categories based on their ore controls: structurally-controlled and stratiform deposits. Hypogene zinc minerals include the main ore, willemite $\left(\mathrm{Zn}_{2} \mathrm{SiO}_{4}\right)$, franklinite $\left(\mathrm{ZnFe}_{2} \mathrm{O}_{4}\right)$, and zincite $(\mathrm{ZnO})$ (Hitzman et al., 2003). Structurally controlled deposits, such as the Beltana-Aroona willemite deposits and the Vazante Northern Extension deposits are hosted within brecciated carbonate rocks (Rigobello et al, 1988; 
Monteiro et al., 1999; Dardenne, 2000; Dardenne and Schobbenhaus, 2001; Groves et al., 2003; Monteiro et al., 2006; 2007).

The Vazante Northern Extension trend is one of the largest hypogene zinc silicate deposits with estimated reserves of $18 \mathrm{Mt}$ at $19 \% \mathrm{Zn}$ in the main Vazante Mine and additional reserves of $850 \mathrm{Kt}$ of ore at $16 \% \mathrm{Zn}$ in its Northern Extension (Votorantim, pers. commun., 2011). It is located in the southern region of the Brasília Fold Belt near the town of Vazante, Minas Gerais, Brazil. Production in the area started with artisanal mining since the 1950's when the area was mined for the secondary "calamine" (hemimorphite and smithsonite) zinc ore. In the Northern Extension, it has been estimated that around $8 \mathrm{Mt}$ of calamine ore were mined (Votorantim, pers. commun., 2011). In 1969 and in 1983, open pit and underground mining commenced, respectively (Monteiro et al., 1999), mining mainly the hypogene ore.

The Vazante deposit has been studied by Rigobello et al. (1988); Dardenne and FreitasSilva (1999); Dardenne (2000); Monteiro (et al., 1999, 2006, 2007); Misi et al. (2005); Cunha et al. (2007) and Rostirolla et al. (2007). Rigobello et al. (1988) and Dardenne (2000) first described the stratigraphy and host rocks as well as the structural setting of the Vazante Mine and their tectonic setting. Rostirolla et al. (2007) conducted regional studies in order to establish a metamorphic history of the Vazante region, and identified 5 major structural events in Vazante. Monteiro (et al., 1999, 2006, 2007) studied the mineralization, alteration, and stable isotopes in the Vazante Mine and the nature of the mineralizing hydrothermal fluids. They suggested that the Vazante mineralization formed by the mixing of an oxygen-enriched, hot, sulfur deficient metalliferous fluid with oxygen-depleted, cool, meteoric water. Dardenne and Freitas-Silva (1999) also investigated the fluids associated with mineralization and concluded that the fluids had salinities of $3-15 \mathrm{Wt} \%$ eq. $\mathrm{NaCl}$ and homogenization temperatures ranging from $65-180$ ${ }^{\circ} \mathrm{C}$. Lead isotope investigations by Dardenne and Freitas-Silva (1999), Misi et al. (2005), and Cunha et al. (2007) revealed that the ages of the galenas to range from $1.8 \mathrm{Ga}$ to $1.2 \mathrm{Ga}$, 
interpreted the lead sources are probably from the São Francisco Craton and the Goiás Massif, and suggested that the lead from Vazante deposit was from the same sources as other lead-zinc sulfide deposits in the Vazante Group. However, major questions remain related to the processes by which these mineralized zones formed, whether they are associated with a major mineralizing event or represent various events of fluid-rock interaction and mineralization, and whether the processes that formed the main Vazante mine orebodies are similar to those that formed the Northern Extension. This study is focused on the characterization of the geology, mineralogy, and geochemistry of the Northern Extension of the Vazante Mine in order to further understanding of this style of mineralization.

\subsection{Regional Geology}

The Brasília Fold Belt is roughly $1000 \mathrm{~km}$ long and is part of the Tocantins Province, which is composed of three fold belts: the Araguaia Belt to the east of the Amazonia Craton, the Paraguay Belt on the southeastern margin of the Amazonia Craton, and the Brasília Fold Belt along the western edge of the São Francisco craton (Fig. 3.1; Almeida et al., 1981; Pimentel et al., 2001; Valeriano et al., 2008; Rodrigues et al., 2010). The Paraguay and Araguaia fold belts are thrust towards the Amazonia Craton to the west; however, the central Brasília Fold Belt is thrust to the east onto the São Francisco Craton (Valeriano et al., 2008).

The Brasília Fold Belt was created through a series of tectonic events starting with continental rifting of the São Francisco Craton c.a. 1700Ma and ending with the Brasiliano Orogeny (Misi et. al, 2005; Martins-Neto, 2009). The continental rift system evolved into a passive margin setting along the western edge of the São Francisco Craton, allowing for the deposition of intracratonic siliciclastic sediments and platform carbonates represented primarily by the Canastra-Paranoá-Vazante groups at around 1200-900 Ma (Valeriano et al., 2008; Martins-Neto, 2009). The latter is the host of the Vazante zinc silicate deposit. 


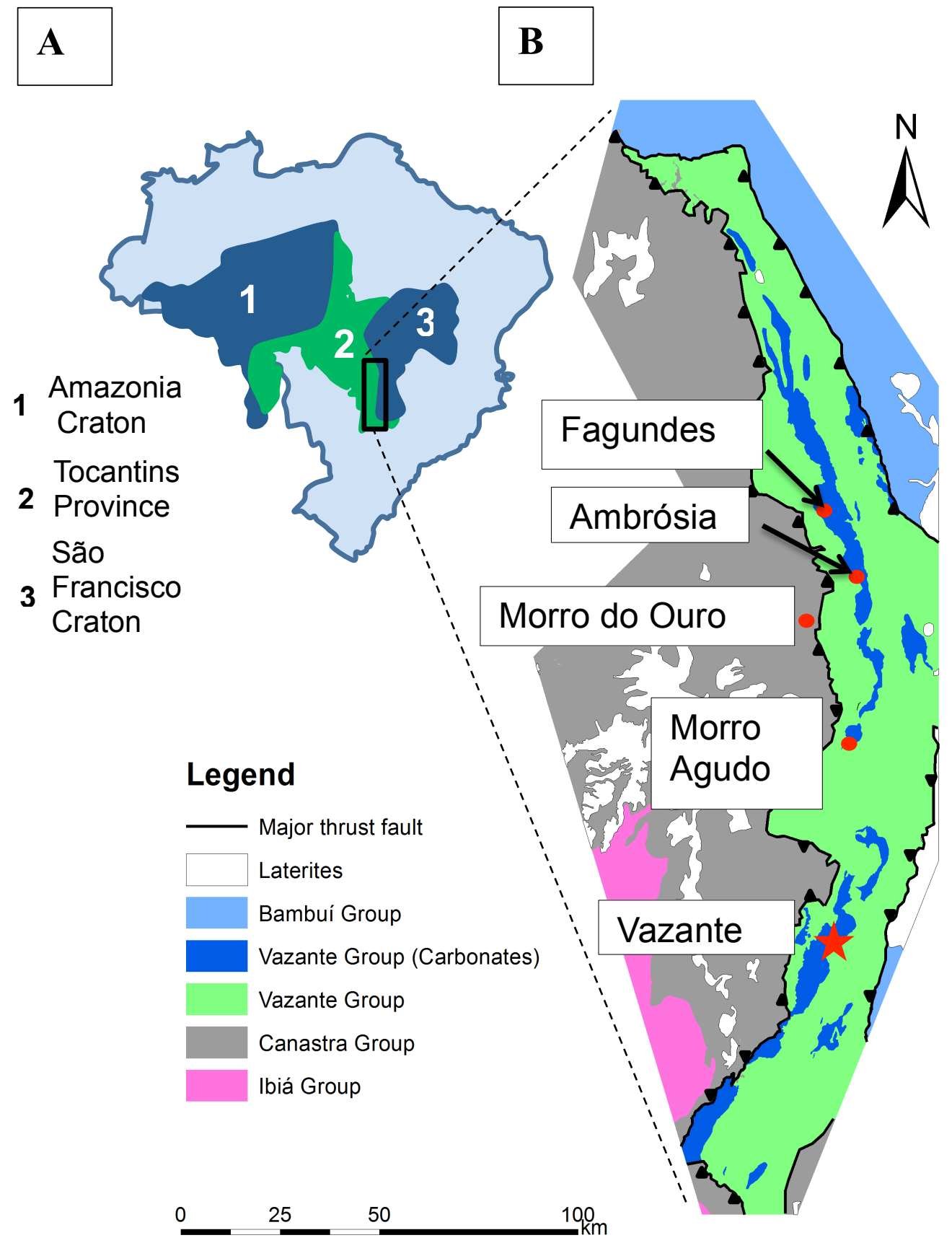

Fig. 3.1 A) Geological map of Brazil with the location of the Tocantins Province. B) Map of the southeastern part of the Brasília Fold Belt, highlighting the major units and mineral deposits in the region. 
Subduction of oceanic crust from the Brazilide Ocean beneath the São Francisco Craton began around $900 \mathrm{Ma}$, causing magmatism that would lead to the formation of the Goiás magmatic arc (Dardenne, 2000; Valeriano et al., 2008; Martins-Neto, 2009). Continuing subduction lead to the convergence of the Goiás magmatic arc and the São Francisco Craton around $850-750 \mathrm{Ma}$, the start of the Brasiliano orogeny. During this collision, the passive margin sediments were thrusted as thin-skinned nappes onto the São Francisco Craton (Dardenne, 2000; Valeriano et al., 2008, Martins-Neto, 2009), and reached its metamorphic peak around 650 Ma (Martins-Neto, 2009). Dardenne (2000) also notes geochemical and isotopic evidence of a late Brasiliano collisional event c.a. $630-610 \mathrm{Ma}$, interpreting it as the latest stage of the convergence of the Goiás magmatic arc with the São Francisco Craton.

The southern region of the Brasília Fold Belt, which comprises the Vazante, Canastra, Araxá, and Ibiá groups, was metamorphosed up to the greenschist facies and generally displays more intense tectonic deformation than the northern region. The Vazante, Canastra, Araxá, and Ibiá groups form a complex system of nappes that are thrust onto the Bambuí Group sediments to the east (Dardenne, 2000). The Canastra, Vazante, and Bambuí groups are the most prominent sedimentary units in the Vazante region.

The Canastra Group is considered to be the southern Brasília Fold Belt equivalent sedimentary group to the Paranoá Group located in the northern BFB (Dardenne, 1978, 2000). The age of the Canastra Group is estimated to be between 1200 - 900 Ma based on stromatolites (Dardenne, 2000), and Pimentel et al. (2001) proposed an age of about 1000 Ma based on Sm$\mathrm{Nd}$ dating. It is composed primarily of phyllites and quartzites that have been metamorphosed up to the greenschist facies. The Canastra Group sits unconformably below the Ibiá Group and is tectonically thrust on top of the Vazante Group (Dardenne, 2000). It comprises three formations from the bottom to the top: the Serra do Landim, Paracatú, and Chapada dos Pilões. The Serra do Landim Formation makes up the lowest section of the Canastra Group according to Freitas- 
Silva and Dardenne (1994); however, Madalosso and Valle (1978) have described the Serra do Landim as being the uppermost formation of the Vazante Group. The Serra do Landim is a pale green to grey-green calc-phyllite to calc-schist (Freitas-Silva and Dardenne, 1994; Dardenne, 2000). The Paracatú Formation is composed of grey carbonaceous phyllite with quartzite lenses (Almeida, 1969; Freitas-Silva and Dardenne, 1994; Dardenne, 2000) and hosts the Morro do Ouro gold mine located in Paracatú, Minas Gerais (Dardenne, 2000). The Chapada dos Pilões Formation lies on top of the Paracatú Formation, and it is made up of intercalated phyllites and quartzites, which have been interpreted as rhythmites and turbidites (Dardenne, 2000).

The Vazante Group is located in the northwest region of Minas Gerais, and it stretches for nearly $250 \mathrm{~km} \mathrm{~N}-\mathrm{S}$. It is primarily composed of intercalated phyllites and platform carbonates that have undergone metamorphism up to the greenschist facies. The Vazante Group lies tectonically below the Canastra Group and is thrust onto the top of the younger Bambuí Group (Dardenne, 2000; Pimentel et al., 2001; Monteiro et al., 2007). The age of the Vazante Group is controversial and poorly constrained due to the absence of igneous rocks and well constrained detrital zircon dating.

The Vazante Group (Fig. 3.2) is divided into 7 formations, which are from the bottom to the top: Retiro (also referred to as Santo Antônio do Bonito Formation; Cunha et al., 2007; Misi et al., 2007), Rocinha, Lagamar, Serra do Garrote, Serra do Poço Verde, Morro do Calcario, and Serra da Lapa (Dardenne, 2000). The Retiro formation consists of quartzite, conglomerate, diamictite (i.e.,debris flows from glacio-marine environments), and slate (Dardenne, 2000; Cunha et al., 2007). It is host to the Coromandel phosphorite mineralization (Dardenne, 2000). The Retiro Formation grades upwards into the Rocinha Formation, which consists of pelite, siltstone rhythmites as well as phosphatic layers that host the Rocinha and Lagamar phosphorite deposits (Dardenne, 2000; Cunha et al., 2007). The top of the Rocinha Formation is a thick sequence of slate and metasiltstone that grades into carbonaceous and pyrite-bearing slate (Dardenne, 2000; 
Pimentel et al., 2001). These pyrite-bearing slates contain phosphorites and are host to the Rocinha phosphate deposit. The upper part of the formation contains both quartzites and siltstones that host the Lagamar phosphate deposit (Dardenne, 2000). Above the Rocinha Formation is the Lagamar Formation which is a metapsamo-pelitic unit with a basal metaconglomerate. Overlaying these clastic units are dolomitic breccias, dark gray limestones, and light pink stromatolitic bioherms with columnar Conophyton and Jacutophyton stromatolites (Dardenne, 2000; Monteiro et al. 2006; Cunha et al., 2007). Above the Lagamar Formation is the Serra do Garrote Formation, which is made up of dark gray to green-gray phyllites with localized pyritic zones (Dardenne, 2000; Cunha et al. 2007; this study).

Sitting atop the Serra do Garrote Formation is the Serra do Poço Verde Formation, which hosts the Vazante zinc silicate mineralization. The Serra do Poço Verde Formation (Fig. 3.2) can further be subdivided into four members from the bottom to the top: Lower Morro do Pinheiro, Upper Morro do Pinheiro, Lower Pamplona, and Middle Pamplona (Dardenne, 2000). The Lower Morro do Pinheiro member consists of light grey and pink dolomite with stromatolites intercalated with oncolitic dolarenites and intraformational dolomite breccias and hosts the Vazante deposit. The Upper Morro do Pinheiro Member is a dark grey dolomite with bird's eyes and minor carbonaceous phyllites. The Lower Pamplona Member consists of grey to pink laminated dolomites intercalated with green and maroon phyllites. The Middle Pamplona Member consists of light grey to pink dolomite with algal laminations and dolarenites with stromatolites (Dardenne, 2000; this study).

The Morro do Calcário Formation, also known as the Upper Pamplona Member according to Rigobello et al. (1988), rests on the top of the Serra do Poço Verde Formation, and is comprised of carbonaceous shale, dolarenites, breccias, and stromatolitic bioherm facies (Dardenne, 2000; this study). It hosts of the Morro Agudo Pb-Zn Mine (Dardenne, 2000), Fagundes zinc sulfide deposit, and Ambrósia zinc sulfide deposit (Monteiro et. al., 2006). 


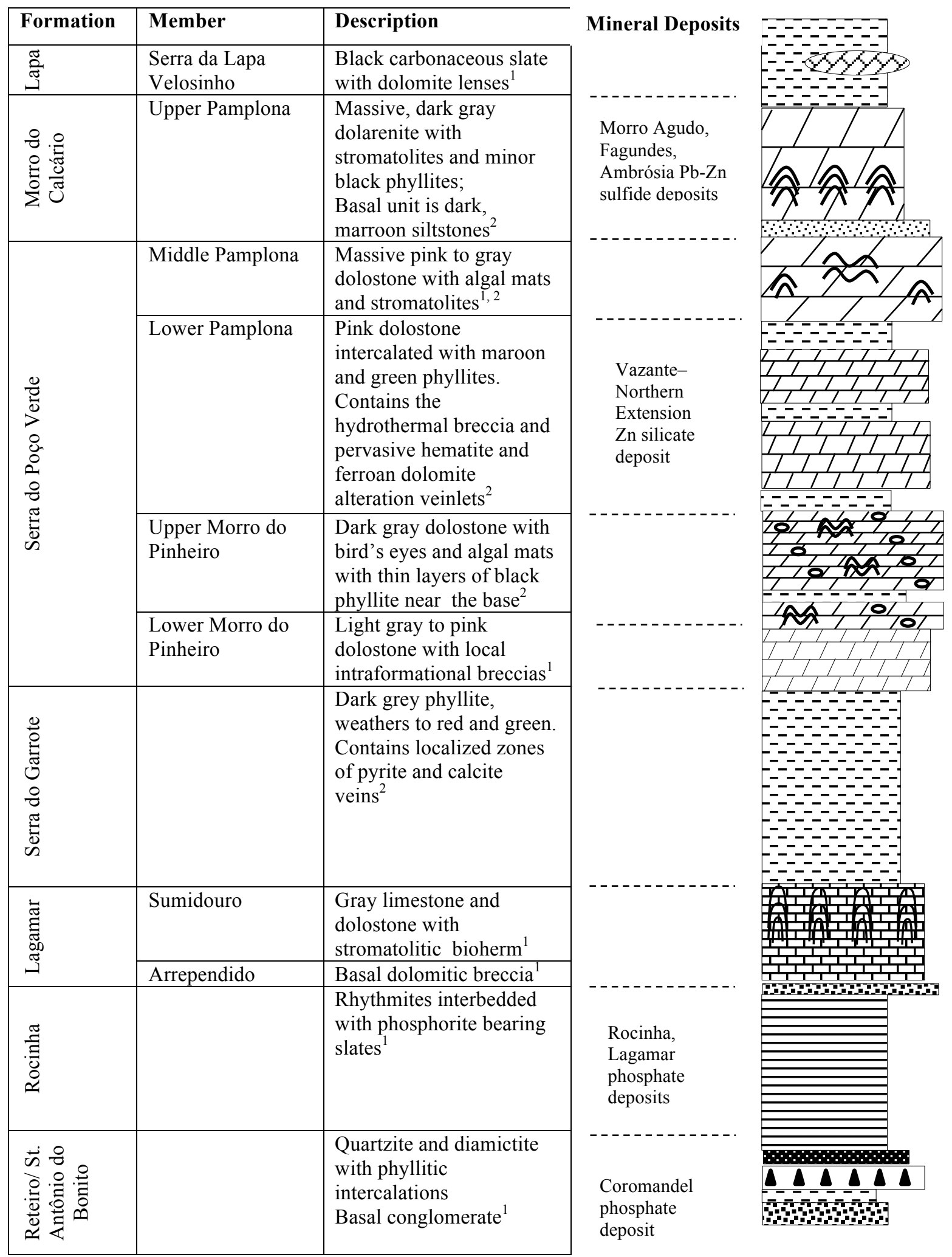

Fig. 3.2 Stratigraphy the Vazante region including the Northern Extension (modified from ${ }^{1}$ Dardenne, 2000; ${ }^{2}$ this study) 
The upper most formation in the Vazante Group is the Lapa Formation, which sits unconformably on top of the Morro do Calcário formation. It is composed of black, carbonaceous rhythmites with intercalated slates and phyllites, sericite-chlorite phyllites, carbonate-bearing metasiltstones with dolomite and quartz lenses (Dardenne, 2000; Monteiro et al., 2006).

\subsection{Geology of the Vazante Mine}

The Vazante Mine (Fig. 3.3) zinc silicate mineralization is hosted in a hydrothermal shear breccia found near the contact between the Lower Pamplona and Upper Morro do Pinheiro members of the Serra do Poço Verde Formation. Metamorphosed dikes are tectonically imbricated with the carbonates and hydrothermal breccias (Rigobello et al., 1988; Monteiro et al., 1999, 2006, 2007; Cunha et al., 2007). Mineralization within the breccia is found along a fault that strikes 230 and dips 60-70 NW (Rigobello et al., 1988; Dardenne and Fritas-Silva, 1999; Monteiro et al., 1999; Dardenne, 2000; Monteiro, et al., 2006, 2007). The fault is interpreted as an early synsedimentary growth fault that underwent multiple reactivations during the Brasiliano Orogeny, including reverse, transcurrent fault during compression that later evolved into a normal, sinistral fault at the end of the orogenic event (Pinho, 1990; Monteiro, 1999; Dardenne, 2000). The shear zone in the Vazante Mine displays features typical of both brittle and ductile deformation, represented by brecciation and S-C foliation, respectively (Monteiro et al., 1999). The Vazante ore body is nearly $5 \mathrm{~km}$ long and variably $10 \mathrm{~s}$ of meters thick, extending over $400 \mathrm{~m}$ below surface (Votorantim, pers. commun., 2010). 


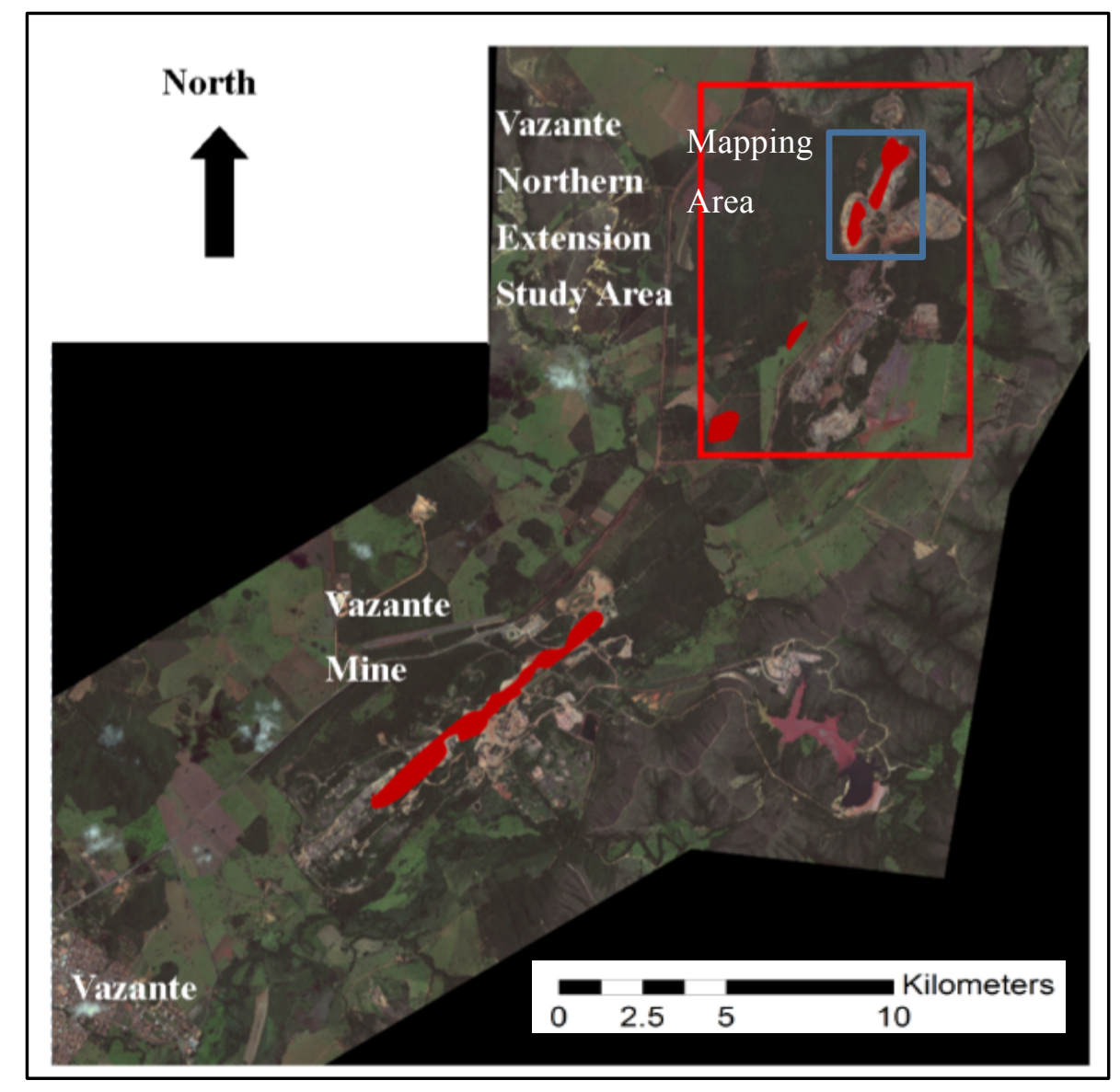

Fig. 3.3 Aerial photographs showing the location of the Vazante Mine and Northern Extension deposit, MG, Brazil. (Photo provided by Votorantim Metals)

The hydrothermal alteration within the Vazante district is largely controlled by the structures related to brecciation. Siderite, ankerite, quartz and hematite are common alteration minerals found in veins and filling spaces between breccia clasts. Rare gahnite also occurs in some veinlets proximal to the ore (Monteiro et al., 1999). Alteration in the surrounding host rocks of the breccia includes a color change from gray to pink dolostones caused by hematite alteration and silicification of dolostone (Monteiro et al., 1999; Dardenne, 2000; Monteiro et al., 2006 ; 2007)

Willemite $\left(\mathrm{Zn}_{2} \mathrm{SiO}_{4}\right)$ is the main zinc ore in the Vazante mine. It occurs as pods, veinlets, and meter-wide veins within the tectonic-hydrothermal breccia and is controlled by antithetic 
faults in the deposit (Monteiro et al., 1999; Dardenne, 2000; Hitzman et al., 2003; Monteiro et al., 2006; 2007). According to Monteiro et al. (1999), small sulfide bodies consisting of Fe-poor sphalerite with minor galena are tectonically imbricated with the willemite, metabasites, and dolostones. The main willemite ore rarely contains sulfide mineralization, but is commonly associated with specular hematite, which is the most abundant mineral associated with the ore; franklinite, smithsonite, zincite, barite, and quartz occur in minor amounts in the ore zone (Monteiro et al., 1999; 2007).

Fluid inclusion studies conducted on the willemite at Vazante by Dardenne and FreitasSilva (1999) revealed salinities of $3-15 \mathrm{Wt} \%$ eq. $\mathrm{NaCl}$ and homogenization temperatures of 65 $-180{ }^{\circ} \mathrm{C}$. Sphalerite fluid inclusion studies conducted by Cunha et al. (2007) show salinities around $15-23 \mathrm{Wt} \%$ eq. $\mathrm{NaCl}$ and homogenization temperatures ranging from $201-232{ }^{\circ} \mathrm{C}$. Sulfur isotopic investigation is limited in Vazante due to the lack of sulfide minerals within the deposit; however, few isotopic data reported by Misi et al. (2005) yield values ranging from $+11.8 \%$ to $16.4 \%$. These results were interpreted to represent sulfur that was sourced from seawater.

Appold and Monteiro (2009) proposed the formation of the Vazante deposit as being the result of mixing between a hot, saline, reducing, acidic, metalliferous fluid and cooler, oxidizing, meteoric water. The ore would then have precipitated out as a result of an increase in $\mathrm{pH}$ and possibly a small decrease in temperature (Brugger et al., 2003; Appold and Monteiro, 2009).

\subsection{The Northern Extension}

\subsubsection{Geologic Units}

The Northern Extension mineralization is hosted by the Vazante Shear Zone that cuts through (Figs. 3.3, 3.4, 3.5 \& 3.6) the Serra do Poço Verde and Morro do Calcário formations of the Vazante Group, and this structure is truncated by the tectonic contact with the phyllites from 


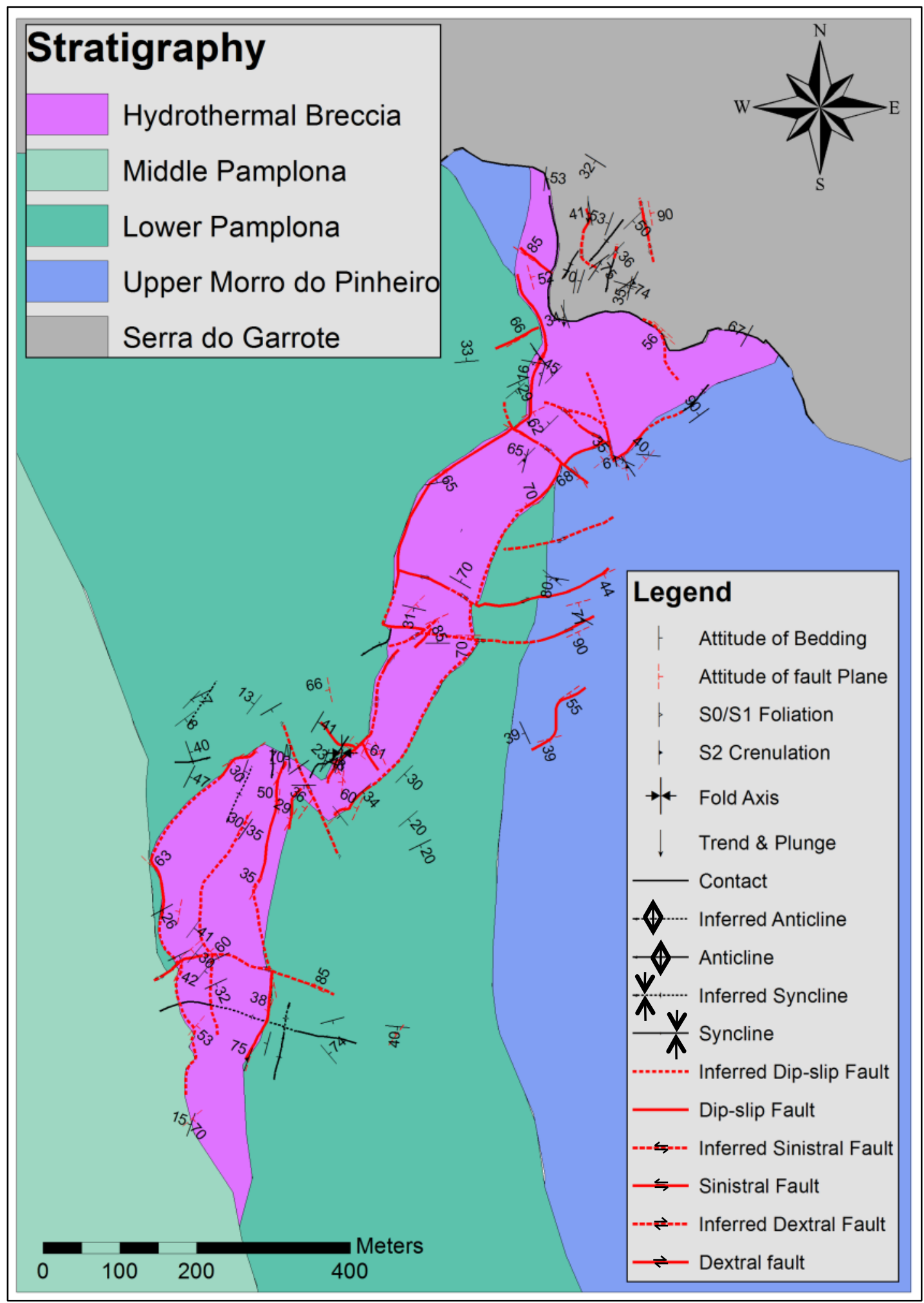

Fig. 3.4 Geological map of Cavas 5A, 6A, and 7A in the northernmost section of the Northern Extension (modified from Votorantim base map; this study) 


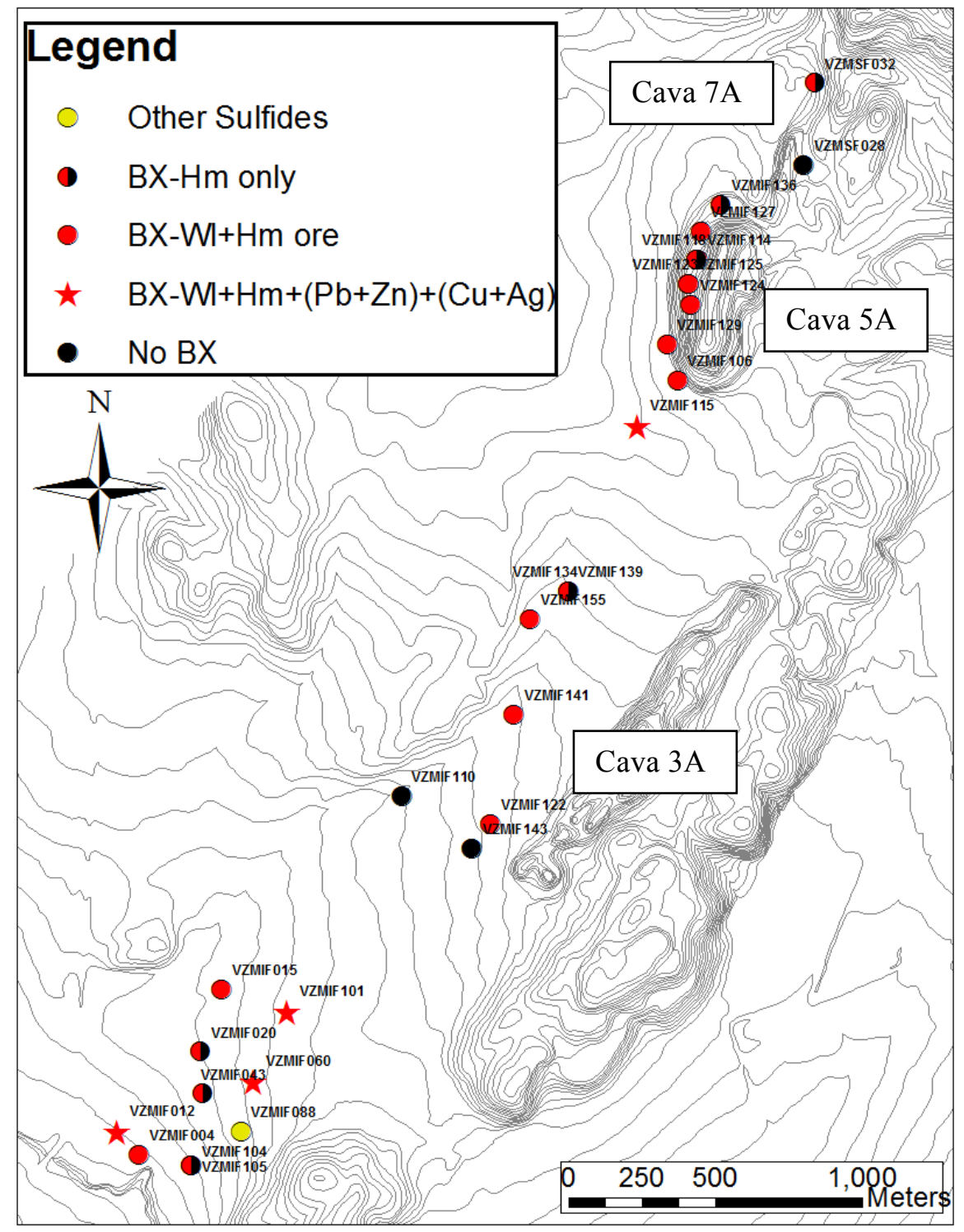

Fig. 3.5 Topographic map of the Vazante Northern Exenstion region with locations of re-logged and sampled drillholes. "BX" is short for "breccia" and "Cava" is Portuguese for "pit" (modified from Votorantim base map; this study).

Serra do Garrote Formation. Each of these formations has been metamorphosed to subgreenschist facies, though metamorphic assemblages are most noticeable in the Serra do Garrote Formation.

In the proximity of the zinc mineralized zones, the Serra do Garrote Formation (Fig. 3.7A) comprises a sedimentary marine sequence of laminated dark gray to black phyllites with 
local zones containing chlorite alteration, pyrite boudins, and calcite. It is commonly exposed in topographic highs in the Vazante region, and it is intensely deformed by folding and faulting with the dominant folding phase $\left(\mathrm{D}_{2}\right)$ striking north-south and having associated crenulations $\left(\mathrm{S}_{2}\right)$ overprinting the $S_{1}$ foliation. The Serra do Garrote has a sharp tectonic contact with the overlying Serra do Poço Verde Formation.

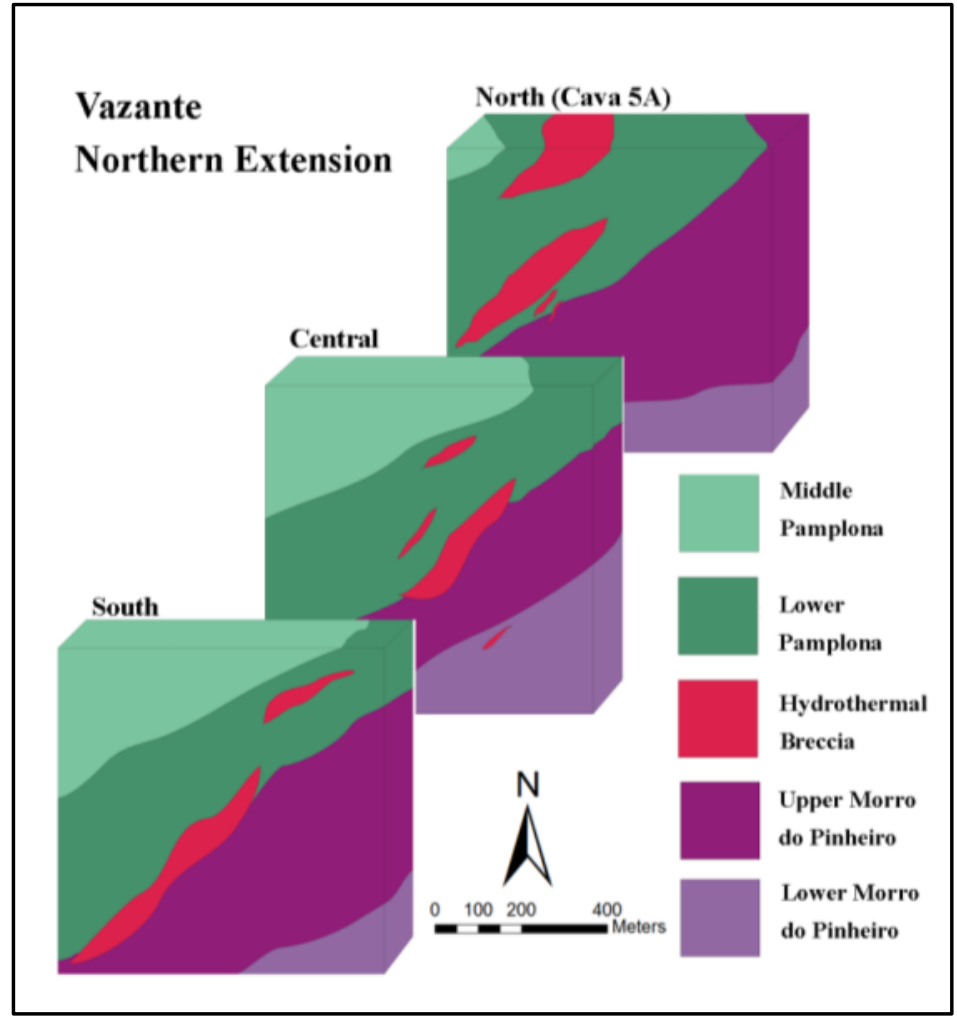

Fig. 3.6 Block diagram of the Northern Extension depicting the morphology of the tectonic-hydrothermal breccia in addition to the dip and thickness of local stratigraphic units.

The four members of the

Serra Poço Verde Formation were identified in the field during mapping and drill core logging: the Lower Morro do Pinheiro, Upper Morro do Pinheiro (Fig. 3.7B), Lower Pamplona (Fig. 3.7C), and Middle Pamplona (Fig. 3.7D). The Lower Morro do Pinheiro Member was not observed in the field and is projected to the surface from the interpretation of drill logs. In drill cores, the unit is gray to dark gray with algal layering and brecciated lenses (Fig. 3.7B). The contact between the Lower Morro do Pinheiro and underlying Serra do Garrote was not observed at depth. The Upper Morro do Pinheiro Member is composed of dark gray dolomite with bird's eyes and some algal mat layering. A few meters of fine-grained black phyllites were also observed. According to Warren (2006), these lithofacies indicate subtidal to intertidal facies. The Upper Morro do Pinheiro member typically has silica filled tension fractures and silica 
selvage alteration, and occasionally dolomitic overgrowths with some veins containing pyrite and bitumen. The Upper Morro do Pinheiro is usually denoted by the presence of bird's eye structures and has gradational contact with the Lower Pamplona Member.

The Lower Pamplona Member constitutes intercalated green to maroon phyllites and pink to light gray dolomites, primarily dolarenites with some dolrudites (Fig 3.7C). These rocks suggest an intertidal to supratidal facies (Warren, 2006). The Lower Pamplona dolostone is generally identifiable in the field, as it forms well indurate outcrops that weather to beige or an orange brown color. Iron carbonate veinlets and silica-filled vugs are common throughout the dolostones in the Lower Pamplona. The phyllites exhibit recognizable North-South trending parasitic folds and crenulations, similar to those found in the Serra do Garrote Formation. In thin section, small amounts of chalcocite and trace covellite mineralization were observed in the maroon laminations from samples 043-191 and M124-297. Iron carbonate and hematite veins and veinlets are common in all rock types of the Lower Pamplona.

The Middle Pamplona Member is comprised of a massive pink and gray dolomite with moderate silica alteration in the form of veins and chalcedony-filled vugs very similar to Lower Pamplona dolomites; however, the carbonate rocks are not interbedded with phyllites and contain some algal mat layering as well as stromatolites, indicative of a subtidal environment (Warren, 2006). Collapse breccias filled with carbonates and sulfides (Fig. 3.7D) are also present and are described in the subsequent section.

The Upper Pamplona member of the Morro do Calcário Formation (Fig. 3.7E) is composed of light gray dolarenite with stromatolitic bioherms. The contact between the Morro do Calcário Formation and the Middle Pamplona Member in characterized by a dark, reddishgray silty marker bed, which has been described by Votorantim staff to be observed in core throughout the Northern Extension region. 


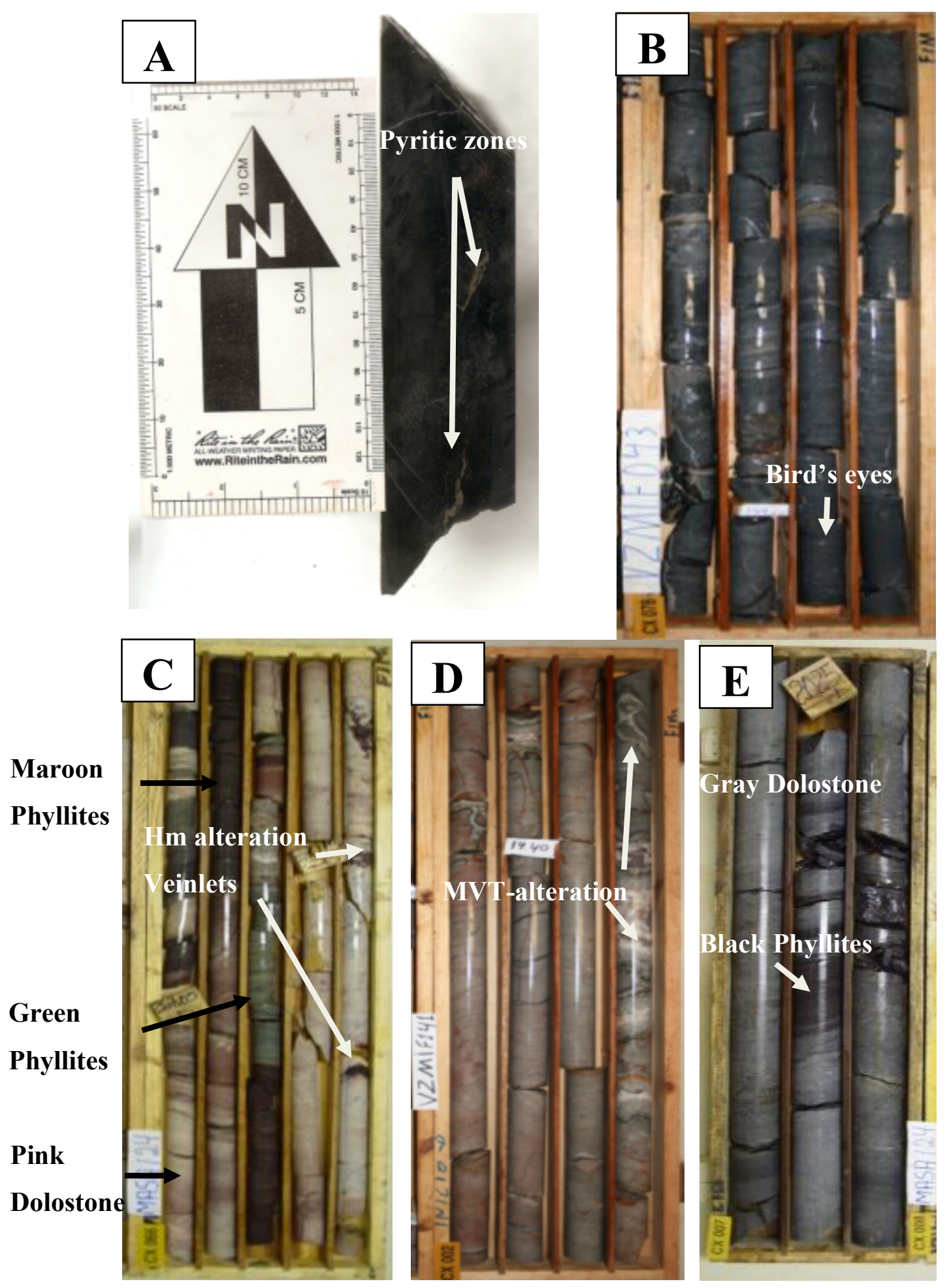

Fig. 3.7 Drill core samples of the geological units in the Northern Extension. A) Black phyllites with pyrite veinlets typical of the Serra do Garrote Fm. B) Upper Morro do Pinheiro Member with dark grey dolostone and bird's eyes C) Lower Pamplona Member with green and maroon phyllites interbedded with pink dolostone that contains hematite alteration veinlets D) Massive pink and gray dolostone of the Middle Pamplona Member with some collapse breccias filled with white ferroan dolomite and sphalerite. E) Massive gray dolostone (with minor black phyllites) of the Upper Pamplona Member of Morro do Calcário Formation (Photos B-E courtesy of Votorantim). Bird's Eyes=small, oval filling texture, MVT=Mississippi Valley-type, Hm=hematite. 


\subsubsection{Structures}

The carbonate and clastic units in the Northern Extension are deformed during $\mathrm{D}_{1}$ and exhibit an $\mathrm{S}_{1}$ cleavage that is subparallel to bedding $\left(\mathrm{S}_{0}\right)$. These $\mathrm{S}_{0} / \mathrm{S}_{1}$ surfaces are openly folded in the

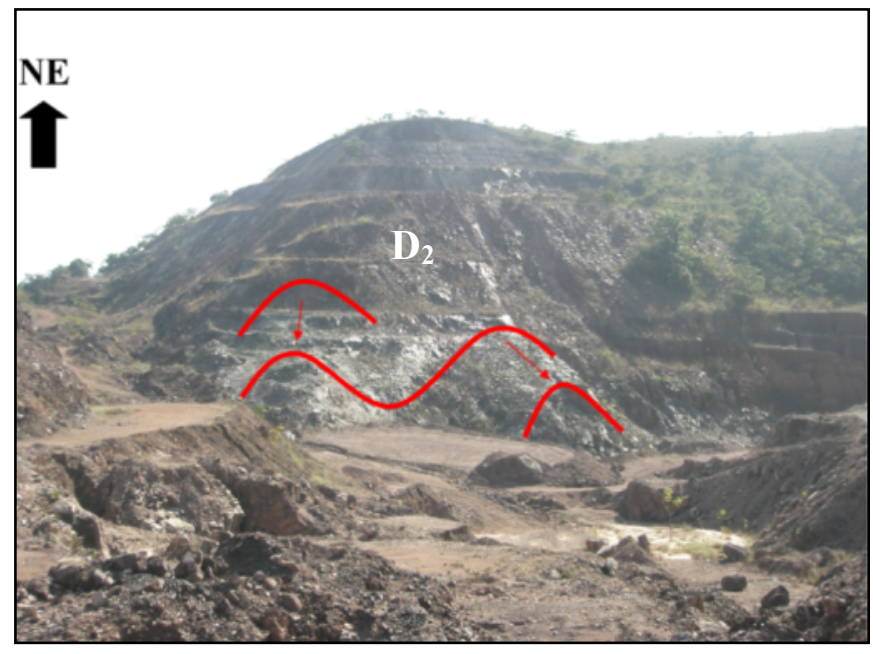

Fig. 3.8 Photograph looking to the Northeast showing the plunging antiform-synform complex in the northern wall of Cava 7A in the Serra do Garrote Fm. (Photo taken by G. Olivo) carbonate units and formed open to tight folds in the less competent phyllite units of the Serra do Garrote (Fig. 3.8, 9A) during $\mathrm{D}_{2}$ deformation. The fold axes have a trend and plunge of approximately $210 \rightarrow 30 \mathrm{SE}$.

This second deformational event $\left(D_{2}\right)$ generated a crenulation cleavage $\left(\mathrm{S}_{2}\right)$ that strikes SSW ranging from 200 to $225^{\circ}$ with dips of 30 to $70 \mathrm{NW} . \mathrm{S}_{2}$ is

predominantly observed in the phyllites of the Serra do Garrote exposed in Cava 7A (Fig. 3.9 A \& B) and was not observed in the carbonate units in the Northern Extension.

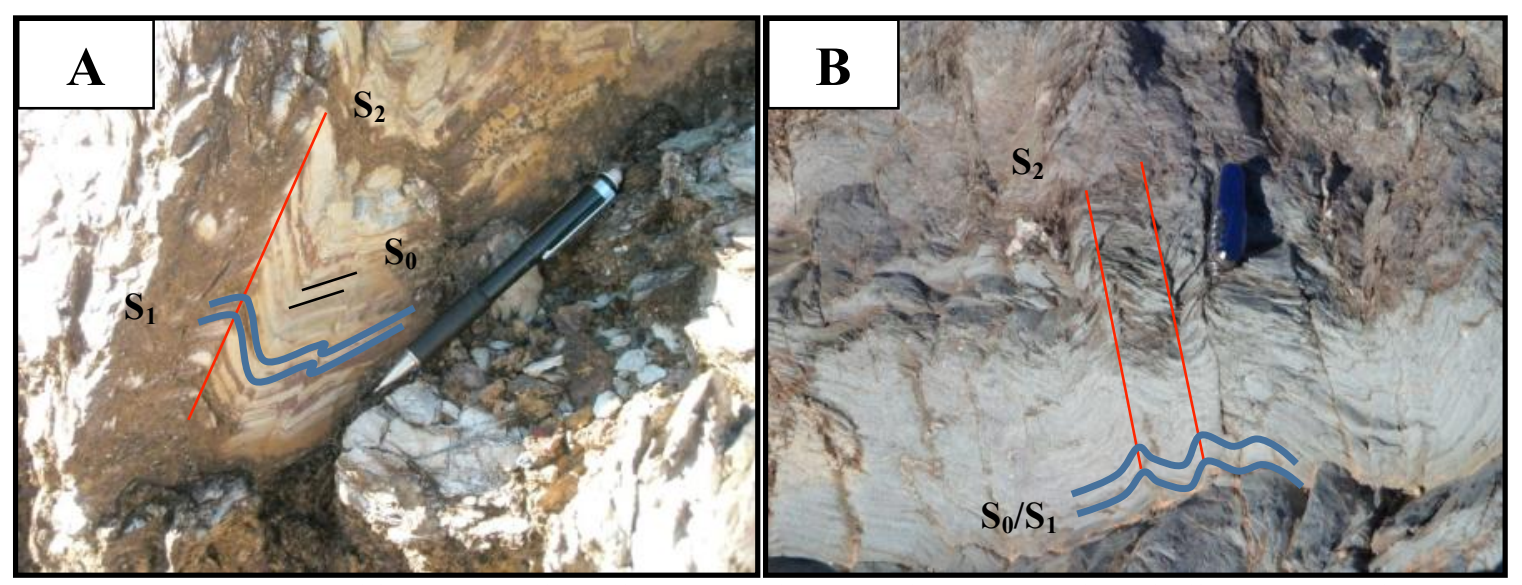

Fig. 3.9 $\mathrm{S}_{0}$ compositional layering with $\mathrm{S}_{1}$ cleavage and $\mathrm{S}_{2}$ crenulations found in phyllitic units in the Lower Pamplona Member (A) and Serra do Garrote Formation (B). (Photo A taken by P. Slezak. Photo B taken by G. Olivo) 
The Northern Extension Fault Zone is controlled by two conjectural oblique dip-slip faults having strikes and dips of $210,30^{\circ} \mathrm{NW}$ and $030,55^{\circ} \mathrm{SE}$. The overall trend of the ore-bearing breccia zone is bounded by these faults (Fig 4). They are observed at surface for about $600 \mathrm{~m}$ and are covered by lateritic soils in the southern limit of the North Zone property and truncated in the North at the tectonic contact of Serra do Pamplona Formation with Serra do Garrote Formation (Fig. 3.4). These faults appear to have been active for an extensive period of time and show evidence of multiple motions as indicated by several sets of slicken lines as well as brecciation of multiple willemite ore phases; the latter is described in the subsequent section. Regionally, these sets of NNE faults are interpreted to be formed by reactivation during the Brasiliano Orogeny of normal faults created by extension during the initial rifting of the São Francisco Craton (Valeriano et al., 2008; Martins-Neto, 2009). As they have a complex evolution, various models were proposed to interpret their geometry and movement. Valeriano et al. (2008) and MartinsNeto (2009) proposed that they were reactivated as oblique reverse faults during compression caused by the Brasiliano

Orogeny. However, Rostirolla et al. (2002) interpreted them as being transverse, sinistral faults and Rigobello et al. (1988) and Dardenne (2000) reported them as both sinistral and normal faults. Slickensides (Fig. 3.10) observed in this study indicate that the latest movement is normal.

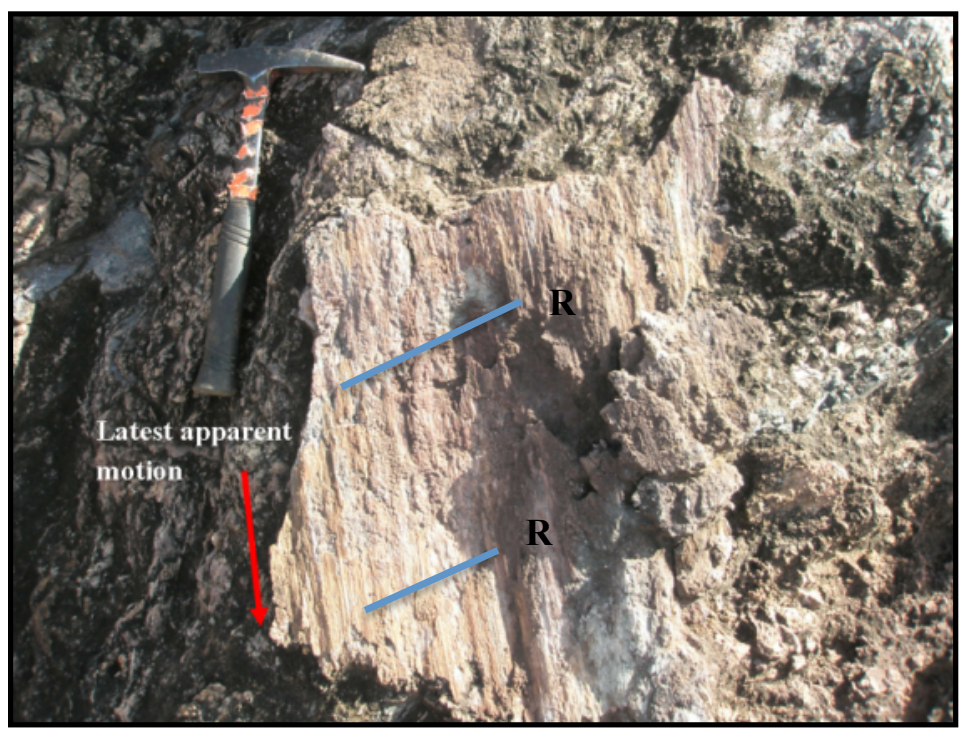

Fig. 3.10 Riedel shears with slickensides indicating the normal fault motion in the ore breccia. (Photo taken and edited by P. Slezak) 
The ore-bearing tectonic-hydrothermal breccias hosted in the fault zone do not cross-cut the folded $\left(D_{2}\right)$ contact with the Serra do Garrote Formation (Fig. 3.4), suggesting that they were emplaced prior to or during early $\mathrm{D}_{2}$ deformation. Moreover, the breccia trend is subparallel to axial planes that contain the $\beta$-axis $(212 \rightarrow 11)$ related to the $\mathrm{D}_{2}$ folding event (Fig. 3.11A \& $\mathrm{B}$ ), supporting then the interpretation that the breccia formed in the early stages of the $\mathrm{D}_{2}$ folding event.

Late sinistral and dextral transverse faults (with strikes ranging from 065 to 170 dipping 30-70 $\mathrm{SW} / \mathrm{SE}$; Fig. 3.11B) cut and displaced by several meters the major fault structures bounding the tectonic-hydrothermal breccia as well as the contact with Serra do Garrote and the large anticline found in the northern pit wall of Cava 7A (Fig 3.8). The secondary (supergene) calamine ore was mainly controlled by and can be found in these faults. Small NW-SE trending joints were also observed cutting the bounding faults of the tectonic-hydrothermal breccia as well as the transverse faults.
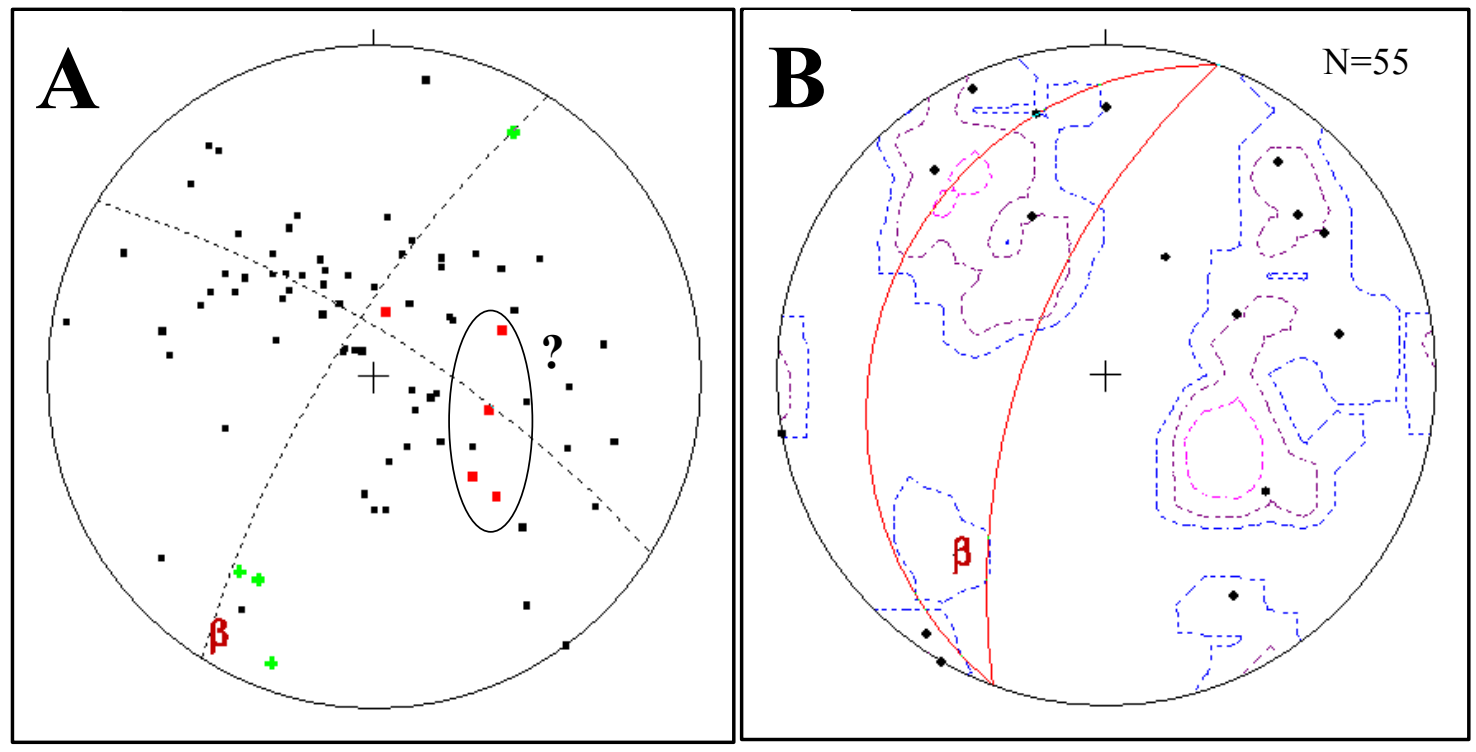

Fig. 3.11 A) Plots of the poles of $S_{0} / S_{1}$ compositional layering/foliation in black, $S_{2}$ crenulation cleavage in red, trend and plunges of major fold axes in green, and the average fold axis denoted by the $\beta$ and plane of $212,79 \mathrm{NW}$. The circled red squares represent $\mathrm{S}_{2}$ cleavages from a fault block, indicating that they may be out of place. B) Plots of the poles of the conjugate fault planes bounding the tectonic-hydrothermal breccia are outlined in blues, purples, and pinks (from lowest to highest concentration of points). In black are fault 
plane poles to the later E-W transverse faults. In red solid lines is the average strike of the tectonichydrothermal breccia with its ranges in dip.

\subsubsection{The Tectonic-hydrothermal breccias and ore zones}

The Northern Extension ore zone is comprised mainly of willemite (in variable amount reaching up to $40 \%$ ) and specular hematite (varying from $1 \%$ to $50 \%$ ) with minor amounts of franklinite. These ore zones form discontinuous lenses (tens of meters in length and width) within laterally and vertically discontinuous tectonic-hydrothermal breccias, which vary greatly in thickness from a few meters to nearly $100 \mathrm{~m}$ (Fig. 3.6). The tectonic-hydrothermal breccia lenses within the shear zone gently plunges to the south. This structural zone has been reactivated during the evolution of the deposit and may have served as the main conduit for the hydrothermal fluids, as it hosts a variety of hydrothermal breccias types. Four main types of breccias were identified based on their overall composition and relative position to the ore lenses. Type 1 Breccia is the least altered and the most distal from the ore breccias (referred to as Type 4 Breccia). Breccia Types 2 and 3 occur between breccias 1 and 4 . All of the breccias are composed mainly of gray,

pink, red, and white dolostone fragments and minor phyllite fragments from the Lower Pamplona Member. Many of the clasts are rimmed by recrystallized red and/or white dolomite. The cement between clasts is composed of various proportions of dolomite, ferroan dolomite, zincian dolomite, quartz, and hematite, depending on the breccias type.

Type 1 Breccia: Weakly to moderately brecciated light gray to white dolostone units with red ferroan dolomite alteration veinlets (Figs. 3.12 and 3.13A). These breccias are commonly located between the unbrecciated host rocks and the intensively ferroan dolomite altered breccias (Type 2) and is interpreted to be formed during the initial phases of brecciation. Dolomites from veins and veinlets have 1.29 wt. \% FeO and 0.13 wt. \% $\mathrm{ZnO}$ (Fig. 3.12; Table 3.1A); the latter being lower than carbonate in the breccias proximal to the ore. The red 
hydrothermal dolomites typically contain hematite inclusions; although high iron contents were observed in white hydrothermal dolomites as well.
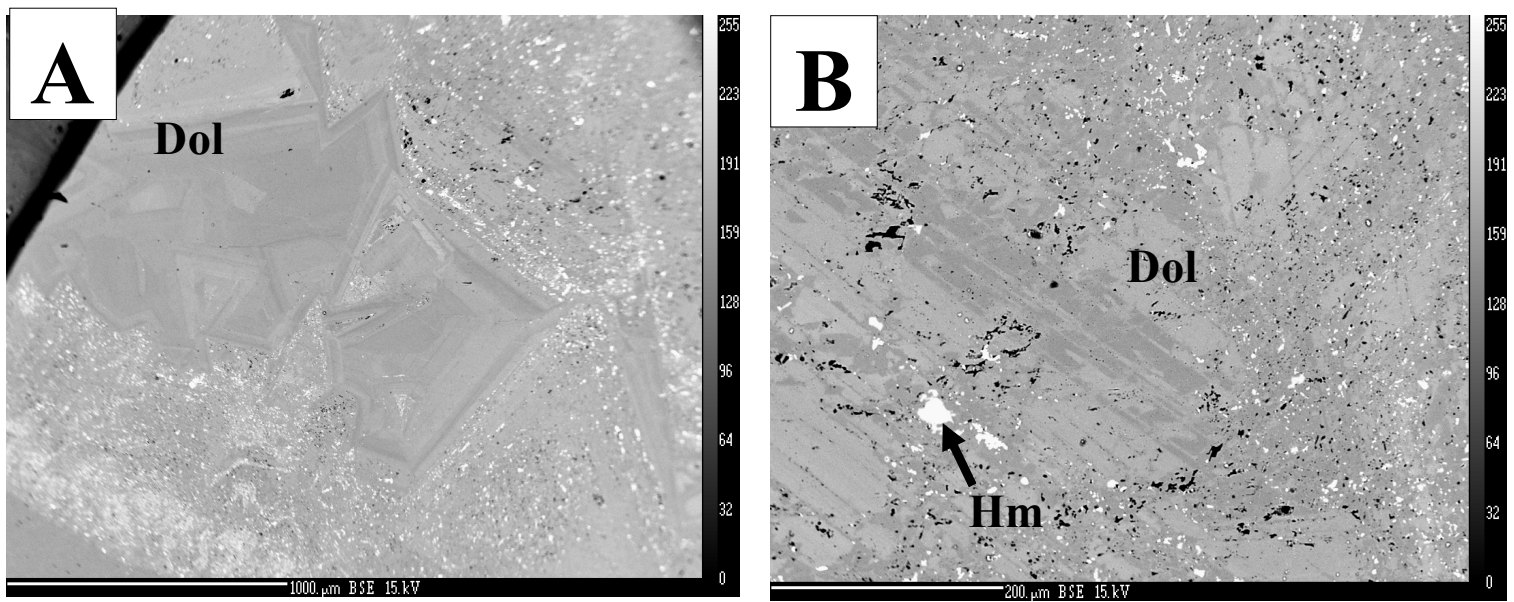

Fig. 3.5 A) Backscattered Electronnic Image (BSEI) from sample 141- 61. A) Ferroan dolomite growths in carbonate alteration veinlets from sample. The dark zones shows areas of higher iron content. (BSEI is 250 $\mu \mathrm{m}$ in diameter). B) Ferroan dolomite with hematite inclusions in dolomite alteration veinlets. The lighter zones are more iron-rich. (BSEI is $600 \mu \mathrm{m}$ in diameter). $\mathrm{Dol}=$ Dolomite. $\mathrm{Hm}=$ Hematite.

Type 2 Breccia: Strongly altered dolostone units with substantial amounts of reddish orange to white ferroan ( 1.5 to $3.3 \mathrm{wt}$. $\% \mathrm{FeO})$ and zincian dolomite $(0.3$ to $1.25 \% \mathrm{ZnO})$ veinlets ( $3 \mathrm{~mm}$ to $2 \mathrm{~cm}$ wide) and red dolomite alteration selvages (Fig. 3.13B; Table 3.1A). Sparry, zoned euhedral dolomite occurs in the center of the veins and hematite is found mainly on the vein margins or as small inclusions within the red dolomite. These breccias are generally proximal to the mineralized Type 4 (willemite) and Type 3 (hematite) breccias and overprint features observed in the Type 1 Breccia.

Type 3 Breccia (hematite-rich breccias): Deep red breccia composed predominantly of hematite with trace willemite (Fig. 3.13C). These breccias are commonly found in the margins of the main ore (Type 4) breccia or as isolated breccia zones (1-3 m wide) in the tectonichydrothermal breccias. Hematite occurs replacing carbonates from the cement and clasts and in 
veinlets cross-cutting the first two breccia types, which indicates that these hematite-rich breccias formed after the major Fe-dolomite hydrothermal episode.

Type 4 Breccia (willemite ore breccia): The ore breccias are commonly found in the core of the tectonic-hydrothermal breccia structure, where the early hydrothermal breccias have been replaced by willemite ( $>10 \%$ to up to $50 \%)$ as well as some hematite $(5-20 \%)$ and minor franklinite (Fig. 3.13D). The presence of hematite is not necessarily ubiquitous throughout the willemite ore zones. Willemite and hematite minerals are associated with ferroan $(\sim 1.99 \mathrm{wt} \%$ $\mathrm{FeO})$ and zincian $(\sim 2.65 \mathrm{wt} \% \mathrm{ZnO})$ dolomite (Table 3.1A) and minor quartz alteration and are locally cut by small sulfide veinlets.

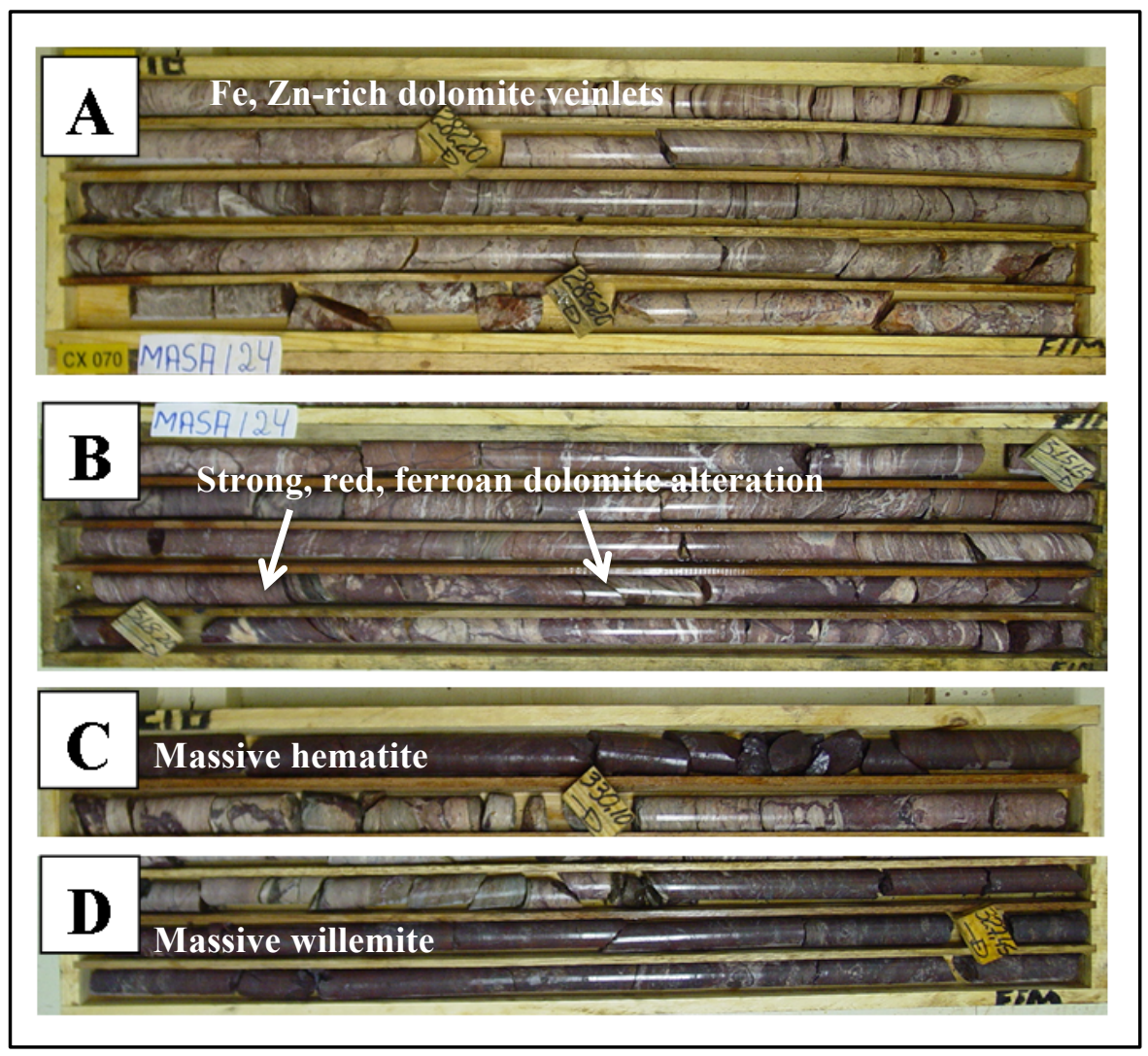

Fig. 3.6 Photographs of drill cores illustrating the various types of breccias. A) Type 1 - Light-colored, weakly brecciated dolostone with ferroan and zincian-dolomite veinlets. B) Type 2 - Strong hydrothermal ferroan and zincian-dolomite alteration in dolostone. C) Type 3 - Breccia replaced by hematite $(>40 \% \mathrm{Fe})$. B) Type 4-Main ore breccia replaced by willemite and hematite. (Photos taken from drill hole MASA 124, courtesy of Votorantim Metals) 
Willemite (0.1 to $0.75 \mathrm{~mm}$ ) occurs as hexagonal crystal masses (Fig. 3.14A) filling in the breccia, lath-shaped crystals (Fig. 3.14B), and small, discontinuous veins that cut across many clasts in the breccia zone (Fig. 3.14C). It commonly replaces carbonates as well as quartz clasts and crystals. The lath-shaped willemite overgrows the earliest, hexagonal willemite. The small (100-200 $\mu \mathrm{m}$ wide), discontinuous willemite veins cut through both the fibrous laths and the hexagonal willemite, indicating that they formed during the last stages of mineralization. The willemite composition varies little throughout all three generations described above. Its average $\mathrm{FeO}$ content is 0.4 wt. \%, however, various grains from generations 1 and 2 yielded about 1 wt. \% $\mathrm{FeO}$ due to $\mathrm{Fe}$ contamination from hematite inclusions. Some grains contain trace amounts of chromium and rare manganese as seen in Table 3.1B.

Hematite $(0.01$ to $1 \mathrm{~mm})$ commonly occurs as either aggregates of fine-grained blades filling in between clasts (Fig. 3.14F), or disseminated, larger crystals replacing willemite, carbonates, and quartz. Euhedral laths are also found included in willemite and in the margins of late sulfide veins.

Franklinite is most commonly found as small octahedral inclusions associated with hematite (Fig. 3.14E) in willemite-rich domains; however, it is also be found included in willemite and carbonate, as very small (1 to $10 \mu \mathrm{m})$ disseminated crystals in the breccia matrix and associated with hematite in the margins of the sulfide veinlets.

Small, $10-50 \mathrm{~mm}$, galena-rich veinlets cross-cut the main willemite ore (Type 4 Breccia) and contain small amounts of sphalerite, stromeyerite $\left(\mathrm{Cu}_{1-\mathrm{x}} \mathrm{Ag}_{1-\mathrm{x}} \mathrm{S}\right)$, chalcocite $\left(\mathrm{Cu}_{2} \mathrm{~S}\right)$, and covellite $(\mathrm{CuS})($ Fig. 3.15A-C). Galena is massive within the veinlets and is altered to anglesite $\left(\mathrm{PbSO}_{4}\right)$ on the veinlet margins (Fig. 3.15A \& B). Sphalerite in the Type 4 Breccia (Table 3.1C) is Cd - rich $(2.88-3.10 \mathrm{wt} \%)$ and iron - poor $(0.01-0.02 \mathrm{wt} \%)$, similar to the sphalerite reported by Monteiro et al. (2006) in the Vazante Mine, and is found as irregular, semi- 
massive crystals within the galena. The stromeyerite forms irregular, disseminated crystals, generally alters to bladed covellite, and rarely to chalcocite (Fig. 3.15C).

Silver-bearing sulfide veinlets (10-100 mm wide) were found to cross-cut the willemite ore in one drill hole. These silver-bearing sulfide veinlets are similar in morphology to galena veinlets described above. The veinlets are primarily composed of chalcocite, native silver, stromeyerite, franklinite, hematite, and minor galena. Barite $\left(\mathrm{BaSO}_{4}\right)$ and phosphohedyphane $\left(\mathrm{Ca}_{2} \mathrm{~Pb}_{3}\left(\mathrm{PO}_{4}\right)_{3} \mathrm{Cl}\right)$ occur in small amounts as disseminated anhedral to subhedral crystals ranging in size from $20-50 \mu \mathrm{m}$. In back-scattered electronic images (BSEI), chalcocite shows a complex texture of light and dark gray zoning (Fig. 3.15D), with the lighter gray sections containing silver $(1.1-1.5 \% \mathrm{Ag})$ and the darker gray sections containing trace amounts or no silver.

One drill hole, VZMIF 088 (Fig. 3.5), intersected a 5 to $7 \mathrm{~m}$ - wide brecciated zone which contains sphalerite, pyrite, dolomite, quartz and bitumen, and are cut by galena veinlets with trace covellite (Fig. 3.15 E \& F), similar to the sulfide veinlets that cut the Type 4 Breccia. This zone exhibits various stages of open filling and brecciation. Dolomite and coarse-grained pyrite grains are deformed and the fractures are filled with late quartz, carbonate and sphalerite. Unlike the cadmium-rich sphalerite found in the sulfide veinlets cross-cutting the willemite ore Breccia Type 4, the sphalerite in this zone contains minor cadmium $(0.2 \mathrm{wt} \% \mathrm{Cd})$ and more iron $(0.2 \mathrm{wt} \% \mathrm{Fe})$.

Furthermore, sphalerite veinlets cut collapse breccias in the Middle and Upper Pamplona members (VZMIF 141 at $16 \mathrm{~m}$ and MASA 124 at $97 \mathrm{~m})$. Sphalerite contains little cadmium $(0.2$ wt. \% Cd; Table 3.1) and higher iron ( 1 wt. \% Fe; Table 3.1C)), has inclusions of pyrite and is associated with sparry, euhedral ferroan dolomite (1 - $2 \mathrm{wt} \% \mathrm{FeO}$; Table 3.1C). It exhibits textural features similar to Mississippi Valley-type deposits; however their paragenetic relationships could not be established with respect to the other Northern Extension mineralizing events. 
Table 3.1 EMP analyses conducted on core samples of dolomite, willemite, and sphalerite in the Northern Extension.

A) Dolomite analysis, recalculated to \% oxide

\begin{tabular}{|c|c|c|c|c|c|c|c|c|c|c|c|}
\hline Comment & Description & $\mathrm{CO}_{2}$ & MgO & $\mathrm{CaO}$ & $\mathrm{SiO}_{2}$ & MnO & FeO & $\mathrm{ZnO}$ & SrO & $\mathbf{B a O}$ & Total \\
\hline $124-75-018$ & type 4 breccia & 46.46 & 20.10 & 30.51 & 0.02 & 0.11 & 0.51 & 1.91 & 0.00 & 0.39 & 100.00 \\
\hline $124-75-019$ & type 4 breccia & 45.55 & 16.94 & 33.02 & 0.02 & 0.06 & 1.83 & 2.55 & 0.00 & 0.00 & 99.96 \\
\hline $124-75-020$ & type 4 breccia & 46.75 & 19.49 & 29.54 & 0.08 & 0.10 & 1.07 & 2.62 & 0.05 & 0.31 & 100.00 \\
\hline $60-136 \mathrm{~B}-033$ & type 4 breccia & 46.54 & 19.64 & 29.62 & 0.00 & 0.27 & 1.68 & 2.15 & 0.00 & 0.00 & 99.89 \\
\hline 60-136B-034 & type 4 breccia & 47.09 & 18.87 & 29.16 & 0.00 & 0.10 & 2.01 & 2.71 & 0.02 & 0.00 & 99.95 \\
\hline $60-136 \mathrm{~B}-035$ & type 4 breccia & 47.18 & 19.76 & 29.25 & 0.00 & 0.08 & 1.44 & 1.91 & 0.07 & 0.31 & 100.00 \\
\hline 60-136B-036 & type 4 breccia & 47.15 & 19.42 & 29.19 & 0.00 & 0.16 & 2.10 & 1.82 & 0.02 & 0.00 & 99.87 \\
\hline $127-108.5-028$ & type 4 breccia & 46.96 & 18.11 & 28.41 & 0.04 & 0.18 & 2.21 & 4.02 & 0.02 & 0.00 & 99.95 \\
\hline $127-108.5-029$ & type 4 breccia & 45.49 & 18.57 & 27.89 & 0.20 & 0.18 & 5.24 & 2.37 & 0.05 & 0.00 & 99.98 \\
\hline $127-108.5-030$ & type 4 breccia & 46.78 & 18.37 & 28.34 & 0.01 & 0.09 & 1.86 & 4.44 & 0.05 & 0.00 & 99.93 \\
\hline $127-108.5-031$ & type 4 breccia & 48.14 & 19.90 & 27.61 & 0.50 & 0.13 & 1.57 & 1.97 & 0.09 & 0.10 & 100.00 \\
\hline $127-108.5-032$ & type 4 breccia & 46.01 & 18.88 & 28.50 & 0.38 & 0.10 & 2.39 & 3.36 & 0.10 & 0.28 & 100.00 \\
\hline 141-16-09 & alteration vein & 46.92 & 20.62 & 30.24 & 0.00 & 0.08 & 1.91 & 0.21 & 0.00 & 0.00 & 99.98 \\
\hline $141-16-010$ & alteration vein in MP & 46.37 & 21.14 & 30.55 & 0.53 & 0.08 & 0.93 & 0.11 & 0.01 & 0.29 & 100.00 \\
\hline $141-16-011$ & alteration vein in MP & 46.50 & 20.29 & 30.59 & 0.01 & 0.27 & 2.18 & 0.01 & 0.05 & 0.00 & 99.91 \\
\hline $141-16-012$ & alteration vein in MP & 45.74 & 20.78 & 30.08 & 2.27 & 0.08 & 0.80 & 0.15 & 0.00 & 0.07 & 99.97 \\
\hline $141-16-013$ & alteration vein in MP & 47.22 & 21.38 & 30.20 & 0.00 & 0.08 & 1.04 & 0.00 & 0.00 & 0.00 & 99.92 \\
\hline $141-16-016$ & alteration vein in MP & 46.62 & 21.04 & 31.01 & 0.04 & 0.07 & 1.01 & 0.02 & 0.00 & 0.00 & 99.80 \\
\hline $141-16-017$ & alteration vein in MP & 46.71 & 20.67 & 30.34 & 0.09 & 0.06 & 1.24 & 0.76 & 0.01 & 0.14 & 100.00 \\
\hline $141-16-018$ & alteration vein in MP & 47.15 & 20.05 & 30.12 & 0.03 & 0.07 & 1.89 & 0.63 & 0.06 & 0.00 & 100.00 \\
\hline
\end{tabular}




\begin{tabular}{|c|c|c|c|c|c|c|c|c|c|c|c|}
\hline Comment & Description & $\mathrm{CO}_{2}$ & MgO & $\mathrm{CaO}$ & $\mathrm{SiO}_{2}$ & MnO & $\mathbf{F e O}$ & $\mathrm{ZnO}$ & SrO & $\mathbf{B a O}$ & Total \\
\hline $141-16-020$ & alteration vein in MP & 44.73 & 20.28 & 29.71 & 4.04 & 0.06 & 0.97 & 0.07 & 0.00 & 0.00 & 99.86 \\
\hline $141-16-021$ & alteration vein in MP & 46.44 & 19.88 & 30.10 & 0.02 & 0.06 & 2.15 & 1.25 & 0.05 & 0.00 & 99.95 \\
\hline $141-16-022$ & alteration vein in MP & 46.09 & 20.46 & 30.42 & 0.00 & 0.08 & 2.22 & 0.70 & 0.00 & 0.00 & 99.96 \\
\hline $141-16-023$ & alteration vein in MP & 46.53 & 20.34 & 30.34 & 0.00 & 0.08 & 1.76 & 0.70 & 0.00 & 0.24 & 99.98 \\
\hline 141-16-024 & alteration vein in MP & 46.42 & 21.31 & 30.08 & 1.28 & 0.05 & 0.50 & 0.10 & 0.00 & 0.21 & 99.96 \\
\hline $141-16-025$ & alteration vein in MP & 46.67 & 21.29 & 31.16 & 0.13 & 0.08 & 0.47 & 0.09 & 0.00 & 0.02 & 99.92 \\
\hline $141-16-026$ & alteration vein in MP & 46.33 & 21.45 & 31.35 & 0.00 & 0.06 & 0.54 & 0.13 & 0.00 & 0.05 & 99.92 \\
\hline $141-16-027$ & alteration vein in MP & 46.76 & 20.76 & 30.35 & 0.03 & 0.18 & 1.50 & 0.04 & 0.00 & 0.26 & 99.87 \\
\hline $141-16-028$ & alteration vein in MP & 46.69 & 21.62 & 31.55 & 0.01 & 0.08 & 0.00 & 0.00 & 0.00 & 0.00 & 99.94 \\
\hline 141-16-029 & alteration vein in MP & 46.43 & 20.39 & 30.28 & 0.00 & 0.19 & 2.32 & 0.00 & 0.02 & 0.36 & 99.99 \\
\hline $141-16-030$ & alteration vein in MP & 47.17 & 20.47 & 30.42 & 0.01 & 0.16 & 1.58 & 0.12 & 0.00 & 0.00 & 99.93 \\
\hline $141-16-031$ & alteration vein in MP & 47.00 & 20.67 & 31.54 & 0.00 & 0.19 & 0.43 & 0.06 & 0.03 & 0.10 & 100.00 \\
\hline $141-16-032$ & alteration vein in MP & 46.86 & 20.82 & 30.57 & 0.00 & 0.10 & 1.25 & 0.05 & 0.04 & 0.00 & 99.69 \\
\hline $141-61-01$ & veinlet in MP & 46.90 & 21.37 & 30.31 & 0.00 & 0.11 & 1.03 & 0.04 & 0.02 & 0.00 & 99.79 \\
\hline $141-61-02$ & veinlet in MP & 46.54 & 21.52 & 31.04 & 0.00 & 0.03 & 0.56 & 0.00 & 0.02 & 0.00 & 99.71 \\
\hline $141-61-03$ & veinlet in MP & 47.57 & 20.87 & 30.20 & 0.00 & 0.10 & 1.04 & 0.11 & 0.01 & 0.00 & 99.91 \\
\hline $141-61-04$ & veinlet in MP & 47.16 & 21.19 & 30.77 & 0.00 & 0.08 & 0.72 & 0.00 & 0.00 & 0.02 & 99.94 \\
\hline $141-61-05$ & veinlet in MP & 47.28 & 21.45 & 30.54 & 0.01 & 0.05 & 0.54 & 0.00 & 0.02 & 0.00 & 99.89 \\
\hline $141-61-06$ & veinlet in MP & 46.54 & 21.56 & 30.80 & 0.01 & 0.04 & 0.50 & 0.00 & 0.06 & 0.00 & 99.50 \\
\hline $141-61-07$ & veinlet in MP & 46.58 & 21.67 & 31.01 & 0.00 & 0.05 & 0.48 & 0.03 & 0.00 & 0.12 & 99.93 \\
\hline $141-61-08$ & veinlet in MP & 46.50 & 20.99 & 30.88 & 0.00 & 0.14 & 1.23 & 0.05 & 0.02 & 0.19 & 99.99 \\
\hline 141-61-09 & veinlet in MP & 46.91 & 21.55 & 30.95 & 0.00 & 0.16 & 0.18 & 0.06 & 0.00 & 0.10 & 99.91 \\
\hline $141-61-014$ & veinlet in MP & 46.28 & 20.94 & 30.82 & 0.00 & 0.17 & 1.61 & 0.04 & 0.05 & 0.00 & 99.90 \\
\hline 141-61-015 & veinlet in MP & 46.54 & 20.94 & 30.54 & 0.00 & 0.18 & 1.72 & 0.03 & 0.04 & 0.00 & 100.00 \\
\hline
\end{tabular}




\begin{tabular}{|c|c|c|c|c|c|c|c|c|c|c|c|}
\hline Comment & Description & $\mathrm{CO}_{2}$ & MgO & $\mathrm{CaO}$ & $\mathrm{SiO}_{2}$ & MnO & $\mathbf{F e O}$ & $\mathrm{ZnO}$ & SrO & $\mathbf{B a O}$ & Total \\
\hline 141-61-016 & veinlet in MP & 46.29 & 21.61 & 31.28 & 0.00 & 0.18 & 0.44 & 0.02 & 0.00 & 0.00 & 99.80 \\
\hline $141-61-017$ & veinlet in MP & 41.50 & 23.70 & 34.13 & 0.01 & 0.17 & 0.18 & 0.08 & 0.06 & 0.17 & 100.00 \\
\hline $141-61-018$ & veinlet in MP & 46.68 & 21.37 & 31.09 & 0.00 & 0.20 & 0.60 & 0.01 & 0.04 & 0.00 & 99.99 \\
\hline 141-61-019 & veinlet in MP & 46.28 & 20.82 & 31.06 & 0.04 & 0.21 & 1.50 & 0.04 & 0.00 & 0.00 & 99.95 \\
\hline $141-61-020$ & veinlet in MP & 46.04 & 20.50 & 30.74 & 0.00 & 0.28 & 1.93 & 0.02 & 0.00 & 0.40 & 99.92 \\
\hline 20-295-01 & type 2 breccia & 46.97 & 19.30 & 30.15 & 0.00 & 0.12 & 3.31 & 0.10 & 0.01 & 0.02 & 99.99 \\
\hline $20-295-02$ & type 2 breccia & 46.79 & 19.17 & 30.24 & 0.00 & 0.16 & 3.25 & 0.15 & 0.11 & 0.12 & 99.98 \\
\hline $20.295 .-03$ & type 2 breccia & 46.52 & 19.32 & 30.59 & 0.00 & 0.14 & 3.21 & 0.06 & 0.04 & 0.12 & 99.99 \\
\hline $20-295-04$ & type 2 breccia & 47.25 & 20.91 & 30.64 & 0.00 & 0.18 & 0.79 & 0.14 & 0.00 & 0.00 & 99.89 \\
\hline $20-295-05$ & type 2 breccia & 43.53 & 22.94 & 33.23 & 0.01 & 0.02 & 0.13 & 0.05 & 0.00 & 0.05 & 99.95 \\
\hline $20-295-06$ & type 2 breccia & 46.94 & 21.62 & 30.72 & 0.00 & 0.05 & 0.18 & 0.24 & 0.05 & 0.19 & 99.99 \\
\hline $20-295-07$ & type 2 breccia & 47.80 & 20.91 & 29.99 & 0.00 & 0.16 & 1.02 & 0.05 & 0.00 & 0.05 & 99.97 \\
\hline $20-295-08$ & type 2 breccia & 47.04 & 21.32 & 30.29 & 0.01 & 0.09 & 0.91 & 0.16 & 0.04 & 0.14 & 100.00 \\
\hline 20-295-09 & type 2 breccia & 47.43 & 20.97 & 30.27 & 0.00 & 0.09 & 1.17 & 0.00 & 0.04 & 0.02 & 99.99 \\
\hline $20-295-010$ & type 2 breccia & 47.21 & 20.98 & 30.66 & 0.00 & 0.12 & 0.45 & 0.52 & 0.00 & 0.02 & 99.96 \\
\hline 20-295-013 & type 2 breccia & 47.56 & 20.58 & 30.54 & 0.00 & 0.05 & 0.88 & 0.34 & 0.04 & 0.00 & 99.99 \\
\hline 20-295-014 & type 2 breccia & 46.98 & 20.81 & 30.67 & 0.01 & 0.06 & 0.96 & 0.44 & 0.00 & 0.05 & 99.97 \\
\hline 20-295-015 & type 2 breccia & 46.53 & 20.70 & 31.38 & 0.00 & 0.06 & 0.75 & 0.44 & 0.02 & 0.00 & 99.88 \\
\hline 20-295-016 & type 2 breccia & 46.86 & 21.34 & 31.33 & 0.02 & 0.07 & 0.27 & 0.01 & 0.02 & 0.07 & 100.00 \\
\hline 20-295-017 & type 2 breccia & 47.05 & 21.42 & 31.14 & 0.01 & 0.05 & 0.11 & 0.08 & 0.00 & 0.12 & 99.96 \\
\hline $141-18-01$ & veinlet in MP & 46.31 & 20.71 & 30.68 & 0.00 & 0.15 & 1.97 & 0.00 & 0.04 & 0.00 & 99.85 \\
\hline $141-18-02$ & veinlet in MP & 46.71 & 21.29 & 30.89 & 0.00 & 0.14 & 0.74 & 0.02 & 0.00 & 0.00 & 99.78 \\
\hline $141-18-03$ & veinlet in MP & 45.93 & 20.04 & 30.77 & 0.00 & 0.06 & 2.91 & 0.00 & 0.00 & 0.00 & 99.71 \\
\hline
\end{tabular}




\begin{tabular}{llrrrrrrrrrr}
\hline Comment & Description & $\mathbf{C O}_{2}$ & $\mathbf{M g O}$ & $\mathbf{C a O}$ & $\mathbf{S i O}_{2}$ & $\mathbf{M n O}$ & $\mathbf{F e O}$ & $\mathbf{Z n O}$ & $\mathbf{S r O}$ & $\mathbf{B a O}$ & Total \\
\hline $141-18-04$ & veinlet in MP & 46.42 & 20.51 & 30.71 & 0.01 & 0.04 & 2.09 & 0.12 & 0.03 & 0.07 & 100.00 \\
$141-18-05$ & veinlet in MP & 46.48 & 20.61 & 30.60 & 0.00 & 0.04 & 2.00 & 0.07 & 0.01 & 0.19 & 99.99 \\
$141-18-06$ & veinlet in MP & 45.92 & 19.76 & 30.49 & 0.01 & 0.06 & 3.58 & 0.04 & 0.00 & 0.14 & 100.00 \\
$141-18-07$ & veinlet in MP & 45.53 & 20.16 & 30.93 & 0.01 & 0.01 & 3.10 & 0.01 & 0.02 & 0.24 & 100.00 \\
$141-18-08$ & veinlet in MP & 46.40 & 21.39 & 31.01 & 0.03 & 0.16 & 0.87 & 0.06 & 0.00 & 0.07 & 99.99 \\
\hline
\end{tabular}


Table 3.1 B) Willemite - all willemite samples were taken from the Type 4 Breccia;

Gen.$=$ Generation

\begin{tabular}{|c|c|c|c|c|c|c|c|}
\hline Comment & Description & $\mathrm{SiO}_{2}$ & $\mathrm{ZnO}$ & $\mathrm{Cr}_{2} \mathrm{O}_{3}$ & MnO & FeO & Total \\
\hline 101-138-03 & Gen. 1 & 27.51 & 71.04 & 0.05 & 0.00 & 0.18 & 98.78 \\
\hline 101-138-04 & Gen. 1 & 27.17 & 72.41 & 0.03 & 0.01 & 0.11 & 99.72 \\
\hline $101-138-05$ & Gen. 1 & 27.40 & 72.89 & 0.02 & 0.00 & 0.16 & 100.47 \\
\hline $101-138-06$ & Gen. 1 & 27.63 & 72.39 & 0.00 & 0.00 & 0.18 & 100.19 \\
\hline $101-138-07$ & Gen. 1 & 27.27 & 72.41 & 0.01 & 0.00 & 0.14 & 99.83 \\
\hline $101-138-08$ & Gen. 1 & 27.35 & 72.67 & 0.00 & 0.00 & 0.56 & 100.59 \\
\hline 101-138-09 & Gen. 1 & 27.44 & 72.31 & 0.00 & 0.04 & 0.45 & 100.24 \\
\hline $101-138-10$ & Gen. 1 & 26.93 & 72.26 & 0.03 & 0.00 & 0.68 & 99.90 \\
\hline 101-138-11 & Gen. 1 & 27.18 & 72.75 & 0.03 & 0.00 & 0.49 & 100.44 \\
\hline $124-75-01$ & Gen. 1 & 27.34 & 73.05 & 0.00 & 0.00 & 0.20 & 100.60 \\
\hline $124-75-02$ & Gen. 1 & 27.26 & 72.93 & 0.01 & 0.00 & 0.02 & 100.22 \\
\hline $124-75-03$ & Gen. 1 & 27.43 & 72.86 & 0.00 & 0.00 & 0.26 & 100.54 \\
\hline $124-75-05$ & Gen. 1 & 27.28 & 72.21 & 0.00 & 0.00 & 0.11 & 99.60 \\
\hline $124-75-06$ & Gen. 1 & 27.45 & 72.79 & 0.00 & 0.01 & 0.13 & 100.37 \\
\hline $124-75-07$ & Gen. 1 & 27.45 & 73.00 & 0.00 & 0.00 & 0.08 & 100.53 \\
\hline $124-75-08$ & Gen. 1 & 27.28 & 73.67 & 0.00 & 0.00 & 0.16 & 101.11 \\
\hline $124-75-09$ & Gen. 1 & 27.39 & 72.76 & 0.00 & 0.00 & 0.07 & 100.23 \\
\hline $124-75-010$ & Gen. 1 & 27.51 & 72.77 & 0.04 & 0.00 & 0.13 & 100.45 \\
\hline $124-75-011$ & Gen. 1 & 27.50 & 72.54 & 0.01 & 0.01 & 0.25 & 100.30 \\
\hline $124-75-012$ & Gen. 1 & 27.01 & 71.54 & 0.00 & 0.02 & 0.13 & 98.69 \\
\hline $124-75-013$ & Gen. 1 & 27.25 & 71.83 & 0.02 & 0.00 & 0.15 & 99.24 \\
\hline $124-75-014$ & Gen. 1 & 27.48 & 72.48 & 0.00 & 0.00 & 0.11 & 100.07 \\
\hline $124-75-015$ & Gen. 1 & 27.12 & 71.81 & 0.00 & 0.00 & 0.09 & 99.01 \\
\hline $124-75-016$ & Gen. 1 & 27.50 & 72.61 & 0.00 & 0.00 & 0.10 & 100.22 \\
\hline $124-75-017$ & Gen. 1 & 27.62 & 72.71 & 0.00 & 0.00 & 0.07 & 100.39 \\
\hline $127-108.5-022$ & Gen. 1 & 27.17 & 70.48 & 0.00 & 0.00 & 1.55 & 99.20 \\
\hline $127-108.5-023$ & Gen. 1 & 27.16 & 71.73 & 0.00 & 0.00 & 1.63 & 100.52 \\
\hline $127-108.5-024$ & Gen. 1 & 27.22 & 71.43 & 0.02 & 0.00 & 2.17 & 100.83 \\
\hline $127-108.5-025$ & Gen. 1 & 27.25 & 71.30 & 0.00 & 0.00 & 1.15 & 99.70 \\
\hline $127-108.5-026$ & Gen. 1 & 27.39 & 71.03 & 0.00 & 0.00 & 1.54 & 99.96 \\
\hline $127-108.5-027$ & Gen. 1 & 27.29 & 72.00 & 0.03 & 0.00 & 1.20 & 100.52 \\
\hline $101-138-01$ & Gen. 2 & 27.10 & 71.17 & 0.00 & 0.00 & 0.23 & 98.51 \\
\hline $101-138-02$ & Gen. 2 & 27.27 & 71.67 & 0.00 & 0.00 & 0.16 & 99.09 \\
\hline 60-136B-01 & Gen. 2 & 27.37 & 72.48 & 0.00 & 0.00 & 0.35 & 100.20 \\
\hline 60-136B-02 & Gen. 2 & 27.45 & 72.37 & 0.00 & 0.00 & 0.30 & 100.13 \\
\hline 60-136B-03 & Gen. 2 & 27.26 & 72.18 & 0.00 & 0.00 & 0.39 & 99.83 \\
\hline
\end{tabular}




\begin{tabular}{|c|c|c|c|c|c|c|c|}
\hline Comment & Description & $\mathrm{SiO}_{2}$ & $\mathrm{ZnO}$ & $\mathrm{Cr}_{2} \mathrm{O}_{3}$ & MnO & FeO & Total \\
\hline 60-136B-04 & Gen. 2 & 27.46 & 72.49 & 0.00 & 0.00 & 0.40 & 100.36 \\
\hline 60-136B-05 & Gen. 2 & 27.30 & 72.01 & 0.00 & 0.00 & 0.37 & 99.68 \\
\hline 60-136B-06 & Gen. 2 & 27.31 & 72.53 & 0.02 & 0.00 & 0.26 & 100.12 \\
\hline 60-136B-07 & Gen. 2 & 27.40 & 72.60 & 0.00 & 0.00 & 0.18 & 100.19 \\
\hline 60-136B-08 & Gen. 2 & 27.37 & 73.21 & 0.00 & 0.00 & 0.15 & 100.73 \\
\hline 60-136B-09 & Gen. 3 & 27.46 & 73.16 & 0.00 & 0.05 & 0.13 & 100.80 \\
\hline 60-136B-10 & Gen. 3 & 27.30 & 72.95 & 0.00 & 0.00 & 0.21 & 100.46 \\
\hline 60-136B-11 & Gen. 3 & 27.29 & 72.64 & 0.00 & 0.00 & 0.19 & 100.11 \\
\hline 60-136B-12 & Gen. 2 & 27.44 & 73.04 & 0.01 & 0.00 & 0.19 & 100.67 \\
\hline $60-136 \mathrm{~B}-13$ & Gen. 2 & 27.43 & 73.19 & 0.00 & 0.00 & 0.22 & 100.84 \\
\hline 60-136B-14 & Gen. 2 & 27.55 & 72.98 & 0.00 & 0.00 & 0.20 & 100.73 \\
\hline 60-136B-15 & Gen. 2 & 27.60 & 72.73 & 0.01 & 0.00 & 0.21 & 100.55 \\
\hline 60-136B-16 & Gen. 2 & 27.30 & 72.47 & 0.00 & 0.00 & 0.25 & 100.02 \\
\hline 60-136B-17 & Gen. 2 & 27.17 & 72.42 & 0.00 & 0.00 & 0.59 & 100.18 \\
\hline 60-136B-18 & Gen. 2 & 27.54 & 72.52 & 0.00 & 0.00 & 0.31 & 100.37 \\
\hline 60-136B-19 & Gen. 2 & 27.51 & 72.58 & 0.00 & 0.00 & 0.27 & 100.35 \\
\hline $60-136 \mathrm{~B}-20$ & Gen. 2 & 27.49 & 72.20 & 0.01 & 0.00 & 0.28 & 99.97 \\
\hline 60-136B-21 & Gen. 2 & 27.44 & 72.03 & 0.03 & 0.00 & 0.16 & 99.66 \\
\hline $60-136 \mathrm{~B}-22$ & Gen. 2 & 27.23 & 71.64 & 0.00 & 0.00 & 0.18 & 99.06 \\
\hline 60-136B-23 & Gen. 2 & 27.20 & 71.75 & 0.02 & 0.00 & 0.21 & 99.18 \\
\hline 60-136B-24 & Gen. 2 & 27.24 & 71.29 & 0.01 & 0.00 & 0.13 & 98.66 \\
\hline $60-136 \mathrm{~B}-25$ & Gen. 2 & 27.15 & 71.23 & 0.04 & 0.00 & 0.19 & 98.61 \\
\hline 60-136B-26 & Gen. 2 & 27.24 & 71.99 & 0.00 & 0.00 & 0.15 & 99.38 \\
\hline 60-136B-27 & Gen. 2 & 27.36 & 72.66 & 0.00 & 0.00 & 0.29 & 100.31 \\
\hline 60-136B-28 & Gen. 2 & 27.35 & 72.84 & 0.00 & 0.00 & 0.29 & 100.47 \\
\hline 60-136B-29 & Gen. 2 & 27.55 & 71.99 & 0.02 & 0.00 & 1.25 & 100.81 \\
\hline 60-136B-30 & Gen. 2 & 27.13 & 72.39 & 0.03 & 0.00 & 0.97 & 100.51 \\
\hline 60-136B-31 & Gen. 2 & 27.26 & 72.81 & 0.01 & 0.00 & 0.66 & 100.73 \\
\hline 60-136B-32 & Gen. 2 & 27.30 & 73.17 & 0.00 & 0.00 & 0.08 & 100.55 \\
\hline $60-136 \mathrm{~B}-33$ & Gen. 2 & 27.32 & 73.02 & 0.01 & 0.00 & 0.43 & 100.78 \\
\hline 60-136A-011 & Gen. 2 & 27.17 & 71.83 & 0.02 & 0.00 & 0.73 & 99.76 \\
\hline $60-136 \mathrm{~A}-012$ & Gen. 2 & 27.30 & 71.68 & 0.00 & 0.00 & 0.59 & 99.57 \\
\hline $60-136 \mathrm{~A}-013$ & Gen. 2 & 27.21 & 72.58 & 0.02 & 0.00 & 0.50 & 100.31 \\
\hline $127-108.5-01$ & Gen. 3 & 27.22 & 72.96 & 0.00 & 0.00 & 0.34 & 100.53 \\
\hline $127-108.5-03$ & Gen. 3 & 27.10 & 72.37 & 0.00 & 0.00 & 0.33 & 99.80 \\
\hline $127-108.5-04$ & Gen. 3 & 27.23 & 72.66 & 0.00 & 0.00 & 0.31 & 100.20 \\
\hline $127-108.5-05$ & Gen. 3 & 27.50 & 72.71 & 0.00 & 0.00 & 0.18 & 100.39 \\
\hline $127-108.5-06$ & Gen. 3 & 27.27 & 72.42 & 0.00 & 0.00 & 0.22 & 99.91 \\
\hline $127-108.5-07$ & Gen. 3 & 27.32 & 72.74 & 0.00 & 0.00 & 0.33 & 100.38 \\
\hline
\end{tabular}




\begin{tabular}{lccccccc}
\hline Comment & Description & $\mathbf{S i O}_{\mathbf{2}}$ & $\mathbf{Z n O}$ & $\mathbf{C r}_{\mathbf{2}} \mathbf{O}_{\mathbf{3}}$ & $\mathbf{M n O}$ & $\mathbf{F e O}$ & Total \\
\hline $127-108.5-08$ & Gen. 3 & 26.98 & 72.45 & 0.02 & 0.00 & 0.31 & 99.75 \\
$127-108.5-09$ & Gen. 3 & 27.35 & 72.54 & 0.00 & 0.00 & 0.30 & 100.18 \\
$127-108.5-010$ & Gen. 3 & 27.10 & 72.76 & 0.01 & 0.00 & 0.08 & 99.95 \\
$127-108.5-011$ & Gen. 3 & 27.45 & 72.43 & 0.00 & 0.00 & 0.41 & 100.29 \\
$127-108.5-012$ & Gen. 3 & 27.21 & 72.15 & 0.00 & 0.00 & 0.41 & 99.77 \\
$127-108.5-013$ & Gen. 3 & 27.45 & 72.56 & 0.00 & 0.00 & 0.40 & 100.41 \\
$127-108.5-014$ & Gen. 3 & 27.22 & 72.64 & 0.00 & 0.04 & 0.36 & 100.26 \\
$127-108.5-015$ & Gen. 3 & 27.31 & 72.72 & 0.00 & 0.01 & 0.30 & 100.34 \\
$127-108.5-016$ & Gen. 3 & 27.51 & 72.12 & 0.00 & 0.00 & 0.36 & 99.99 \\
$127-108.5-017$ & Gen. 3 & 27.42 & 72.92 & 0.01 & 0.00 & 0.59 & 100.94 \\
$127-108.5-018$ & Gen. 3 & 27.42 & 72.77 & 0.01 & 0.01 & 0.33 & 100.54 \\
$127-108.5-019$ & Gen. 3 & 27.43 & 72.48 & 0.00 & 0.00 & 0.35 & 100.26 \\
$127-108.5-020$ & Gen. 3 & 27.22 & 72.30 & 0.02 & 0.00 & 0.33 & 99.87 \\
$127-108.5-021$ & Gen. 3 & 27.16 & 71.92 & 0.00 & 0.00 & 0.66 & 99.73 \\
\hline
\end{tabular}


Table 3.1 C) Sphalerite analysis

\begin{tabular}{|c|c|c|c|c|c|c|c|}
\hline Comment & Description & $\mathbf{S}$ & Zn & Mn & $\mathbf{F e}$ & Cd & Total \\
\hline 60-136A-01 & type 4 breccia & 32.42 & 63.37 & 0.00 & 0.02 & 3.04 & 98.85 \\
\hline 60-136A-02 & type 4 breccia & 32.44 & 63.59 & 0.00 & 0.02 & 2.91 & 98.95 \\
\hline 60-136A-03 & type 4 breccia & 32.28 & 63.36 & 0.00 & 0.05 & 2.88 & 98.56 \\
\hline 60-136A-04 & type 4 breccia & 32.23 & 63.22 & 0.00 & 0.03 & 3.01 & 98.49 \\
\hline 60-136A-05 & type 4 breccia & 32.55 & 62.16 & 0.00 & 0.02 & 2.91 & 97.64 \\
\hline 60-136A-06 & type 4 breccia & 32.19 & 63.59 & 0.00 & 0.01 & 3.01 & 98.80 \\
\hline 60-136A-07 & type 4 breccia & 32.51 & 63.00 & 0.00 & 0.02 & 2.88 & 98.41 \\
\hline 60-136A-08 & type 4 breccia & 32.44 & 63.36 & 0.00 & 0.00 & 3.09 & 98.89 \\
\hline $60-136 A-09$ & type 4 breccia & 32.41 & 62.82 & 0.00 & 0.03 & 2.93 & 98.19 \\
\hline 60-136A-010 & type 4 breccia & 32.39 & 63.00 & 0.00 & 0.07 & 3.10 & 98.55 \\
\hline $88-68-01$ & sulfide breccia & 32.92 & 66.87 & 0.00 & 0.22 & 0.24 & 100.25 \\
\hline $88-68-02$ & sulfide breccia & 32.89 & 67.26 & 0.00 & 0.23 & 0.22 & 100.59 \\
\hline $88-68-03$ & sulfide breccia & 32.86 & 66.98 & 0.00 & 0.24 & 0.24 & 100.32 \\
\hline $88-68-04$ & sulfide breccia & 33.07 & 67.16 & 0.00 & 0.21 & 0.19 & 100.62 \\
\hline $88-68-05$ & sulfide breccia & 32.90 & 67.13 & 0.00 & 0.24 & 0.21 & 100.48 \\
\hline $88-68-06$ & sulfide breccia & 32.91 & 67.07 & 0.00 & 0.21 & 0.17 & 100.36 \\
\hline $88-68-07$ & sulfide breccia & 33.04 & 67.08 & 0.00 & 0.25 & 0.24 & 100.61 \\
\hline $88-68-08$ & sulfide breccia & 32.64 & 67.49 & 0.00 & 0.23 & 0.21 & 100.56 \\
\hline $88-68-09$ & sulfide breccia & 32.92 & 67.37 & 0.00 & 0.19 & 0.21 & 100.69 \\
\hline $88-68-010$ & sulfide breccia & 33.02 & 66.85 & 0.00 & 0.19 & 0.21 & 100.27 \\
\hline $88-68-011$ & sulfide breccia & 33.01 & 67.24 & 0.00 & 0.29 & 0.16 & 100.69 \\
\hline $88-68-012$ & sulfide breccia & 33.02 & 67.23 & 0.00 & 0.20 & 0.25 & 100.70 \\
\hline $141-16-02$ & alteration vein in MP & 33.48 & 66.29 & 0.00 & 1.10 & 0.39 & 101.26 \\
\hline $141-16-03$ & alteration vein in MP & 32.69 & 66.01 & 0.00 & 1.19 & 0.31 & 100.19 \\
\hline $141-16-04$ & alteration vein in MP & 32.92 & 66.43 & 0.00 & 1.14 & 0.30 & 100.79 \\
\hline $141-16-05$ & alteration vein in MP & 32.84 & 66.27 & 0.00 & 1.17 & 0.33 & 100.62 \\
\hline $141-16-06$ & alteration vein in MP & 32.83 & 66.56 & 0.00 & 1.17 & 0.34 & 100.90 \\
\hline $141-16-07$ & alteration vein in MP & 32.87 & 66.28 & 0.00 & 1.17 & 0.34 & 100.66 \\
\hline $141-16-08$ & alteration vein in MP & 32.93 & 66.17 & 0.00 & 1.13 & 0.30 & 100.54 \\
\hline
\end{tabular}



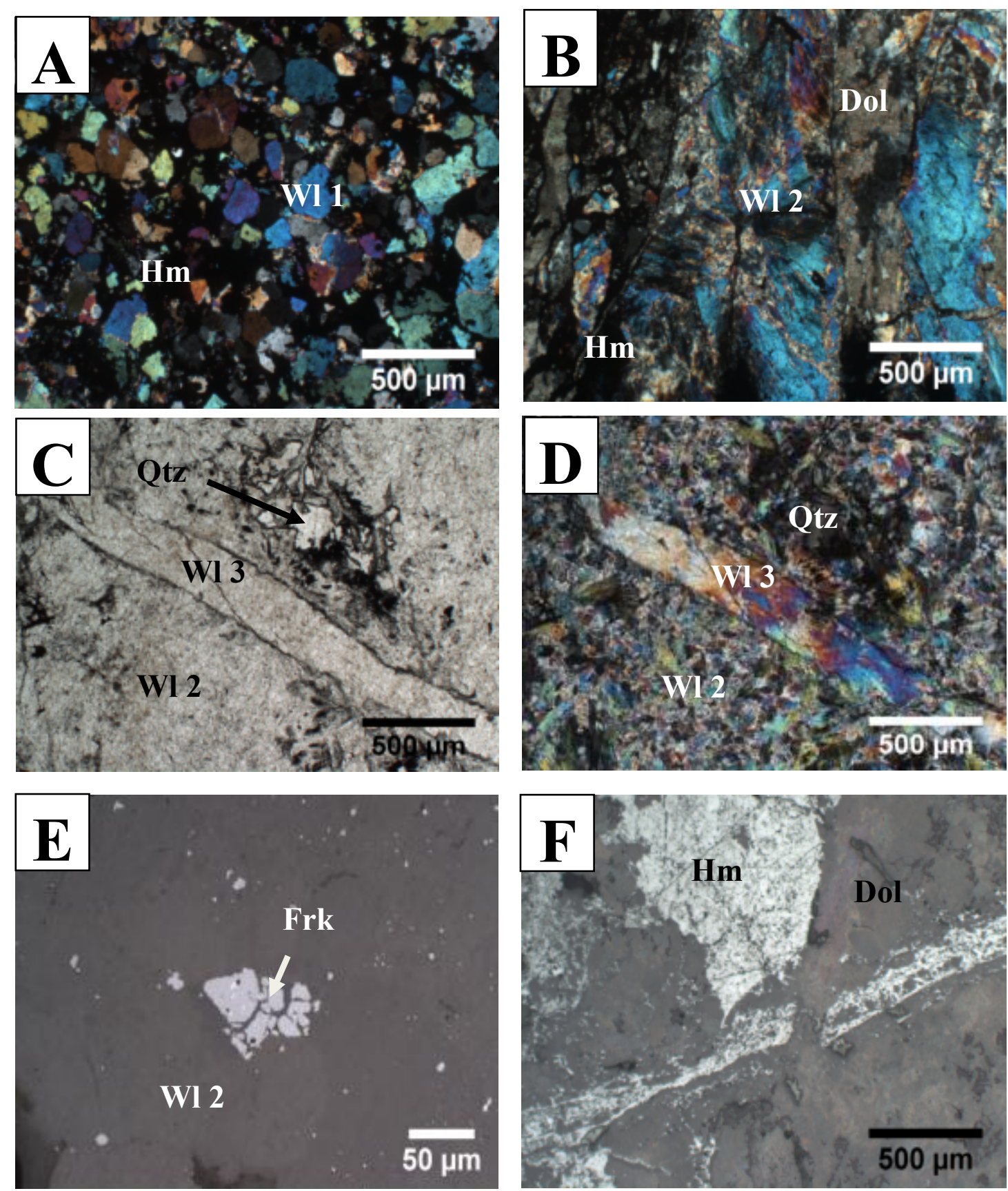

Fig. 3.14 Photomicrographs showing the minerals and their textural relationships in the Type 4

Breccia. A) Sample 106-122, euhedral, generation 1 willemite in a hematite matrix. Willemite is being replaced by hematite. B) Sample 060-136B, generation 2 willemite as radiating, fibrous crystal laths replacing Fe-Zn rich dolomite breccia fragments. C) Sample 060-136B, fibrous generation 3 willemite veinlets cutting generation 2 willemite. Quartz is being replaced by generation 2 willemite (PPL). D) Sample 060-136B, fibrous generation 3 willemite veinlet cutting generation 2 willemite (XPL). E) Sample 106-122, franklinite associated with generation 2 willemite in reflected light. F) Sample 106122, hematite cutting Fe-Zn dolomite and filling in open space within the breccia. $\mathrm{Wl}=$ Willemite, Frk $=$ Franklinite, $\mathrm{Hm}=$ Hematite. Dol $=$ Dolomite. $\mathrm{Qtz}=$ Quartz. 

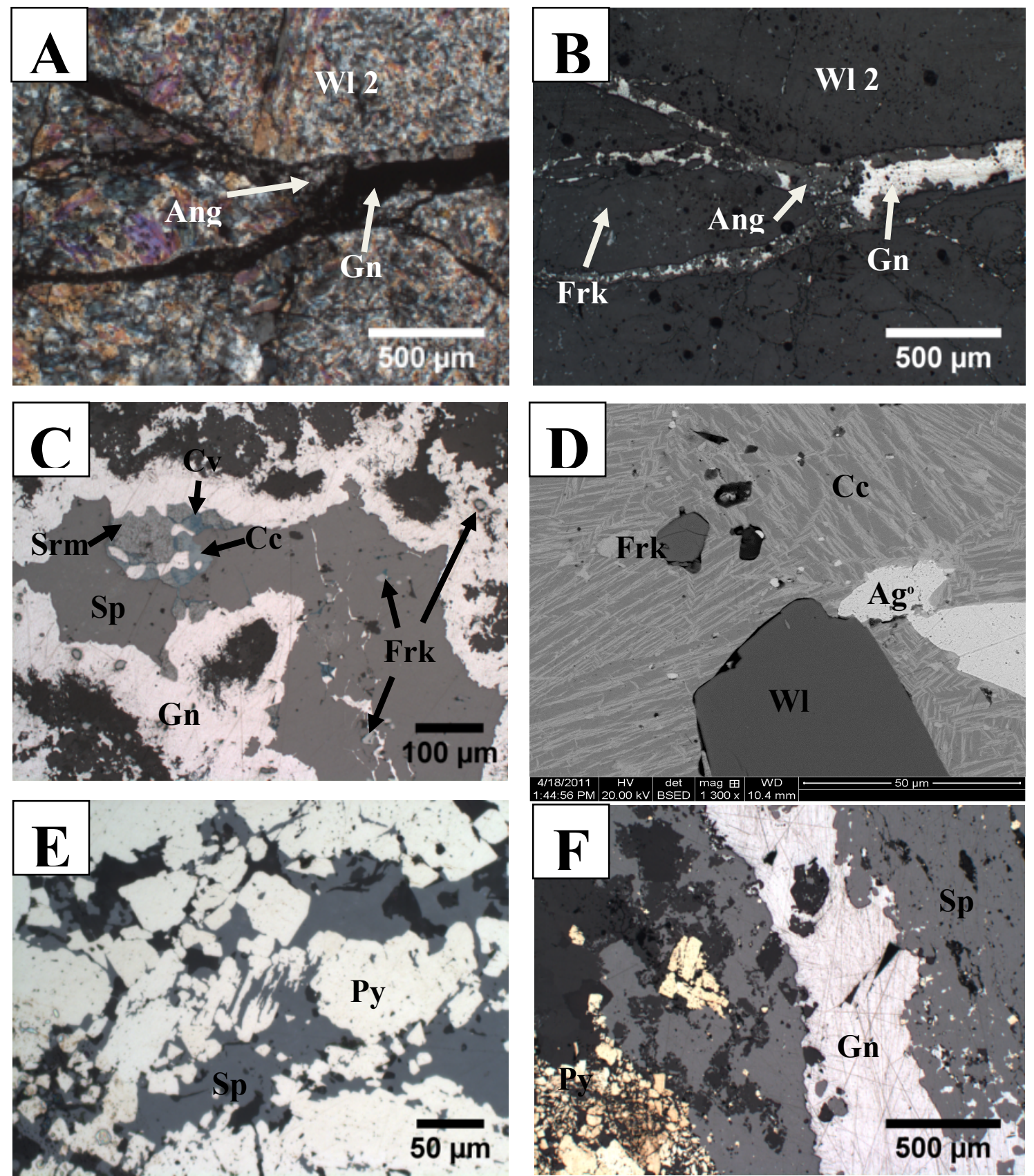

Fig. 3.15 Photomicrographs and BSEI showing the sulfide veins and veinlets. A) Sample 060-136A, galena (replaced by anglesite) veinlets cross cutting $2^{\text {nd }}$ generation willemite (XPL). B) Sample 060$136 \mathrm{~A}$, galena and anglesite veinlets cross cutting $2^{\text {nd }}$ generation willemite (reflected PPL). Some franklinite crystals are included in the willemite. C) Sample 060-136A, sphalerite-galena veinlets with stromeyerite altering to covellite and chalcocite (reflected PPL). D) Sample 012-347A, Cu-Ag sulfide veinlets with Ag-rich intergrowths in chalcocite (lighter color). Also present willemite, franklinite, and native silver (BSEI). E) Sample 088-71, sphalerite-pyrite mineralization in hole VZMIF 088 where sphalerite is replacing pyrite (reflected PPL). F) Sample 088-72, galena in $\mathrm{Pb}-\mathrm{Zn}$ sulfide veinlet cutting sphalerite-pyrite mineralization in hole VZMIF 088 (reflected PPL).

Py $=$ Pyrite, $\mathrm{Sp}=$ Sphalerite, $\mathrm{Gn}=$ Galena, Ang = Anglesite, $\mathrm{Wl}=$ Willemite, Frk = Franklinite, $\mathrm{Srm}=$ Stromeyerite, $\mathrm{Cc}=$ Chalcocite. $\mathrm{Cv}=$ Covellite, $\mathrm{Hm}=$ Hematite, $\mathrm{Ag}^{\mathrm{O}}=$ Native Silver. 
Based on the field and textural relationships described above, the interpreted paragenesis to the Northern Extension mineralization is presented in Figure 3.16 and summarized below:

Stage 1 (pre-ore): Early brecciation of the Serra do Poço Verde dolostones and precipitation of Fe-bearing dolomite (types 1 and 2 breccias)

Stage 2 (syn-main ore): Formation of the hematite-rich Type 3 Breccia and the willemite-rich Type 4 Breccia with multiple episodes of precipitation of willemite, hematite, franklinite, ferroan and zincian dolomites filling and replacing the previous breccias. The isolated Sp-Py mineralization in VZMIF 088 was hypothesized to be contemporaneous with the willemite ore formation because it was cross-cut by the galena-rich veinlets.

Stage 3: (post- main ore): Subsequent to the main ore stage, two distinct sulfide veinlets (i.e. $\mathrm{Pb}$ rich and Ag-rich veinlets) formed and cross-cut the main ore stage minerals.

As for the isolated sphalerite and pyrite veins, they formed prior to the galena and silverrich veinlets, but it was not impossible to determine their timing of formation relative to the Type 4 willemite breccia.

\subsection{Lithogeochemistry}

\subsubsection{Analytical Methods}

Whole rock geochemical analyses of samples from each unit, alteration and mineralization zones were conducted in order to determine the geochemical signatures related to the various styles of mineralization and alteration as well as the mass change involved in the alteration process. The geochemical analyses were carried out by the ALS Laboratory Group in Canada on 74 samples including the least altered and most representative samples of each of the local geologic units, the non-ore breccias, and the willemite ore samples. 


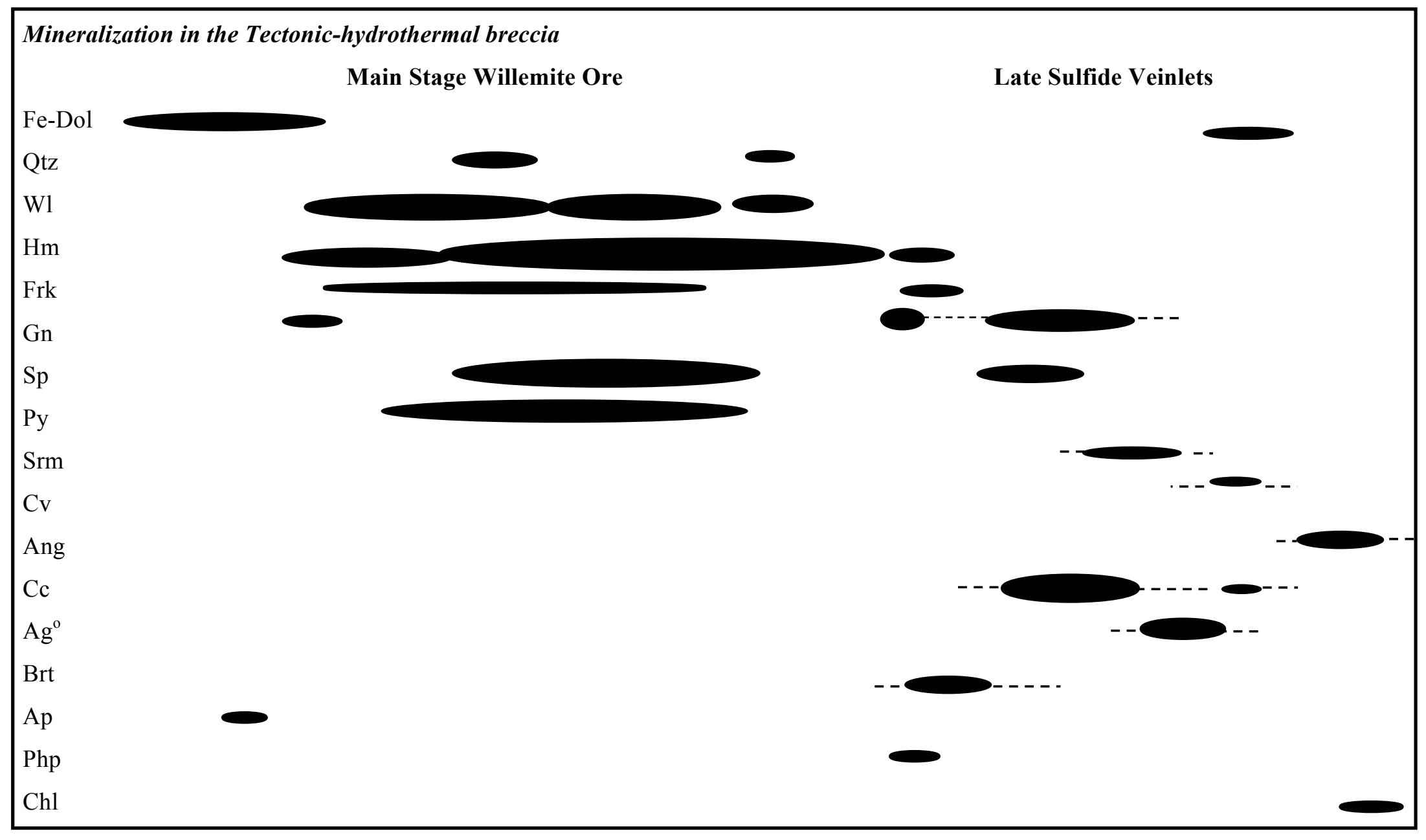

Fig. 3.16 Paragenetic sequence of the mineralized zone in the Northern Extension. Fe-Dol $=$ Ferroan dolomite, $\mathrm{Qtz}=\mathrm{Qtz}, \mathrm{Wl}=$ willemite, $\mathrm{Hm}=\mathrm{Hematite}$, Frk $=$ Franklinite, $\mathrm{Gn}=$ Galena, $\mathrm{Sp}=$ Sphalerite, $\mathrm{Py}=$ Pyrite, $\mathrm{Srm}=$ Stromeyerite, $\mathrm{Cv}=$ Covellite, $\mathrm{Ang}=$ Anglesite $, \mathrm{Cc}=\mathrm{Chalcocite}, \mathrm{Ag}^{\circ}=\mathrm{Native}$ Silver, $\mathrm{Brt}=$ Barite, $\mathrm{Ap}=$ Apatite, $\mathrm{Php}=$ Phosphohedyphane, $\mathrm{Chl}=$ Chlorite 
They were prepared using 4-acid digestion then analyzed for the elements $\mathrm{Ag}, \mathrm{Al}, \mathrm{Ba}, \mathrm{Be}, \mathrm{Bi}, \mathrm{Ca}$, Cd, Ce, Co, Cr, Cu, Cs, Fe, Ga, Ge, Hf, In, K, La, Li, Mg, Mn, Mo, Na, Nb, Ni, P, Pb, Rb, Re, S, Sb, Sc, Se, Sn, Sr, Ta, Te, Th, Ti, Tl, U, V, W, Y, Zn, Zr by inductively coupled plasma mass spectrometry (ICP-MS). The element As in addition to samples containing greater than $100 \mathrm{ppm}$ $\mathrm{Ag}$ and greater than 10,000 $\mathrm{ppm} \mathrm{Pb}$ were prepared using 4-acid digestion and analyzed using inductively coupled plasma absorption emission spectrometry (ICP-AES) to eliminate interferences and increase the upper detection limits. Mercury was analyzed by 4-acid digestion with trace vapor/atomic absorption spectrometry (AAS). Samples containing more than 10,000 ppm Zn were analyzed by titration. Standards and duplicates were used by ALS to maintain quality control and quality assurance.

A subset of 39 of those samples were analyzed for whole rock geochemistry using X-ray fluorescence (XRF), and include representative samples of the various alteration styles and of the least altered samples from the host rock types.

Major and trace elements selected from the four types of tectonic-hydrothermal breccias and their host rocks were plotted in box and whiskers diagrams using ioGAS to evaluate their variation among rock types (Fig. 3.17). Furthermore, isocons diagrams (based on Grant, 2000) were constructed to identify and compare the elemental enrichment and depletion of selected elements among the various breccia types and least altered host rocks. The mass balance equation from Grant (2000) is defined by $\Delta M=\left[\left(C_{A} / C_{I}\right)-1\right] * 100$ where $\Delta M$ is the slope, and in this study for the various isocon plots, $C_{I}$ was considered as the less altered sample and $C_{A}$ the more altered sample (e.g., least altered dolostone was plotted against Breccia Type 1, and the latter was potted against Breccia Type 2, etc.). The immobile elements necessary for the isocon line were determined to be $\mathrm{Ce}, \mathrm{La}, \mathrm{Sc}, \mathrm{Ti}, \mathrm{Y}$, and $\mathrm{Zr}$ based on their high correlation coefficients to each other $(>0.9)$ as well as their repeated near linear plots in the isocon diagrams. 


\subsubsection{Results}

The results of the whole rock geochemical analyses can be found in Appendix D. The box and whiskers plots for selected elements related to the ore-forming process (Fig. 3.17) show that the variations among the compositions of least altered samples and Type 1 and 2 breccias are minor; however, the Type 3 and 4 breccias show significant variations when compared with the least altered host rock and the Type 1 and 2 breccias. Types 3 and 4 breccias yielded the highest averages in As, Ba, Be, Bi, Fe, Ge, In, Mo, Ni, S, Sb, U, V, and W, with Type 3 being the most enriched in these elements. Breccia Type 4 has significantly higher average concentrations of $\mathrm{Zn}$, $\mathrm{Ag}, \mathrm{Hg}, \mathrm{Se}$, and $\mathrm{SiO}_{2}$, and it also contains anomalously high concentrations of $\mathrm{Cu}$ and $\mathrm{Pb}$. Types 3 and 4 breccias have the lowest average concentrations of $\mathrm{Ca}$ and $\mathrm{Mg}$, which is consistent with the field and petrographic observations that hematite and willemite replace carbonate clasts and carbonate matrix.

Figures $3.18,3.19,3.20$ and 3.21 are isocon plots that represent the evolution of element gains and losses when a more altered breccia type is plotted again a less altered sample (e.g. Type 1 vs. Unaltered Lower Pamplona dolostone, Type 3 Breccia vs. Type 4 Breccia). The isocon line is plotted as a line of best fit through the immobile elements $\mathrm{Ce}, \mathrm{La}, \mathrm{Sc}, \mathrm{Ti}, \mathrm{Y}$, and $\mathrm{Zr}$. The isocon plot illustrating the unaltered Lower Pamplona dolostone with the Type 1 Breccia (Fig. 3.18) reveals that the latter is significantly enriched in $\mathrm{Ag}, \mathrm{Cd}, \mathrm{Pb}, \mathrm{Zn}$ and moderately enriched in $\mathrm{Bi}, \mathrm{Co}, \mathrm{Cu}, \mathrm{Fe}, \mathrm{In}, \mathrm{Mn}, \mathrm{Mo}, \mathrm{Ni}, \mathrm{P}$, and $\mathrm{Sb}$. When the Type 2 Breccia is compared to the Type 1 Breccia (Fig. 3.19), it is shown that the Type 2 Breccia is enriched in the same suite of elements as the Type 1 Breccia, except for Bi and In, which are aligned on the isocon. 


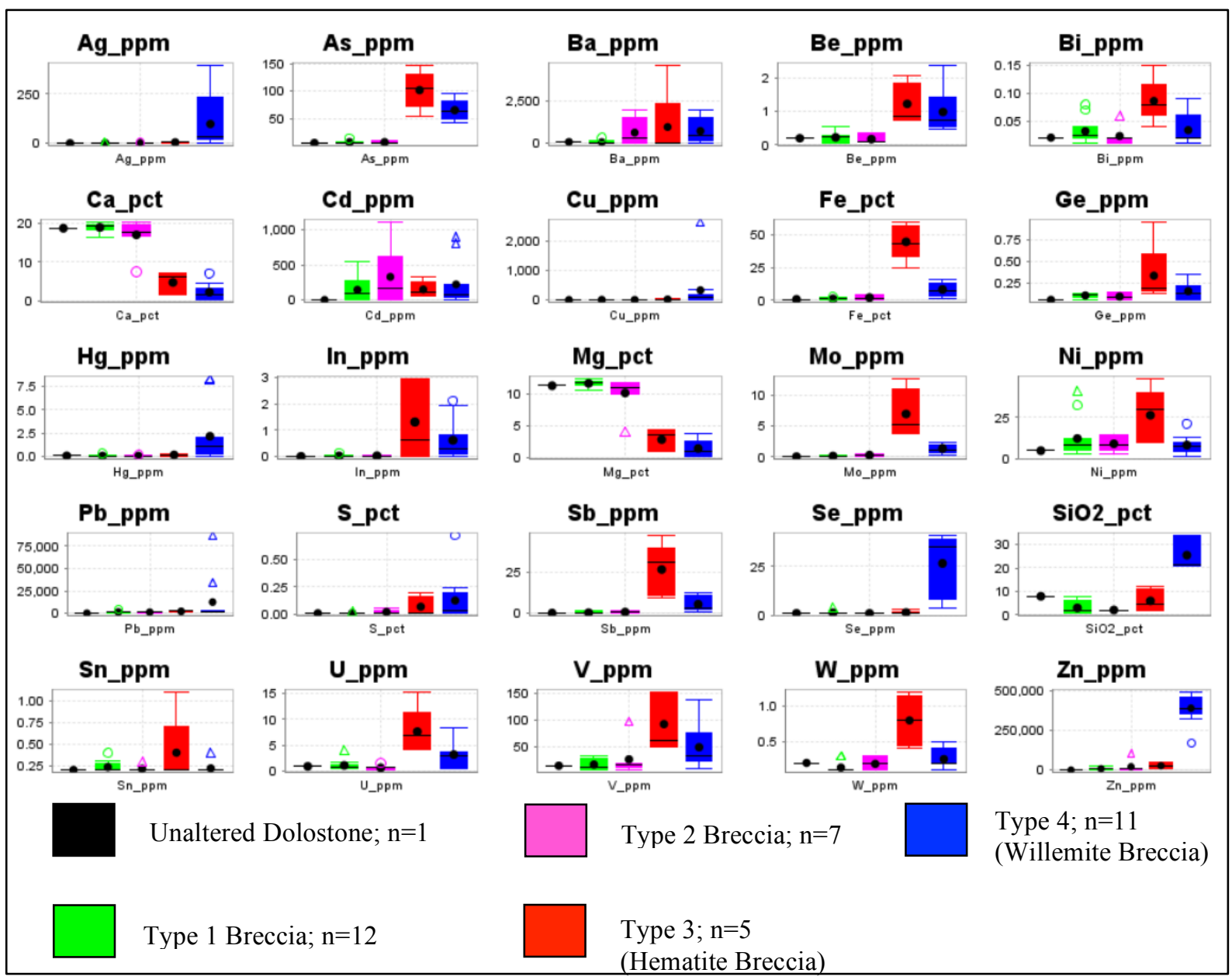

Fig. 3.17 Box and whisker plots showing the variation in composition of the least altered host rocks and various breccia types. 
The Type 2 Breccia is also enriched in $\mathrm{Ba}, \mathrm{V}, \mathrm{S}$, and W. Figure 3.20 compares the Type 3

Breccia composed mainly of hematite with the Type 2 Breccia. This graph shows that the Type 3

Breccia is enriched in the same elements as breccia Type 1 and Type 2, as well as in As, Ga, Ge,

$\mathrm{Hg}$, In, $\mathrm{Pb}, \mathrm{Se}, \mathrm{SiO}_{2}, \mathrm{U}$, and $\mathrm{Zn}$. The Type 3 Breccia is depleted in $\mathrm{Ca}, \mathrm{K}$, and $\mathrm{Mg}$ compared to

the Type 2 Breccia. Figure 3.21, the isocon comparing the Type 3 Breccia with the Type 4

Breccia (willemite ore) shows that the Type 4 Breccia is enriched in $\mathrm{Ag}, \mathrm{Cd}, \mathrm{Cu}, \mathrm{Hg}, \mathrm{S}, \mathrm{Se}, \mathrm{SiO}_{2}$,

$\mathrm{Sr}, \mathrm{Pb}$ and $\mathrm{Zn}$. Many of the elements previously enriched in the other breccias are aligned along the isocon and show no change or are slightly depleted compared to the Type 3 Breccia.

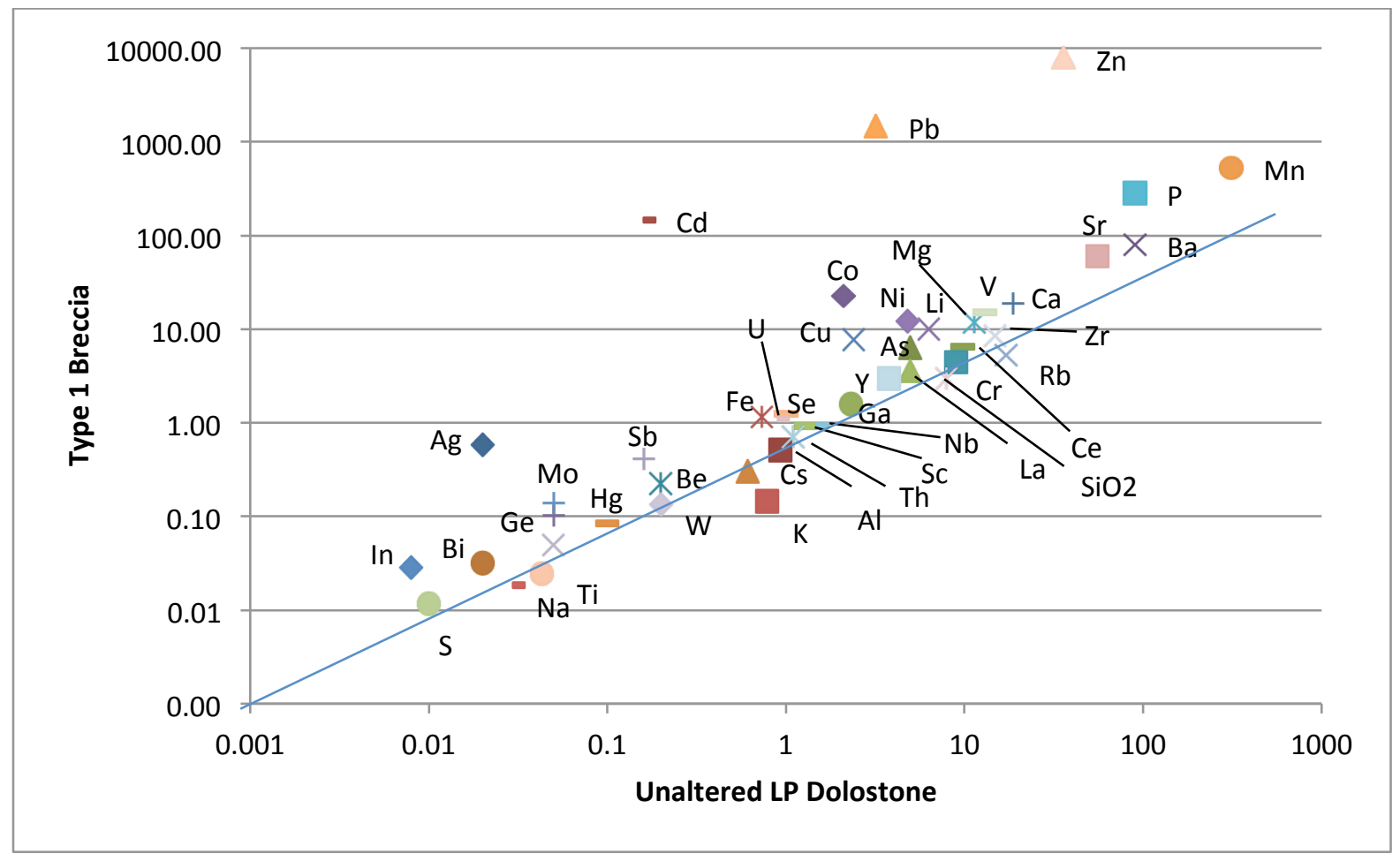

Fig. 3.18 Isocon plot of Type 1 Breccia vs. unaltered dolostone from the Lower Pamplona Member, showing the gains and losses during the hydrothermal event (see text for further explanation). 


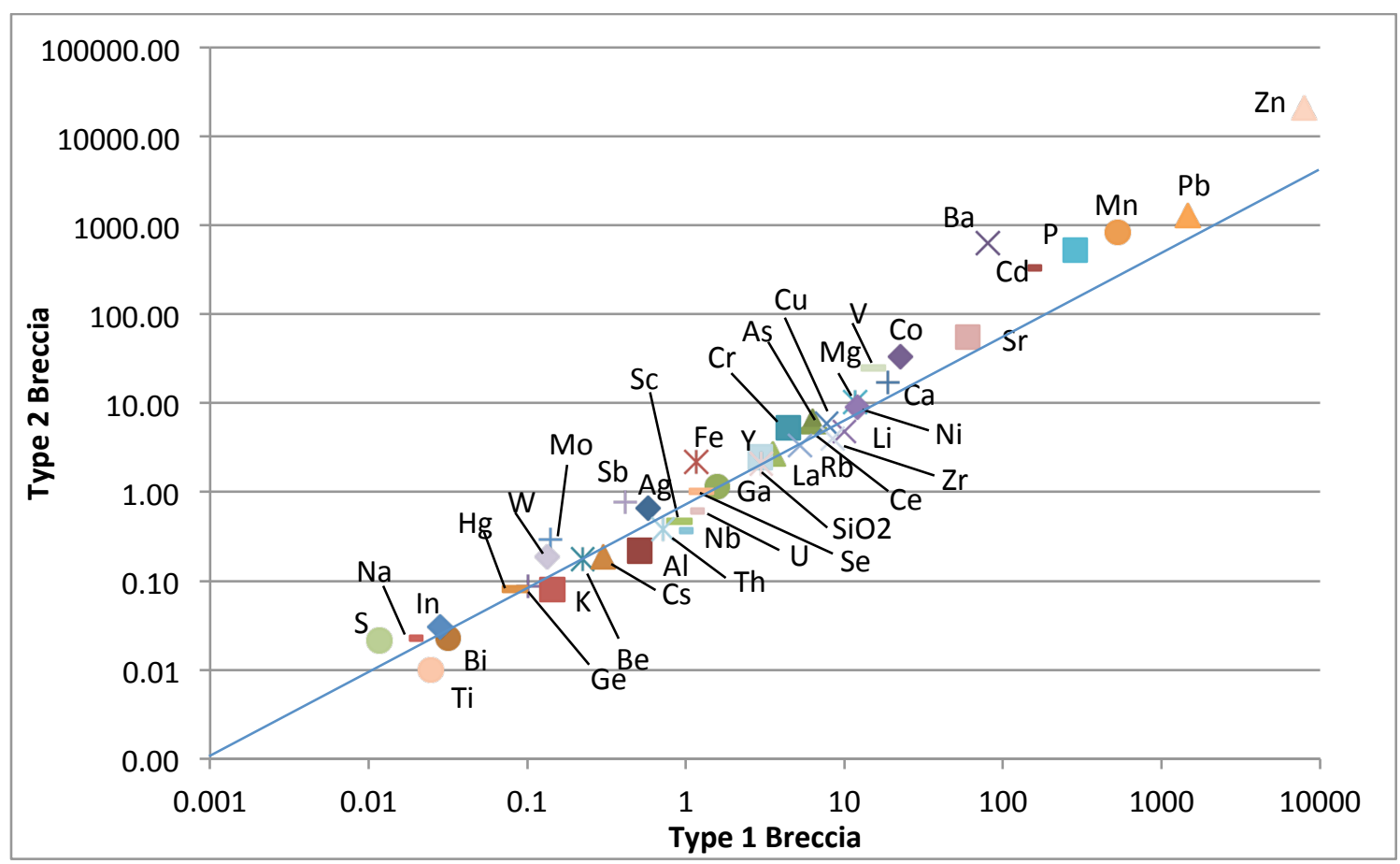

Fig. 3.19 Isocon plot of Type 1 Breccia vs. Type 2 Breccia showing the gains and losses during the hydrothermal event (see text for further explanation).

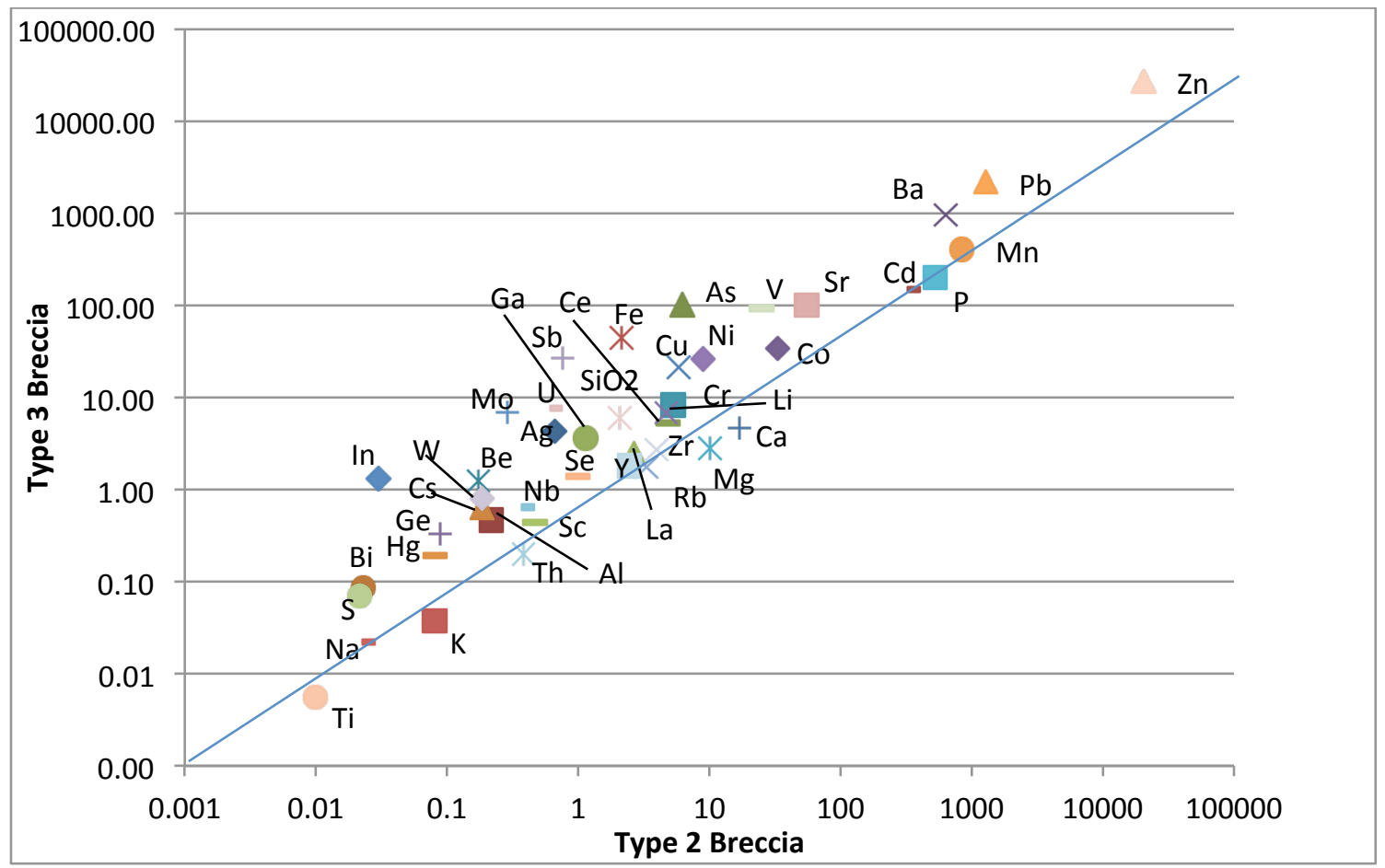

Fig. 3.20 Isocon plot of Type 2 Breccia vs. Type 3 Breccia showing the gains and losses during the hydrothermal event (see text for further explanation). 


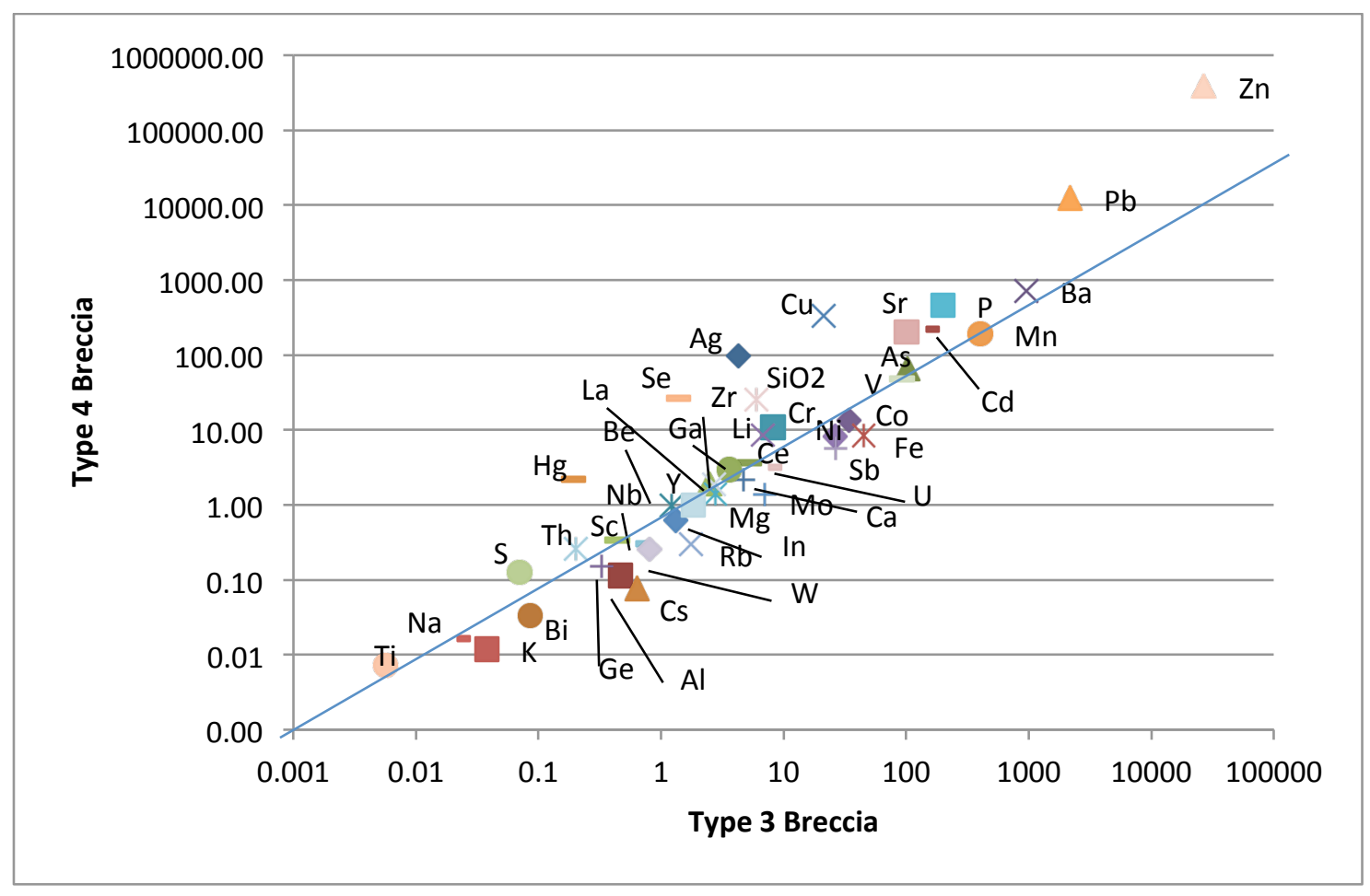

Fig. 3.21 Isocon plot of Type 3 (hematite) Breccia vs. Type 4 (willemite) Breccia showing the gains and losses during the hydrothermal event (see text for further explanation).

\subsection{Discussion}

\subsubsection{Evolution of the Northern Extension}

The evolution of the Vazante Group provided favorable conditions for the formation of a zinc silicate deposit as discussed below. After deposition and dolomitization of the passive margin carbonates in the Serra do Poço Verde Formation c.a 900-1200 Ma, tectonism began with the onset of the Brasiliano Orogeny around 850 Ma. Deformation related to the beginning of the Brasiliano Orogeny $\left(D_{1}\right)$ created a regional foliation $\left(S_{1}\right)$ subparallel to bedding $\left(S_{0}\right)$ within the units of the Vazante Group. Continued deformation $\left(D_{2}\right)$ of the region folded the $S_{0} / S_{1}$ foliation, resulting in an $\mathrm{S}_{2}$ crenulation cleavage, regional folds, and the SE-striking conjugate faults of the Vazante Northern Extension. This event is most likely coeval with the formation of the thinskinned nappes and low-grade metamorphism as described by Dardenne (2000), Valeriano et al. 
(2008), and Martins-Neto (2009). These structures focused the hydrothermal fluid flow, leading to the formation of the hydrothermal breccias and ore mineralization.

The hydrothermal fluids were most likely a metalliferous, low temperature, acidic, relatively saline fluid with low S content (Appold and Monteiro, 2009), in which $\mathrm{Zn}$ is transported as $\mathrm{ZnCl}_{4}{ }^{2-}$ and $\mathrm{Fe}$ as $\mathrm{FeCl}_{3}$ according to Riveros and Dutruziac, (1997) and Brugger et al. (2003). These fluids would have moved through the Northern Extension Fault Zone, creating through hydraulic fracturing the Type 1 Breccia, which is characterized by weak brecciation; Fedolomite alteration and veinlets; substantial enrichments in $\mathrm{Ag}, \mathrm{Cd}, \mathrm{Pb}, \mathrm{Zn}$; and moderate enrichments in $\mathrm{Bi}, \mathrm{Co}, \mathrm{Cu}, \mathrm{Fe}, \mathrm{In}, \mathrm{Mn}, \mathrm{Mo}, \mathrm{Ni}, \mathrm{P}$, and $\mathrm{Sb}$. Enduring basinal fluid flow caused further brecciation and alteration, creating the strongly Fe-dolomite altered Type 2 Breccia, which exhibit similar geochemical signature as Type 1 Breccia. After the formation of the Type 2 Breccia, mixing of the basinal brine with a cooler, oxidizing, meteoric fluid in the Northern Extension may have induced further brecciation and mineralization, leading to the formation of the types 3 and 4 breccias, dominated by hypogene hematite and willemite, respectively. This interpretation is based on the fact that the main alteration minerals changed from Fe-bearing carbonate to hematite in Type 3 Breccia, denoting interaction with more oxidizing fluids. These fluids would have also added $\mathrm{As}, \mathrm{Bi}, \mathrm{Ga}, \mathrm{Ge}, \mathrm{Hg}, \mathrm{In}, \mathrm{Pb}, \mathrm{Se}, \mathrm{SiO}_{2}, \mathrm{U}$, and $\mathrm{Zn}$, as the Type 3 and 4 breccias are more enriched in these elements. Mixing between the basinal brine and the cooler meteoric fluids in the tectonic breccia would have diluted and cooled the system to maintain willemite precipitation, which in turn would have created a stable environment for hematite. A possible reaction for the formation of hematite from an Fe-bearing carbonate would be:

$$
\begin{array}{ll}
\text { Reaction 1) } & 8 \mathrm{Ca}\left(\mathrm{Mg}_{0.5}, \mathrm{Fe}_{0.5}\right)\left(\mathrm{CO}_{3}\right)_{2}+12 \mathrm{FeCl}_{3}+24 \mathrm{H}^{+}+6 \mathrm{H}_{2} \mathrm{O}+\mathrm{O}_{2}=8 \mathrm{Fe}_{2} \mathrm{O}_{3}+ \\
& 36 \mathrm{HCl}+8 \mathrm{Ca}^{+2}+4 \mathrm{Mg}^{+2}+16 \mathrm{CO}_{2}
\end{array}
$$

As the system became cooler and more dilute leading to increase in $\mathrm{pH}$, concentrations of $\mathrm{Zn}$ and $\mathrm{SiO}_{2}$ became substantial enough to precipitate out willemite in addition to hematite under the following reaction: 


$$
\text { Reaction 2) } \begin{aligned}
2 \mathrm{Ca}\left(\mathrm{Mg}_{0.5}, \mathrm{Fe}_{0.5}\right)\left(\mathrm{CO}_{3}\right)_{2}+3 \mathrm{FeCl}_{3}+4 \mathrm{ZnCl}_{4}^{-2}+2 \mathrm{SiO}_{2}+14 \mathrm{H}^{+}+11 \mathrm{H}_{2} \mathrm{O} \\
=2 \mathrm{Fe}_{2} \mathrm{O}_{3}+2 \mathrm{Zn}_{2} \mathrm{SiO}_{4}+25 \mathrm{HCl}+2 \mathrm{Ca}^{+2}+2 \mathrm{Mg}^{+2}+4 \mathrm{CO}_{2}+5 \mathrm{O}_{2}
\end{aligned}
$$

The production of $\mathrm{O}_{2}$ may have kept the system oxidized enough to maintain hematite stability. In addition, these reactions account for the elemental losses in $\mathrm{Ca}$ and $\mathrm{Mg}$ as well as the gains in $\mathrm{Fe}, \mathrm{Zn}$, and $\mathrm{SiO}_{2}$; however, it is also possible for hematite and willemite to precipitate without interacting with the host carbonates, as shown in the following reactions:

$$
\begin{array}{ll}
\text { Reaction 3) } & \text { Willemite: } 2 \mathrm{ZnCl}_{4}^{-2}+\mathrm{SiO}_{2}+2 \mathrm{H}^{+}+3 \mathrm{H}_{2} \mathrm{O}=\mathrm{Zn}_{2} \mathrm{SiO}_{4}+8 \mathrm{HCl}+1 / 2 \mathrm{O}_{2} \\
\text { Reaction 4) } & \text { Hematite: } 2 \mathrm{FeCl}_{3}+3 \mathrm{H}_{2} \mathrm{O}=\mathrm{Fe}_{2} \mathrm{O}_{3}+6 \mathrm{HCl}
\end{array}
$$

\begin{tabular}{|c|c|c|}
\hline Factor & Willemite $(\mathrm{Wl})$ & Sphalerite (Sp) \\
\hline Temperature $(T)$ & Precipitation not affected & As $T$ decreases, Sp precipitates \\
\hline $\mathrm{pH}$ & $\begin{array}{l}\text { More sensitive to } \mathrm{pH} \text { than } \mathrm{Sp} \text {, } \\
\text { as } \mathrm{pH} \text { increases, Wl } \\
\text { precipitates }\end{array}$ & $\begin{array}{l}\text { Less sensitive to } \mathrm{pH} \text { than } \mathrm{Wl} \text {, } \\
\text { as } \mathrm{pH} \text { increases, } \mathrm{Sp} \text { will } \\
\text { precipitate but to a lesser } \\
\text { extent than } \mathrm{Wl}\end{array}$ \\
\hline Salinity & $\begin{array}{l}\text { A decrease in salinity } \\
\text { precipitates } \mathrm{Wl} \text { and Sp (favors } \\
\text { neither) }\end{array}$ & $\begin{array}{l}\text { A decrease in salinity } \\
\text { precipitates W1 and Sp (favors } \\
\text { neither) }\end{array}$ \\
\hline $\log f \mathrm{O}_{2}$ & No effect & $\begin{array}{l}\text { As } \log f \mathrm{O}_{2} \text { increases, sulfides } \\
\text { converted to sulfates }\end{array}$ \\
\hline
\end{tabular}

This interpretation is in agreement with the models for precipitation of willemite proposed by Brugger et al. (2003) and Appold and Monteiro (2009) and summarized in Table 1. The scarcity in sphalerite is explained by the low $\mathrm{S}$ content in the hydrothermal fluids.

Table 3.2 Summary of the factors controlling willemite and sphalerite precipitation from a fluid with temperatures ranging from $100-300{ }^{\circ} \mathrm{C}$, at a $\mathrm{pH}$ of 4.5 , and $\log f \mathrm{O}_{2}=-40.5$ (Summarized from Brugger et al. (2003) and Appold and Monteiro (2009). Wl = willemite, $\mathrm{Sp}=$ sphalerite

After the formation of the Type 4 Breccia, another basinal fluid containing some reduced $\mathrm{S}$, base metals, and $\mathrm{Ag}^{\mathrm{o}}$ moved through the Type 4 Breccia and precipitated some minor sulfide upon cooling. Much later, when the willemite was exposed to weather, supergene processes 
would have oxidized the willemite, creating hydrozincite, smithsonite, and other zinc-oxides in the late structures.

When compared to the research conducted by Monteiro et al. $(1999,2006,2007)$ on the Vazante Mine, the Northern Extension shows many similarities. Both deposits are hosted in the carbonates of the Lower Pamplona Member, structurally controlled, dominated by willemite mineralization, and have a strong $\mathrm{Ag}, \mathrm{Cd}, \mathrm{Pb}, \mathrm{Zn}$ geochemical signature (Monteiro et al., 1999, 2006, 2007; this study).

Unlike the Vazante Mine, the Northern Extension deposit is located closer to the surface, outcrops in the very northern region of the deposit, and shows a greater influence of brittle deformation instead of the ductile deformation recorded by Monteiro et al. (1999) in the Vazante Mine. Monteiro et al. (1999), Hitzman et al (2003), and Monteiro et al. (2006, 2007) documented sphalerite in pods that were imbricated with the willemite ore, but very little sphalerite was observed in the Northern Extension except for small amounts located in late sulfide veinlets, indicating that the ore fluid in the Northern Extension may have evolved into a cooler, higher $\mathrm{pH}$ fluid, or was poorer in $\mathrm{S}$. In addition to the strong $\mathrm{Ag}, \mathrm{Cd}, \mathrm{Pb}, \mathrm{Zn}$ signature, Monteiro et al. (2007) also documented gains in $\mathrm{La}, \mathrm{P}$, and $\mathrm{Rb}$ in the mineralized breccia; however, no substantial gains in La or P were observed in the Northern Extension, and it was observed that $\mathrm{Rb}$ was actually lost in the types 3 and 4 breccias in this study.

The carbonate units of the Serra do Poço Verde Formation show a decreasing $\delta^{13} \mathrm{C}$ signature from $+2 \%$ to $-2.3 \%$ at the top of the Middle Pamplona Member followed by a positive $\delta^{13} \mathrm{C}$ excursion to $+3.9 \%$ in the Morro do Calcário Formation, indicating that the Morro do Calcário may be a cap carbonate for the Sturtian snowball Earth event that occurred during the deposition of the Serra do Poço Verde Formation (Azmy et al., 2001; Misi et al., 2005).

Extensive glaciation could have provided the oxygenated meteoric fluid that mixed with the basinal brine in the Vazante Northern Extension trend. 


\subsubsection{Comparison with Other Deposits}

The Vazante Northern Extension deposit has some similarities with other hypogene zinc silicate deposits and their characteristics are summarized in Table 2. The Franklin-Sterling Hill deposit is metamorphosed to a very high grade so the original alteration and ore minerals are no longer preserved. As for all the other known zinc silicate deposits, they are hosted in carbonate rocks that formed during the Neoproterozoic to Cambrian, contain willemite and hematite as the major minerals in the ore zone, are enriched in $\mathrm{Be}, \mathrm{Cd}, \mathrm{Cu}, \mathrm{Ge}, \mathrm{Pb}$ and $\mathrm{V}$, and are interpreted to have been formed by low temperature fluids $\left(50\right.$ to $\left.180{ }^{\circ} \mathrm{C}\right)$ of variable salinities $(3-15$ eq. wt $\% \mathrm{NaCl})$. In the Vazante Northern Extension and Kabwe deposits, brecciation is interpreted to be related to major faults (Dardenne, 2000; Kamona and Friedrich, 2007, this study); whereas the breccias at Beltana are associated with karst (Groves et al., 2003). 


\begin{tabular}{|c|c|c|c|c|c|c|c|c|c|c|c|}
\hline Deposits & Location & $\begin{array}{l}\text { Size } \\
\text { (Mt) }\end{array}$ & $\begin{array}{l}\text { Grade } \\
\text { (\% Zn) }\end{array}$ & Host rocks & Host rock Age & $\begin{array}{l}\text { Related } \\
\text { Structures }\end{array}$ & Ore minerals & Alteration & $\begin{array}{l}\mathrm{T}_{H} \\
\left({ }^{0} \mathrm{C}\right)\end{array}$ & $\begin{array}{l}\text { Salinity } \\
\text { (eq. wt } \\
\% \mathrm{NaCl})\end{array}$ & $\begin{array}{l}\text { Associated } \\
\text { Elements }\end{array}$ \\
\hline Beltana $^{1}$ & Australia & 0.86 & 37.9 & $\begin{array}{l}\text { limestone, } \\
\text { dolostone }\end{array}$ & Cambrian & $\begin{array}{l}\text { Beltana Fault, } \\
\text { district scale } \\
\text { folds, breccia, } \\
\text { karst }\end{array}$ & $\begin{array}{l}\text { Willemite, } \\
\text { coronadite, } \\
\text { hedyphane, } \\
\text { mimetite, } \\
\text { smithsonite }\end{array}$ & $\begin{array}{l}\text { Ferroan } \\
\text { dolomite, } \\
\text { hematite, } \\
\text { quartz }\end{array}$ & $\begin{array}{l}50- \\
170 \\
(w 1)\end{array}$ & $4-14$ & $\begin{array}{l}\mathrm{As}, \mathrm{Be}, \\
\mathrm{Cd}, \mathrm{Cu}, \\
\mathrm{Fe}, \mathrm{Ge}, \\
\mathrm{Mn}, \mathrm{Pb}, \\
\mathrm{Sb}, \mathrm{V}\end{array}$ \\
\hline Franklin ${ }^{2}$ & U.S.A. & 24 & 19.6 & marble & Neoproterozoic & $\begin{array}{l}\text { Zero Fault, } \\
\text { district scale } \\
\text { folds }\end{array}$ & $\begin{array}{l}\text { franklinite, } \\
\text { willemite, } \\
\text { zincite, } \\
\text { tephroite, }\end{array}$ & $\begin{array}{l}\text { rhodonite, } \\
\text { magnetite, } \\
\text { calcsilicates }\end{array}$ & $\begin{array}{l}650- \\
700\end{array}$ & $\mathrm{n} / \mathrm{a}$ & $\begin{array}{l}\mathrm{As}, \mathrm{B}, \\
\mathrm{Co}, \mathrm{Cu}, \\
\mathrm{Fe}, \mathrm{Mn}, \\
\mathrm{Ni}, \mathrm{Pb}, \\
\mathrm{V}, \mathrm{W}\end{array}$ \\
\hline Kabwe $^{3}$ & Zambia & $13^{8}$ & $20-25^{8}$ & dolostone & Neoproterozoic & $\begin{array}{l}\text { Mine Club } \\
\text { fault zone, } \\
\text { district scale } \\
\text { folds, minor } \\
\text { breccia }\end{array}$ & $\begin{array}{l}\text { sphalerite, } \\
\text { willemite, } \\
\text { galena }\end{array}$ & $\begin{array}{l}\text { Quartz, } \\
\text { hematite, } \\
\text { smithsonite, } \\
\text { cerussite }\end{array}$ & $\begin{array}{l}98- \\
178 \\
(\mathrm{sp})\end{array}$ & 11.5 & $\begin{array}{l}\mathrm{Ag}, \mathrm{Be}^{8}, \\
\mathrm{Cd}, \mathrm{Cu}, \\
\mathrm{Ga}, \mathrm{Ge}, \\
\mathrm{In}, \mathrm{V}, \mathrm{Pb}\end{array}$ \\
\hline $\begin{array}{l}\text { Vazante- } \\
\text { North } \\
\text { Extension } \\
\text { Trend }\end{array}$ & Brazil & $18^{4}$ & $19^{4}$ & dolostone & Neoproterozoic & $\begin{array}{l}\text { Vazante Shear } \\
\text { Zone, district } \\
\text { scale folds, } \\
\text { breccia }^{4}\end{array}$ & $\begin{array}{l}\text { willemite, } \\
\text { sphalerite, } \\
\text { franklinite, } \\
\text { sphalerite }^{4,5}\end{array}$ & $\begin{array}{l}\text { Quartz, } \\
\text { ferroan and } \\
\text { zincian } \\
\text { dolomite, } \\
\text { hematite }^{4}\end{array}$ & $\begin{array}{l}65- \\
180^{6} \\
(w 1)\end{array}$ & $3-15^{6}$ & $\begin{array}{l}\mathrm{Ag}, \mathrm{As}, \\
\mathrm{Be}, \mathrm{Bi}, \\
\mathrm{Cd}, \mathrm{Cu}, \\
\mathrm{Ge}^{7}, \mathrm{Fe}, \\
\mathrm{Hg}, \mathrm{In}, \\
\mathrm{Mo}, \mathrm{Pb}, \\
\mathrm{Sb}, \mathrm{Se}, \\
\mathrm{V}^{4}\end{array}$ \\
\hline
\end{tabular}

Table 3.3 Temperatures are taken from fluid inclusions hosted in the cited mineral, except for Franklin where the temperatures were estimated based on the equilibrium assemblage of $\mathrm{Fe}_{3} \mathrm{O}_{4}-\mathrm{Mn}_{3} \mathrm{O}_{4}-\mathrm{ZnFe}_{2} \mathrm{O}_{4}$.

${ }^{1}$ (Groves et al., 2003); ${ }^{2}$ (Frondel and Baum, 1974); ${ }^{3}$ (Kamona and Friedrich, 2007); ${ }^{4}$ (this study); ${ }^{5}$ ( Monteiro et al., 1999); ${ }^{6}$ (Dardenne and Freitas-Silva, 1999); ${ }^{7}$ (Monteiro et al., 2007), ${ }^{8}$ (Boni et al., 2011). 


\subsubsection{Guidelines for Exploration}

Based on the characteristics of the hypogene zinc silicate deposits studied in the Vazante region and those ones for other deposits described in the literature, the follow guidelines are proposed for their exploration. Hypogene zinc silicate deposits should be explored for in Neoproterozoic to Cambrian carbonate rocks that formed in epicontinental basins along shallow passive margins. These basins must have then subsequently undergone deformation during major orogenic events forming tectonic belts (e.g., Brasília Fold Belt, Zambezi Belt, and Adelaide Geosyncline). The orogenic event creates and reactivates structures suitable as ore fluid conduits and traps for fluid mixing.

On a district and local scale, shallow brittle fault zones and breccias associated with regional folds and faults should be targeted as they create a favorable environment for mixing between basinal brines and meteoric waters. Stratigraphic contacts may also be important for controlling fluid flow and mineral precipitation. With respect to Vazante, distal alteration containing enrichment in $\mathrm{Ag}, \mathrm{Cd}, \mathrm{Pb}$, and $\mathrm{Zn}$ is characterized by iron-carbonates and hematite in the areas surrounding the ore breccia. Geochemical signatures that show high concentrations of $\mathrm{Ag}, \mathrm{As}, \mathrm{Ba}, \mathrm{Be}, \mathrm{Bi}, \mathrm{Cu}, \mathrm{Fe}, \mathrm{Ge}, \mathrm{Hg}$, In, Mo, Ni, Pb, S, Sb, U, V, W, an d Zn are indicative of hydrothermal alteration of hematite breccias proximal to the willemite dominated breccias.

\subsection{Conclusions}

- The Northern Extension is hosted in dolomitized, passive margin carbonates.

- The Northern Extension has undergone several phases of brittle deformation, the tectonic-hydrothermal breccia forming in Vazante Northern Extension Shear Zone, interpreted to be coeval with the $\mathrm{D}_{2}$ folds observed in the less competent phyllitic units. 
These structures acted as fluid conduits and control the hydrothermal alteration and ore deposition.

- The tectonic-hydrothermal breccia can be classified into four distinct breccia types and from the earliest to latest form:

- Type 1 - distally located from the willemite ore, weakly brecciated with some Fe-carbonate alteration.

- Type 2 - Strong Fe-carbonate alteration, intense brecciation.

○ Type 3 - Hematite replacement breccia, usually proximal to the Type 4 Breccia.

○ Type 4 - willemite ore breccia, containing multiple willemite generations as well as hematite and franklinite.

- The main mineralizing event contains hypogene willemite ore; however, late $\mathrm{Pb}-\mathrm{Zn}$ and Ag-Cu sulfide veinlets were documented cross-cutting the Type 4 Breccia.

- The hydrothermally altered zones at the Northern Extension contains distinctly higher concentrations of $\mathrm{Ag}, \mathrm{Cd}, \mathrm{Pb}, \mathrm{Zn}$ and the willemite breccia has additionally high concentrations of $\mathrm{Cu}, \mathrm{Hg}, \mathrm{Pb}, \mathrm{Se}$, and $\mathrm{SiO}_{2}$ that correlate to losses in $\mathrm{Ca}$ and $\mathrm{Mg}$ due to replacement of carbonates in the system.

- The willemite ore is interpreted to be formed by mixing between metalliferous, low temperature, acidic, relatively saline fluid with low $\mathrm{S}$ content with more oxidizing meteoric fluids, leading the precipitation of abundant hematite and willemite. 


\section{Chapter 3 References}

Almeida, F.F.M., 1969, Evolucao tectônica do Centro-Oeste Brasileiro: Anais da Academia Brasileira de Ciências, p. 280-296.

Almeida, F.F.M., Hasui, Y., Brito Neves, B.B., and Fuck, R.A., 1981, Brazilian structural provinces: an introduction: Earth-Science Reviews, v. 17, p. 1-29.

Appold, M.S. and Monteiro, L.V.S., 2009, Numerical modeling of the hydrothermal zinc silicate and sulfide mineralization in the Vazante deposit, Brazil: Geofluids, v. 9, p. 96-115.

Azmy, K. Veizer, J., Misi, A., Oliveira, T.F., Sanches, A.L., and Dardenne, M.A., 2001, Dolomitization and isotope stratigraphy of the Vazante Formation, São Francisco Basin, Brazil: Precambrian Research, v. 112, p. 303-329.

Boni, M. and Large, D., 2003, Nonsulfide Zinc mineralization in Europe: An overview: Economic Geology, v. 98, p. 715-729.

Boni, M., Rosario, T., Balassone, G., Gleeson, S.A., and Matthews, A., 2011, The carbonatehosted willemite prospects of the Zambezi Metamorphic Belt, Zambia: Minerlia Deposita, v. 46, p. 707-729.

Brugger, J., McPhail, D.C., Wallace, M., and Waters, J., 2003, Formation of willemite in hydrothermal environments: Economic Geology, v. 98, p. 819-835.

Cunha, I.A., Misi, A., Babinski, M., and Iyer, S.S.S., 2007, Lead isotope constraints on the genesis of $\mathrm{Pb}-\mathrm{Zn}$ deposits in the Neoproterozoic Vazante Group, Minas Gerais, Brazil: Gondwana Research, v. 11, p. 382-395.

Dardenne, M.A., 1978, Geologia da Região de Morro Agudo, Minas Gerais. s.l., Sociedade Brasilera de Geologia, p. 68-84.

Dardenne, M.A., 2000, The Brasília Fold Belt, in: The tectonic evolution of South America: Rio de Janeiro, 31st International Geological Congress, p. 231-263.

Dardenne, M.A. and Freitas-Silva, F.H., 1999, Pb-Zn ore deposits of Bambuí and Vazante groups, in the São Francisco Craton and Brasília Fold Belt, Brazil, in: Base metal deposits of Brazil: Salvador, Ernesto von Sperling, p. 75-83. 
Dardenne, M.A. and Schobbenhaus, C., 2001, Metalogenese do Brasil: Brasília, University of Brasília.

Freitas-Silva, F.H. and Dardenne, M.A., 1994, Proposta de subdiviSão estratigrafica formal para o Grupo Canastra no oeste de Minas Gerais e leste de Goias: Goiana, Sociedade Brasilera de Geologia, p. 161-163.

Frondel, C. and Baum, J.L., 1974, Structure and mineralogy of the Franklin-zinc-iron-manganese deposit, New Jersey: Economic Geology, v. 69, p. 157-180.

Groves, I.M., Carman, C.E., and Dunlap, W.J., 2003, Geology of the Beltana Willemite Deposit, Flinders Range, South Australia: Economic Geology, v. 98, p. 797-818.

Hitzman, M.W., Reynolds, N.A., Sangster, D.F., Allen, C.R., and Carman, C.E., 2003, Classification, genesis, and exploration guides for nonsulfide zinc deposits: Economic Geology, v. 98, p. 685-714.

Kamona, A.F. and Friedrich, G.H., 2007, Geology, mineralogy, and stable isotopes of the Kabwe carbonate-hosted Pb-Zn deposit, Central Zambia: Ore Geology Reviews, v. 30, p. 217-243.

Madalosso, A. and Valle, C.R.O., 1978, Consideraçoẽs sobre a estratigrafia e sedimentologia do Grupo Bambuí na região de Paracatú - Morro Agudo, MG: s.l., Sociedade Brasileira de Geociências, p. 622-634.

Martins-Neto, M.A., 2009, Sequence stratigraphic framework of Proterozoic successions in eastern Brazil: Marine and Petroleum Geology, V. 26, p. 163-176.

Misi, A., Iyer, S.S.S., Coelho, C.E.S., Tassinari, C.C.G., Franca-Rocha, W.J.S., Cunha, I.A., Gomes, A.S.R., Oliveira, T.F., Teixeira, J.B.G., and Filho, V.M.C., 2005, Sediment hosted lead-zinc deposits of the Neoproterozoic Bambuí Group and correlative sequences, São Francisco Craton, Brazil: A review and a possible metallogenic evolution model: Ore Geology Reviews, v. 26, p. 263-304.

Misi, A., Kaufman, A.J., Veizer, J., Powis, K., Azmy, K., Boggiani, P.C., Gaucher, C., Teixeira, J.B.G., Sanches, A.L., and Iyer, S.S.S., 2007, Chemostratigraphic correlation of Neoproterozoic successions in South America: Chemical Geology v. 237, p. 143-167. 
Monteiro, J.V.S., Bettencourt, J.S., Spiro, B., Graca, R., and Oliveira, T.F., 1999, The Vazante zinc mine, Minas Gerais, Brazil: constraints on willemitic mineralization and fluid evolution: Exploration Mining Geology, v. 8, p. 21-42.

Monteiro, L.V.S., Bettencourt, J.S., Juliani, C., and Oliveira, T.F., 2006, Geology, petrography, and mineral chemistry of the Vazante non-sulfide and Ambrosia and Fagundes sulfide-rich carbonate-hosted Zn-(Pb) deposits, Minas Gerais, Brazil: Ore Geology Reviews, v. 28, p. 201-234.

Monteiro, L.V.S., Bettencourt, J.S., Juliani, C., and Oliveira, T.F., 2007, Nonsulfide and sulfiderich zinc mineralizations in the Vazante, Ambrosia and Fagundes deposits, Minas Gerais, Brazil: Mass balance and stable isotope characteristics of the hydrothermal alterations: Gondwana Research, v. 11, p. 362-381.

Pimentel, M.M., Dardenne, M.A., Fuck, R.A., Viana, M.G., Junges, S.L., Fischel, D.P., Seer, H.J., and Dantas, E.L., 2001, Nd isotopes of the provenance of detrital sediments of the Neoproterozoic Brasília Belt, central Brazil: Journal of South American Earth Sciences, v. 14, p. 571-585.

Pinho, J.M.M., 1990, Evolução tectônica da mineralizacao de zinco de Vazante, Brasília: Master's thesis, Brasília, Universidade de Brasília.

Rigobello, A.E., Branquinho, J.A., Silva Dantas, M.G., Oliveira, T.F., and Neves Filho, W., 1988, Mina de zinco de Vazante, Minas Gerais, in: Principais Depositos Minerais do Brasil: Brasília, DNPM, p. 101-110.

Riveros, P.A. and Dutrizac, J.E., 1997, The precipitation of hematite from ferric chloride media: Hydrometallurgy, v. 46, p. 85-104.

Rodrigues, J.B., Pimentel, M.M., Dardenne, M.A., and Armstrong, R.A., 2010, Age, provenance and tectonic setting of the Canastra and Ibiá Groups (Brasília Belt, Brazil): Implications for the age of a Neoproterozoic glacial event in central Brazil: Journal of South American Earth Sciences, v. 29, p. 512-521.

Rostirolla, S.P., Mancini, F., Reis Neto, J.M., Figueira, E.G., Araujo, E.C., 2002, Analise estrutural da mina de Vazante e adjacencias: Geometria, cinematica, e implicacoes para a hidrogeologia: Revista Brasileira de Geociências, v. 32, p. 59-68. 
U.S. Geological Survey, Tolcin, A.C., 2011, Minerals Yearbook: Zinc

(http://minerals.er.usgs.gov/minerals/pubs/commodity/zinc/myb1-2009-zinc.pdf)

Valeriano, C.M., Pimentel, M.M., Heilbron, M., Almeida, J.C.H., and Trouw, R.A.J., 2008, Tectonic evolution of the Brasília Belt, Central Brazil, and early assembly of Gondwana: Geological Society, London, Special Publications, v. 294, p. 197-210.

Warren, J.K., 2006, Evaporites: sediments, resources, and hydrocarbons: Sturtz, Springer. 


\section{Chapter 4}

\section{Final Remarks}

\subsection{Discussion and Conclusions}

The Northern Extension willemite ore is hosted in dolomitized, passive margin carbonates that have undergone greenschist facies metamorphism and several phases of ductile and brittle deformation. The tectonic-hydrothermal breccia that hosts the ore is controlled by the VazanteNorthern Extension Shear Zone in the contact between the Upper Morro do Pinheiro and Upper Pamplona members of the Serra do Poço Verde Formation. This structure is interpreted to have formed during the $\mathrm{D}_{2}$ phase along with the major folds observed in the less competent phyllitic units and acted as a conduit for the hydrothermal fluids. Four breccia types (Fig. 1) were identified in the North Vazante Shear zone and they range from the initial, weakly altered Type 1 Breccia, to the more strongly altered Type 2 , to the Type 3 hematite and Type 4 willemite replacement breccias. The willemite mineralization is associated with hematite and franklinite, and is characterized by early hexagonal crystalline willemite ( $1^{\text {st }}$ generation $)$ that has been overgrown by later lath-shaped willemite ( $2^{\text {nd }}$ generation $)$, which, in turn, is being overgrown by the $3^{\text {rd }}$ generation, of fibrous willemite veinlets. In addition to the hypogene willemite ore, late $\mathrm{Pb}-\mathrm{Zn}$ and $\mathrm{Ag}-\mathrm{Cu}$ sulfide veinlets were documented to have cut the Type 4 Breccia. The various breccia types at the Northern Extension contains distinctly higher concentrations of $\mathrm{Ag}, \mathrm{Cd}, \mathrm{Pb}$, $\mathrm{Zn}$ as well as moderate gains of $\mathrm{Bi}, \mathrm{Co}, \mathrm{Cu}, \mathrm{Fe}, \mathrm{In}, \mathrm{Mn}, \mathrm{Mo}, \mathrm{Ni}, \mathrm{P}$, and $\mathrm{Sb}$. The willemite breccia has additionally high concentrations of $\mathrm{Cu}, \mathrm{Hg}, \mathrm{Pb}, \mathrm{Se}$, and $\mathrm{SiO}_{2}$ that correlate to losses in $\mathrm{Ca}$ and $\mathrm{Mg}$ due to replacement of carbonates in the system. 
Fig. 4.1: Genetic Model for the Vazante-Northern Extension

A) Stage 1
Initial brecciation
from tectonism and
hydraulic pumping of
basinal brine,
forming Type 1
Breccia

B) Stage 2 Continued brecciation from hydraulic pumping and hydrothermal alteration, forming Type 2 Breccia

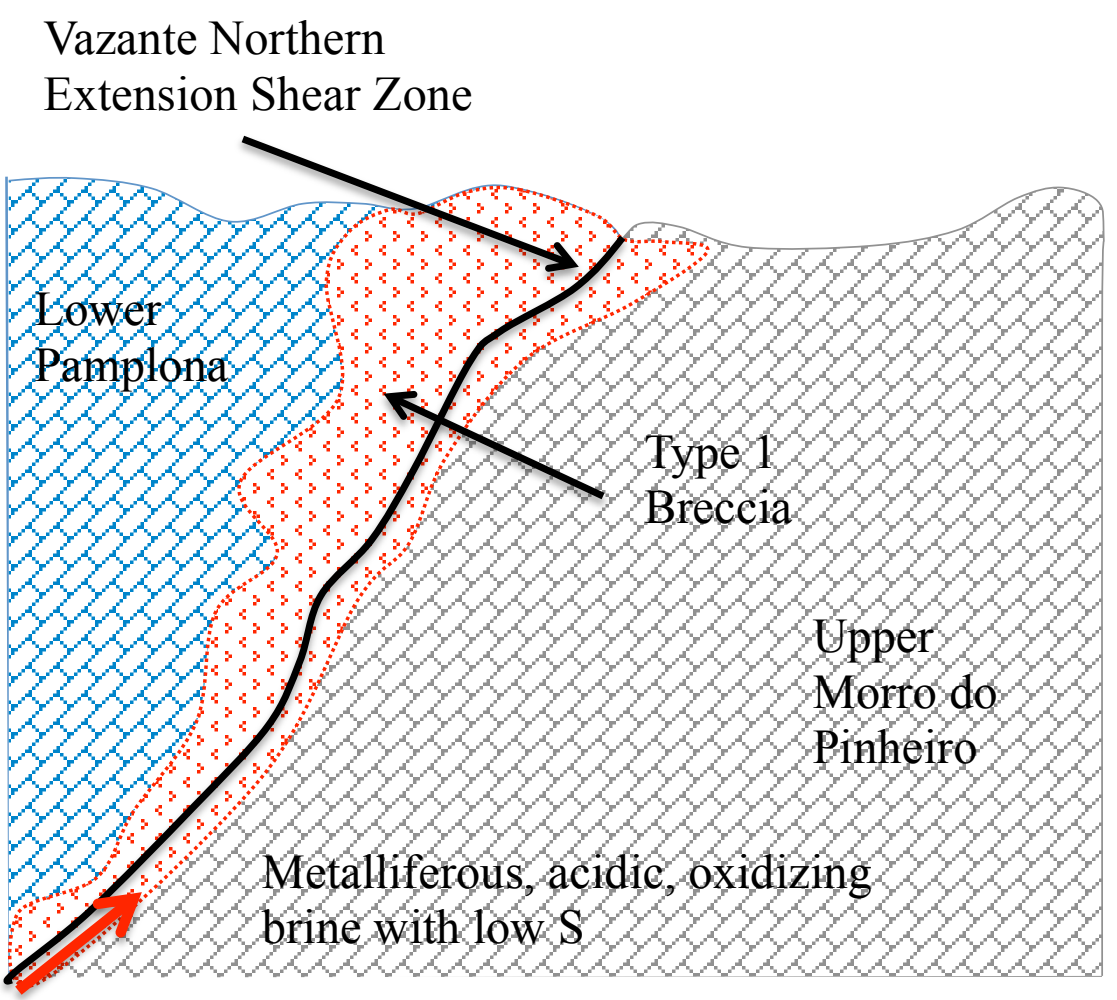

\section{Vazante-Northern \\ Extension Shear Zone}

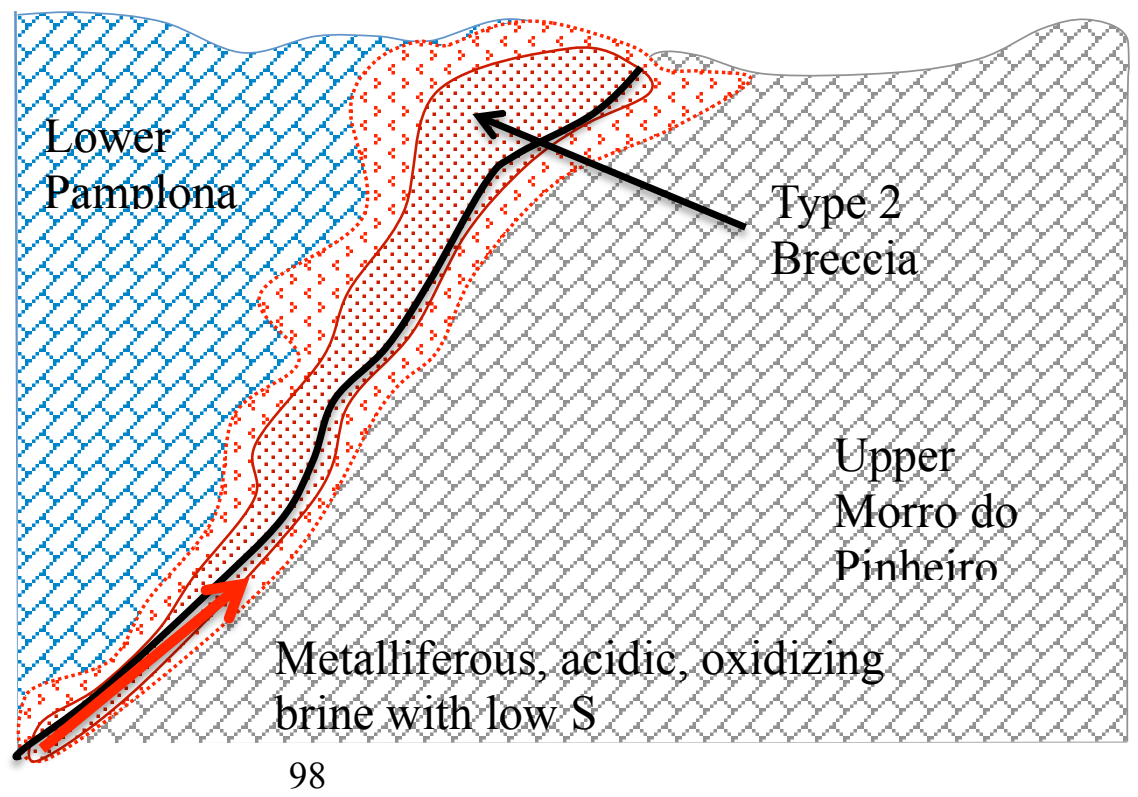


C) Stage 3 Mixing of cool, oxidizing, meteoric water with basinal brine, forming Type 3 Breccia (red)

\section{D) Stage 4}

Further mixing of cool, oxidizing, meteoric water with basinal brine, resulting in dilution and $\mathrm{pH}$ increase, forming Type 4 Breccia (blue)

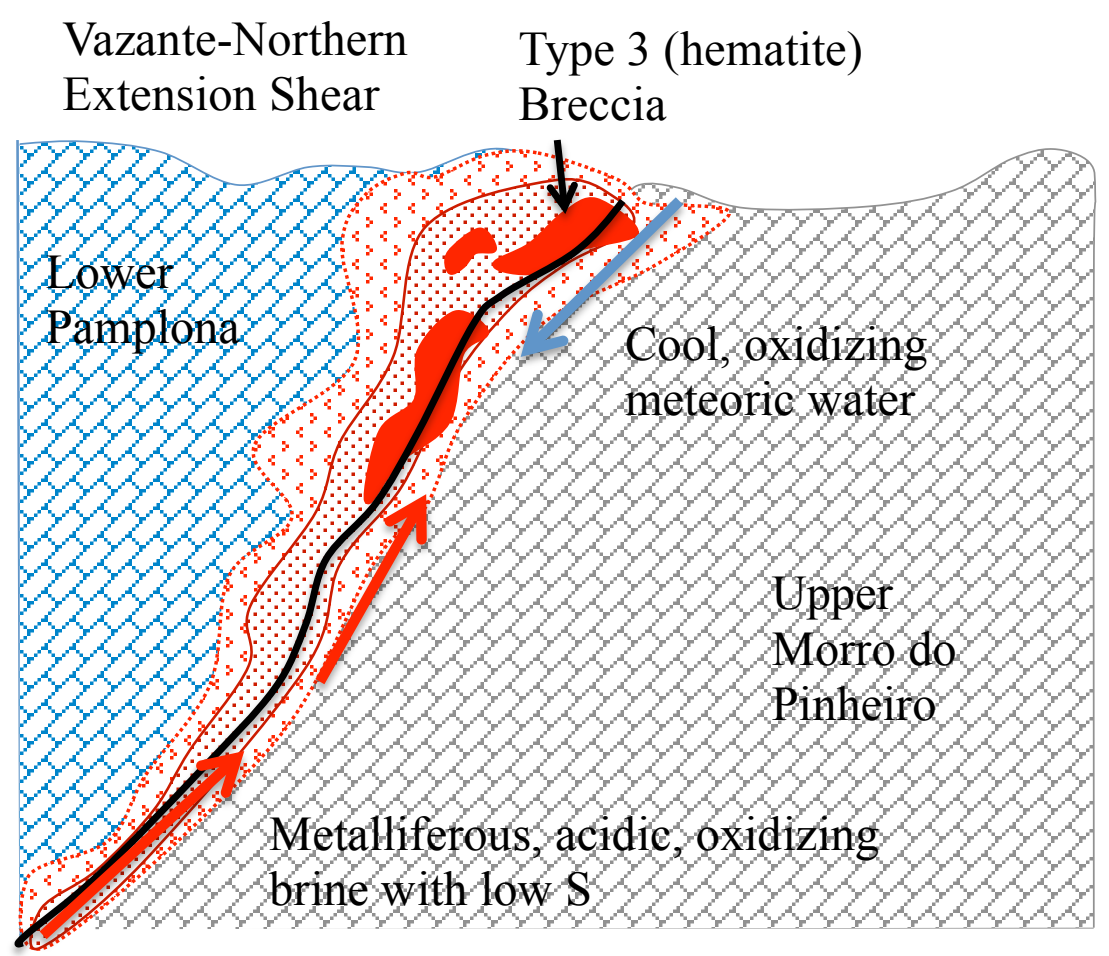
Vazante-Northern
Type 4 (willemite)
Extension Shear
Breccia

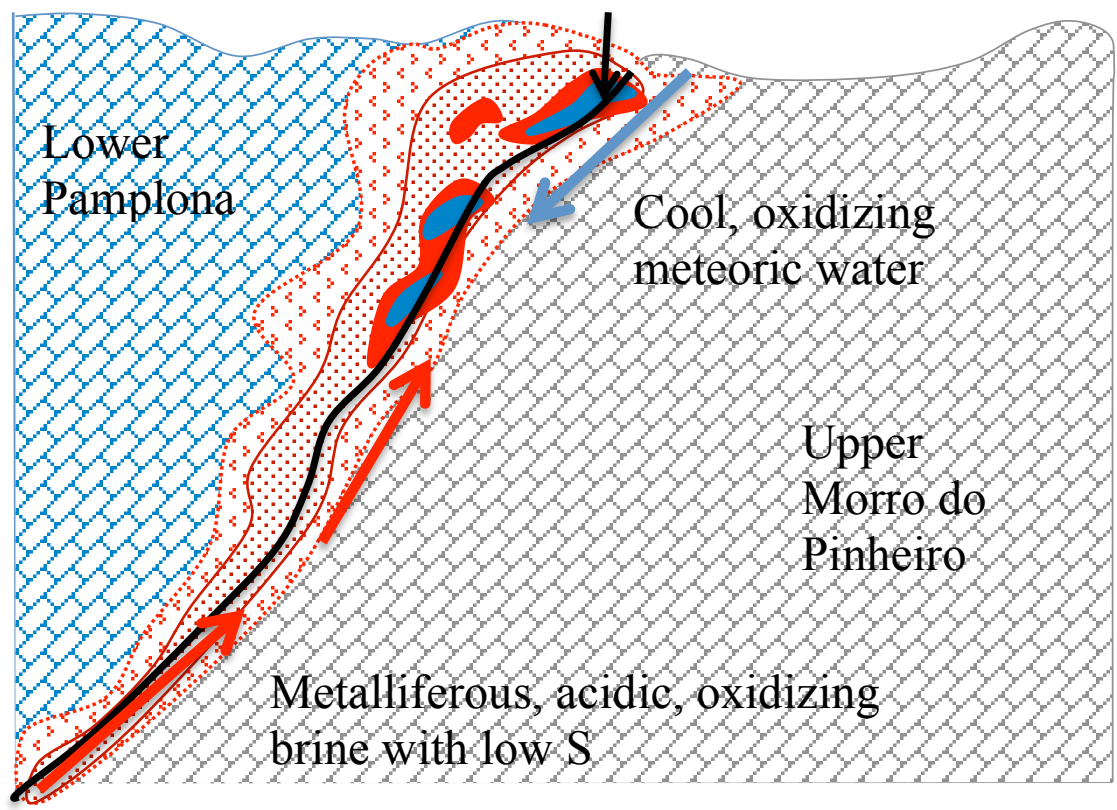


As discussed in this study, the Vazante-Northern Extension is a zinc silicate deposit, which is quite different from the other zinc deposits in the Brasília Fold Belt, particularly those in the Vazante Formation. The Morro Agudo Mine is a $\mathrm{Pb}-\mathrm{Zn}$ sulfide Irish-type deposit located in the northern part of the Vazante Group and also hosted in the carbonates of the Serra do Poço Verde Formation (Dardenne and Freitas-Silva, 1999; Dardenne, 2000; Monteiro et al, 2006; 2007). There are several possible scenarios to form two deposits that contain similar metals with different ore mineralogy (sulfide vs. silicate): 1) both the silicate and sulfide deposits originate from similar basinal brines, but they interact with different fluids in the site of deposition, prompting different minerals to precipitate or 2) the original metal-bearing brine is completely different in the amount of sulfur available.

With the first scenario, both deposits would have had similar basinal fluids that were relatively warm, metalliferous, oxidizing, and acidic. These fluids may have been similar to those found in MVT, Irish-type, or SEDEX deposits (Groves et al, 2003). In the case of the Morro Agudo deposit (sulfide), the basin fluids are focused into a fault, acting as a conduit, where they interact with S-bearing, reducing fluids containing reductants such as organic matter or methane, causing reduction of the fluid and the precipitation of $\mathrm{Zn}$ and $\mathrm{Pb}$ sulfides. With respect to the Vazante-Northern Extension deposit (silicate), the basin fluid does not mix with a S-bearing, reducing fluid; rather it interacts with the low temperature, meteoric water (as proposed in Chapter 3), where dilution and $\mathrm{pH}$ decrease cause willemite to precipitate (Appold and Monteiro, 2009).

In the second scenario, the amount of available $\mathrm{S}$ present in the basinal fluid could have been substantial for Morro Agudo and negligible for the Vazante-Northern Extension, causing sphalerite to have preferentially precipitated in Morro Agudo due to reduction of the $\mathrm{S}$ in the site 
of deposition; whereas willemite precipitated in Vazante during mineralization due to absence of reduced $\mathrm{S}$ and the presence of conditions ideal for the precipitation of $\mathrm{Zn}$ as a silicate.

When compared to the research conducted by Monteiro et al. $(1999,2006,2007)$ on the Vazante Mine, the Northern Extension shows many similarities. Both deposits are hosted in the carbonates of the Lower Pamplona Member, structurally controlled, dominated by willemite mineralization, and have a strong $\mathrm{Ag}, \mathrm{Cd}, \mathrm{Pb}, \mathrm{Zn}$ geochemical signature (Monteiro et al., 1999, 2006, 2007; this study).

Unlike the Vazante Mine, the Northern Extension deposit is located closer to the surface, outcrops in the very northern region of the deposit, and shows a greater influence of brittle deformation instead of the ductile deformation in recorded by Monteiro et al. (1999) in the Vazante Mine. Monteiro et al. $(1999,2006,2007)$ documented sphalerite in pods that were imbricated with the willemite ore, but very little sphalerite was observed in the Northern Extension except for small amounts located in late sulfide veinlets. In addition to the strong $\mathrm{Ag}$, $\mathrm{Cd}, \mathrm{Pb}, \mathrm{Zn}$ signature, Monteiro et al. (2007) also documented gains in La, $\mathrm{P}$, and $\mathrm{Rb}$ in the mineralized breccia; however, no substantial gains in La or P were observed in the Northern Extension, and it was observed that $\mathrm{Rb}$ was actually lost in the types 3 and 4 breccias in this study.

The Vazante-North Extension deposit has some similarities with other silicate deposits such as Beltana, Australia and Kabwe, Namibia (Groves et al, 2003; Kamona and Friedrich, 2007). They are hosted in carbonate rocks that formed during the Neoproterozoic to Cambrian, contain willemite and hematite as the major minerals in the ore zone, are enriched in $\mathrm{Cd}, \mathrm{Cu}, \mathrm{Ge}$, $\mathrm{Pb}$ and $\mathrm{V}+/-\mathrm{Be}$, and are interpreted to have been formed by low temperature fluids (50 to 180 
${ }^{\circ} \mathrm{C}$ ) of variable salinities (3-15 eq. wt \% NaCl) (Dardenne, 2000; Kamona and Friedrich, 2007, this study). Therefore they may have been formed by similar processes.

\subsection{Remaining Questions and Future Work}

As previously discussed in Chapter 3, the characterization of nonsulfide zinc deposits was well established in the Vazante-Northern Extension with respect to host rocks, structures, mineral paragenesis, and geochemistry (Monteiro et al 1999; 2006; 2007; Appold and Monteiro, 1999; this study); however, open questions still remain such as:

- What geologic processes may affect the precipitation of zinc silicates over zinc sulfides?

- What are the ages of zinc mineralization in the deposit and how does this compare to the other base metal sulfide deposits found in the Vazante Group?

- Does this trend of $\mathrm{Pb}-\mathrm{Zn}$ deposits in the Serra do Poço Verde Formation share a common fluid source?

- What are the different processes related to the formation of the sulfide deposits such as Morro Agudo compared to the Vazante Northern Extension?

- What are the isotopic characteristics of the willemite ore, and how it can help in identify the source of fluids and conditions of deposition?

- Why these deposits seem to be confined to a specific geological time (hosted in Neoproterozoic to Cambrian carbonate rocks)? 
In order to address these questions the following future work is recommended:

1) Correlative studies between the ages of known nonsulfide zinc deposits with respect to specific time periods and events, such as glaciation and changes in global oxygen levels

2) Age dating of the Serra do Poço Verde Formation using detrital zircon analysis or another robust geochronological system

3) Dating of the ore deposits in the Serra do Poço Verde Formation

a. Age determination of the hypogene ore in the Vazante-Northern Extension using $\mathrm{Rb}-\mathrm{Sr}$ and Sm-Nd dating techniques

b. Age determination of the sphalerite using Rb-Sr and $\mathrm{Sm}-\mathrm{Nd}$ in the Morro Agudo Mine as well as the Fagundes and Ambrôsia mineralizations

4) Fluid inclusion analysis to better compare the relationships between ore fluids in the BFB

a. In willemite for the Vazante Northern Extension

b. In sphalerite for the Morro Agudo Mine

5) More stable isotope analyses of the $\delta^{18} \mathrm{O}$ from willemite to create a more robust data set of formation temperatures and fluid sources 


\section{Chapter 4 References}

Appold, M.S. and Monteiro, L.V.S., 2009, Numerical modeling of the hydrothermal zinc silicate and sulfide mineralization in the Vazante deposit, Brazil: Geofluids, v. 9, p. 96-115.

Dardenne, M.A., 2000, The Brasília Fold Belt, in: The tectonic evolution of South America: Rio de Janeiro, 31st International Geological Congress, p. 231-263.

Dardenne, M.A. and Freitas-Silva, F.H., 1999, Pb-Zn ore deposits of Bambuí and Vazante groups, in the São Francisco Craton and Brasília Fold Belt, Brazil, in: Base metal deposits of Brazil: Salvador, Ernesto von Sperling, p. 75-83.

Groves, I.M., Carman, C.E., and Dunlap, W.J., 2003, Geology of the Beltana Willemite Deposit, Flinders Range, South Australia: Economic Geology, v. 98, p. 797-818.

Kamona, A.F. and Friedrich, G.H., 2007, Geology, mineralogy, and stable isotopes of the Kabwe carbonate-hosted Pb-Zn deposit, Central Zambia: Ore Geology Reviews, v. 30, p. 217-243.

Monteiro, J.V.S., Bettencourt, J.S., Spiro, B., Graca, R., and Oliveira, T.F., 1999, The Vazante zinc mine, Minas Gerais, Brazil: constraints on willemitic mineralization and fluid evolution: Exploration Mining Geology, v. 8, p. 21-42.

Monteiro, L.V.S., Bettencourt, J.S., Juliani, C., and Oliveira, T.F., 2006, Geology, petrography, and mineral chemistry of the Vazante non-sulfide and Ambrosia and Fagundes sulfide-rich carbonate-hosted Zn- $(\mathrm{Pb})$ deposits, Minas Gerais, Brazil: Ore Geology Reviews, v. 28, p. 201-234.

Monteiro, L.V.S., Bettencourt, J.S., Juliani, C., and Oliveira, T.F., 2007, Nonsulfide and sulfiderich zinc mineralizations in the Vazante, Ambrosia and Fagundes deposits, Minas Gerais, Brazil: Mass balance and stable isotope characteristics of the hydrothermal alterations: Gondwana Research, v. 11, p. 362-381. 


\section{Appendix A Field Notes and Measurements}

Field Measurements were taken using a Brunton Pocket Transit. The measurements were taken using azimuth and the "right-hand rule" for dip (e.g. when oriented along strike, the dip is measured $90^{\circ}$ perpendicular to strike on the right-hand side). Comments were recorded with respect to compositional layering, foliations, folds, faults, etc., in addition to the locations of each measurement.

\section{A.1 Field Measurements: Cavas 5A, 6A, 7A}

\begin{tabular}{|c|c|c|c|c|c|c|}
\hline Easting & Northing & $\frac{\text { elevation }}{\underline{(\mathbf{m})}}$ & $\underline{\text { strike }}$ & dip & $\stackrel{\operatorname{dip}}{\text { direction }}$ & $\underline{\text { notes }}$ \\
\hline 308039 & 8019190 & $\overline{649}$ & 224 & $\overline{43}$ & NW & bx layering (S0) \\
\hline 308030 & 8019193 & 659 & 30 & 45 & SE & fault plane \\
\hline 308021 & 8019192 & 670 & 196 & 30 & NW & bx layering (S0) \\
\hline 308012 & 8019210 & 675 & 145 & 24 & SW & \\
\hline 308012 & 8019210 & 675 & 140 & 42 & SW & S1 foliation \\
\hline 308005 & 8019221 & 683 & 240 & 66 & NW & fault \\
\hline 308001 & 8019199 & 686 & 100 & 16 & S & bx layering (S0) \\
\hline 307988 & 8019160 & 698 & 50 & 40 & SE & \\
\hline 307992 & 8019109 & 690 & 300 & 90 & & fault \\
\hline 308029 & 8019121 & 677 & 225 & 35 & NW & compositional layering (S0) \\
\hline 308017 & 8019101 & 684 & 65 & 62 & SE & fault plane \\
\hline 308017 & 8019101 & 684 & & & & $320 \rightarrow 55$ on previous fault plane \\
\hline 307999 & 8019079 & 690 & 225 & 54 & NW & compositional layering (S0) \\
\hline 307999 & 8019079 & 690 & 195 & 65 & NW & S1 foliation \\
\hline 308028 & 8018993 & 691 & 250 & 70 & NW & fault \\
\hline 308071 & 8019040 & 685 & 147 & 68 & SW & fault \\
\hline 308104 & 8019079 & 683 & 230 & 35 & NW & fault \\
\hline 308104 & 8019079 & 683 & 140 & 65 & SW & fault \\
\hline 308126 & 8019069 & 695 & 116 & 20 & SW & compositional layering (S0) \\
\hline 308126 & 8019069 & 695 & 145 & 52 & SW & S1 foliation \\
\hline 308126 & 8019069 & 695 & 170 & 61 & SW & fault \\
\hline 308159 & 8019081 & 701 & 275 & 29 & $\mathrm{~S}$ & compositional layering (S0) \\
\hline 308159 & 8019081 & 701 & 220 & 40 & NW & fault \\
\hline 307990 & 8019171 & 691 & 65 & 29 & SE & compositional layering (S0) \\
\hline
\end{tabular}




\begin{tabular}{|c|c|c|c|c|c|c|}
\hline \multirow{2}{*}{ Easting } & \multicolumn{2}{|c|}{ elevation } & \multicolumn{3}{|r|}{$\underline{\operatorname{dip}}$} & \multirow[b]{2}{*}{$\underline{\text { notes }}$} \\
\hline & Northing & $\underline{(\mathbf{m})}$ & $\underline{\text { strike }}$ & dip & direction & \\
\hline 308224 & 8019132 & 707 & 234 & 90 & & $\begin{array}{l}\text { compositional layering (S0) } \\
\text { and fault surface }\end{array}$ \\
\hline 380197 & 8019141 & 700 & 236 & 72 & NW & bx foliation $(\mathrm{S} 0)$ \\
\hline 308285 & 8019234 & 680 & 210 & 67 & NW & Saprolite \\
\hline 308194 & 8019239 & 671 & 132 & 75 & SW & fault \\
\hline 308173 & 8019236 & 670 & 130 & 56 & SW & fault \\
\hline 308137 & 8019295 & 680 & 12 & 56 & SE & fold limb \\
\hline 308137 & 8019295 & 680 & 26 & 74 & $\mathrm{SE}$ & $\begin{array}{l}\mathrm{S} 1 \text { foliation in fold limb } \\
\text { compositional layering ( } \mathrm{S} 0) \text {, }\end{array}$ \\
\hline 308130 & 8019298 & 686 & 54 & 31 & $\mathrm{SE}$ & fold limb \\
\hline 308128 & 8019298 & 686 & 106 & 35 & SW & $\begin{array}{l}\text { compositional layering (SO), } \\
\text { fold limb }\end{array}$ \\
\hline 308049 & 8019258 & 688 & 160 & 34 & SW & $\begin{array}{l}\text { compositional layering (S0) } \\
030 \rightarrow 30 \text { on preveious }\end{array}$ \\
\hline 308049 & 8019258 & 688 & & & & compositional layer \\
\hline 308061 & 8019305 & 701 & 189 & 39 & NW & compositional layering (S0) \\
\hline 308069 & 8019305 & 708 & 197 & 70 & NW & compositional layering (S0) \\
\hline 308092 & 8019318 & 711 & 34 & 46 & SE & compositional layering (S0) \\
\hline 308096 & 8019328 & 716 & 54 & 75 & SE & compositional layering (S0) \\
\hline 308118 & 8019342 & 720 & 45 & 36 & SE & compositional layering (S0) \\
\hline 308166 & 8019386 & 731 & 350 & 90 & & fault \\
\hline 308140 & 8019382 & 738 & 46 & 50 & SE & compositional layering (S0) \\
\hline 308106 & 8019381 & 734 & 200 & 53 & NW & compositional layering (S0) \\
\hline 308096 & 8019391 & 736 & 170 & 41 & $\mathrm{~W}$ & fault $w / 35^{\circ}$ rake \\
\hline 308096 & 8019391 & 736 & & & & $330 \rightarrow 29$ on previous fault \\
\hline 308089 & 8019460 & 740 & 122 & 32 & SW & compositional layering (S0) \\
\hline 308027 & 8019438 & 704 & 6 & 53 & $\mathrm{E}$ & compositional layering (S0) \\
\hline 308013 & 8019336 & 696 & 305 & 85 & $\mathrm{NE}$ & fault \\
\hline 308011 & 8019303 & 695 & 345 & 52 & $\mathrm{NE}$ & fault \\
\hline 307922 & 8019203 & 700 & 265 & 33 & $\mathrm{~S}$ & compositional layering (S0) \\
\hline 307883 & 8019045 & 717 & 260 & 21 & $\mathrm{~S}$ & compositional layering (S0) \\
\hline 307892 & 8019049 & 717 & 60 & 65 & SE & fault \\
\hline 307915 & 8018915 & 695 & 300 & 70 & $\mathrm{NE}$ & compositional layering (S0) \\
\hline 307885 & 8018836 & 680 & 270 & 33 & $\mathrm{~N}$ & compositional layering (S0) \\
\hline 307878 & 8018859 & 678 & 65 & 85 & $\mathrm{SE}$ & fault \\
\hline 307853 & 8018877 & 682 & 45 & 57 & $\mathrm{SE}$ & fault \\
\hline 307853 & 8018877 & 682 & 103 & 31 & SW & compositional layering (S0) \\
\hline 307996 & 8018716 & 680 & 155 & 39 & SW & compositional layering (S0) \\
\hline 308039 & 8018769 & 683 & 75 & 39 & SE & fault \\
\hline 308059 & 8018786 & 693 & 55 & 55 & SW & fault \\
\hline 308062 & 8018842 & 697 & 65 & 90 & & fault \\
\hline
\end{tabular}




\begin{tabular}{|c|c|c|c|c|c|c|}
\hline Easting & Northing & $\frac{\text { levation }}{\underline{(\mathrm{m})}}$ & $\underline{\text { strike }}$ & dip & $\underset{\text { direction }}{\underline{\operatorname{dip}}}$ & $\underline{\text { notes }}$ \\
\hline 308072 & 8018863 & 702 & 120 & 34 & SW & compositional layering (S0) \\
\hline 308096 & 8018917 & 706 & 65 & 44 & SE & fault \\
\hline 308061 & 8018881 & 702 & 75 & 71 & $\mathrm{SE}$ & shear zone \\
\hline 308032 & 8018917 & 698 & 130 & 40 & SW & compositional layering (S0) \\
\hline 308032 & 8018917 & 698 & 100 & 80 & $\mathrm{~S}$ & S1 foliation \\
\hline 307931 & 8018838 & 686 & 90 & 70 & $\mathrm{~S}$ & fault \\
\hline 307598 & 8018426 & 637 & 245 & 19 & SE & compositional layering (S0) \\
\hline 307596 & 8018429 & 638 & 315 & 60 & $\mathrm{NE}$ & fault \\
\hline 307583 & 8018405 & 639 & 39 & 10 & SE & compositional layering (S0) \\
\hline 307569 & 8018461 & 640 & 37 & 41 & SE & compositional layering (S0) \\
\hline 307571 & 8018428 & 645 & 40 & 30 & SE & compositional layering (S0) \\
\hline 307596 & 8018388 & 656 & 65 & 32 & SE & compositional layering (S0) \\
\hline 307559 & 8018428 & 662 & 50 & 70 & SE & fault \\
\hline 307549 & 8018425 & 666 & 64 & 45 & SE & compositional layering (S0) \\
\hline 307540 & 8018481 & 667 & 190 & 66 & $\mathrm{E}$ & fault \\
\hline 307545 & 8018495 & 666 & 61 & 26 & SE & compositional layering (S0) \\
\hline 307532 & 8018544 & 671 & 315 & 63 & $\mathrm{NE}$ & fault \\
\hline 307563 & 8018659 & 680 & 27 & 47 & SE & compositional layering (S0) \\
\hline 307564 & 8018692 & 688 & 341 & 40 & NE & compositional layering (S0) \\
\hline 307644 & 8018756 & 693 & 218 & 13 & NW & compositional layering (S0) \\
\hline 307578 & 8018765 & 701 & 64 & 7 & SE & compositional layering (S0) \\
\hline 307566 & 8019758 & 703 & 44 & 10 & SE & compositional layering (S0) \\
\hline 307557 & 8018733 & 703 & 53 & 8 & SE & compositional layering (S0) \\
\hline 307668 & 8018746 & 696 & 60 & 43 & SE & compositional layering (S0) \\
\hline 307739 & 8018780 & 695 & 169 & 66 & $\mathrm{~W}$ & fault \\
\hline 307755 & 8018607 & 666 & 142 & 24 & SW & compositional layering (S0) \\
\hline 307768 & 8018624 & 667 & 225 & 32 & NW & fault \\
\hline 307781 & 8018626 & 674 & 205 & 60 & NW & fault \\
\hline 307787 & 8018639 & 676 & 49 & 34 & SE & compositional layering (S0) \\
\hline 307848 & 8018603 & 680 & 50 & 20 & SE & compositional layering (S0) \\
\hline 307843 & 8018661 & 680 & 45 & 30 & SE & compositional layering (S0) \\
\hline 307804 & 8018696 & 680 & 155 & 36 & SW & fault \\
\hline 307758 & 8018660 & 656 & 185 & 90 & & fault \\
\hline 307754 & 8018681 & 655 & 200 & 10 & NW & compositional layering (S0) \\
\hline 307785 & 8018697 & 673 & 117 & 33 & SW & fault \\
\hline 307792 & 8018696 & 675 & 25 & 61 & SE & fault \\
\hline 307770 & 8018695 & 664 & 129 & 72 & SW & fault \\
\hline 307763 & 8018692 & 656 & 40 & 30 & SE & fault \\
\hline 307760 & 8018678 & 653 & 46 & 70 & SE & comp layering (fold limb) \\
\hline
\end{tabular}




\begin{tabular}{|c|c|c|c|c|c|c|}
\hline & & levation & & & & \\
\hline Easting & Northing & $\underline{(\mathbf{m})}$ & $\underline{\text { strike }}$ & dip & direction & notes \\
\hline 307760 & 8018678 & 653 & 40 & 30 & SE & $\begin{array}{l}\text { comp layering (fold limb) } \\
t \rightarrow p \text { offold axis for previous }\end{array}$ \\
\hline 307760 & 8018678 & 653 & $20 \rightarrow 9$ & & & 2 measurements \\
\hline 307771 & 8018680 & 655 & 150 & 50 & SW & fault \\
\hline 307729 & 8018730 & 666 & 31 & 41 & SE & compositional layering (S0) \\
\hline 307705 & 8018670 & 640 & 55 & 72 & SE & comp layering in shear zone \\
\hline 307692 & 8018668 & 628 & 212 & 34 & NW & fault \\
\hline 307688 & 8018686 & 628 & 10 & 85 & $\mathrm{E}$ & left fold limb (comp layering) \\
\hline 307688 & 8018686 & 628 & 172 & 60 & $\mathrm{~W}$ & right fold limb (comp layering) \\
\hline 307689 & 8018638 & 630 & 183 & 50 & $\mathrm{~W}$ & fault? Or part of previous fold \\
\hline 307740 & 8018680 & 645 & 22 & 48 & SE & comp layering (fold limb) \\
\hline 307740 & 8018680 & 645 & 224 & 23 & NW & comp layering (fold limb) \\
\hline 307710 & 8018646 & 634 & 90 & 22 & $\mathrm{~S}$ & compositional layering (S0) \\
\hline 307642 & 8018676 & 634 & 54 & 29 & SE & fault \\
\hline 307635 & 8018666 & 637 & 20 & 26 & SE & compositional layering (S0) \\
\hline 307625 & 8018675 & 656 & 60 & 30 & SE & fault \\
\hline 307714 & 8018626 & 642 & 215 & 36 & NW & fault \\
\hline 307717 & 8018606 & 644 & 210 & 29 & NW & fault \\
\hline 307685 & 8018398 & 645 & 170 & 29 & $\mathrm{~W}$ & fault \\
\hline 307676 & 8018363 & 636 & 199 & 38 & NW & fault \\
\hline 307660 & 8018313 & 630 & 195 & 16 & NW & compositional layering (S0) \\
\hline 307650 & 8018297 & 627 & 198 & 35 & NW & fault \\
\hline 307650 & 8018297 & 627 & 200 & 75 & NW & S1 foliation \\
\hline 307586 & 8018332 & 632 & 40 & 53 & $\mathrm{SE}$ & fault \\
\hline 307569 & 8018401 & 648 & 24 & 42 & SE & fault \\
\hline 307663 & 8018537 & 628 & 210 & 35 & NW & fault \\
\hline 307632 & 8018593 & 632 & 33 & 35 & SE & fault \\
\hline 307632 & 8018593 & 632 & 215 & 30 & NW & compositional layering (S0) \\
\hline 307863 & 8018569 & 728 & 64 & 20 & $\mathrm{SE}$ & compositional layering (S0) \\
\hline 307832 & 8018332 & 723 & 216 & 50 & NW & fault \\
\hline 307832 & 8018332 & 723 & 95 & 40 & $\mathrm{~S}$ & compositional layering (S0) \\
\hline 307749 & 8018385 & 720 & 216 & 50 & NW & fault \\
\hline 307748 & 8018340 & 715 & 75 & 26 & $\mathrm{SE}$ & compositional layering (S0) \\
\hline 307745 & 8018300 & 709 & 320 & 74 & NE & compositional layering (S0) \\
\hline 307561 & 8018208 & 690 & 200 & 15 & NW & compositional layering (S0) \\
\hline 307561 & 8018208 & 690 & 50 & 70 & SE & fault \\
\hline 307689 & 8018682 & 615 & 185 & 70 & $\mathrm{~W}$ & Masa ore zone orientation \\
\hline
\end{tabular}




\section{B.2 Field Measurements Cava 3A}

\begin{tabular}{|c|c|c|c|c|c|c|}
\hline Easting & Northing & $\frac{\text { elevation }}{\underline{(\mathbf{m})}}$ & strike & dip & $\underset{\text { direction }}{\text { dip }}$ & $\underline{\text { notes }}$ \\
\hline 307032 & 8016594 & 677 & 255 & 20 & NW & compositional layering (S0) \\
\hline 307000 & 8016564 & 671 & 225 & 8 & NW & compositional layering (S0) \\
\hline 307000 & 8016564 & 671 & 60 & 54 & SE & fault \\
\hline 306994 & 8016528 & 659 & 215 & 23 & NW & strike and dip fold (N plunge?) \\
\hline 307020 & 8016546 & 657 & 239 & 35 & NW & strike and dip fold (N plunge?) \\
\hline 307029 & 8016542 & 663 & 230 & 25 & NW & strike and dip fold (N plunge?) \\
\hline 307036 & 8016522 & 665 & 40 & 90 & & compositional layering (S0) \\
\hline 307024 & 8016497 & 677 & 40 & 57 & SE & strike and dip, fault \\
\hline 307007 & 8036477 & 583 & 123 & 16 & SW & compositional layering (S0) \\
\hline 307031 & 8016416 & 682 & 45 & 11 & $\mathrm{SE}$ & compositional layering (S0) \\
\hline 307062 & 8016442 & 685 & 110 & 46 & SW & compositional layering (S0) \\
\hline 307072 & 8016430 & 686 & 20 & 83 & SE & fault \\
\hline 307072 & 8016430 & 686 & 229 & 55 & NW & compositional layering (S0) \\
\hline 307086 & 8016429 & 687 & 47 & 25 & $\mathrm{SE}$ & compositional layering (S0) \\
\hline 307127 & 8016454 & 688 & 230 & 20 & NW & compositional layering (S0) \\
\hline 307104 & 8016453 & 684 & 345 & 45 & $\mathrm{NE}$ & strike and dip, fault \\
\hline 307093 & 8016454 & 682 & 115 & 27 & SW & compositional layering (S0) \\
\hline 307087 & 8016487 & 666 & 359 & 83 & $\mathrm{E}$ & fault \\
\hline 307049 & 8016514 & 668 & 80 & 19 & SE & compositional layering (S0) \\
\hline 307060 & 8016528 & 668 & 335 & 23 & $\mathrm{NE}$ & strike and dip, fold limb \\
\hline 307053 & 8016533 & 668 & 205 & 11 & NW & strike and dip, fold limb \\
\hline 307044 & 8016549 & 670 & 210 & 50 & NW & strike and dip, fold limb \\
\hline 307049 & 8016554 & 671 & 226 & 25 & NW & compositional layering (S0) \\
\hline 307073 & 8016574 & 678 & 260 & 25 & NW & $\begin{array}{l}\text { compositional layering (S0) } \\
\text { strike and dip, fold limb }\end{array}$ \\
\hline 307074 & 8016540 & 676 & 220 & 5 & NW & $\begin{array}{l}\text { (N plunge) } \\
\text { strike and dip, fold limb }\end{array}$ \\
\hline 307077 & 8016550 & 678 & 52 & 31 & SE & (N plung) \\
\hline 307141 & 8016698 & 677 & 235 & 23 & NW & compositional layering (S0) \\
\hline 307154 & 8016659 & 671 & 247 & 20 & NW & compositional layering (S0) \\
\hline 307161 & 8016625 & 673 & 32 & 35 & SE & compositional layering (S0) \\
\hline 307171 & 8016636 & 675 & 40 & 72 & $\mathrm{SE}$ & compositional layering (S0) \\
\hline 307178 & 8016642 & 677 & 320 & 73 & $\mathrm{NE}$ & $\begin{array}{l}\text { compositional layering (S0) } \\
82 \rightarrow 55 \text { Trend and plunge of }\end{array}$ \\
\hline 307185 & 8016649 & 679 & & & & fold axis \\
\hline 307157 & 8016620 & 676 & 66 & 40 & SE & compositional layering (S0) \\
\hline 307136 & 8016628 & 672 & 260 & 55 & NW & compositional layering (S0) \\
\hline 307096 & 8016624 & 666 & 321 & 28 & NE & compositional layering (S0) \\
\hline 307052 & 8016607 & 679 & 28 & 30 & SE & compositional layering (S0) \\
\hline
\end{tabular}




\begin{tabular}{|c|c|c|c|c|c|c|}
\hline & & levation & & & & \\
\hline Easting & Northing & $\underline{(\mathbf{m})}$ & $\underline{\text { strike }}$ & dip & direction & $\underline{\text { notes }}$ \\
\hline 307100 & 8016614 & 674 & & & & $\begin{array}{l}011 \rightarrow 60 \text { trend and plunge } \\
\text { of fold axis }\end{array}$ \\
\hline 307212 & 8016620 & 698 & 51 & 0 & & compositional layering (S0) \\
\hline 307209 & 8016601 & 706 & 55 & 4 & $\mathrm{SE}$ & compositional layering (S0) \\
\hline 307163 & 8016583 & 706 & 348 & 65 & $\mathrm{NE}$ & reverse fault, $\sim 10 \mathrm{~m}$ wide bx zone \\
\hline 307160 & 8016583 & 707 & 212 & 34 & NW & strike and dip fold limb \\
\hline 307163 & 8016576 & 708 & 58 & 14 & SE & strike and dip fold limb \\
\hline 307163 & 8016576 & 708 & & & & $\begin{array}{l}220 \rightarrow 10 \text { trend and plunge } \\
\text { strike and dip fold limb }\end{array}$ \\
\hline 307232 & 8016661 & 666 & 255 & 48 & NW & $\begin{array}{l}\text { (N plunge) } \\
\text { strike and dip fold limb }\end{array}$ \\
\hline 307219 & 8016672 & 665 & 15 & 35 & SE & (N plunge) \\
\hline 307215 & 8016672 & 665 & 222 & 89 & NW & compositional layering (S0) \\
\hline 307271 & 8016722 & 668 & 128 & 240 & SW & compositional layering (S0) \\
\hline 307273 & 8016729 & 664 & 202 & 50 & NW & compositional layering (S0) \\
\hline 307270 & 8016723 & 665 & 189 & 68 & $\mathrm{~W}$ & fault \\
\hline 307273 & 8016746 & 665 & 198 & 29 & NW & compositional layering (S0) \\
\hline 307335 & 8016758 & 669 & 230 & 15 & NW & compositional layering (S0) \\
\hline 307234 & 8016771 & 673 & 235 & 35 & NW & compositional layering (S0) \\
\hline 307401 & 8016778 & 656 & 15 & 17 & SE & compositional layering (S0) \\
\hline 307401 & 8016778 & 656 & 311 & $\sim 90$ & & fault \\
\hline 307370 & 8016793 & 654 & 87 & 32 & $S$ & compositional layering (S0) \\
\hline 307394 & 8016789 & 669 & 232 & 87 & NW & shear zone $\sim 1 \mathrm{~m}$ wide \\
\hline 307396 & 8016785 & 668 & 201 & 40 & NW & $\begin{array}{l}\text { strike and dip, right fold limb } \\
35 \rightarrow 29 \text { trend and plunge }\end{array}$ \\
\hline 307396 & 8016785 & 668 & & & & of fold axis \\
\hline 307396 & 8016785 & 668 & 234 & 30 & NW & strike an dip, left fold limb \\
\hline 307352 & 8016830 & 655 & 231 & 30 & NW & compositional layering (S0) \\
\hline 307374 & 8016843 & 660 & 220 & 11 & NW & compositional layering (S0) \\
\hline 307395 & 8016850 & 664 & 235 & 10 & NW & compositional layering (S0) \\
\hline 307396 & 8016848 & 666 & 40 & 62 & SE & fault \\
\hline 307433 & 8016867 & 672 & 239 & 38 & NW & compositional layering (S0) \\
\hline 307474 & 8016860 & 691 & 219 & 16 & NW & compositional layering (S0) \\
\hline 307467 & 8016924 & 676 & 203 & 26 & NW & compositional layering (S0) \\
\hline 307498 & 8016932 & 684 & 57 & 15 & SE & compositional layering (S0) \\
\hline 307355 & 8016986 & 632 & 81 & 3 & $\mathrm{~S}$ & compositional layering (S0) \\
\hline 307412 & 8012037 & 636 & 335 & 29 & NE & compositional layering (S0) \\
\hline 307441 & 8017048 & 640 & 202 & 18 & NW & compositional layering (S0) \\
\hline 307461 & 8017657 & 645 & 194 & 44 & NW & compositional layering (S0) \\
\hline 307462 & 8017035 & 649 & 222 & 26 & NW & compositional layering (S0) \\
\hline 307475 & 8016985 & 653 & 115 & $\begin{array}{l}43 \\
110\end{array}$ & SW & compositional layering (S0) \\
\hline
\end{tabular}




\begin{tabular}{|c|c|c|c|c|c|c|}
\hline Easting & Northing & $\frac{\text { elevation }}{\underline{(\mathbf{m})}}$ & strike & dip & $\stackrel{\operatorname{dip}}{\text { direction }}$ & $\underline{\text { notes }}$ \\
\hline 307484 & 8017000 & 656 & 185 & 67 & W & compositional layering (S0) \\
\hline 307496 & 8016998 & 665 & 165 & 68 & SW & compositional layering (S0) \\
\hline 307501 & 8016980 & 674 & 179 & 55 & $\mathrm{~W}$ & compositional layering (S0) \\
\hline 307532 & 8017018 & 679 & 29 & 23 & SE & compositional layering (S0) \\
\hline 307502 & 8017038 & 670 & 207 & 24 & NW & $\begin{array}{l}\text { compositional layering (S0) } \\
\text { strike and dip of very tight }\end{array}$ \\
\hline 307575 & 8017086 & 672 & 120 & 22 & SW & $\begin{array}{l}\text { fold limb } \\
\text { strike and dip of very }\end{array}$ \\
\hline 307579 & 8017075 & 681 & 286 & 55 & NE & tight fold limb \\
\hline 307576 & 8017143 & 676 & 204 & 47 & NW & compositional layering (S0) \\
\hline 307649 & 8017126 & 714 & 51 & 68 & SE & strike and dip of tight fold limb \\
\hline 307649 & 8017143 & 714 & 214 & 68 & NW & strike and dip of tight fold limb \\
\hline 307649 & 8017143 & 714 & & & & $210 \rightarrow 16 \mathrm{~T} \& \mathrm{P}$ \\
\hline 307674 & 8017202 & 710 & 84 & 46 & $\mathrm{~S}$ & compositional layering (S0) \\
\hline 307690 & 8017261 & 701 & 172 & 34 & $\mathrm{~W}$ & compositional layering (S0) \\
\hline 307715 & 8017285 & 702 & 107 & 37 & SW & compositional layering (S0) \\
\hline 307719 & 8017322 & 694 & 86 & 51 & $\mathrm{~S}$ & compositional layering (S0) \\
\hline 307721 & 8017332 & 691 & 10 & 8 & $\mathrm{E}$ & compositional layering (S0) \\
\hline 307718 & 8017348 & 689 & 20 & 26 & SE & compositional layering (S0) \\
\hline 307746 & 8017386 & 685 & 18 & 19 & SE & compositional layering (S0) \\
\hline 307804 & 8017389 & 706 & 128 & 50 & SW & compositional layering (S0) \\
\hline 307808 & 8017398 & 704 & 207 & 15 & NW & compositional layering (S0) \\
\hline 307796 & 8017424 & 696 & 202 & 16 & NW & compositional layering (S0) \\
\hline 307759 & 8017451 & 679 & 144 & 48 & SW & compositional layering (S0) \\
\hline 307773 & 8017461 & 684 & 76 & 30 & $\mathrm{~W}$ & compositional layering (S0) \\
\hline 307711 & 8017359 & 676 & 179 & 10 & $\mathrm{~W}$ & compositional layering (S0) \\
\hline
\end{tabular}




\section{Appendix B Drill Logs}

The drill logs were compiled in a similar manner with the same headings as used by the Votorantim Exploration Team at Vazante, MG. All intervals are recorded in meters.

\section{B.1 Drill Core Logs}

\begin{tabular}{|c|c|c|c|c|c|c|c|}
\hline Hole & $\underline{\text { From }}$ & $\underline{\text { To }}$ & Rock Type & $\underline{\text { Geologic Unit }}$ & $\underline{\text { Color }}$ & $\underline{\text { Texture }}$ & $\underline{\text { Alteration }}$ \\
\hline VZMSF 032 & 0 & 5.3 & altered bx & Hydrothermal Breccia & brown & bx & weathered bx \\
\hline VZMSF 032 & 5.3 & 38.4 & dol bx & Hydrothermal Breccia & red & sheared bx & slightly wthd, hm alt \\
\hline VZMSF 032 & 38.4 & 52.6 & dol bx & Hydrothermal Breccia & brown-red & sheared bx & hydrothermal/wthg? \\
\hline VZMSF 032 & 52.6 & 53.75 & dol bx & Hydrothermal Breccia & red-pink & soil & hydrothermal/wthg? \\
\hline VZMSF 032 & 53.75 & 73.7 & dol bx & Hydrothermal Breccia & gray & soil & hydrothermal/wthg? \\
\hline VZMSF 032 & 73.7 & 94.3 & dol bx & Hydrothermal Breccia & pink-violet & sheared bx & hydrothermal/wthg? \\
\hline VZMSF 032 & 94.3 & 96.5 & dol bx & Hydrothermal Breccia & brown & soil & hydrothermal/wthg? \\
\hline VZMSF 032 & 96.5 & 97.4 & phyllite & Serra do Garrote & gray & soil & hydrothermal/wthg? \\
\hline VZMSF 032 & 97.4 & 103.8 & phyllite & Serra do Garrote & dark gray & foliated & lesser hydrothermal alt \\
\hline VZMSF 032 & 103.8 & 106.95 & dol bx & Hydrothermal Breccia & pink & bx (minor shear) & \\
\hline VZMSF 032 & 106.95 & 122 & phyllite & Serra do Garrote & black & laminated shear & \\
\hline VZMSF 032 & 122 & 157.4 & dol bx & Hydrothermal Breccia & pink & bx & $\mathrm{hm}$ and Fe-carb \\
\hline VZMSF 032 & 157.4 & 200 & phyllite & Serra do Garrote & black & laminated shear & \\
\hline
\end{tabular}




\section{$\underline{P y} \underline{\text { Po }} \quad \underline{\text { pp }} \underline{\text { Sp }} \quad \underline{\text { Ga }} \underline{\text { Cl }} \underline{\text { Wl }}$ Shear $\underline{\text { Observations }}$}

\section{brown weathered breccia}

$\pm \quad$ broken sheared bx (red to pink, low recovery)

$\mathrm{x} \quad$ heavily altered bx to dirt and cobbles; silica and hm rich clasts more resistant to alteration

x hm-rich bx

$\mathrm{x} \quad$ altered $\mathrm{bx}$

$\mathrm{x} \quad$ well indurated bx with Fe-carb alteration

$\mathrm{x} \quad$ brown hm-rich dirt

$\mathrm{x} \quad$ altered phyllite (wthg?)

$\mathrm{x} \quad$ gray phyllite, part of minor fold limb of Serra do Garrote

$\mathrm{x}$ dolomite in center of minor fold ( Hydrothermal Breccia/Lower Morro do Pinhero?)

$\mathrm{x} \quad$ black phyllite (major fold limb)

$\mathrm{x}$ dolomite in center of major fold (Hydrothermal BrecciaLower Morro do Pinhero?)

$\mathrm{x}$ black shale on other side of fold limb, faulted and boudinaged 


\begin{tabular}{|c|c|c|c|c|c|c|c|c|}
\hline Hole & Area & From & $\underline{\text { To }}$ & Rock Type & Geologic Unit & Color & Texture & Alteration \\
\hline VZMSF 028 & Masa & 0 & 2.3 & soil & & pink-brown & & \\
\hline VZMSF 028 & Masa & 2.3 & 3.05 & bx & Lower Morro do Pinheiro? & red-gray & bx & Fe-carb alt \\
\hline VZMSF 028 & Masa & 3.05 & 4.35 & soil/bx & Lower Morro do Pinheiro? & brown & soil & wthg/hydrothermal alt \\
\hline VZMSF 028 & Masa & 4.35 & 8.6 & $\mathrm{dt}$ & Lower Morro do Pinheiro? & red-gray & bx & $\mathrm{hm}$ \\
\hline VZMSF 028 & Masa & 8.6 & 11.4 & dol & Lower Morro do Pinheiro? & red-gray & bx & $\mathrm{hm}$ \\
\hline VZMSF 028 & Masa & 11.4 & 13.3 & dol & Lower Morro do Pinheiro? & pink & bx & Fe-carb alt \\
\hline VZMSF 028 & Masa & 13.3 & 14.4 & dol & Lower Morro do Pinheiro? & gray & $\mathrm{bx}$ & minor hm \\
\hline VZMSF 028 & Masa & 14.4 & 21.15 & dol & Lower Morro do Pinheiro? & pink-red & bx & $\mathrm{hm}$ \\
\hline VZMSF 028 & Masa & 21.15 & 25.7 & soil/bx & Lower Morro do Pinheiro? & gray-brown & bx/soil & weathering $/ \mathrm{hm}$ \\
\hline VZMSF 028 & Masa & 25.7 & 39.65 & dol & Lower Morro do Pinheiro? & red-pink & $\mathrm{bx}$ & $\mathrm{hm}$ \\
\hline VZMSF 028 & Masa & 39.65 & 47.6 & dol & Lower Morro do Pinheiro? & pink & $\mathrm{bx}$ & Fe-carb alt \\
\hline VZMSF 028 & Masa & 47.6 & 57.2 & dol & Lower Morro do Pinheiro? & gray & bx & Fe-carb alt \\
\hline VZMSF 028 & Masa & 57.2 & 71.95 & dol & Lower Morro do Pinheiro? & pink-gray & $b x$ & Fe-carb alt \\
\hline VZMSF 028 & Masa & 71.95 & 87.5 & dol & Lower Morro do Pinheiro? & gray & bx & Fe-carb alt \& minor kaolinite \\
\hline VZMSF 028 & Masa & 87.5 & 108.1 & $\mathrm{dt}$ & Lower Morro do Pinheiro? & red-gray & dolorudite/bx & some Fe-carb \\
\hline
\end{tabular}




\section{$\underline{P y} \underline{\text { Po }} \quad \underline{\text { p }} \underline{\text { Sp }} \quad \underline{\text { Ga }} \underline{\text { l }} \underline{\text { Wl }} \underline{\text { Shear }} \underline{\text { Observations }}$}

red to gray bx with minor shear

dolorudite w/ some bx and Fe-carb veins

dolmitic bx w/ heavy hm and Fe-carb alteration

sheared dol bx (lot of core loss)

sheared gray dol bx

sheared bx with increasing hm as dept increases

gray to brown diret with bx from 24-24.5; lot of hm in soil

pink dol $\mathrm{w} / \mathrm{hm}$ bands and alteration

pink dol with gray dol clasts

gray dol bx w/ fault

pink and gray mottled dol bx

dark to light gray dol bx; 80.4-80.6 (kaol alt)

pink, red \& gray dolorudite with some bx 


\begin{tabular}{|c|c|c|c|c|c|c|c|}
\hline$\underline{\text { Hole }}$ & $\underline{\text { From }}$ & $\underline{\text { To }}$ & $\underline{\text { Rock Type }}$ & Geologic Unit & $\underline{\text { Color }}$ & $\underline{\text { Texture }}$ & $\underline{\text { Alteration }}$ \\
\hline VZMIF 141 & 0 & 10.25 & soil & Middle Pamplona? & brown & & \\
\hline VZMIF 141 & 10.25 & 15.25 & dol & Middle Pamplona? & gray & laminated $\mathrm{w} / \mathrm{bx}$ & \\
\hline VZMIF 141 & 15.25 & 18.5 & dol & Middle Pamplona? & gray & $b x$ & \\
\hline VZMIF 141 & 18.5 & 28.6 & dol & Middle Pamplona? & gray & mottled & kaol at $22.1 \& 24.4-24.95$ \\
\hline VZMIF 141 & 28.6 & 34.85 & dol & Middle Pamplona? & $\begin{array}{l}\text { gray } \\
\text { orange- }\end{array}$ & laminated & very minor silicic \\
\hline VZMIF 141 & 34.85 & 47.35 & soil & Middle Pamplona? & brown & & \\
\hline VZMIF 141 & 47.35 & 60.55 & dol & Middle Pamplona? & gray & laminated & silicic; some hm veins \\
\hline VZMIF 141 & 60.55 & 62.95 & dol & Middle Pamplona? & gray & dolarenite/dolorudite & \\
\hline VZMIF 141 & 62.95 & 68.5 & dol & Middle Pamplona? & gray & $\mathrm{bx}$ & minor hm veinlets \\
\hline VZMIF 141 & 68.5 & 71.3 & dol & Middle Pamplona? & gray & dolorudite \& mottled & \\
\hline VZMIF 141 & 71.3 & 79.9 & dol & Middle Pamplona? & pink & laminated & Fe-carb alt \\
\hline VZMIF 141 & 79.9 & 93.1 & dol & Lower Pamplona & gray & laminated & Fe-carb alt w/ hm \\
\hline VZMIF 141 & 93.1 & 123.25 & dol & Lower Pamplona & pink/gray & laminated w/ qtz vugs \& veins & silica w/ minor Fe-carb alt \\
\hline VZMIF 141 & 123.25 & 153.75 & dol & Upper Morro do Pinheiro & pink & bird's eyes & Fe-carb \\
\hline VZMIF 141 & 153.75 & 182.65 & dol bx & Hydrothermal Breccia & red & bx & Fe-carb w/ hm veins \\
\hline VZMIF 141 & 182.65 & 212.6 & dol/ phyllite & Upper Morro do Pinheiro & gray & laminated & \\
\hline VZMIF 141 & 212.6 & 220.7 & dol & Upper Morro do Pinheiro & pink & mottled & Fe-carb \& minor silica \\
\hline VZMIF 141 & 220.7 & 229.85 & dol & Upper Morro do Pinheiro & pink/gray & mottled w/ bird's eyes & Fe-carb \& minor silica \\
\hline
\end{tabular}




\section{$\underline{P y} \underline{\text { Po }} \underline{\mathrm{Cp}} \quad \underline{\mathrm{Sp}} \underline{\mathrm{Ga}} \underline{\mathrm{Cl}} \underline{\mathrm{Wl}} \underline{\text { Shear }} \underline{\text { Observations }}$}

$\mathrm{X}$

$\mathrm{X}$

$\mathrm{x}$

$\mathrm{X} ?$
$\mathrm{X}$

$\mathrm{x}$ ?

\section{bx $13.45-13.6$}

dol bx w/ minor hm \& sulfides

dolarenite $\mathrm{w} / \mathrm{kaol}$ alt; $26-27.35$ pink dolarenite $\mathrm{w} / \mathrm{hm}$ veins

gray dolarenite $w /$ vugs at $33.23 \& 34.4-34.85$

soil

gray dolarenite $\&$ massive dol

gray dolarenite \& dolorudite

intercalated laminated dolarenite \& mottled dolorudite; 63.2, 64.7,66.8 \& 71.0 are tectonic bx's mottled dolorudite \& micritic dol w/ tectonic \& sed bx

laminated pink dol, sheared at 78 , bx at $78.25 \& 78.75$

intercalated gray dol w/ greenish-red phyllites

pink \& gray dol w/ silicified vugs w/ Fe-carb (hm?) alt

pink dol w/ bird's eye texture; congl at 136-136.22; hm vein at 153.3

hydrothermal bx (Hydrothermal Breccia) w/ Zn mineralization

intercalated gray dol \& darker gray phyllites (phyllites)

mottled pink dol w/ silica \& Fe-carb filled vugs

mottled pink dol \& laminated; bird's eyes dolarenite (dk gray color) 


\begin{tabular}{|c|c|c|c|c|c|c|c|}
\hline Hole & From & $\underline{\text { To }}$ & Rock Type & Geologic Unit & Color & $\underline{\text { Texture }}$ & $\underline{\text { Alteration }}$ \\
\hline VZMIF 012 & 0 & 65 & dol & Middle? Pamplona & lt gray & laminated & slight wthg \\
\hline VZMIF 012 & 65 & 89.2 & dol & Middle? Pamplona & lt pink & laminated & \\
\hline VZMIF 012 & 89.2 & 108.6 & phyllite & Lower Pamplona & pink/green & laminated & minor silicic \\
\hline VZMIF 012 & 108.6 & 113.3 & phyllite & Lower Pamplona & green & laminated/bx & \\
\hline VZMIF 012 & 113.3 & 123.2 & phyllite & Lower Pamplona & pink/green & laminated minor bx & \\
\hline VZMIF 012 & 123.2 & 127.1 & phyllite & Lower Pamplona & gray & $\mathrm{bx}$ & highly silicic \\
\hline VZMIF 012 & 127.1 & 133.9 & dol \& phyllite & Lower Pamplona & red & $b x$ & minor silicic \\
\hline VZMIF 012 & 133.9 & 298.15 & dol \& phyllite & Lower Pamplona & white/green & laminated & \\
\hline VZMIF 012 & 298.15 & 336.1 & dol \& phyllite & Lower Pamplona & pink/green & laminated minor bx & minor Fe-carb \\
\hline VZMIF 012 & 336.1 & 398 & dol bx & Hydrothermal Breccia & pink/green & bx & $\mathrm{hm}$ veins \\
\hline VZMIF 012 & 398 & 430.1 & dol bx & Upper Morro do Pinheiro & pink/gray & bx & minor hm \\
\hline Hole & $\underline{\text { From }}$ & $\underline{\text { To }}$ & $\underline{\text { Rock Type }}$ & Geologic Unit & $\underline{\text { Color }}$ & $\underline{\text { Texture }}$ & $\underline{\text { Alteration }}$ \\
\hline VZMIF 127 & 0 & 20.65 & soil & $?$ & It red-brown & & wthg \\
\hline VZMIF 127 & 20.65 & 24.7 & soil & $?$ & beige & & wthg \\
\hline VZMIF 127 & 24.7 & 34.75 & soil & $?$ & beige & & wthg \\
\hline VZMIF 127 & 34.75 & 87.1 & dol & $\begin{array}{l}\text { Lower Pamplona } \\
\text { Hydrothermal Breccia (Lower }\end{array}$ & red \& gray & laminated & kaol, Fe-carb \\
\hline VZMIF 127 & 87.1 & 112.6 & dol bx & $\begin{array}{l}\text { Pamplona) } \\
\text { Hydrothermal Breccia (Upper }\end{array}$ & red \& gray & $\begin{array}{l}\text { bx } \\
\text { bx w/ oncolites and }\end{array}$ & hm \& Fe-carb alt \\
\hline VZMIF 127 & 112.6 & 150.3 & dol bx & $\begin{array}{l}\text { Morro do Pinheiro) } \\
\text { Hydrothermal Breccia (Upper } \\
\text { Morro do Pinheiro) }\end{array}$ & pink \& gray & $\begin{array}{l}\text { bird's eyes } \\
\text { bx w/ laminated \& } \\
\text { oncolite clastic } \\
\text { intervals }\end{array}$ & minor Fe-carb \\
\hline
\end{tabular}




\section{$\underline{\text { Py }} \underline{\text { Po }} \underline{\mathrm{Cp}} \underline{\mathrm{Sp}} \underline{\mathrm{Ga}} \underline{\mathrm{Cl}} \underline{\text { Wl }} \underline{\text { Shear }} \underline{\text { Observations }}$}

broken dol, minor kalo and silica

$\mathrm{x} \quad$ pink dol w/ green phyllites

fault marled

$\mathrm{x} \quad$ interbedded dolarenites and phyllites

$\mathrm{X}$

$\mathrm{x} \quad$ red dol bx

$\mathrm{x} \quad$ interbedded wht dolarenites and red-green phyllites

$\mathrm{x} \quad$ pink dol w/ green phyllites

$\mathrm{X}$

$\mathrm{x} \quad \mathrm{x}$ ?

$\mathrm{X}$

pink \& gray bx

alternating pink and gray bx

\section{$\underline{\text { Py }} \underline{\text { Po }} \underline{\text { Cp }} \underline{\text { Sp }} \underline{\text { Ga }} \underline{\text { Cl }} \underline{\text { Wl }} \underline{\text { Shear }}$ Observations}

x calamine zone

$\mathrm{X} \quad$ dol at $21.45 ; 27.75-28.0$

$\mathrm{x} \quad$ intercalated red \& gray dol w/ dk red phyllites; intermittent wthg zones to clay

Hydrothermal Breccia, very vuggy w/ core loss; lot of hm near ore zone (107.5-109.7);

sharp contact with $\mathrm{hm}$ and dol

$\mathrm{x} \quad$ alternating gray \& pink dol bx; uniform; oncolites in pink dol bx and bird's eyes in gray clasts 


\begin{tabular}{|c|c|c|c|c|c|c|c|}
\hline Hole & $\underline{\text { From }}$ & $\underline{\text { To }}$ & $\underline{\text { Rock Type }}$ & Geologic Unit & $\underline{\text { Color }}$ & $\underline{\text { Texture }}$ & Alteration \\
\hline VZMIF 136 & 0 & 27.97 & soil & & brown & & \\
\hline VZMIF 136 & 27.97 & 70.05 & dol/phyllite & Lower Pamplona & pink/lt gray & Algal laminated & kaol wthg/ minor Fe-carb \\
\hline VZMIF 136 & 70.05 & 74.4 & $\mathrm{dol} /$ soil & Lower Pamplona & pink/brown & vuggy & wthg to soil \\
\hline VZMIF 136 & 74.4 & 108.25 & dol & Lower Pamplona & pink & dolarenitic & hm \& Fe-carb (minor) \\
\hline VZMIF 136 & 108.25 & 117.1 & dol bx & Lower Pamplona & pink & bx & hm veins \\
\hline VZMIF 136 & 117.1 & 138.1 & dol bx & Hydrothermal Breccia & pink \& gray & bx & minor hm; silica; Fe-carb \\
\hline VZMIF 136 & 138.1 & 205.75 & dol & Hydrothermal Breccia & pink \& gray & bx & Fe-carb \\
\hline Hole & $\underline{\text { From }}$ & $\underline{\text { To }}$ & Rock Type & Geologic Unit & $\underline{\text { Color }}$ & $\underline{\text { Texture }}$ & $\underline{\text { Alteration }}$ \\
\hline VZMIF 143 & 0 & 74.3 & soil & Lower Pamplona? & & & \\
\hline VZMIF 143 & 74.3 & 77.05 & dol & Lower Pamplona? & lt gray & algal laminated & minor Fe-carb; minor wthg \\
\hline VZMIF 143 & 77.05 & 77.7 & soil & Lower Pamplona? & brown & & \\
\hline VZMIF 143 & 77.7 & 80.23 & dol & Lower Pamplona? & lt pink & algal laminated & minor Fe-carb; minor wthg \\
\hline VZMIF 143 & 80.23 & 81.6 & soil & Lower Pamplona? & & & \\
\hline VZMIF 143 & 81.6 & 96.8 & phyllite/dol & Lower Pamplona & red-gray to lt pink & $\begin{array}{l}\text { algal laminated } \\
\text { laminated phyllite; }\end{array}$ & trace Fe-carb veinlets \\
\hline VZMIF 143 & 96.8 & 120.7 & phyllite/dol & Lower Pamplona & lt gray to gray & dolarenite & \\
\hline VZMIF 143 & 120.7 & 150.95 & phyllite/dol & Lower Pamplona & gray/white & $\begin{array}{l}\text { laminated phyllite; marble } \\
\text { laminated phyllite; dol has }\end{array}$ & \\
\hline VZMIF 143 & 150.95 & 186.55 & phyllite/dol & Lower Pamplona & gray/pink & algal laminations & trace $\mathrm{Fe}$-carb veinlets \\
\hline
\end{tabular}




\section{$\underline{\text { Py }} \underline{\text { Po }} \underline{\text { Cp }} \underline{\text { Sp }} \underline{\text { Ga }} \underline{\text { Cl }} \underline{\text { Wl }}$ Shear $\underline{\text { Observations }}$}

$\mathrm{x} \quad$ vuggy, lt gray to lt pink dol intercalated $\mathrm{w} /$ red-brown phyllite

$\mathrm{x} \quad$ dol wthg to soil $\mathrm{w} /$ vugs at $72.95-74.4$

$\mathrm{x} \quad$ pink dol w/ Fe-carb \& hm veinlets; minor crackle bx

$\mathrm{x} \quad$ pink dol bx w/ massive hm veins at 133.4-113.55; 114.4-115.31

$\mathrm{x} \quad$ 125.55-125.7 (fault $\mathrm{w} /$ chlorite); pink and gray dol bx, specularite veins

$\mathrm{x} \quad$ pink \& gray dol bx with diamictite at $160-160.35 \& 160.75-161$; some silicic clasts and bird's eyes

\section{$\underline{P y} \quad \underline{P 0} \quad \underline{\mathrm{Cp}} \quad \underline{\mathrm{Sp}} \quad \underline{\mathrm{Ga}} \quad \underline{\mathrm{Cl}} \underline{\mathrm{Wl}} \underline{\text { Shear }} \underline{\text { Observations }}$}

lt gray dol, minor bx at 76.15

lt pink dol

soil $\mathrm{w} / \mathrm{hm}$

intercalated red-gray phyllites w/ pink dol

white to light gray dolarenite marbles intercalated $\mathrm{w} /$ gray phyllites $\mathrm{w} /$ trace py

intercalated gray shales/phyllites w/ white marble

intercalated gray shales/phyllites w/ pink \& gray dolomite 


\begin{tabular}{|c|c|c|c|c|c|c|c|}
\hline Hole & From & $\underline{\text { To }}$ & $\underline{\text { Rock Type }}$ & Geologic Unit & Color & Texture & Alteration \\
\hline VZMIF 122 & 0 & 70.8 & soil & & brown & & wthg \\
\hline VZMIF 122 & 79.3 & 86.55 & dol breccia & Hydrothermal Breccia & pink & dol bx & silica; hm \\
\hline VZMIF 122 & 188.55 & 193.85 & dol/phyllite & Upper Morro do Pinheiro & gray & bird's eyes & minor Fe-carb veins \\
\hline Hole & $\underline{\text { From }}$ & $\underline{\text { To }}$ & $\underline{\text { Rock Type }}$ & Geologic Unit & $\underline{\text { Color }}$ & Texture & Alteration \\
\hline VZMIF 110 & 17.8 & 34.6 & dol & Upper Pamplona & gray & mottled dolarenite & kaol wthg; vuggy \\
\hline VZMIF 110 & 34.6 & 107.4 & dolarenite & Upper Pamplona & gray & lightly mottled & silica; trace Fe-carb \\
\hline VZMIF 110 & 107.4 & 159.82 & dolorudite & Middle Pamplona & dark gray & algal laminated & silica; minor Fe-carb \\
\hline VZMIF 110 & 159.82 & 223.9 & dolarenite & Middle Pamplona & lt pink \& lt gray & mottled \& algal laminated & silica; trace hm vns \\
\hline VZMIF 110 & 223.9 & 344.25 & phyl/dolarenite & Lower Pamplona & gray \& lt gray & mottled \& algal laminated & minor Fe-carb \\
\hline VZMIF 020 & 42.15 & 83.83 & dolarenite & Middle Pamplona & gray & $\begin{array}{l}\text { massive dolarenite } \\
\text { laminated. minor aloal mats. }\end{array}$ & \\
\hline VZMIF 020 & 83.83 & 273 & phyll/ dol & Lower Pamplona & black/gray & sed bx & \\
\hline VZMIF 020 & 273 & 335.35 & dol breccia & Hydrothermal Breccia & red & brecciated & \\
\hline VZMIF 020 & 335.35 & 388.55 & dol & Upper Morro do Pinheiro & dark gray & bird's eyes \& algal layering & \\
\hline
\end{tabular}




\section{$\underline{\text { Py }} \underline{\text { Po }} \underline{\text { Cp }} \underline{\text { Sp }} \underline{\text { Ga }} \underline{\text { Cl }} \underline{\text { Wl }} \underline{\text { Shear }} \underline{\text { Observations }}$}

$\pm \quad \pm$

pink dol bx w/ some hm veins

pink \& gray dolarenite interbedded $w /$ ruddy brown phyllite

dk gray dol w/ a few Fe-carb veins; bx at contact w/ L.P.

\section{$\underline{\text { Py }} \underline{\text { Po }} \quad \underline{\text { Cp }} \quad \underline{\text { Sp }} \quad \underline{\text { Ga }} \quad \underline{\mathrm{Cl}} \underline{\text { Wl }} \underline{\text { Shear }} \underline{\text { Observations }}$}

mottled lt and dk gray dolarenite

$\mathrm{x}$ ? gray, lightly mottled dolarenite

$\mathrm{x}$ dark gray dolorudite interbedded with some dolarenite

$\mathrm{x} \quad$ light pink to light gray dolarenite w/ silicified and hm veins

$\mathrm{x} \quad$ intercalated gray phyllites with lt gray and lt pink dolarenites; some orange areas; bx (291.8-292.8) dol with bird's eyes; vuggy, missing some intervals

\section{$\underline{\text { Py }} \underline{\text { Po }} \quad \underline{\text { Cp }} \quad \underline{\text { Sp }} \quad \underline{\text { Ga }} \quad \underline{\text { Cl }} \quad \underline{\text { Wl }} \underline{\text { Shear Observations }}$}

massive gray dolarinte $\mathrm{w} /$ small sedimentary bx near contact intercalated black shale and gray dolarenite

red dolomite bx (some bx phyllites) $\mathrm{Wl} \leq 1 \%$; high Bar (323.25-324.25)

dark gray dolomite $\mathrm{w} /$ bird's eys at end is crackle breccia 


\begin{tabular}{|c|c|c|c|c|c|c|c|}
\hline Hole & $\underline{\text { From }}$ & $\underline{\text { To }}$ & $\underline{\text { Rock Type }}$ & Geologic Unit & $\underline{\text { Color }}$ & $\underline{\text { Texture }}$ & $\underline{\text { Alteration }}$ \\
\hline VZMIF 114 & 0 & 0.8 & soil & Lower Pamplona & pinkish brown & & \\
\hline VZMIF 114 & 0.8 & 25.55 & dol & Lower Pamplona & pink & laminated with some bx & Fe-carb \& silica \\
\hline VZMIF 114 & 25.55 & 42.5 & dol & Lower Pamplona & buff & kaol wthg & \\
\hline VZMIF 114 & 42.5 & 42.75 & $\mathrm{hm}$ & Lower Pamplona & red & vuggy hm & $\mathrm{hm}$ \\
\hline VZMIF 114 & 42.75 & 49.55 & dol & Lower Pamplona & pink & laminated vuggy & Fe-carb \& hm veinlets \\
\hline VZMIF 114 & 49.55 & 51 & vug & Lower Pamplona & & & \\
\hline VZMIF 114 & 51 & 51.4 & soil & Lower Pamplona & brown & & \\
\hline VZMIF 114 & 51.4 & 54.4 & vug & Lower Pamplona & & & \\
\hline VZMIF 114 & 54.4 & 67.5 & dol & Lower Pamplona & pink & laminated vuggy & Fe-carb \& hm veinlets \\
\hline VZMIF 114 & 67.5 & 74.75 & vug & Lower Pamplona & & & \\
\hline VZMIF 114 & 74.75 & 88.85 & dol breccia & Hydrothermal Breccia & reddish gray & vuggy bx & $\mathrm{hm}$ and silica \\
\hline VZMIF 114 & 88.85 & 94.55 & dol breccia & Hydrothermal Breccia & pink to red & bx & Fe-carb \& hm \\
\hline VZMIF 114 & 94.55 & 115 & dol & Upper Morro do Pinhero & gray & laminated, crackle bx & trace Fe-carb \\
\hline
\end{tabular}




\section{$\underline{P y} \underline{\text { Po }} \quad \underline{\text { pp }} \underline{\text { Sp }} \quad \underline{\text { Ga }} \underline{\text { Cl }} \underline{\text { Wl }}$ Shear $\underline{\text { Observations }}$}

$\mathrm{x} \quad$ vuggy pink dolomite with silica alteration

$\mathrm{x} \quad$ orange to buff weathered dolomite with Mn dendrites

x vuggy; hm

$\mathrm{x} \quad$ pink dolomite with Fe-carb alteratioin $\& \mathrm{hm}$ veinlets; bx at 50.3

x vuggy

dol replaced by $\mathrm{Wm} \& \mathrm{hm}$, vugs increase at end of run (75.0-76.0 high $\mathrm{Ag}, \mathrm{Cu}, \mathrm{Pb}, \mathrm{Zn}$ ) end of thorough Fe-carb alteration in Hydrothermal Breccia zone

dark gray dol with faults and gouge; cataclasite appears after fault (downhole) 


\begin{tabular}{|c|c|c|c|c|c|c|c|}
\hline Hole & $\underline{\text { From }}$ & $\underline{\text { To }}$ & Rock Type & Geologic Unit & $\underline{\text { Color }}$ & $\underline{\text { Texture }}$ & $\underline{\text { Alteration }}$ \\
\hline VZMIF 115 & 0 & 49.25 & soil & Middle Pamplona & reddish brown & & \\
\hline VZMIF 115 & 49.25 & 52.55 & dol & Middle Pamplona & lt gray & kharst & kaol \& dissolution \\
\hline VZMIF 115 & 52.55 & 62.85 & dol & Middle Pamplona & pink \& gray & bird's eyeys \& vugs & silica alt \\
\hline VZMIF 115 & 62.85 & 87.3 & dol & Middle Pamplona & gray & laminated, minor bird's eyes & silica alt; minor hm \\
\hline VZMIF 115 & 87.3 & 90.6 & dol & Middle Pamplona & gray & bird's eye's \& kharst & kaol \& dissolution \\
\hline VZMIF 115 & 90.6 & 94.2 & dol & Middle Pamplona & gray & bird's eyes \& kharst & kaol \& dissolution \\
\hline VZMIF 115 & 94.2 & 122.3 & dol & Lower Pamplona & pink \& gray & bird's eyes \& bx & silica $\pm \mathrm{hm}$ \\
\hline VZMIF 115 & 122.3 & 123.85 & phyllite & Lower Pamplona & brown & laminated & carb (non-ferrous) \\
\hline VZMIF 115 & 123.85 & 169.2 & dol breccia & Hydrothermal Breccia & pink & bx & Fe-carb \& silica \\
\hline VZMIF 115 & 169.2 & 205.5 & dol & Lower Pamplona & pink \& gray & laminated & Fe-carb \& silica \\
\hline VZMIF 115 & 205.5 & 212.6 & dol & Lower Pamplona & red & bx \& dolarenite & $\mathrm{hm} \pm$ silica \\
\hline VZMIF 115 & 212.6 & 226.05 & dol breccia & Hydrothermal Breccia & red & $\mathrm{bx}$ & hm \& silica \\
\hline VZMIF 115 & 226.05 & 227.05 & dol breccia & Hydrothermal Breccia & pink & bx & silicia \\
\hline VZMIF 115 & 227.05 & 236.65 & dol breccia & $\begin{array}{l}\text { Hydrothermal Breccia } \\
\text { Lower Pamplona/ }\end{array}$ & pink & bx & silica, secondary dol \\
\hline VZMIF 115 & 236.65 & 244.75 & dol bx & $\begin{array}{l}\text { Hydrothermal Breccia } \\
\text { Lower Pamplona/ }\end{array}$ & gray \& pink & laminated w/ some bx & minor hm \\
\hline VZMIF 115 & 244.75 & 274 & dol bx & Hydrothermal Breccia & pink & lamellear w/ some bx & Fe-carb \\
\hline VZMIF 115 & 274 & 300.3 & dol & Upper Morro do Pinheiro & gray \& pink & algal layered, some bird's eyes & Fe-carb \\
\hline
\end{tabular}




\section{$\underline{P y} \quad \underline{P o} \quad \underline{C p} \quad \underline{\text { p }} \quad \underline{\text { Ga }} \underline{\mathrm{Cl}} \underline{\text { Wl }} \underline{\text { Shear }} \underline{\text { Observations }}$}

heavy silica alteration of dol

66.3-66.4 blk dol with qtz

minor silica alt at 88.1

minor silica alt

pink dol with bird's eyes; 105.55-108.35 bx with dark layers (algal?); hm alt scattered

$\mathrm{X}$

$\mathrm{X}$

$\mathrm{x}$

$\mathrm{X}$

bx w/ minor intervals of laminated dol pink \& gray dol w/ minor bx and gray-brown phyllites pink dol $\mathrm{b}$ w/ red hm alteration

Hydrothermal Breccia with sulfide mineralization (*TS)

Hydrothermal Breccia, minor fractures with silica overgrowths intercalated gray laminated dol w/ pink dol bx (secondary dol filling in bx) pink dol bx w/ gray spots and minor hm in bx; carb alteration at 265.7 gray algal layered dol w/ pink laminated dol and minor shear bx 


\begin{tabular}{|c|c|c|c|c|c|c|c|}
\hline$\underline{\text { Hole }}$ & From & $\underline{\text { To }}$ & Rock Type & Geologic Unit & Color & $\underline{\text { Texture }}$ & Alteration \\
\hline VZMIF 129 & 0 & 18.05 & soil & Middle Pamplona & reddish brown & & \\
\hline VZMIF 129 & 18.05 & 26.75 & dol & Middle Pamplona & lt gray & massive & Fe-carb veinlets \& silica \\
\hline VZMIF 129 & 26.75 & 29.3 & soil & Middle Pamplona & reddish brown & massive & \\
\hline VZMIF 129 & 29.3 & 48.1 & dol & Middle Pamplona & gray & massive & silica \\
\hline VZMIF 129 & 48.1 & 76.7 & dol & Middle Pamplona & pink & motled-massive & Fe-carb, minor silica \\
\hline VZMIF 129 & 76.7 & 158 & dol & Lower Pamplona & gray \& pink & dolarenite; vuggy & Fe-carb \& silica veins \\
\hline VZMIF 129 & 158 & 163.75 & dol breccia & Hydrothermal Breccia & pink & mottled w/ some dolarenite & Fe-carb w/ hm \\
\hline VZMIF 129 & 163.75 & 168.75 & dol breccia & Hydrothermal Breccia & pink & mineralized mottling \& veins & hm veins \\
\hline VZMIF 129 & 168.75 & 174.8 & dol breccia & Hydrothermal Breccia & pink & dol bx (no mins) & Fe-carb \pm hm veinlets \\
\hline VZMIF 129 & 174.8 & 187.35 & dol & Lower Pamplona & pink & slightly mottled & Fe-carb veins \\
\hline VZMIF 129 & 187.35 & 221 & dol & Lower Pamplona & gray \& pink & mottled & some Fe-carb veins \\
\hline VZMIF 129 & 221 & 232.9 & dol & Lower Pamplona & pink & bx & $\mathrm{Fe}$-carb \\
\hline VZMIF 129 & 232.9 & 250.1 & dol & Upper Morro do Pinheiro & gray & bird's eye \& algal laminated & trace Fe-carb, blanching \\
\hline
\end{tabular}




\section{$\underline{P y} \underline{\text { Po }} \quad \underline{\text { Cp }} \underline{\text { Sp }} \quad \underline{\text { Ga }} \underline{\text { Cl }} \underline{\text { Wl }} \underline{\text { Shear }} \underline{\text { Observations }}$}

light gray massive dol

$\mathrm{x} \quad$ gray dolomite $\mathrm{w} /$ soil at $44.2-44.45$

x pink dolarenite with vugs; soil at 49.9-50.25; , some minor clay alt

$\mathrm{x} \quad$ pink \& gray dolomite interbedded with red-brown phyllites; some silica alteration; wthg to soil

$\mathrm{x}$ pink dolomite $\mathrm{bx}$

$\mathrm{x} \quad \mathrm{x} \quad$ dolomite bx replaced by $\mathrm{Wm} \& \mathrm{Hm}(\mathrm{Hm}$ in veins)

$\pm \quad \mathrm{x} \quad$ little Zn mineralization; substantial Fe-carb alt

$\mathrm{x} \quad$ pink dolomite thoroughly veined with Fe-carb

$\mathrm{x} \quad$ gray and pink dolomite $\mathrm{w} /$ some shear fractures \& bx's, lots of mottling

$\mathrm{x} \quad$ pink dolomite bx w/ Fe-carb veins

$\mathrm{x} \quad$ gray dolomite $\mathrm{w} /$ minor $\mathrm{bx}$ 


\begin{tabular}{|c|c|c|c|c|c|c|c|}
\hline Hole & $\underline{\text { From }}$ & $\underline{\text { To }}$ & $\underline{\text { Rock Type }}$ & Geologic Unit & Color & $\underline{\text { Texture }}$ & $\underline{\text { Alteration }}$ \\
\hline VZMIF 124 & 0 & 34.35 & dol & $\begin{array}{l}\text { Hydrothermal Breccia } \\
\text { overprinting Lower Pamplona } \\
\text { Hydrothermal Breccia }\end{array}$ & pink & algal laminated & lots of Fe-carb veining \\
\hline VZMIF 124 & 34.35 & 40.1 & dol/soil & $\begin{array}{l}\text { overprinting Lower Pamplona } \\
\text { Hydrothermal Breccia }\end{array}$ & pink/brown & algal laminated & Fe-carb veins, soil wth'd \\
\hline VZMIF 124 & 40.1 & 47.1 & dol & $\begin{array}{l}\text { overprinting Lower Pamplona } \\
\text { Hydrothermal Breccia }\end{array}$ & pink & $\begin{array}{l}\text { algal laminated, vuggy } \\
\text { algal laminated, w/ }\end{array}$ & Fe-carb veins; minor wth'g \\
\hline VZMIF 124 & 47.1 & 51.2 & dol & $\begin{array}{l}\text { overprinting Lower Pamplona } \\
\text { Hydrothermal Breccia }\end{array}$ & red & some bx & heavey Fe-carb alt; hm veins \\
\hline VZMIF 124 & 51.2 & 59.9 & $\mathrm{dol} / \mathrm{bx}$ & overprinting Lower Pamplona & pink & bx w/ algal layering & Fe-carb alt \\
\hline VZMIF 124 & 59.9 & 62.9 & dol breccia & Hydrothermal Breccia & pink & bx & Fe-carb alt; minor silicic \\
\hline VZMIF 124 & 62.9 & 65.3 & dol breccia & Hydrothermal Breccia & red & bx & heavey Fe-carb alt; hm veins \\
\hline VZMIF 124 & 65.3 & 67.6 & dol breccia & Hydrothermal Breccia & pink & $\mathrm{bx}$ & Fe-carb alt; minor silicic \\
\hline VZMIF 124 & 67.6 & 73.15 & dol breccia & Hydrothermal Breccia & red & bx & heavey Fe-carb alt; hm veins \\
\hline VZMIF 124 & 73.15 & 79.45 & dol breccia & Hydrothermal Breccia & reddish-gray & mottled gray and red bx & hm \& Wl (Fe \& silicic) \\
\hline VZMIF 124 & 79.45 & 87.33 & dol breccia & Hydrothermal Breccia & pink & $\mathrm{bx}$ & Fe-carb alt; hm veins \\
\hline VZMIF 124 & 87.33 & 102.03 & dol/phyllite & Lower Pamplona & pink/gray & bx & Fe-carb veins \\
\hline VZMIF 124 & 102.03 & 103.85 & dol & Upper Morro do Pinheiro & $\begin{array}{l}\text { gray } \\
\text { gray with }\end{array}$ & bird's eyes & minor Fe-carb nodules \\
\hline VZMIF 124 & 103.85 & 130.15 & dol & Upper Morro do Pinhero & some pink & bx \& laminated & minor Fe-carb alt along fractures \\
\hline
\end{tabular}




\section{$\underline{\text { Py }} \quad \underline{\text { Po }} \quad \underline{C p} \quad \underline{S p} \quad \underline{G a} \quad \underline{\text { Cl }} \quad \underline{\text { Wl }} \quad \underline{\text { Shear }} \underline{\text { Observations }}$}

pink dol w/ Fe-carb alt, some hydrothermal bx and sed bx scattered throughout pink dol alternating $\mathrm{w} /$ brown soil; some core loss

pink dol w/ some wthg and small vugs

red dol w/ bx similar in appearance to Hydrothermal Breccia at 47.1-49.0 (thinner than BH) pink dol w/ periodic small bx's (no mineralization)

pink dol bx w/ minor hm (no wl); silicic alt $\rightarrow$ why lighter and less altered?

red dol w/ lot of Fe-carb alt \& hm shear veins (> $2 \mathrm{~cm}$ )

pink dol bx w/ minor hm (no wl); silicic alt $\rightarrow$ why lighter and less altered?

$\mathrm{X}$

red dol w/ lot of Fe-carb alt $\&$ hm shear veins (> $2 \mathrm{~cm}$ )

$\mathrm{X}$ bx w/ hm shear veins \& mottled gray Wl

$\mathrm{x} \quad$ pink dol bx w/ minor hm (no wl); silicic alt $\rightarrow$ why lighter and less altered?

$\mathrm{x} \quad$ pink \& gray dol bx intercalated w/ reddish-gray phyllites; some black shales also present (fold limb?)

$\mathrm{x} \quad$ gray dol w/ bird's eyes \& Fe-carb nodular alt $(\sim 3-5 \mathrm{~cm})$

gray \& pink laminated dolarenite $\mathrm{w} / \mathrm{dol}$ bx scattered throughout; some pelitic layers towards end 


\begin{tabular}{|c|c|c|c|c|c|c|c|}
\hline Hole & From & $\underline{\text { To }}$ & Rock Type & Geologic Unit & Color & Texture & $\underline{\text { Alteration }}$ \\
\hline VZMIF 134 & 0 & 17.6 & soil & Lower Pamplona & & & \\
\hline VZMIF 134 & 17.6 & 96.4 & dol/dolorudite & Lower Pamplona & $\begin{array}{l}\text { gray } \\
\text { dark }\end{array}$ & algal laminated & silica vugs and veins; some Fe-carb alt \\
\hline VZMIF 134 & 96.4 & 112.2 & dol & Upper Morro do Pinheiro & gray & bird's eyes & trace Fe-carb veinlets \\
\hline VZMIF 134 & 112.2 & 127 & dol bx & Hydrothermal Breccia & red & $\begin{array}{l}\text { sheared bx } \\
\text { bird's eyes; crackle }\end{array}$ & Fe-carb alt and hm veins \\
\hline VZMIF 134 & 127 & 160.6 & dol & Upper Morro do Pinheiro & gray & bx & some Fe-carb alt and carb alt (in bx) \\
\hline VZMIF 134 & 160.6 & 162.7 & phyllite & Upper Morro do Pinheiro & $\begin{array}{l}\text { black } \\
\text { dark }\end{array}$ & laminated & \\
\hline VZMIF 134 & 162.7 & 201.1 & dol & Lower Morro do Pinhero & gray & algal mottled & Fe-carb veins $\mathrm{w} / \mathrm{hm}$ \\
\hline
\end{tabular}

$\underline{\text { Py }} \underline{\text { Po }} \quad \underline{\text { Cp }} \quad \underline{\text { Sp }} \quad \underline{\text { Ga }} \quad \underline{\text { Cl }} \quad \underline{\text { Wl }}$ Shear Observations

$\mathrm{X}$

\author{
$\pm \quad$ light gray to gray dolarenite and dolorudite w/ algal layering, some minor algal mottling \\ $\pm \quad$ dark gray dol $\mathrm{w} /$ some dolorudite $\mathrm{w} /$ bird's eyes \\ $\pm \quad \mathrm{x} \quad$ red shear bx w/ hm veins, minor $\mathrm{Zn}$ (high Cd) \\ $\mathrm{x}$ crackle bx near Hydrothermal Breccia contact, dark gray dol w/ bird's eyes \\ $\mathrm{x} \quad$ black shale at base of UMdP, contains minor Py \\ dark gray dol w/ sporadic small bx lenses $(\sim 30-50 \mathrm{~cm})$; mottled algal texture
}




\begin{tabular}{|c|c|c|c|c|c|c|c|}
\hline Hole & $\underline{\text { From }}$ & $\underline{\text { To }}$ & Rock Type & Geologic Unit & $\underline{\text { Color }}$ & Texture & $\underline{\text { Alteration }}$ \\
\hline VZMIF 139 & 0 & 13.6 & soil & Middle Pamplona? & red-brown & & \\
\hline VZMIF 139 & 13.6 & 40.95 & dolarenite & Middle Pamplona? & $\begin{array}{l}\text { pink-gray } \\
\text { red-brown/ }\end{array}$ & $\begin{array}{l}\text { laminated dolarenite; } \\
\text { some bx } \\
\text { laminated dolarenite; }\end{array}$ & minor kaol; Fe-carb w/ hm; silica alt \\
\hline VZMIF 139 & 40.95 & 50.85 & soil/dol & Middle Pamplona? & gray & $\begin{array}{l}\text { vuggy } \\
\text { algal dolarenite \& }\end{array}$ & minor Fe-carb veinlets \\
\hline VZMIF 139 & 50.85 & 92.46 & dolarenite/dolorudite & Middle Pamplona? & $\begin{array}{l}\text { gray } \\
\text { dark }\end{array}$ & dolorudite & trace Fe-carb veinlets \\
\hline VZMIF 139 & 92.46 & 101.95 & phyllite/dol & $\begin{array}{l}\text { Lower Pamplona } \\
\text { Upper Morro do }\end{array}$ & gray/gray & $\begin{array}{l}\text { laminated } \\
\text { bird's eys w/ minor }\end{array}$ & wthg to kaol \\
\hline VZMIF 139 & 101.95 & 149.9 & dol & $\begin{array}{l}\text { Pinheiro } \\
\text { Upper Morro do }\end{array}$ & gray & $\begin{array}{l}\text { algal layering } \\
\text { algal laminated; some }\end{array}$ & silica veins \& filled vugs, some Fe-carb \\
\hline VZMIF 139 & 149.9 & 159.15 & phyllite/dol & $\begin{array}{l}\text { Pinheiro? } \\
\text { Upper Morro do }\end{array}$ & brown/gray & $\begin{array}{l}\text { sed bx } \\
\text { sheared bx (splay of } \\
\text { Hydrothermal }\end{array}$ & minor Fe-carb; chl alt \\
\hline VZMIF 139 & 159.15 & 205 & dol & $\begin{array}{l}\text { Pinheiro? } \\
\text { Upper Morro do }\end{array}$ & redish pink & Breccia?) & Fe-carb alt \\
\hline VZMIF 139 & 205 & 220.7 & dol & $\begin{array}{l}\text { Pinheiro? } \\
\text { Lower Morro do }\end{array}$ & dark gray & $\begin{array}{l}\text { bx w/ bird's eyes } \\
\text { algal laminated; }\end{array}$ & Fe-carb alt in bx \\
\hline VZMIF 139 & 220.7 & 262.75 & dol & $\begin{array}{l}\text { Pinheiro? } \\
\text { Lower Morro do }\end{array}$ & dark gray & $\begin{array}{l}\text { crackle bx } \\
\text { bx; dol }\end{array}$ & $\mathrm{Fe}$-carb \\
\hline VZMIF 139 & 262.75 & 301.7 & dol bx & $\begin{array}{l}\text { Pinheiro? } \\
\text { Hydrothermal }\end{array}$ & pink-gray & recrystallization & Fe-carb w/ hm veins \\
\hline VZMIF 139 & 301.7 & 318.05 & dol bx & Breccia & red & sheared bx & Fe-carb alt w/ hm \& silica alt \\
\hline
\end{tabular}




\section{$\underline{\mathbf{P y}} \quad \underline{\mathbf{P o}} \quad \underline{\mathrm{Cp}} \quad \underline{\mathrm{Sp}} \quad \underline{\mathrm{Ga}} \quad \underline{\mathrm{Cl}} \quad \underline{\text { Wl }} \quad \underline{\text { Shear }} \underline{\text { Observations }}$}

pink-gray dolarenite w/ minor bx's w/ sulfides in silicified bx; bx at 19.5-20.55 \& 25.5-28.5 alternating gray dol and red soil

intercalated dolarenite \& dolorudite; vuggy spots in veins and fractures w/ some kaol zones intercalted gray dol w/ gray phyllites, some wthg to light pink in phyllites gray dolarenite w/ bird's eyes \& algal layering; silica vugs and Fe-carb veinlets

$\mathrm{X}$

$\mathrm{X}$

$\mathrm{x}$

$\pm$ intercalated gray-brown dol (w/ sed bx's) and red-green phyllites pink to red sheared dol bx w/ minor spots of sheared dark red phyllites; faults also present pink \& gray bx (w/ bird's eyes) grading into dark gray dol w/ bird's eyes crackled gray dol w/ alagal laminations \& Fe-carb alt dol bx w/ red/white/gray clasts and dol overgrowths gray to mostly Hydrothermal Breccia; heavily fractures \& filled w/ hm and Fe-carb alt; some Wl 


\begin{tabular}{|c|c|c|c|c|c|c|c|}
\hline$\underline{\text { Hole }}$ & From & $\underline{\text { To }}$ & Rock Type & Geologic Unit & Color & $\underline{\text { Texture }}$ & Alteration \\
\hline VZMIF 123 & 0 & 5.7 & soil & Lower Pamplona & yellow-brown & & \\
\hline VZMIF 123 & 5.7 & 17.7 & dol/soil & Lower Pamplona & pink/brown & laminated & Fe-carb veins; kaol wthg \\
\hline VZMIF 123 & 17.7 & 19.7 & hm & Lower Pamplona & red & $\begin{array}{l}\text { vuggy } \\
\text { oncolites; some hm } \\
\text { veins; minor bx: }\end{array}$ & $\mathrm{hm}$ \\
\hline VZMIF 123 & 19.7 & 38.4 & dol/soil & Lower Pamplona & pink & vuggy & carb dissolution; Fe-carb w/ hm \\
\hline VZMIF 123 & 38.4 & 56.85 & dol/soil & Lower Pamplona & pink & laminated & some Fe-carb veinlets \\
\hline VZMIF 123 & 56.85 & 72.6 & soil/hm & Hydrothermal Breccia & brown/red & vuggy & $\mathrm{hm} \&$ dol wthd to soil \\
\hline VZMIF 123 & 72.6 & 79.55 & $\mathrm{hm} /$ silicates & Hydrothermal Breccia & red & massive hm \& wl & $\mathrm{hm}$ \\
\hline VZMIF 123 & 79.55 & 87.2 & Dol bx & Hydrothermal Breccia & pink/brown & bx & Fe-carb alt \\
\hline VZMIF 123 & 87.2 & 93.9 & soil & Hydrothermal Breccia? & white to brown & & \\
\hline VZMIF 123 & 93.9 & 129.36 & Do bx & Hydrothermal Breccia & pink/gray & bx & $\mathrm{hm}$ veins; Fe-carb alt \\
\hline
\end{tabular}




\section{$\underline{P y} \underline{\text { Po }} \quad \underline{\text { p }} \underline{\text { Sp }} \underline{\text { Ga }} \underline{\text { Cl }} \underline{\text { Wl }} \underline{\text { Shear }}$ Observations}

intercalted pink to ruddy brown dol w/ brown soil

$\mathrm{hm}$ replacement of dol, very vuggy

pink dol w/ thin intervals of soil; some hm veins; very vuggy (core loss)

pink dol w/ Fe-carb \& hm veinlets

$\mathrm{x} \quad$ massive $\mathrm{hm} \mathrm{w} / \mathrm{vugs}$ and altered to soil [vugs come after hm replacement]

$\mathrm{x}$

$\mathrm{X}$

dol replaced by hm \& wl (mod-high grade $15-40 \% \mathrm{Zn}$ ); very vuggy

$\mathrm{x} \quad$ dol bx (Hydrothermal Breccia) no mineralization

white soil that darkens in color with depth; possible elluvial horizon or hydrothermal alteration?

$\pm$ 


\begin{tabular}{|c|c|c|c|c|c|c|c|}
\hline Hole & From & $\underline{\text { To }}$ & $\frac{\text { Rock }}{\text { Type }}$ & Geologic Unit & Color & Texture & Alteration \\
\hline VZMIF 118 & 0 & 1.8 & soil & Middle Pamplona & brown & & \\
\hline VZMIF 118 & 1.8 & 27.45 & dol & Middle Pamplona & pink \& gray & algal laminated $w /$ minor bx & minor Fe-carb alt $\&$ wthg \\
\hline VZMIF 118 & 27.45 & 39.9 & dol/soil & Middle Pamplona & pink & algal laminated & minor Fe-carb alt \& wthg \\
\hline VZMIF 118 & 39.9 & 81.55 & dol & Middle Pamplona & pink & algal laminated & minor Fe-carb alt $\&$ wthg \\
\hline VZMIF 118 & 81.55 & 97.05 & soil & Middle Pamplona & shades of brown & & supergene calmine \\
\hline VZMIF 118 & 97.05 & 119.1 & dol & Middle Pamplona & pink & bx; oncolites; algal layering & minor Fe-carb vns \\
\hline VZMIF 118 & 119.1 & 126.62 & dol & Middle Pamplona & pink & algal layering and oncolites & Fe-carb veins \\
\hline VZMIF 118 & 126.62 & 135.3 & dol bx & Middle Pamplona & gray & bx; stromatolites & Fe-carb veins \\
\hline VZMIF 118 & 135.3 & 137.18 & dol & Upper Morro do Pinheiro & dark gray & bird's eyes & \\
\hline VZMIF 118 & 137.18 & 139.05 & shale & Upper Morro do Pinheiro & black & laminated & calcite veinlets \\
\hline VZMIF 118 & 139.05 & 162.8 & dol & Upper Morro do Pinheiro & pink & laminated $\mathrm{w} /$ some bx & Fe-carb veins \\
\hline VZMIF 118 & 162.8 & 163.8 & shale & Upper Morro do Pinheiro & black & laminated & calcite veinlets \\
\hline VZMIF 118 & 163.8 & 165.2 & dol & Lower Morro do Pinheiro & dark gray & mottled & hydrothermal dolomite \\
\hline
\end{tabular}




\section{$\underline{\text { Py }} \underline{\text { Po }} \underline{\text { Cp }} \underline{\text { Sp }} \underline{\text { Ga }} \underline{\text { Cl }} \underline{\text { Wl }} \underline{\text { Shear }} \underline{\text { Observations }}$}

intercalated pink \& gray dol (sheared); minor weathering to kaolinite in spots intercalated vuggy dol \& soil

intercalated pink $\&$ gray dol (sheared); minor weathering spots; very vuggy ( $\sim 75 \%$ core loss) calamine

pink dol w/ minor bx \& some oncolites

pink dol bx (still part of Upper Pamplona?)

gray w/ some pink dol bx w/ stromatolites

dark gray dol w/ bird's eyes (UMdP)

x black shales, similar to Serra do Garrote BUT probably blk sh at base of UMdP

$\mathrm{x} \quad$ pink \& gray dol w/ some bx zones \& laminated sections

x black shales, similar to Serra do Garrote BUT probably blk sh at base of UMdP

large mottled dark gray dol 


\begin{tabular}{|c|c|c|c|c|c|c|c|}
\hline Hole & $\underline{\text { From }}$ & $\underline{\text { To }}$ & $\underline{\text { Rock }}$ & Geologic Unit & $\underline{\text { Color }}$ & $\underline{\text { Texture }}$ & $\underline{\text { Alteration }}$ \\
\hline VZMIF 125 & 0 & 20.95 & soil & Upper Pamplona? & lt to dk brown & & \\
\hline VZMIF 125 & 20.95 & 29.4 & dol & Upper Pamplona? & light pink & bird's eyes w/ minor bx & slight kaol wthg; Fe-carb veins \\
\hline VZMIF 125 & 29.4 & 31.7 & soil & Upper Pamplona? & brown & & \\
\hline VZMIF 125 & 31.7 & 41.9 & dol/phyll & Upper Pamplona? & red & $\begin{array}{l}\text { algal lamellae w/ bird's } \\
\text { eyes }\end{array}$ & Fe-carb \& hm veins \\
\hline VZMIF 125 & 41.9 & 64.45 & dol bx & Hydrothermal Breccia? & red & $\mathrm{bx}$ & Fe-carb \& hm veins \\
\hline VZMIF 125 & 64.45 & 70.65 & dol & Middle Pamplona? & white & bird's eyes w/ marbling & minor Fe-carb alt \\
\hline VZMIF 125 & 70.65 & 75.65 & dol bx & Hydrothermal Breccia? & red & $\mathrm{bx}$ & Fe-carb alt, moderate hm \\
\hline VZMIF 125 & 75.65 & 82 & dol & Middle Pamplona? & pink & laminated & Fe-carb alt, moderate hm \\
\hline VZMIF 125 & 82 & 88.4 & dol bx & Hydrothermal Breccia? & gray & bx & minor Fe-carb and hm alt \\
\hline VZMIF 125 & 88.4 & 107.05 & dol & Middle Pamplona? & light gray & laminated $\mathrm{w} /$ minor $\mathrm{bx}$ & minor Fe-carb and hm alt \\
\hline VZMIF 125 & 107.05 & 108.95 & dol & Middle Pamplona? & red & bx, vuggy & hm veins, high Fe-carb alt \\
\hline VZMIF 125 & 108.95 & 127.85 & dol & Middle Pamplona? & pink \& gray & laminated $\mathrm{w} /$ minor $\mathrm{bx}$ & hm veinlets \\
\hline VZMIF 125 & 127.85 & 130.3 & dol bx & Middle Pamplona? & pink & & Fe-carb \& hm veins \\
\hline VZMIF 125 & 130.3 & 154.95 & dol & Middle Pamplona? & pink \& gray & $\begin{array}{l}\text { laminated, minor bx, } \\
\text { bird's eyes }\end{array}$ & minor Fe-carb alt \\
\hline
\end{tabular}




\section{$\underline{\text { Py }} \underline{\text { Po }} \underline{\mathrm{Cp}} \underline{\mathrm{Sp}} \underline{\mathrm{Ga}} \underline{\mathrm{Cl}} \underline{\mathrm{Wl}} \underline{\text { Shear }} \underline{\text { Observations }}$}

x pink dol w/ minor Fe-carb alt \& soil alteration

$\pm$

intercalated dol w/ dark red phyllites, some hm \& Fe-carb veins

hydrothermal dol bx w/ massive hm veins $(5 \mathrm{~cm} w / 1-2 \% \mathrm{Zn})$

whit dolomite w/ Fe-carb veins, no bx, no mineralization

red hydrothermal bx w/ moderate hm (less than previous bx)

some bx in pink dol (not Hydrothermal Breccia)

dol bx w/ little Fe-carb and hm alteration

light gray dol w/ some bx, little mineralization; minor phyllite intercalations

dol w/ lots of hm and vugs, lot of Fe-carb alt and veins

pink \& gray dol w/ some bx; Fe-carb \& hm veinlets

pink dol bx w/ stromatolites at end of run

intercalted pink \& gray dol w/ lamellae \& bird's eyes, shearing w/ some hm, some minor bx 


\begin{tabular}{|c|c|c|c|c|c|c|c|}
\hline Hole & $\underline{\text { From }}$ & $\underline{\text { To }}$ & $\underline{\text { Rock Type }}$ & Geologic Unit & Color & Texture & $\underline{\text { Alteration }}$ \\
\hline VZMIF 106 & 0 & 82.75 & soil & Lower Pamplona & brown & & \\
\hline VZMIF 106 & 82.75 & 87.1 & dol & Lower Pamplona & light pink & massive & wthg to clays; minor Fe-carb veins \\
\hline VZMIF 106 & 87.1 & 88.25 & soil & Lower Pamplona & brown & & \\
\hline VZMIF 106 & 88.25 & 104.8 & $\begin{array}{l}\text { dol } \\
\text { No }\end{array}$ & Lower Pamplona & pink to white & $\begin{array}{l}\text { massive w/ specks of } \\
\mathrm{hm}\end{array}$ & minor hm \& Fe-carb alt \\
\hline VZMIF 106 & 104.8 & 118.25 & Recovery & Hydrothermal Breccia & & & \\
\hline VZMIF 106 & 118.25 & 120.3 & dol bx & Hydrothermal Breccia & reddish-gray & bx; vuggy & Fe-carb, hm alt \\
\hline VZMIF 106 & 120.3 & 122.15 & dol bx & Hydrothermal Breccia & reddish-gray & bx; vuggy & Fe-carb, hm alt, silica Wl alt \\
\hline VZMIF 106 & 122.15 & 126.6 & soil/ dol bx & Hydrothermal Breccia & brown & & wthg w/ supergene calamine \\
\hline VZMIF 106 & 126.6 & 134.2 & dol bx & Hydrothermal Breccia & dark red & $\mathrm{bx} \mathrm{w} / \mathrm{hm}$ veins & hm veins $\mathrm{w} / \mathrm{some}$ wl \\
\hline VZMIF 106 & 134.2 & 149.9 & dol & Lower Pamplona? & $\begin{array}{l}\text { gray \& pink } \\
\text { brownish }\end{array}$ & mottled bx & minor Fe-carb \& hm alt \\
\hline VZMIF 106 & 149.9 & 167.25 & soil/dol & Lower Pamplona? & gray & soil \& dol bx & wthg to soil \\
\hline VZMIF 106 & 167.25 & 211.95 & dol & $\begin{array}{l}\text { Lower Pamplona? } \\
\text { Upper Morro do }\end{array}$ & red & bx & minor Fe-carb \& hm alt \\
\hline VZMIF 106 & 211.95 & 217.5 & dol & Pinheiro & gray & bird's eyes, dolarenite & trace Fe-carb veinlets \\
\hline
\end{tabular}




\section{$\underline{\text { Py }} \underline{\text { Po }} \underline{\text { Cp }} \underline{\text { Sp }} \underline{\text { Ga }} \underline{\text { Cl }} \underline{\text { Wl }} \underline{\text { Shear }} \underline{\text { Observations }}$}

pink dol, very chewed up, altering to soil

pink to white dol w/ hm specks; some spots altering to soil

No Recovery, vugs?

vuggy dol bx w/ some hm (low recovery)

dol bx replaced by $\mathrm{hm}$ veins and $\mathrm{Wl}$

$\pm \quad x$

Calamine in orange-brown soil (not enough soil for sample)

$\mathrm{X}$

$\mathrm{X}$ $\mathrm{X}$

$\mathrm{X}$

dol replaced by hm, vuggy

dol bx w/ gray and pink clasts, mottling

alternating soil and gray dol bx

red dol bx w/ Fe-carb veins \& minor hm veinsk some algal laminated \& dolarenite clasts;green phyllites

gray dol/dolarenite w/ bird's eyes \& minor bx; trace Fe-carb alt 


\begin{tabular}{|c|c|c|c|c|c|c|c|}
\hline Hole & From & $\underline{\text { To }}$ & $\underline{\text { Rock Type }}$ & Geologic Unit & $\underline{\text { Color }}$ & $\underline{\text { Texture }}$ & Alteration \\
\hline VZMIF 155 & 0 & 48.8 & No recovery & & & & \\
\hline VZMIF 155 & 48.8 & 49.05 & dol & Middle Pamplona? & pink & mottled & silica \& Fe-carb alt \\
\hline VZMIF 155 & 49.05 & 50.28 & dol & Middle Pamplona? & pink & bx & silica \& hm veins $(<1 \mathrm{~cm})$ \\
\hline VZMIF 155 & 50.28 & 61.2 & dol & Middle Pamplona? & pink & mottled, minor bx & silica $\& \mathrm{hm}$ veins $(<1 \mathrm{~cm})$ \\
\hline VZMIF 155 & 61.2 & 63.65 & dol & Middle Pamplona? & pink \& gray & bx & mottled kaol wthg \\
\hline VZMIF 155 & 63.65 & 66.15 & dolarenite & Middle Pamplona? & gray & stromatolites $\mathrm{w} /$ dolarenite & silica; kaol \\
\hline VZMIF 155 & 66.15 & 69.15 & dolarenite & Middle Pamplona? & gray & dolarenite & minor silicic alt \\
\hline VZMIF 155 & 69.15 & 59.7 & dol/phyllite & Lower Pamplona & gray & $\begin{array}{l}\text { phyllite } \\
\text { intercalated dolarenite \& }\end{array}$ & hm banding \\
\hline VZMIF 155 & 59.7 & 72.95 & dol/phyllite & Lower Pamplona & light to dark gray & phyllite & $\mathrm{hm}$ alt in phyllites \\
\hline VZMIF 155 & 72.95 & 75.15 & phyllite & Lower Pamplona & brownish gray & laminated, minor mottling & silica alt \\
\hline VZMIF 155 & 75.15 & 83.95 & dol & Lower Pamplona & pink \& gray & laminated & silica alt in bx \\
\hline VZMIF 155 & 83.95 & 93.85 & dol & Lower Pamplona & gray & laminated; bx & silica \& hydrothermal dol \\
\hline VZMIF 155 & 93.85 & 94.43 & dol & Lower Pamplona & dark gray & bx & silicified bx \\
\hline VZMIF 155 & 94.43 & 100.85 & dol & Lower Pamplona & gray & algal laminated; some bx & silicified bx \\
\hline VZMIF 155 & 100.85 & 108.8 & dol & Lower Pamplona & gray & algal laminated; some bx & minor silicic alt \\
\hline VZMIF 155 & 108.8 & 121.85 & dol & Lower Pamplona & dark gray & mottled, some bx & silica \& hydrothermal dol \\
\hline VZMIF 155 & 121.85 & 129.05 & dol & Lower Pamplona & gray & semi-massive dol & minor silicic alt \& $\mathrm{hm}$ \\
\hline VZMIF 155 & 129.05 & 130.75 & dol & Lower Pamplona & brownish gray & laminated & minor silicic alt \\
\hline VZMIF 155 & 130.75 & 132.65 & dol & Lower Pamplona & pink \& gray & laminated & $\mathrm{hm}$ alt \\
\hline VZMIF 155 & 132.65 & 151.7 & dol & Lower Pamplona & pink \& gray & laminated \pm bird's eyes & silica veins \\
\hline VZMIF 155 & 151.7 & 191.3 & dol & $\begin{array}{l}\text { Lower Pamplona } \\
\text { Hydrothermal }\end{array}$ & pink \& gray & laminated \pm bird's eyes & silica veins \\
\hline VZMIF 155 & 191.3 & 193.3 & dol bx & Breccia & red & bx & Fe-carb \& hm \\
\hline VZMIF 155 & 193.3 & 206.5 & dol & Lower Pamplona & pink & bx; laminated & Fe-carb \& hm \\
\hline VZMIF 155 & 206.5 & 213.65 & dol/phyllite & Lower Pamplona & light pink & laminated & \\
\hline
\end{tabular}




\begin{tabular}{|c|c|c|c|c|c|c|c|}
\hline VZMIF 155 & 213.65 & 215.4 & phyllite & Lower Pamplona & reddish brown & laminated & Fe-carb \& hm \\
\hline VZMIF 155 & 215.4 & 232.58 & dol & Lower Pamplona & pink \& gray & bx, minor laminations & silicic \& $\mathrm{hm}$ alt \\
\hline VZMIF 155 & 232.58 & 233.45 & phyllite & Lower Pamplona & brown & laminated & chl alt \\
\hline VZMIF 155 & 235.45 & 235.75 & dol & $\begin{array}{l}\text { Lower Pamplona } \\
\text { Hydrothermal }\end{array}$ & pink & wavy laminated & minor silicic \\
\hline VZMIF 155 & 235.75 & 256.15 & dol bx & Breccia & reddish pink & bx w/ 2nd carb & Fe-carb \& hm \\
\hline VZMIF 155 & 256.15 & 262.85 & dol & Lower Pamplona & pink & laminated & minor silicic \\
\hline VZMIF 155 & 262.85 & 263.85 & phyllite & Lower Pamplona & reddish brown & laminated & silica \\
\hline VZMIF 155 & 263.85 & 265.85 & dol & $\begin{array}{l}\text { Lower Pamplona } \\
\text { Hydrothermal }\end{array}$ & pink \& gray & laminated & $\mathrm{hm}$ \\
\hline VZMIF 155 & 265.85 & 337.7 & dol bx & Breccia & reddish pink & bx & Fe-carb \& hm \\
\hline VZMIF 155 & 337.7 & 349.15 & dol & Lower Pamplona & red & laminated $\mathrm{w} /$ minor bx & silicic?; hm \\
\hline
\end{tabular}

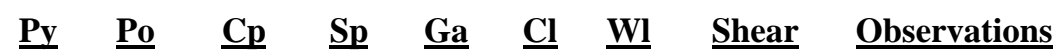

hm filled fractures at $47.6 \& 48.03$

hm veins \& fractures throughout; kaol alt spotty

small vugs filled $\mathrm{w} /$ secondary dol

at 68.5-68.62 recrystallized dol w/ py; 68.7-69.15 intercalated hm alt

70.98-71.05 bx in pelite; 71.18-71.66 chl alt in phyllites; $72.15-72.95 \mathrm{hm}$ alt 72.95-73.65 silica layers \& bx; 74.95-75.15 hm veins, fault?

layered bx at 79; oncolites at 83 ; minor silica $\& \mathrm{hm}$ fractures

hydrothermal bx at 87.05, 90.9 vert fracture; algal mats at 91.65-91.8, styolites at 92.65

93.6-93.85 hydrothermal bx, 93.85-94 laminated/algal bx

98-99.85 bx, minor hm staining around veins 
bx at $102.6 \&$ py at 107.14

dark gray laminated to mottled dolarenite intercalated w/ lighter hydrothermal bx

intercalated brown-gray dol and dark gray phyllite; Fe-carb alt \& minor bx's present

interlayered pink to gray dol w/ laminated \& bird's eyes, multiple dark gray qtz veins 166.85-169.30 ( bx w/ qtz infill, silica alt); 169.3-191.3 massive dol w/ layers of bird's eyes texture dark red hydrothermal breccia; Fe-carb alt

dol w/ thin pelitic layers, bird's eyes @ 212.5?

phyllite w/ Fe-carb alt

gry to mostly pink dol, various small bx's

marroon phyllite

dark pink dol

$?$

red to pink Hydrothermal Breccia w/ qtz, hm, \& Fe-carb veins

interlayered dol \& phyllite

pink \& gray dol

pink to red Hydrothermal Breccia; 295.85-298.45 heavy hm alt, Wm in bx

intercalated laminated dol \& dolomitic Hydrothermal Breccia w/ silicic alteration 


\begin{tabular}{|c|c|c|c|c|c|c|c|}
\hline Hole & From & $\underline{\text { To }}$ & Rock Type & Geologic Unit & Color & $\underline{\text { Texture }}$ & Alteration \\
\hline VZMIF 060 & 0 & 57.05 & destroyed & Lower Pamplona & & & \\
\hline VZMIF 060 & 57.05 & 98.85 & phyllite/dol & Lower Pamplona & pink \& gray & $\begin{array}{l}\text { laminated } \\
\text { phyllite/dolarenite }\end{array}$ & Fe-carb, hm veins in small bx \\
\hline VZMIF 060 & 98.85 & 119.3 & phyllite/dol & Hydrothermal Breccia & red & $\begin{array}{l}\mathrm{bx} \mathrm{w} / \mathrm{hm} \text { veins } \\
\text { massive } \mathrm{Wl} \mathrm{w} / \mathrm{hm}\end{array}$ & Fe-carb, silica alt, \pm hm \\
\hline VZMIF 060 & 119.3 & 122.95 & dol bx & Hydrothermal Breccia & dark red & $\begin{array}{l}\text { veins } \\
\text { massive } \mathrm{Wl} \mathrm{w} / \mathrm{hm}\end{array}$ & Fe-carb, hm veins, Wl \\
\hline VZMIF 060 & 122.95 & 135.15 & dol bx & Hydrothermal Breccia & red & $\begin{array}{l}\text { veins } \\
\text { massive } \mathrm{Wl} \mathrm{w} / \mathrm{hm}\end{array}$ & Fe-carb, silica alt, $\pm \mathrm{hm}$ \\
\hline VZMIF 060 & 135.15 & 137.5 & dol bx & Hydrothermal Breccia & dark red & veins & Fe-carb, hm veins, Wl \\
\hline VZMIF 060 & 137.5 & 155.45 & dol bx & $\begin{array}{l}\text { Hydrothermal Breccia } \\
\text { Upper Morro do }\end{array}$ & reddish pink & $\mathrm{bx} w / \mathrm{hm}$ alt veins & Fe-carb \& silica alt veins \\
\hline VZMIF 060 & 155.45 & 161.75 & dol & Pinheiro? & dark gray & $\begin{array}{l}\text { crackle bx } \\
\text { laminated }\end{array}$ & Fe-carb \& silica veins \\
\hline VZMIF 060 & 161.75 & 209 & phyllite/dol & Lower Pamplona? & pink \& gray & $\begin{array}{l}\text { phyllite/dolarenite } \\
\text { bird's eyes, bx near }\end{array}$ & Fe-carb, hm veins in small bx \\
\hline VZMIF 060 & 209 & 214.7 & dol & Upper Morro do Pinheiro & dark gray & contact & graphite veins \\
\hline Hole & From & $\underline{\text { To }}$ & $\underline{\text { Rock Type }}$ & Geologic Unit & Color & Texture & $\underline{\text { Alteration }}$ \\
\hline VZMIF 004 & 0 & 42.45 & destroyed & Middle Pamplona & & & \\
\hline VZMIF 004 & 42.45 & 64.5 & dol & Middle Pamplona & gray & massive marble & silica alt \& some Fe-carb veins \\
\hline VZMIF 004 & 64.5 & 232.2 & phyllite/dol & Lower Pamplona & Brn-red/gray & laminated dolarenite & some hm veins; some marbling \\
\hline VZMIF 004 & 232.2 & 242.1 & dol bx & $\begin{array}{l}\text { Hydrothermal Breccia } \\
\text { Lower Pamplona? or }\end{array}$ & $\begin{array}{l}\text { red } \\
\text { light pink \& }\end{array}$ & $\mathrm{bx}$ & Fe-carb alt \\
\hline VZMIF 004 & 242.1 & 250.6 & dol & UMdP? & white & marble & minor Fe-carb alt \\
\hline VZMIF 004 & 250.6 & 294.5 & dol & Upper Morro do Pinheiro & gray \& pink & bird's eyes \& bx & $\begin{array}{l}\text { Fe-carb alt } \\
\text { dolomite recrystallization }\end{array}$ \\
\hline VZMIF 004 & 294.5 & 421.1 & dol & Upper Morro do Pinheiro & dark gray & bird's eyes & (hydrothermal?) \\
\hline
\end{tabular}




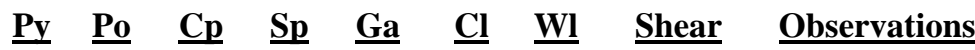

intercalated light pink to light gray dolarenite \& red-gray phyllite;

blanching proximal to Hydrothermal Breccia

red \& white Hydrothermal Breccia w/ Fe-carb \& silica altertion

red Hydrothermal Breccia w/ hm in shear veins; Wl

red \& pink bx $\pm \mathrm{Wl}$; lighter than surrounding bx

gray-red Hydrothermal Breccia w/ high Zn \& Cd, some high Ag values

pink to red Hydrothermal Breccia

dark gray dol w/ crackle breccia (UMdP?)

fault at 17.45-167.7 (thrust? Setting UMdP on LP?)

dark gray dol w/ bx near contact (also graphite veins) \& bird's eyes

\section{$\underline{P y} \quad$ Po $\quad \underline{\text { Cp }} \quad \underline{\mathrm{Sp}} \quad \underline{\mathrm{Ga}} \quad \underline{\mathrm{Cl}} \quad \underline{\text { Wl }} \underline{\text { Shear Observations }}$}

gray dol \& dolarenite w/ some Fe-carb veins w/ vugs \& a lot of silica alteration white-gray dolarenite intercalated $\mathrm{w} /$ red-brown phyllites red bx w/ Fe-carb alt; Wl w/ less massive hm; red color fades at end of bx light pink to white dolomite marble below Hydrothermal Breccia light pink dolomite w/ Fe-carb veins intercalated w/ gray dolomite w/ bird's eyes; bx from 264-267.1 (at end of bx is fault w/ chlorite)

$\mathrm{x} \quad$ dark gray dol w/ bird's eyes \& minor bx's throughout, some shear veins 


\begin{tabular}{|c|c|c|c|c|c|c|c|}
\hline Hole & From & $\underline{\text { To }}$ & $\underline{\text { Rock Type }}$ & Geologic Unit & Color & Texture & $\underline{\text { Alteration }}$ \\
\hline VZMIF 101 & 0 & 68.2 & destroyed & $?$ & & & \\
\hline VZMIF 101 & 68.2 & 122.58 & phyllite/ dol & Lower Pamplona & gray/pink & mottled & Fe-carb veins; hm shear v \\
\hline VZMIF 101 & 122.58 & 150.5 & dol bx & Hydrothermal Breccia & dark red & $\begin{array}{l}\text { sheared bx } \\
\text { laminated phyll/ }\end{array}$ & Fe-carb alt; hm shear veir \\
\hline VZMIF 101 & 150.5 & 242.1 & phyllite/ dol & $\begin{array}{l}\text { Lower Pamplona } \\
\text { Upper Morro do }\end{array}$ & gray/pink & dolarenite & $\begin{array}{l}\text { Fe-carb alt that increases } \\
\text { dolomite recrystallization }\end{array}$ \\
\hline VZMIF 101 & 242.1 & 247.7 & dol & Pinheiro & gray & botryoidal \& bx & (hydrothermal?) \\
\hline$\underline{\text { Hole }}$ & $\underline{\text { From }}$ & $\underline{\text { To }}$ & $\underline{\text { Rock Type }}$ & Geologic Unit & $\underline{\text { Color }}$ & $\underline{\text { Texture }}$ & $\underline{\text { Alteration }}$ \\
\hline VZMIF 043 & 0 & 45.5 & destroyed & Middle Pamplona? & & & \\
\hline VZMIF 043 & 45.5 & 58 & dol & Middle Pamplona & pinkish gray & $\begin{array}{l}\text { massive dolarenite } \\
\text { laminated phyllite; } \\
\text { dolarenite \& }\end{array}$ & hm veinlets \\
\hline VZMIF 043 & 58 & 198.65 & phyll/dA \& dR & Lower Pamplona & red-brown/ gray & dolorudite & Fe-carb \& hm alt veins \\
\hline VZMIF 043 & 198.65 & 259.45 & dol bx & $\begin{array}{l}\text { Hydrothermal Breccia } \\
\text { Upper Morro do }\end{array}$ & pink & $\begin{array}{l}\text { bx w/ alteration veins } \\
\text { bird's eyes \& algal }\end{array}$ & Fe-carb veins, minor hm \\
\hline VZMIF 043 & 259.45 & 349.45 & dol & Pinheiro & dark gray & layering & \\
\hline$\underline{\text { Hole }}$ & $\underline{\text { From }}$ & $\underline{\text { To }}$ & $\underline{\text { Rock Type }}$ & $\underline{\text { Geologic Unit }}$ & $\underline{\text { Color }}$ & $\underline{\text { Texture }}$ & $\underline{\text { Alteration }}$ \\
\hline VZMIF 104 & 0 & 46 & destroyed & & & & \\
\hline VZMIF 104 & 46 & 66.7 & phyllite/dol & Lower Pamplona & $\begin{array}{l}\text { reddish green- } \\
\text { brown/pink \& } \\
\text { white }\end{array}$ & $\begin{array}{l}\text { laminated phyllite \& } \\
\text { dolarenite }\end{array}$ & $\begin{array}{l}\text { treace } h m \text { veinlets } \& \& \\
\text { Fe-carb alt }\end{array}$ \\
\hline VZMIF 104 & 66.7 & 71.3 & dol & Lower Pamplona & $\begin{array}{l}\text { gray-orange } \\
\text { reddish green- }\end{array}$ & dolarenite & weathering to soil \\
\hline VZMIF 104 & 71.3 & 81.2 & phyllite/dol & Lower Pamplona & $\begin{array}{l}\text { brown/pink \& } \\
\text { white }\end{array}$ & $\begin{array}{l}\text { laminated phyllite \& } \\
\text { dolarenite }\end{array}$ & $\begin{array}{l}\text { treace } \mathrm{hm} \text { veinlets \& } \\
\text { Fe-carb alt }\end{array}$ \\
\hline VZMIF 104 & 81.2 & 88.8 & dol bx & Hydrothermal Breccia & $\begin{array}{l}\text { red-gray } \\
\text { reddish green- }\end{array}$ & $\mathrm{bx}$ w/ veins & Fe-carb, slicica alt \\
\hline VZMIF 104 & 88.8 & 131.8 & phyllite/dol & Lower Pamplona & $\begin{array}{l}\text { brown/pink \& } \\
\text { white }\end{array}$ & $\begin{array}{l}\text { laminated phyllite \& } \\
\text { dolarenite }\end{array}$ & $\begin{array}{l}\text { treace } h m \text { veinlets \& } \\
\text { Fe-carb alt }\end{array}$ \\
\hline \multicolumn{8}{|c|}{148} \\
\hline
\end{tabular}




\section{$\underline{\text { Py }} \underline{\text { Po }} \quad \underline{\text { Cp }} \quad \underline{\text { Sp }} \underline{\text { Ga }} \underline{\text { Cl }} \underline{\text { Wl }} \underline{\text { Shear }} \underline{\text { Observations }}$}

intercalated gray phyllite $\&$ pink dolarenite w/ Fe-carb \& hm veins

$\mathrm{x} \quad$ (some sheared) increasing in density towards Hydrothermal Breccia

$\mathrm{x} \quad \mathrm{x} \quad \mathrm{x} \quad$ dark red sheared dolomite Hydrothermal Breccia (lighter on margins w/ Wl \& $\mathrm{hm} \pm \mathrm{Cd} \& \mathrm{Ba}$

$\mathrm{x} \quad$ intercalted pink \& gray dolarenite w/ gray phyllite; Fe-carb alt decreases with depth; minor bx

? $\quad$ gray dol w/ some bx \& carbonate (dol) overgrowths; no Fe-alt

\section{$\underline{\underline{P y}} \quad \underline{\text { Po }} \quad \underline{\underline{C}} \quad \underline{\mathrm{Sp}} \quad \underline{\mathrm{Ga}} \quad \underline{\mathrm{Cl}} \quad \underline{\mathrm{Wl}} \quad \underline{\text { Shear }} \underline{\text { Observations }}$}

pinkish gray massive dolarenite $\mathrm{w} / \mathrm{hm} \pm$ Py in veinlets

interclated phyllites \& dolarenite (w/ dolorudite) w/ increased shear towards

Hydrothermal Breccia contact; at 189 m blanching of phyllite \& dolomite begins

x Hydrothermal Breccia, but with little hm veins, some minor $\mathrm{Wl}$; one high $0.7 \% \mathrm{Cu}$ interval dark gray dol w/ black phyllites (characteristic of base of UMdP)

at $332.45-332.75 ; 339.5-339.78 ; 342.6-342.9$; blanching at 265.65

\section{$\underline{\text { Py }} \quad \underline{\text { Po }} \quad \underline{\text { pp }} \quad \underline{\text { Sp }} \quad \underline{\text { Ga }} \quad \underline{\text { Cl }} \quad \underline{\text { Wl }} \quad \underline{\text { Shear }}$ Observations}

red-brown \& green gray phyllites intercalted with pink \& white dolarenite gray dolarenite weathering to osil in spots; vuggy red-brown \& green gray phyllites intercalted with pink \& white dolarenite; blanching w/in $10 \mathrm{~m}$ of Hydrothermal Breccia

Hydrothermal Breccia w/ Fe-carb alt \& some hm veins; high $\mathrm{Wl}+\mathrm{Cd}$ red-brown \& green gray phyllites intercalted with pink \& white dolarenite;

$\mathrm{x} \quad$ blanching \& pink dol w/ in $10 \mathrm{~m}$ of Hydrothermal Breccia; hm \& Fe-carb alt dissipate downhole 


\begin{tabular}{|c|c|c|c|c|c|c|c|}
\hline Hole & $\underline{\text { From }}$ & $\underline{\text { To }}$ & Rock Type & Geologic Unit & $\underline{\text { Color }}$ & $\underline{\text { Texture }}$ & $\underline{\text { Alteration }}$ \\
\hline VZMIF 105 & 0 & 44 & destroyed & & & & \\
\hline VZMIF 105 & 44 & 58.35 & dol & Lower Pamplona & gray & mottled & Fe-carb veinlets; some weathering \\
\hline VZMIF 105 & 58.35 & 81.2 & dol bx & Hydrothermal Breccia & pink & $\begin{array}{l}\text { vuggy bx } \\
\text { laminated phyllite }\end{array}$ & Fe-carb; some small hm veinlets \\
\hline VZMIF 105 & 81.2 & 96 & phyllite/ dol & Lower Pamplona & dark gray/pink & $\begin{array}{l}\text { \& dolarenite } \\
\text { sheared laminated } \\
\text { phyllite/ }\end{array}$ & some hm alt \& hm stains \\
\hline VZMIF 105 & 106.2 & 167.8 & phyllite/ dol & $\begin{array}{l}\text { Lower Pamplona } \\
\text { Upper Morro do }\end{array}$ & dark gray/pink & $\&$ dolarenite & some hm alt \& hm stains \\
\hline VZMIF 105 & 167.8 & 170.2 & dol & Pinheiro & dark gray & bird's eyes & trace silica \\
\hline Hole & $\underline{\text { From }}$ & $\underline{\text { To }}$ & $\underline{\text { Rock Type }}$ & $\underline{\text { Geologic Unit }}$ & $\underline{\text { Color }}$ & $\underline{\text { Texture }}$ & $\underline{\text { Alteration }}$ \\
\hline VZMIF 015 & 0 & 41.6 & destroyed & & & & \\
\hline VZMIF 015 & 114.65 & 253.6 & phyll/dol & Lower Pamplona & $\begin{array}{l}\text { white } \\
\text { red, gray \& }\end{array}$ & laminated & trace Fe-carb veins \\
\hline VZMIF 015 & 253.6 & 389.9 & phyll/dol & Lower Pamplona & white & laminated & trace Fe-carb veins \\
\hline VZMIF 015 & 389.9 & 414.95 & bx & $\begin{array}{l}\text { Hydrothermal Breccia } \\
\text { Upper Morro do }\end{array}$ & red & $\begin{array}{l}\text { bx } \\
\text { bird's eyes \& algal }\end{array}$ & Fe-carb veins w/ hm veins \\
\hline VZMIF 015 & 414.95 & 452.8 & dol & Pinheiro & gray & laminated & minor blanching \\
\hline
\end{tabular}




\section{$\underline{P y} \underline{\text { Po }} \quad \underline{\text { p }} \quad \underline{\text { pp }}$ Ga $\underline{\text { Cl }} \underline{\text { Wl }}$ Shear $\underline{\text { Observations }}$}

gray dolomite/dolorudite w/ very minor Fe-carb alt

pink dol bx(distal or secondary Hydrothermal Breccia?);

not sheared like Hydrothermal Breccia nor as heavily brecciated; less Fe-carb alt then next interval;

some minor blanching near this Hydrothermal Breccia? $(<1 \mathrm{~m})$

x $\quad$ intercalated dark gray phyllites \& pink-gray dolarenites some $\mathrm{hm}$ alt

$\mathrm{X}$

$\mathrm{x}$

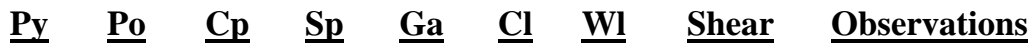

intercalated gray-green phyllites \& gray boudinaged dolomites \& dolarenites; heavily sheared intercalated dark gray phyllites \& pink-gray dolarenites some $\mathrm{hm}$ alt $\mathrm{w} /$ some bx 158.5-160 w/ py \& hm

dark gray dol $\mathrm{w} / \mathrm{py}$ in some fractures gray dolarenite $\mathrm{w} /$ silica $\& \mathrm{hm} \pm$ py vugs $\&$ veins mostly red-gray phyllites intercalted w/ white dolarenite (similar to wht dol in VZMIF 012) same as previous entry but with $\mathrm{hm}$ strains \& vugs (closer to weathering surface?)

Hydrothermal Breccia w/ some Wl; very vuggy; lot of core loss

gray dolomite w/ minor blanching; vuggy; lot of core loss 


\begin{tabular}{|c|c|c|c|c|c|c|c|}
\hline Hole & $\underline{\text { From }}$ & $\underline{\text { To }}$ & $\underline{\text { Rock Type }}$ & Geologic Unit & $\underline{\text { Color }}$ & $\underline{\text { Texture }}$ & Alteration \\
\hline VZMIF 088 & 0 & 45.5 & destroyed & & & & \\
\hline VZMIF 088 & 45.5 & 66.7 & phyll/dol & $\begin{array}{l}\text { Lower Pamplona } \\
\text { Hydrothermal }\end{array}$ & $\begin{array}{l}\text { gray- } \\
\text { green/pink }\end{array}$ & $\begin{array}{l}\text { laminated } \\
\text { dolarenite }\end{array}$ & some hm veinlets; \& Fe-carb alt in bx \\
\hline VZMIF 088 & 66.7 & 72.7 & dol bx & $\begin{array}{l}\text { Breccia? } \\
\text { Hydrothermal }\end{array}$ & gray & sheared bx & specularite veins; some Fe-carb alt \\
\hline VZMIF 088 & 72.7 & 83.7 & dol bx & Breccia? & $\begin{array}{l}\text { red \& white } \\
\text { gray- }\end{array}$ & sheared bx & specularite veins; some Fe-carb alt \\
\hline VZMIF 088 & 83.7 & 140.7 & phyll/dol & Lower Pamplona & green/pink & $\begin{array}{l}\text { laminated } \\
\text { algal }\end{array}$ & chloritic alt in phyllite \\
\hline VZMIF 088 & 140.7 & 173.35 & phyll/dol & $\begin{array}{l}\text { Lower Pamplona } \\
\text { Upper Morro do }\end{array}$ & gray \& white & $\begin{array}{l}\text { laminated } \\
\text { secondary dol }\end{array}$ & trace Fe-carb; chlorite alt in phyll; silica alt \\
\hline VZMIF 088 & 173.35 & 183.4 & dol bx & Pinheiro & dark gray & overgrowths & carbonate \& silica alt \\
\hline
\end{tabular}

$\underline{P y} \quad \underline{P o} \quad \underline{\text { p }} \quad \underline{\text { Sp }} \quad \underline{\text { Ga }} \quad \underline{\mathrm{Cl}} \underline{\text { Wl }} \underline{\text { Shear }} \underline{\text { Observations }}$

$\mathrm{X}$

$\mathrm{X}$

$\mathrm{X}$ intercalated green-gray phyll w/ pink dolarenite; hm stains; shear increases \& color blanches w/ proximity to Hydrothermal Breccia gray Hydrothermal Breccia w/ sulfides: py, sp \& specularite instead of hm \& Wl red \& white Hydrothermal Breccia, no sulfides intercalated green-gray phyll w/ chlorite alt; light pink to gray dolarenite intercalted gray phyllite \& white algal layered dolomite dark gray dol w/ marroon phyllite at 179.9-180.5;

bx w/ carb overgrowths; algal laminated texture where not brecciated 


\begin{tabular}{|c|c|c|c|c|c|c|c|}
\hline Hole & From & $\underline{\text { To }}$ & Rock Type & Geologic Unit & Color & $\underline{\text { Texture }}$ & $\underline{\text { Alteration }}$ \\
\hline Masa 124 & 0 & 6.8 & soil & Upper Pamplona & yellow-orange & & \\
\hline Masa 124 & 6.8 & 32.3 & phyll/dol & Upper Pamplona & blk/dark gray & $\begin{array}{l}\text { evaporitic } \\
\text { texture }\end{array}$ & \\
\hline Masa 124 & 32.3 & 48.2 & dolarenite & Upper Pamplona & It gray to lt pink & $\begin{array}{l}\text { dolarenite } \\
\text { massive }\end{array}$ & silicified \\
\hline Masa 124 & 48.2 & 85.45 & dol & Upper Pamplona & dark gray & dolostone & silicified \\
\hline Masa 124 & 85.45 & 87.35 & fault & Upper Pamplona & & $\begin{array}{l}\text { boxworks } \\
\text { massive }\end{array}$ & clay alt \\
\hline Masa 124 & 87.35 & 96.55 & dol & Upper Pamplona & dark gray & dolostone & silicified \\
\hline Masa 124 & 96.55 & 96.95 & fault & Upper Pamplona & orange red & $\begin{array}{l}\text { minor fractures } \\
\text { massive }\end{array}$ & $\mathrm{Fe}$-carb and $\mathrm{Hm}$ alt \\
\hline Masa 124 & 96.95 & 112.85 & dol & Upper Pamplona & lt gray & $\begin{array}{l}\text { dolostone } \\
\text { dolstone } \mathrm{w} /\end{array}$ & silicified \\
\hline Masa 124 & 112.85 & 115.2 & dol/phyll & Upper Pamplona & gray & $\begin{array}{l}\text { minor } \\
\text { dissolution }\end{array}$ & $\begin{array}{l}\text { silicified vugs, carb dissolution, } \\
\text { Fe-carb alt veinlets }\end{array}$ \\
\hline Masa 124 & 115.2 & 128.4 & phyllite & Upper Pamplona & dark gray-brown & & \\
\hline Masa 124 & 128.4 & 168 & dol/phyll & Middle Pamplona & $\begin{array}{l}\text { pinkish gray } \\
\text { pinkish gray w/ }\end{array}$ & $\begin{array}{l}\text { dissolution } \\
\text { vugs }\end{array}$ & $\begin{array}{l}\text { minor Fe-carb, slicified vugs } \\
\text { and selvages }\end{array}$ \\
\hline Masa 124 & 168 & 207.7 & dolorudite & Lower Pamplona & $\begin{array}{l}\text { green marls } \\
\text { maroon \& green }\end{array}$ & & minor Fe-carb \\
\hline Masa 124 & 207.7 & 267 & dol/phyll & Lower Pamplona & $\begin{array}{l}\text { phyll w/ lt gray dol } \\
\text { pink dol and red }\end{array}$ & & \\
\hline Masa 124 & 267 & 283 & dol/phyll & Lower Pamplona & phyll & & \\
\hline Masa 124 & 283 & 297.45 & $\begin{array}{l}\text { dol bx } \\
\text { dol bx w/ }\end{array}$ & Hydrothermal Breccia & red & & Fe-carb \\
\hline Masa 124 & 297.45 & 304 & phyll & Hydrothermal Breccia & pink to lt red & & less Fe-carb than previous \\
\hline Masa 124 & 304 & 308.9 & $\begin{array}{l}\text { dol bx } \\
\text { dol bx w/ }\end{array}$ & Hydrothermal Breccia & dark red & & hematite replacement \\
\hline Masa 124 & 308.9 & 326.2 & phyll & Hydrothermal Breccia & lt red to pink & & Fe-carb and Hm replacement \\
\hline Masa 124 & 326.2 & 329.75 & dol bx & Hydrothermal Breccia & dark red & & $\mathrm{Hm}+\mathrm{Wl}$ \\
\hline & & & & & 153 & & \\
\hline
\end{tabular}




\begin{tabular}{|c|c|c|c|c|c|c|}
\hline Masa 124 & 329.75 & 336.3 & dol bx & Hydrothermal Breccia & lt pink to gray & Fe-carb \\
\hline Masa 124 & 336.3 & 344.95 & dol bx & Hydrothermal Breccia & lt pink & Fe-carb \\
\hline Masa 124 & 344.95 & 345.5 & dol bx & Hydrothermal Breccia & red bx & Fe-carb \\
\hline Masa 124 & 345.5 & 352.6 & dol bx & Hydrothermal Breccia & white and red bx & Fe-carb \\
\hline Masa 124 & 352.6 & 354.85 & dol bx & Hydrothermal Breccia & dark red & Hm replacement \\
\hline Masa 124 & 354.85 & 356.65 & dol bx & Hydrothermal Breccia & lt pink to lt gray & Fe-carb \\
\hline Masa 124 & 356.65 & 372.9 & dol & $\begin{array}{l}\text { Upper Morro do } \\
\text { Pinheiro }\end{array}$ & gray & \\
\hline
\end{tabular}

\section{$\underline{\mathbf{P y}} \quad \underline{\mathbf{P}} \quad \underline{\mathrm{Cp}} \quad \underline{\mathrm{Sp}} \quad \underline{\mathrm{Ga}} \quad \underline{\mathrm{Cl}} \quad \underline{\mathrm{Wl}} \quad \underline{\text { Shear }} \quad \underline{\text { Observations }}$}

dk gray dolarenites intercalated w/ black shale (phyllites); vermiform veinlet texure (evaporites?); 18.7-19.1 dolarenite $\mathrm{w} /$ styolites and clasts

lt gray and pink dolarenites; 42.55-42.8 dissolution bx

$\mathrm{dk}$ gray silicified dolostone w/ minor dissolution bx and Fe-veinlets; 56.8-57.24 silicified dolorudite fault w/ metoric alteration; has Fe-stained halo

$\mathrm{dk}$ gray silicified dolostone w/ minor dissolution bx and Fe-veinlets

$\pm \quad$ Fe-alteration strong, proximal to fault

some minor faults w/ Fe-association; very silicified; "snow in the roof" bx w/ sphalerite gray silicifed dolostone w/ minor gray phyllite and dissolution bx; stromatolites at $107 \mathrm{~m}$ phyllitic/silty marker bed b/w UP and MP

gray dol w/ Fe-carb alt vugs and red-marroon phyllite w/ Hm alteration pink dol w/ porcelin white silica vugs

pink dolarenite w/ dissolution vugs and sedimentary carbonate conglomerate; some green marls pink dolostone intercalated with marroon and green phyllites

transition into Hydrothermal Breccia; Fe-carb alteration, some dolostones and marls (still in LP) 
less breccia, more bleached and cohesive dolostone unit within Hydrothermal Breccia Fe-carbonate bx w/ Hm-rich zones

pink dolostone inercalated with phyllites all with Fe-carb alt veinlets; dolostone is weakly brecciated massive $\mathrm{Wl}( \pm \mathrm{Hm})$ in Hydrothermal Breccia (ore)

brecciated dolostone w/ Fe-carb alteration; bleached higher $\mathrm{SiO} 2$

breccia w/ Fe-carb alteration veinlets

breccia w/ Fe-carb alteration veinlets

Fe-carbonate bx w/ silicified intervals

$\mathrm{Wl}+\mathrm{Hm}$ replacing Fe-carbonate in hydrothermal bx (Hydrothermal Breccia)

Fe-carbonate veinlets common in dolostone bx; end of Hydrothermal Breccia

dark gray dolostone w/ vuggy dissolution of algal mats replaced by Fe-carb 


\section{Appendix C Thin Section Descriptions}

Geologic Unit: Hydrothermal Breccia

Lithological Unit (Votorantim): Disseminated Silicates (SD) in BH

Sample Photograph

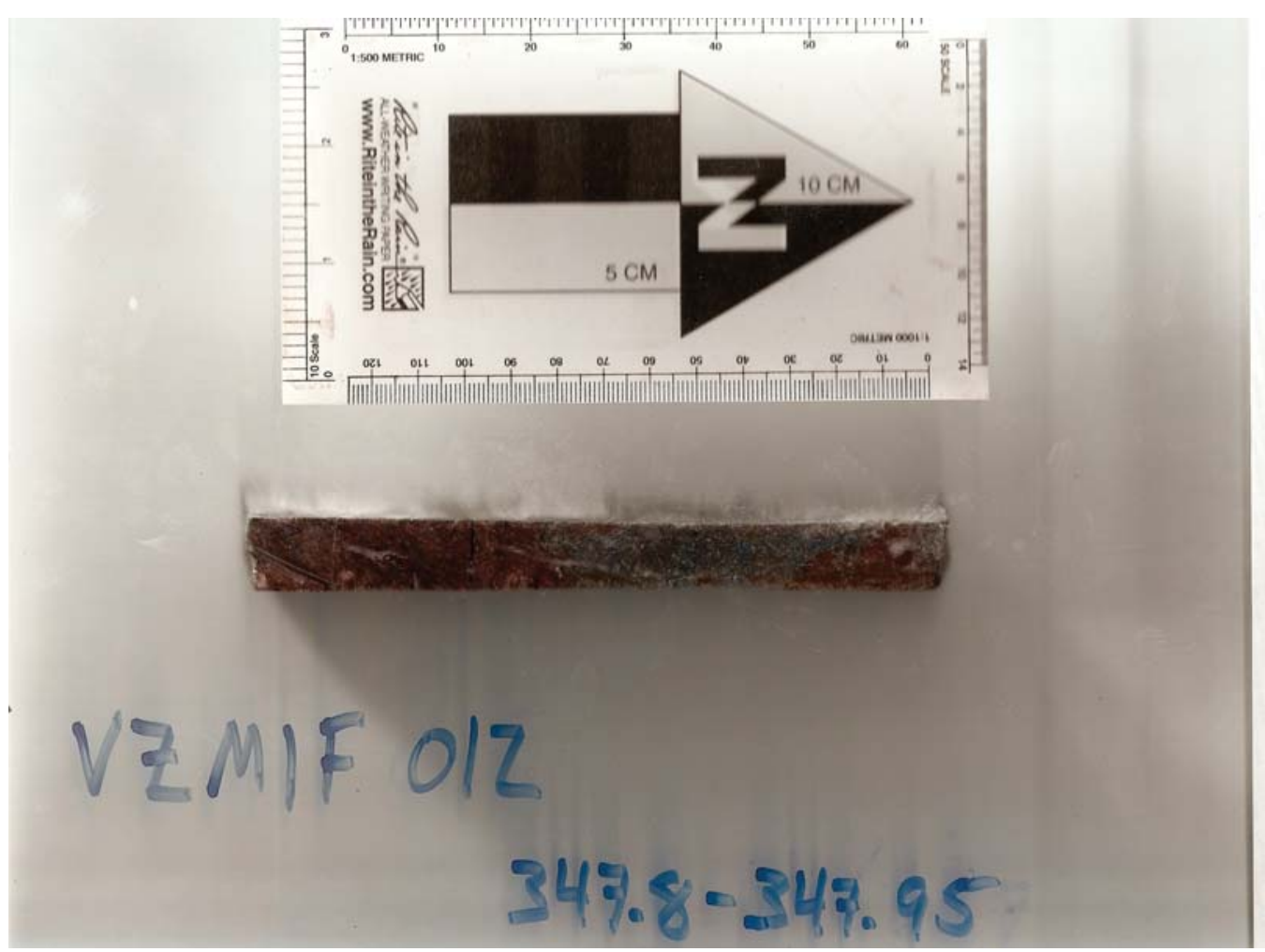

Other Sample Information: Sample from Gilberto Batista. 31\% Zn in sample interval *1685 ppm Ag 


\begin{tabular}{|c|c|c|c|}
\hline minerals & Dolomite & Willemite & quartz \\
\hline percentage & 2 & 56 & 14 \\
\hline morphology & Large sparry xtals & $\begin{array}{l}2 \text { xtal types: } \\
\text { 1) Sm to lg euhedral hexag- } \\
\text { onal xtals } \\
\text { 2) Large, fibrous laths }\end{array}$ & $\begin{array}{l}\text { Anhedral around wl, subhe- } \\
\text { dral in sulfide veins }\end{array}$ \\
\hline texture & $\begin{array}{l}\text { Being replaced by hm }+ \text { frk } \\
\text { and overgrown by wl }\end{array}$ & $\begin{array}{l}\text { Hexagonal xtals massive. } \\
\text { Fibrous xtals filling in open } \\
\text { space }\end{array}$ & $\begin{array}{l}\text { Disseminated, found in } \\
\text { some veins }\end{array}$ \\
\hline $\begin{array}{l}\text { transmitted opti- } \\
\text { cal properties }\end{array}$ & & & \\
\hline $\begin{array}{l}\text { reflected optical } \\
\text { properties }\end{array}$ & & & \\
\hline $\begin{array}{l}\text { contact/ } \\
\text { relationships with } \\
\text { other minerals }\end{array}$ & $\begin{array}{l}\text { Irregular contacts with hm } \\
\text { and frk. Straight edges with } \\
\text { large, euhedral wl xtals. } \\
\text { Some irregular contacts w/ } \\
\text { small wl xtals }\end{array}$ & $\begin{array}{l}\text { Hexagonal xtals have irregu- } \\
\text { lar contacts, replacing dol, } \\
\text { qtz. Fribrous xtals replacing } \\
\text { dol, qtz, and some granular } \\
\text { wl. Hm + frk replacing wl } \\
\text { (found as blebs in wl and has } \\
\text { irregular contacts around } \\
\text { xtal margins }\end{array}$ & $\begin{array}{l}\text { Being replaced by wl, } \\
\text { hm+frk. Qtz in veins being } \\
\text { replaced by wl and Un- } \\
\text { known Ag-Cu Sulfide } \pm \text { sp }\end{array}$ \\
\hline $\begin{array}{l}\text { other (growth di- } \\
\text { rection/zoning) }\end{array}$ & & & \\
\hline
\end{tabular}




\begin{tabular}{|c|c|c|c|}
\hline minerals & Native Silver & Hematite & Frnaklinite \\
\hline percentage & 3 & 4 & trace \\
\hline morphology & $\begin{array}{l}\text { Irregular blobs on Unknown } \\
\text { Ag-Cu Sulfide }\end{array}$ & $\begin{array}{l}\text { Euhederal xtals associated } \\
\text { with Unknown } \mathrm{Ag}-\mathrm{Cu} \text { Sul- } \\
\text { fide }\end{array}$ & Euhedral xtals \\
\hline texture & disseminated & disseminated & disseminated \\
\hline $\begin{array}{l}\text { transmitted opti- } \\
\text { cal properties }\end{array}$ & opaque & opaque & \\
\hline $\begin{array}{l}\text { reflected optical } \\
\text { properties }\end{array}$ & $\begin{array}{l}\text { Yellow with orange tarnish } \\
\text { (happens quickly); soft } \\
\text { Weakly? anisotropic }\end{array}$ & $\begin{array}{l}\text { White, brighter than Un- } \\
\text { known Ag-Cu Sulfide } \\
\text { Deep carmine red internal } \\
\text { reflections } \\
\text { Weak to moderate anisotro- } \\
\text { py; no bireflectance (not hm) }\end{array}$ & \\
\hline $\begin{array}{l}\text { contact/ } \\
\text { relationships with } \\
\text { other minerals }\end{array}$ & $\begin{array}{l}\text { Irregular with Unknown Ag- } \\
\text { Cu Sulfide, exosolution from } \\
\text { Unknown Ag-Cu Sulfide? } \\
\text { Straight contacts with sp and } \\
\text { white Ag-min }\end{array}$ & $\begin{array}{l}\text { Straight contact edge with } \\
\text { Unknown Ag-Cu Sulfide. } \\
\text { Some embayments with sp, } \\
\text { sp found in mineral } \\
\text { (replacing Ag-min). Straight } \\
\text { contact with yellow Ag-min }\end{array}$ & $\begin{array}{l}\text { Some xtals included in Un- } \\
\text { known Ag-Cu Sulfide, but } \\
\text { have straight contacts } \\
\text { (coeval). Embayed into } \\
\text { white Ag-min, replacing Ag. }\end{array}$ \\
\hline $\begin{array}{l}\text { other (growth di- } \\
\text { rection/zoning) }\end{array}$ & Tarnished & & \\
\hline
\end{tabular}




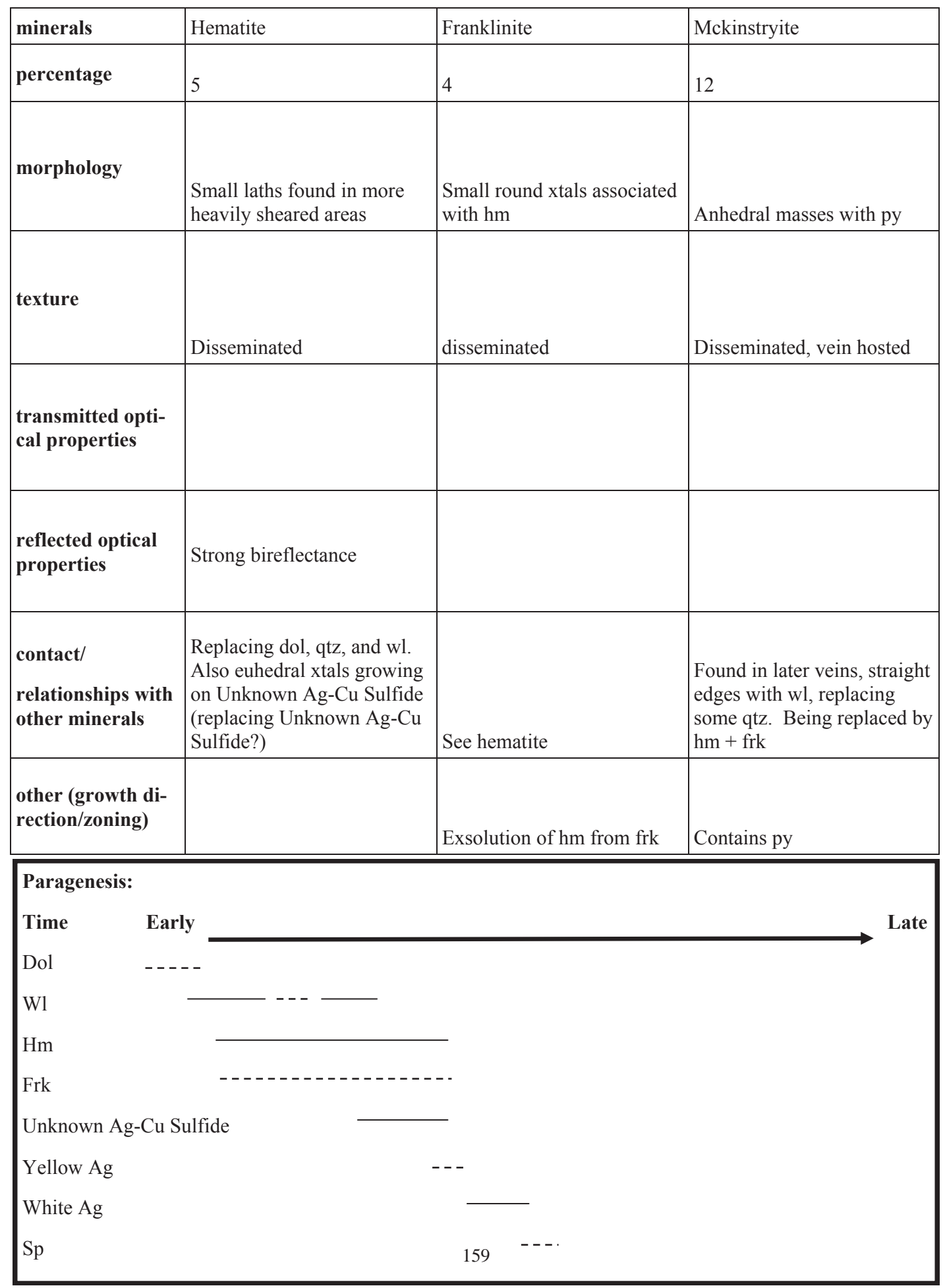


Structures: Areas with small $\mathrm{wl}$ and $\mathrm{hm}$ are associated with shearing. Fractures after main mineralization are also present and filled with Unknown $\mathrm{Ag}-\mathrm{Cu}$ Sulfide. Ore mineralization was still occurring, but at a decreased rate. Hm and Frk may be on Unknown Ag-Cu Sulfide, need to view on SEM.

The minerals are more likely Ag-mins, but unsure as of 3/18/2011

Paragenetic Sequence: $\mathrm{dol} \rightarrow \mathrm{Wl}+\mathrm{Qtz} \rightarrow \mathrm{Hm}+\mathrm{Frk} \rightarrow$ Unknown Ag-Cu Sulfide $\rightarrow \mathrm{Ag}$ mins $\rightarrow \mathrm{Sp}$ ?

Paragenetic Reasoning: Cross-cutting relationships and overgrowths of minerals

Sample Highlights: Substantial presence of Unknown Ag-Cu Sulfide, with lots of sulfosalts? 
Geologic Unit: Hydrothermal Breccia

Lithological Unit (Votorantim): Disseminated Silicate (in BH)

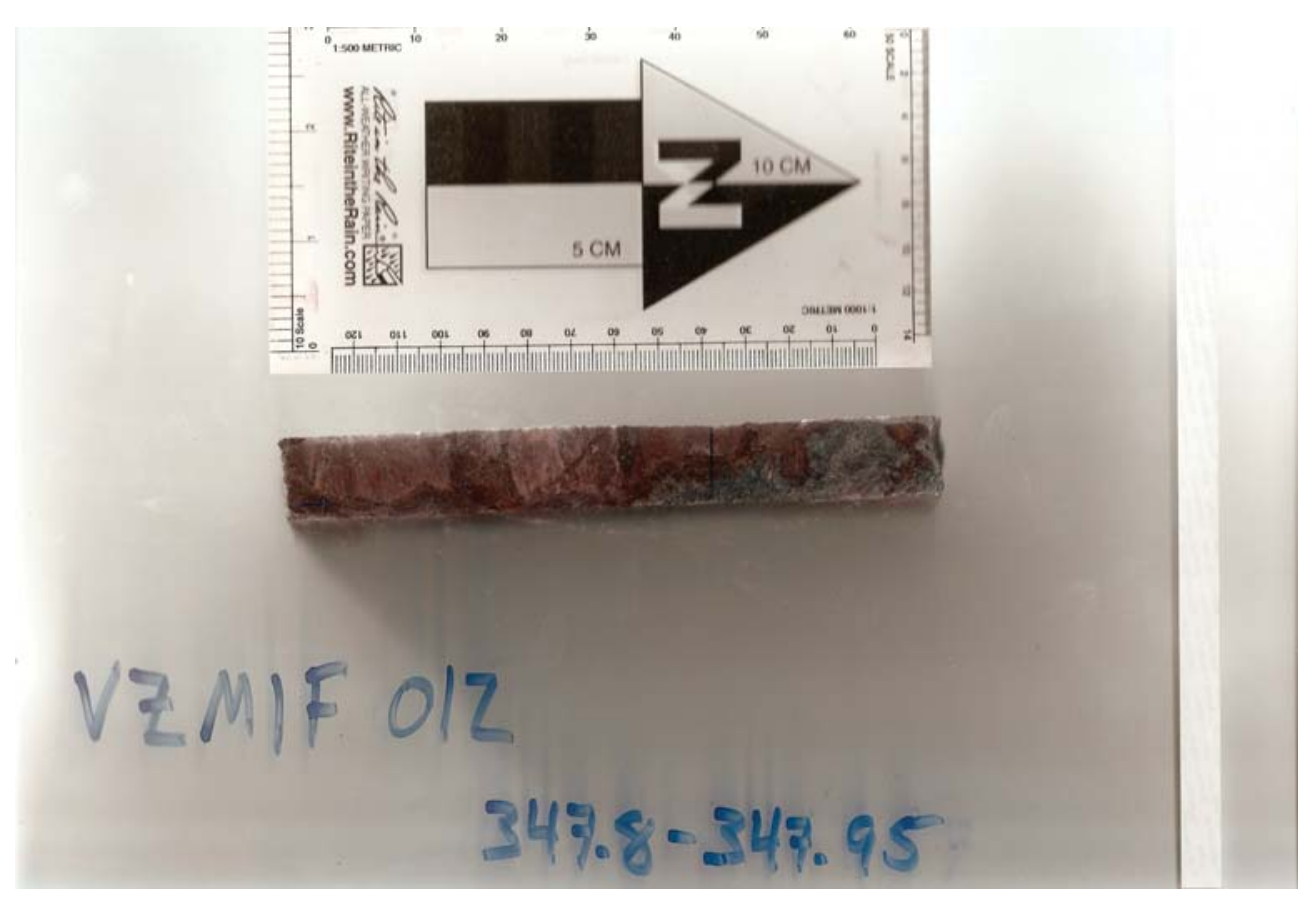

Other Sample Information: Same as 012-347A, but with more qtz and galena at the expense of willemite 
Geologic Unit: Hydrothermal Breccia

Lithological Unit (Votorantim): Disseminated Silicate (SD) in BH

\section{Sample Photograph}

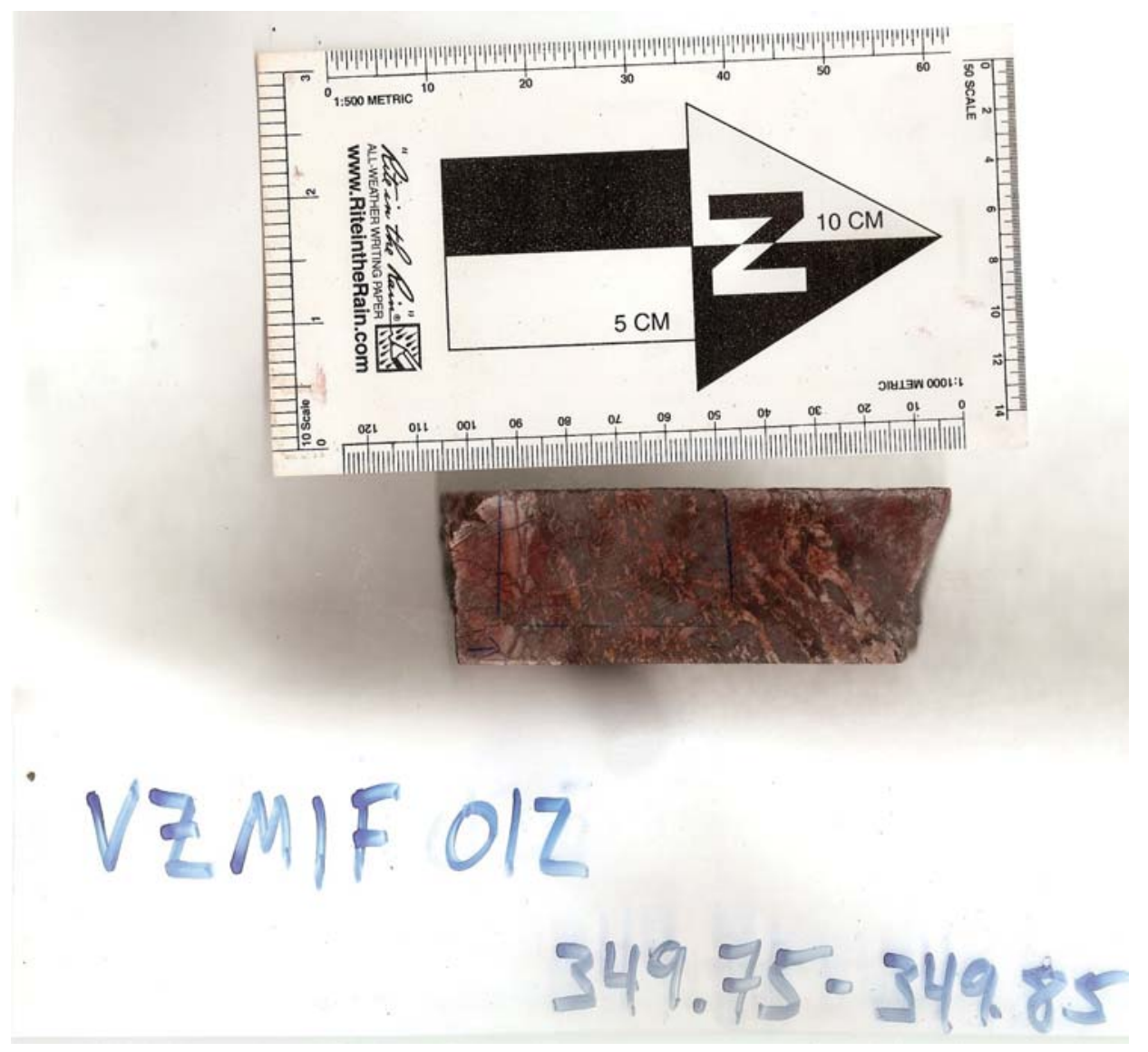

Other Sample Information: $\sim 2 \% \mathrm{Zn}$ and $12 \% \mathrm{Fe}$

** Similar to $125-130 * *$ 


\begin{tabular}{|c|c|c|c|}
\hline minerals & Dolomite & Hematite & quartz \\
\hline percentage & 42 & 55 & 3 \\
\hline morphology & Euhdral sparry dol & Veins and euhedral xtal laths & Anhedral replacement xtals \\
\hline texture & $\begin{array}{l}\text { Brecciated, being replaced } \\
\text { by hm }\end{array}$ & Semi-massive & Veins, being replaced \\
\hline $\begin{array}{l}\text { transmitted opti- } \\
\text { cal properties }\end{array}$ & & & \\
\hline $\begin{array}{l}\text { reflected optical } \\
\text { properties }\end{array}$ & & & \\
\hline $\begin{array}{l}\text { contact/ } \\
\text { relationships with } \\
\text { other minerals }\end{array}$ & $\begin{array}{l}\text { Being replaced by } \mathrm{hm} \text { and } \\
\text { qtz. Irregular contacts with } \\
\text { both mins }\end{array}$ & $\begin{array}{l}\text { Shredded, irregular contacts } \\
\text { with dol and qtz }\end{array}$ & $\begin{array}{l}\text { Being replaced by } \mathrm{hm} \text { and } \\
\text { Fe-dol in vein }\end{array}$ \\
\hline $\begin{array}{l}\text { other (growth di- } \\
\text { rection/zoning) }\end{array}$ & & & \\
\hline
\end{tabular}




\begin{tabular}{|l|l|l|l|}
\hline minerals & Franklinite & & \\
\hline percentage & trace & & \\
\hline morphology & Very minute blebs & & \\
\hline texture & & & \\
\hline $\begin{array}{l}\text { transmitted opti- } \\
\text { cal properties }\end{array}$ & Sparsely disseminated & & \\
\hline $\begin{array}{l}\text { reflected optical } \\
\text { properties }\end{array}$ & & & \\
\hline $\begin{array}{l}\text { contact/ } \\
\text { relationships with } \\
\text { other minerals }\end{array}$ & & & \\
\hline $\begin{array}{l}\text { other (growth di- } \\
\text { rection/zoning) }\end{array}$ & Difficult to find & & \\
\hline $\begin{array}{l}\text { Coeval with hm } \\
\text { Pang }\end{array}$ & & & \\
\hline
\end{tabular}

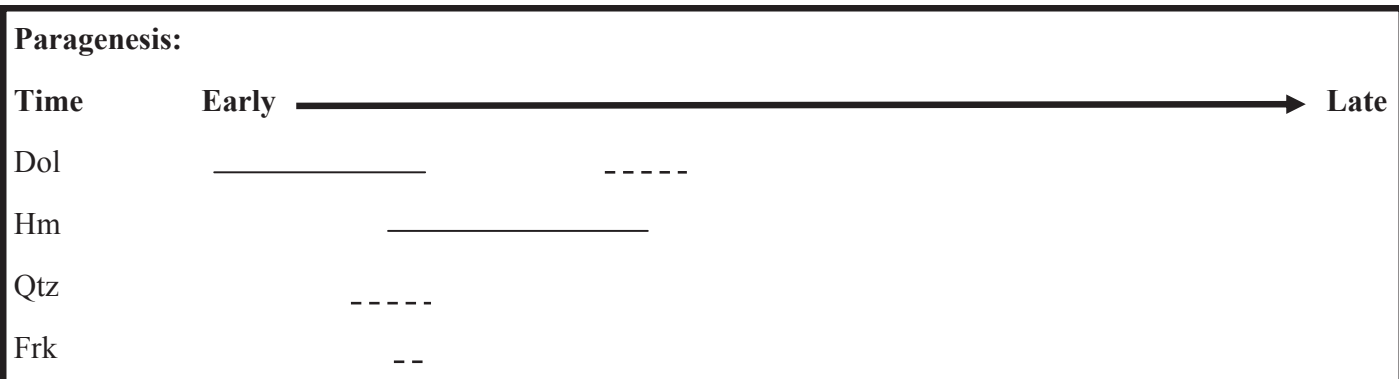


Structures: There are some dolomite veins that are orthogonal to the main shear (later even tension gashes?) The hm composes the main shear in this sample

Paragenetic Sequence: See chart

Paragenetic Reasoning: cross-cutting of minerals and irregular contacts

Sample Highlights: see 125-130 
Geologic Unit: Hydrothermal Breccia

Lithological Unit (Votorantim): Hydrothermal Breccia

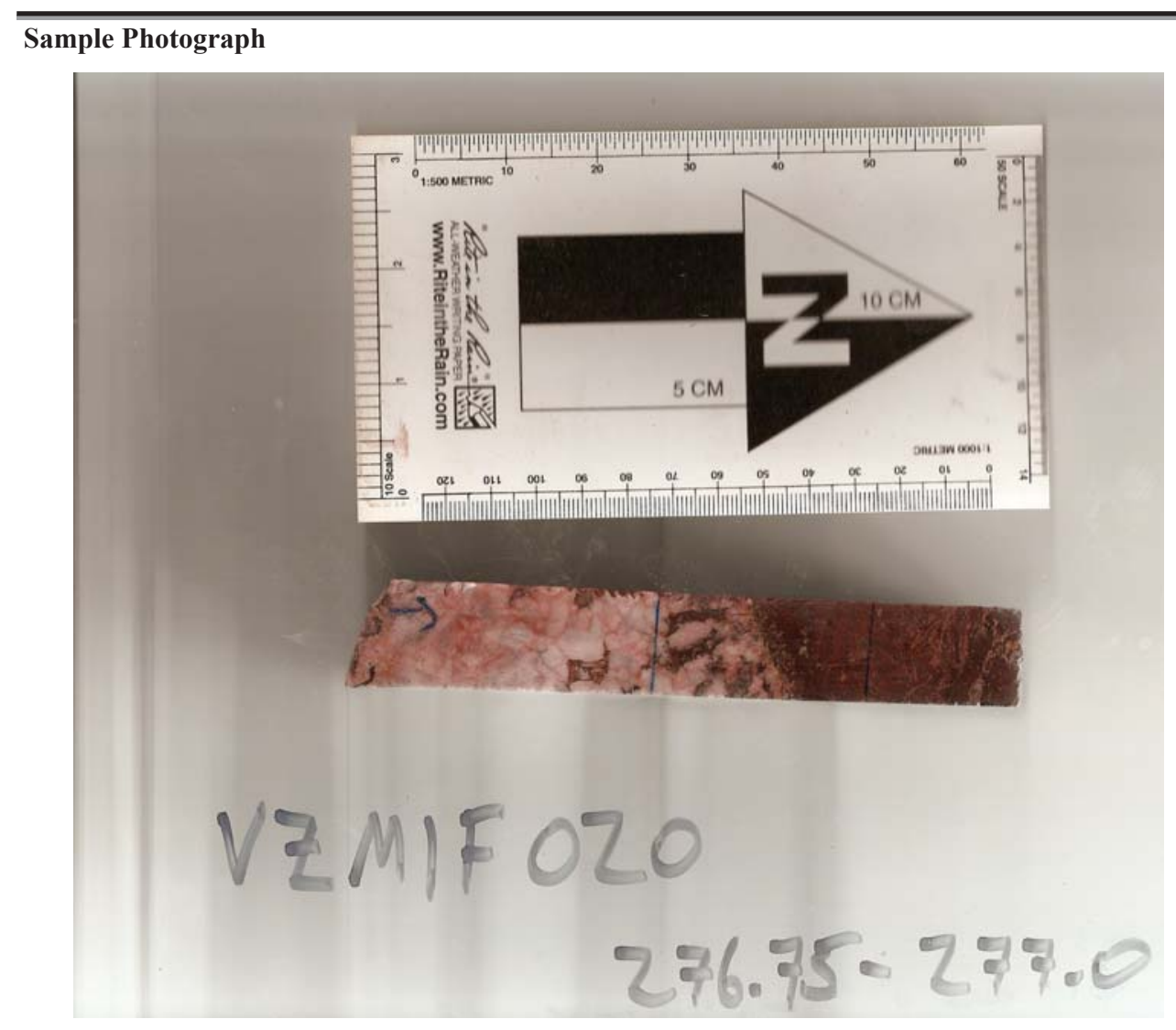

Other Sample Information: No wl in sample or in drill hole 


\begin{tabular}{|c|c|c|c|}
\hline minerals & Dolomite & Hematite & quartz \\
\hline percentage & 70 & 20 & 10 \\
\hline morphology & $\begin{array}{l}\text { Large euhedral xtals, some } \\
\text { smaller 2ndary dol xtals } \\
\text { growing on larger ones }\end{array}$ & $\begin{array}{l}\text { Subhedral laths to semi- } \\
\text { massive veins }\end{array}$ & $\begin{array}{l}\text { Anhedral, follows fractures } \\
\text { and dol xtal boundaries, as- } \\
\text { sociated with hm }\end{array}$ \\
\hline texture & Neomorphic carbonate & $\begin{array}{l}\text { Netted texture in veins (b/w } \\
\text { specular and non specular } \\
\mathrm{hm} \text { ). Replacement similar to }\end{array}$ & $\begin{array}{l}\text { Replacement texture along } \\
\text { dol xtal boundaries and in } \\
\text { hm-rich zones }\end{array}$ \\
\hline \multicolumn{4}{|l|}{$\begin{array}{l}\text { transmitted opti- } \\
\text { cal properties }\end{array}$} \\
\hline \multicolumn{4}{|l|}{$\begin{array}{l}\text { reflected optical } \\
\text { properties }\end{array}$} \\
\hline $\begin{array}{l}\text { contact/ } \\
\text { relationships with } \\
\text { other minerals }\end{array}$ & $\begin{array}{l}\text { Sharp contacts with dol. } \\
\text { More irregular contacts with } \\
\text { qtz. Embayments and hm } \\
\text { overgrowths on dol contacts }\end{array}$ & $\begin{array}{l}\text { Lath shaped overgrowths on } \\
\text { dol and qtz }\end{array}$ & $\begin{array}{l}\text { Wavy contacts with hm and } \\
\text { dol, replaced by hm }\end{array}$ \\
\hline $\begin{array}{l}\text { other (growth di- } \\
\text { rection/zoning) }\end{array}$ & & & \\
\hline
\end{tabular}

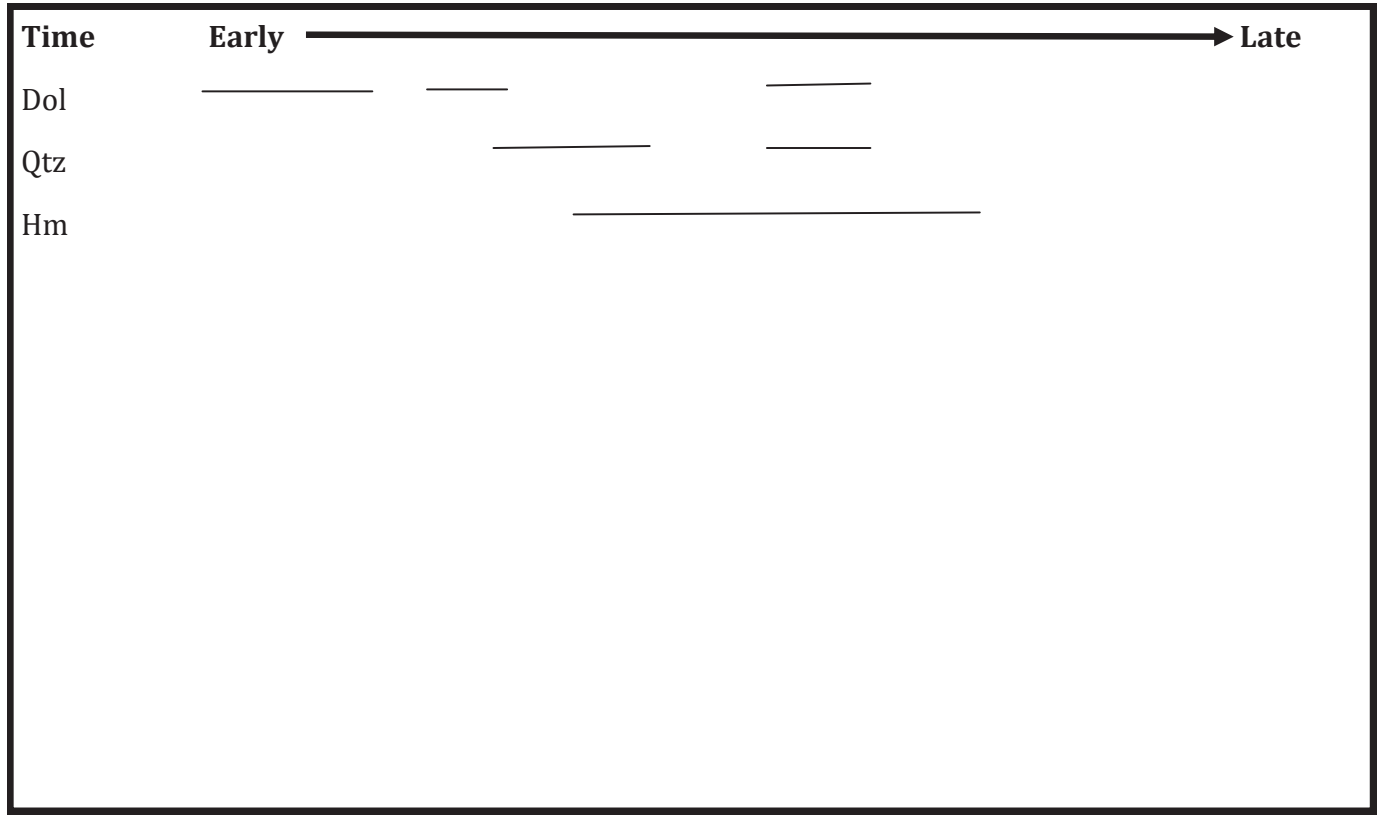


Structures: $\sim 3$ dol and qtz veins that run through dol and hm zones. Veins being replaced by hm when in hm and dol when in dol (after dol, but during hm events)

Paragenetic Sequence: dol $\rightarrow$ 2ndary dol $\rightarrow \mathrm{qtz} \rightarrow \mathrm{hm}+\mathrm{qtz} \rightarrow$ dol/qtz veins $\rightarrow \mathrm{hm}$

(hm is continuous until end)

Paragenetic Reasoning: dol recrystallized and is being replaced in the center of crystals by smaller dol xtals. Qtz is replacing dol along xtal boundaries but is being replaced by $\mathrm{hm}$. $\mathrm{Hm}$ is replacing dol as well. Dol/qtz veins cut across existing $\mathrm{hm}$ and dol, but have some $\mathrm{hm}$ laths overgrowing the veins, suggesting that hm is coeval with later qtz and the veining.

Sample Highlights: dol veins 
Geologic Unit: Hyrothermal Breccia

Lithological Unit (Votorantim): Hyrothermal Breccia

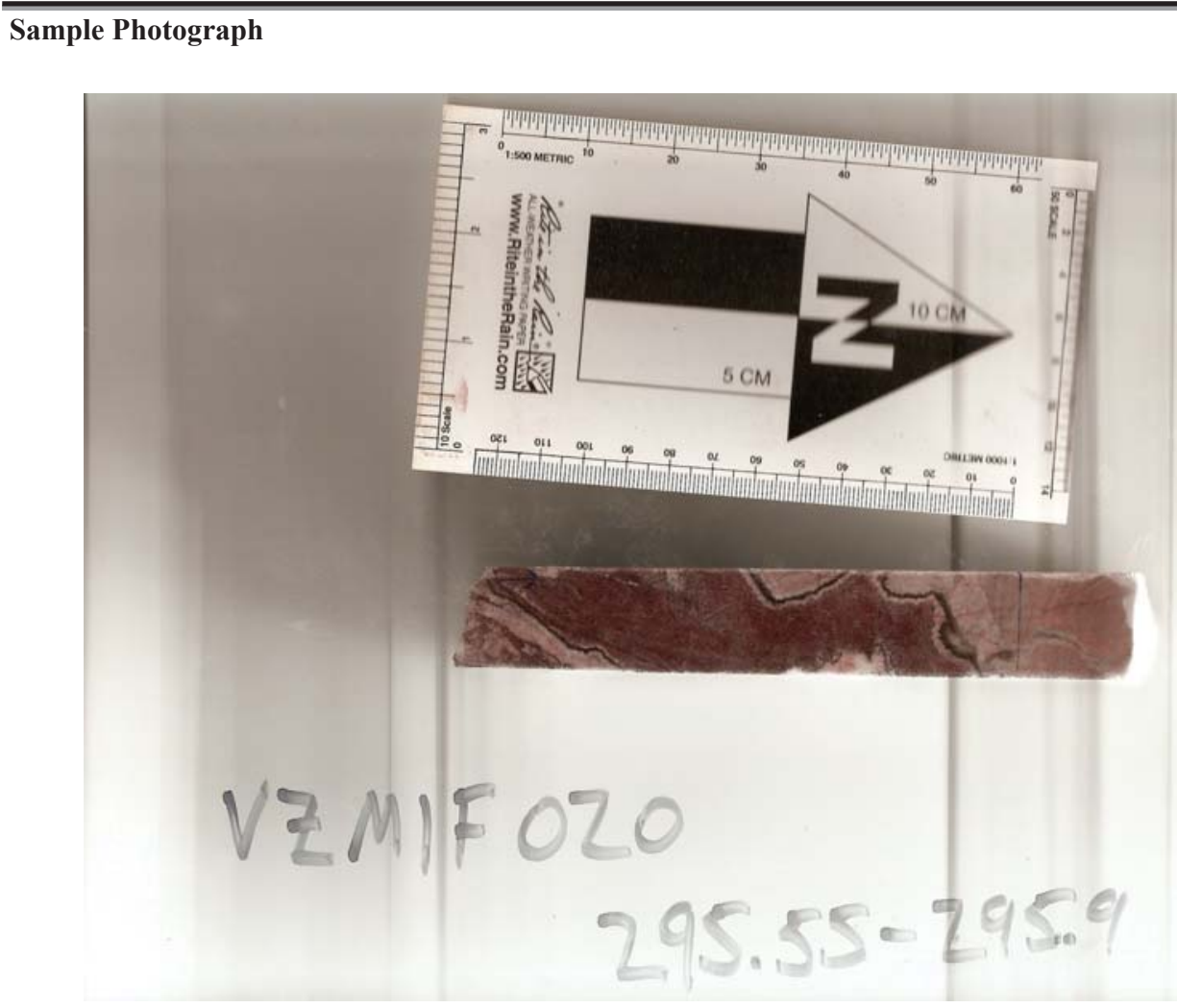

Other Sample Information: Large Fe-carbonate vein 


\begin{tabular}{|l|l|l|l|}
\hline minerals & Dolomite & Fe-rich dol & quartz \\
\hline percentage & 30 & 55 & 10 \\
\hline morphology & $\begin{array}{l}\text { Small xtals neomorphosing } \\
\text { to large euhdreal xtals, }\end{array}$ & See dolomite & Amorphous, small to larger \\
xtals
\end{tabular}




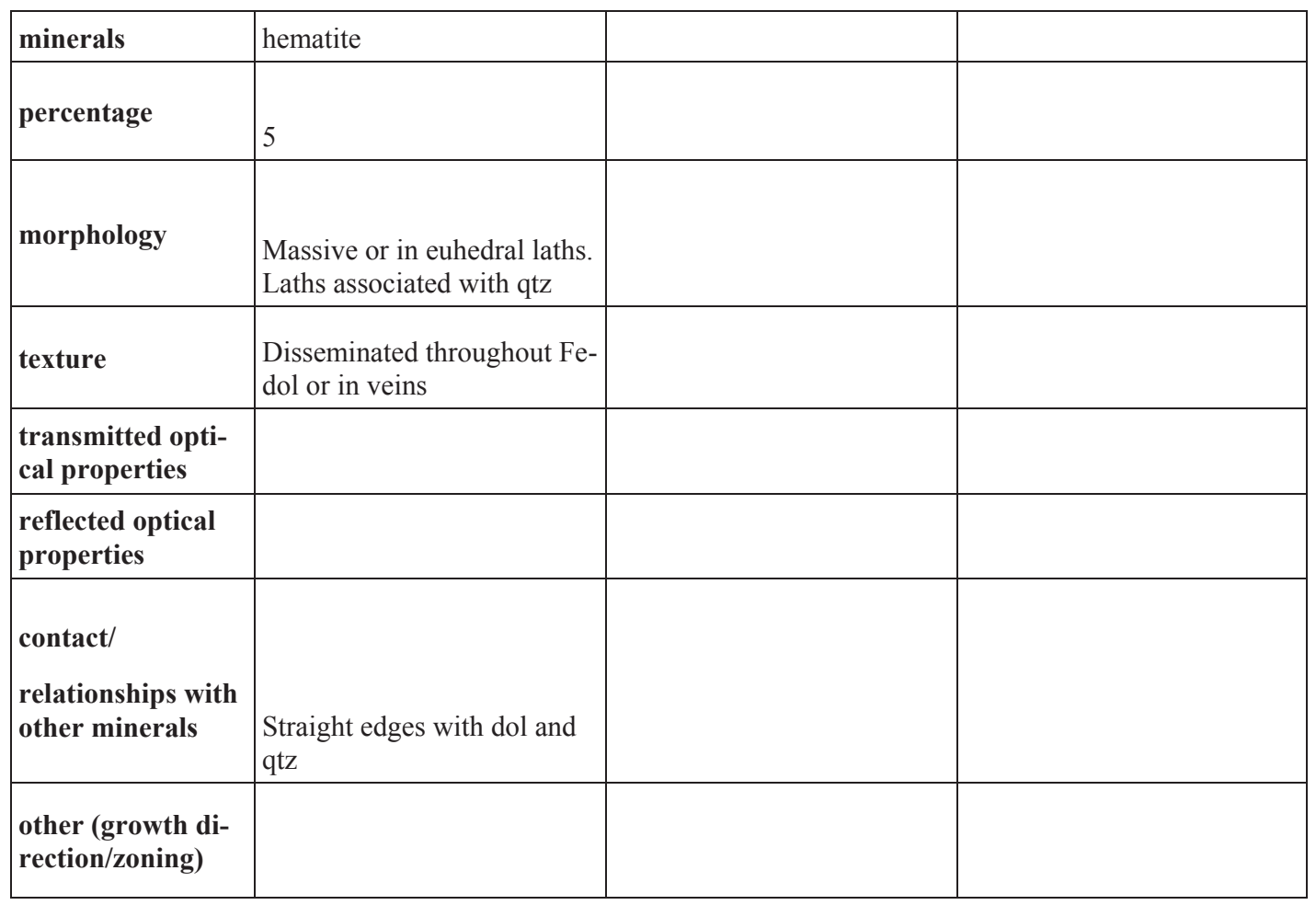

Paragenesis:

Time

Early

Late

Dol

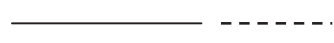

Qtz

Hm

Fe-Dol 
Structures: Qtz vein being cut by regular dol. Qtz vein is folded. Some qtz being replaced by dol

Paragenetic Sequence: $\mathrm{Dol} \rightarrow \mathrm{qtz} \rightarrow \mathrm{hm}$

Paragenetic Reasoning: qtz contains fragments of dol and is being overgrown by $\mathrm{hm}$

Sample Highlights: Sample is an example of Fe-carb alteration. Do microprobe and/or SEM analysis for further data. 
Geologic Unit: Hydrothermal Breccia

Lithological Unit (Votorantim): Disseminated Silicate (SD in BH)

Sample Photograph
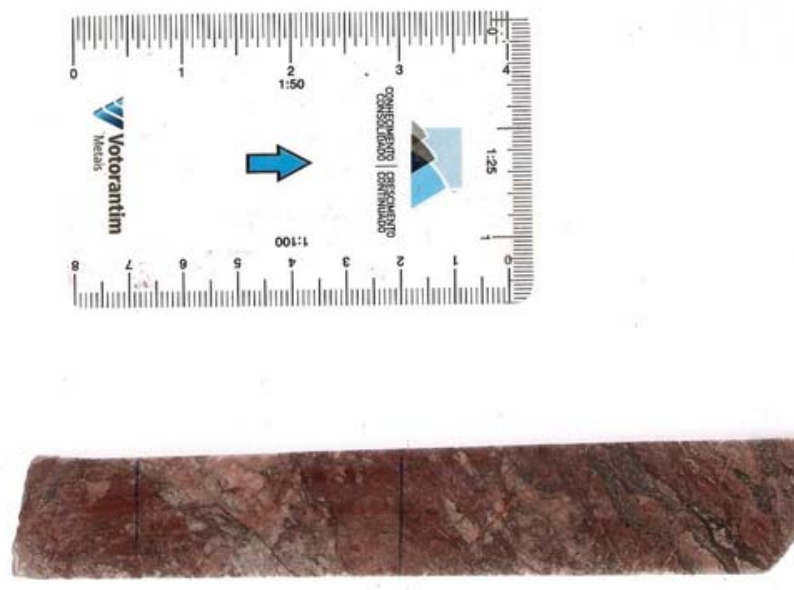

\section{VEMIF OZO}

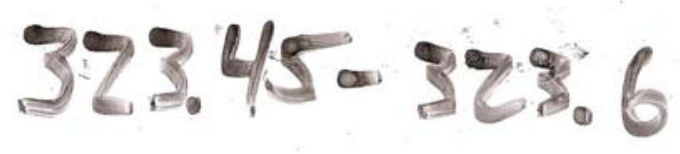

Other Sample Information: Contains $\mathrm{Sp}^{* * *}$ 


\begin{tabular}{|c|c|c|c|}
\hline minerals & Fe-carb & Quartz & Sphalerite \\
\hline percentage & 75 & 5 & 10 \\
\hline morphology & $\begin{array}{l}\text { Hydrothermal sparite, very } \\
\text { large xtals }\end{array}$ & Anhedral grains & veinlet \\
\hline texture & Rock forming, brecciated & $\begin{array}{l}\text { Disseminated, being re- } \\
\text { placed by Fe-carb and Hm }\end{array}$ & Semi-massive in veinlets \\
\hline $\begin{array}{l}\text { transmitted opti- } \\
\text { cal properties }\end{array}$ & & & Transparent in ppl \\
\hline $\begin{array}{l}\text { reflected optical } \\
\text { properties }\end{array}$ & & & \\
\hline $\begin{array}{l}\text { contact/ } \\
\text { relationships with } \\
\text { other minerals }\end{array}$ & $\begin{array}{l}\text { Irregular, replacing qtz, be- } \\
\text { ing replaced by late carb and } \\
\mathrm{hm}\end{array}$ & $\begin{array}{l}\text { Very irregular boundaries } \\
\text { with Fe-carb and hm (these } \\
\text { mins replacing qtz) }\end{array}$ & $\begin{array}{l}\text { Irregular contacts w/ Fe- } \\
\text { carb, cutting Fe-carb, } \\
\text { straight contacts with Hm } \\
\text { inclusions }\end{array}$ \\
\hline $\begin{array}{l}\text { other (growth di- } \\
\text { rection/zoning) }\end{array}$ & & & $\begin{array}{l}\text { Contains disseminated } \mathrm{hm} \\
\text { crystals }\end{array}$ \\
\hline
\end{tabular}




\begin{tabular}{|c|c|c|}
\hline minerals & Hematite & Late Carb veinlets \\
\hline percentage & 7 & 3 \\
\hline morphology & $\begin{array}{l}\text { Crystal laths, specular and } \\
\text { nonspecular } \\
\text { Disseminated in Sp }\end{array}$ & Fine-grained carb \\
\hline texture & Veinlets and disseminated & veinlets \\
\hline $\begin{array}{l}\text { transmitted opti- } \\
\text { cal properties }\end{array}$ & & \\
\hline $\begin{array}{l}\text { reflected optical } \\
\text { properties }\end{array}$ & & \\
\hline $\begin{array}{l}\text { contact/ } \\
\text { relationships with } \\
\text { other minerals }\end{array}$ & $\begin{array}{l}\text { Irregular with qtz and Fe- } \\
\text { carb }\end{array}$ & $\begin{array}{l}\text { Cut all other minerals, re- } \\
\text { places Fe-carb }\end{array}$ \\
\hline $\begin{array}{l}\text { other (growth di- } \\
\text { rection/zoning) }\end{array}$ & & \\
\hline
\end{tabular}

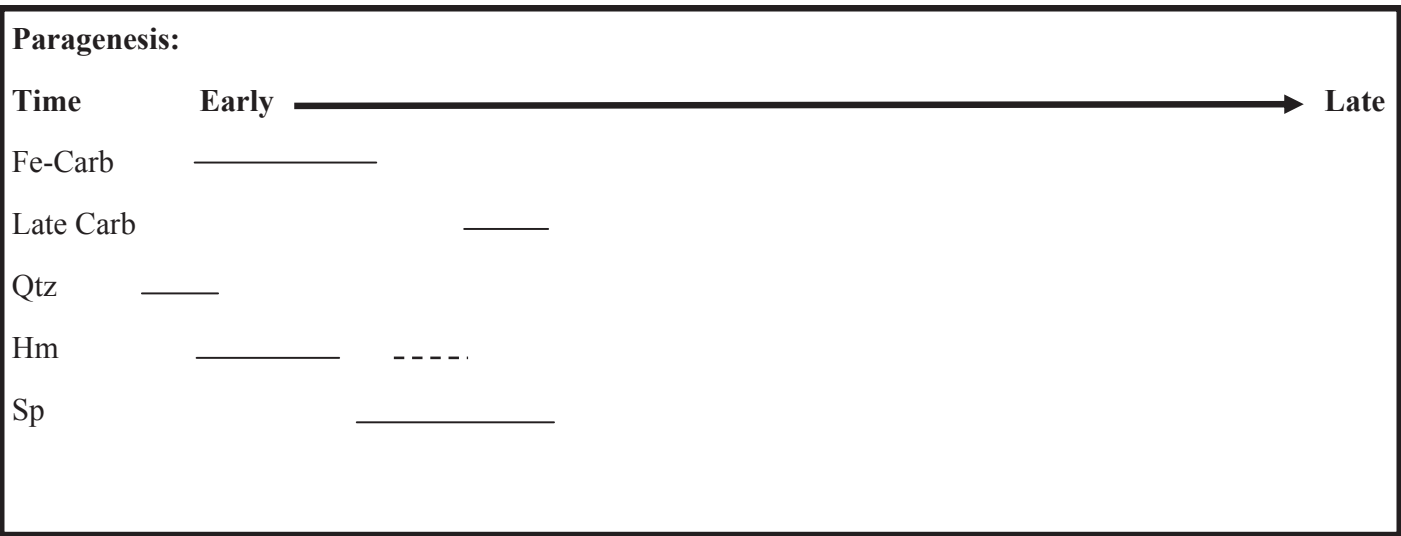


Structure: Sheared $\mathrm{Sp}$ in sample

Paragenetic Sequence:

Paragenetic Reasoning: Sp in a shear vein, cutting Fe-carb alteration

Sample Highlights: 
Geologic Unit: Hydrothermal Breccia

Lithological Unit (Votorantim): Pink dolomite (DO)

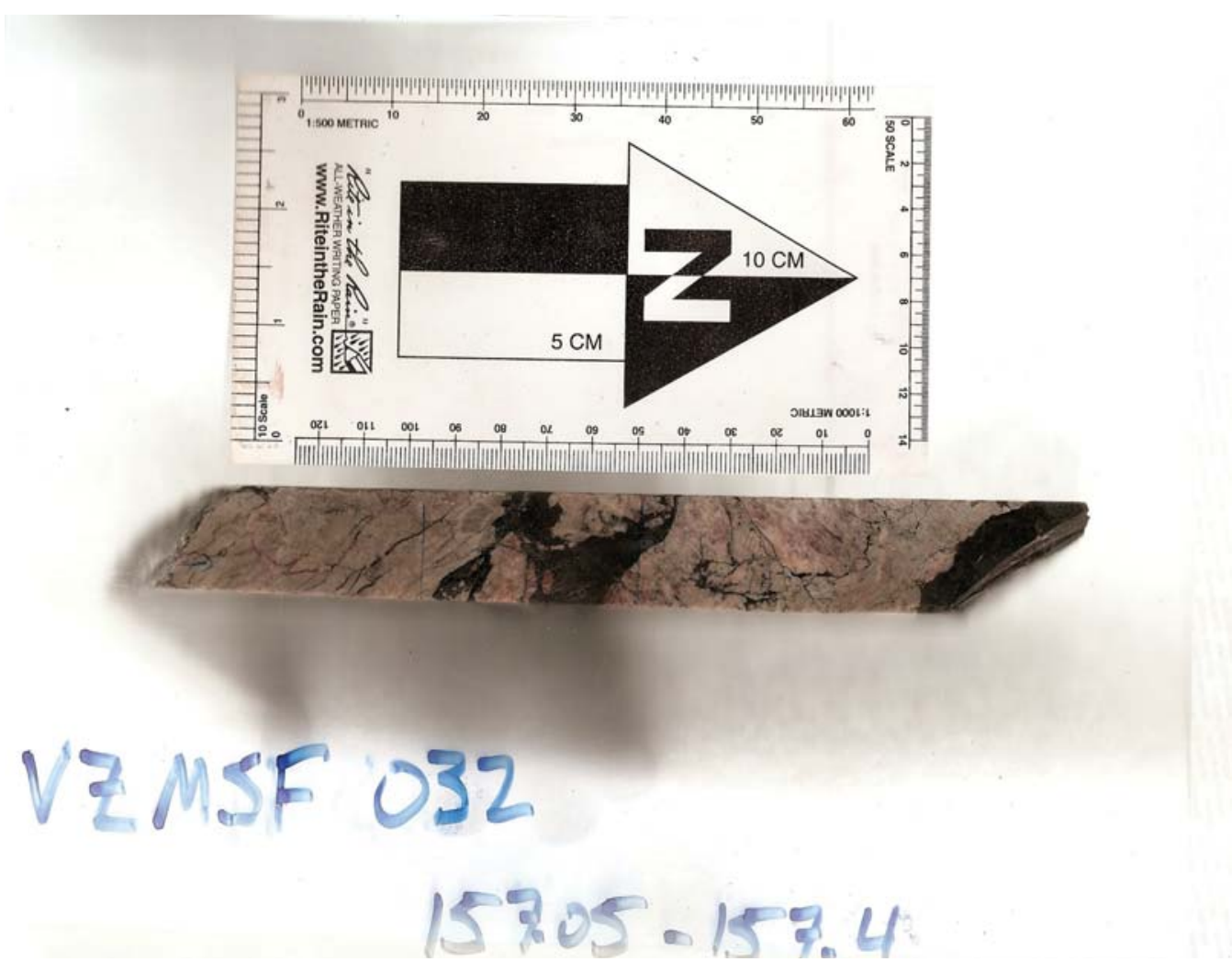

Other Sample Information: Magnetite vein in bx, near contact between BH and Serra do Garrote *V high in this sample 


\begin{tabular}{|c|c|c|c|}
\hline minerals & Dolomite & Magnetite & Hematite \\
\hline percentage & 35 & 20 & 25 \\
\hline morphology & $\begin{array}{l}\text { Euhedral xtals, small in ma- } \\
\text { trix, some larger neomor- } \\
\text { phosed xtals }\end{array}$ & $\begin{array}{l}\text { Euhdedral xtals forming } \\
\text { massive vein }\end{array}$ & $\begin{array}{l}\text { Pseudomorphs of mt. also } \\
\text { found in thin veins running } \\
\text { through chlorite }\end{array}$ \\
\hline texture & Sparry matrix & Vein filling texture & Replacing mt, veins in chl \\
\hline $\begin{array}{l}\text { transmitted opti- } \\
\text { cal properties }\end{array}$ & & & \\
\hline $\begin{array}{l}\text { reflected optical } \\
\text { properties }\end{array}$ & & & \\
\hline $\begin{array}{l}\text { contact/ } \\
\text { relationships with } \\
\text { other minerals }\end{array}$ & $\begin{array}{l}\text { Straight edges with chlorite. } \\
\text { Replacement by iron oxides, } \\
\text { embayments, xtal replace- } \\
\text { ments of dol by mt. Found } \\
\text { as euhedral xtals in chl }\end{array}$ & $\begin{array}{l}\text { Fuzzy contact with hm. Ir- } \\
\text { regular contacts with chl and } \\
\text { dol }\end{array}$ & $\begin{array}{l}\text { Veins are straight contacts } \\
\text { w/ chl. Fuzzy edges w/ mt. } \\
\text { Some irregular edges with } \\
\text { dol and chl }\end{array}$ \\
\hline $\begin{array}{l}\text { other (growth di- } \\
\text { rection/zoning) }\end{array}$ & & $\begin{array}{l}2 \text { types of mt? brn-grey and } \\
\text { dark grey? }\end{array}$ & $\begin{array}{l}2 \text { stages of hm: veins (early) } \\
\text { and replacement of } \mathrm{mt} \\
\text { (later) }\end{array}$ \\
\hline
\end{tabular}




\begin{tabular}{|c|c|c|c|}
\hline minerals & Chlorite & Goethite & Unknown \\
\hline percentage & 15 & 2 & trace \\
\hline morphology & Long fibrous xtals & Thin veins & Euhedral center of mt \\
\hline texture & Sheared, vein forming & fuzzy & Being replaced by hm \\
\hline $\begin{array}{l}\text { transmitted opti- } \\
\text { cal properties }\end{array}$ & Blue in xpl & & \\
\hline $\begin{array}{l}\text { reflected optical } \\
\text { properties }\end{array}$ & & Bluish-grey, darker than hm & $\begin{array}{l}\text { Dark grey compared to hm } \\
\text { and more grey than } \mathrm{mt} . \\
\text { Weakly anisotropic }\end{array}$ \\
\hline $\begin{array}{l}\text { contact/ } \\
\text { relationships with } \\
\text { other minerals }\end{array}$ & $\begin{array}{l}\text { Some irregular contacts with } \\
\text { mt, straight contacts with dol } \\
\text { and hm veins }\end{array}$ & $\begin{array}{l}\text { Cloudy edges with sphalerite } \\
\text { (oxidizing), shreddy edges } \\
\text { with chl, (reflection of goe } \\
\text { and chl morphology?), re- } \\
\text { placing mt }\end{array}$ & $\begin{array}{l}\text { Wavy edges and embay- } \\
\text { ments with hm }\end{array}$ \\
\hline $\begin{array}{l}\text { other (growth di- } \\
\text { rection/zoning) }\end{array}$ & & & \\
\hline
\end{tabular}

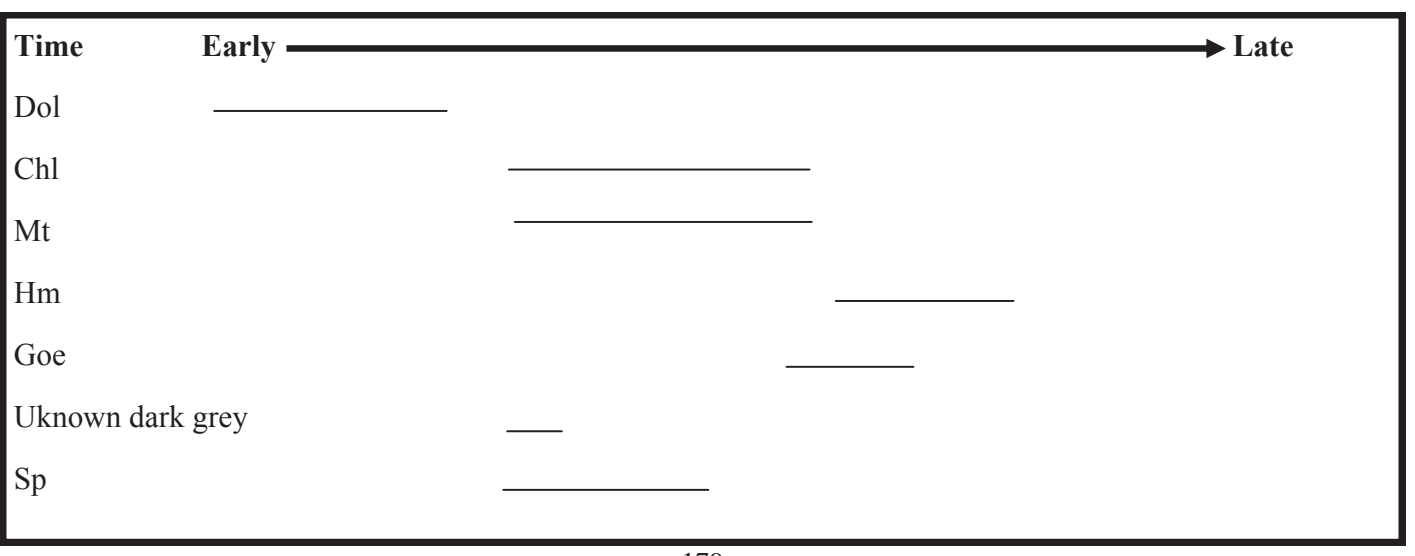




\begin{tabular}{|c|c|c|c|}
\hline minerals & Sphalerite & & \\
\hline percentage & 3 & & \\
\hline morphology & $\begin{array}{l}\text { Hydrothermal veins and as } \\
\text { disseminated xtals }\end{array}$ & & \\
\hline texture & $\begin{array}{l}\text { Small veins and xtals in chl, } \\
\text { being replaced by goe }\end{array}$ & & \\
\hline $\begin{array}{l}\text { transmitted opti- } \\
\text { cal properties }\end{array}$ & & & \\
\hline $\begin{array}{l}\text { reflected optical } \\
\text { properties }\end{array}$ & & & \\
\hline $\begin{array}{l}\text { contact/ } \\
\text { relationships with } \\
\text { other minerals }\end{array}$ & $\begin{array}{l}\text { Irregular contacts with goe } \\
\text { and chl }\end{array}$ & & \\
\hline $\begin{array}{l}\text { other (growth di- } \\
\text { rection/zoning) }\end{array}$ & & & \\
\hline
\end{tabular}

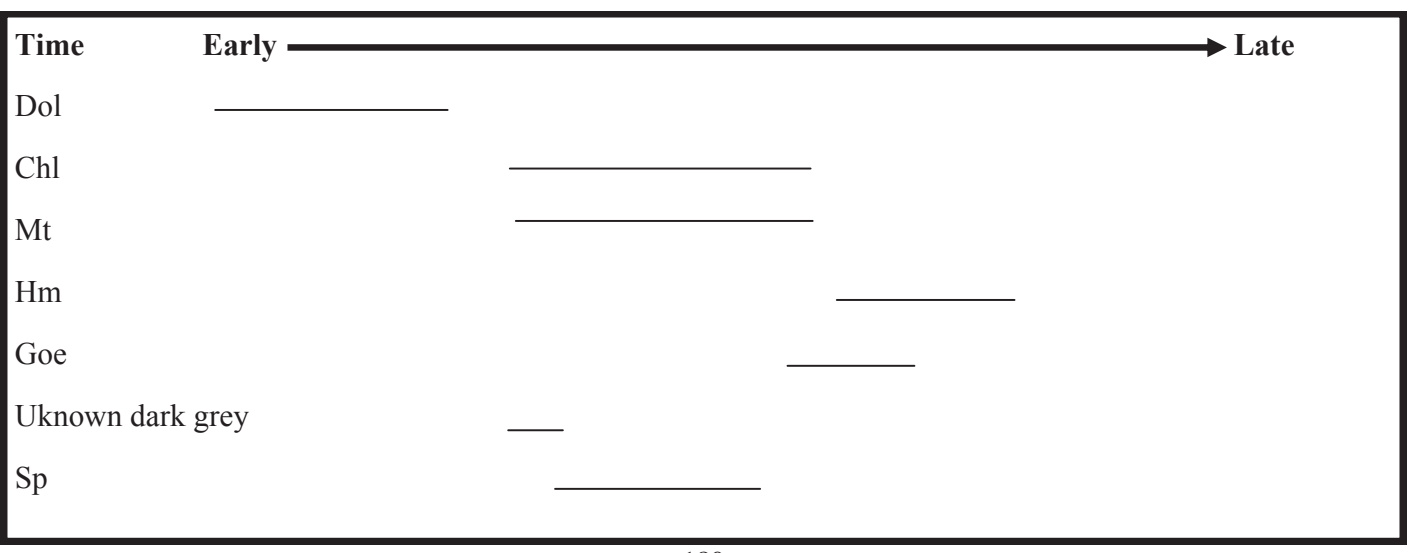


Structures: Shear vein filled with chl, sp, goe, and $\mathrm{mt}$. Goe appears to be altered from chl and possibly sp.

Paragenetic Sequence: dol $\rightarrow \mathrm{mt}+\mathrm{chl}+($ unknown $) \rightarrow \mathrm{hm}$

Paragenetic Reasoning: dol has been fractured and filled with chl and $\mathrm{mt}$. The $\mathrm{mt}$ is being replaced by $\mathrm{hm}$, as seen by the embayments, fuzzy edges, and irregular contacts $\mathrm{b} / \mathrm{w} \mathrm{mt}$ and $\mathrm{hm}$.

Sample Highlights: magnetite, unknown mins, chl, high V content 
Sample \#: 32-177

Hole \#: VZMSF 032 Sample Interval: 177.3-177.5 m

Geologic Unit: Serra do Garrote

Lithological Unit (Votorantim): Carbonaceous phyllites (FC)

Sample Photograph
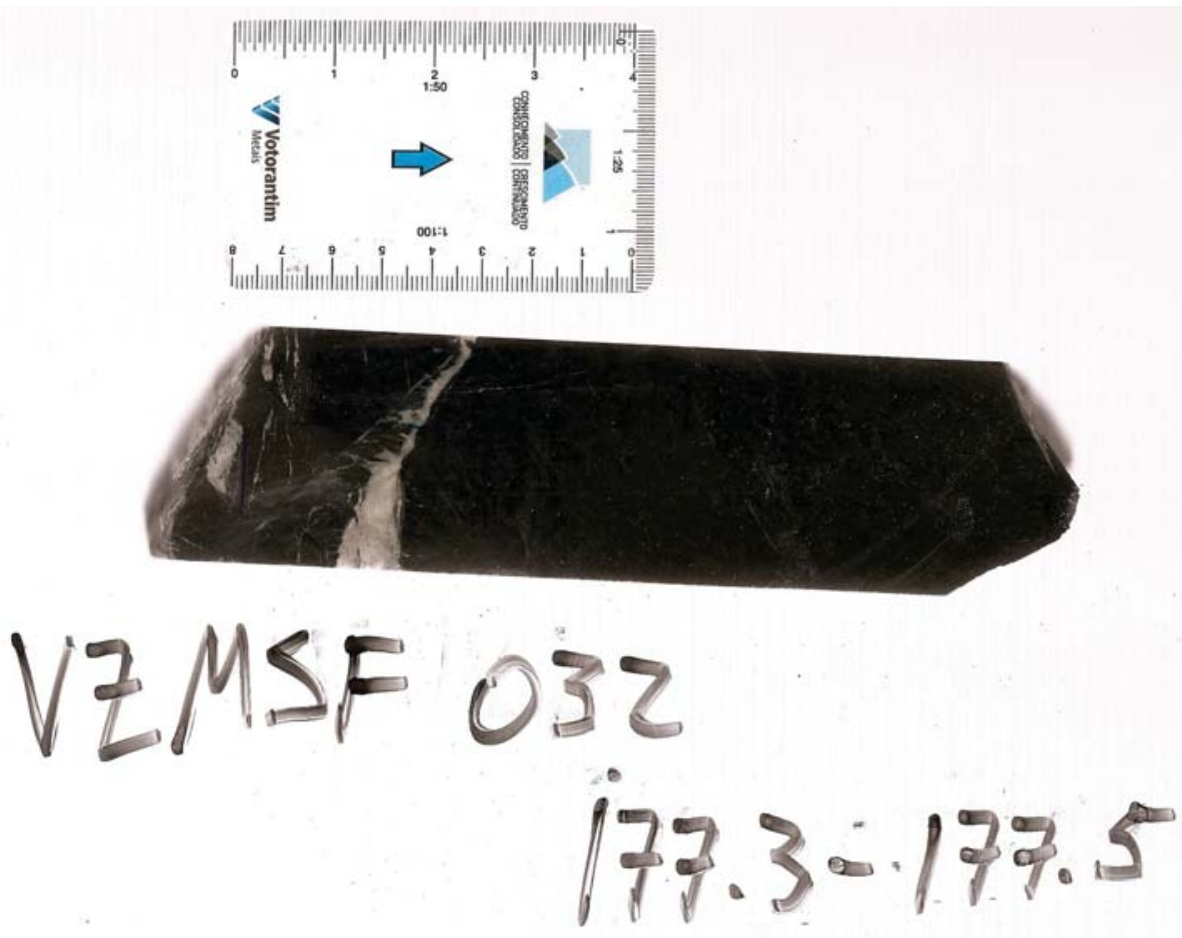

Other Sample Information:

182 


\begin{tabular}{|c|c|c|c|}
\hline minerals & Fine-grained silicates & Carbonate (calcite?) & pyrite \\
\hline percentage & 70 & 23 & 4 \\
\hline morphology & $\begin{array}{l}\text { Foliated, mostly composed } \\
\text { of quartz with some dark, } \\
\text { non-reflective opaques } \\
\text { (carbon?) }\end{array}$ & $\begin{array}{l}\text { Large, subhedral xtals with } \\
\text { minor qtz associations (1\%) }\end{array}$ & $\begin{array}{l}\text { Small crystals in fine- } \\
\text { grained silicates } \\
\text { Discontinuous veinlets next } \\
\text { to Sp (more granular than } \\
\mathrm{Sp} \text { ) }\end{array}$ \\
\hline texture & Foliated, rock matrix & $\begin{array}{l}\text { Vein-forming, hydrother- } \\
\text { mal? }\end{array}$ & $\begin{array}{l}\text { Foliated with silicate matrix; } \\
\text { some found within } \mathrm{Sp} \text { as dis- } \\
\text { seminations }\end{array}$ \\
\hline $\begin{array}{l}\text { transmitted opti- } \\
\text { cal properties }\end{array}$ & & & \\
\hline $\begin{array}{l}\text { reflected optical } \\
\text { properties }\end{array}$ & & & \\
\hline $\begin{array}{l}\text { contact/ } \\
\text { relationships with } \\
\text { other minerals }\end{array}$ & $\begin{array}{l}\text { Rock matrix, being cut by } \\
\text { carbonate vein }\end{array}$ & $\begin{array}{l}\text { Cutting rock matrix, next to } \\
\text { Sp }+ \text { Cpy veinlet }\end{array}$ & $\begin{array}{l}\text { Veinlet xtals being replaced } \\
\text { by Sp } \\
\text { Within Sp and rock matrix } \\
\text { some minor replacement }\end{array}$ \\
\hline $\begin{array}{l}\text { other (growth di- } \\
\text { rection/zoning) }\end{array}$ & & & \\
\hline
\end{tabular}




\begin{tabular}{|c|c|c|c|}
\hline minerals & Sphalerite & Chalcopyrite & Arsenopyrite \\
\hline percentage & 1 & 1 & Very trace \\
\hline morphology & Semi-massive in veinlet & $\begin{array}{l}\text { disseminated throughout } \mathrm{Sp} \\
\text { veinlet }\end{array}$ & $\begin{array}{l}\text { Very disseminated through- } \\
\text { out Sp veinlet }\end{array}$ \\
\hline texture & Veinlet-forming & $\begin{array}{l}\text { Veinlet-forming, } \\
\text { Cpy disease }\end{array}$ & Very disseminated in SP \\
\hline $\begin{array}{l}\text { transmitted opti- } \\
\text { cal properties }\end{array}$ & & & \\
\hline $\begin{array}{l}\text { reflected optical } \\
\text { properties }\end{array}$ & & & Pinkish tint \\
\hline $\begin{array}{l}\text { contact/ } \\
\text { relationships with } \\
\text { other minerals }\end{array}$ & Replacing py and some cpy & Being exsolved from $\mathrm{sp}$ & Within Sp, smooth contacts \\
\hline $\begin{array}{l}\text { other (growth di- } \\
\text { rection/zoning) }\end{array}$ & & & \\
\hline
\end{tabular}

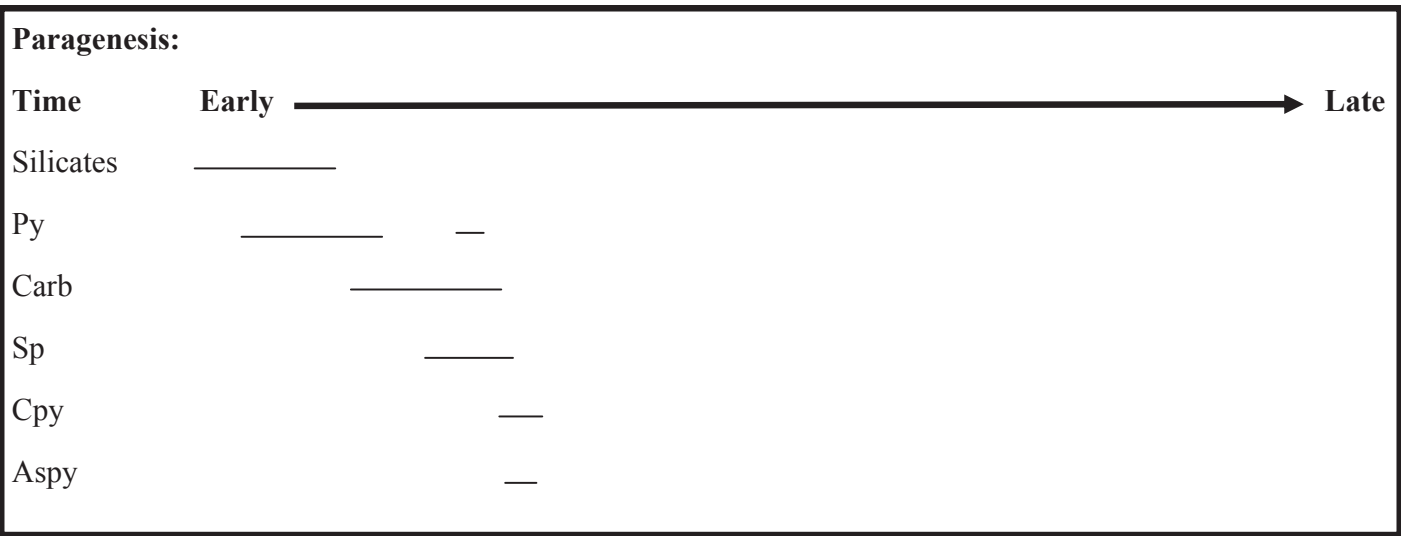


Structures: Silicate rock matrix is foliated, only one observed. Carbonate veinlet cuts foliation and caused local brecciation. Pyrite is include with silicate matrix and larger xtals associated with car-

bonate veinlet. Sp and Py veinlets are subparallel to foliation 


\section{Geologic Unit: Serra do Garrote}

Lithological Unit (Votorantim): Carbonaceous Phyllite (FC)

Sample Photograph

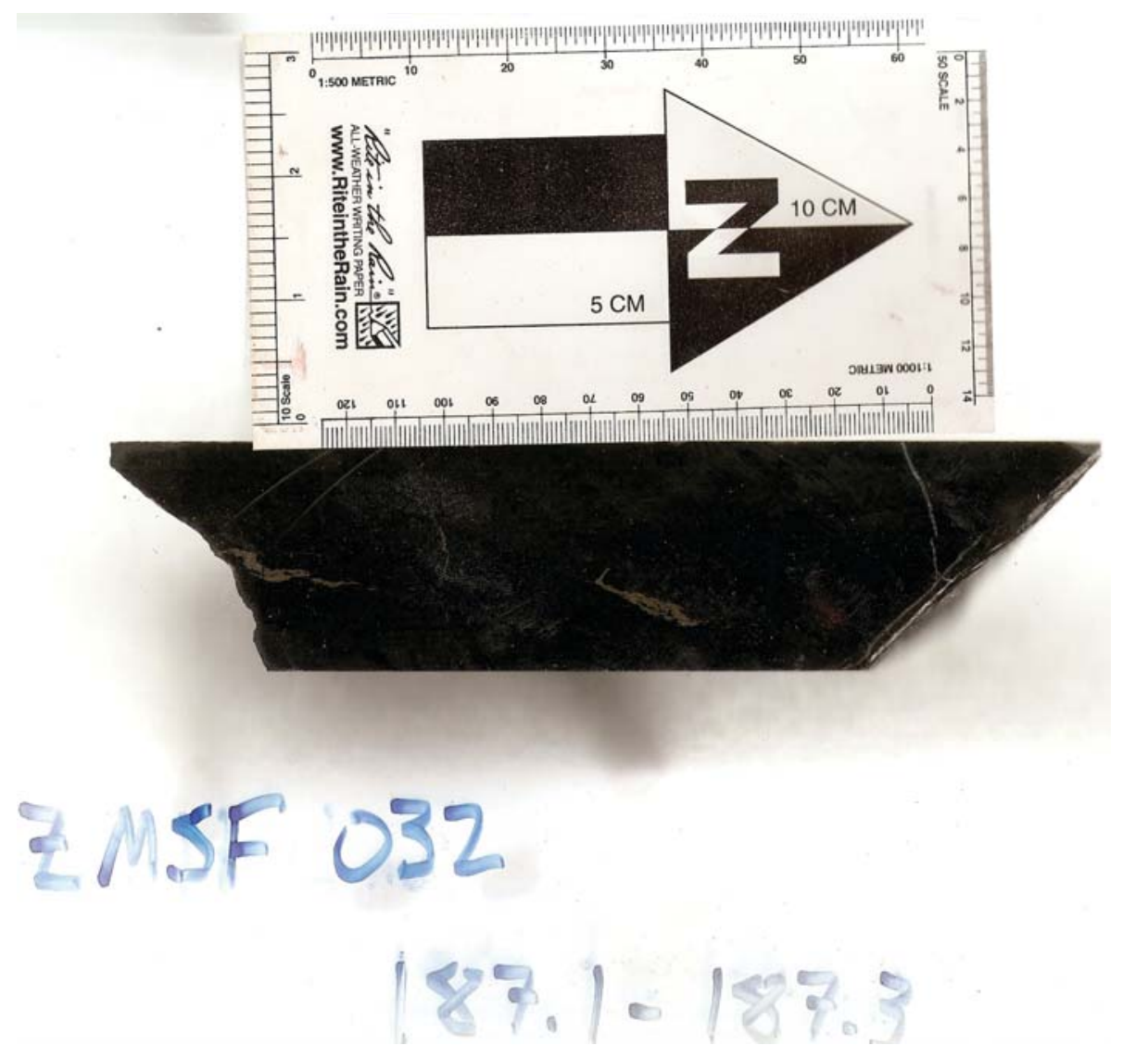

Other Sample Information: 


\begin{tabular}{|l|l|l|l|}
\hline minerals & Quartz & Mica and Clays & Calcite \\
\hline percentage & 40 & 24 & 15 \\
\hline morphology & Very fine anhedral grains & Very fine, subhedral laths & $\begin{array}{l}\text { Very fine subhedral xtals, } \\
\text { larger xtals in veins }\end{array}$ \\
\hline $\begin{array}{l}\text { texture } \\
\text { transmitted opti- } \\
\text { cal properties }\end{array}$ & Foliated rock matrix & Foliated rock matrix & $\begin{array}{l}\text { Foliated rock matrix and } \\
\text { vein }\end{array}$ \\
\hline $\begin{array}{l}\text { reflected optical } \\
\text { properties }\end{array}$ & & & \\
\hline $\begin{array}{l}\text { contact/ } \\
\text { relationships with } \\
\text { other minerals } \\
\text { rection/zoning) }\end{array}$ & & & \\
\hline & & & \\
\hline
\end{tabular}




\begin{tabular}{|c|c|c|c|}
\hline minerals & Pyrite & Sphalerite & chalcopyrite \\
\hline percentage & 15 & 5 & 1 \\
\hline morphology & Anhedral grains & Anhedral grains & Anhedral grains \\
\hline texture & $\begin{array}{l}\text { Disseminated, small grains. } \\
\text { Large vein }\end{array}$ & Disseminated, larger in vein. & $\begin{array}{l}\text { Cpy disease in sl, associated } \\
\text { with some py }\end{array}$ \\
\hline $\begin{array}{l}\text { transmitted opti- } \\
\text { cal properties }\end{array}$ & & & \\
\hline $\begin{array}{l}\text { reflected optical } \\
\text { properties }\end{array}$ & & & \\
\hline $\begin{array}{l}\text { contact/ } \\
\text { relationships with } \\
\text { other minerals }\end{array}$ & $\begin{array}{l}\text { Rounded grains in matrix. } \\
\text { Blebs with sl embayments in } \\
\text { py-vein }\end{array}$ & $\begin{array}{l}\text { Wavy contacts with matrix } \\
\text { and py, replacing py? }\end{array}$ & $\begin{array}{l}\text { Mostly cpy disease in sl. } \\
\text { Some cpy with wavy con- } \\
\text { tacts in py. }\end{array}$ \\
\hline $\begin{array}{l}\text { other (growth di- } \\
\text { rection/zoning) }\end{array}$ & & & \\
\hline
\end{tabular}

\begin{tabular}{|l|l|}
\hline Time & Early $\longrightarrow$ Late \\
Py & $\longrightarrow$ \\
Spy & \\
& \\
\end{tabular}


Structures: Pyrite and calcite veins. Large py vein is folded (follow small py foliation). Foliation in phyllite

Paragenetic Sequence: $p y \rightarrow s l \rightarrow c p y$

Paragenetic Reasoning: Py is embayed by sl, which contains cpy disease

Sample Highlights: foliation, sulfides 


\section{Geologic Unit: Serra do Garrote}

Lithological Unit (Votorantim): Filito Carbonacão (FC)

\section{Sample Photograph}

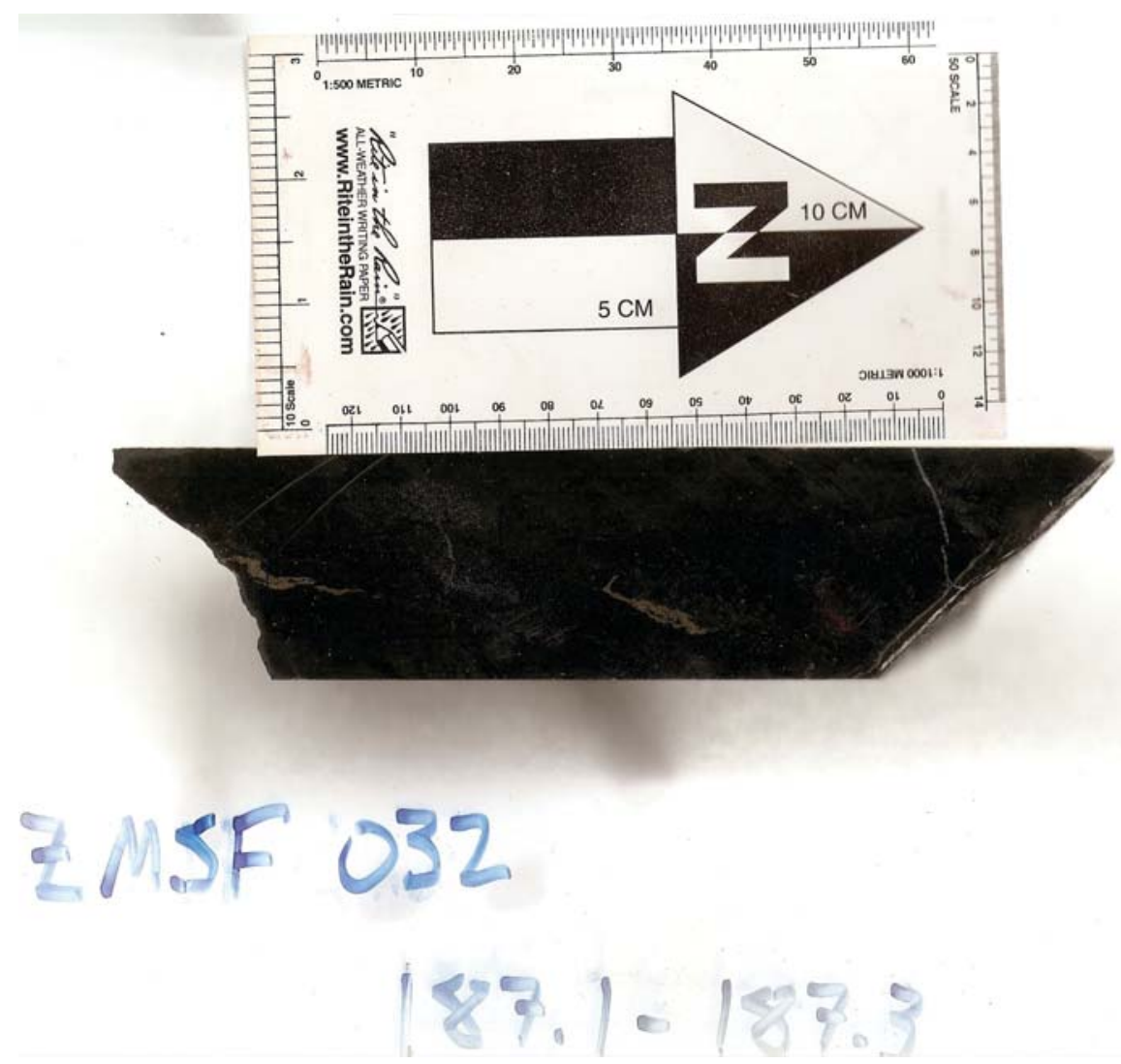

Other Sample Information: Same as 032-187A, but with trace Pyrrhotite 


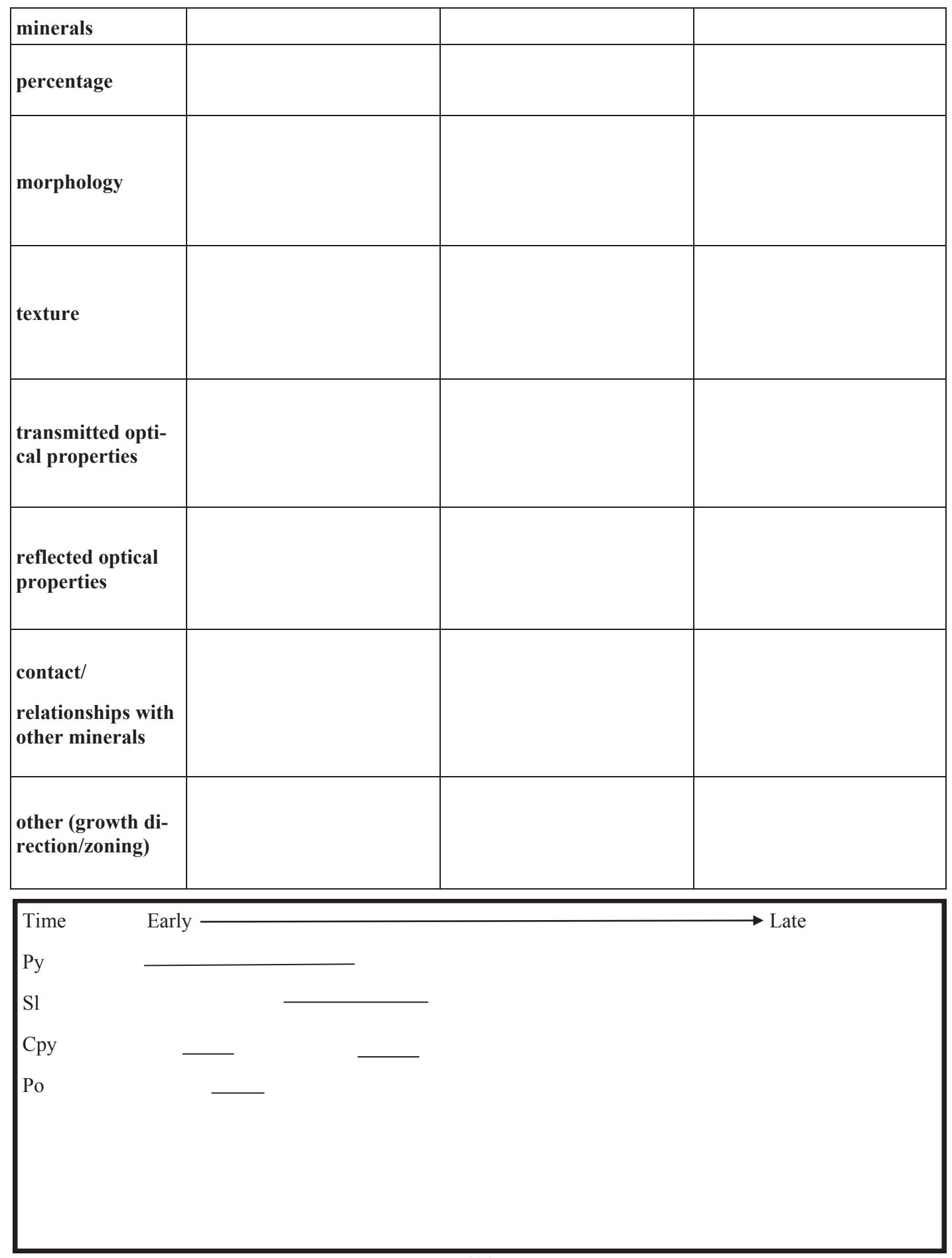


Geologic Unit: Serra do Garrote

Lithological Unit (Votorantim): Carbonaceous phyllites (FC)

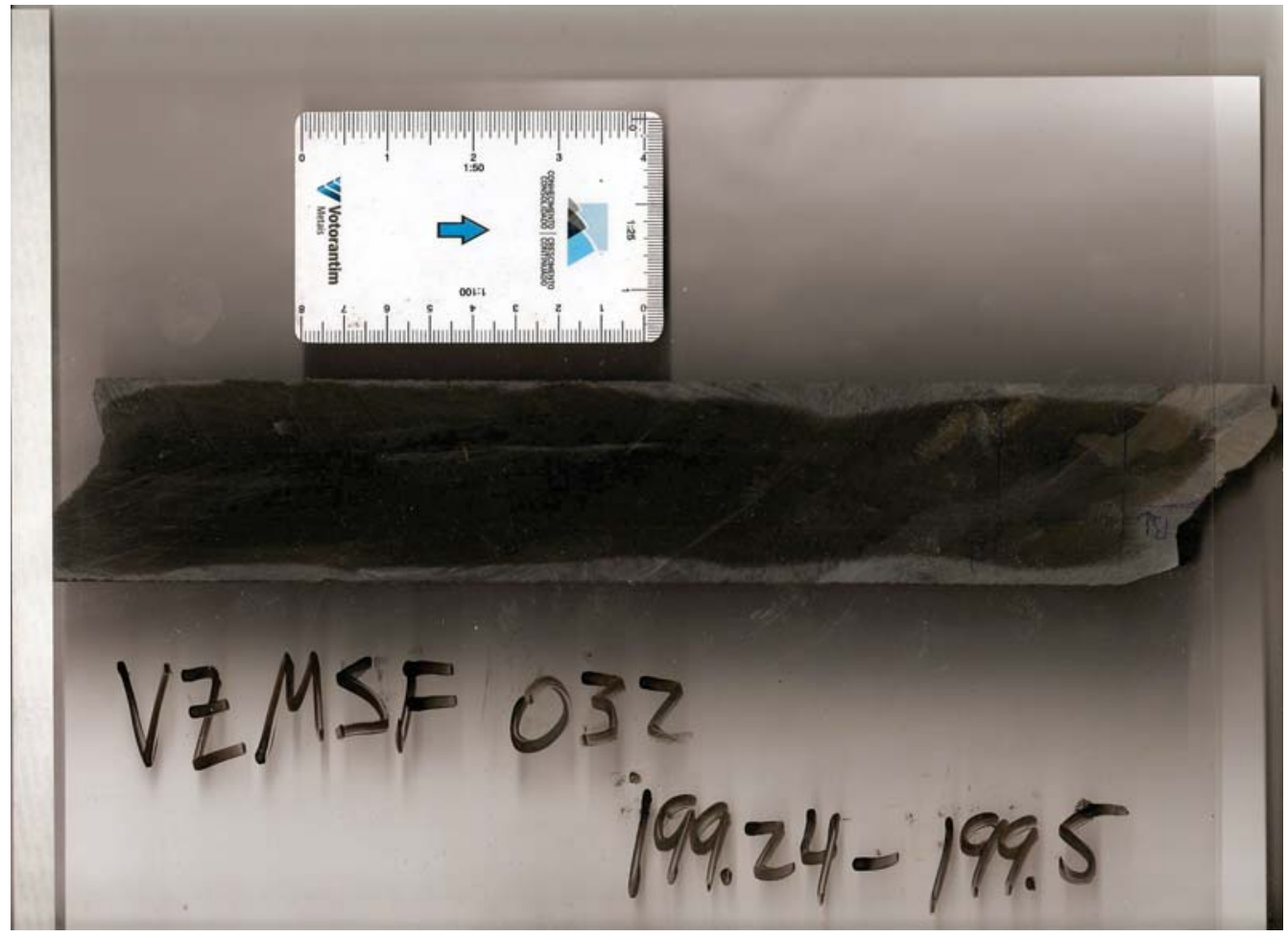

Other Sample Information:

Yellow part of vein is Py, grey part is carbonate 


\begin{tabular}{|c|c|c|c|}
\hline minerals & Fine-grained siliciclastic & Carbonate & Pyrite \\
\hline percentage & 30 & 25 & 45 \\
\hline morphology & Foliated, slightly elongated & Localized in a pod/veinlet & $\begin{array}{l}\text { Foliated, composed most of } \\
\text { rock matrix }\end{array}$ \\
\hline texture & Foliated rock forming matrix & $\begin{array}{l}\text { Foliated, grains include } \\
\text { some qtz and are sheared } \\
\text { (sense difficult to tell, left } \\
\text { lateral?) }\end{array}$ & $\begin{array}{l}\text { Disseminated throughout } \\
\text { siliciclastic mins, composed } \\
\text { majority of rock matrix } \\
\text { Some zones have larger eu- } \\
\text { hedral py }\end{array}$ \\
\hline $\begin{array}{l}\text { transmitted opti- } \\
\text { cal properties }\end{array}$ & & & \\
\hline $\begin{array}{l}\text { reflected optical } \\
\text { properties }\end{array}$ & & & \\
\hline $\begin{array}{l}\text { contact/ } \\
\text { relationships with } \\
\text { other minerals }\end{array}$ & $\begin{array}{l}\text { Straight edges with py, gra- } \\
\text { dational to sharp contact } \\
\text { with carbonate }\end{array}$ & $\begin{array}{l}\text { Ranges from a sharp to gra- } \\
\text { dational contact with dark } \\
\text { silicates and Py }\end{array}$ & $\begin{array}{l}\text { Straight contacts with all } \\
\text { (diagenetic?) }\end{array}$ \\
\hline $\begin{array}{l}\text { other (growth di- } \\
\text { rection/zoning) }\end{array}$ & & Poor in pyrite & $\begin{array}{l}\text { Some zones more rich in } \\
\text { pyrite when associated with } \\
\text { siliciclastics }\end{array}$ \\
\hline
\end{tabular}




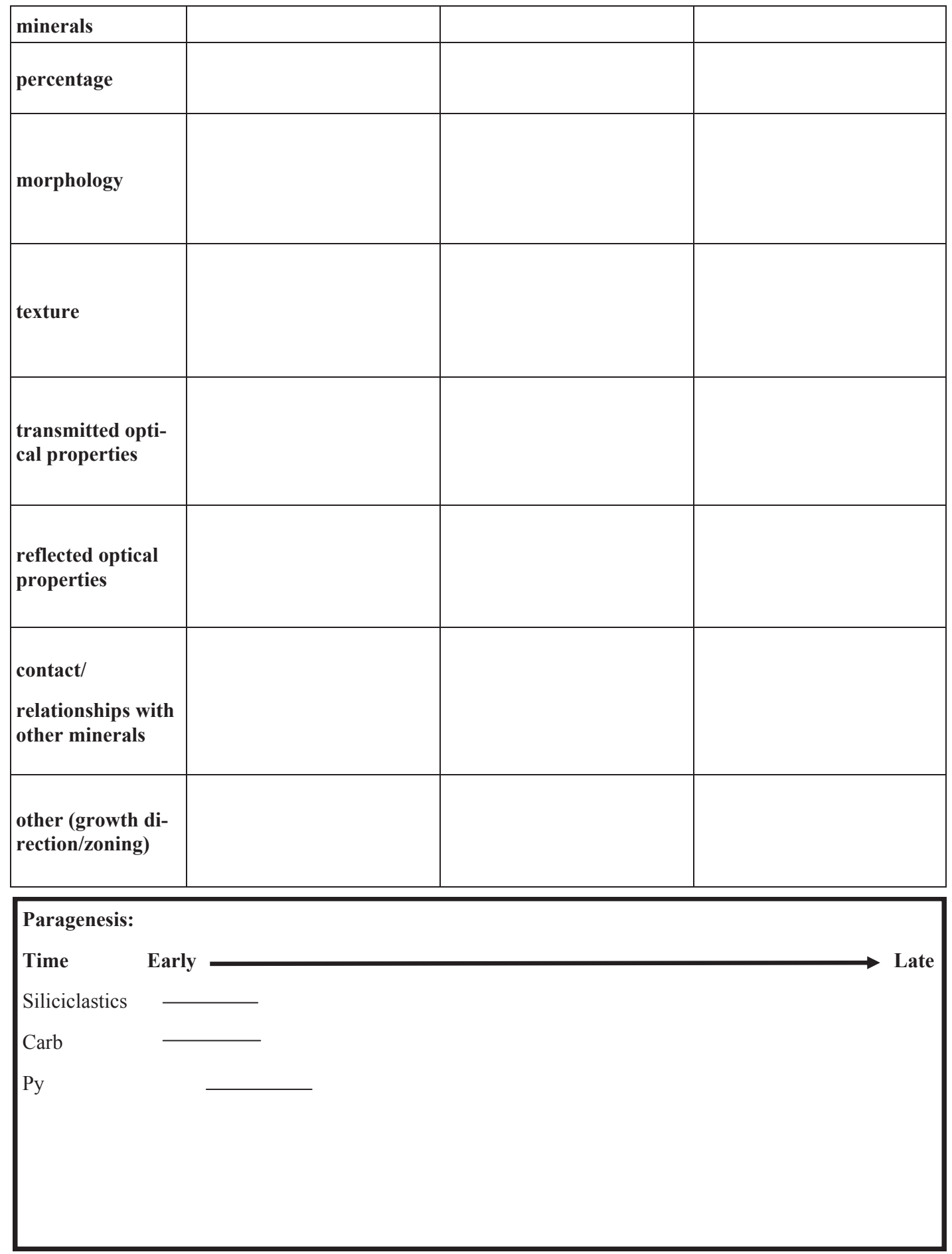


Geologic Unit: Lower Pamplona

Lithological Unit (Votorantim): Pink dolomite with phyllite intercalations (DG)

Sample Photograph
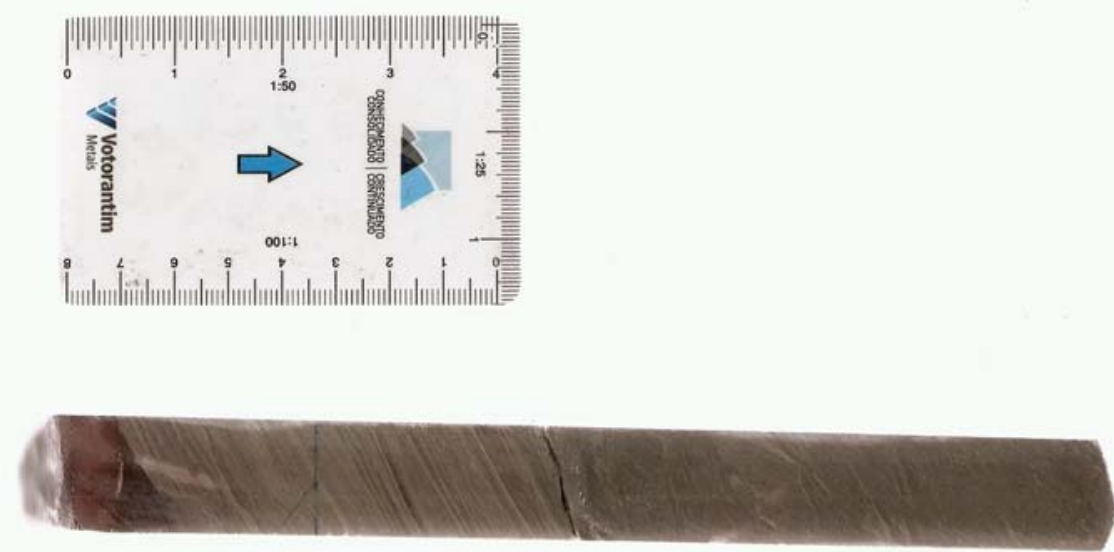

VZMIF O4S

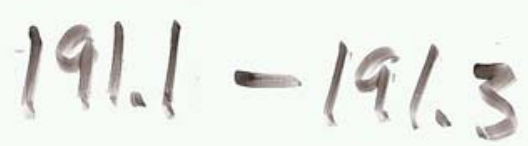

Other Sample Information: Maroon phyllite is replaced by hematite, green phyllite contains very very fine chlorite 


\begin{tabular}{|c|c|c|c|}
\hline minerals & Dolomite & Chlorite? & Quartz \\
\hline percentage & 40 & 30 & 20 \\
\hline morphology & micritic & Ultra fine crystals & $\begin{array}{l}\text { Subangular to subrounded } \\
\text { grains } \\
\text { Minor qtz cement in some } \\
\text { veins and vugs }\end{array}$ \\
\hline texture & $\begin{array}{l}\text { Fine laminations with quartz } \\
\text {-rich layers and chl dominat- } \\
\text { ed layers }\end{array}$ & $\begin{array}{l}\text { Replacement of dol? Finely } \\
\text { laminated with dol and dol + } \\
\text { qtz }\end{array}$ & $\begin{array}{l}\text { Disseminated grains found } \\
\text { in lenses within dol }\end{array}$ \\
\hline $\begin{array}{l}\text { transmitted opti- } \\
\text { cal properties }\end{array}$ & & Pleochroic in ppl & \\
\hline $\begin{array}{l}\text { reflected optical } \\
\text { properties }\end{array}$ & & & \\
\hline $\begin{array}{l}\text { contact/ } \\
\text { relationships with } \\
\text { other minerals }\end{array}$ & $\begin{array}{l}\text { Diffuse and fuzzy contact } \\
\text { with chl, being replaced by } \\
\text { chl }\end{array}$ & See dol* & $\begin{array}{l}\text { Straight grain boundaries } \\
\text { with dol and hm } \\
\text { Later open spaced-filling qtz } \\
\text { cement }\end{array}$ \\
\hline $\begin{array}{l}\text { other (growth di- } \\
\text { rection/zoning) }\end{array}$ & & Turns phyllite green & \\
\hline
\end{tabular}




\begin{tabular}{|c|c|c|c|}
\hline minerals & Hematite & Muscovite & Chalcocite \\
\hline percentage & 10 & trace & Trace \\
\hline morphology & $\begin{array}{l}\text { Replacing carbonate and chl, } \\
\text { intercalated contact with chl- } \\
\text { rich part of rock }\end{array}$ & $\begin{array}{l}\text { Small crystal laths and nee- } \\
\text { dles }\end{array}$ & Very small irregular crystals \\
\hline texture & $\begin{array}{l}\text { Flat lath crystals (foliated?) } \\
\text { containing qtz laminations; } \\
\text { similar role to dol }\end{array}$ & disseminated & Very disseminated \\
\hline $\begin{array}{l}\text { transmitted opti- } \\
\text { cal properties }\end{array}$ & & & \\
\hline $\begin{array}{l}\text { reflected optical } \\
\text { properties }\end{array}$ & & & \\
\hline & & & Steel blue-gray tint \\
\hline $\begin{array}{l}\text { contact/ } \\
\text { relationships with } \\
\text { other minerals }\end{array}$ & $\begin{array}{l}\text { Replacing dol and chl, inter- } \\
\text { calated contact with chl-rich } \\
\text { rock zone }\end{array}$ & & $\begin{array}{l}\text { Smooth contacts with minor } \\
\text { embayments with surround- } \\
\text { ing clay minerals }\end{array}$ \\
\hline $\begin{array}{l}\text { other (growth di- } \\
\text { rection/zoning) }\end{array}$ & & & \\
\hline
\end{tabular}

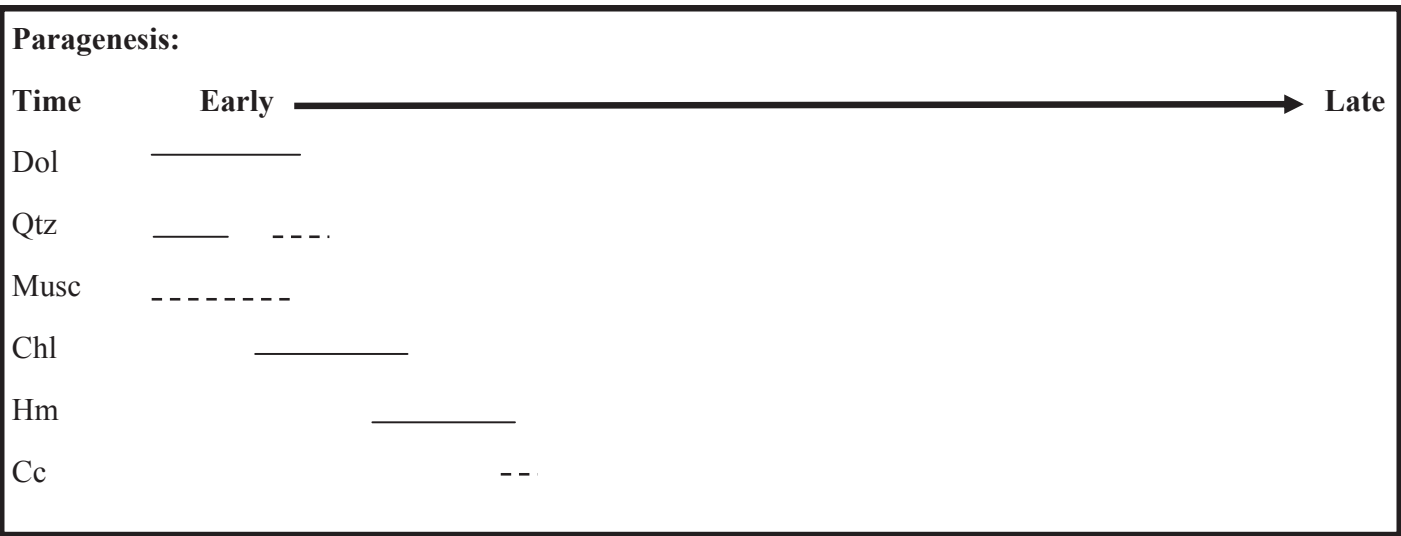


Geologic Unit: Upper Morro do Pinheiro

Lithological Unit (Votorantim): Grey Dolomite (DO)

Sample Photograph
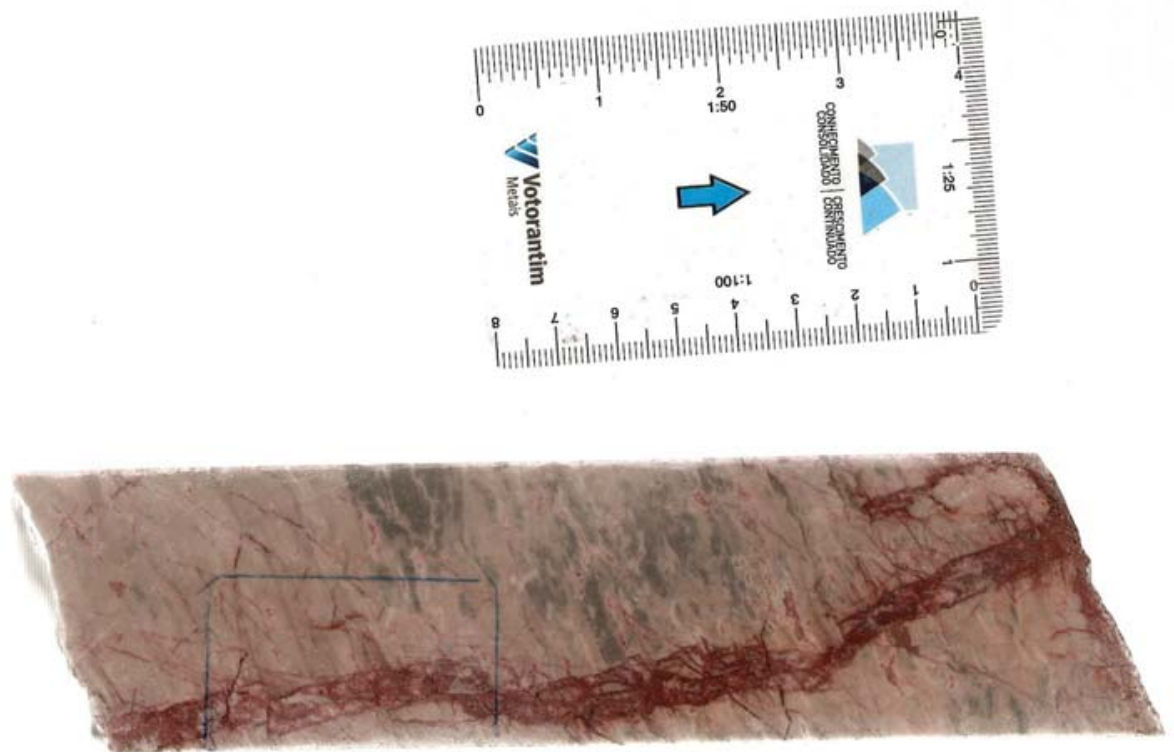

VZMIF O49

$264.25=264.99$

Other Sample Information: 


\begin{tabular}{|c|c|c|c|}
\hline minerals & Dolomite & Fe-carb & Hematite \\
\hline percentage & 80 & 20 & trace \\
\hline morphology & $\begin{array}{l}\text { Micritic boundstone with } \\
\text { sparry carbonate cement }\end{array}$ & Sparry carbonate & Irregular crystals blebs \\
\hline texture & $\begin{array}{l}\text { Algal layering with dissolu- } \\
\text { tion features filled with car- } \\
\text { bonate cement }\end{array}$ & Veinlet & $\begin{array}{l}\text { Disseminated in Fe-carb } \\
\text { veinlet }\end{array}$ \\
\hline $\begin{array}{l}\text { transmitted opti- } \\
\text { cal properties }\end{array}$ & & & \\
\hline $\begin{array}{l}\text { reflected optical } \\
\text { properties }\end{array}$ & & & \\
\hline $\begin{array}{l}\text { contact/ } \\
\text { relationships with } \\
\text { other minerals }\end{array}$ & $\begin{array}{l}\text { Primary structure in host } \\
\text { rock }\end{array}$ & $\begin{array}{l}\text { Cutting boundstone, tiny } \\
\text { veinlet nets with hm on mar- } \\
\text { gins }\end{array}$ & Replacing Fe-carb \\
\hline $\begin{array}{l}\text { other (growth di- } \\
\text { rection/zoning) }\end{array}$ & & & \\
\hline
\end{tabular}

Paragenesis:

Time

Early

Dol

Fe-carb

$\mathrm{Hm}$ 
Geologic Unit: Upper Morro do Pinheiro

Lithological Unit (Votorantim): Grey Dolomite (DO)

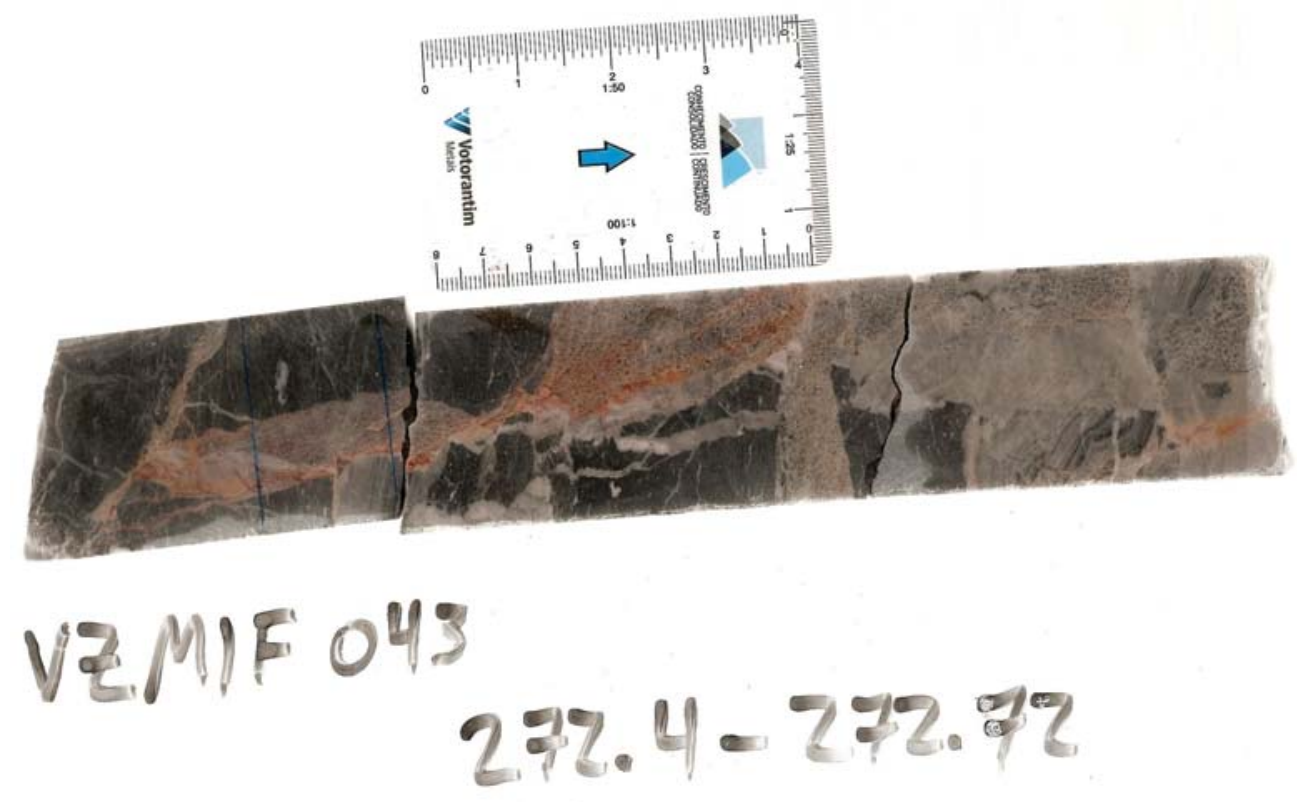

Other Sample Information: 


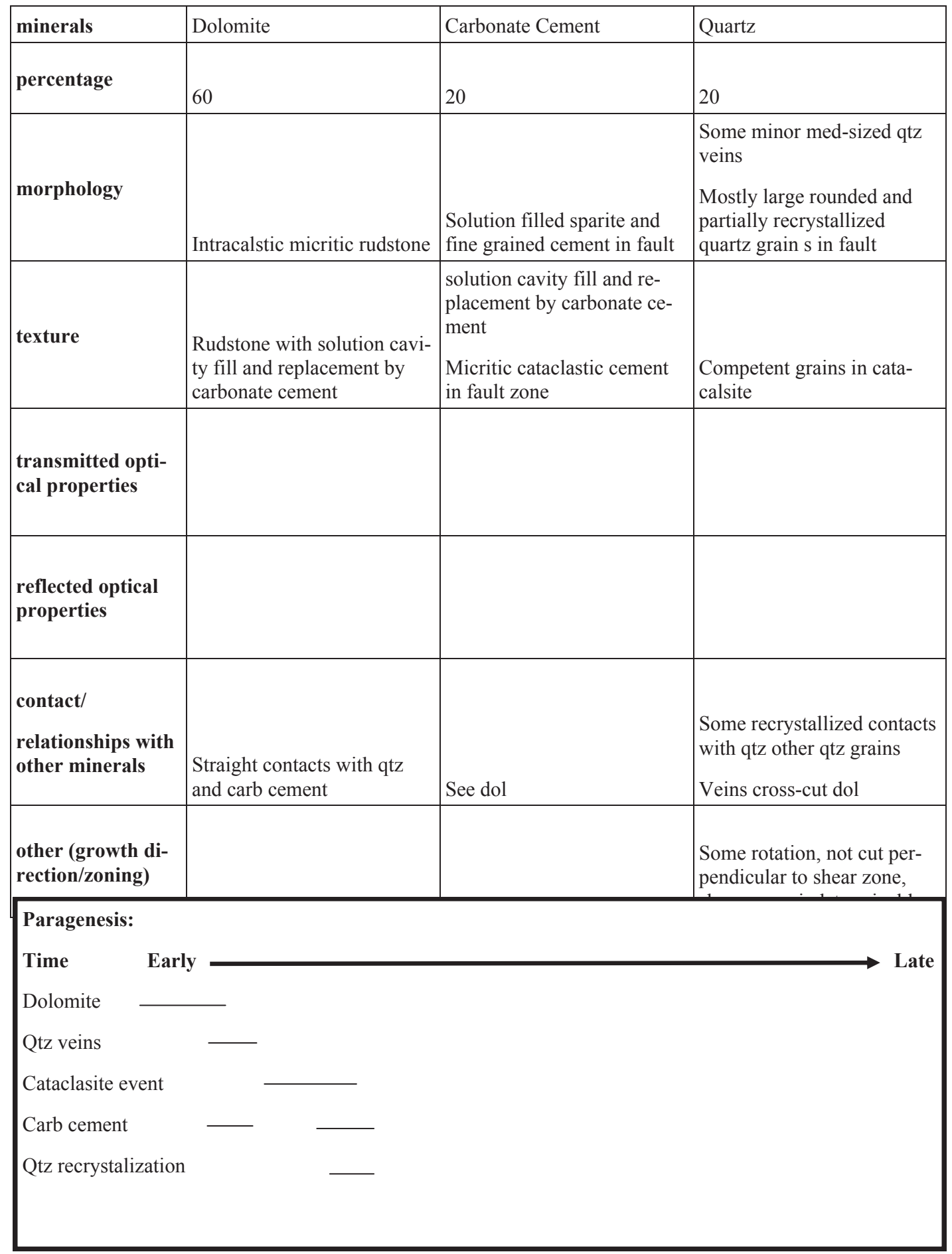


Geologic Unit: Middle Pamplona
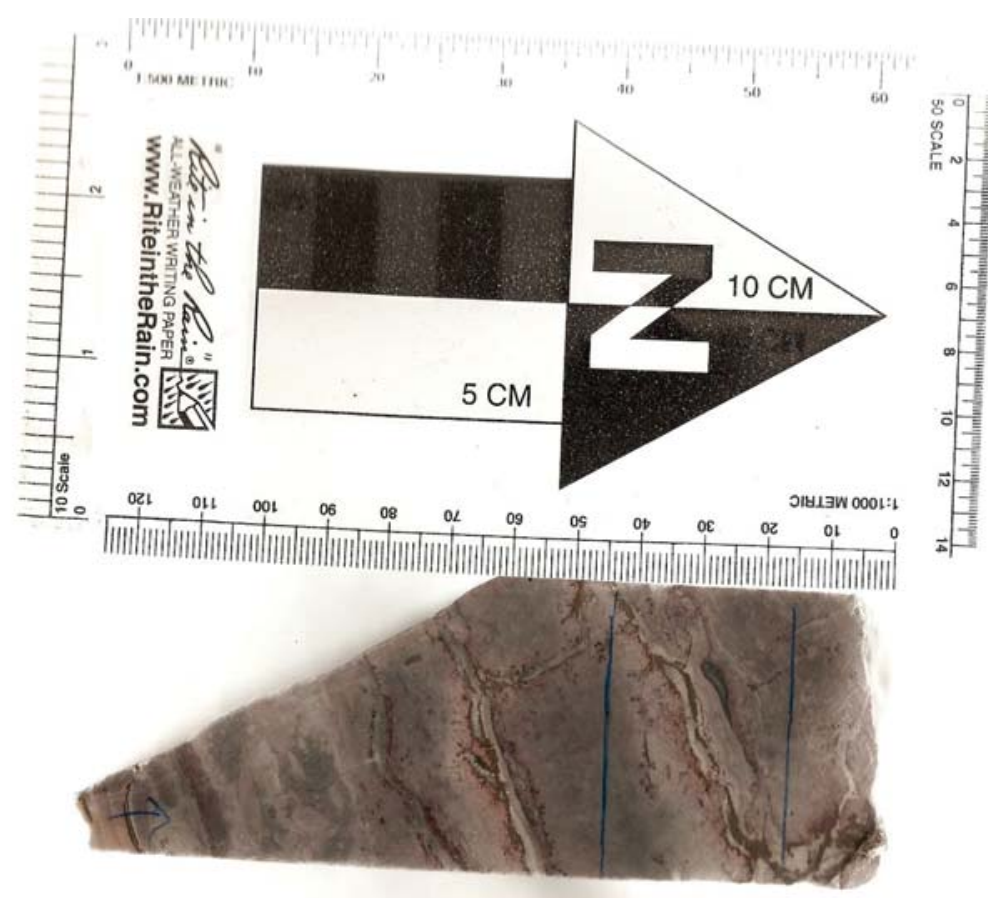

\section{5}

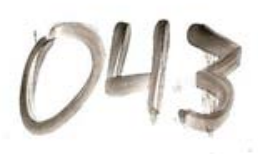

$55.9 .5 \cdot 51.2 \%$

Other Sample Information: Pink, massive dolarenite with py/ hm veins 


\begin{tabular}{|c|c|c|c|}
\hline minerals & dolomite & quartz & pyrite \\
\hline percentage & 70 & 10 & 20 \\
\hline morphology & $\begin{array}{l}\text { Small, subedral to euhedral } \\
\text { grains. Larger recrystallized } \\
\text { euhedral grains in veins }\end{array}$ & $\begin{array}{l}\text { occurs in irregular lenses. } \\
\text { Some larger anhedral grains } \\
\text { in dol matrix. Filling voids }\end{array}$ & $\begin{array}{l}\text { Angular grains with botryoi- } \\
\text { dal overgrowhts in vein }\end{array}$ \\
\hline texture & Rock matrix, & Replacing dolomite & $\begin{array}{l}\text { Massive/semi massive in } \\
\text { vein }\end{array}$ \\
\hline $\begin{array}{l}\text { transmitted opti- } \\
\text { cal properties }\end{array}$ & & & opaque \\
\hline $\begin{array}{r}\text { color } \\
\text { ppl } \\
\mathrm{xpl}\end{array}$ & $\begin{array}{l}\text { Clear } \\
\text { Pastels }\end{array}$ & $\begin{array}{l}\text { Clear } \\
\text { Black and white }\end{array}$ & \\
\hline pleochroism & $\mathrm{n} / \mathrm{a}$ & $\mathrm{N} / \mathrm{a}$ & \\
\hline birefringence & 3 rd order & 1 st order & \\
\hline $\begin{array}{l}\text { twinning/ } \\
\text { cleavage }\end{array}$ & Rhomobhedral twinning & Undulatory extinction & \\
\hline other & & High relief & \\
\hline $\begin{array}{l}\text { reflected optical } \\
\text { properties }\end{array}$ & $\mathrm{N} / \mathrm{a}$ & $\mathrm{n} / \mathrm{a}$ & \\
\hline color & & & Light yellow \\
\hline bireflectance & & & $\mathrm{n} / \mathrm{a}$ \\
\hline anisotropy & & & Faint color change if present \\
\hline internal reflections & & & $\mathrm{n} / \mathrm{a}$ \\
\hline other & & & \\
\hline $\begin{array}{l}\text { contact/ } \\
\text { relationships with } \\
\text { other minerals }\end{array}$ & $\begin{array}{l}\text { Being replaced by qtz. Irreg- } \\
\text { ular contact with py over- } \\
\text { growths }\end{array}$ & $\begin{array}{l}\text { Irregular and blobby contact } \\
\text { with dolomite, replacing do- } \\
\text { lomite. }\end{array}$ & $\begin{array}{l}\text { Overgrowing dolomite in } \\
\text { vein }\end{array}$ \\
\hline $\begin{array}{l}\text { other (growth di- } \\
\text { rection/zoning) }\end{array}$ & Filling in fracture & Replacement lenses & Outward zoning \\
\hline
\end{tabular}




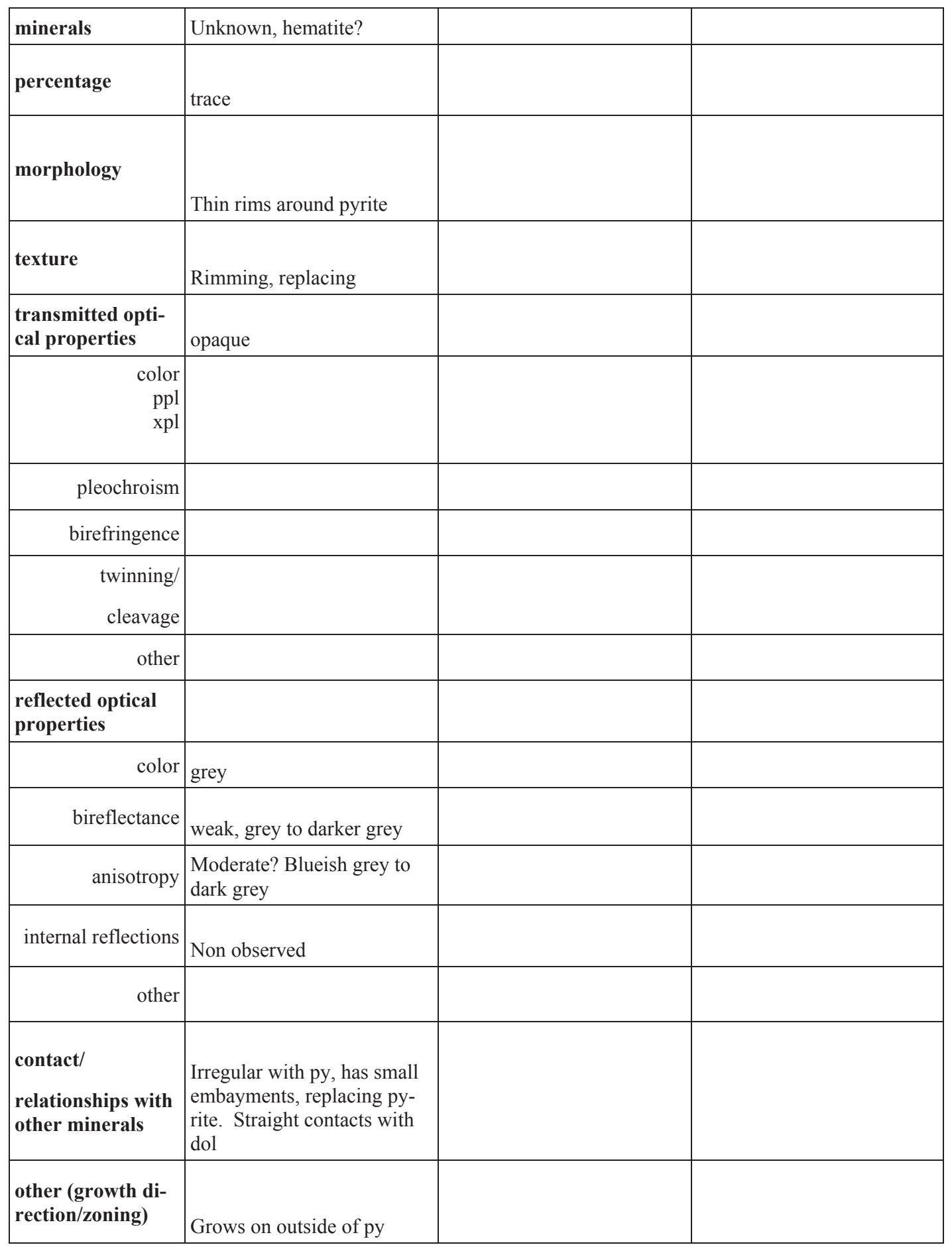


Structures: Fracture filled in by dolomite and pyrite

Paragenetic Sequence: dol $\rightarrow$ fracture $\rightarrow$ dol recrystallization $\rightarrow p y+h m$ ? (slightly later than $p y) \rightarrow q t z$

Paragenetic Reasoning: Dolomite crystals are large around the edges of the fracture, suggesting recrystallization of the host rock into the vein. The py and $\mathrm{hm}$ are intergrown and both growing on top of the dolomite (Fig 2) and are in the center of the vein. The contacts between the pyrite and quartz are fairly straight, suggesting little interaction. There are a few specs of py in the qtz, indicating that the pyritic fluid was at an end when silicification occurred. The qtz is late, replacing dolomite.

*No qtz in fracture with dol and py*

Sample Highlights: Pyrite overgrowths with interim hematite 
Geologic Unit: Lower Pamplona

Lithological Unit (Votorantim): Pink Dolomite (DG)

Sample Photograph

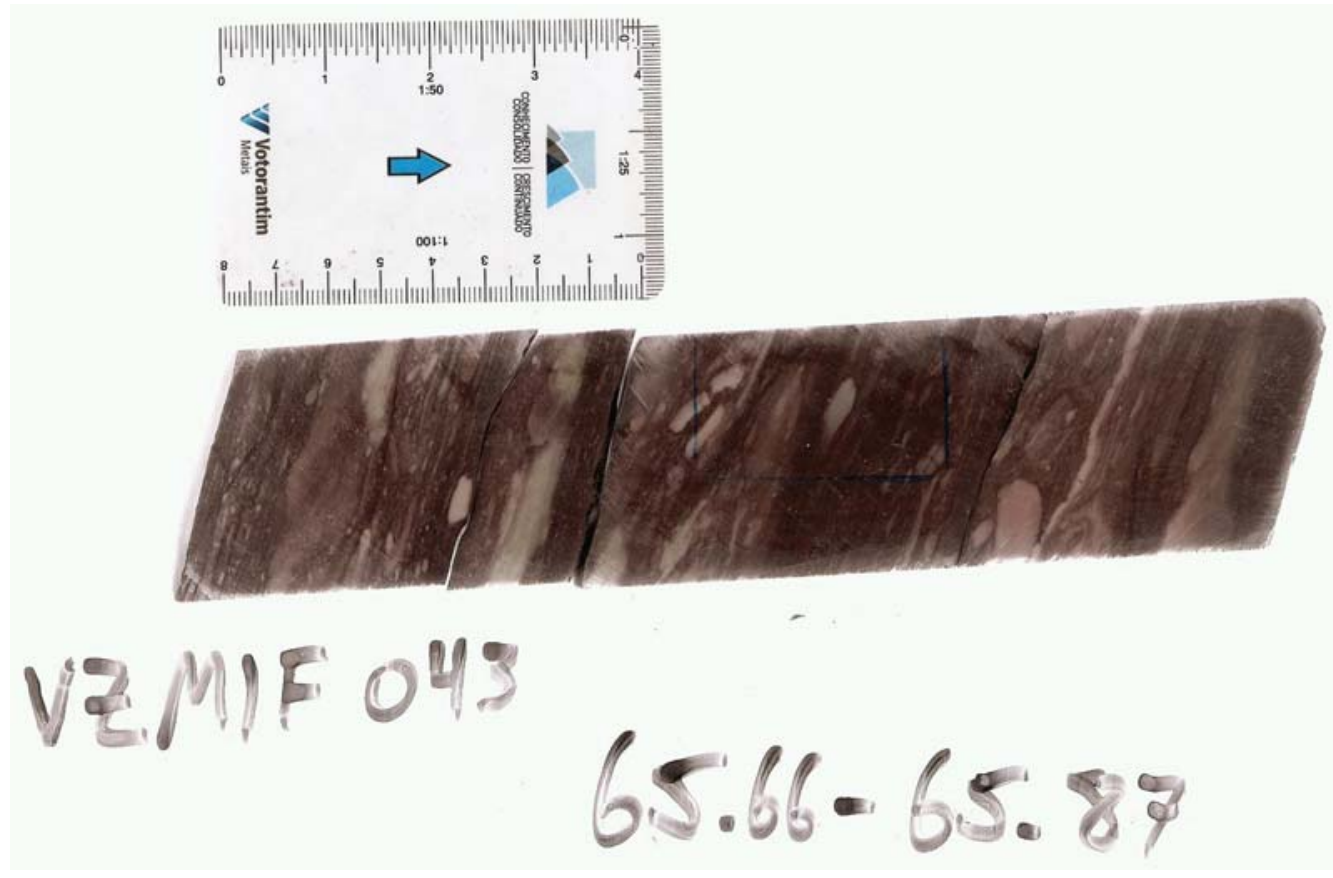

Other Sample Information: maroon staining contains more hematite 


\begin{tabular}{|c|c|c|c|}
\hline minerals & Dolomite & Quartz & Muscovite \\
\hline percentage & 80 & 17 & 3 \\
\hline morphology & Micritic clasts and matrix & $\begin{array}{l}\text { Subangular to subrounded } \\
\text { grains }\end{array}$ & Flattened laths and needles \\
\hline texture & $\begin{array}{l}\text { Composes clasts (with mud- } \\
\text { cracks); host mineral fabric } \\
\text { to qtz and musc; some pres- } \\
\text { sure shadows }\end{array}$ & $\begin{array}{l}\text { Foliated and rotated grains } \\
\text { indicating shear }\end{array}$ & $\begin{array}{l}\text { Oriented and flattened grains } \\
\text { indicating shear }\end{array}$ \\
\hline $\begin{array}{l}\text { transmitted opti- } \\
\text { cal properties }\end{array}$ & & & \\
\hline $\begin{array}{l}\text { reflected optical } \\
\text { properties }\end{array}$ & & & \\
\hline $\begin{array}{l}\text { contact/ } \\
\text { relationships with } \\
\text { other minerals }\end{array}$ & $\begin{array}{l}\text { Irregular contacts with } \mathrm{hm} \text {, } \\
\text { replacing } \mathrm{hm}\end{array}$ & $\begin{array}{l}\text { Straight edges with musc } \\
\text { and dol }\end{array}$ & $\begin{array}{l}\text { Straight edges with qtz and } \\
\text { dol }\end{array}$ \\
\hline $\begin{array}{l}\text { other (growth di- } \\
\text { rection/zoning) }\end{array}$ & & & \\
\hline
\end{tabular}




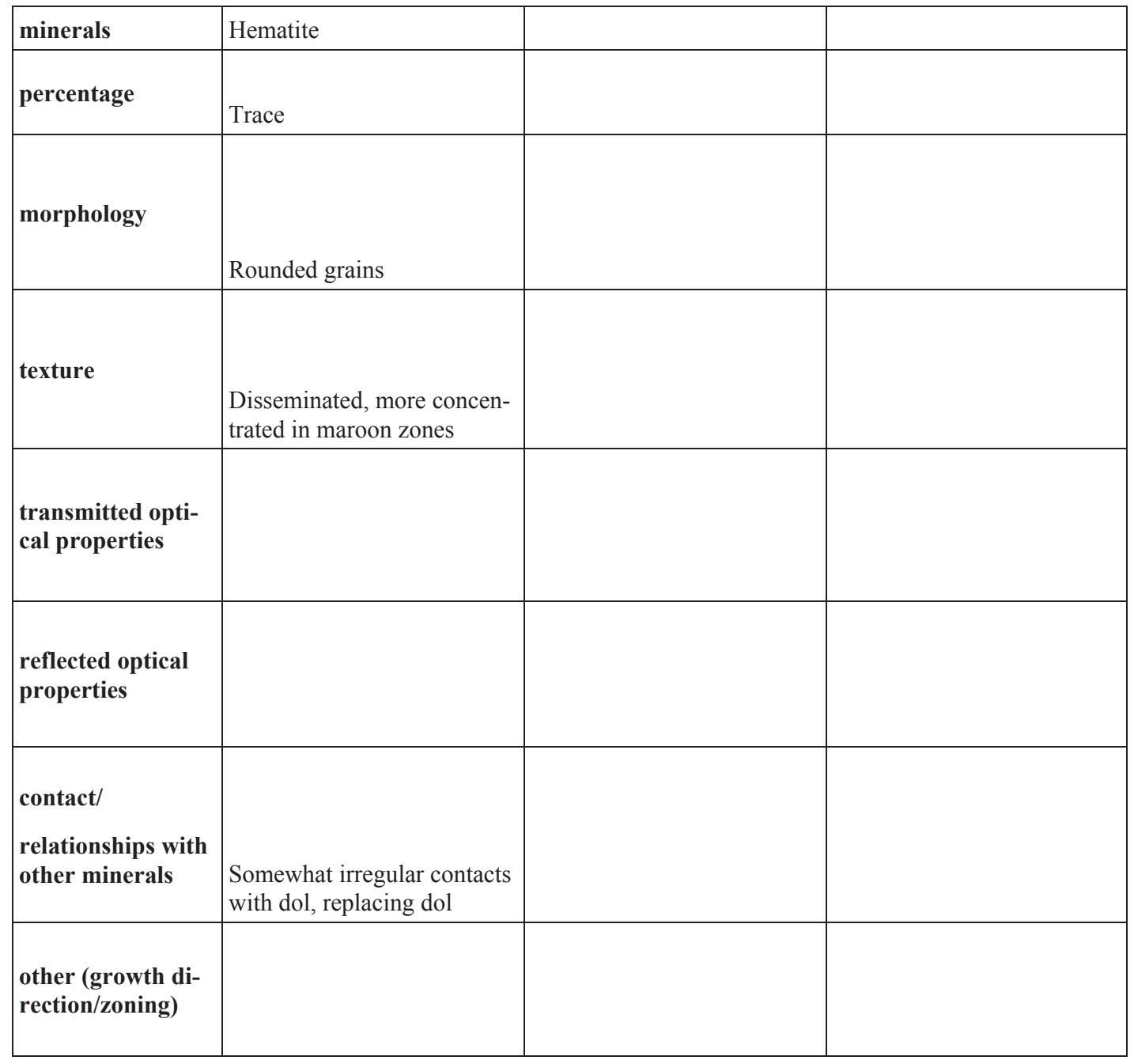

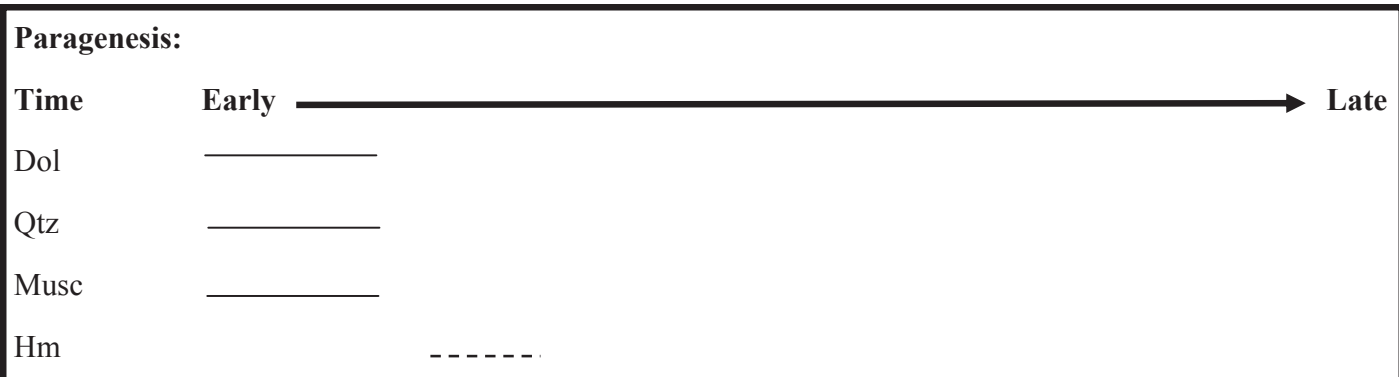


Geologic Unit: Lower Pamplona

Lithological Unit (Votorantim): Pink dolomite (DG)

Sample Photograph

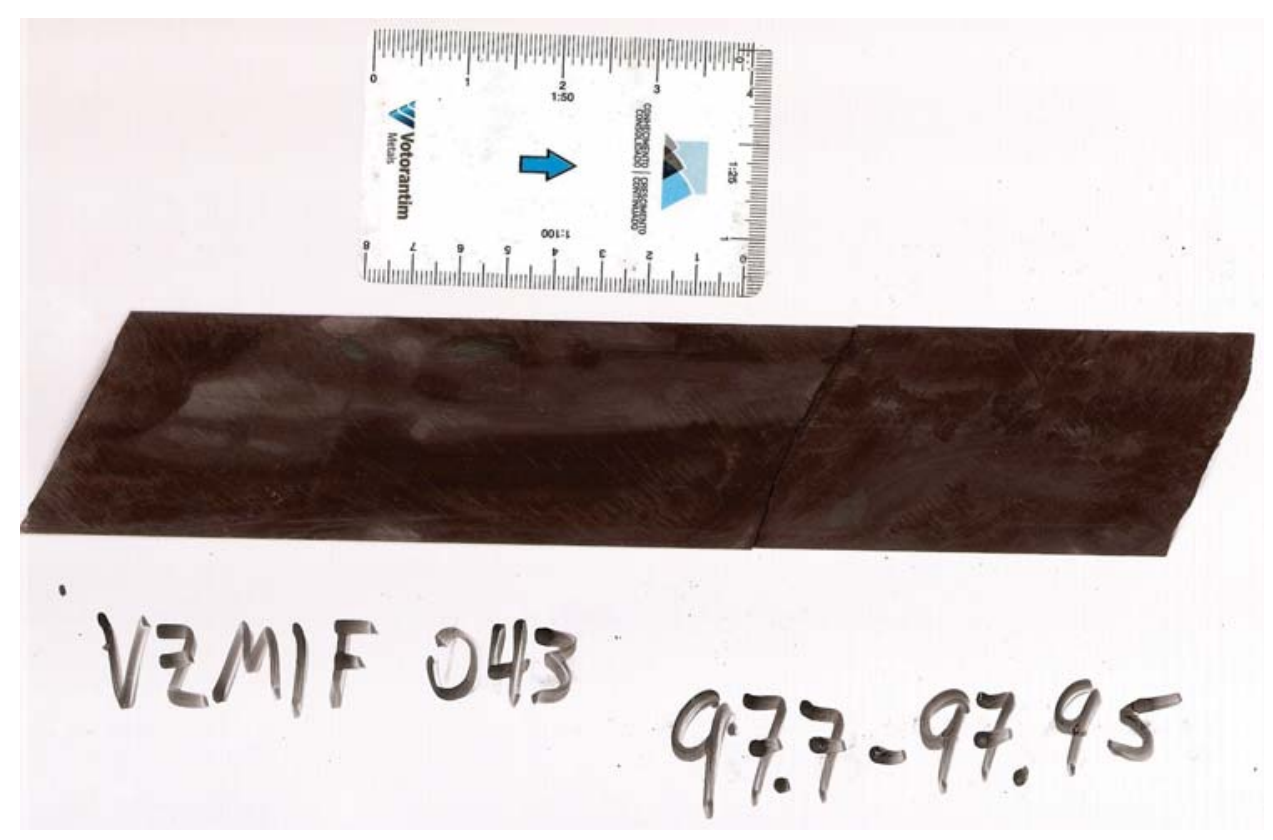

Other Sample Information: 


\begin{tabular}{|c|c|c|c|}
\hline minerals & Fe-Chlorite & Quartz & Muscovite \\
\hline percentage & 85 & 5 & 3 \\
\hline morphology & Very fine crystalline & $\begin{array}{l}0.1-0.2 \mathrm{~mm} \text { sized grains, } \\
\text { subangular to rounded }\end{array}$ & $0.3-0.4 \mathrm{~mm}$ crystal laths \\
\hline texture & Same extinction & disseminated & disseminated \\
\hline $\begin{array}{l}\text { transmitted opti- } \\
\text { cal properties }\end{array}$ & & & \\
\hline $\begin{array}{l}\text { reflected optical } \\
\text { properties }\end{array}$ & & & \\
\hline $\begin{array}{l}\text { contact/ } \\
\text { relationships with } \\
\text { other minerals }\end{array}$ & $\begin{array}{l}\text { Straight contacts with all } \\
\text { mins except } \mathrm{hm} \text {, hem replac- } \\
\text { ing all minerals }\end{array}$ & $\begin{array}{l}\text { Straight contacts with all } \\
\text { mins except hm, hem replac- } \\
\text { ing all minerals }\end{array}$ & $\begin{array}{l}\text { Straight contacts with all } \\
\text { mins except hm, hem replac- } \\
\text { ing all minerals }\end{array}$ \\
\hline $\begin{array}{l}\text { other (growth di- } \\
\text { rection/zoning) }\end{array}$ & & & \\
\hline
\end{tabular}




\begin{tabular}{|c|c|c|c|}
\hline minerals & Dolomite & Hematite & \\
\hline percentage & 3 & 4 & \\
\hline morphology & Rounded grains & Irregular crystals & \\
\hline texture & disseminated & disseminated & \\
\hline $\begin{array}{l}\text { transmitted opti- } \\
\text { cal properties }\end{array}$ & & & \\
\hline $\begin{array}{l}\text { reflected optical } \\
\text { properties }\end{array}$ & & & \\
\hline $\begin{array}{l}\text { contact/ } \\
\text { relationships with } \\
\text { other minerals }\end{array}$ & $\begin{array}{l}\text { Straight contacts with all } \\
\text { mins except hm, hem replac- } \\
\text { ing all minerals }\end{array}$ & $\begin{array}{l}\text { Straight contacts with all } \\
\text { mins except hm, hem replac- } \\
\text { ing all minerals }\end{array}$ & $\begin{array}{l}\text { Straight contacts with all } \\
\text { mins except hm, hem replac- } \\
\text { ing all minerals }\end{array}$ \\
\hline $\begin{array}{l}\text { other (growth di- } \\
\text { rection/zoning) }\end{array}$ & & & \\
\hline
\end{tabular}

Paragenesis:

Time Early

Uknown Clay-silicate

Dol

Qtz

Musc

$\mathrm{Hm}$

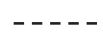


Geologic Unit: Hydrothermal Breccia

Lithological Unit (Votorantim): Massive Silicate in Hydrothermal Breccia

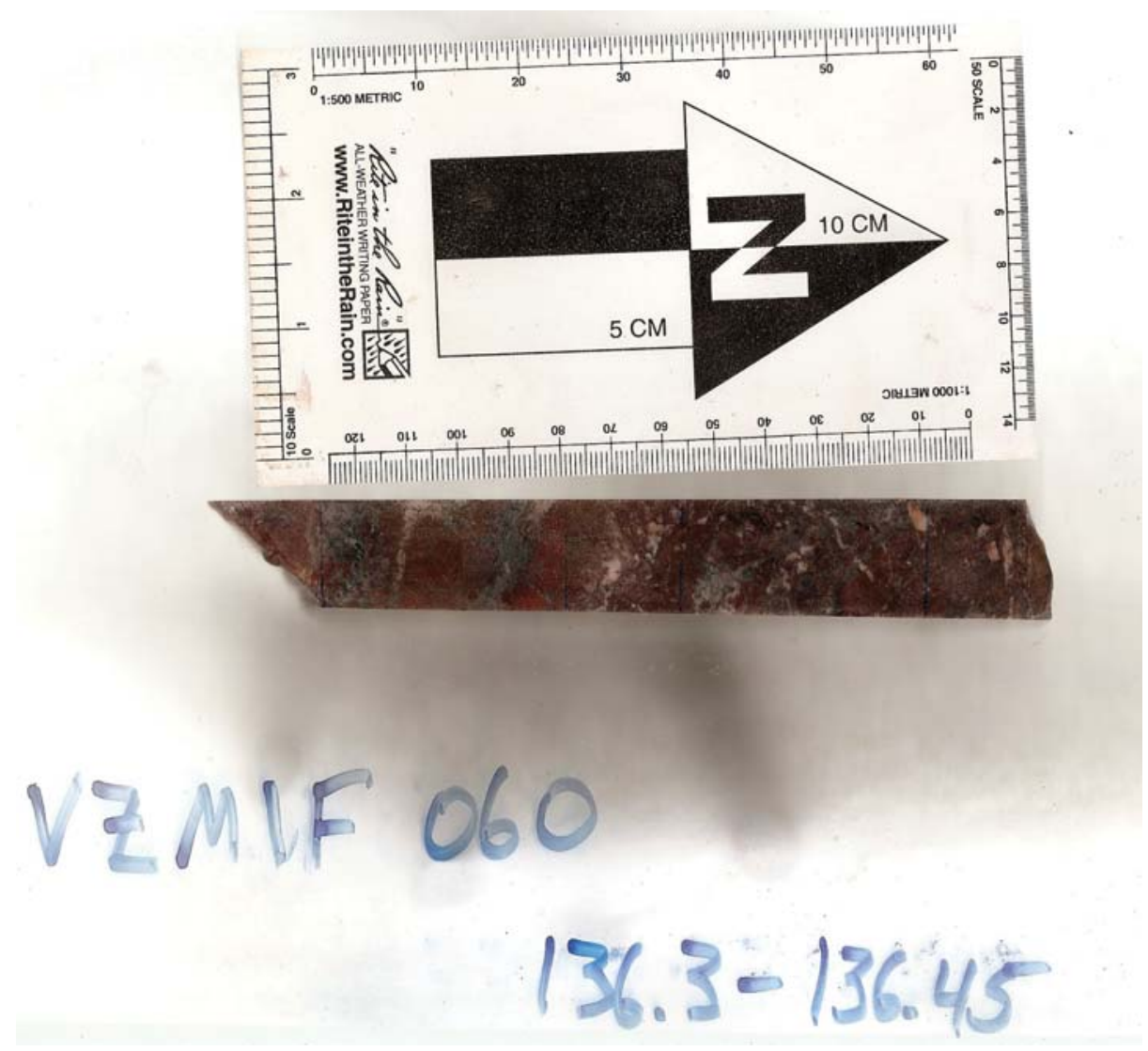

Other Sample Information: 


\begin{tabular}{|c|c|c|c|}
\hline minerals & Dolomite & Willemite & Hematite \\
\hline percentage & 15 & 72 & 2 \\
\hline morphology & $\begin{array}{l}\text { Large, euhedral crystals and } \\
\text { minor amounts of small an- } \\
\text { hedral Fe-dol overgrowing } \\
\text { euhedral dol }\end{array}$ & $\begin{array}{l}\text { Small }(0.1-0.2 \mathrm{~mm}) \text { granular } \\
\text { euhedral crystals as well as } \\
\text { lesser amounts of larger }(0.5 \\
-1 \mathrm{~mm}) \text { euhedral laths }\end{array}$ & $\begin{array}{l}\text { Some euhedral crystal laths, } \\
\text { mostly irregular crystals }\end{array}$ \\
\hline texture & $\begin{array}{l}\text { Growing in dol veins, minor } \\
\text { amounts in late, sulfide } \\
\text { veins }\end{array}$ & Massive & Sheared, semi-massive \\
\hline $\begin{array}{l}\text { transmitted opti- } \\
\text { cal properties }\end{array}$ & & & \\
\hline $\begin{array}{l}\text { reflected optical } \\
\text { properties }\end{array}$ & & & \\
\hline $\begin{array}{l}\text { contact/ } \\
\text { relationships with } \\
\text { other minerals }\end{array}$ & $\begin{array}{l}\text { Dol has mostly straight con- } \\
\text { tacts with wl, being over- } \\
\text { grown/replaced by wl. Fe- } \\
\text { dol is replacing dol and wl }\end{array}$ & $\begin{array}{l}\text { Somewhat irregular contacts } \\
\text { with dol filling in fractures, } \\
\text { beign replaced by Fe-dol. } \\
\text { Lath-shaped wl overgrowing } \\
\text { granular wl }\end{array}$ & $\begin{array}{l}\text { Straight contacts with frk, } \\
\text { replacing dol }\end{array}$ \\
\hline $\begin{array}{l}\text { other (growth di- } \\
\text { rection/zoning) }\end{array}$ & Large dol filling vein & & \\
\hline
\end{tabular}




\begin{tabular}{|c|c|c|c|}
\hline minerals & Franklinite & Galena & Sphalerite \\
\hline percentage & 5 & 5 & trace \\
\hline morphology & $\begin{array}{l}\text { Subhedral to euhedral crys- } \\
\text { tals that increase in size in } \\
\text { proximity to galena }\end{array}$ & Irregular crystals veins & Irregular xtals \\
\hline texture & $\begin{array}{l}\text { Disseminated with some } \\
\text { semi-massive clusters }\end{array}$ & Vein forming & $\begin{array}{l}\text { Surrounded by gn in vein } \\
\text { and vug }\end{array}$ \\
\hline $\begin{array}{l}\text { transmitted opti- } \\
\text { cal properties }\end{array}$ & & & \\
\hline $\begin{array}{l}\text { reflected optical } \\
\text { properties }\end{array}$ & & & \\
\hline $\begin{array}{l}\text { contact/ } \\
\text { relationships with } \\
\text { other minerals }\end{array}$ & $\begin{array}{l}\text { Straight contacts with gn and } \\
\mathrm{hm}\end{array}$ & $\begin{array}{l}\text { Has straight contacts with } \\
\text { frk, unknown Ag min and } \\
\text { srm. Cuts through sp and is } \\
\text { replacing sp }\end{array}$ & Being replaced by gn \\
\hline $\begin{array}{l}\text { other (growth di- } \\
\text { rection/zoning) }\end{array}$ & Exsolving hm & & \\
\hline
\end{tabular}




\begin{tabular}{|c|c|c|c|}
\hline minerals & Stromeyerite & Chalcocite & Covelite \\
\hline percentage & trace & Very trace & trace \\
\hline morphology & Irregular xtals & Irregular xtals & Small fibers \\
\hline texture & Growing on gn & Growing on gn & Growing on srm, cc and gn \\
\hline $\begin{array}{l}\text { transmitted opti- } \\
\text { cal properties }\end{array}$ & & & \\
\hline $\begin{array}{l}\text { reflected optical } \\
\text { properties }\end{array}$ & $\begin{array}{l}\text { Slight violet tinge to gray in } \\
\text { ppl } \\
\text { Strong blue-indigo anisotro- }\end{array}$ & Steel blue & $\begin{array}{l}\text { Blue in ppl } \\
\text { Orange-yellow anisotropy }\end{array}$ \\
\hline $\begin{array}{l}\text { contact/ } \\
\text { relationships with } \\
\text { other minerals }\end{array}$ & $\begin{array}{l}\text { Growing on gn, irregular } \\
\text { contacts with cv and cc be- } \\
\text { ing replaced by cv and cc }\end{array}$ & $\begin{array}{l}\text { Irregular contact with cv, } \\
\text { being replaced by cv }\end{array}$ & $\begin{array}{l}\text { Replacing srm and cc, jag- } \\
\text { ged contacts with both mins }\end{array}$ \\
\hline $\begin{array}{l}\text { other (growth di- } \\
\text { rection/zoning) }\end{array}$ & & & \\
\hline
\end{tabular}




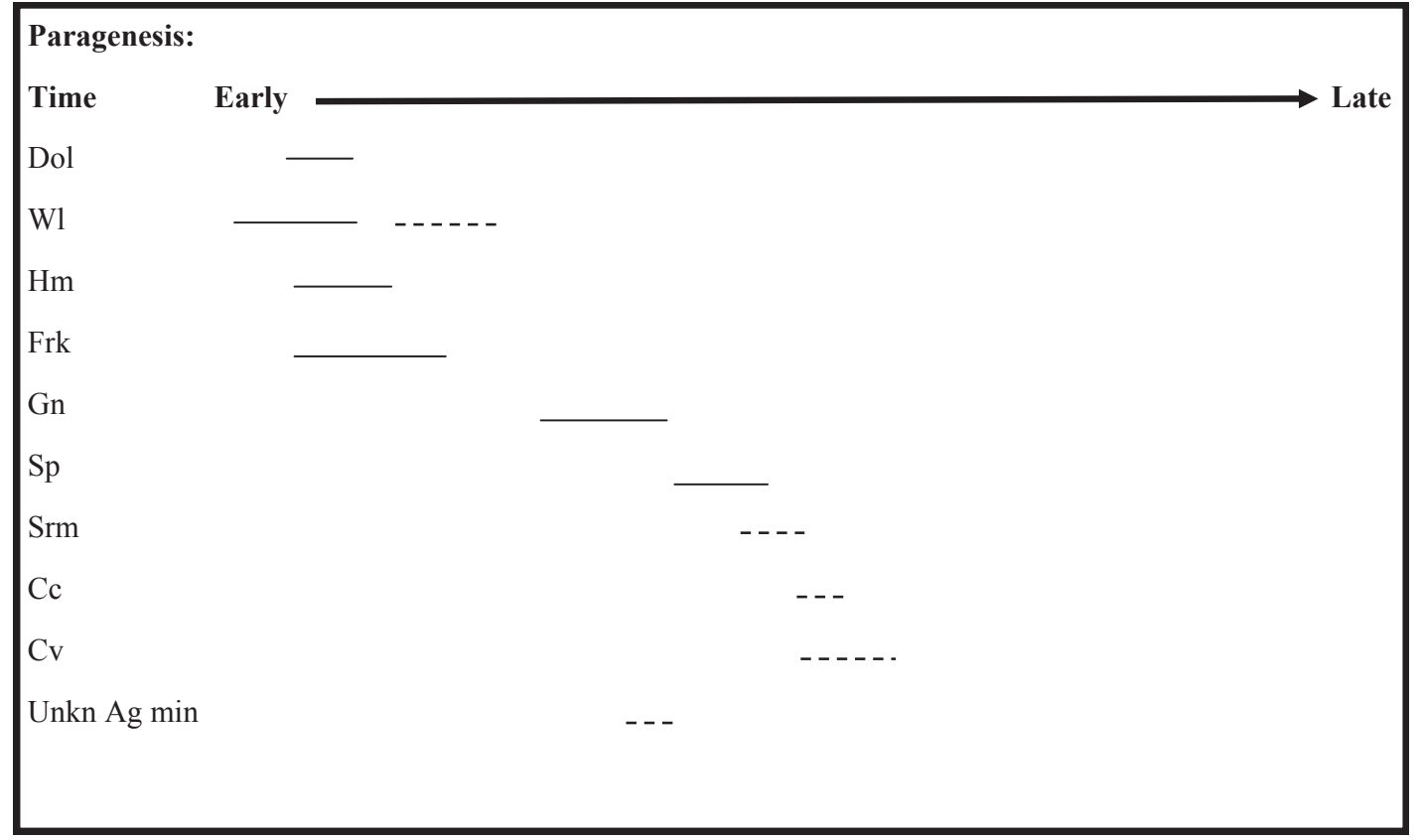


Geologic Unit: Hydrothermal Breccia

Lithological Unit (Votorantim): Massive Silicate (SM) in the Hydrothermal Breccia (BH)

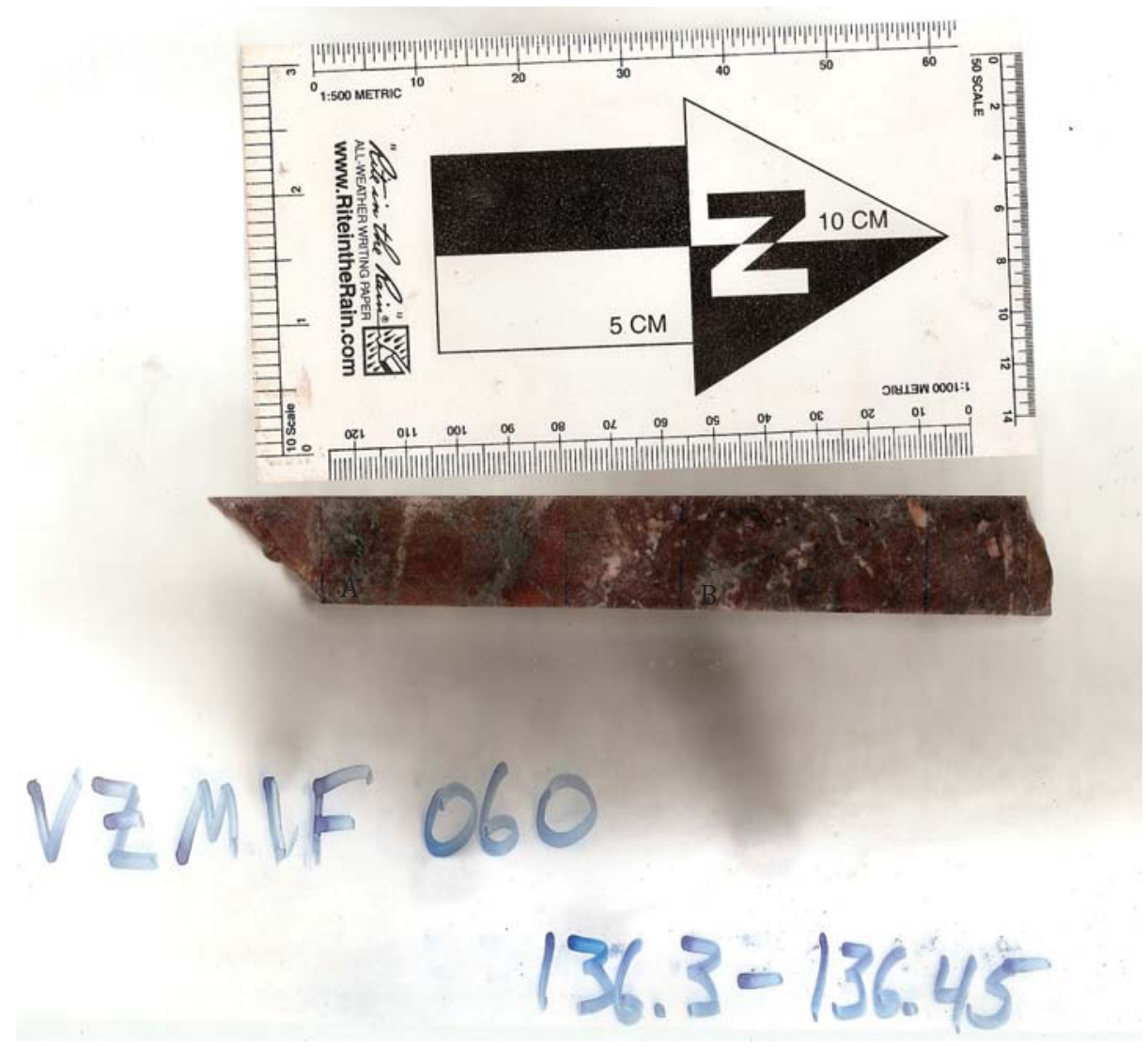

Other Sample Information: No sulfides like in 60-136A 


\begin{tabular}{|c|c|c|c|}
\hline minerals & Dolomite & Willemite & Quartz \\
\hline percentage & 20 & 60 & 10 \\
\hline morphology & $\begin{array}{l}\text { Large euhedral hydrothermal } \\
\text { dol xtals with later minor } \\
\text { fine xtals }\end{array}$ & $\begin{array}{l}\text { Large xtal laths and some } \\
\text { smaller finer granular xtals }\end{array}$ & Large anhedral xtals \\
\hline texture & brecciated & Massive, has been brecciated & Disseminated/brecciated \\
\hline $\begin{array}{l}\text { transmitted opti- } \\
\text { cal properties }\end{array}$ & & & \\
\hline $\begin{array}{l}\text { reflected optical } \\
\text { properties }\end{array}$ & & & \\
\hline $\begin{array}{l}\text { contact/ } \\
\text { relationships with } \\
\text { other minerals }\end{array}$ & $\begin{array}{l}\text { Large dol xtals have irregu- } \\
\text { lar contacts and being re- } \\
\text { placed by qtz, wl, late fine } \\
\text { dol and } \mathrm{hm} / \mathrm{frk}\end{array}$ & $\begin{array}{l}\text { Lath-shaped xtals are later, } \\
\text { growing in qtz, large dol, } \\
\text { and granular wl. Granular } \\
\text { wl replaced qtz and dol. Ir- } \\
\text { regular contacts with hm/frk } \\
\text { which are replacing wl }\end{array}$ & $\begin{array}{l}\text { Irregular contacts with all } \\
\text { minerals, being replaced by } \\
\text { all minerals }\end{array}$ \\
\hline $\begin{array}{l}\text { other (growth di- } \\
\text { rection/zoning) }\end{array}$ & $\begin{array}{l}\text { Original zoning may be pre- } \\
\text { sent, but fully replaced by wl }\end{array}$ & & $\begin{array}{l}\text { Original grain may be pre- } \\
\text { sent but pseudomorphed by } \\
\text { wl }\end{array}$ \\
\hline
\end{tabular}




\begin{tabular}{|c|c|c|}
\hline minerals & Hematite & Franklinite \\
\hline percentage & 5 & 5 \\
\hline morphology & $\begin{array}{l}\text { Sheared veins with hm xtal } \\
\text { laths }\end{array}$ & $\begin{array}{l}\text { Semi-massive amalgama- } \\
\text { tions of granular xtals }\end{array}$ \\
\hline texture & Shear veins & Associated with hm \\
\hline $\begin{array}{l}\text { transmitted opti- } \\
\text { cal properties }\end{array}$ & & \\
\hline $\begin{array}{l}\text { reflected optical } \\
\text { properties }\end{array}$ & & $\begin{array}{l}\text { Grey, lighter than } \mathrm{hm} \text {, darker } \\
\text { than sp }\end{array}$ \\
\hline $\begin{array}{l}\text { contact/ } \\
\text { relationships with } \\
\text { other minerals }\end{array}$ & Irregular, replacing all mins & Irregular replacing all mins, \\
\hline $\begin{array}{l}\text { other (growth di- } \\
\text { rection/zoning) }\end{array}$ & & $\begin{array}{l}\text { replacing wl on old dol clvg } \\
\text { planes }\end{array}$ \\
\hline
\end{tabular}

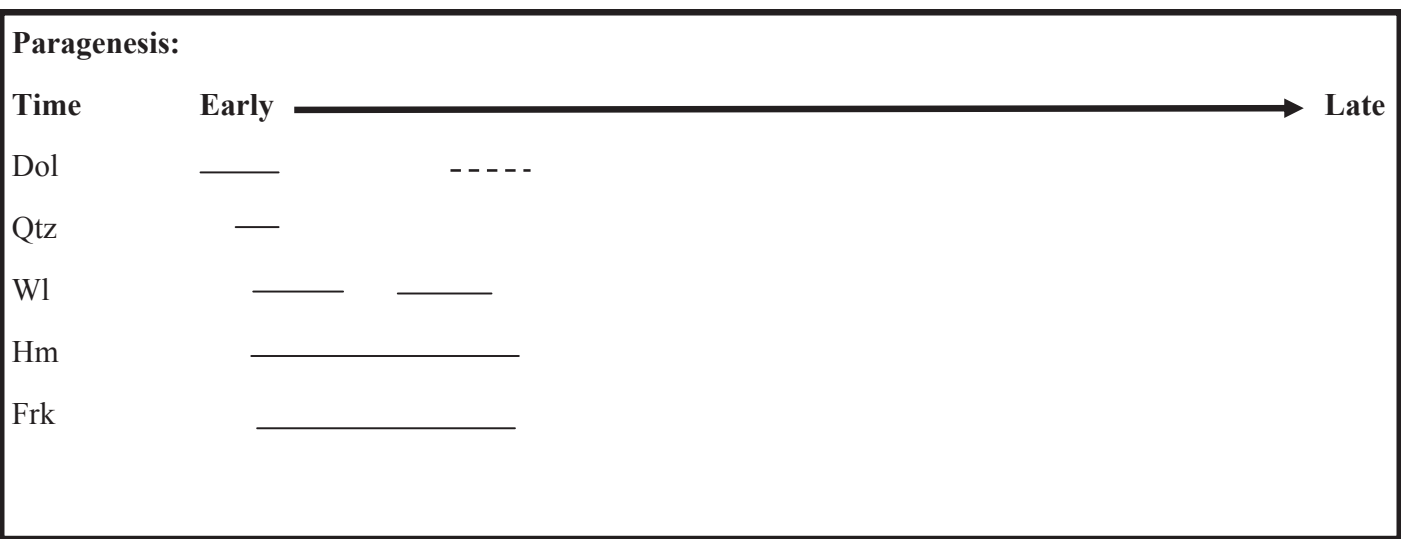


Geologic Unit: Upper Morro do Pinheiro

Lithological Unit (Votorantim): Grey Dolomite (DO)

Sample Photograph

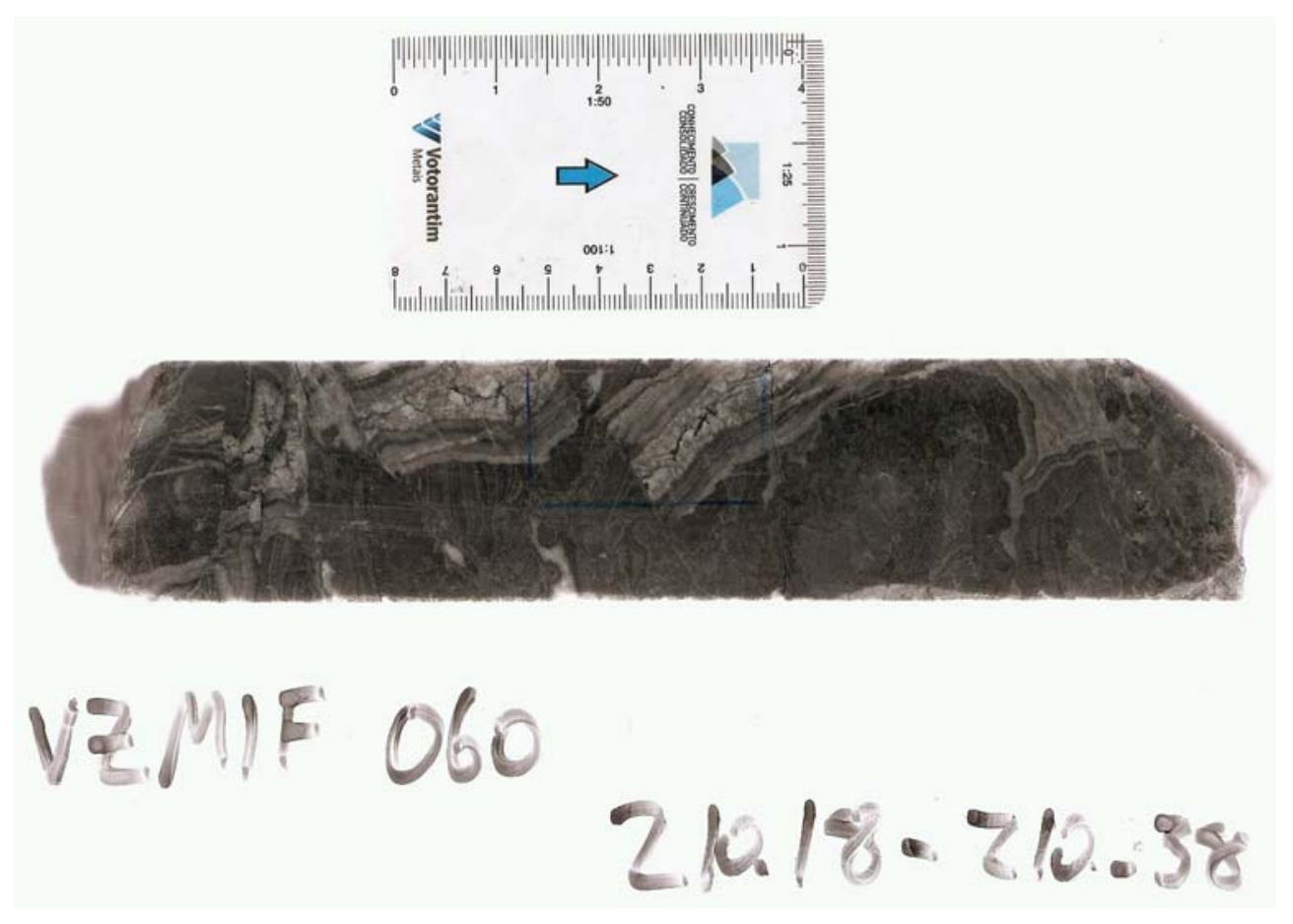

Other Sample Information: 


\begin{tabular}{|c|c|c|c|}
\hline minerals & Dolomite & Hydrothermal Carbonate & Quartz \\
\hline percentage & 25 & 30 & 40 \\
\hline morphology & Crystalline carbonate & euhedral & Euhedral \\
\hline texture & Medium spar & Megaspar in vein & Megacrystalline in vein \\
\hline $\begin{array}{l}\text { transmitted opti- } \\
\text { cal properties }\end{array}$ & & & \\
\hline $\begin{array}{l}\text { reflected optical } \\
\text { properties }\end{array}$ & & & \\
\hline $\begin{array}{l}\text { contact/ } \\
\text { relationships with } \\
\text { other minerals }\end{array}$ & Host rock & $\begin{array}{l}\text { Growing out from host rock, } \\
\text { transition into quartz; brecci- } \\
\text { ated }\end{array}$ & $\begin{array}{l}\text { Growing out from host and } \\
\text { transition into carbonate; } \\
\text { sheared }\end{array}$ \\
\hline $\begin{array}{l}\text { other (growth di- } \\
\text { rection/zoning) }\end{array}$ & & & \\
\hline
\end{tabular}




\begin{tabular}{|c|c|c|c|}
\hline minerals & Pyrite & Sphalerite & Hematite \\
\hline percentage & 4 & 1 & trace \\
\hline morphology & Anhedral granular xtals & Crystalline, anhedral & Crystalline, anhedral \\
\hline texture & In veinlets & In open space in breccia & Fracture filling in breccia \\
\hline $\begin{array}{l}\text { transmitted opti- } \\
\text { cal properties }\end{array}$ & & & \\
\hline $\begin{array}{l}\text { reflected optical } \\
\text { properties }\end{array}$ & & & \\
\hline $\begin{array}{l}\text { contact/ } \\
\text { relationships with } \\
\text { other minerals }\end{array}$ & $\begin{array}{l}\text { Straight contacts with qtz, } \\
\text { some fractures }\end{array}$ & Straight contacts with carb & $\begin{array}{l}\text { Irregular contacts with carb, } \\
\text { minor replacement }\end{array}$ \\
\hline $\begin{array}{l}\text { other (growth di- } \\
\text { rection/zoning) }\end{array}$ & & & \\
\hline
\end{tabular}

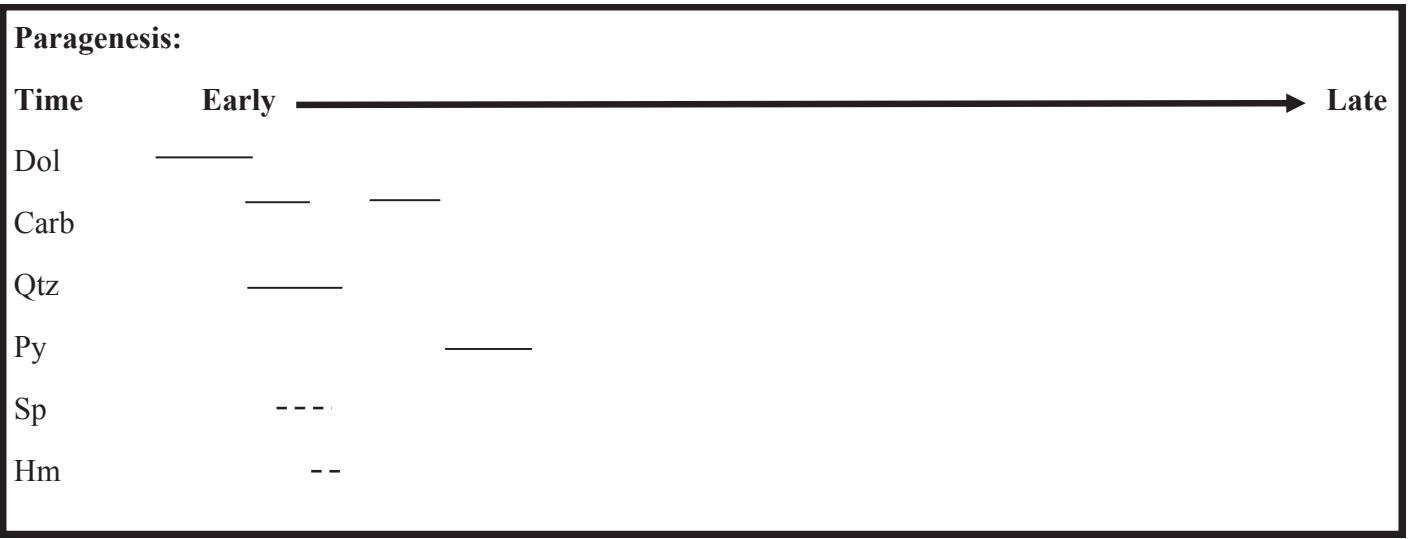


Geologic Unit: Lower Pamplona

Lithological Unit (Votorantim): Pink dolomite (DG)

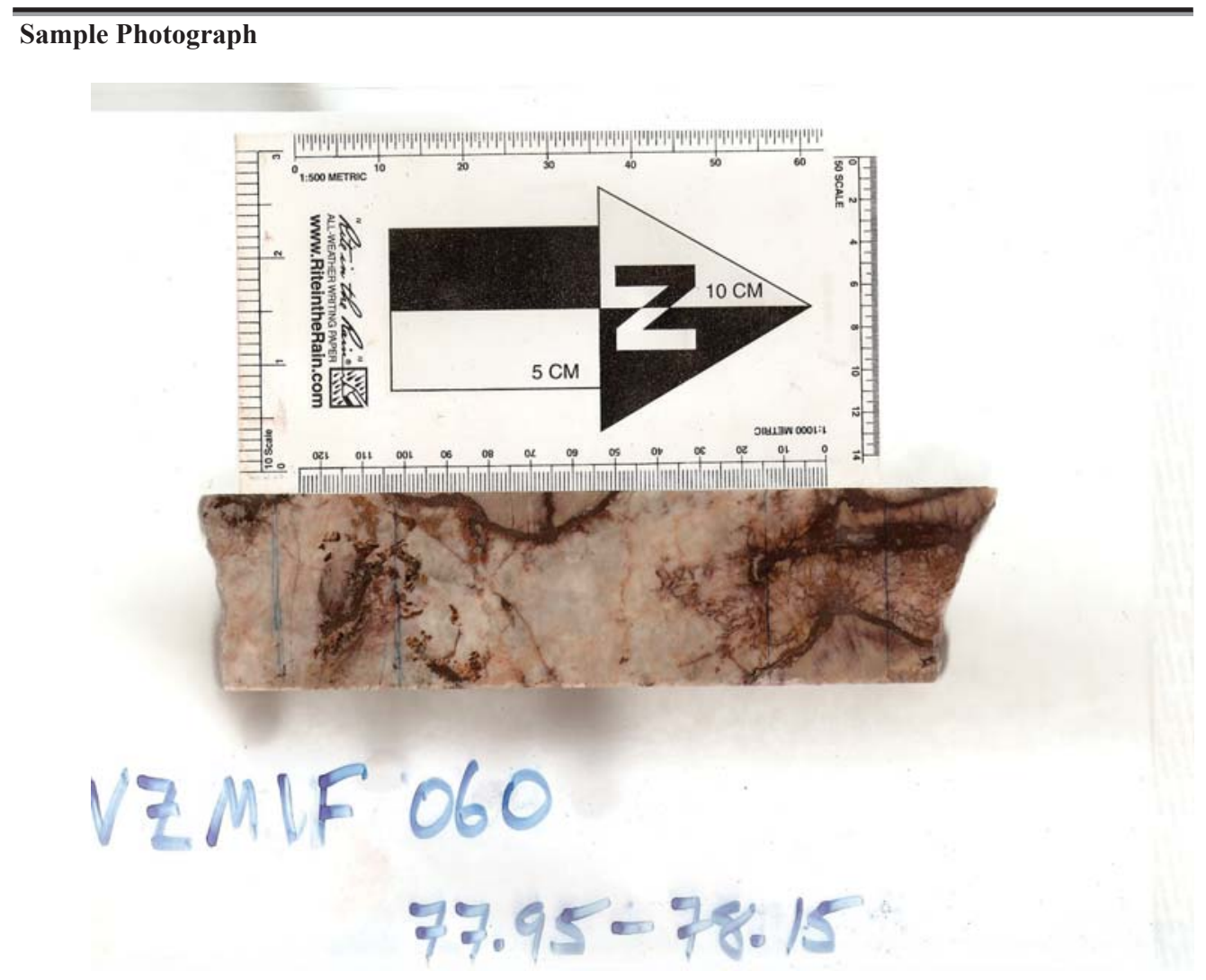

Other Sample Information: Fe-carbonate alteration in a minor bx in the Lower Pamplona 


\begin{tabular}{|c|c|c|c|}
\hline minerals & Dolomite & Hydrothermal dolomite & Quartz \\
\hline percentage & 30 & 45 & 5 \\
\hline morphology & $\begin{array}{l}\text { Subhedral crystalline dolar- } \\
\text { enite }\end{array}$ & Sparry, euhedral & Elongate, anhedral xtals \\
\hline texture & Composes host rock & Vein-filling & $\begin{array}{l}\text { Replacing interstices be- } \\
\text { tween large hydrothermal } \\
\text { dol xtals }\end{array}$ \\
\hline $\begin{array}{l}\text { transmitted opti- } \\
\text { cal properties }\end{array}$ & & & \\
\hline $\begin{array}{l}\text { reflected optical } \\
\text { properties }\end{array}$ & & & \\
\hline $\begin{array}{l}\text { contact/ } \\
\text { relationships with } \\
\text { other minerals }\end{array}$ & $\begin{array}{l}\text { Straight contacts with itself, } \\
\text { fault/brecciated contact with } \\
\mathrm{hm} \text { and hydrothermal dol }\end{array}$ & $\begin{array}{l}\text { Contains some hm inclu- } \\
\text { sions, being replaced by hm } \\
\text { at bx margins, being re- } \\
\text { placed by qtz }\end{array}$ & Replacing hydrothermal dol \\
\hline $\begin{array}{l}\text { other (growth di- } \\
\text { rection/zoning) }\end{array}$ & & $\begin{array}{l}\text { Growing towards vein cen- } \\
\text { ters }\end{array}$ & \\
\hline
\end{tabular}




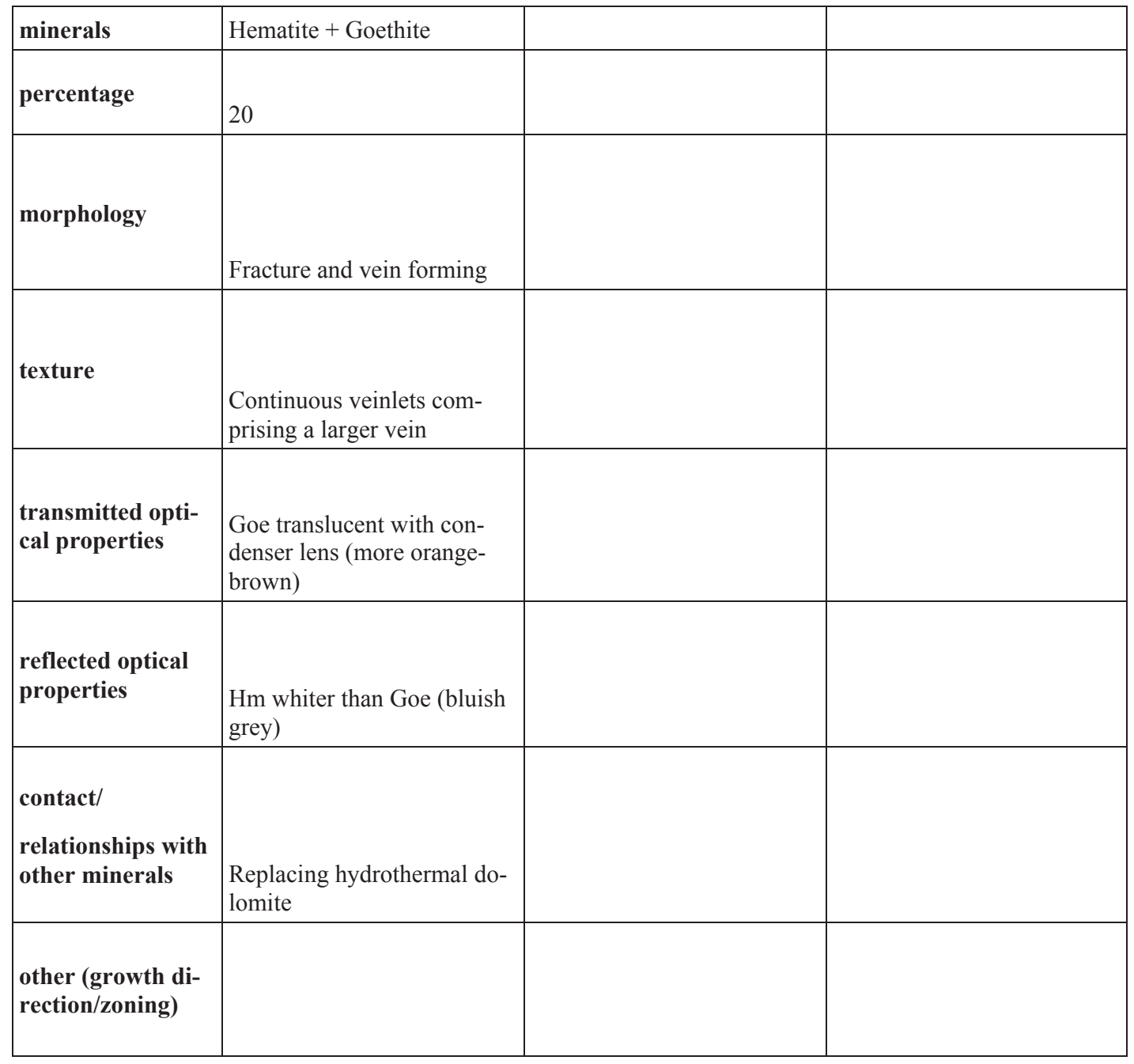

Paragenesis:

Time

Early

Late

Dol

$\downarrow$ vein emplacement

Hydr. Dol

$\mathrm{Hm}$

Qtz 
Geologic Unit: Lower Pamplona

Lithological Unit (Votorantim): Pink dolomite (DG)

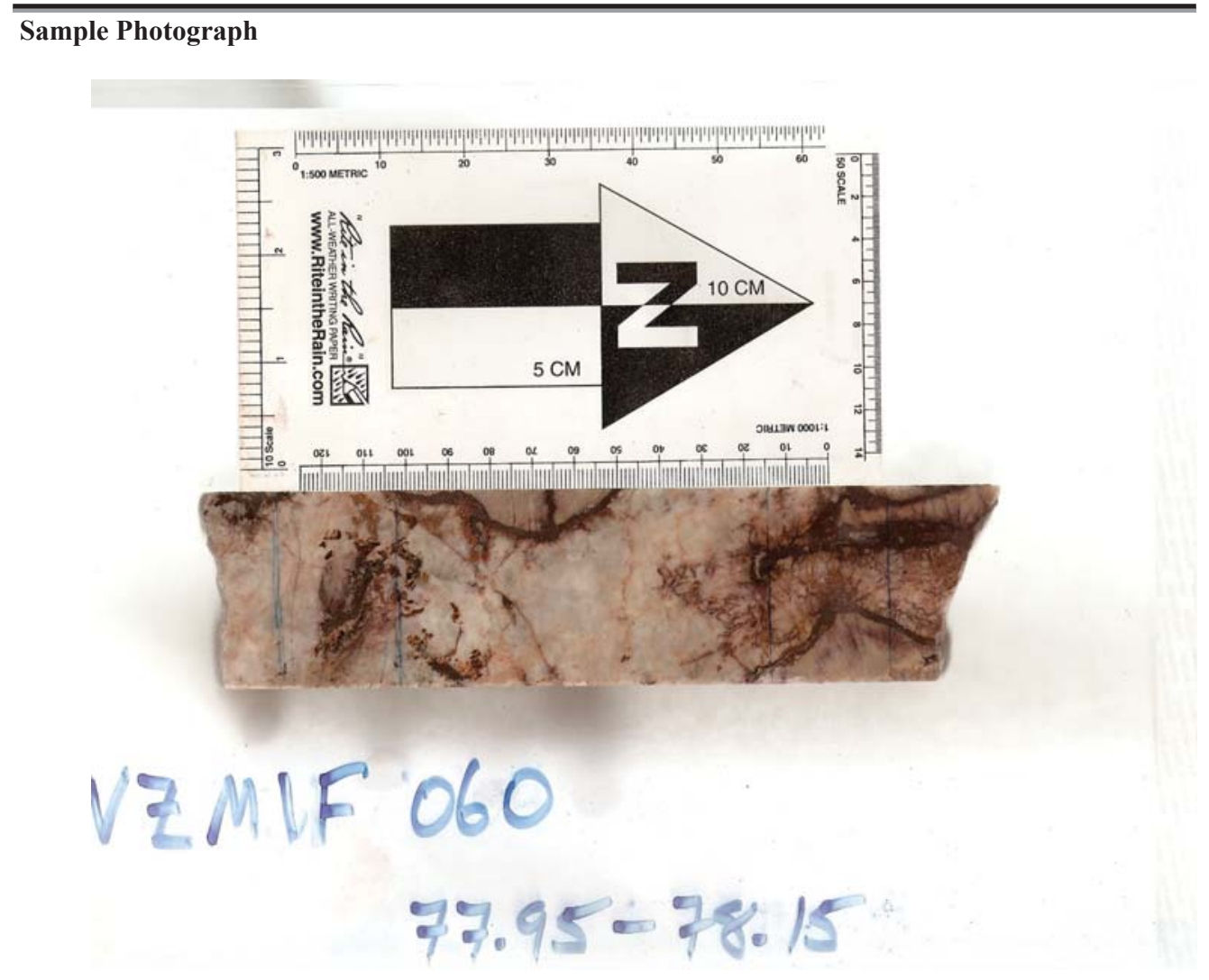

Other Sample Information: Fe-carbonate alteration in a minor bx in the Lower Pamplona * Contains Py instead of Hm in 60-78A 


\begin{tabular}{|c|c|c|c|}
\hline minerals & Dolomite & Hydrothermal dolomite & Quartz \\
\hline percentage & 20 & 45 & 15 \\
\hline morphology & $\begin{array}{l}\text { Subhedral crystalline dolar- } \\
\text { enite }\end{array}$ & Sparry, euhedral & chalcedonic \\
\hline texture & Host rock bx piece & Vein-filling & $\begin{array}{l}\text { Replacing dolomite host } \\
\text { rock, minor occurrences } \\
\text { with hydrothermal dol } \\
\text { (replacing h. dol) }\end{array}$ \\
\hline $\begin{array}{l}\text { transmitted opti- } \\
\text { cal properties }\end{array}$ & & & \\
\hline $\begin{array}{l}\text { reflected optical } \\
\text { properties }\end{array}$ & & & \\
\hline $\begin{array}{l}\text { contact/ } \\
\text { relationships with } \\
\text { other minerals }\end{array}$ & $\begin{array}{l}\text { Straight contacts with itself, } \\
\text { fault/brecciated contact with } \\
\text { hm and hydrothermal dol. } \\
\text { Being replaced by qtz }\end{array}$ & $\begin{array}{l}\text { Contains some hm inclu- } \\
\text { sions, being replaced by hm } \\
\text { at bx margins, being re- } \\
\text { placed by qtz }\end{array}$ & $\begin{array}{l}\text { Replacing dol host rock and } \\
\text { hydrothermal dol }\end{array}$ \\
\hline $\begin{array}{l}\text { other (growth di- } \\
\text { rection/zoning) }\end{array}$ & & $\begin{array}{l}\text { Growing towards vein cen- } \\
\text { ters }\end{array}$ & \\
\hline
\end{tabular}




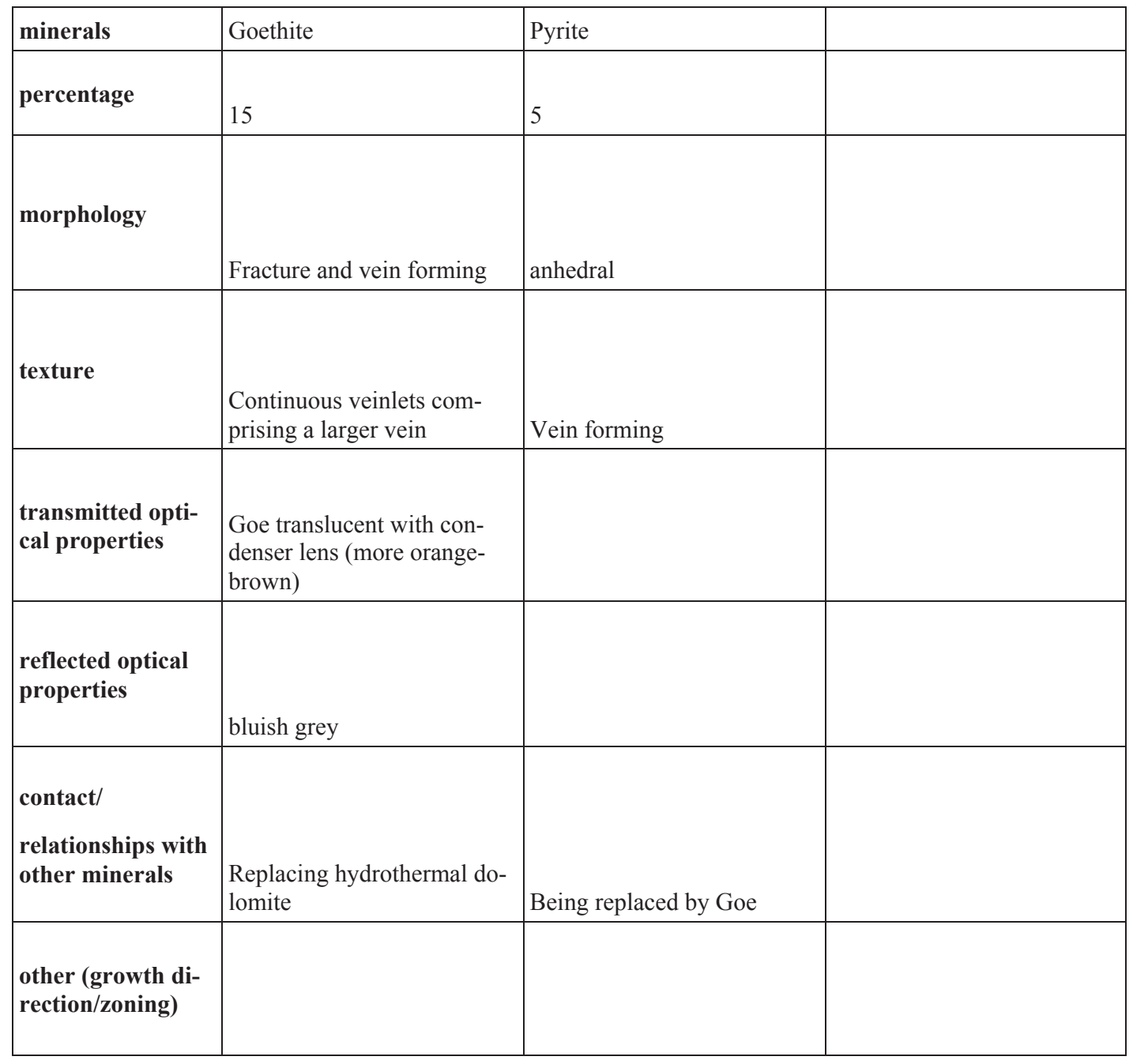

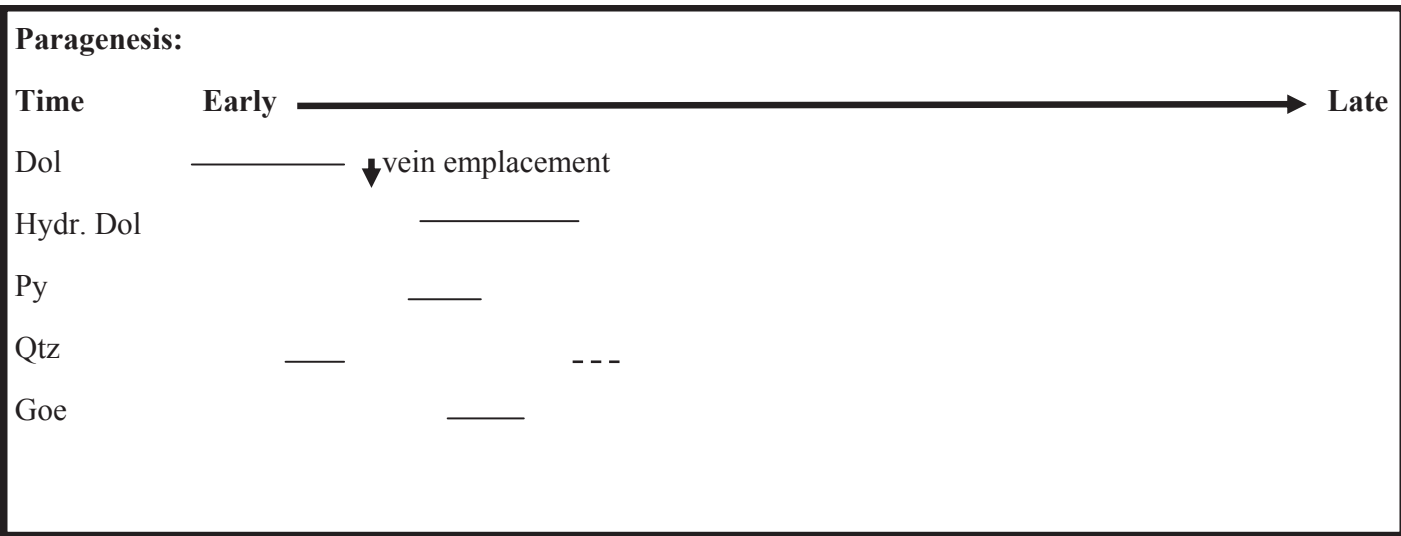


Geologic Unit: Lower Pamplona

Lithological Unit (Votorantim): Phyllite and pink dolomite (DG)

Sample Photograph

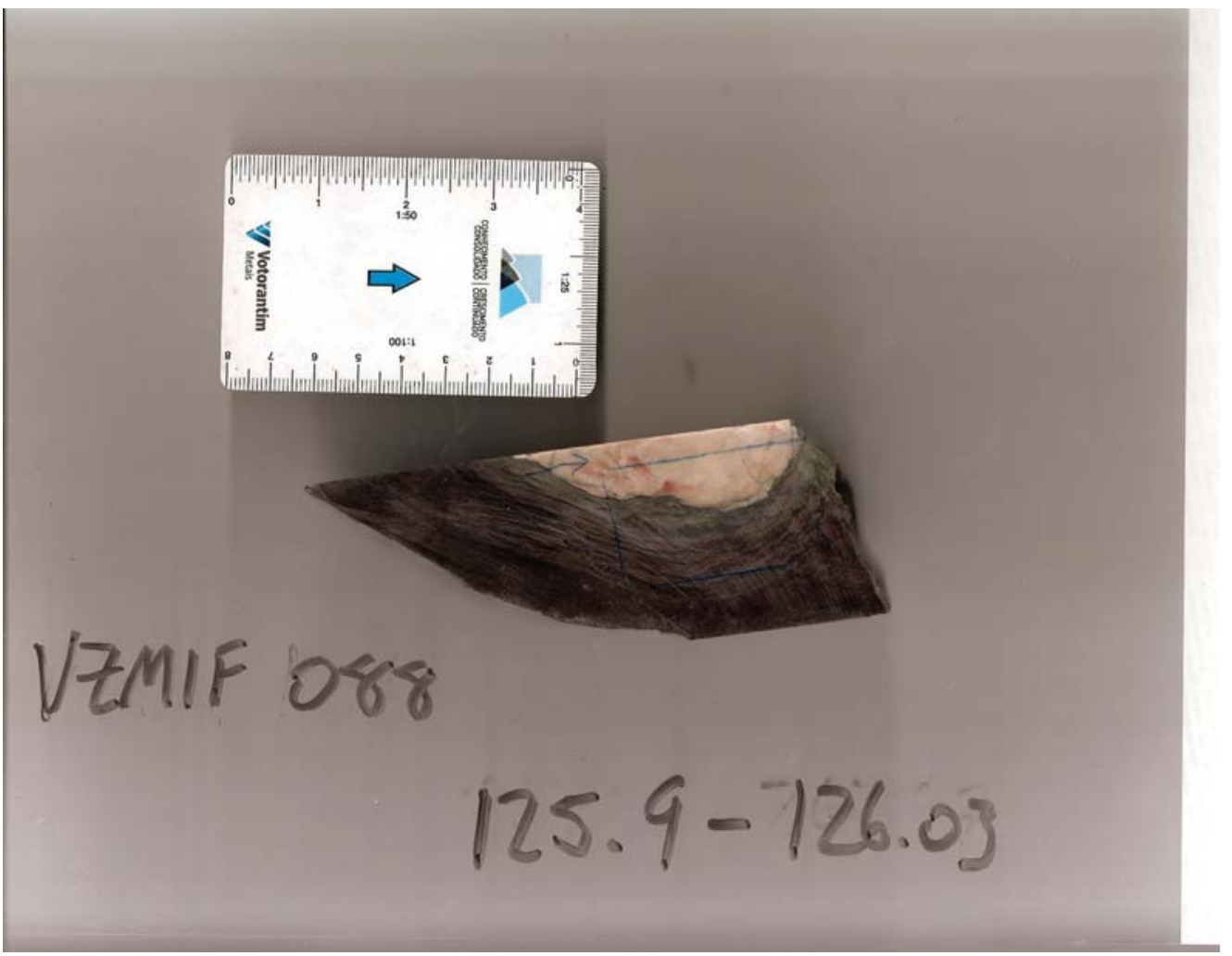

Other Sample Information: 


\begin{tabular}{|l|l|l|l|}
\hline minerals & Dolomite & Quartz & Unknown-Chl? \\
\hline percentage & 20 & 35 & 45 \\
\hline morphology & Clast in phyllite & Subangular to subrounded & platy \\
\hline $\begin{array}{l}\text { texture } \\
\text { transmitted opti- } \\
\text { cal properties }\end{array}$ & boundstone & & \\
\hline $\begin{array}{l}\text { reflected optical } \\
\text { properties }\end{array}$ & laminar & Very fine \\
\hline $\begin{array}{l}\text { contact/ } \\
\text { relationships with } \\
\text { other minerals } \\
\text { rection/zoning) }\end{array}$ & $\begin{array}{l}\text { Irregular, brittle contact with } \\
\text { qtz and phyllite rock }\end{array}$ & $\begin{array}{l}\text { Smooth contacts with phyl- } \\
\text { lite host rock, some qtz vein- } \\
\text { lets growing in between carb } \\
\text { and phyllite }\end{array}$ & Coeval with qtz and micas \\
\hline & & & \\
\hline
\end{tabular}




\begin{tabular}{|c|c|c|}
\hline minerals & White Mica & Hematite \\
\hline percentage & trace & trace \\
\hline morphology & Euhedral & Irregular crystalline \\
\hline texture & Disseminated & disseminated \\
\hline $\begin{array}{l}\text { transmitted opti- } \\
\text { cal properties }\end{array}$ & & \\
\hline $\begin{array}{l}\text { reflected optical } \\
\text { properties }\end{array}$ & & \\
\hline $\begin{array}{l}\text { contact/ } \\
\text { relationships with } \\
\text { other minerals }\end{array}$ & Composes host rock & $\begin{array}{l}\text { Replacement of grains in } \\
\text { phyllite }\end{array}$ \\
\hline $\begin{array}{l}\text { other (growth di- } \\
\text { rection/zoning) }\end{array}$ & & \\
\hline
\end{tabular}

Paragenesis:

Time

Early

Late 
Geologic Unit: Lower Pamplona

Lithological Unit (Votorantim): Pink Dolomite

Sample Photograph

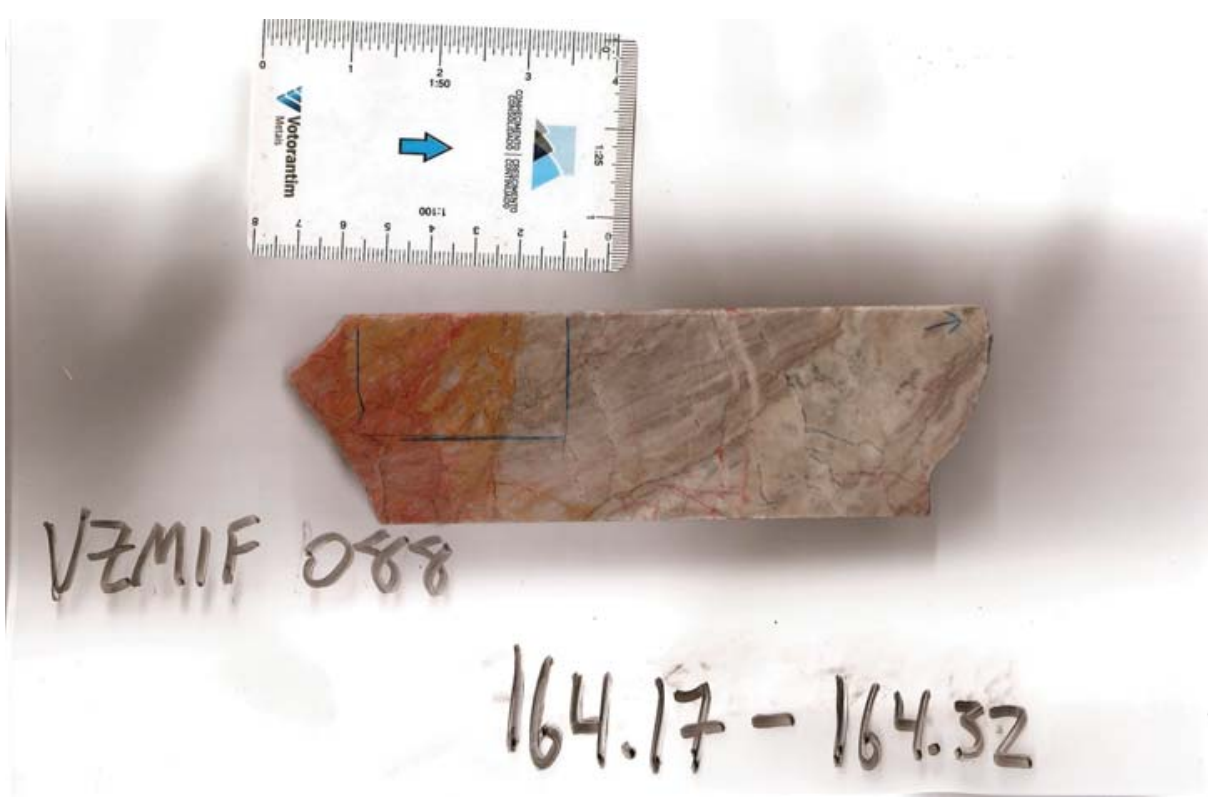

Other Sample Information: 


\begin{tabular}{|c|c|c|c|}
\hline minerals & Grey dolomite & Orange dolomite & Hematite \\
\hline percentage & 50 & 50 & trace \\
\hline morphology & Crystalline carbonate & Crystalline carbonate & Irregular xtals \\
\hline texture & Some neomorphic vugs & Some neomorphic vugs & Disseminated in orange dol \\
\hline $\begin{array}{l}\text { transmitted opti- } \\
\text { cal properties }\end{array}$ & & & \\
\hline $\begin{array}{l}\text { reflected optical } \\
\text { properties }\end{array}$ & & & \\
\hline $\begin{array}{l}\text { contact/ } \\
\text { relationships with } \\
\text { other minerals }\end{array}$ & & Fades into regular dol & $\begin{array}{l}\text { Irregular edges with orange } \\
\text { dol, replacing orange dol }\end{array}$ \\
\hline $\begin{array}{l}\text { other (growth di- } \\
\text { rection/zoning) }\end{array}$ & & & \\
\hline
\end{tabular}

Paragenesis:

Time

Early

Late

Grey Dol

Orange Dol

$\mathrm{Hm}$ 
Geologic Unit: Hydrothermal Breccia

Lithological Unit (Votorantim): Hydrothermal Breccia

Sample Photograph

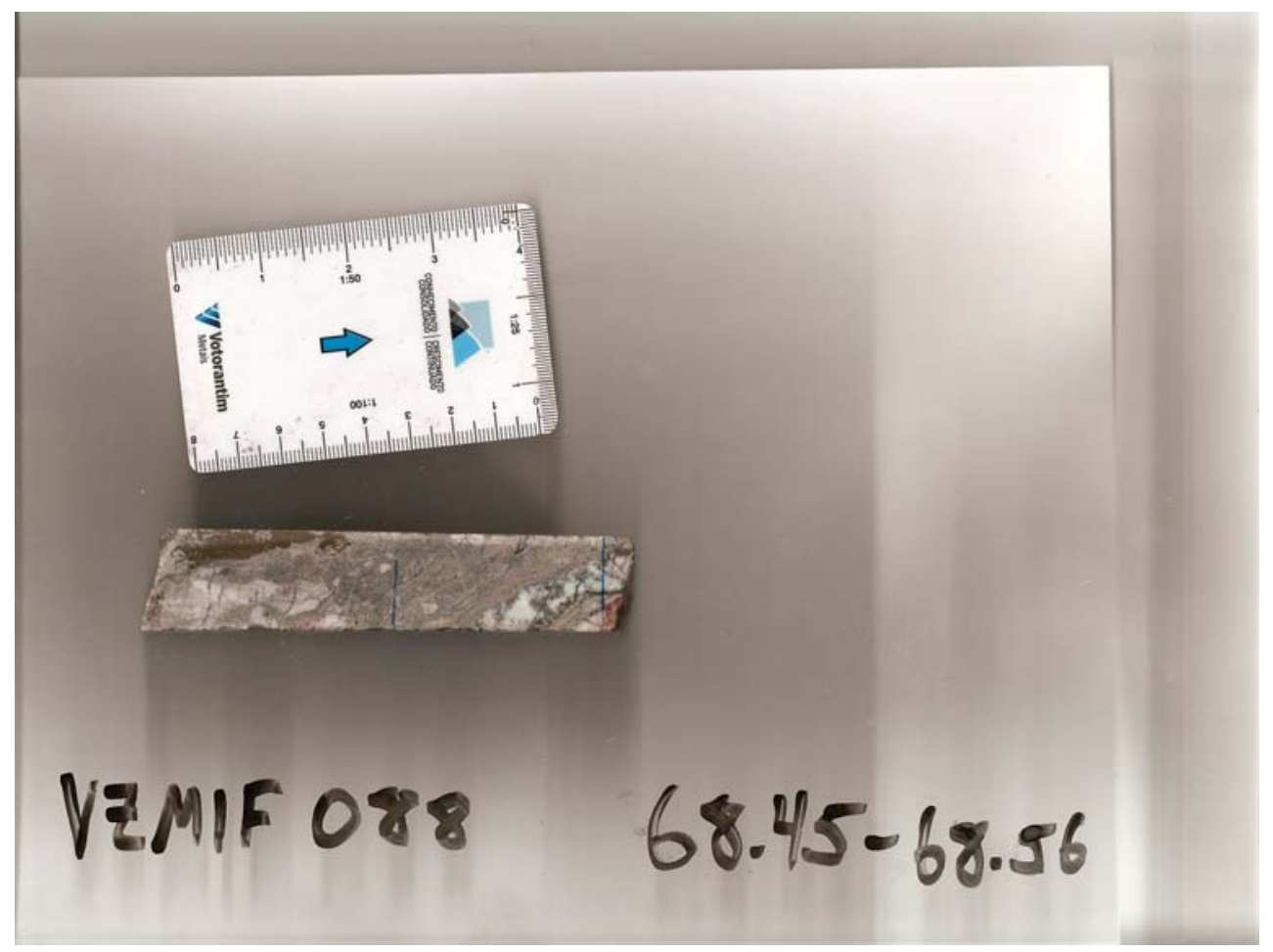

Other Sample Information: 


\begin{tabular}{|l|l|l|l|}
\hline minerals & Carbonate & Quartz & Sphalerite \\
\hline percentage & 20 & 7 & 65 \\
\hline morphology & $\begin{array}{l}\text { Large sparite with late fine- } \\
\text { grained carb }\end{array}$ & Anhedral crystalline & Massive crystalline \\
\hline texture & & & \\
\hline $\begin{array}{l}\text { transmitted opti- } \\
\text { cal properties }\end{array}$ & $\begin{array}{l}\text { Brecciated, fine grain carb is } \\
\text { in veinlets }\end{array}$ & Veinlets that cut sp & Massive and sheared \\
\hline $\begin{array}{l}\text { reflected optical } \\
\text { properties }\end{array}$ & $\begin{array}{l}\text { Sparite is being replaced by } \\
\text { sulfides, late fine carb is re- } \\
\text { placing sp, irregular contacts } \\
\text { and spotty }\end{array}$ & $\begin{array}{l}\text { Irregular contacts with spar- } \\
\text { ite and sp, cutting these } \\
\text { mins, replacing carb }\end{array}$ & $\begin{array}{l}\text { Replacing carb, being cut by } \\
\text { qtz, some inclusions of py } \\
\text { and gn, }\end{array}$ \\
\hline $\begin{array}{l}\text { contact/ } \\
\text { relationships wer (growth di- } \\
\text { other minerals } \\
\text { rection/zoning) }\end{array}$ & & & \\
\hline & & & \\
\hline
\end{tabular}




\begin{tabular}{|c|c|c|}
\hline minerals & Pyrite & Galena \\
\hline percentage & 7 & 1 \\
\hline morphology & Granular crystals & Anhedral inclusions \\
\hline texture & Fractured and disseminated & disseminated \\
\hline $\begin{array}{l}\text { transmitted opti- } \\
\text { cal properties }\end{array}$ & & \\
\hline $\begin{array}{l}\text { reflected optical } \\
\text { properties }\end{array}$ & & \\
\hline $\begin{array}{l}\text { contact/ } \\
\text { relationships with } \\
\text { other minerals }\end{array}$ & $\begin{array}{l}\text { Minor replacement and } \\
\text { exsolution from sphalerite }\end{array}$ & $\begin{array}{l}\text { Round contacts included in } \\
\text { sp }\end{array}$ \\
\hline $\begin{array}{l}\text { other (growth di- } \\
\text { rection/zoning) }\end{array}$ & & \\
\hline
\end{tabular}

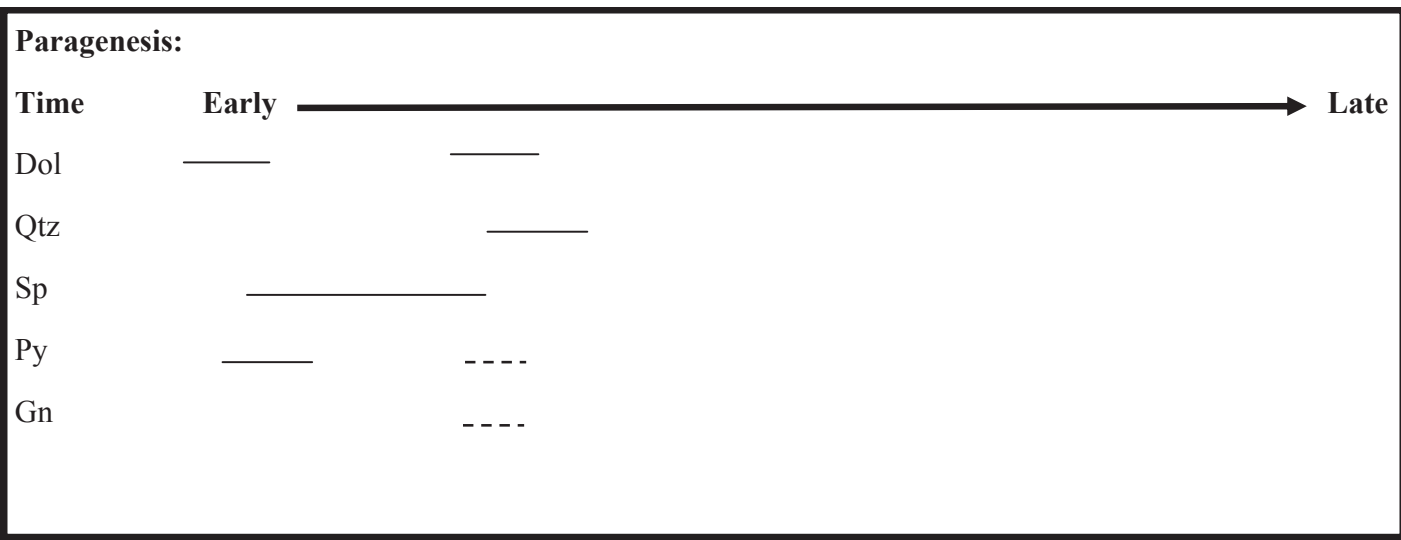


Geologic Unit: Hydrothermal Breccia

Lithological Unit (Votorantim): Disseminated Sulfide/Silicate in BH

Sample Photograph
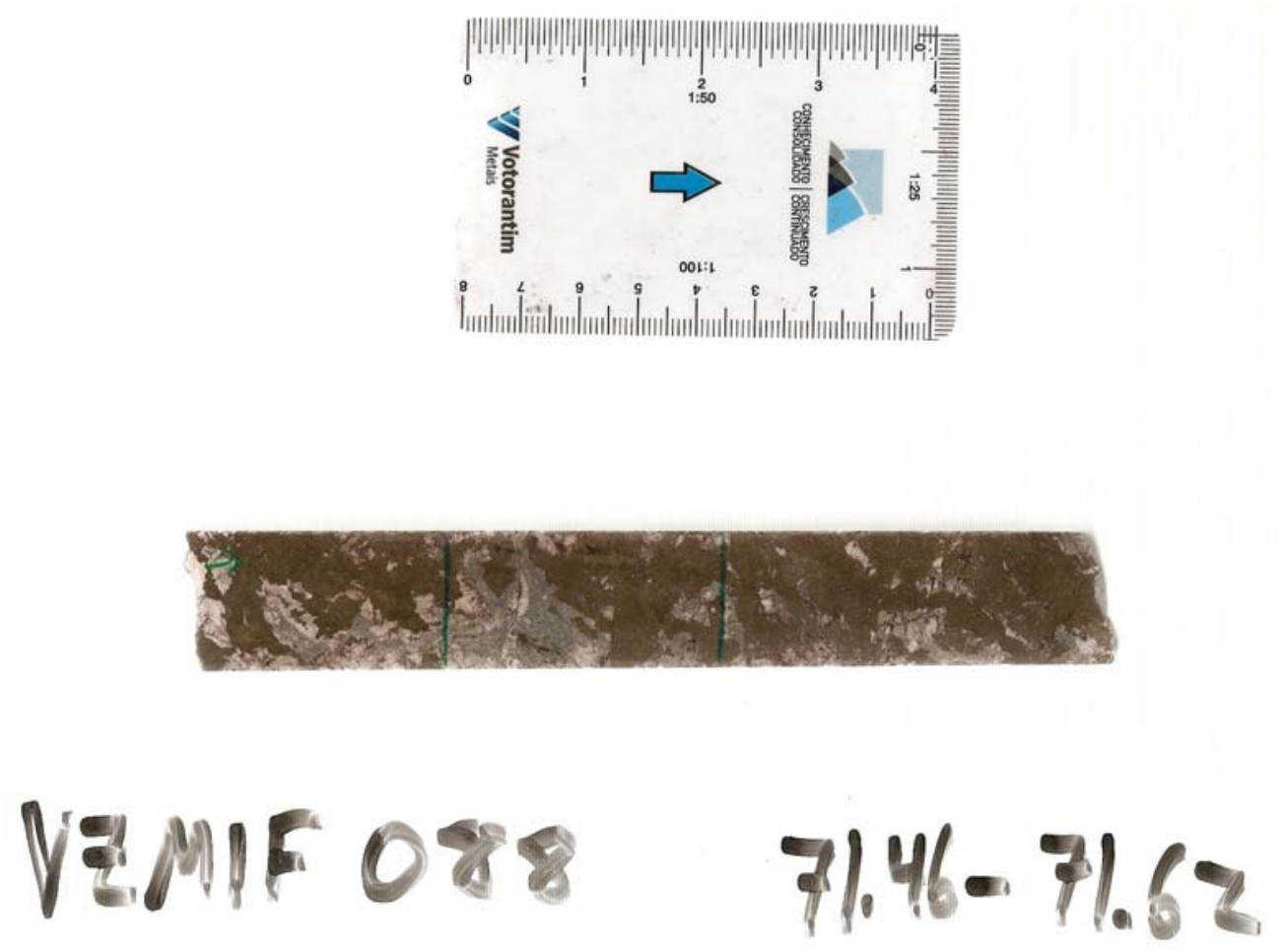

Other Sample Information: 


\begin{tabular}{|c|c|c|c|}
\hline minerals & Pyrite & Sphaerlite & Galena \\
\hline percentage & 60 & 21 & 1 \\
\hline morphology & Euhedral cubes & $\begin{array}{l}\text { Fine crystalline, appears to } \\
\text { 'flow' }\end{array}$ & Semi massive in veinlets \\
\hline texture & Forms massive sulfide & Semi-massive & $\begin{array}{l}\text { Disseminated, discontinuous } \\
\text { veinlets }\end{array}$ \\
\hline $\begin{array}{l}\text { transmitted opti- } \\
\text { cal properties }\end{array}$ & & & \\
\hline $\begin{array}{l}\text { reflected optical } \\
\text { properties }\end{array}$ & & & \\
\hline $\begin{array}{l}\text { contact/ } \\
\text { relationships with } \\
\text { other minerals }\end{array}$ & $\begin{array}{l}\text { Irregular with sp, being re- } \\
\text { placed by sp }\end{array}$ & $\begin{array}{l}\text { Irregular with py, replacing } \\
\text { py }\end{array}$ & $\begin{array}{l}\text { Filling voids in sp, replacing } \\
\text { sp? }\end{array}$ \\
\hline $\begin{array}{l}\text { other (growth di- } \\
\text { rection/zoning) }\end{array}$ & & & \\
\hline
\end{tabular}




\begin{tabular}{|c|c|c|c|}
\hline minerals & Carbonate & Quartz & Barite \\
\hline percentage & 15 & 3 & trace \\
\hline morphology & Hydrothermal carb & anhedral & Anhedral \\
\hline texture & sparry & Replacing carb & $\begin{array}{l}\text { Weakly associated with ga- } \\
\text { lena }\end{array}$ \\
\hline $\begin{array}{l}\text { transmitted opti- } \\
\text { cal properties }\end{array}$ & & & \\
\hline $\begin{array}{l}\text { reflected optical } \\
\text { properties }\end{array}$ & & & \\
\hline $\begin{array}{l}\text { contact/ } \\
\text { relationships with } \\
\text { other minerals }\end{array}$ & $\begin{array}{l}\text { Jagged, irregular contacts, } \\
\text { overgrowths and embay- } \\
\text { ments with sulf; being re- } \\
\text { placed by sulfides and qtz }\end{array}$ & $\begin{array}{l}\text { Being replaced by sulfides, } \\
\text { irregular contacts and em- } \\
\text { bayments }\end{array}$ & $\begin{array}{l}\text { None observed, irregular } \\
\text { contacts with all mins }\end{array}$ \\
\hline $\begin{array}{l}\text { other (growth di- } \\
\text { rection/zoning) }\end{array}$ & & & \\
\hline
\end{tabular}

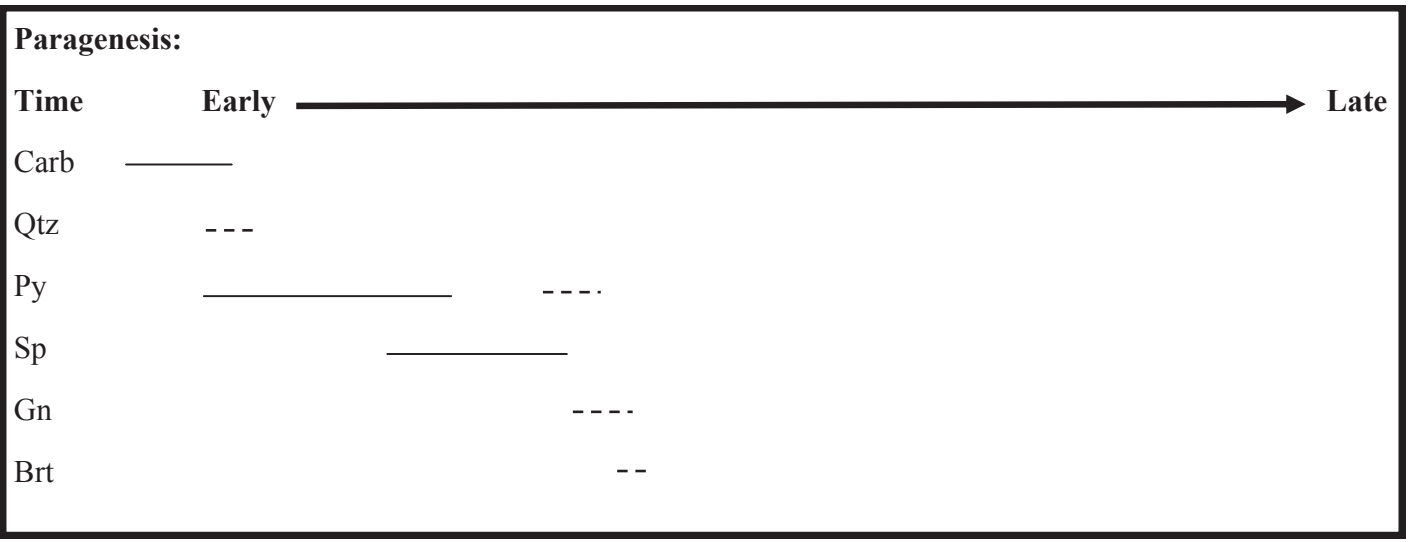


Geologic Unit: Hydrothermal Breccia

Lithological Unit (Votorantim): Hydrothermal Breccia

Sample Photograph

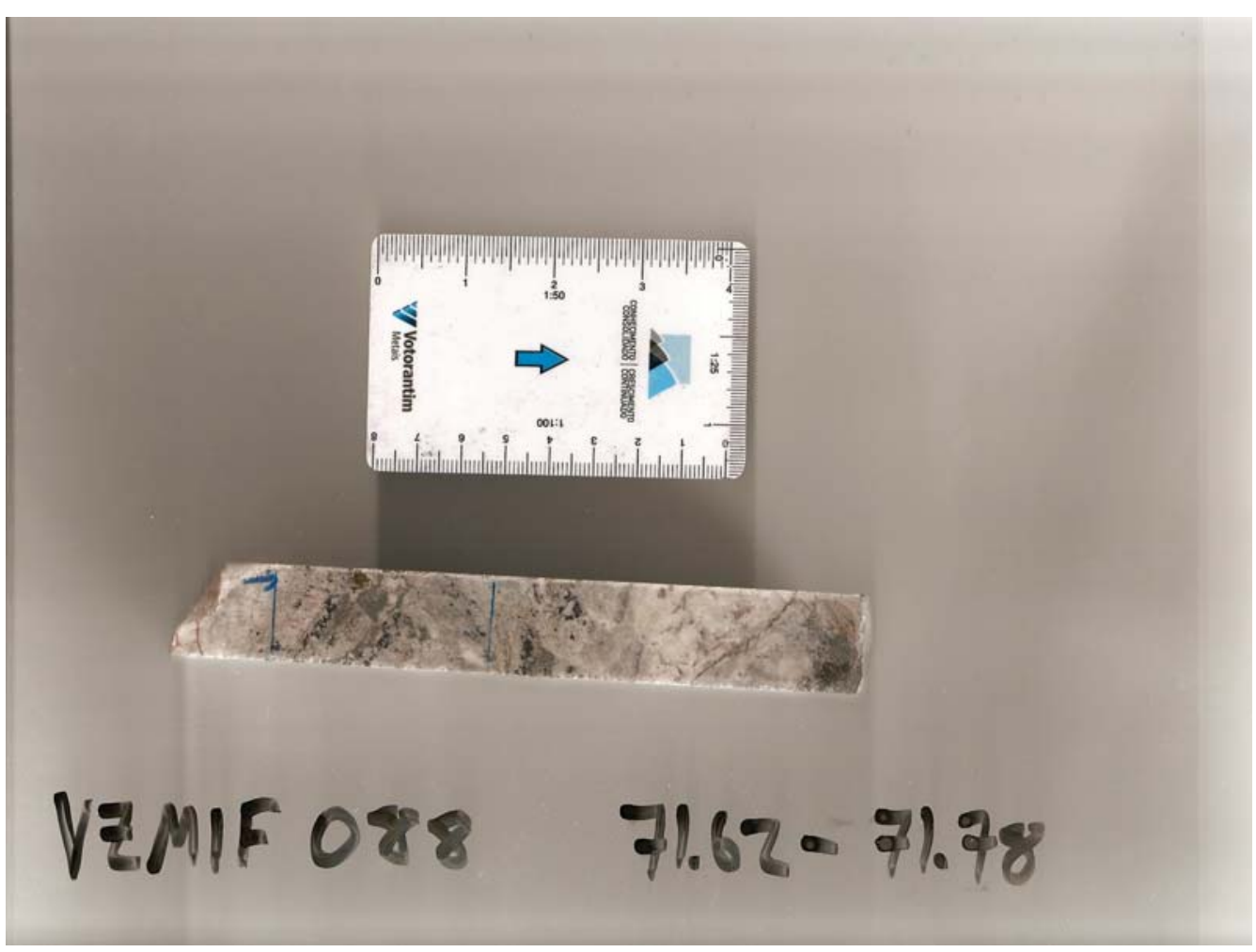

Other Sample Information: Same as 88-71.4 less Py more Sp-rich 
Geologic Unit: Hydrothermal Breccia

Lithological Unit (Votorantim): Disseminated Sulfides (in BH)

\section{Sample Photograph}

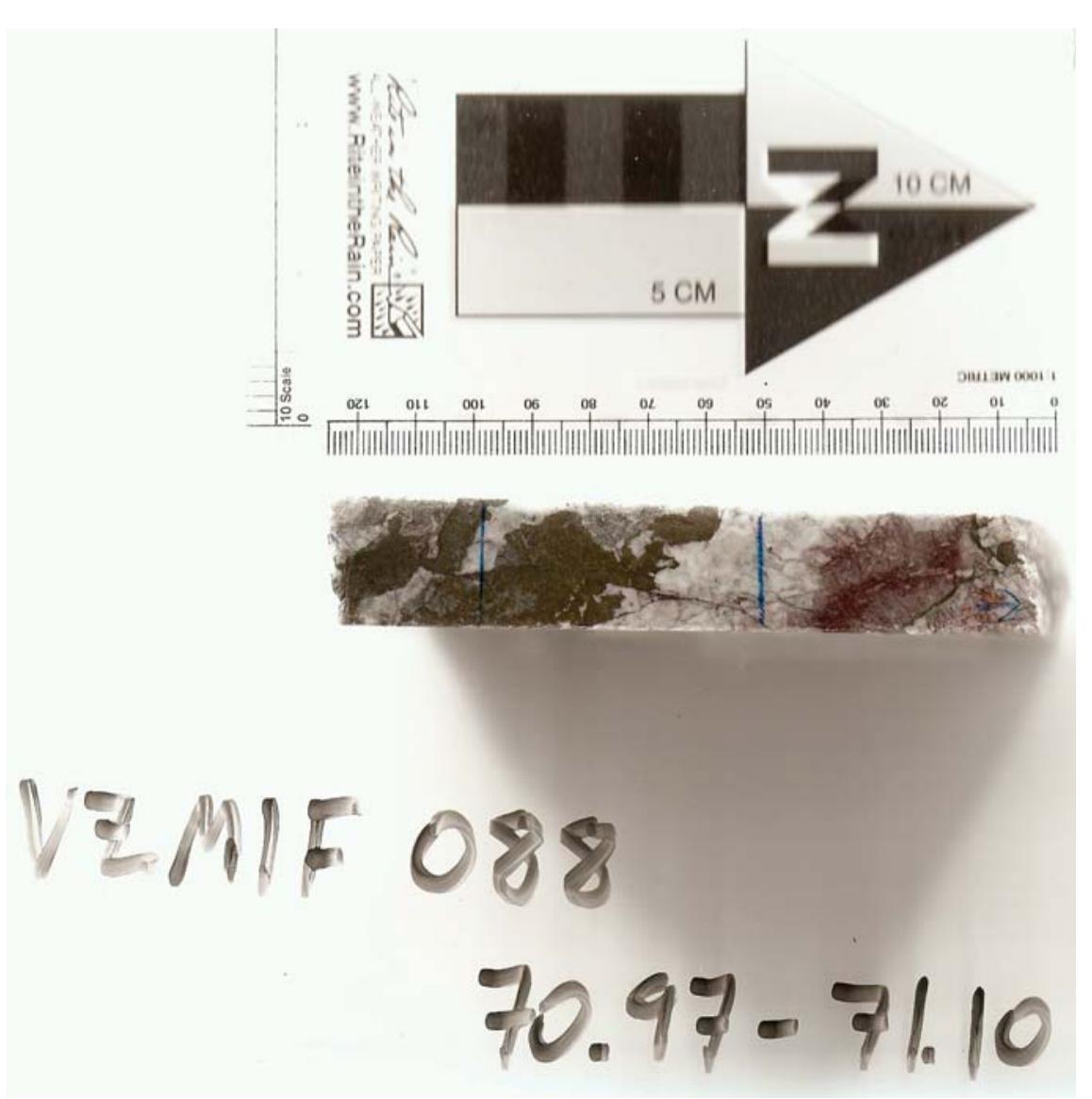

Other Sample Information: 


\begin{tabular}{|c|c|c|c|}
\hline minerals & Dolomite & Pyrite & Sphalerite \\
\hline percentage & 50 & 38 & 10 \\
\hline morphology & $\begin{array}{l}\text { Dolomitic marble, large } \\
\text { sparry xtals, los of twinning, } \\
\text { some micrite in veins }\end{array}$ & Massive & massive \\
\hline texture & $\begin{array}{l}\text { Hydrothermal re- } \\
\text { crystallization }\end{array}$ & brecciated & $\begin{array}{l}\text { Vein/stringer vein; dissemi- } \\
\text { nated with py-disease }\end{array}$ \\
\hline $\begin{array}{l}\text { transmitted opti- } \\
\text { cal properties }\end{array}$ & & & Brown in ppl and xpl \\
\hline $\begin{array}{l}\text { reflected optical } \\
\text { properties }\end{array}$ & & & $\begin{array}{l}\text { Internal reflections are yel- } \\
\text { low to clear, not red }\end{array}$ \\
\hline $\begin{array}{l}\text { contact/ } \\
\text { relationships with } \\
\text { other minerals }\end{array}$ & $\begin{array}{l}\text { Straight contacts, growing in } \\
\text { fractures between py }\end{array}$ & $\begin{array}{l}\text { Straight contacts with dol, } \\
\text { growing over dol. Irregular } \\
\text { contacts with sp (from bx?), } \\
\text { being replaced by sp }\end{array}$ & $\begin{array}{l}\text { Cutting across some py, re- } \\
\text { placement of py }\end{array}$ \\
\hline $\begin{array}{l}\text { other (growth di- } \\
\text { rection/zoning) }\end{array}$ & & & $\begin{array}{l}\text { Occurs in semi-stringer } \\
\text { veins after py. Contains } \\
\text { some py-disease (NOT Cpy) }\end{array}$ \\
\hline
\end{tabular}




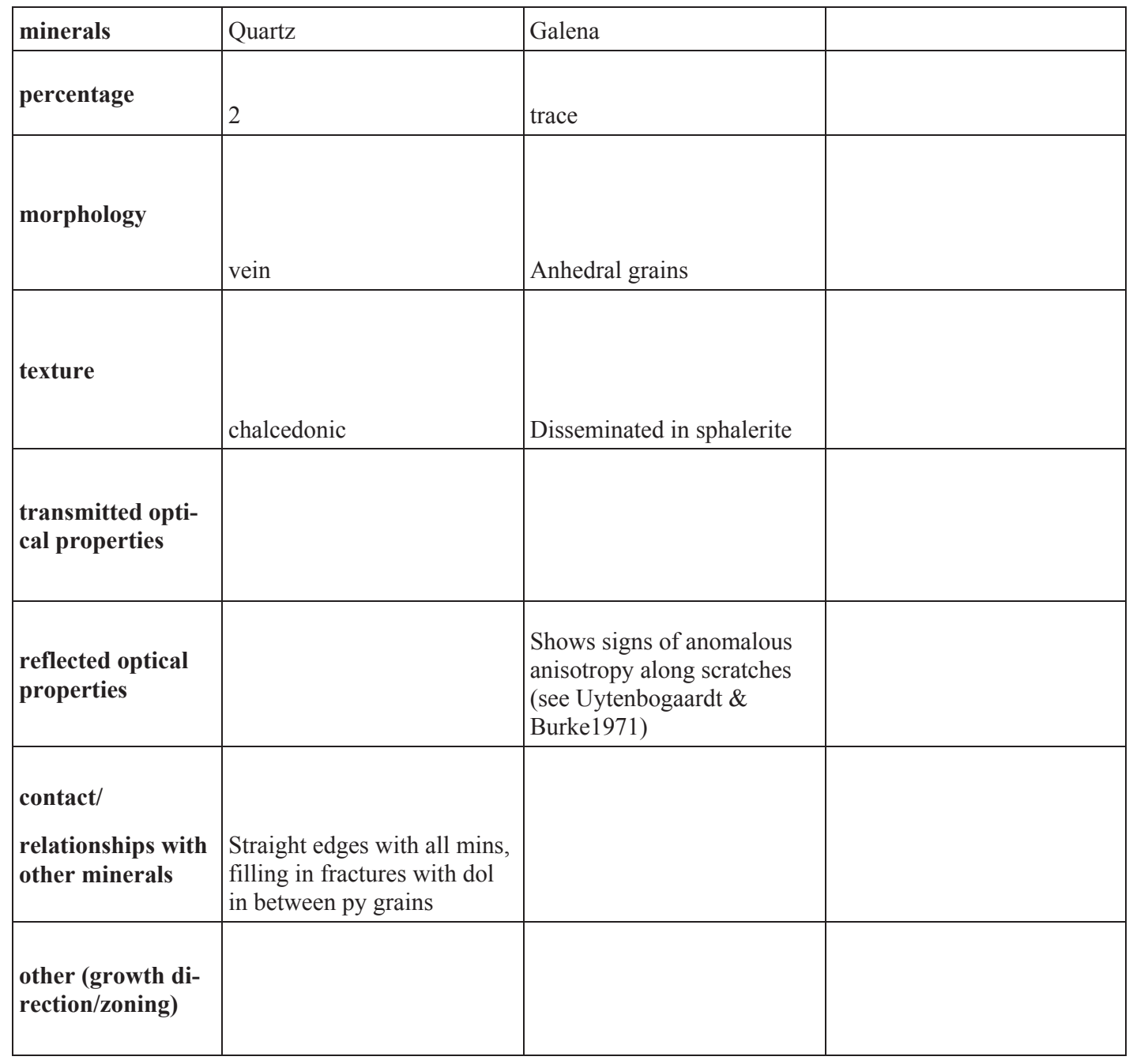

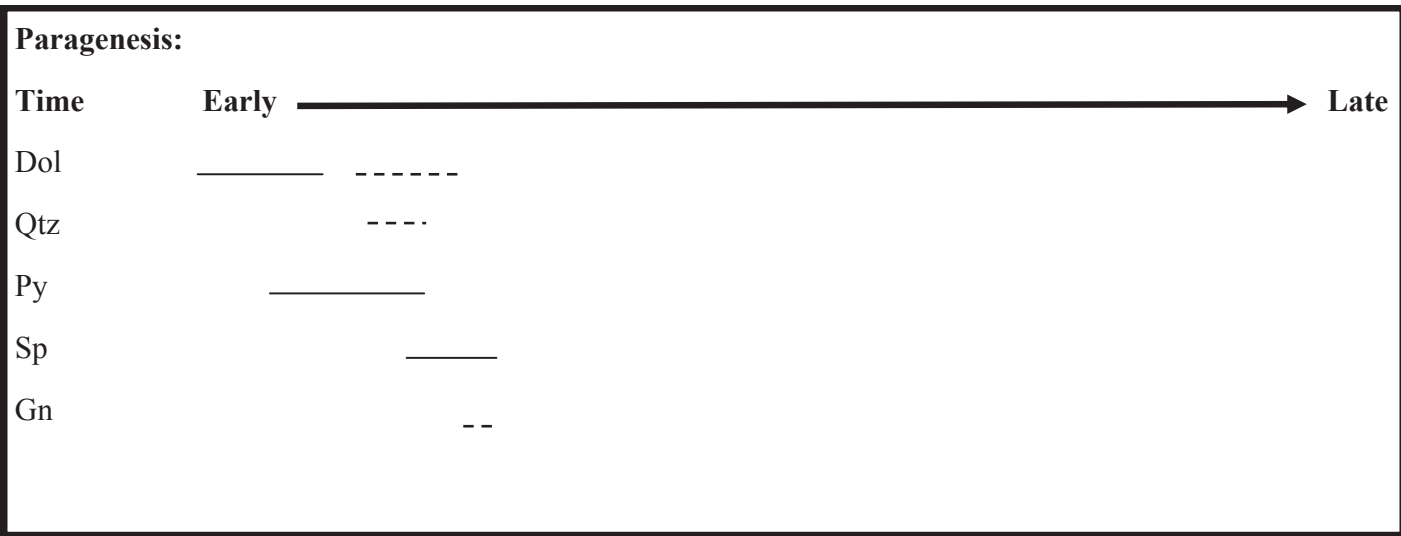


Structures: Sample consists of recrystallized dolomite that was brecciated, filled in with pyrite, then brecciated again. During the pyrite fluid event, a later pulse of $\mathrm{Zn}$-rich sphalerite with minor galena game through replacing some of the pyrite. Euhedral dolomite crystals are in contact with sphalerite, indicating that recrystallization started before sphalerite precipitated.

Paragenetic Sequence: See chart

Paragenetic Reasoning: see structures

Sample Highlights: Paucity of galena, evidence of 2 bx events 
Geologic Unit: Hydrothermal Breccia

Lithological Unit (Votorantim): Hydrothermal Breccia

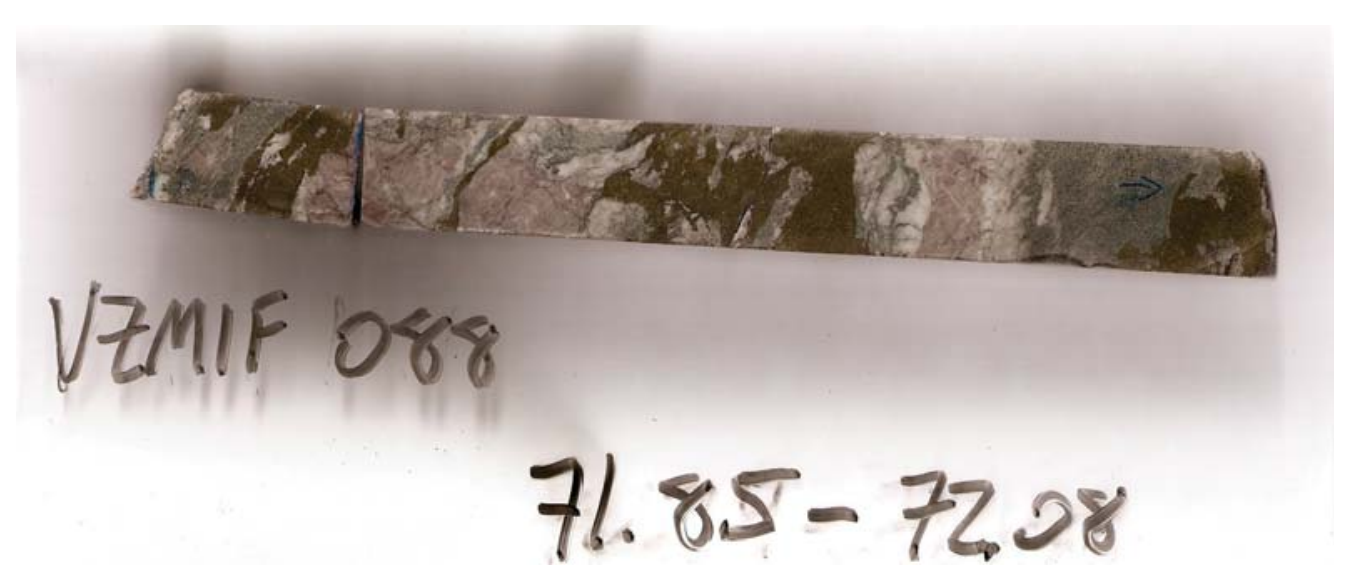

Other Sample Information: Same as 088-71.4 only with slightly more Gn ( 1\%); note Gn is slightly later than $\mathrm{Sp}$ in these samples 
Geologic Unit: Hydrothermal Breccia

Lithological Unit (Votorantim): Hydrothermal Breccia

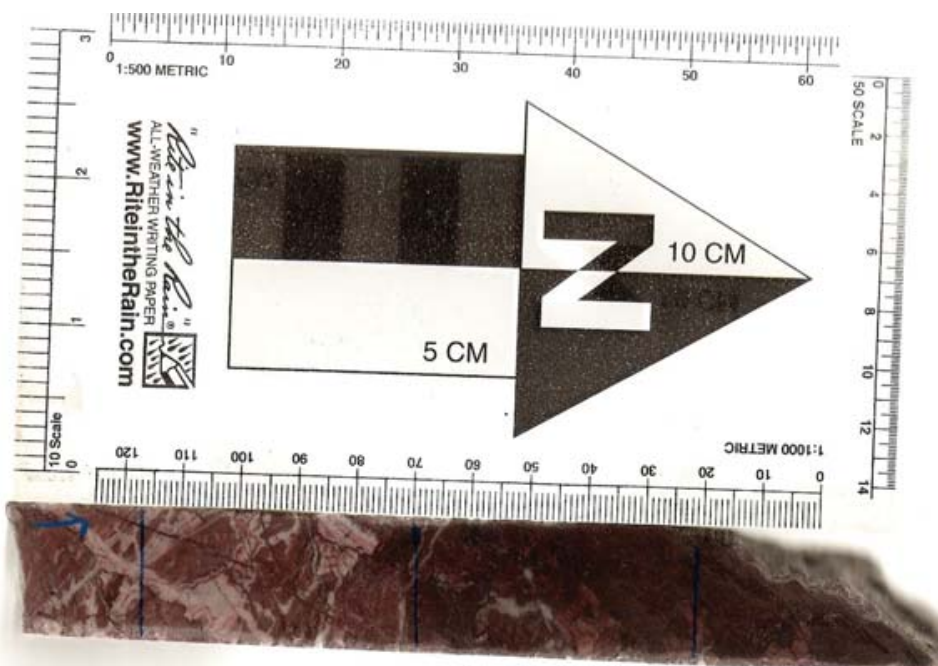

\section{VU 088}

$$
75.19 \cdot 79.81
$$

Other Sample Information: $0.3 \% \mathrm{Zn}$ 


\begin{tabular}{|c|c|c|c|}
\hline minerals & Fe-dolomite & Quartz & Dolomite \\
\hline percentage & 70 & 15 & 10 \\
\hline morphology & $\begin{array}{l}\text { Subhedral to euhedral, spar- } \\
\text { ry dolomite with hematite } \\
\text { inclusions }\end{array}$ & $\begin{array}{l}\text { Chalcedonic, cataclasic, and } \\
\text { anhedral xtals }\end{array}$ & Euhedral xtals \\
\hline texture & brecciated & $\begin{array}{l}\text { In veins with dol and in be- } \\
\text { tween and replacing Fe-dol }\end{array}$ & In veins \\
\hline $\begin{array}{l}\text { transmitted opti- } \\
\text { cal properties }\end{array}$ & & & \\
\hline $\begin{array}{l}\text { reflected optical } \\
\text { properties }\end{array}$ & & & \\
\hline $\begin{array}{l}\text { contact/ } \\
\text { relationships with } \\
\text { other minerals }\end{array}$ & $\begin{array}{l}\text { Somewhat irregular with } \\
\text { quartz, being replaced by } \\
\text { qtz. Being cut by dol }+ \text { qtz }\end{array}$ & $\begin{array}{l}\text { Replacing Fe-dol and being } \\
\text { cut by later dol veins, } \\
\text { straight contacts with dol in } \\
\text { veins }\end{array}$ & $\begin{array}{l}\text { Cutting Fe-dol and cata- } \\
\text { clasic and chalcedonic qtz }\end{array}$ \\
\hline $\begin{array}{l}\text { other (growth di- } \\
\text { rection/zoning) }\end{array}$ & $\begin{array}{l}\text { Some "poker chip" car- } \\
\text { bonate present with over- } \\
\text { growths (alternating Fe-rich } \\
\text { zones) }\end{array}$ & & \\
\hline
\end{tabular}




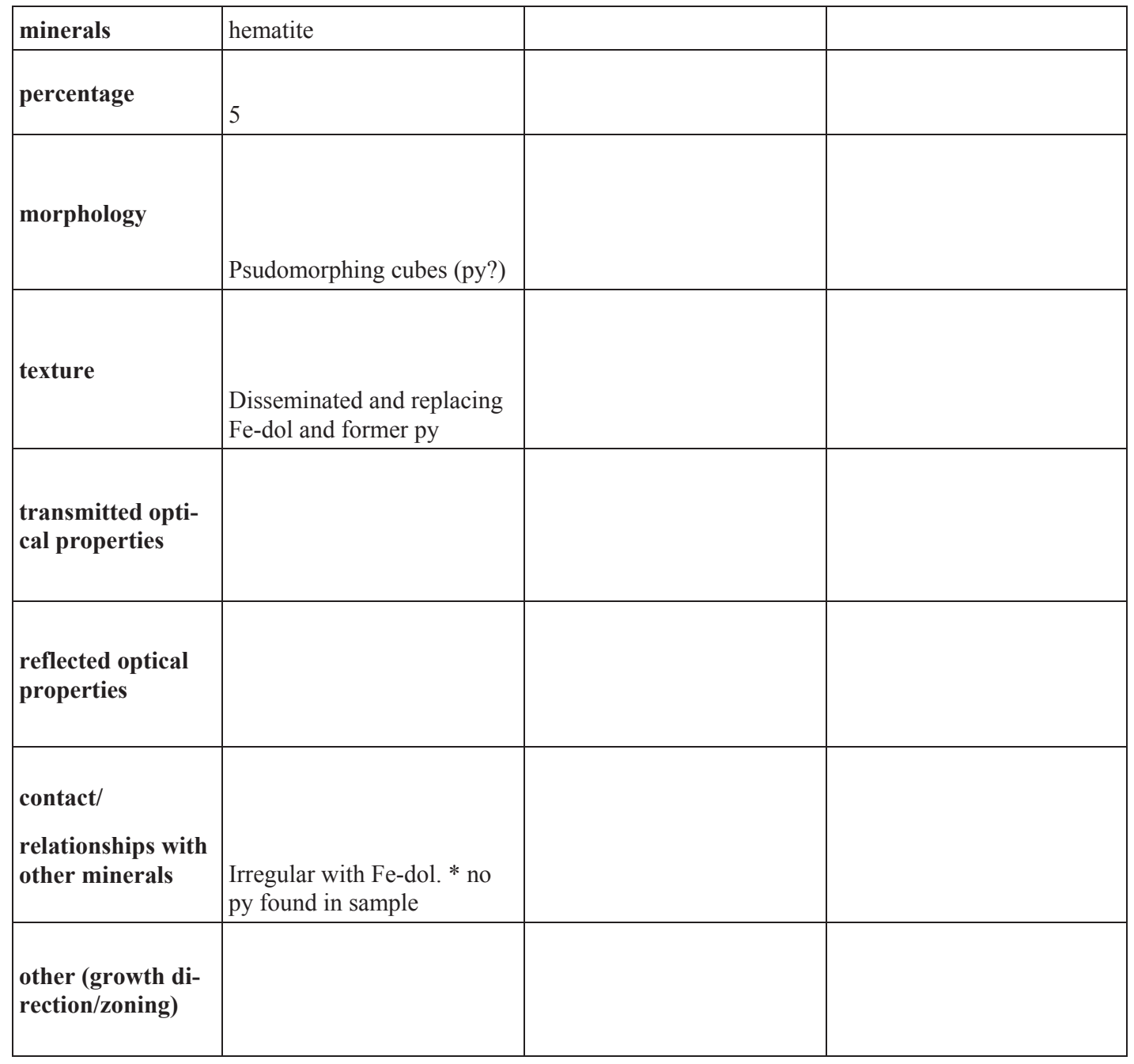

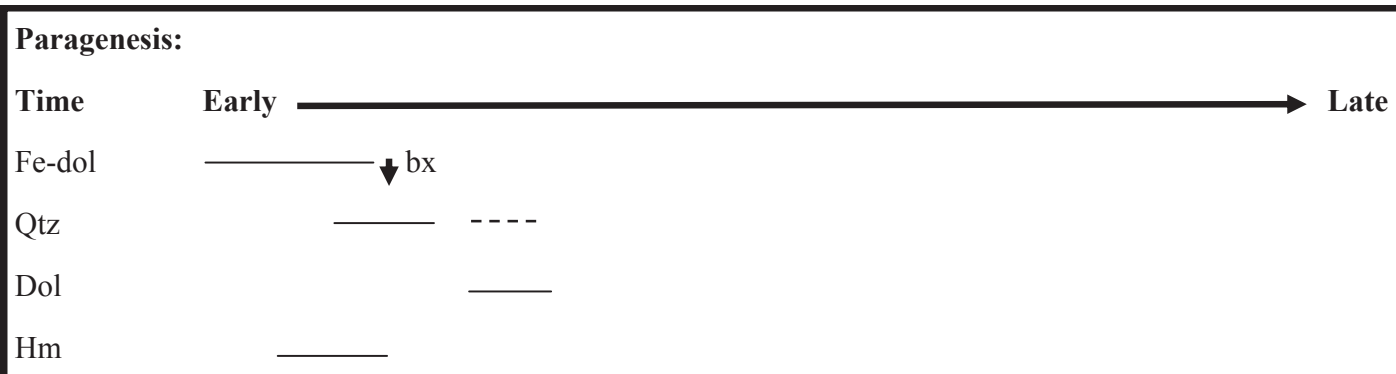


Structures: Cataclasic qtz \pm carb filling in fractures caused by brecciation (and faulting). Late carbonated veins cutting Fe-dol and earlier chalcedonic qtz (replacing Fe-dol) and cataclasic qtz. Hm appears to be pseudomorphing cubic crystals, possibly Py.

Paragenetic Sequence: $\mathrm{Py} ? \rightarrow \mathrm{Fe}-\mathrm{dol}+\mathrm{Hm} \rightarrow$ chalcedonic $\mathrm{qtz} \rightarrow \mathrm{bx}+$ cataclasites $\rightarrow \mathrm{qtz}+$ carb veins

Paragenetic Reasoning: see diagram

Sample Highlights: Possible oxidation of py to hm 
Geologic Unit: Hydrthermal Breccia

Lithological Unit (Votorantim): Hydrothermal Breccia (BH)

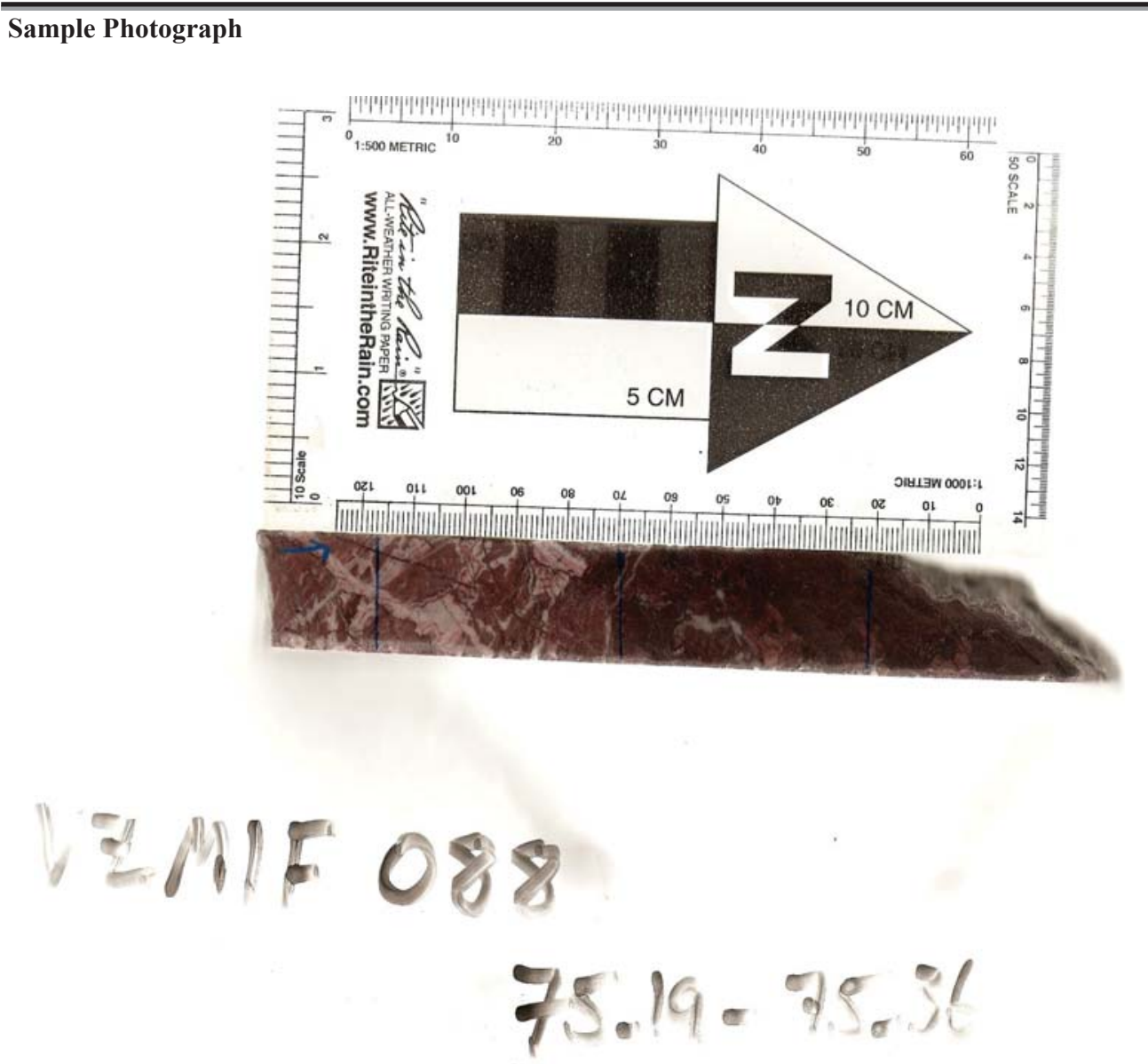

Other Sample Information: Similar to 88-75A, but with less sparry Fe-dol and more micritic Fe-dol. Less $\mathrm{Hm}$ as well. Neomorphic carbonates common.

*(slightly further from main breccia shear than $88-75 \mathrm{~A}$ ) 
Geologic Unit: Hydrothermal Breccia

Lithological Unit (Votorantim): Hydrothermal Breccia

Sample Photograph

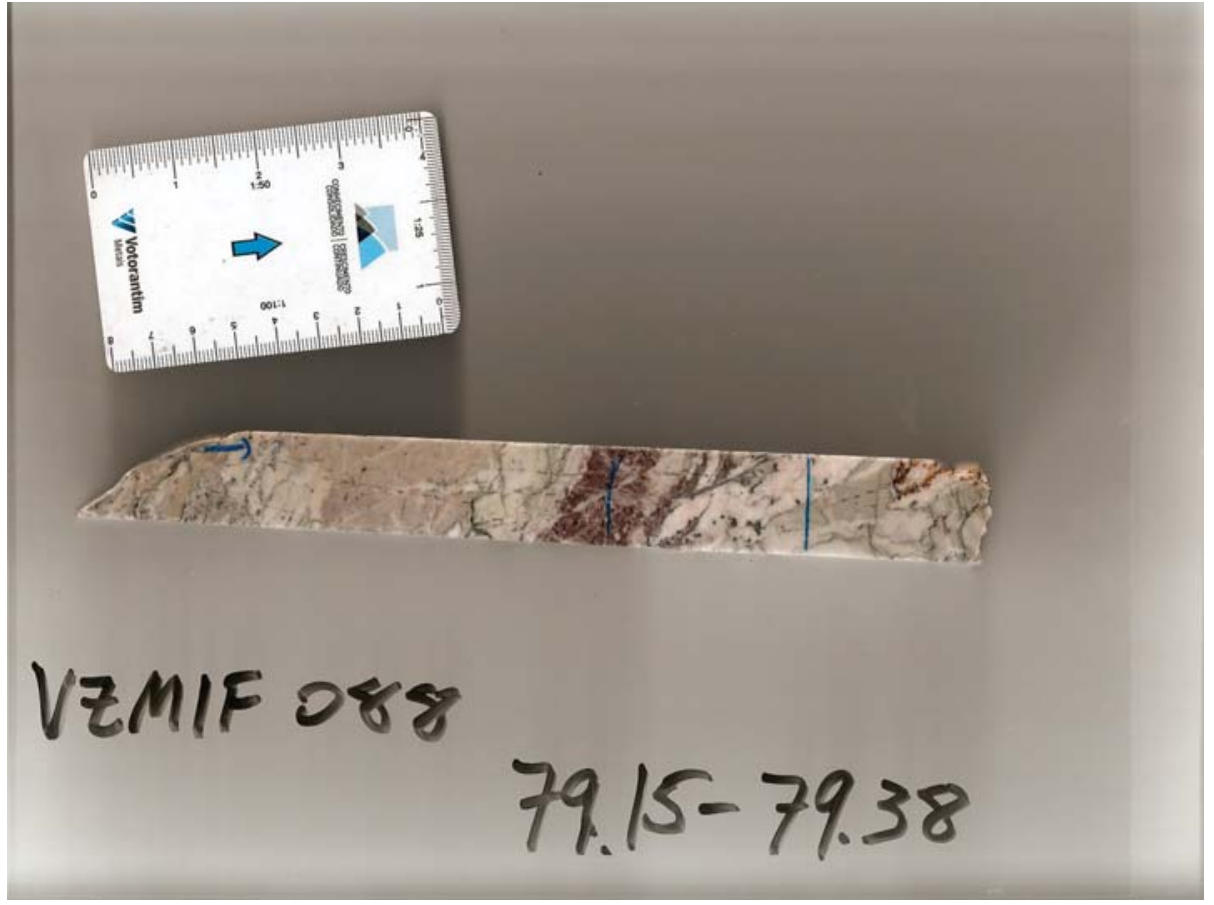

Other Sample Information: 


\begin{tabular}{|c|c|c|c|}
\hline minerals & Recrystallized carb (dol) & Fe-carbonate & Quartz \\
\hline percentage & 50 & 40 & 5 \\
\hline morphology & Large sparry xtals & $\begin{array}{l}\text { Sparry xtals, but looks } \\
\text { "dirty" in ppl (b/c of trace } \\
\text { hm inclusions) }\end{array}$ & Megaquartz xtals \\
\hline texture & Host rock, fractured & Host rock, fractured & Veinlets and disseminations \\
\hline $\begin{array}{l}\text { transmitted opti- } \\
\text { cal properties }\end{array}$ & & & \\
\hline $\begin{array}{l}\text { reflected optical } \\
\text { properties }\end{array}$ & & & \\
\hline $\begin{array}{l}\text { contact/ } \\
\text { relationships with } \\
\text { other minerals }\end{array}$ & $\begin{array}{l}\text { Irregular contacts with qtz, } \\
\text { fades into Fe-carb, being } \\
\text { replaced by sulfides }\end{array}$ & $\begin{array}{l}\text { Irregular contacts with qtz, } \\
\text { fades into carb, being re- } \\
\text { placed by sulfides }\end{array}$ & $\begin{array}{l}\text { Spotty, wavy and irregular } \\
\text { contacts with both car- } \\
\text { bonates, no contact observed } \\
\text { with sulfides }\end{array}$ \\
\hline $\begin{array}{l}\text { other (growth di- } \\
\text { rection/zoning) }\end{array}$ & & & \\
\hline
\end{tabular}




\begin{tabular}{|c|c|c|c|}
\hline minerals & Sphalerite & Galena & Hematite \\
\hline percentage & 5 & 3 & 2 \\
\hline morphology & Semi-massive & $\begin{array}{l}\text { Semi-massive to disseminat- } \\
\text { ed }\end{array}$ & Crystalline laths \\
\hline texture & Vug filling & Veinlet and vug filling & disseminated \\
\hline $\begin{array}{l}\text { transmitted opti- } \\
\text { cal properties }\end{array}$ & & & \\
\hline $\begin{array}{l}\text { reflected optical } \\
\text { properties }\end{array}$ & & & \\
\hline $\begin{array}{l}\text { contact/ } \\
\text { relationships with } \\
\text { other minerals }\end{array}$ & $\begin{array}{l}\text { Some irregular contacts with } \\
\text { gn, replacing gn, irregular } \\
\text { contacts with hm, sp is re- } \\
\text { placing hm, and cv is over- } \\
\text { growing sp }\end{array}$ & $\begin{array}{l}\text { Gn is replacing sphalerite in } \\
\text { some cases, irregular con- } \\
\text { tacts with hm, replacing hm } \\
\text { to a minor extent, being } \\
\text { overgrown my cv }\end{array}$ & $\begin{array}{l}\text { Irregular and corroded con- } \\
\text { tacts with sp and gn, being } \\
\text { replaced by these mins }\end{array}$ \\
\hline $\begin{array}{l}\text { other (growth di- } \\
\text { rection/zoning) }\end{array}$ & & & \\
\hline
\end{tabular}




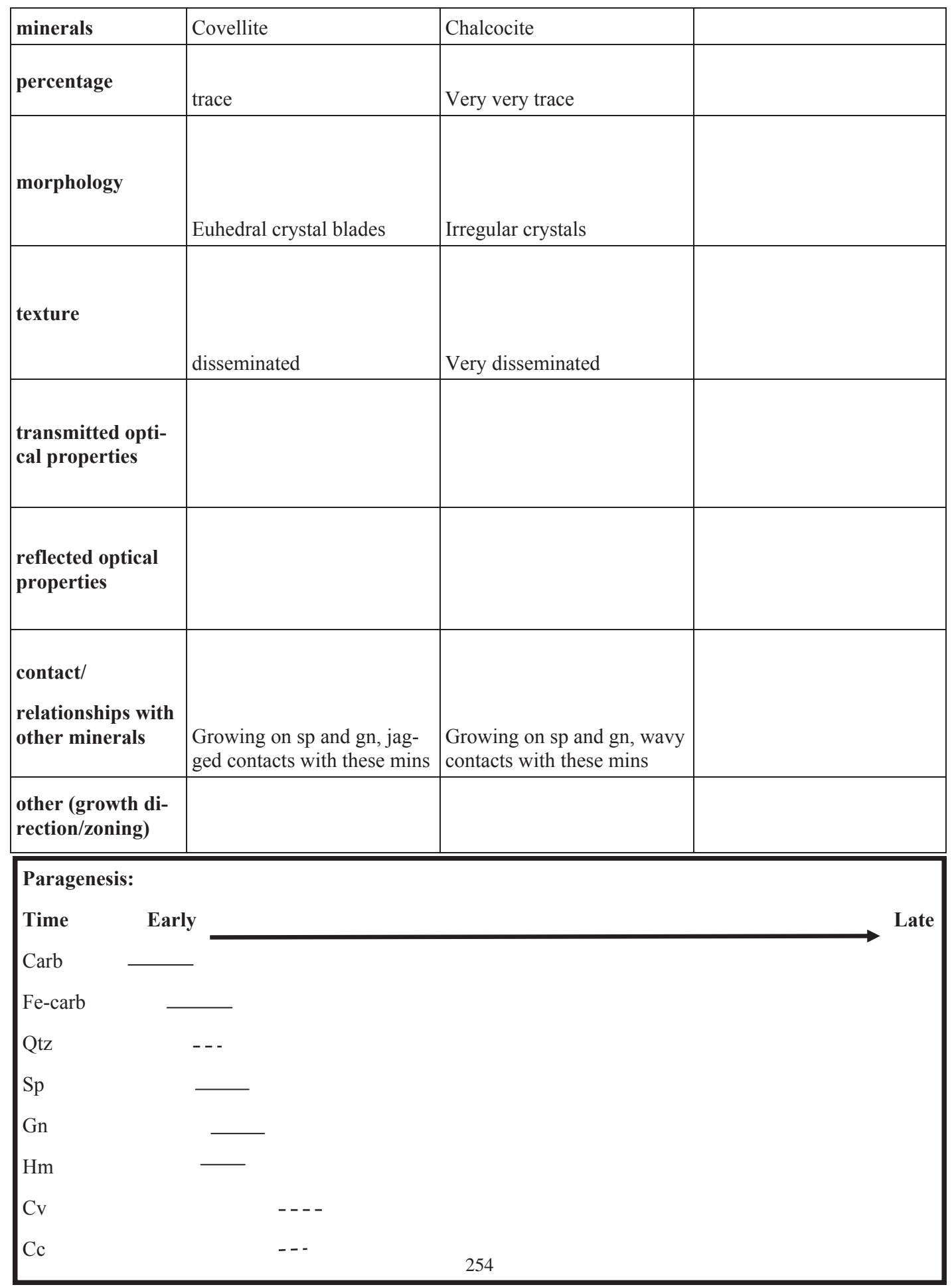


Geologic Unit: Hydrothermal Breccia

Lithological Unit (Votorantim): Hydrothermal Breccia

Sample Photograph

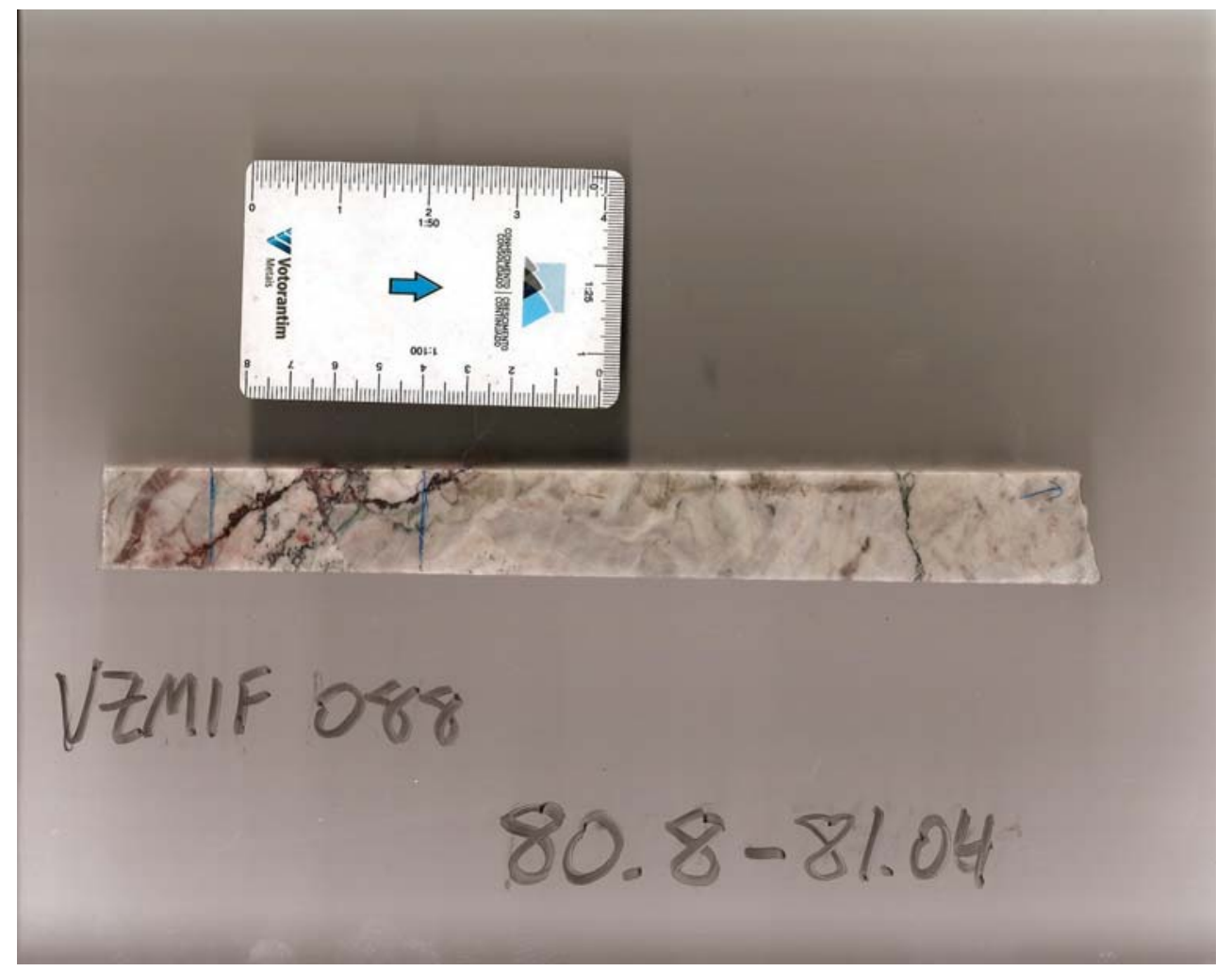

Other Sample Information: Same as 088-79 
Geologic Unit: Hydrothermal Breccia

Lithological Unit (Votorantim): Disseminated Silicate (SD) of BH

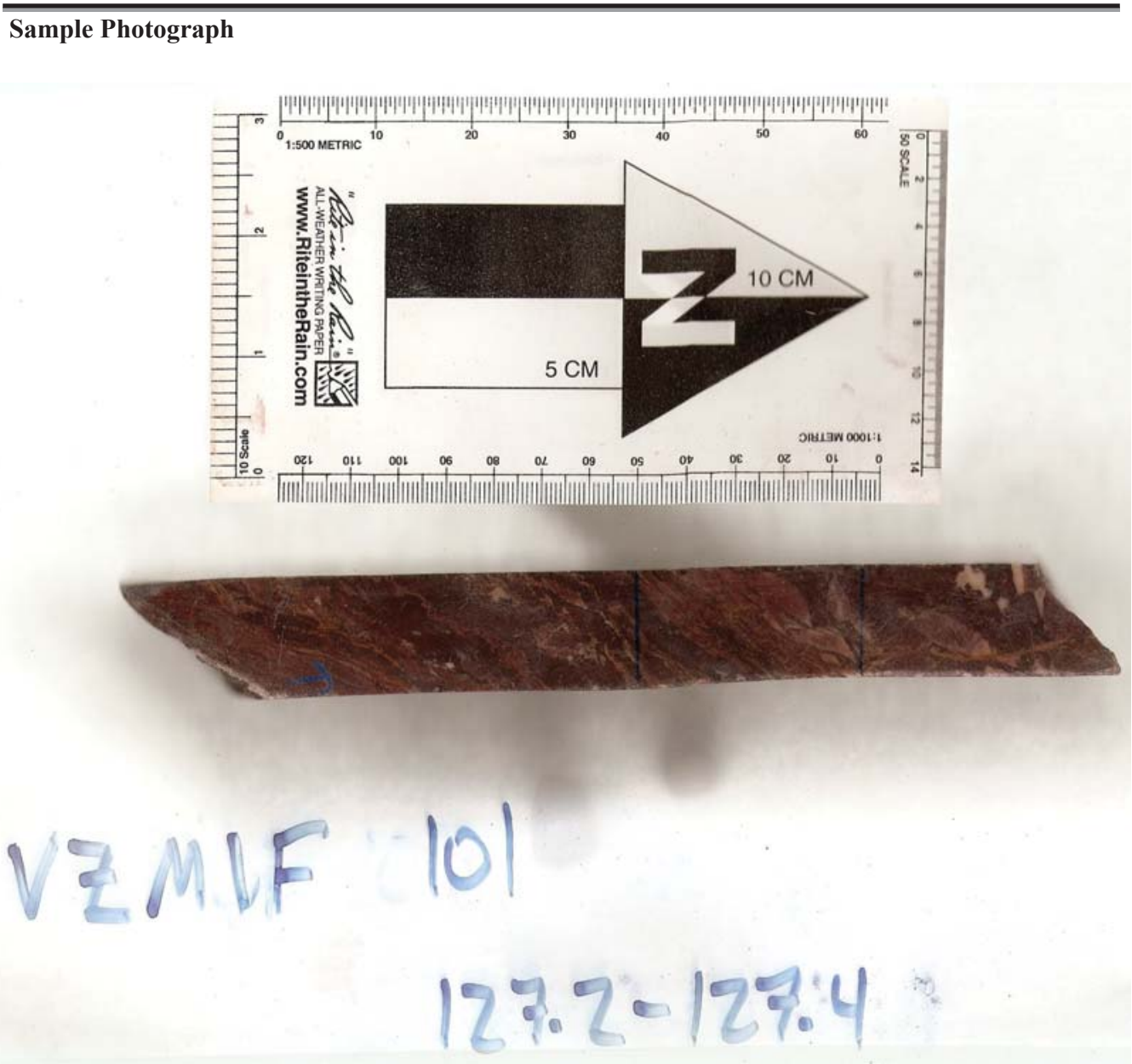

Other Sample Information: Intensely sheared 


\begin{tabular}{|c|c|c|c|}
\hline minerals & Dolomite & Hematite & quartz \\
\hline percentage & 70 & 23 & 5 \\
\hline morphology & $\begin{array}{l}\text { Fine grained where dol com- } \\
\text { poses rock matrix. Larger, } \\
\text { euhedral xtals in between bx } \\
\text { fragments and in veins }\end{array}$ & $\begin{array}{l}\text { Mostly in veins, but also in } \\
\text { disseminated euhedral laths } \\
\text { throughout dol }\end{array}$ & $\begin{array}{l}\text { Euhedral xtals found in } \\
\text { veins }\end{array}$ \\
\hline texture & Sheared breccia & Sheared breccia & Folded veins \\
\hline $\begin{array}{l}\text { transmitted opti- } \\
\text { cal properties }\end{array}$ & & & \\
\hline $\begin{array}{l}\text { reflected optical } \\
\text { properties }\end{array}$ & & & \\
\hline $\begin{array}{l}\text { contact/ } \\
\text { relationships with } \\
\text { other minerals }\end{array}$ & $\begin{array}{l}\text { Straight contacts with qtz } \\
\text { and chl, irregular contact } \\
\text { with hm and wl. }\end{array}$ & $\begin{array}{l}\text { Straight with qtz, wavy with } \\
\text { dol }\end{array}$ & $\begin{array}{l}\text { Straight contacts with dol, } \\
\text { wl, and hm }\end{array}$ \\
\hline $\begin{array}{l}\text { other (growth di- } \\
\text { rection/zoning) }\end{array}$ & $\begin{array}{l}\text { Growing towards center of } \\
\text { veins (were dol veins exist) }\end{array}$ & & $\begin{array}{l}\text { Qtz veinlets cut the hm indi- } \\
\text { cating a small later phase of } \\
\text { qtz }\end{array}$ \\
\hline
\end{tabular}




\begin{tabular}{|c|c|c|}
\hline minerals & Willemite & Chlorite \\
\hline percentage & 1 & 1 \\
\hline morphology & $\begin{array}{l}\text { A few granular xtals over- } \\
\text { growing dol and one fibrous } \\
\text { splay overgrowing qtz }\end{array}$ & In folded veins \\
\hline texture & disseminated & Feathery texture \\
\hline $\begin{array}{l}\text { transmitted opti- } \\
\text { cal properties }\end{array}$ & & \\
\hline $\begin{array}{l}\text { reflected optical } \\
\text { properties }\end{array}$ & & \\
\hline $\begin{array}{l}\text { contact/ } \\
\text { relationships with } \\
\text { other minerals }\end{array}$ & $\begin{array}{l}\text { Straight with qtz, slightly } \\
\text { irregular with dol }\end{array}$ & Irregular contact $\mathrm{w} / \mathrm{hm}$ \\
\hline $\begin{array}{l}\text { other (growth di- } \\
\text { rection/zoning) }\end{array}$ & & \\
\hline
\end{tabular}

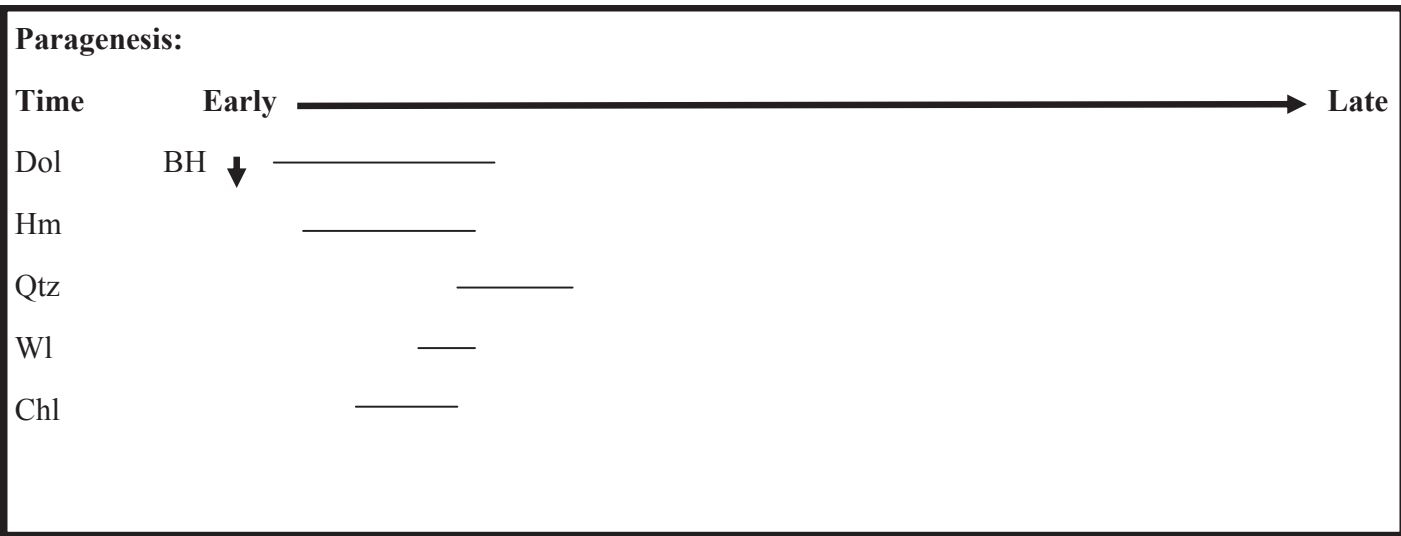


Structures: Folded veins after shear? and general shear of sample. The chlorite and qtz veins are folded and refolded after the main shearing event. The qtz veins cut the hm shear in places.

Paragenetic Sequence: dol $\rightarrow \mathrm{hm}+\mathrm{wl}+\mathrm{chl} \rightarrow \mathrm{qtz}$

Paragenetic Reasoning: The dolostone has been brecciated and recrystallized. During recrystallization, hematite along with chl and wl were deposited in the fractures and the sample began to undergo shearing. During the late stages of shearing, qtz and dol grew into open fractures that cross cut the hm and were further folded.

Sample Highlights: shearing 
Geologic Unit: Hydrothermal Breccia

Lithological Unit (Votorantim): Massive Silicate (SM) of BH

Sample Photograph

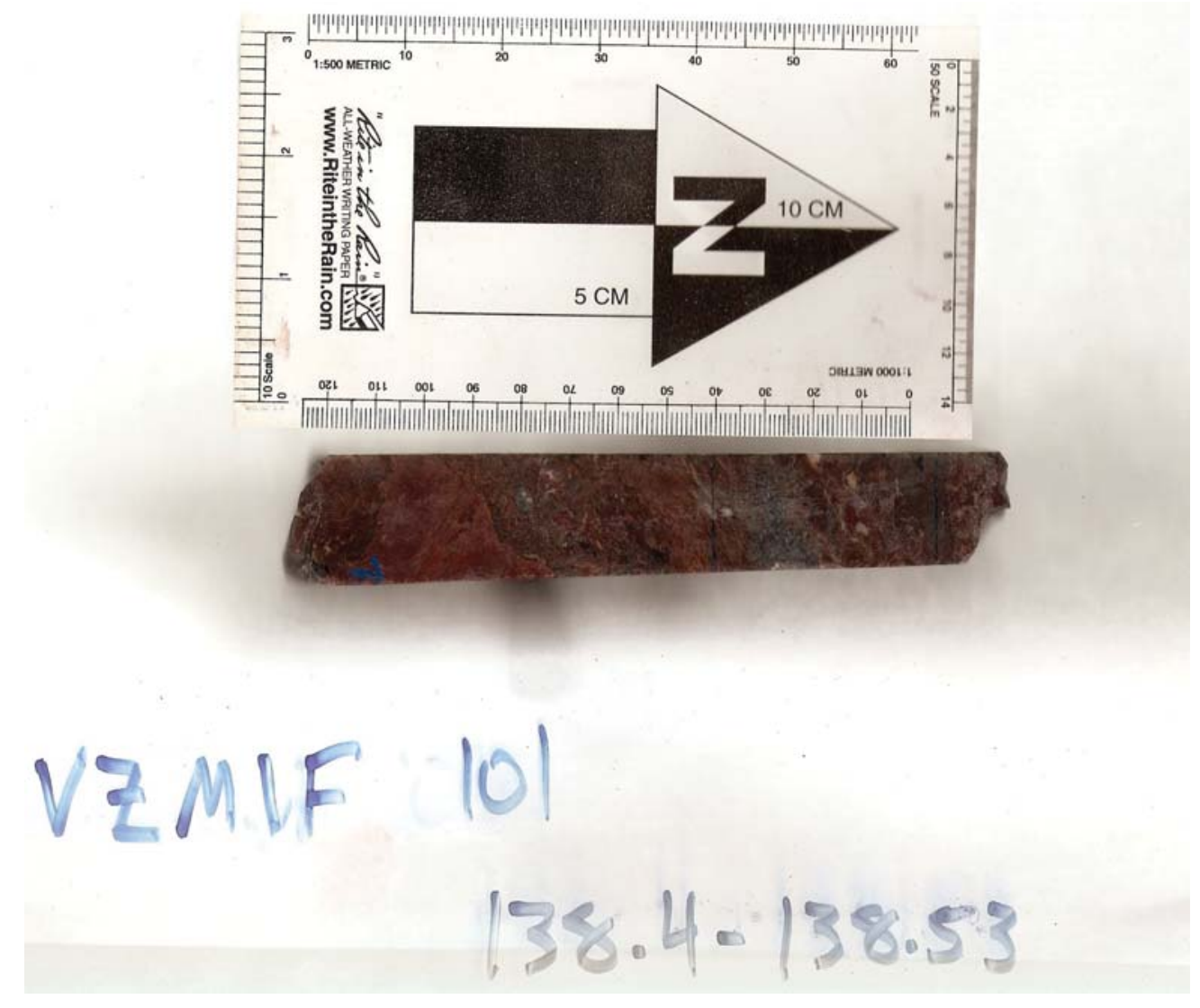

Other Sample Information: 


\begin{tabular}{|c|c|c|c|}
\hline minerals & Dolomite & Willemite & Quartz \\
\hline percentage & 10 & 60 & 15 \\
\hline morphology & anhedral & $\begin{array}{l}\text { Variety of crystal sizes with } \\
\text { respect to both granular and } \\
\text { fibrous crystal habits }\end{array}$ & Subhedral \\
\hline texture & $\begin{array}{l}\text { Being replaced by qtz and } \\
\text { wl }\end{array}$ & massive & $\begin{array}{l}\text { Rimmed by wl, replaced by } \\
\text { hm. disseminated }\end{array}$ \\
\hline $\begin{array}{l}\text { transmitted opti- } \\
\text { cal properties }\end{array}$ & & & \\
\hline $\begin{array}{l}\text { reflected optical } \\
\text { properties }\end{array}$ & & & \\
\hline $\begin{array}{l}\text { contact/ } \\
\text { relationships with } \\
\text { other minerals }\end{array}$ & $\begin{array}{l}\text { Irregular contacts with } \mathrm{hm} \text {, } \\
\text { wl, and qtz, being replaced } \\
\text { by these mins }\end{array}$ & $\begin{array}{l}\text { Irregular and convoluted } \\
\text { contacts with other mins. } \\
\text { replacing dol, sl, and hm but } \\
\text { also being replaced by hm }\end{array}$ & $\begin{array}{l}\text { Straight contacts with wl } \\
\text { (rimming qtz). Has inclu- } \\
\text { sions and wavy contacts of } \\
\text { hm }\end{array}$ \\
\hline $\begin{array}{l}\text { other (growth di- } \\
\text { rection/zoning) }\end{array}$ & & $\begin{array}{l}\text { Rimming some qtz grains, } \\
\text { replacing bx clasts }\end{array}$ & \\
\hline
\end{tabular}




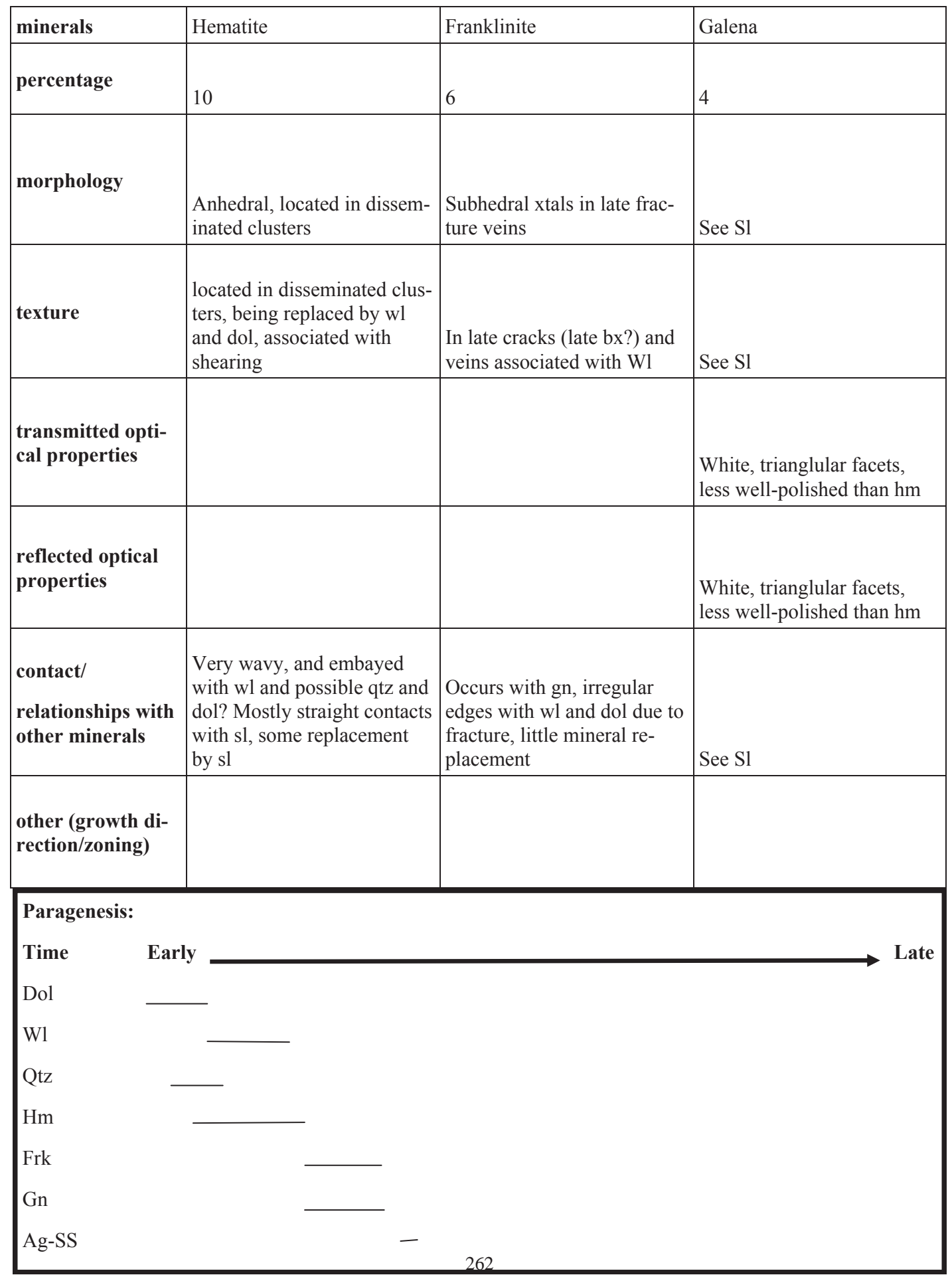


Structures: Sample has been brecciated and the clasts subsequently replaced by wl followed by further brecciation

Paragenetic Sequence: dol $+\mathrm{qtz} \rightarrow \mathrm{wl}+\mathrm{hm} \rightarrow \mathrm{sl}+\mathrm{gn} \rightarrow$ Ag-sulfosalts

Paragenetic Reasoning: Dol and qtz were early. Dol is being replaced by wl, hm, and sl. Qtz grains also exist with wl rimming textures. Wl and $\mathrm{hm}$ seem to be coeval because they are all replacing one another, as seen by mineral inclusions and embayed contacts with each other. Sl and gn are later minerals filling in fractures from a brittle fracture event. S1 and gn are more closely associated with Wl.

The Ag-sulfosalts are after and associated with gn. They are very minute and very late.

Sample Highlights: Lots of sphalerite and willemite

Rimming and replacement textures of willemite

***some minor late sulfides 
Geologic Unit: Hydrothermal Breccia

Lithological Unit (Votorantim): Massive Silicate (SM)

Sample Photograph

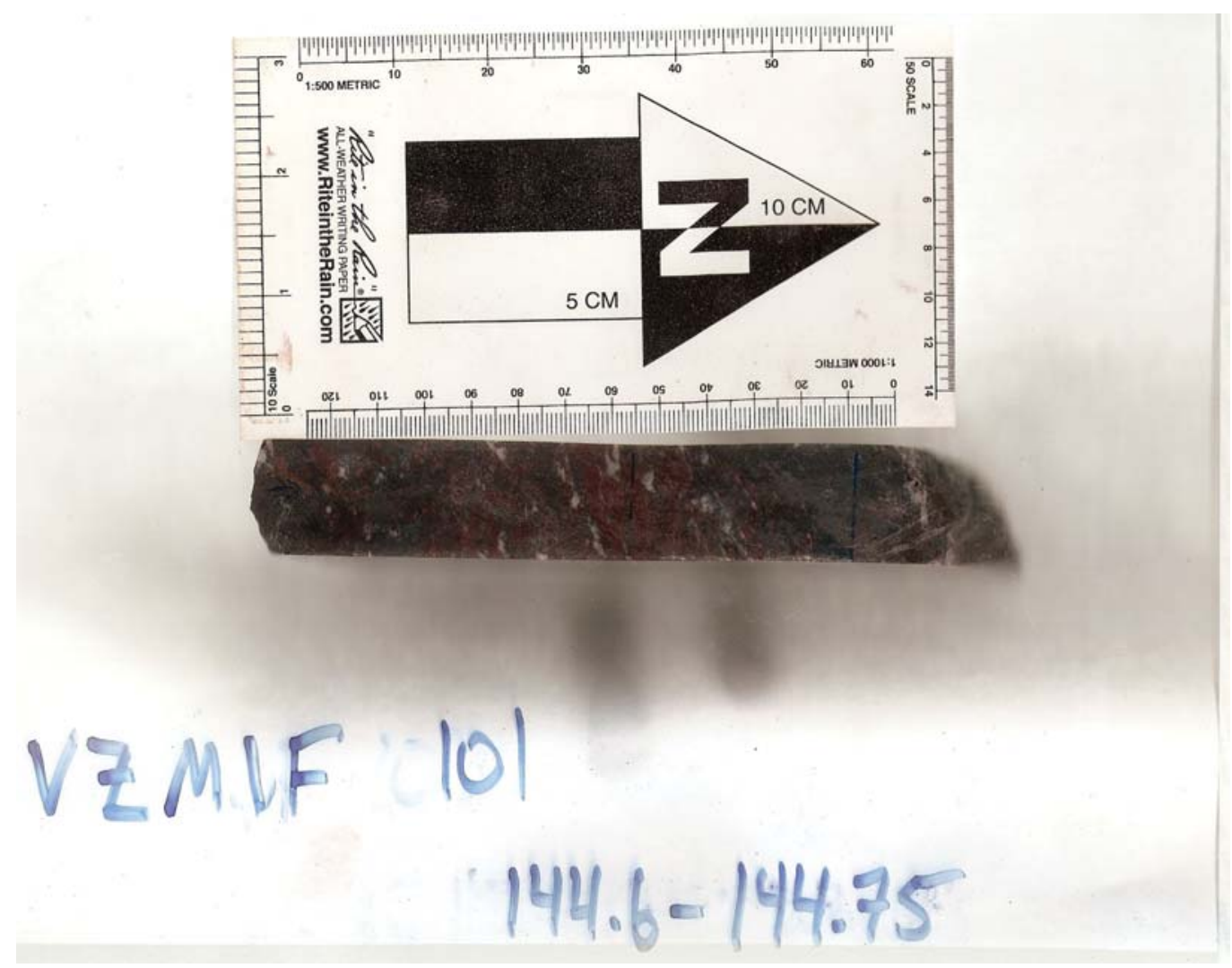

Other Sample Information: $2.22 \% \mathrm{Zn}$ 


\begin{tabular}{|c|c|c|c|}
\hline minerals & Dolomite & Hematite & Fe-rich dolomite \\
\hline percentage & 30 & 50 & 30 \\
\hline morphology & $\begin{array}{l}\text { Large euhdral xtals and } \\
\text { smaller anhedral xtals }\end{array}$ & Euhedral laths & Same as dol \\
\hline texture & $\begin{array}{l}\text { Large dol being replaced by } \\
\text { small dol and } \mathrm{hm}\end{array}$ & $\begin{array}{l}\text { Stringer veins, altering dol } \\
\text { and being altered by } \mathrm{Fe} \text {-dol }\end{array}$ & $\begin{array}{l}\text { Same as dol but with hm } \\
\text { xtals included in dol xtals }\end{array}$ \\
\hline $\begin{array}{l}\text { transmitted opti- } \\
\text { cal properties }\end{array}$ & & & \\
\hline $\begin{array}{l}\text { reflected optical } \\
\text { properties }\end{array}$ & & & \\
\hline $\begin{array}{l}\text { contact/ } \\
\text { relationships with } \\
\text { other minerals }\end{array}$ & $\begin{array}{l}\text { Irregular w/ dol and } \mathrm{hm}(\mathrm{b} / \mathrm{c} \\
\text { of replacement })\end{array}$ & Irregular see dol & Same as dol \\
\hline $\begin{array}{l}\text { other (growth di- } \\
\text { rection/zoning) }\end{array}$ & & Some hm overgrowths & \\
\hline
\end{tabular}

Paragenesis:

Time Early

Dol

$\mathrm{Hm}$

Fe-Dol 
Geologic Unit: Lower Pamplona

Lithological Unit (Votorantim): Pink Dolomite (DG)

Sample Photograph
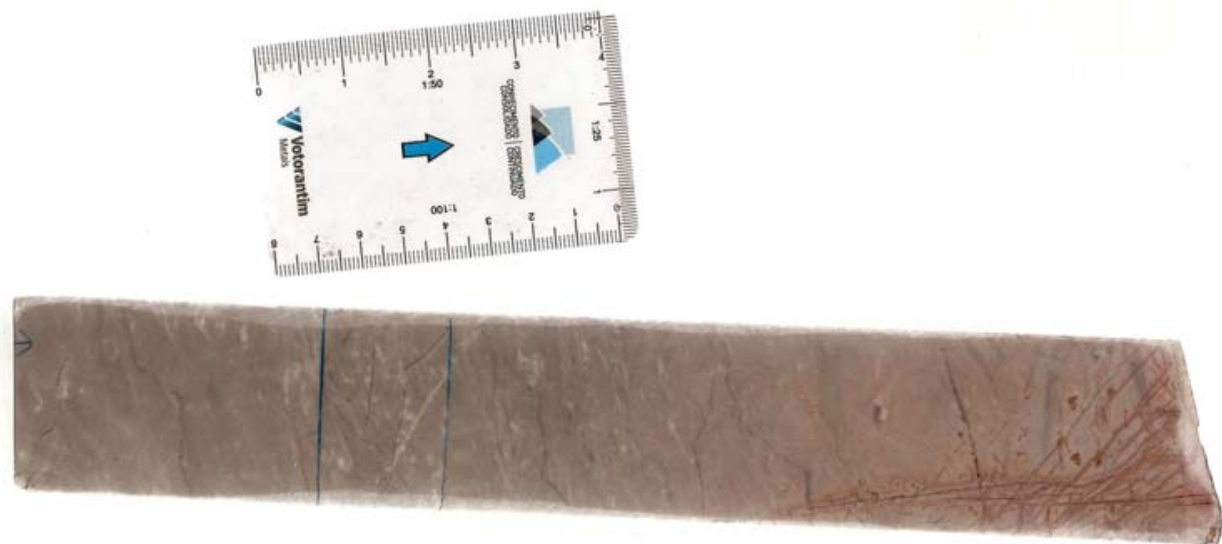

$$
\text { VZMIF } 104
$$

$$
75.5-75.8
$$

Other Sample Information: ** Same as 110-150 
Geologic Unit: Hydrothermal Breccia

Lithological Unit (Votorantim): Hydrothermal Breccia

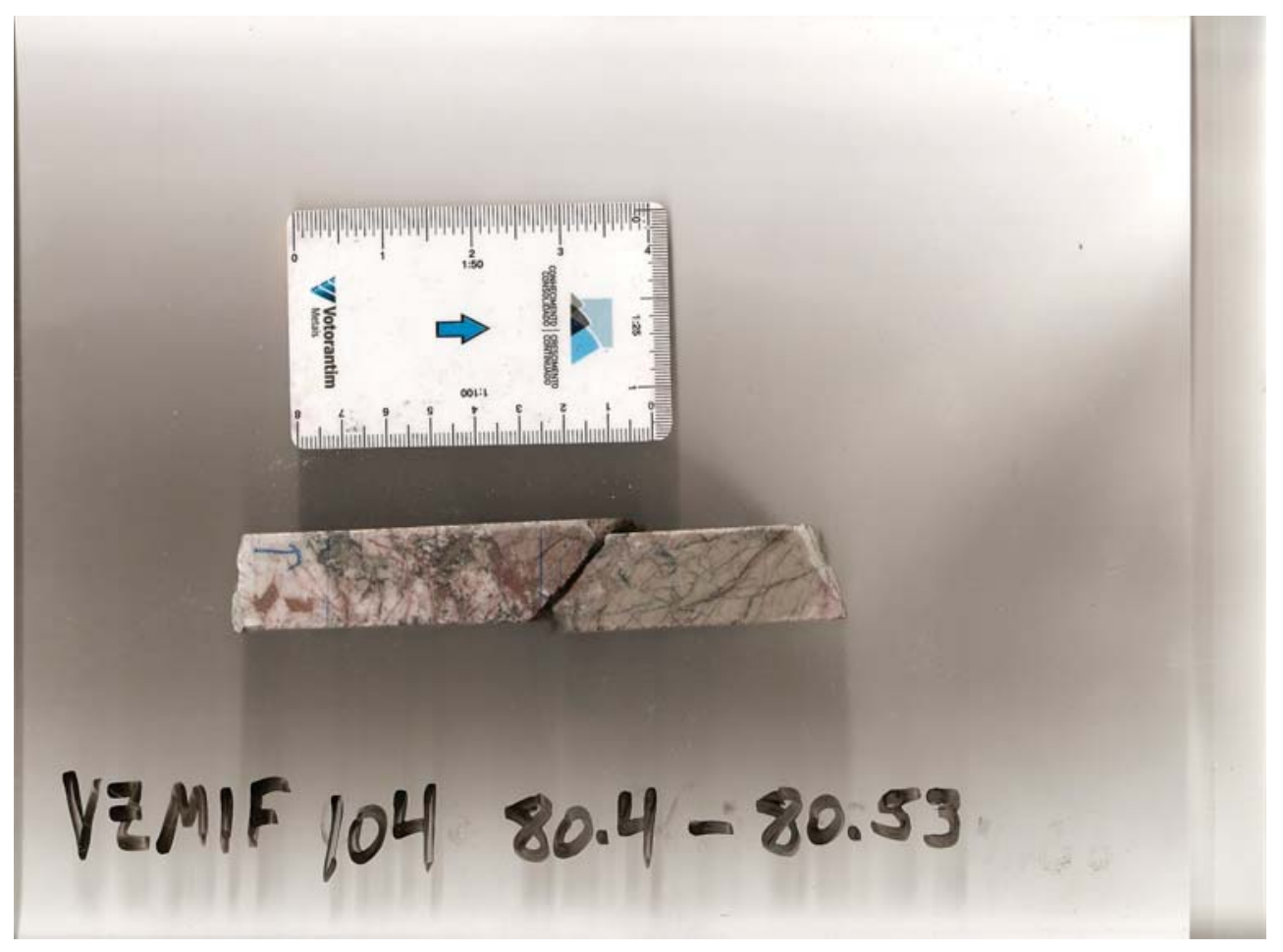

Other Sample Information: 


\begin{tabular}{|l|l|l|l|}
\hline minerals & Carbonate & Quatz & Pyrite \\
\hline percentage & 60 & 20 & 5 \\
\hline morphology & Megacrystalline sparite & Micro and megacrystalline & Anhedral xtals \\
\hline $\begin{array}{l}\text { texture } \\
\text { transmitted opti- } \\
\text { cal properties }\end{array}$ & Brecciated, recrystallized & Replacing carbonate & $\begin{array}{l}\text { Fractured xtals in discontin- } \\
\text { uous veins }\end{array}$ \\
\hline $\begin{array}{l}\text { reflected optical } \\
\text { properties }\end{array}$ & & & \\
\hline $\begin{array}{l}\text { contact/ } \\
\text { relationships with } \\
\text { other minerals }\end{array}$ & $\begin{array}{l}\text { Being replaced by sulfides } \\
\text { and qtz }\end{array}$ & $\begin{array}{l}\text { Replacing carb, being re- } \\
\text { placed by sulf }\end{array}$ & $\begin{array}{l}\text { Replacing carb and qtz, be- } \\
\text { ing replaced by Sp and Fe- } \\
\text { Ox }\end{array}$ \\
\hline rection/zoning) & & & \\
\hline
\end{tabular}




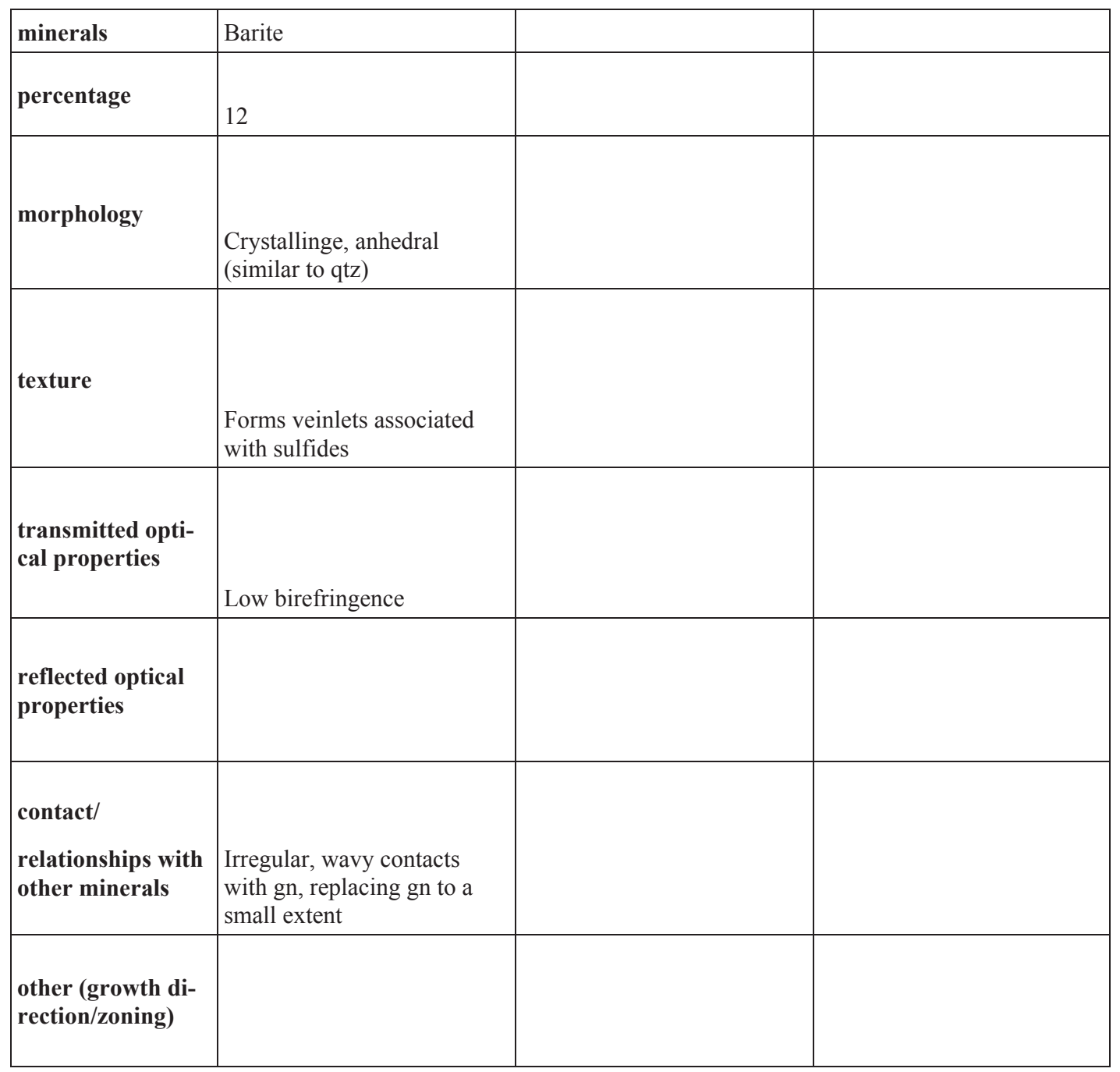




\begin{tabular}{|c|c|c|c|}
\hline minerals & Sphalerite & Galena & Unknown-Chalcocite? \\
\hline percentage & 1 & 2 & trace \\
\hline morphology & Anhdredal blebby xtals & Anhedral xtals & anhedral \\
\hline texture & Disseminated & Disseminated and in veinlets & disseminated \\
\hline $\begin{array}{l}\text { transmitted opti- } \\
\text { cal properties }\end{array}$ & & & opaque \\
\hline $\begin{array}{l}\text { reflected optical } \\
\text { properties }\end{array}$ & & & $\begin{array}{l}\text { Color: light blue-grey } \\
\text { Birefl: weak to mod } \\
\text { Anis: mod pink-grey } \\
\text { IR: none }\end{array}$ \\
\hline $\begin{array}{l}\text { contact/ } \\
\text { relationships with } \\
\text { other minerals }\end{array}$ & $\begin{array}{l}\text { Replacing Py, coeval w/ Gn } \\
\text { (some Gn replacing } \mathrm{Sp} \text { and } \\
\text { vice versa) }\end{array}$ & $\begin{array}{l}\text { Coeval with Sp (some Gn } \\
\text { replacing Sp and vice versa) }\end{array}$ & $\begin{array}{l}\text { Chalcocite? Replacing Sp } \\
\text { and Py }\end{array}$ \\
\hline $\begin{array}{l}\text { other (growth di- } \\
\text { rection/zoning) }\end{array}$ & & & \\
\hline
\end{tabular}

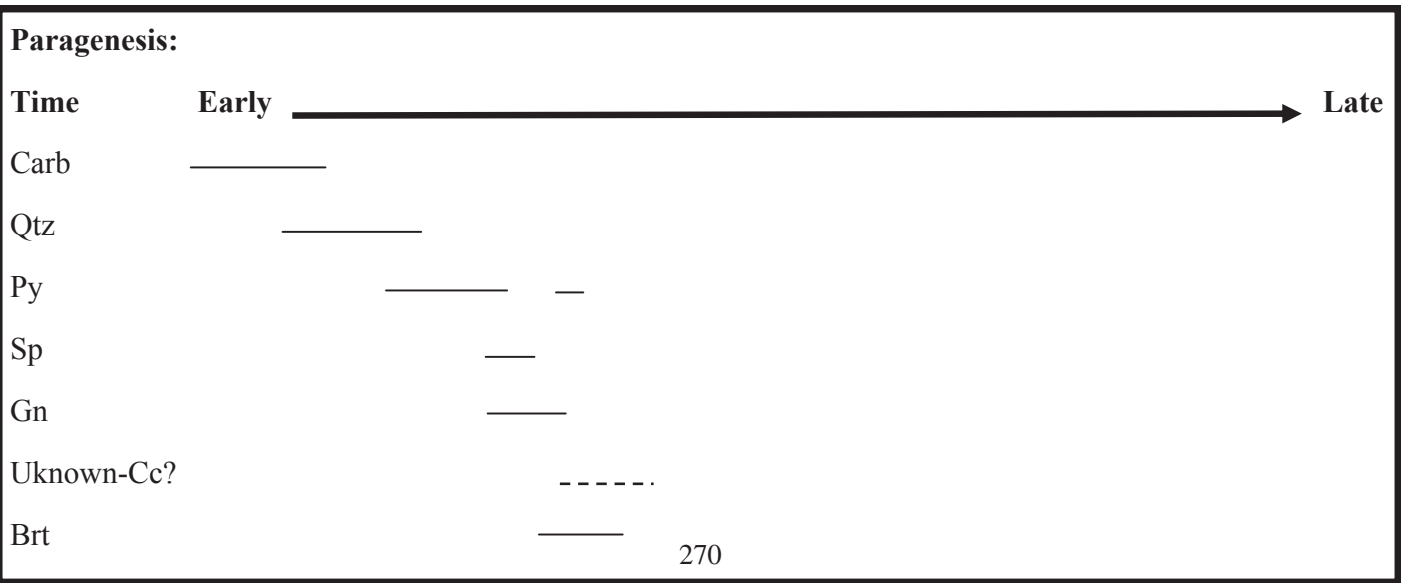


Geologic Unit: Hydrothermal Breccia

Lithological Unit (Votorantim): SM in Hydrothermal Breccia

Sample Photograph

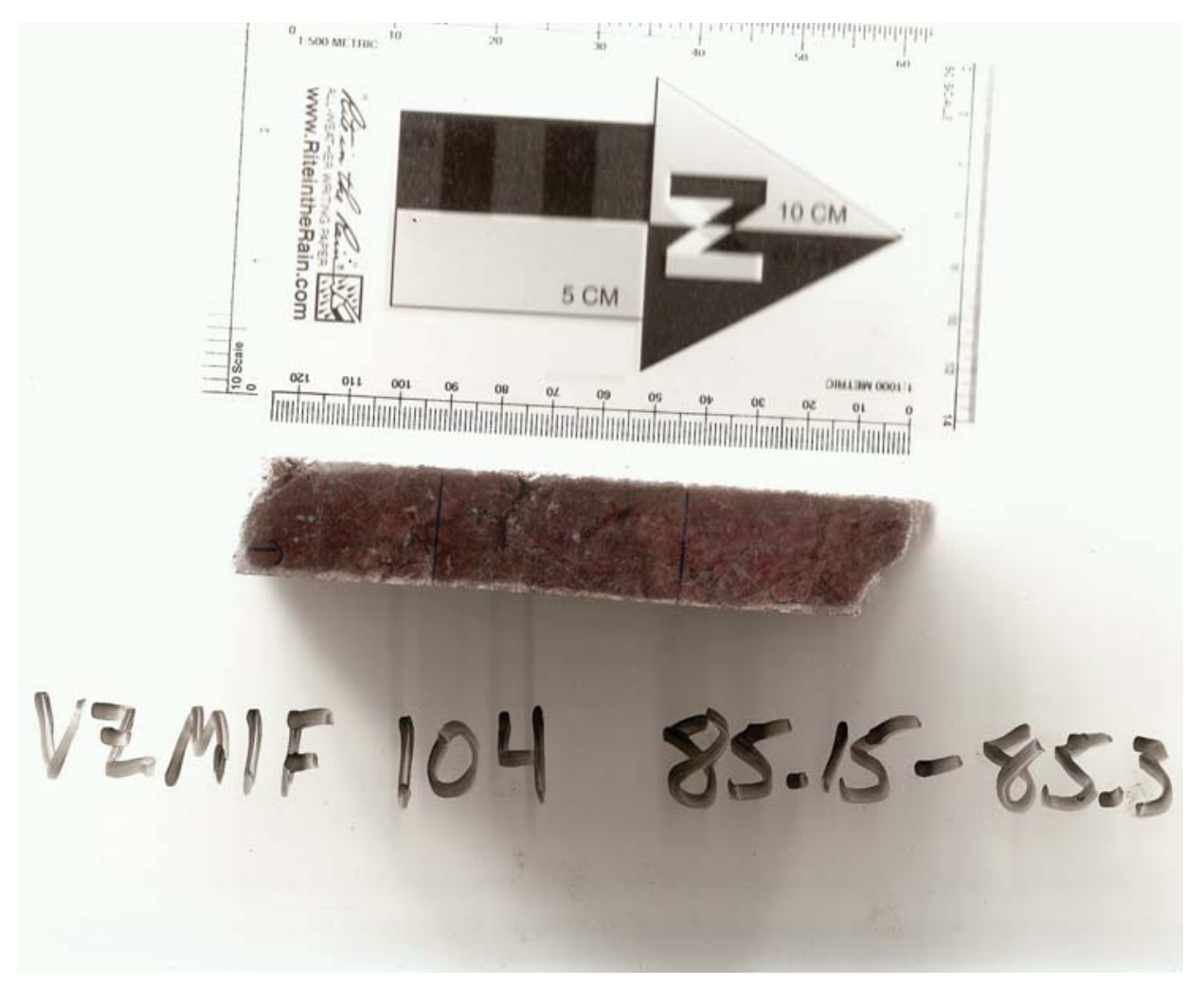

Other Sample Information: From Gilberto Batista 


\begin{tabular}{|c|c|c|c|}
\hline minerals & Dolomite (Ferroan) & Willemite & chlorite \\
\hline percentage & 40 & 27 & 10 \\
\hline morphology & $\begin{array}{l}\text { Anhedral, in fractures in be- } \\
\text { tween wl fragments }\end{array}$ & $\begin{array}{l}\text { Subhedral to euhdral xtals } \\
\text { on breccia fragments }\end{array}$ & $\begin{array}{l}\text { Fibrous xtals in anastomos- } \\
\text { ing veins }\end{array}$ \\
\hline texture & $\begin{array}{l}\text { Fracture filling and replac- } \\
\text { ing wl, minor replacement of } \\
\text { chl. Associated with hm } \\
\text { (being replaced by hm as } \\
\text { well) }\end{array}$ & $\begin{array}{l}\text { Replacing original dol bx } \\
\text { fragments, but being re- } \\
\text { placed by recent dolomitiza- } \\
\text { tion of bx fractures }\end{array}$ & Vein \\
\hline $\begin{array}{l}\text { transmitted opti- } \\
\text { cal properties }\end{array}$ & & & \\
\hline $\begin{array}{l}\text { reflected optical } \\
\text { properties }\end{array}$ & & & \\
\hline $\begin{array}{l}\text { contact/ } \\
\text { relationships with } \\
\text { other minerals }\end{array}$ & $\begin{array}{l}\text { Dol is filling in fractures and } \\
\text { creating small embayments } \\
\text { in wl. Irregular contact with } \\
\mathrm{hm}, \mathrm{hm} \text { replacing dol. }\end{array}$ & $\begin{array}{l}\text { Fractured edges with dol, } \\
\text { being replaced by dol. Hm } \\
\text { blebs on wl with irregular } \\
\text { contacts. }\end{array}$ & $\begin{array}{l}\text { Straight contacts with dol } \\
\text { and wl. Somewhat irregular } \\
\text { edge with sl and hm. Alter- } \\
\text { ing to hm }\end{array}$ \\
\hline $\begin{array}{l}\text { other (growth di- } \\
\text { rection/zoning) }\end{array}$ & & & \\
\hline
\end{tabular}




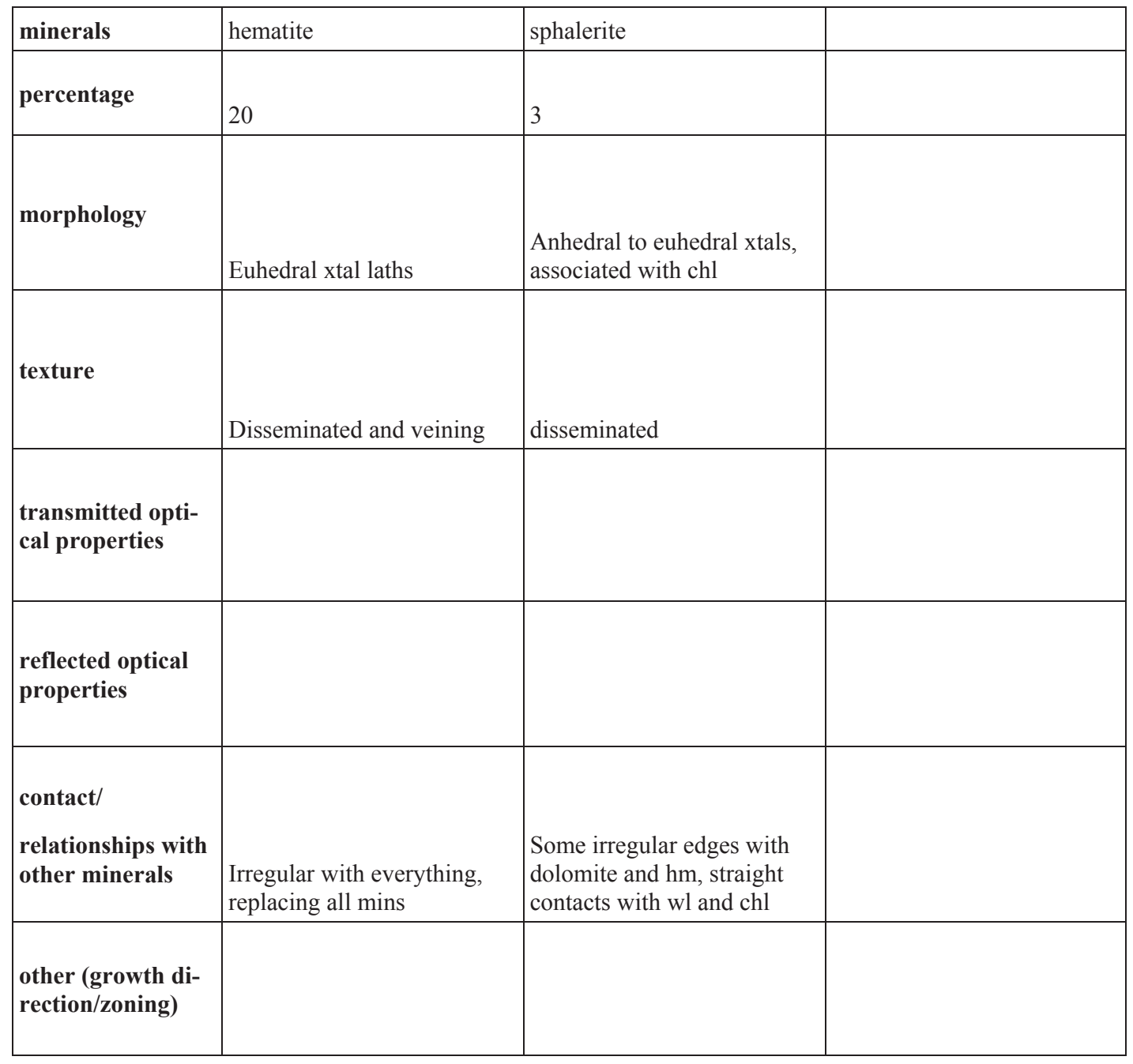

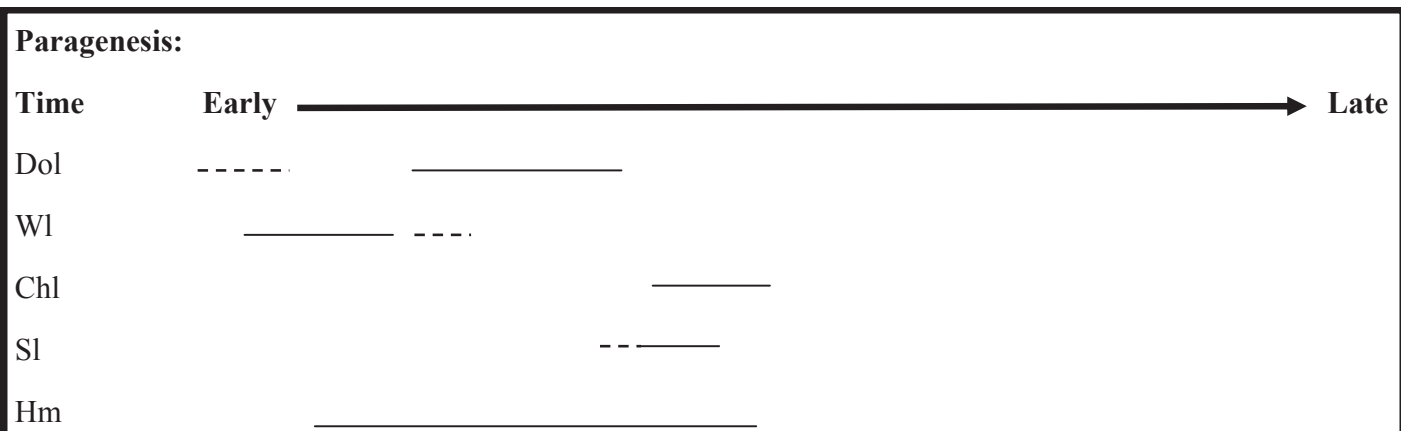


Structures: The sample has been brecciated under brittle conditions during which replacement of dolomite by willemite and minor hematite took place. After brecciation and wl mineralization, $\mathrm{Fe}-$

dolomite filled in the fractures and started to replace wl. The chlorite shear then cut the sample. The

fluid contained chl as well as sl and some hm. Sl seems to be minorly diagentic in the sample as it oc-

curs in some of the wl breccia clasts.

Paragenetic Sequence: see chart* 
Geologic Unit: Hydrothermal Breccia

Lithological Unit (Votorantim): Massive Silicate in BH

Sample Photograph
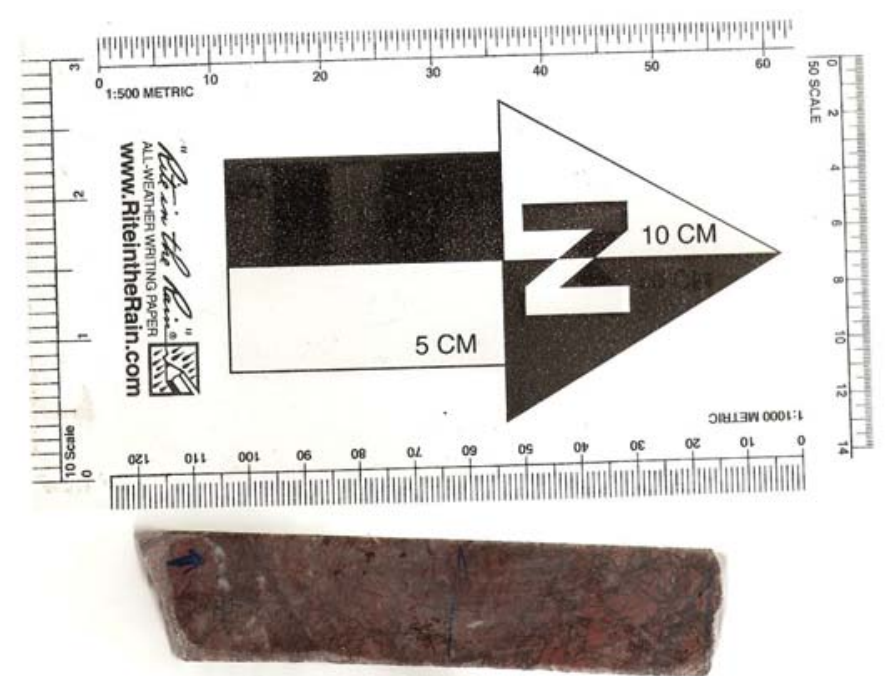

V.1.5 104

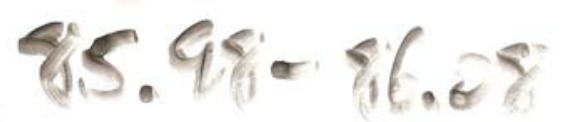

Other Sample Information: 


\begin{tabular}{|c|c|c|c|}
\hline minerals & Dolomite & Hematite & Willemite \\
\hline percentage & 60 & 15 & 22 \\
\hline morphology & Sparry and micritic dol & Euhedral laths & $\begin{array}{l}\text { Subhedral granular, minor } \\
\text { fibrous splays }\end{array}$ \\
\hline texture & $\begin{array}{l}\text { Sheared micritic dol and } \\
\text { sparry vein dol. Original } \\
\text { sparry dol being replaced by } \\
\text { wl and hm }\end{array}$ & $\begin{array}{l}\text { Small xtals in dol and shear } \\
\text { veins b/w bx clasts }\end{array}$ & Make up bx clasts \\
\hline $\begin{array}{l}\text { transmitted opti- } \\
\text { cal properties }\end{array}$ & & & \\
\hline $\begin{array}{l}\text { reflected optical } \\
\text { properties }\end{array}$ & & & \\
\hline $\begin{array}{l}\text { contact/ } \\
\text { relationships with } \\
\text { other minerals }\end{array}$ & $\begin{array}{l}\text { Most sparry dol is being re- } \\
\text { placed by wl, qtz, and hm } \\
\text { (except for sparry dol in } \\
\text { veins) and micritic dol is } \\
\text { replacing wl }\end{array}$ & $\begin{array}{l}\text { Replacing all mins, shreddy } \\
\text { contacts }\end{array}$ & $\begin{array}{l}\text { Replacing sparry dol and } \\
\text { being replaced by hm and } \\
\text { micritic dol (some straight, } \\
\text { mostly irregular edges) }\end{array}$ \\
\hline $\begin{array}{l}\text { other (growth di- } \\
\text { rection/zoning) }\end{array}$ & & $\begin{array}{l}\text { Some exosolution laths in } \\
\text { sphalerite }\end{array}$ & \\
\hline
\end{tabular}




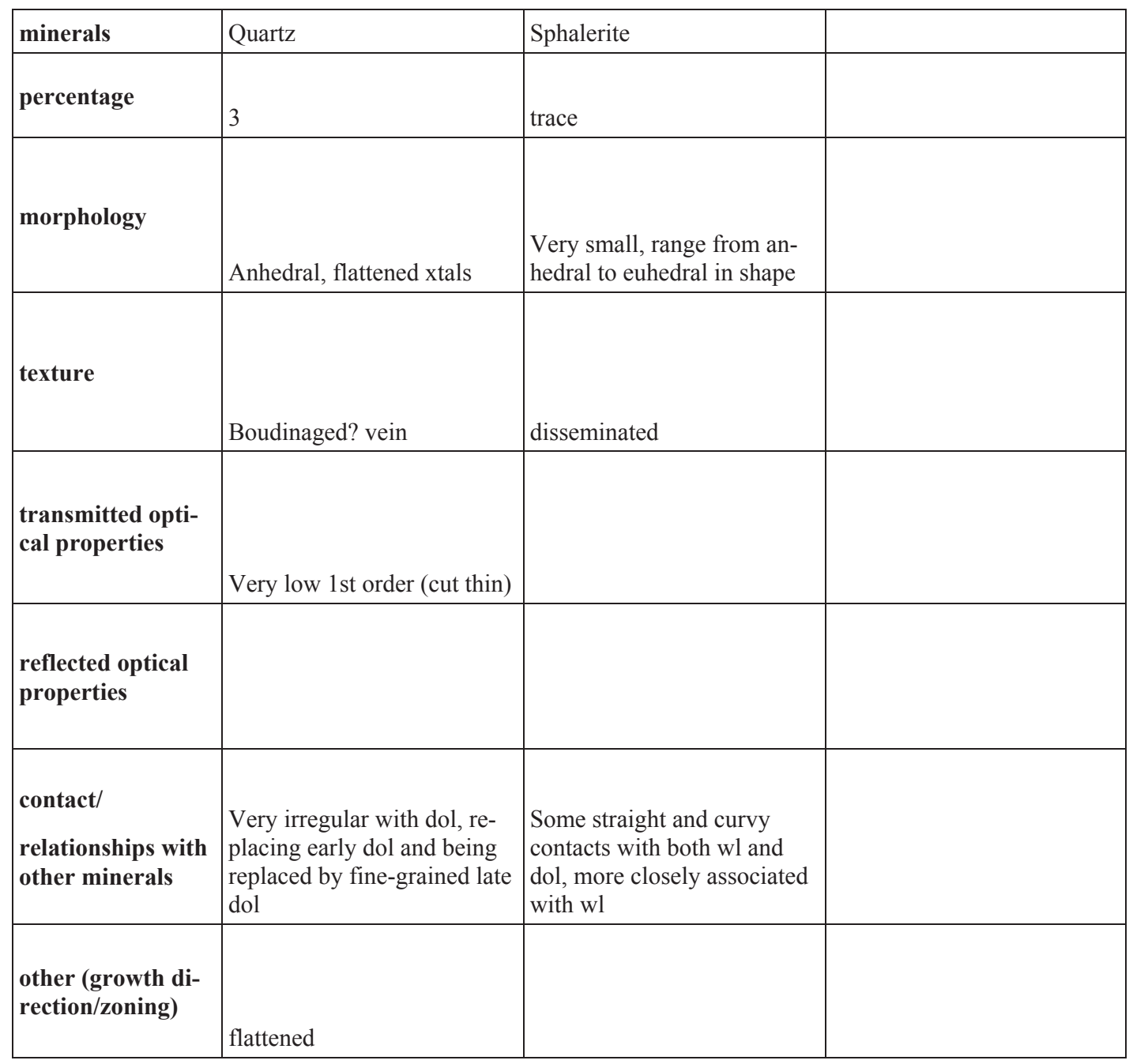

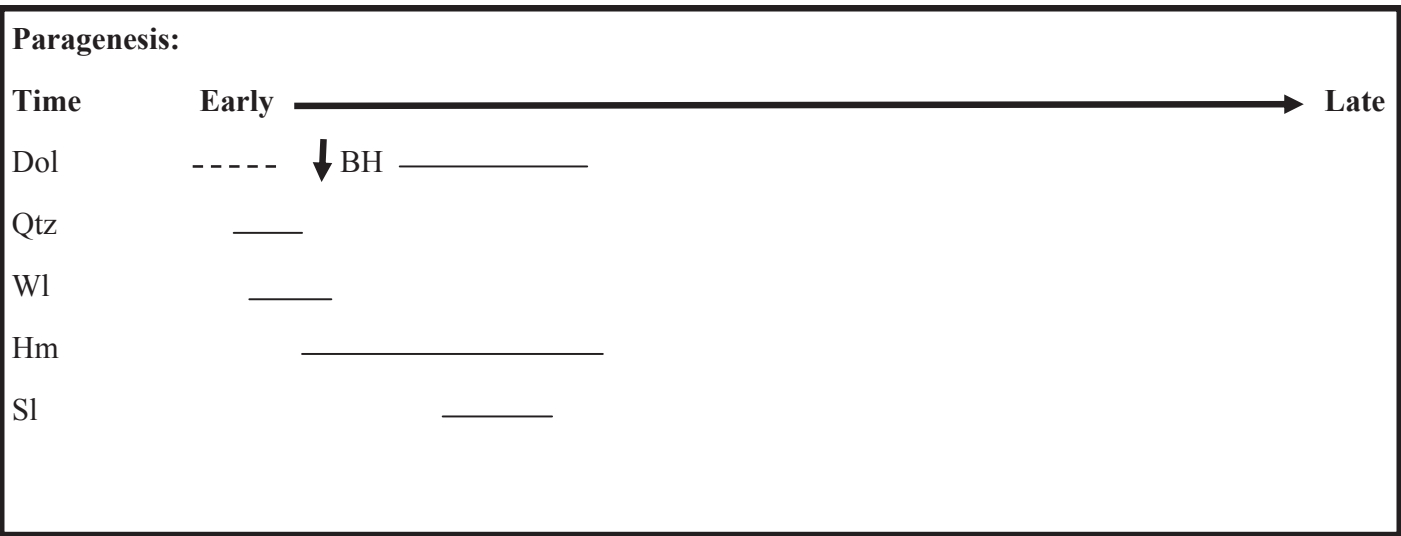


Structures: General brecciation and shearing of sample. Quartz provides evidence for shearing. Willemite/dolomite clasts show brecciation.

Paragenetic Sequence:

Paragenetic Reasoning: Dolomite occurred first as it is being replaced by willemite (second) in breccia clasts floating in hematite (third). 
Geologic Unit: Hydrothermal Breccia

Lithological Unit (Votorantim): Hydrothermal Breccia

\section{Sample Photograph}

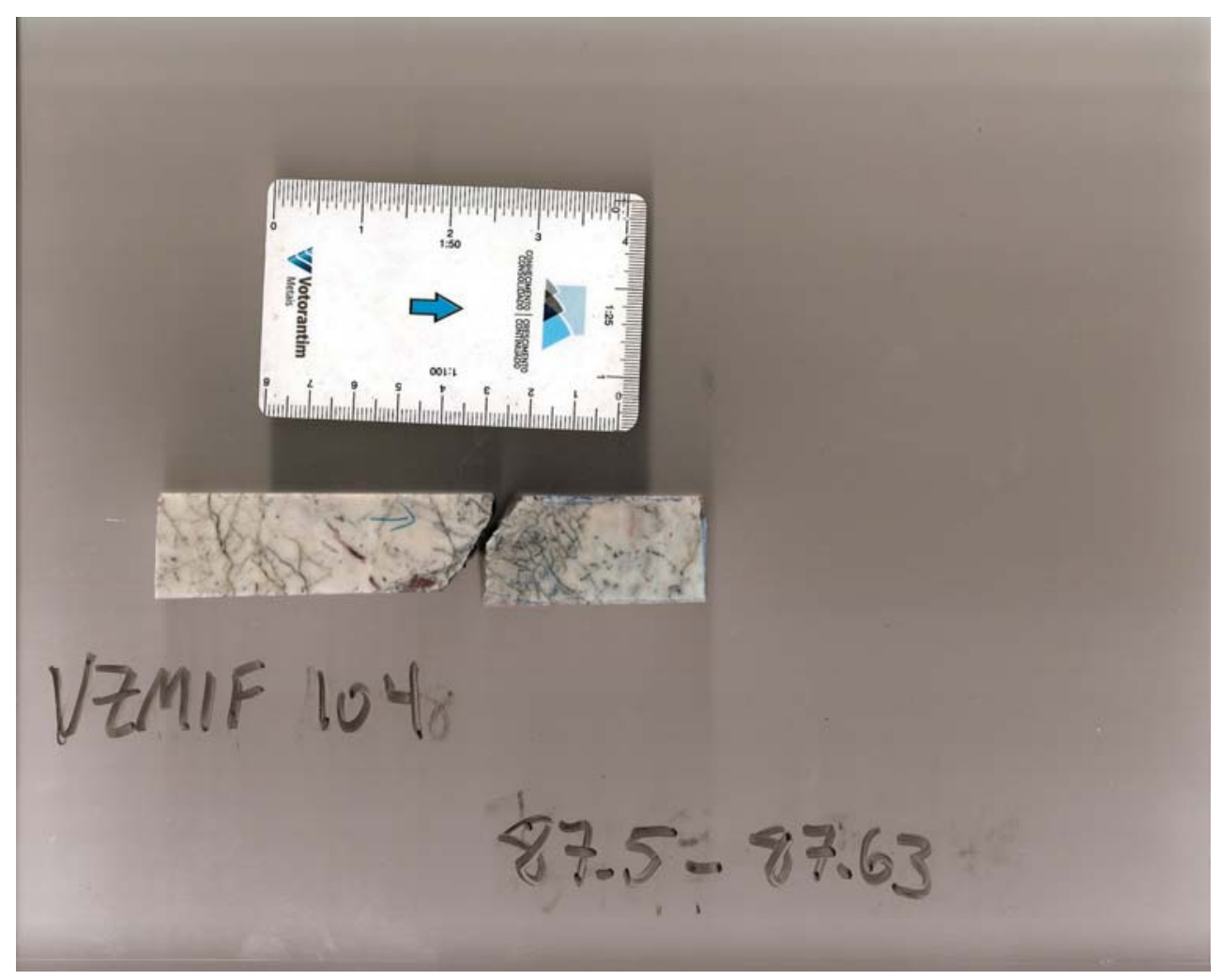

Other Sample Information: Same as 104-80 
Geologic Unit: Lower Pamplona

Lithological Unit (Votorantim): DG-Intercalated phyllites and dolomites

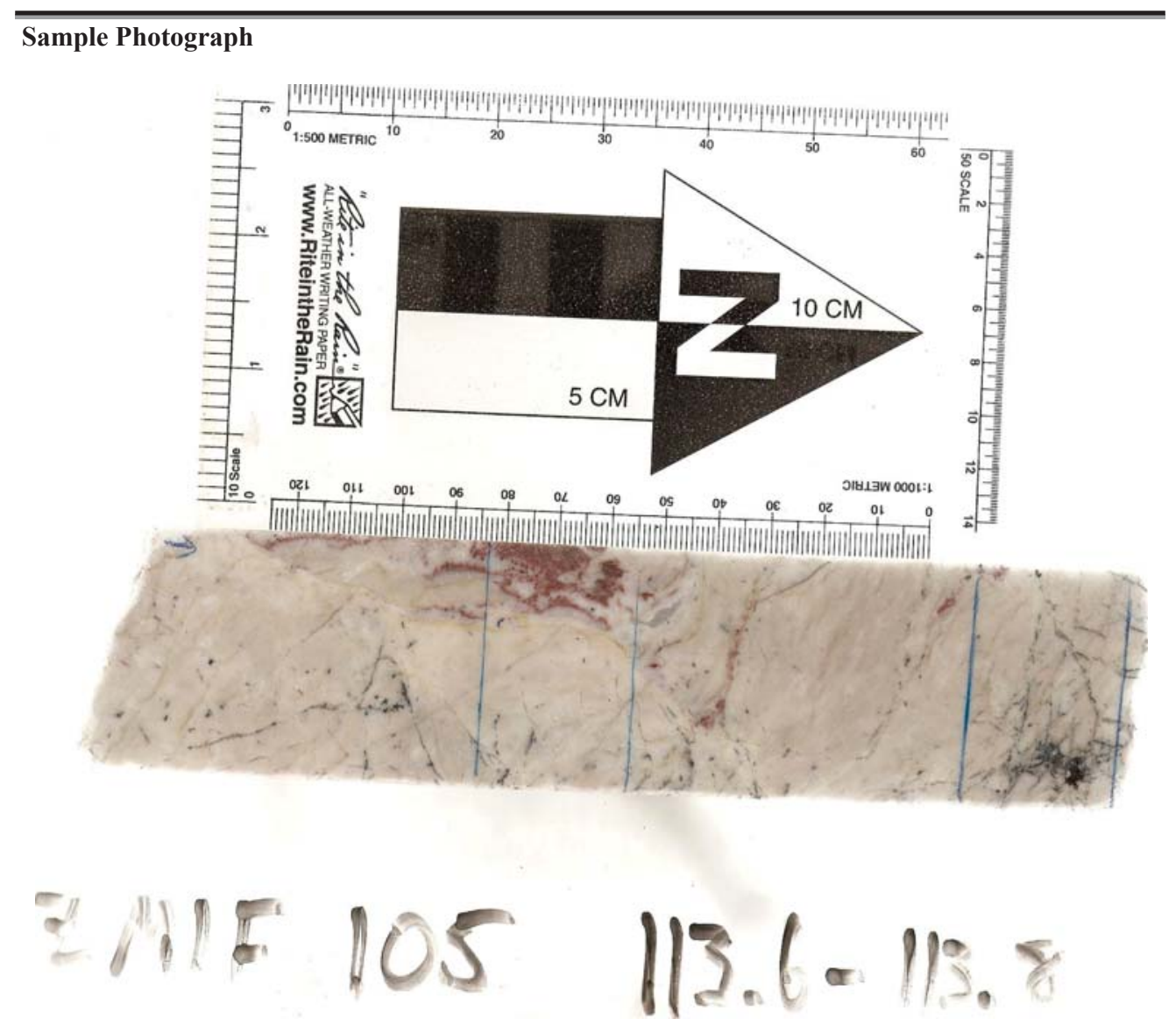

Other Sample Information: Part of dolomite marble in Lower Pamplona 


\begin{tabular}{|c|c|c|c|}
\hline minerals & Dolomite & Quartz & Fe-oxides \\
\hline percentage & 85 & 5 & 1 \\
\hline morphology & Micrite to sparry & $\begin{array}{l}\text { Directional growth during } \\
\text { shearing }\end{array}$ & anhedral \\
\hline texture & $\begin{array}{l}\text { Sheared, annealed, and frac- } \\
\text { tured (in that order) }\end{array}$ & Chalcedonic fibers & Fracture filling \\
\hline $\begin{array}{l}\text { transmitted opti- } \\
\text { cal properties }\end{array}$ & & & \\
\hline $\begin{array}{l}\text { reflected optical } \\
\text { properties }\end{array}$ & & & $\begin{array}{l}\text { Bluish-grey to light purple } \\
\text { with orange tinge }\end{array}$ \\
\hline $\begin{array}{l}\text { contact/ } \\
\text { relationships with } \\
\text { other minerals }\end{array}$ & $\begin{array}{l}\text { Irregular contact b/w qtz and } \\
\text { sparry dol. Irregular and } \\
\text { embayed contacts w/ sul- } \\
\text { fides, dol is being replaced } \\
\text { by sulfides }\end{array}$ & Replacing sparry dol & $\begin{array}{l}\text { Irregular with gn, replacing } \\
\text { gn. Straight edges with } \\
\text { sl.and dol }\end{array}$ \\
\hline $\begin{array}{l}\text { other (growth di- } \\
\text { rection/zoning) }\end{array}$ & & & \\
\hline
\end{tabular}




\begin{tabular}{|c|c|c|c|}
\hline minerals & Sphalerite & Galena & Chalcopyrite \\
\hline percentage & 4 & 5 & trace \\
\hline morphology & $\begin{array}{l}\text { Fracture filling, anhedral to } \\
\text { subhedra }\end{array}$ & See S1 & Anhedral blebls \\
\hline texture & Semi-massive vein fill & & Found in sl and on own \\
\hline $\begin{array}{l}\text { transmitted opti- } \\
\text { cal properties }\end{array}$ & & & \\
\hline $\begin{array}{l}\text { reflected optical } \\
\text { properties }\end{array}$ & & & \\
\hline $\begin{array}{l}\text { contact/ } \\
\text { relationships with } \\
\text { other minerals }\end{array}$ & $\begin{array}{l}\text { Straight contacts with gn and } \\
\text { Ag-SS. Replacement of ear- } \\
\text { ly dol, but straight contacts } \\
\text { with dol in fractures }\end{array}$ & $\begin{array}{l}\text { Irregular contacts with early } \\
\text { dol (same as sl) and Ag-SS. } \\
\text { Straight edges with late dol } \\
\text { and sl }\end{array}$ & $\begin{array}{l}\text { Straight egdes, exosolution } \\
\text { of } \mathrm{Cu}\end{array}$ \\
\hline $\begin{array}{l}\text { other (growth di- } \\
\text { rection/zoning) }\end{array}$ & & & \\
\hline
\end{tabular}

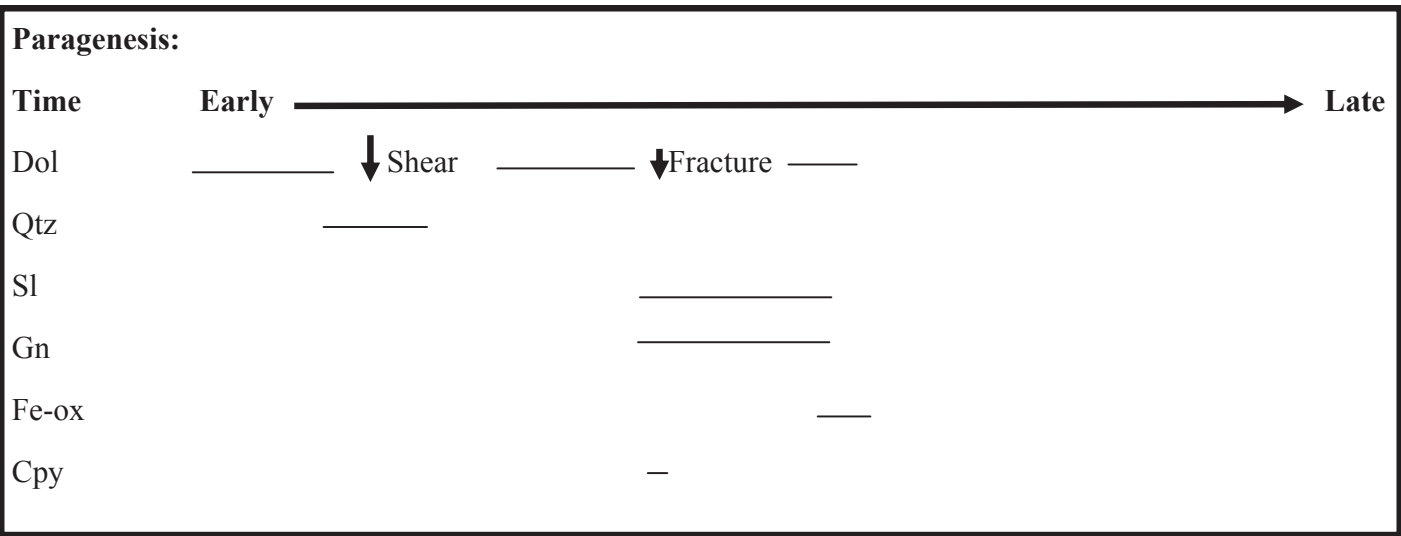


Structures: Sample exhibits dolomitic brecciation and shearing followed by dolomitic annealing. Qtz has replaced some sparry dol during shearing. Later fracture filled with sulfides.

Paragenetic Sequence: dol $\rightarrow \mathrm{qtz} \rightarrow \mathrm{gn}+\mathrm{sl}+\mathrm{dol}+\mathrm{cpy} \rightarrow \mathrm{Fe}-\mathrm{ox}$

Paragenetic Reasoning: Dolomite is sheared and replaced by qtz. Quartz crystals are growing in open space perpendicular? to shear direction. Later brittle fracture of dolomite has been filled in by gn, sl, and dol rhombs, which are all coeval. Gn can be seen replacing dolomite cleavage planes. Cpy present in some sl, very, very minute. 
Geologic Unit: Lower Pamplona

Lithological Unit (Votorantim): DG-Intercalated phyllites and dolomite

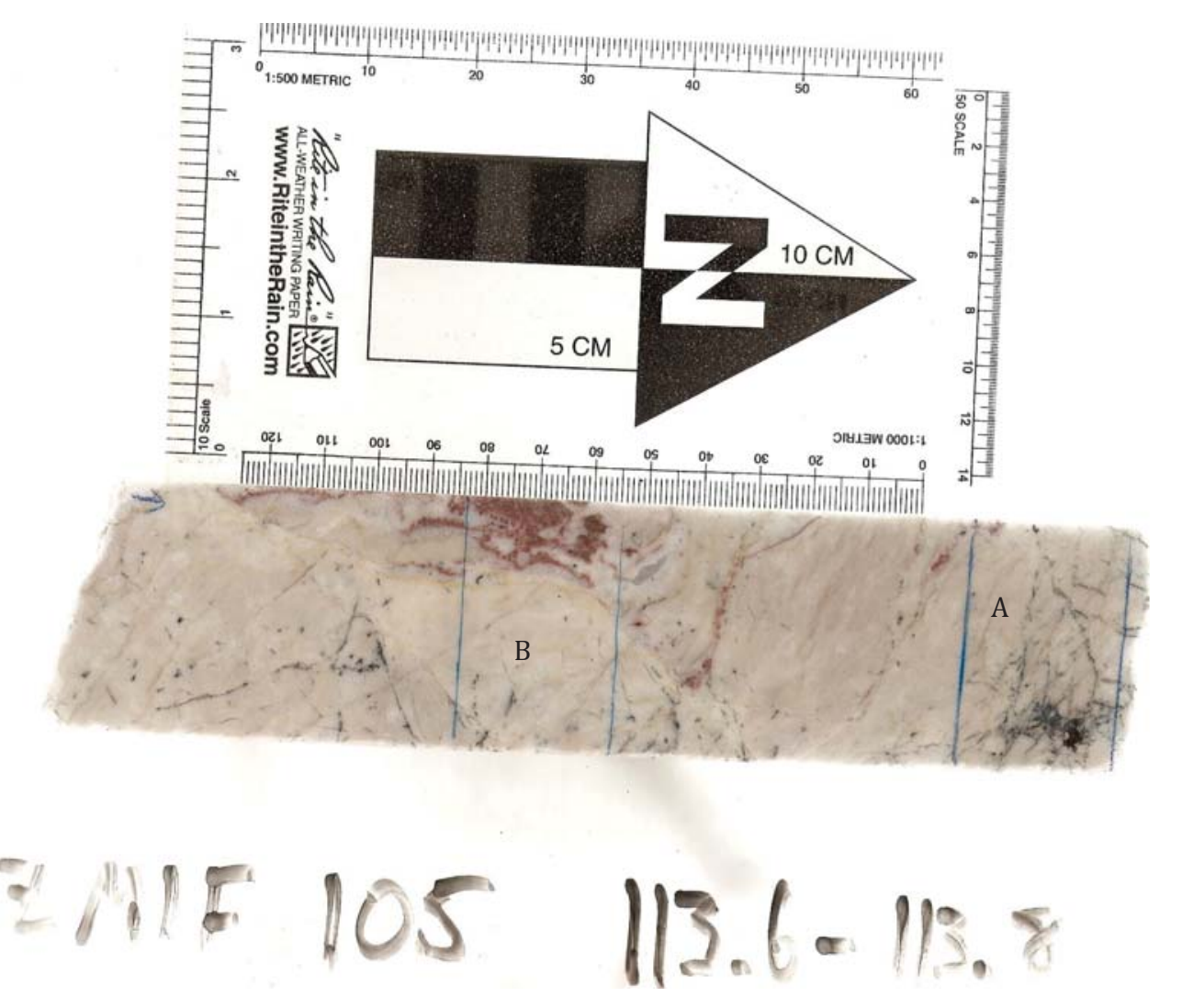

Other Sample Information: Marbled dolomite 


\begin{tabular}{|c|c|c|c|}
\hline minerals & Dolomite & Quartz & Sulfides (gn, sl) \\
\hline percentage & 95 & 5 & trace \\
\hline morphology & $\begin{array}{l}\text { Micritic dol pieces and re- } \\
\text { crystallized sparry dol } \\
\text { (growing into open space } \\
\text { from bx) }\end{array}$ & $\begin{array}{l}\text { Elongated chalcedonic xtals } \\
\text { in direction of shear and lat- } \\
\text { er small qtz vein }\end{array}$ & Minute blebs \\
\hline texture & Micritic and sparry & Replacing dol & disseminated \\
\hline $\begin{array}{l}\text { transmitted opti- } \\
\text { cal properties }\end{array}$ & & & \\
\hline $\begin{array}{l}\text { reflected optical } \\
\text { properties }\end{array}$ & & & \\
\hline $\begin{array}{l}\text { contact/ } \\
\text { relationships with } \\
\text { other minerals }\end{array}$ & $\begin{array}{l}\text { Irregular and blobby con- } \\
\text { tacts with qtz. Sharp con- } \\
\text { tacts with sulfides }\end{array}$ & $\begin{array}{l}\text { See dol. Not in contact with } \\
\text { sulfides }\end{array}$ & $\begin{array}{l}\text { Sharp with dol, gn has fuzzy } \\
\text { boundary with Ag-sulfosalts, } \\
\text { straight contacts with sl }\end{array}$ \\
\hline $\begin{array}{l}\text { other (growth di- } \\
\text { rection/zoning) }\end{array}$ & $\begin{array}{l}\text { Growing into open space left } \\
\text { by brecciation }\end{array}$ & $\begin{array}{l}\text { Xtals elongated in shear di- } \\
\text { rection, some rotation }\end{array}$ & \\
\hline
\end{tabular}

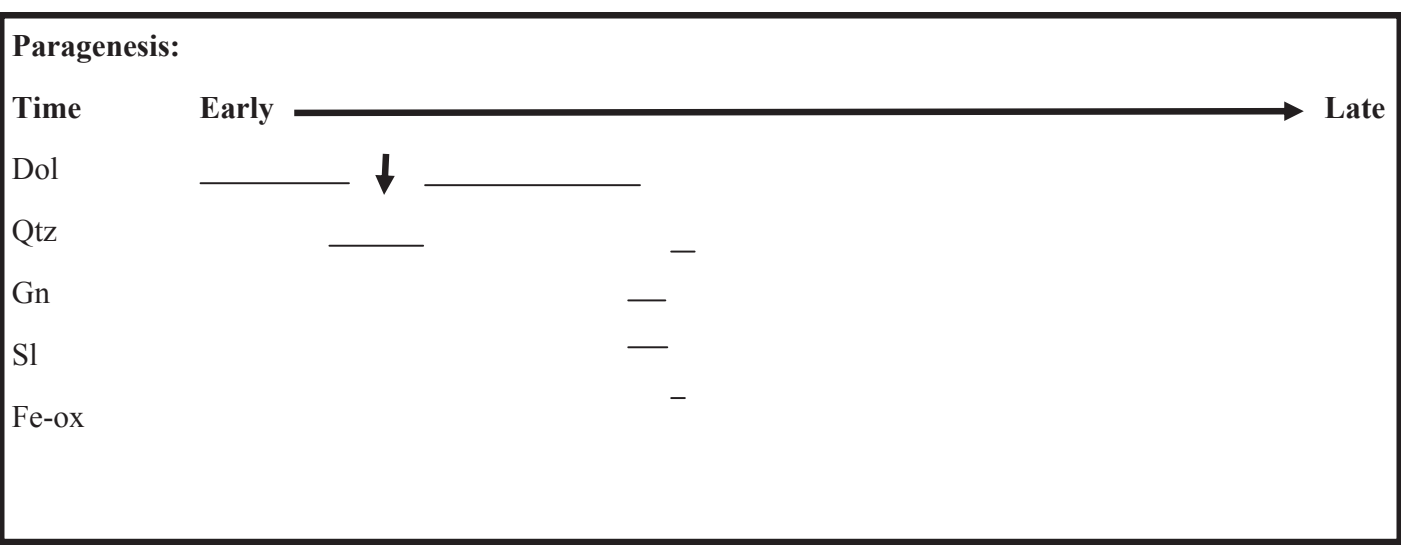


Geologic Unit: Upper Morro do Pinheiro ( \pm bird's eyes)

Lithological Unit (Votorantim): DO - Grey dolomite with fenestrate texture

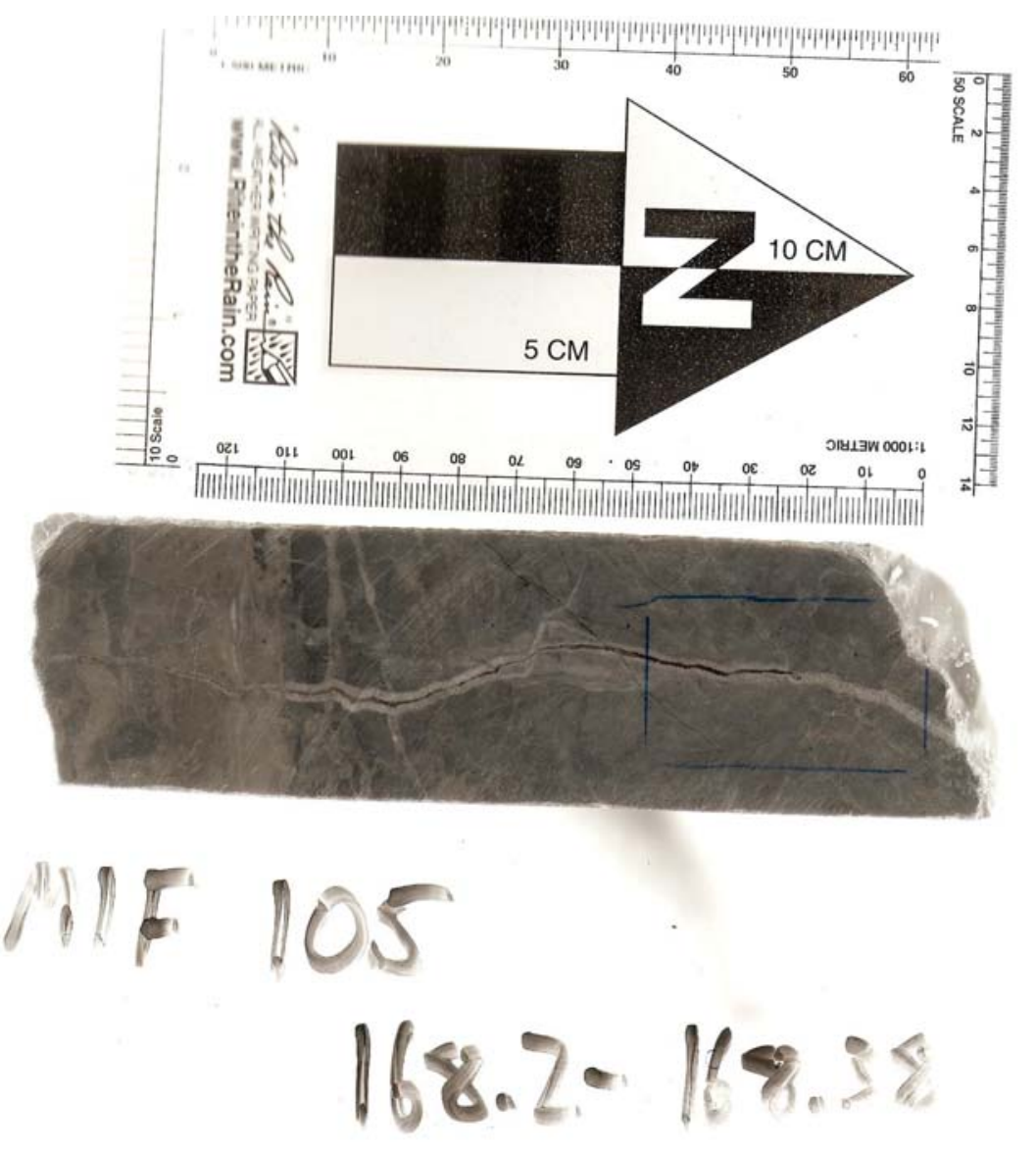

Other Sample Information: Sedimentary Sample 


\begin{tabular}{|c|c|c|c|}
\hline minerals & Dolomite & Quartz & pyrite \\
\hline percentage & 85 & 14 & 1 \\
\hline morphology & $\begin{array}{l}\text { Micritic. Sparry euhedral } \\
\text { xtals in vein }\end{array}$ & $\begin{array}{l}\text { Subhedral grains in micrite. } \\
\text { Chalcedonic qtz in vein }\end{array}$ & Euhedral (diagenetic?) \\
\hline texture & $\begin{array}{l}\text { Rock matrix. Some annealed } \\
\text { bx around vein emplacement }\end{array}$ & $\begin{array}{l}\text { Disseminated, (massive in } \\
\text { vein) }\end{array}$ & $\begin{array}{l}\text { Disseminated, some associ- } \\
\text { ated with microfractures }\end{array}$ \\
\hline $\begin{array}{l}\text { transmitted opti- } \\
\text { cal properties }\end{array}$ & & & \\
\hline $\begin{array}{l}\text { reflected optical } \\
\text { properties }\end{array}$ & & & \\
\hline $\begin{array}{l}\text { contact/ } \\
\text { relationships with } \\
\text { other minerals }\end{array}$ & Straight with all & Straight with all & $\begin{array}{l}\text { Straight edges with dol and } \\
\text { qtz }\end{array}$ \\
\hline $\begin{array}{l}\text { other (growth di- } \\
\text { rection/zoning) }\end{array}$ & Growing towards vein center & Growing in center of vein & \\
\hline
\end{tabular}

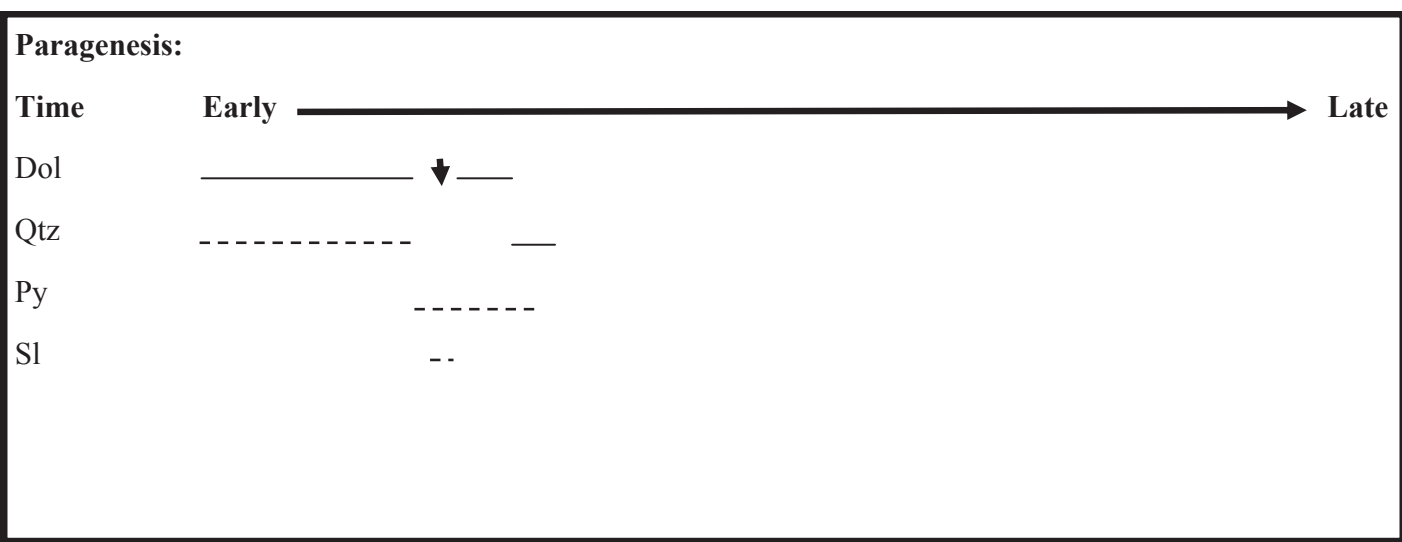




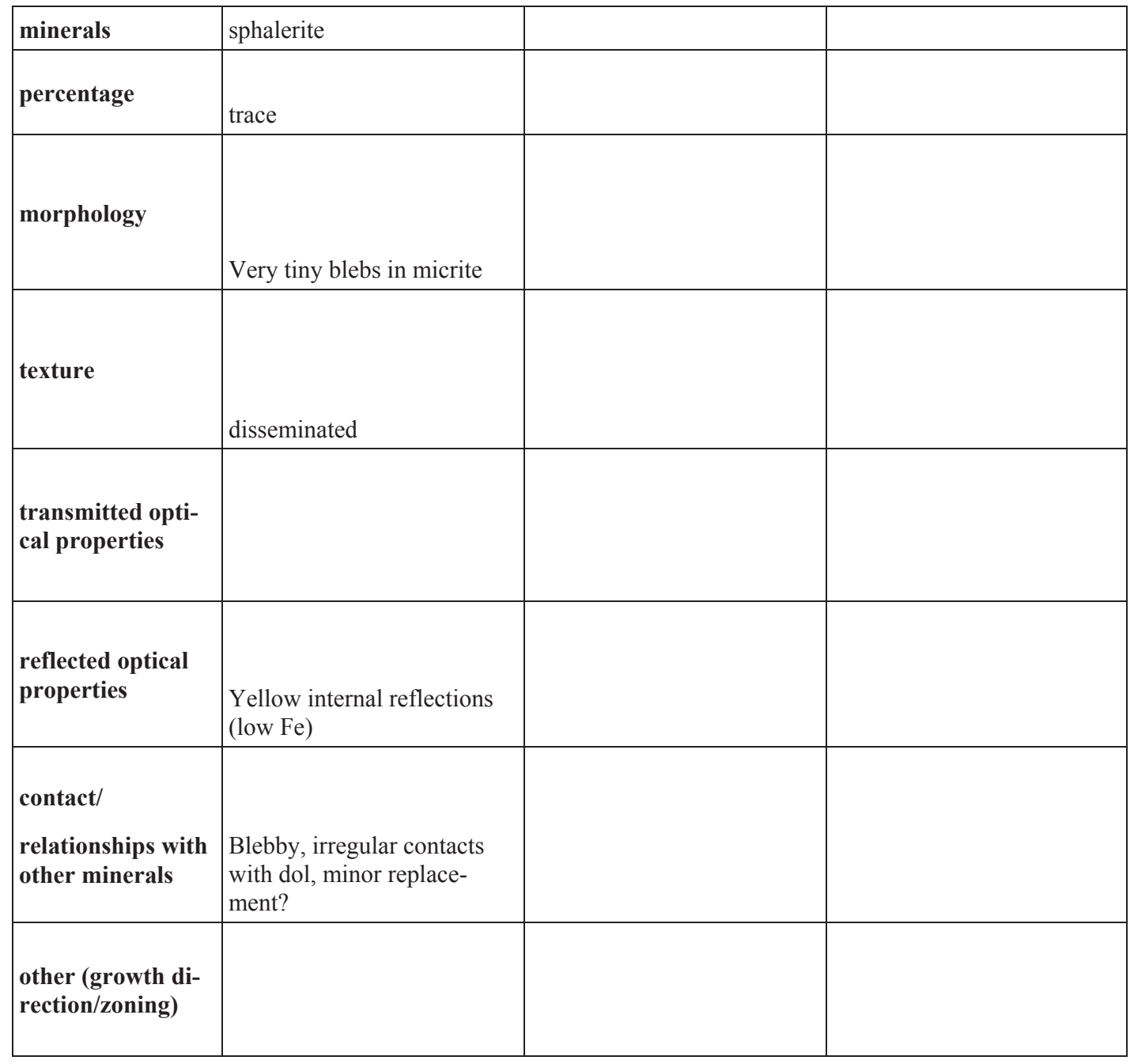

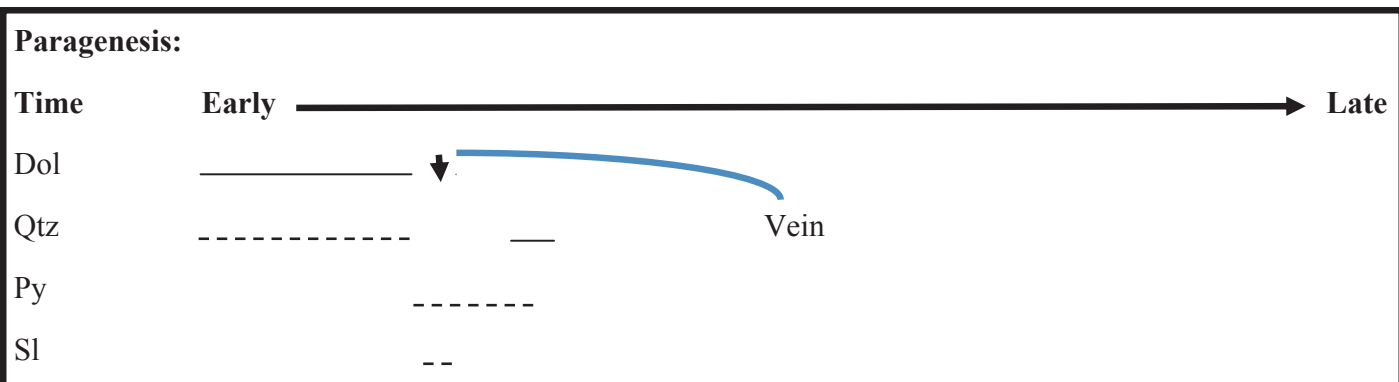


Structures: There is some brecciation around vein, but it has been healed. There are several small fractures, but all are cut by the major vein or simply end (start?) from the vein.

Paragenetic Sequence: dol $\rightarrow \mathrm{qtz} \rightarrow \mathrm{py}+\mathrm{sl}$ ?

Paragenetic Reasoning: Dolomite makes up the host rock and contains fragments of qtz and diagenetic pyrite. A vein runs through the center of the sample and is composed of recrystallized dolomite, then quartz with some minor pyrite in the center and along fractures near the vein margins. Little replacement is present. Sphalerite most likely associated with pyrite

Sample Highlights: Sedimentary sample of UMdP 
Geologic Unit: Hydrothermal Breccia

Lithological Unit (Votorantim): Massive silicate (SM) in Hydrothermal Breccia

Sample Photograph **Not available

Other Sample Information: Not Available 


\begin{tabular}{|c|c|c|c|}
\hline minerals & Willemite & Willemite veins & Specular hematite \\
\hline percentage & 62 & 3 & 35 \\
\hline morphology & Subhedral granular & Finer, subhedral to euhedral & $\begin{array}{l}\text { In interstices of } \mathrm{Wm} \text { and as } \\
\text { large and small veins } \\
\text { (multiple generations) }\end{array}$ \\
\hline texture & Being replaced by $\mathrm{Hm}$ & Cross-cutting all mins & Massive to veined \\
\hline $\begin{array}{l}\text { transmitted opti- } \\
\text { cal properties }\end{array}$ & & See Wm & opaque \\
\hline $\begin{array}{r}\text { color } \\
\mathrm{ppl} \\
\mathrm{xpl}\end{array}$ & $\begin{array}{l}\text { Clear } \\
\text { Beige to blues, greens, \& } \\
\text { oranges, reds }\end{array}$ & & \\
\hline pleochroism & $\begin{array}{l}\text { Faint? Light to slightly } \\
\text { darker grey }\end{array}$ & & \\
\hline birefringence & Mid 1st to low 3rd order & & \\
\hline $\begin{array}{l}\text { twinning/ } \\
\text { cleavage }\end{array}$ & & & \\
\hline other & & & \\
\hline $\begin{array}{l}\text { reflected optical } \\
\text { properties }\end{array}$ & N/A & & \\
\hline color & & & Whitish-grey \\
\hline bireflectance & & & Very weak, lighter to darker \\
\hline anisotropy & & & $\begin{array}{l}\text { Distinct, grey to greyish } \\
\text { blue }\end{array}$ \\
\hline internal reflections & & & Some, red \\
\hline other & & & \\
\hline $\begin{array}{l}\text { contact/ } \\
\text { relationships with } \\
\text { other minerals }\end{array}$ & $\begin{array}{l}\text { Euhdral contact w/ Wm, } \\
\text { brecciated and irregular con- } \\
\text { tact with } \mathrm{Hm}\end{array}$ & $\begin{array}{l}\text { Overgrown on all mins, } \\
\text { smaller veins cut by faults }\end{array}$ & $\begin{array}{l}\text { Hm filling space } \mathrm{b} / \mathrm{w} \mathrm{Wm} \\
\text { grains. Also multiple gener- } \\
\text { ations of veins (see HL) }\end{array}$ \\
\hline $\begin{array}{l}\text { other (growth di- } \\
\text { rection/zoning) }\end{array}$ & & & \\
\hline
\end{tabular}




\begin{tabular}{|c|c|c|c|}
\hline minerals & Hematite & Magnetite? & \\
\hline percentage & Trace & trace & \\
\hline morphology & Minute specs & anhedral grain & \\
\hline texture & Small blebs & broken & \\
\hline $\begin{array}{l}\text { transmitted opti- } \\
\text { cal properties }\end{array}$ & & opaque & \\
\hline $\begin{array}{r}\text { color } \\
\mathrm{ppl} \\
\mathrm{xpl}\end{array}$ & $\begin{array}{l}\text { Red to ruddy brown } \\
\text { Opaque }\end{array}$ & & \\
\hline pleochroism & & & \\
\hline birefringence & & & \\
\hline $\begin{array}{r}\text { twinning/ } \\
\text { cleavage }\end{array}$ & & & \\
\hline other & & & \\
\hline $\begin{array}{l}\text { reflected optical } \\
\text { properties }\end{array}$ & & & \\
\hline color & & Grey with white specs & \\
\hline bireflectance & & none & \\
\hline anisotropy & & isotropic & \\
\hline internal reflections & & none & \\
\hline other & & White specs, Hm? & \\
\hline $\begin{array}{l}\text { contact/ } \\
\text { relationships with } \\
\text { other minerals }\end{array}$ & $\begin{array}{l}\text { On margins and on top of } \\
\mathrm{Wm} \text { and Specular Hm }\end{array}$ & $\begin{array}{l}\text { Irregular boundary w/ Wm } \\
\text { (some growing inside), some } \\
\text { red-brown hm on edges in } \\
\text { ppl }\end{array}$ & \\
\hline $\begin{array}{l}\text { other (growth di- } \\
\text { rection/zoning) }\end{array}$ & & & \\
\hline
\end{tabular}


Structures: Many cross-cutting veins; micro faults; replacement of Wl by Hm

At least 3 sets of Hm veins:

1)wide, run parallel to label, appear to be related to $\mathrm{Hm}$ that replaces $\mathrm{Wl} ; 2$ )narrow, cut \#1, run perpendicular to \# 1; 3)run sub-parallel to \#1 and cut \#2

Paragenetic Sequence: $\mathrm{W} 1+\mathrm{Sl} ? \rightarrow \mathrm{Hm} \mathrm{V} 1+\mathrm{Hm} \rightarrow \mathrm{Hm} \mathrm{V} 2+\mathrm{Hm}$ V3 $\rightarrow \mathrm{W} 1 \mathrm{~V} 1$

Paragenetic Reasoning: The Wl already appears to be in place and is subhedral with smooth shared grain boundaries. The $\mathrm{Hm}$ associated with $\mathrm{Hm} \mathrm{V} 1$ is replacing the massive $\mathrm{Wl}$ boundaries. The $\mathrm{Hm}$

V2 cut and displace Hm V1. Hm V3 cuts Hm V2 and vice versa. W1 V1 cuts all Hm veins and is not replaced by $\mathrm{Hm}$.

Sample Highlights: Possible sphalerite, veins and faults associated with breccia

SEM- for sphalerite and quartz 
Geologic Unit: Middle Pamplona

Lithological Unit (Votorantim): Grey Dolomite (DO)

Sample Photograph

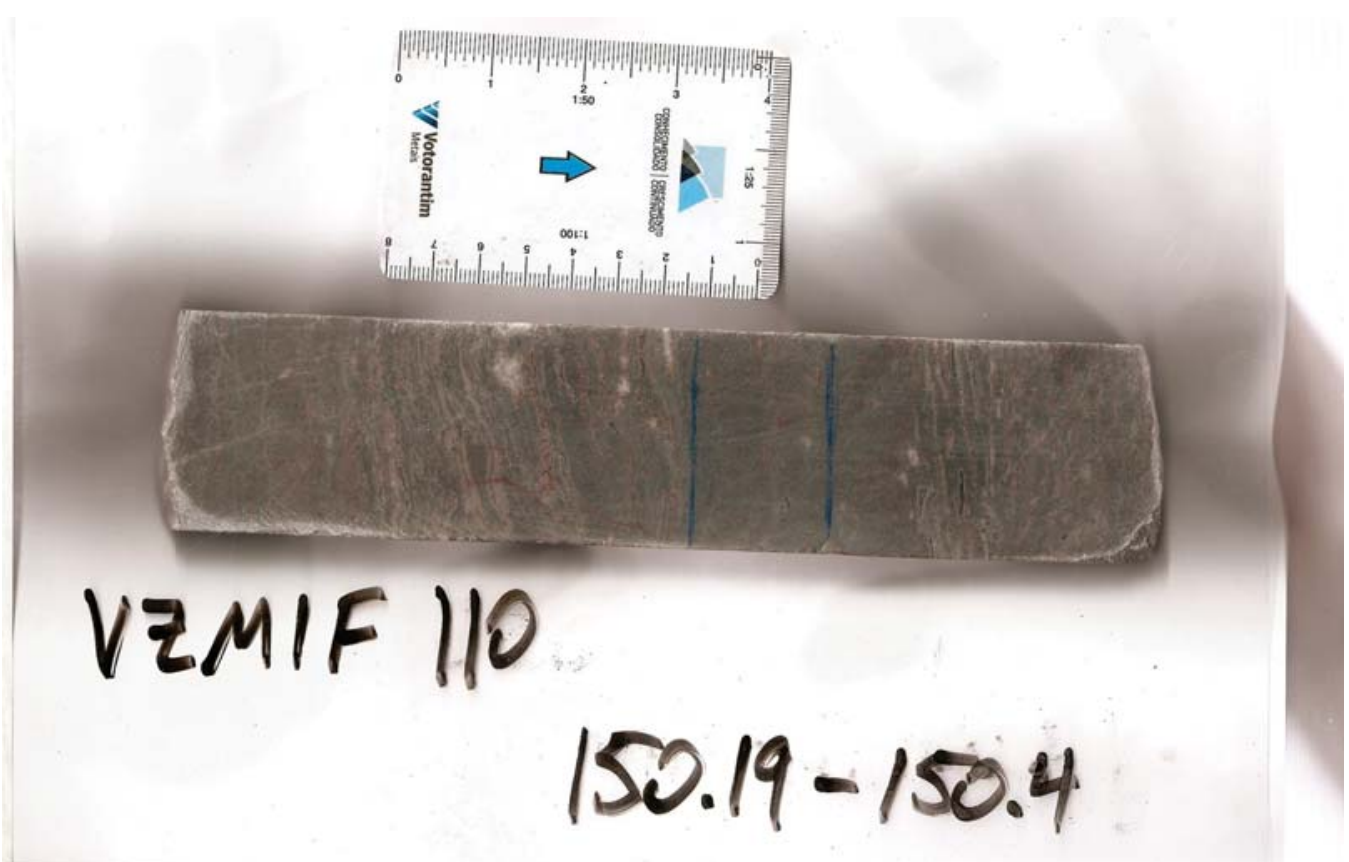

Other Sample Information: 


\begin{tabular}{|c|c|c|c|}
\hline minerals & Dolomite & $\mathrm{Fe}-\mathrm{Carb}$ & Quatz \\
\hline percentage & 90 & 5 & 5 \\
\hline morphology & psuedospar & Euhedral xtals & Euhedral xtals \\
\hline texture & Rock matrix & Vug-filling & Vug-filling \\
\hline $\begin{array}{l}\text { transmitted opti- } \\
\text { cal properties }\end{array}$ & & & \\
\hline $\begin{array}{l}\text { reflected optical } \\
\text { properties }\end{array}$ & & & \\
\hline $\begin{array}{l}\text { contact/ } \\
\text { relationships with } \\
\text { other minerals }\end{array}$ & & $\begin{array}{l}\text { Fillings vugs in dolomite } \\
\text { matrix }\end{array}$ & $\begin{array}{l}\text { Fillings vugs in dolomite } \\
\text { matrix }\end{array}$ \\
\hline $\begin{array}{l}\text { other (growth di- } \\
\text { rection/zoning) }\end{array}$ & & & \\
\hline
\end{tabular}


Geologic Unit: Middle Pamplona

Lithological Unit (Votorantim): Grey Dolomite (DO)

Sample Photograph
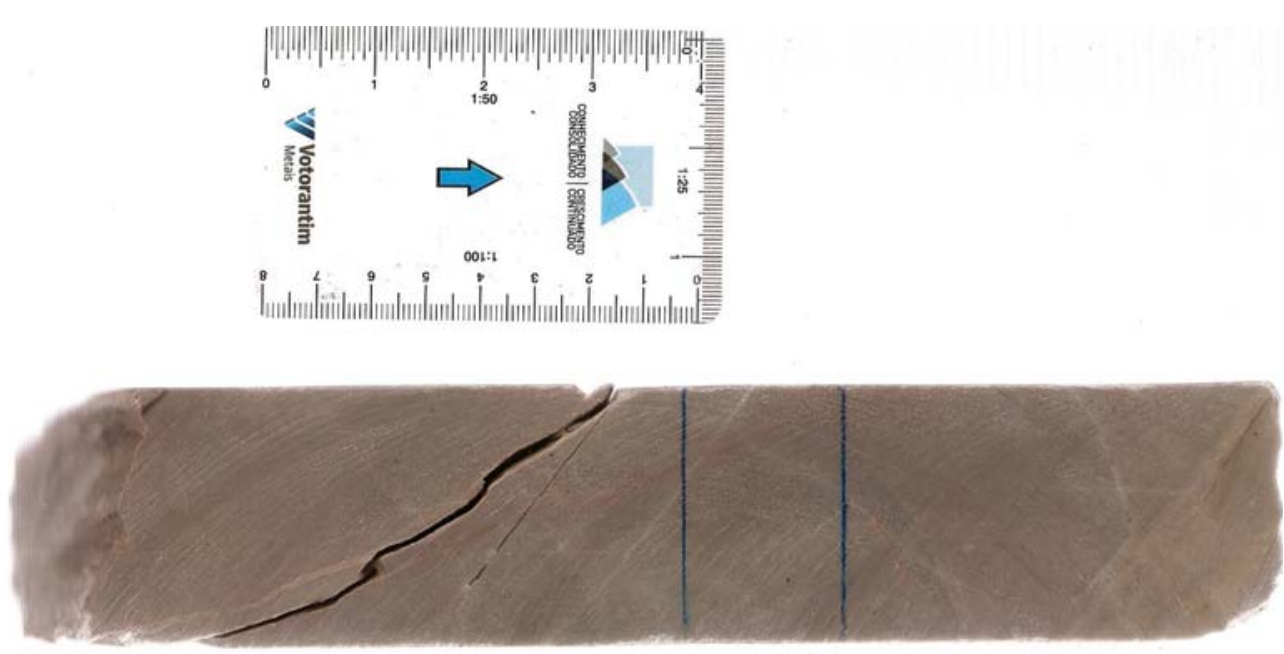

\section{VEMIF 110}

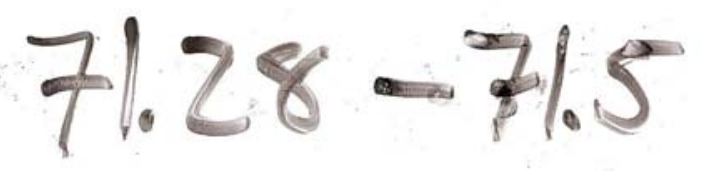

Other Sample Information: 


\begin{tabular}{|l|l|l|l|}
\hline minerals & Dolomite & Hematite & \\
\hline percentage & 100 & Very trace & \\
\hline morphology & micrite & Subangular grains in dol & \\
\hline $\begin{array}{l}\text { texture } \\
\text { transmitted opti- } \\
\text { cal properties }\end{array}$ & & & \\
\hline $\begin{array}{l}\text { Neflected optical } \\
\text { properties }\end{array}$ & & disseminated & \\
\hline $\begin{array}{l}\text { contact/ } \\
\text { relationships with } \\
\text { other minerals }\end{array}$ & & & \\
\hline $\begin{array}{l}\text { other (growth di- } \\
\text { rection/zoning) }\end{array}$ & & & \\
\hline
\end{tabular}


Geologic Unit: Upper Morro do Pinheiro

Lithological Unit (Votorantim): DO — Gray dolomite

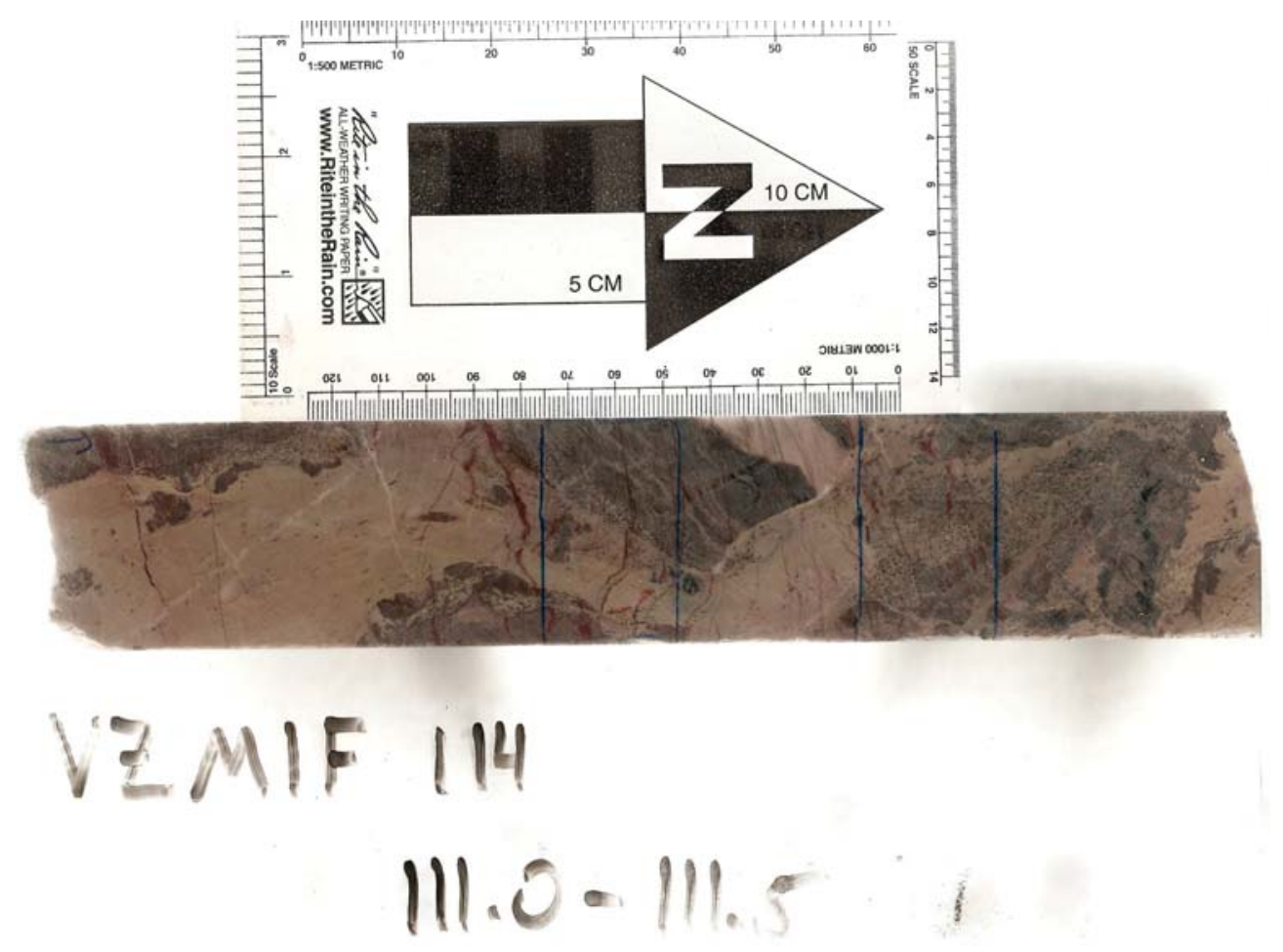

Other Sample Information: Sample taken from fault 


\begin{tabular}{|c|c|c|c|}
\hline minerals & Dolomite & Cataclasite dolomite & Cataclasite matrix \\
\hline percentage & 35 & 50 & 15 \\
\hline morphology & Subhedral sparry dolomite & $\begin{array}{l}\text { Mix of micrite and sparry } \\
\text { dol; elongated xtals }\end{array}$ & $\begin{array}{l}\text { Made of clay and some mi- } \\
\text { nor qtz }\end{array}$ \\
\hline texture & Sparry, granular & Foliated cataclasic & foliated \\
\hline $\begin{array}{l}\text { transmitted opti- } \\
\text { cal properties }\end{array}$ & & & \\
\hline $\begin{array}{l}\text { reflected optical } \\
\text { properties }\end{array}$ & & & \\
\hline $\begin{array}{l}\text { contact/ } \\
\text { relationships with } \\
\text { other minerals }\end{array}$ & $\begin{array}{l}\text { Straight contacts, broken at } \\
\text { xtal boundaries and space } \\
\text { filled in with cataclasite ma- } \\
\text { trix }\end{array}$ & $\begin{array}{l}\text { Straight contacts, minor re- } \\
\text { placement by Fe-dol veins } \\
\text { (veins are syntectonic) }\end{array}$ & $\begin{array}{l}\text { Straight contacts with all } \\
\text { mins }\end{array}$ \\
\hline $\begin{array}{l}\text { other (growth di- } \\
\text { rection/zoning) }\end{array}$ & & & \\
\hline
\end{tabular}




\begin{tabular}{|c|c|c|}
\hline minerals & Hematite & Quartz \\
\hline percentage & trace & trace \\
\hline morphology & anhedral & anhedral \\
\hline texture & $\begin{array}{l}\text { Partial vein and disseminat- } \\
\text { ed }\end{array}$ & $\begin{array}{l}\text { Disseminated, found in cata- } \\
\text { clasite and shear zone }\end{array}$ \\
\hline $\begin{array}{l}\text { transmitted opti- } \\
\text { cal properties }\end{array}$ & & \\
\hline $\begin{array}{l}\text { reflected optical } \\
\text { properties }\end{array}$ & & \\
\hline $\begin{array}{l}\text { contact/ } \\
\text { relationships with } \\
\text { other minerals }\end{array}$ & $\begin{array}{l}\text { Irregular/sheared edges, re- } \\
\text { placing cataclastic dol? }\end{array}$ & \\
\hline $\begin{array}{l}\text { other (growth di- } \\
\text { rection/zoning) }\end{array}$ & & $\begin{array}{l}\text { Straight contacts with all } \\
\text { mins }\end{array}$ \\
\hline
\end{tabular}
Paragenesis:
Time
Early
Late
Dol
Cataclasite
Fe-dol
- - - - 
Structures: Sample is in a fault zone and is a cataclasite. Cataclasite matrix fills in space in between dolomite crystals broken at crystal boundaries. Strong foliation present in dolomitic cataclasite. Fedol veins show some replacement, but are bent by foliation, suggesting they are syngenetic with faulting. 
Geologic Unit: Upper Morro do Pinheiro

Lithological Unit (Votorantim): DO_-Gray dolomite

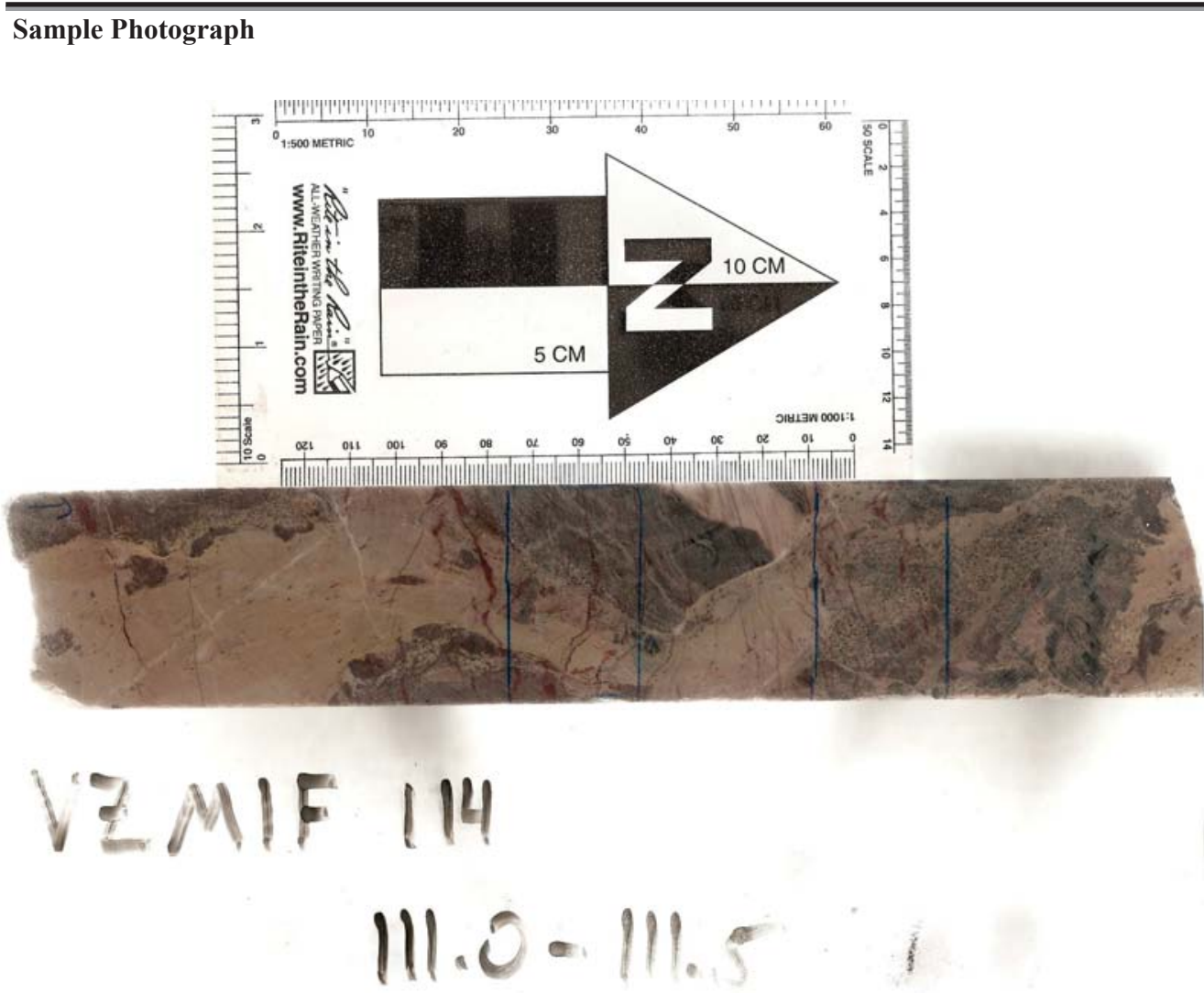

Other Sample Information: Same as 114-111A. Sample is all dolomite cataclasite (dolomite crystals in a clay/carbonate \pm qtz cataclasite matrix). Foliation present as well as minor neomorphism of carbonates. 
Geologic Unit: Upper Morro do Pinheiro

Lithological Unit (Votorantim): DO -Gray dolomite

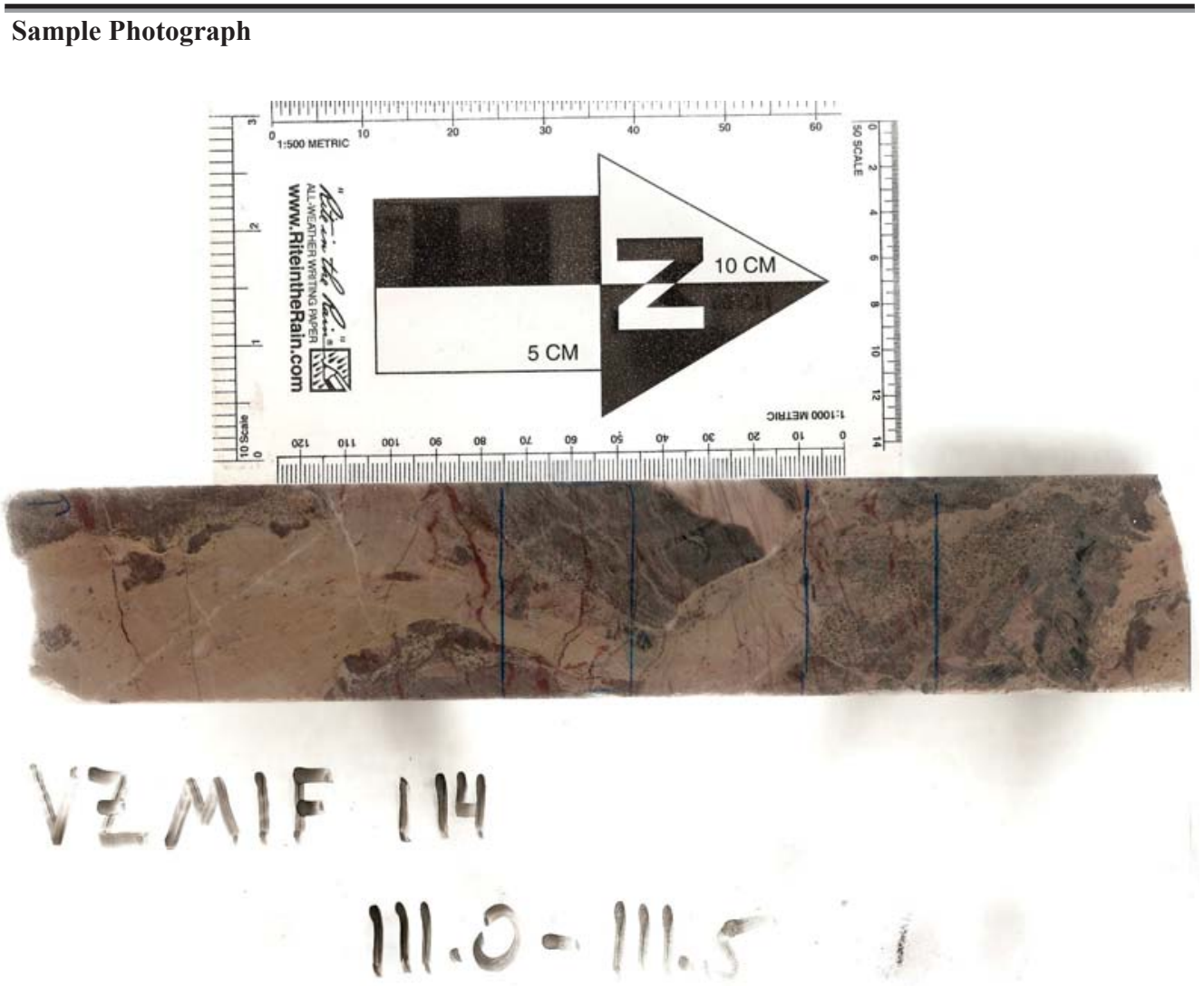

Other Sample Information: Similar to 114-111A \& B. More fault bx and less cataclasite (more distil from fault) 


\begin{tabular}{|c|c|c|}
\hline minerals & Dolomite & Quartz \\
\hline percentage & 98 & 2 \\
\hline morphology & $\begin{array}{l}\text { 1)Sparry, euhedral dol } \\
\text { 2)Micritic dol host rock }\end{array}$ & amorphous \\
\hline texture & $\begin{array}{l}\text { 1)Sparry dol is filling vugs } \\
\text { and cracks } \\
\text { 2)Micrite is part of cata- } \\
\text { clasite and host rock }\end{array}$ & $\begin{array}{l}\text { Chalcedonic layers in be- } \\
\text { tween some sparry dol }\end{array}$ \\
\hline $\begin{array}{l}\text { transmitted opti- } \\
\text { cal properties }\end{array}$ & & \\
\hline $\begin{array}{l}\text { reflected optical } \\
\text { properties }\end{array}$ & & \\
\hline $\begin{array}{l}\text { contact/ } \\
\text { relationships with } \\
\text { other minerals }\end{array}$ & $\begin{array}{l}\text { Straight contacts with all } \\
\text { mins }\end{array}$ & $\begin{array}{l}\text { Straight contacts with all } \\
\text { mins }\end{array}$ \\
\hline $\begin{array}{l}\text { other (growth di- } \\
\text { rection/zoning) }\end{array}$ & & \\
\hline
\end{tabular}
Paragenesis:
Time
Early
Late
Micrite
Fault
Sparrite
Qtz

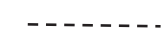


Structures: Sample is from a more "distal" location to the fault in the 111.0-111.5 m interval. The dolostone has been fractured and secondary, hydrothermal dolomite with small chalcedonic quartz intercalations is crystallizing into vugs and fractures. Some of the hydrothermal dolomite is baroque dolomite. Quartz fills the centers of some vugs, but also appears in between dolomite growth zones, indicating small siliceous "pulses", ending with quartz.

Paragenetic Sequence: micrite $\rightarrow$ fault $\rightarrow$ spar + qtz

Paragenetic Reasoning: growth of minerals in veins

Sample Highlights: Fault bx, baroque dolomite 
Geologic Unit: Hydrothermal Breccia

Lithological Unit (Votorantim): Massive Silicated in BH

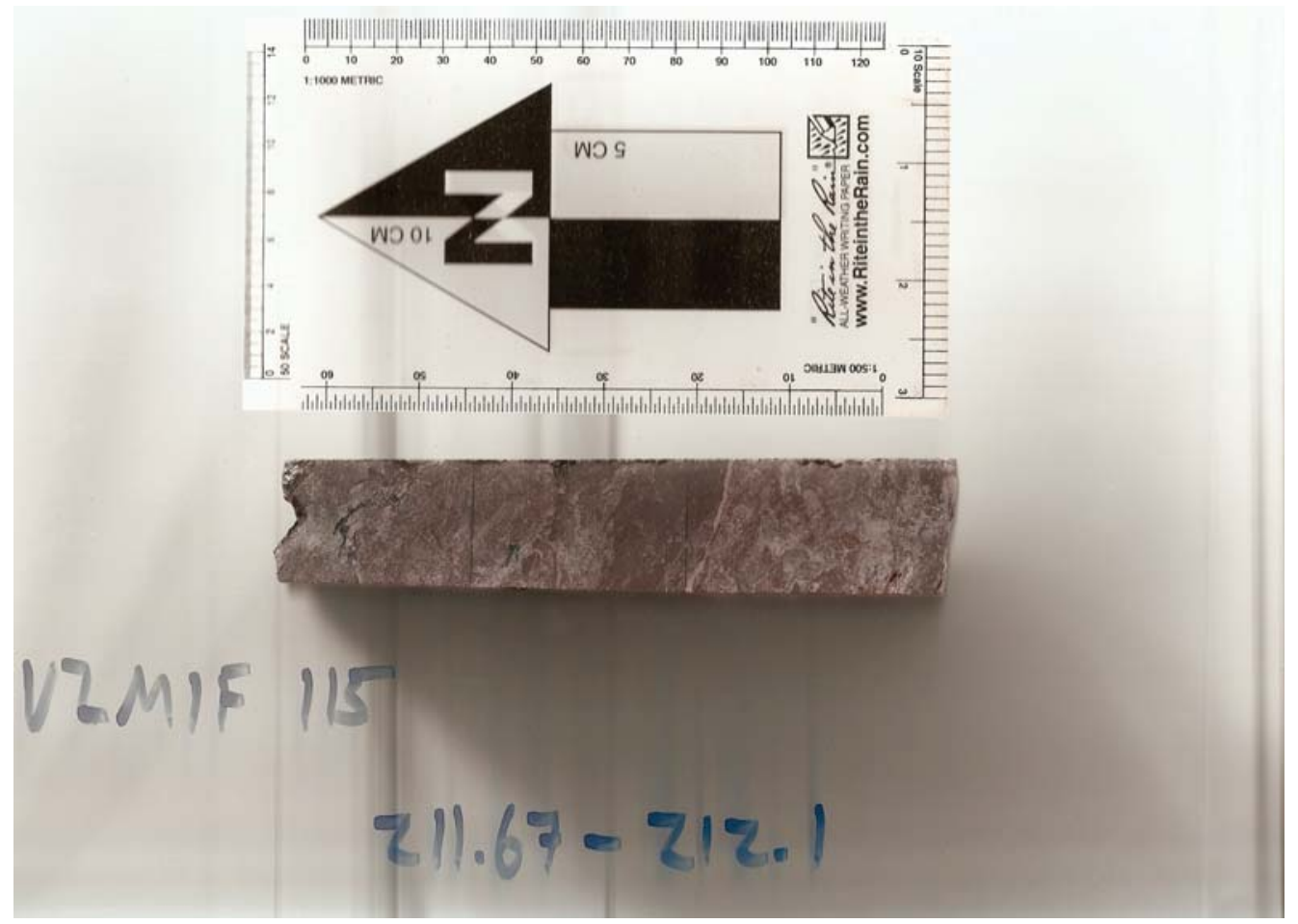

Other Sample Information: 


\begin{tabular}{|c|c|c|c|}
\hline minerals & Dolomite & W1 & Chl \\
\hline percentage & 65 & 10 & 4 \\
\hline morphology & $\begin{array}{l}\text { Fine xtal dol "shreddy" } \\
\text { looking }\end{array}$ & Granular xtals & Fibrous, Fe-rich \\
\hline texture & $\begin{array}{l}\text { Semi-massive, some algal } \\
\text { layering still preserved }\end{array}$ & disseminated & vein \\
\hline $\begin{array}{l}\text { transmitted opti- } \\
\text { cal properties }\end{array}$ & & & \\
\hline $\begin{array}{l}\text { reflected optical } \\
\text { properties }\end{array}$ & & & \\
\hline $\begin{array}{l}\text { contact/ } \\
\text { relationships with } \\
\text { other minerals }\end{array}$ & $\begin{array}{l}\text { Irregular contacts with all } \\
\text { minerals, replacing all min- } \\
\text { erals except hm/frk }\end{array}$ & $\begin{array}{l}\text { Irregular contacts with hm } \\
\text { and dol. Seems to initially } \\
\text { replaced dol, but is now be- } \\
\text { ing replaced by dol. Coeval } \\
\text { with hm? }\end{array}$ & $\begin{array}{l}\text { Cuts all minerals, but is then } \\
\text { truncated by a fault }\end{array}$ \\
\hline $\begin{array}{l}\text { other (growth di- } \\
\text { rection/zoning) }\end{array}$ & Algal layering & $\begin{array}{l}\text { Hollowed-out xtals of wl } \\
\text { filled with hm and surrouned } \\
\text { by hm }\end{array}$ & \\
\hline
\end{tabular}




\begin{tabular}{|c|c|c|c|}
\hline minerals & Qtz & $\mathrm{Hm}$ & Frk \\
\hline percentage & 1 & 20 & trace \\
\hline morphology & Anhedral & $\begin{array}{l}\text { Some "granular" hm, mostly } \\
\text { massive crystalline }\end{array}$ & Small granular xtals \\
\hline texture & disseminated & Massive vein & Associated with hm \\
\hline $\begin{array}{l}\text { transmitted opti- } \\
\text { cal properties }\end{array}$ & & & \\
\hline $\begin{array}{l}\text { reflected optical } \\
\text { properties }\end{array}$ & & & \\
\hline $\begin{array}{l}\text { contact/ } \\
\text { relationships with } \\
\text { other minerals }\end{array}$ & $\begin{array}{l}\text { Irregular, being replaced by } \\
\mathrm{hm}\end{array}$ & Irregular, replacing all mins & See hm \\
\hline $\begin{array}{l}\text { other (growth di- } \\
\text { rection/zoning) }\end{array}$ & & & \\
\hline
\end{tabular}

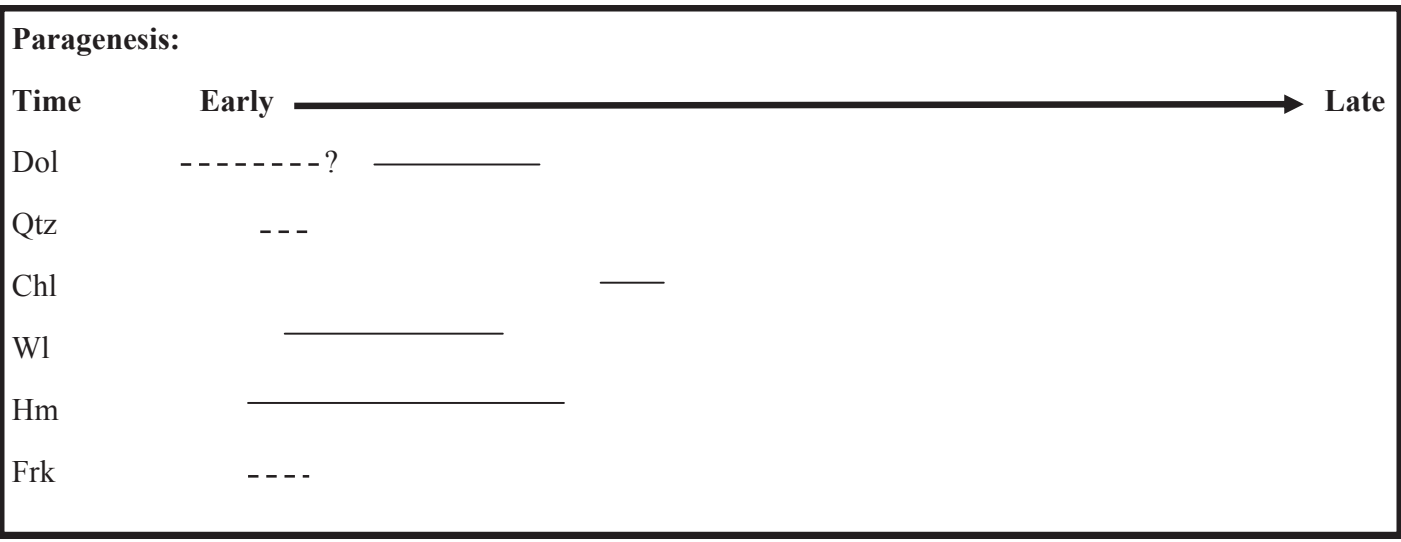


Structures: Sample has both brittle/ductile brecciation/shearing as seen in broken and sheared hm.

Faults truncate late chlorite, indicating that tectonism was still active even during late mineralization.

Dolomite seems to be late and is very fine. 
Geologic Unit: Hydrothermal Breccia

Lithological Unit (Votorantim): Massive Silicate of BH

Sample Photograph

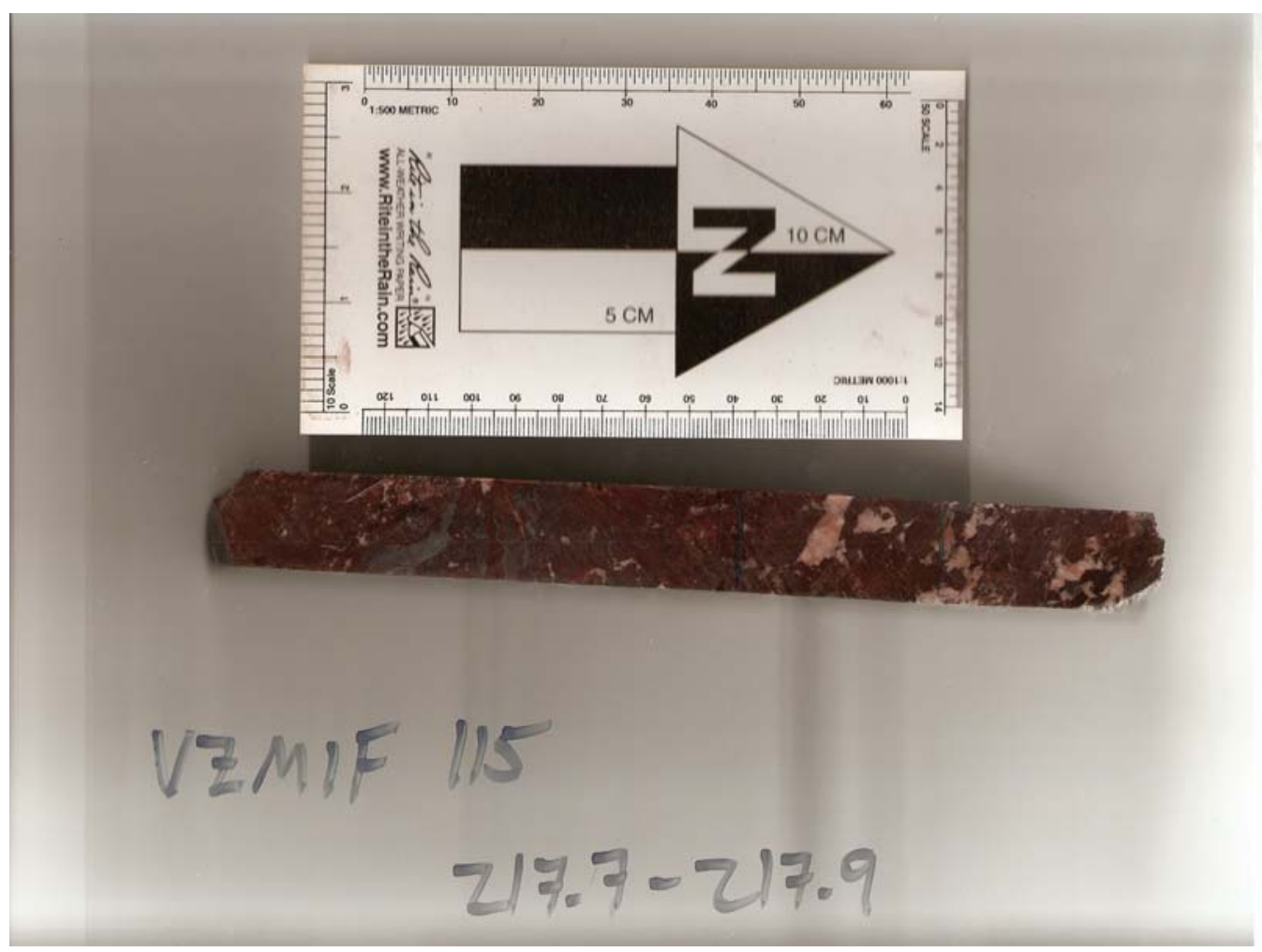

Other Sample Information: 


\begin{tabular}{|c|c|c|c|}
\hline minerals & Dol & $\mathrm{Hm}$ & Frk \\
\hline percentage & 30 & 70 & Very trace \\
\hline morphology & $\begin{array}{l}\text { Wide range of crystal sizes } \\
\text { from small to microcrystal- } \\
\text { line (original dol?) }\end{array}$ & Euhedral crystal laths & Ehudral xtals \\
\hline texture & brecciated & Massive & $\begin{array}{l}\text { Very disseminated, associat- } \\
\text { ed with } \mathrm{hm}\end{array}$ \\
\hline $\begin{array}{l}\text { transmitted opti- } \\
\text { cal properties }\end{array}$ & & & \\
\hline $\begin{array}{l}\text { reflected optical } \\
\text { properties }\end{array}$ & & & \\
\hline $\begin{array}{l}\text { contact/ } \\
\text { relationships with } \\
\text { other minerals }\end{array}$ & $\begin{array}{l}\text { Dol is replacing itself and } \\
\text { being replaced by hm }\end{array}$ & $\begin{array}{l}\text { Irregular, replacing/ } \\
\text { overgrowing dol along non- } \\
\text { specific and specific zones }\end{array}$ & $\begin{array}{l}\text { Straight contacts with all } \\
\text { mins, associated with hm }\end{array}$ \\
\hline $\begin{array}{l}\text { other (growth di- } \\
\text { rection/zoning) }\end{array}$ & $\begin{array}{l}\text { Preferential replacement } \\
\text { along certain zones in dol } \\
\text { and in specific algal layers }\end{array}$ & & $\begin{array}{l}\text { Contains some hm exsolu- } \\
\text { tion texture }\end{array}$ \\
\hline
\end{tabular}

Paragenesis:

Time

Early Late

Dol

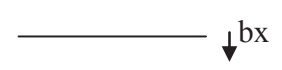

$\mathrm{Hm}$

Frk 
Structures: Sample is both brecciated and sheared. Preferential replacement of certain dolomite zones (don't know why) 
Geologic Unit: Hydrothermal Breccia

Lithological Unit (Votorantim): Massive Silicate in BH

Sample Photograph

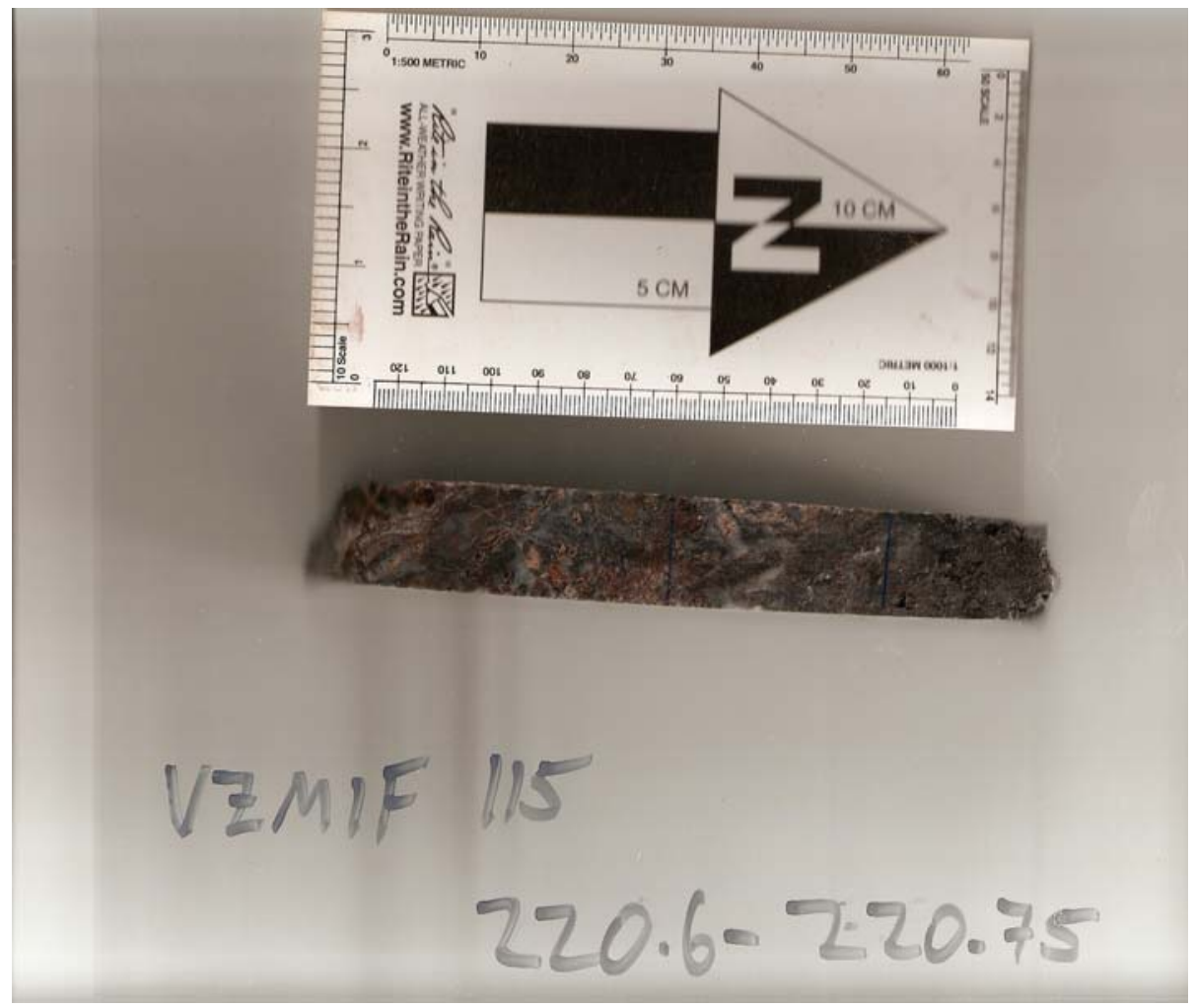

Other Sample Information: SULFIDES PRESENT 


\begin{tabular}{|c|c|c|c|}
\hline minerals & Willemite & Quartz & Hematite \\
\hline percentage & 70 & 10 & 5 \\
\hline morphology & $\begin{array}{l}\text { Euhedral granular and lath } \\
\text { crystals }\end{array}$ & Anhedral crystals & $\begin{array}{l}\text { Massive crystal laths and } \\
\text { small disseminated crystal } \\
\text { grains in gn }\end{array}$ \\
\hline texture & massive & disseminated & $\begin{array}{l}\text { Minor massive shears and } \\
\text { disseminated in gn }\end{array}$ \\
\hline $\begin{array}{l}\text { transmitted opti- } \\
\text { cal properties }\end{array}$ & & & \\
\hline $\begin{array}{l}\text { reflected optical } \\
\text { properties }\end{array}$ & & & \\
\hline $\begin{array}{l}\text { contact/ } \\
\text { relationships with } \\
\text { other minerals }\end{array}$ & $\begin{array}{l}\text { Irregular contacts with qtz, } \\
\text { replacing qtz. Being re- } \\
\text { placed by hm and frk. Cross } \\
\text {-cut by gn }+ \text { frk }+\mathrm{hm}+\mathrm{cv}\end{array}$ & Being replaced by wl and gn & $\begin{array}{l}\text { Straight edges with gn, re- } \\
\text { placing wl and qtz }\end{array}$ \\
\hline $\begin{array}{l}\text { other (growth di- } \\
\text { rection/zoning) }\end{array}$ & & & Being exsolved by frk \\
\hline
\end{tabular}




\begin{tabular}{|c|c|c|c|}
\hline minerals & Franklinite & Covellite & Galena \\
\hline percentage & 5 & trace & 10 \\
\hline morphology & Granular xtals & Small shreddy xtals & massive \\
\hline texture & disseminated & Very disseminated & vein \\
\hline $\begin{array}{l}\text { transmitted opti- } \\
\text { cal properties }\end{array}$ & & & \\
\hline $\begin{array}{l}\text { reflected optical } \\
\text { properties }\end{array}$ & & & Triangular facets \\
\hline $\begin{array}{l}\text { contact/ } \\
\text { relationships with } \\
\text { other minerals }\end{array}$ & $\begin{array}{l}\text { Straight contacts with gn and } \\
\mathrm{hm}\end{array}$ & Shreddy contacts with gn & $\begin{array}{l}\text { Straight contacts with wl, } \\
\text { replacing qtz }\end{array}$ \\
\hline $\begin{array}{l}\text { other (growth di- } \\
\text { rection/zoning) }\end{array}$ & Exsolving hm & & \\
\hline
\end{tabular}

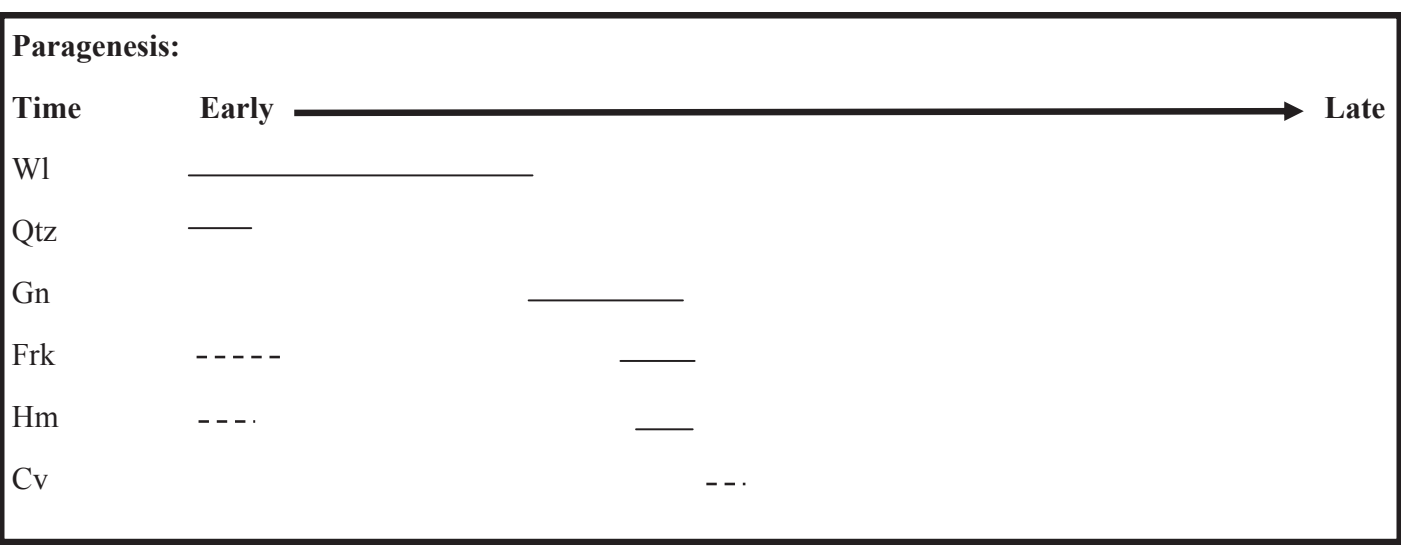


Structures: late sulfide fractures, minor brecciation associated with wl mineralization 
Geologic Unit: Hydrothermal Breccia

Lithological Unit (Votorantim): Massive Silicated of BH

Sample Photograph

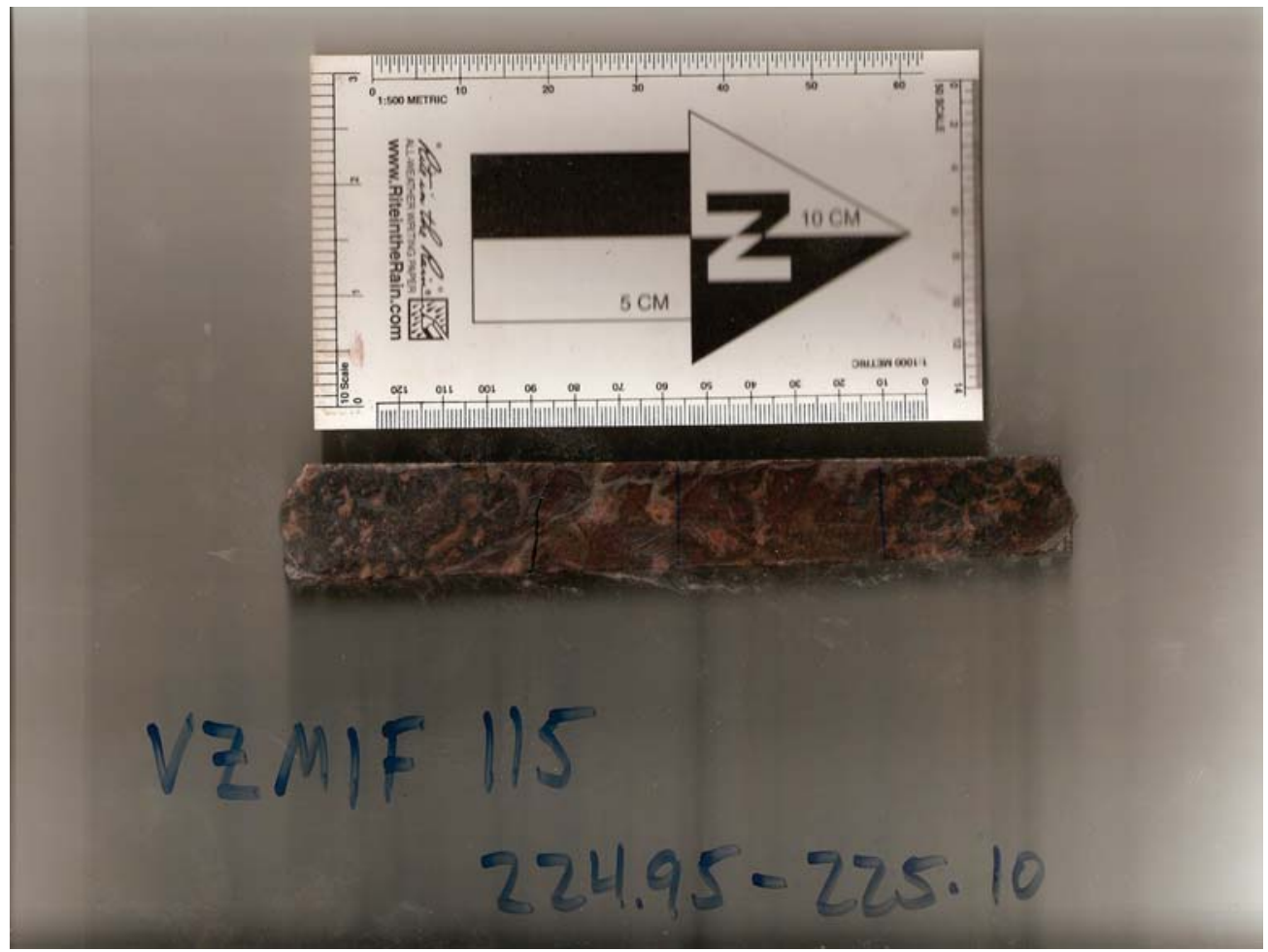

Other Sample Information: Same as 141-166 
Geologic Unit: Upper Morro do Pinheiro

Lithological Unit (Votorantim): Grey Dolomite (DO)

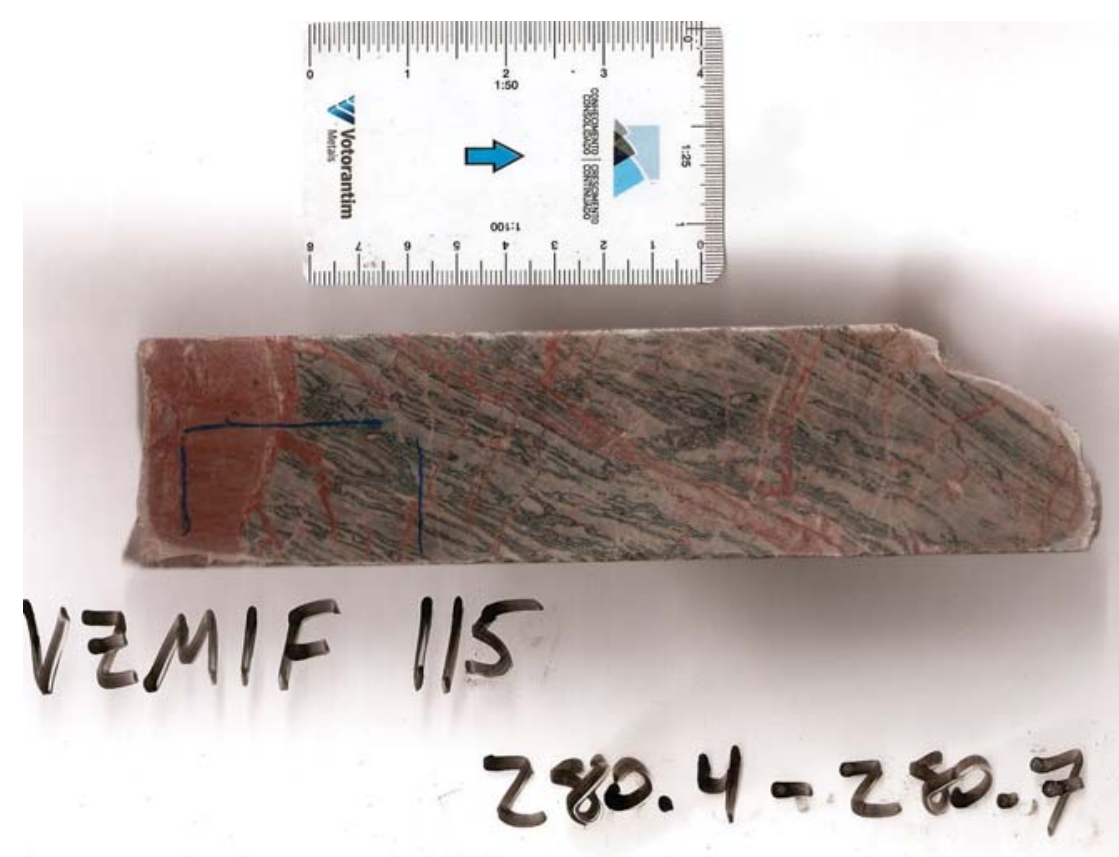

Other Sample Information: 


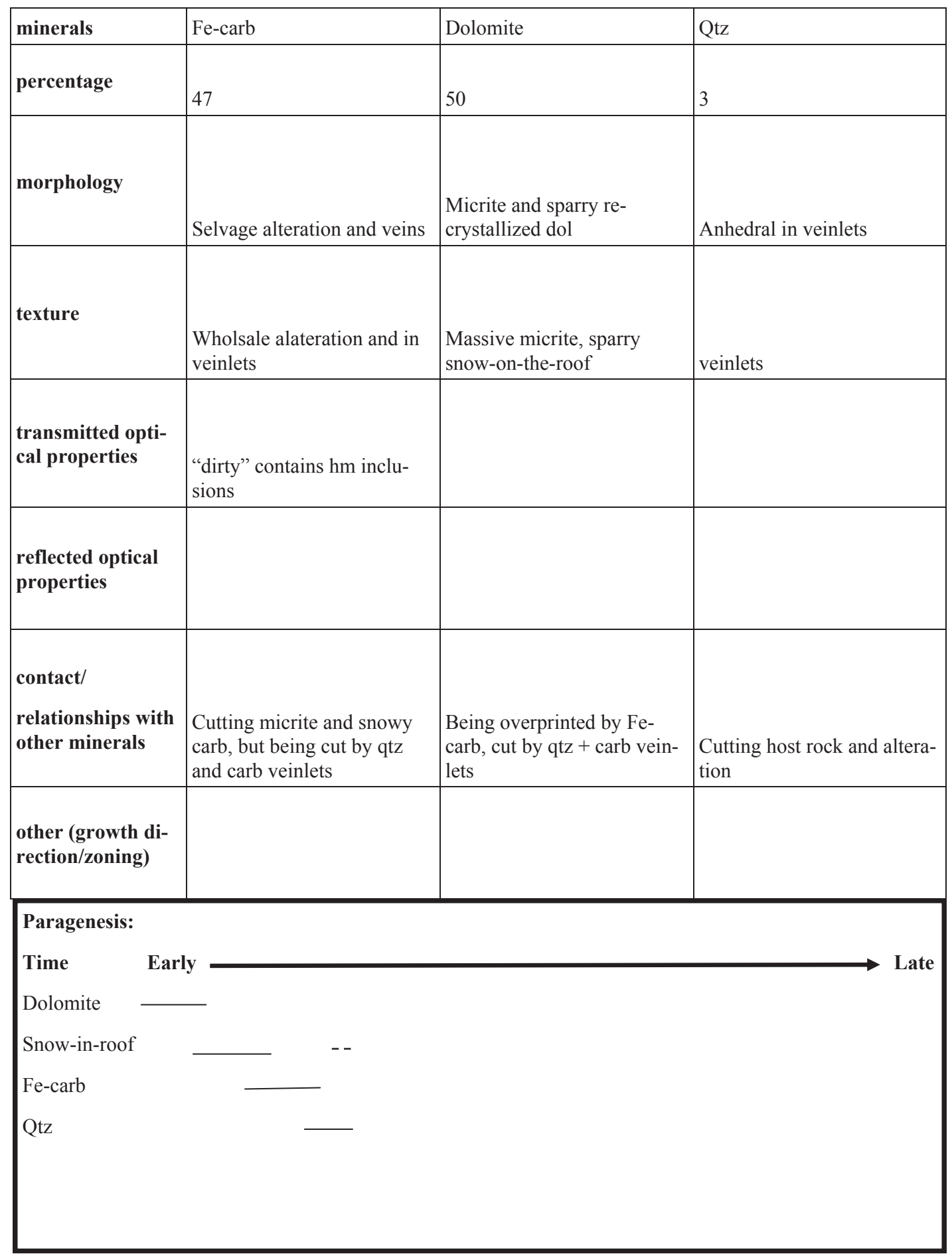




\section{Geologic Unit:}

\section{Lithological Unit (Votorantim):}

Sample Photograph

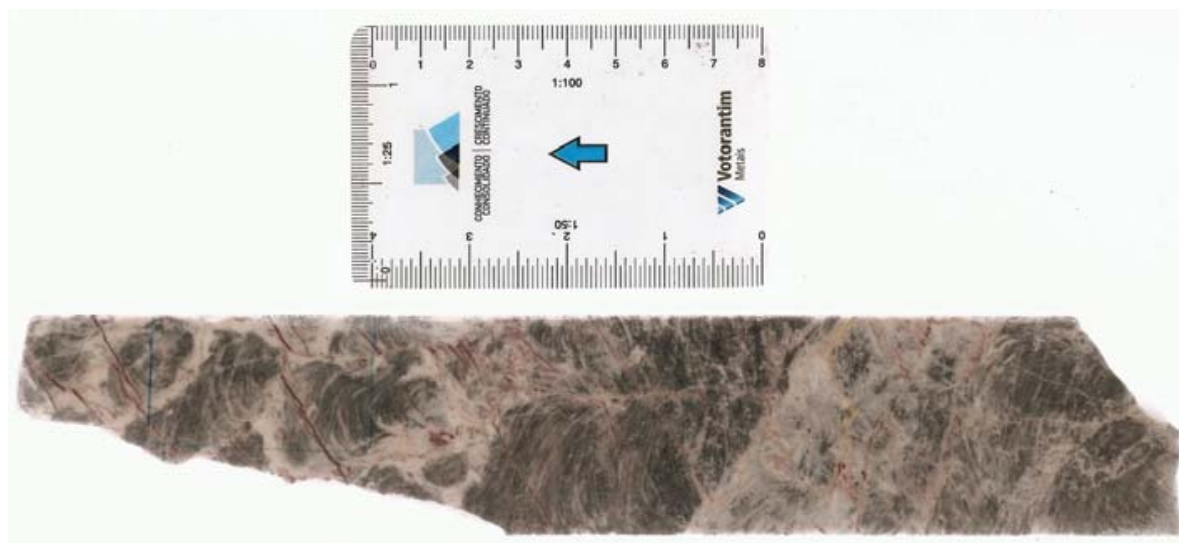

VZMIF $\| 8$

$132.03 \cdot 152.4$

Other Sample Information: 


\begin{tabular}{|c|c|c|c|}
\hline minerals & Dolomite & Fe-carb & Hematite \\
\hline percentage & 95 & 5 & trace \\
\hline morphology & $\begin{array}{l}\text { Micritic stromatolite bound- } \\
\text { stone }\end{array}$ & $\begin{array}{l}\text { Sparite vein with hm inclu- } \\
\text { sions }\end{array}$ & Included in Fe-carb \\
\hline texture & $\begin{array}{l}\text { Stromoatolites with some } \\
\text { small intraformational brec- } \\
\text { cias with carbonate and sili- } \\
\text { ca cement }\end{array}$ & Vein & $\begin{array}{l}\text { Disseminated in Fe-carb } \\
\text { vein }\end{array}$ \\
\hline $\begin{array}{l}\text { transmitted opti- } \\
\text { cal properties }\end{array}$ & & $\begin{array}{l}\text { Drirty looking b/c of hm in- } \\
\text { clusions }\end{array}$ & \\
\hline $\begin{array}{l}\text { reflected optical } \\
\text { properties }\end{array}$ & & & \\
\hline $\begin{array}{l}\text { contact/ } \\
\text { relationships with } \\
\text { other minerals }\end{array}$ & See Fe-carb & $\begin{array}{l}\text { Cuts across boundstone, fair- } \\
\text { ly straight edges (brittle?) }\end{array}$ & $\begin{array}{l}\text { Blebby and irregular, replac- } \\
\text { ing Fe-carb? }\end{array}$ \\
\hline $\begin{array}{l}\text { other (growth di- } \\
\text { rection/zoning) }\end{array}$ & & & \\
\hline
\end{tabular}




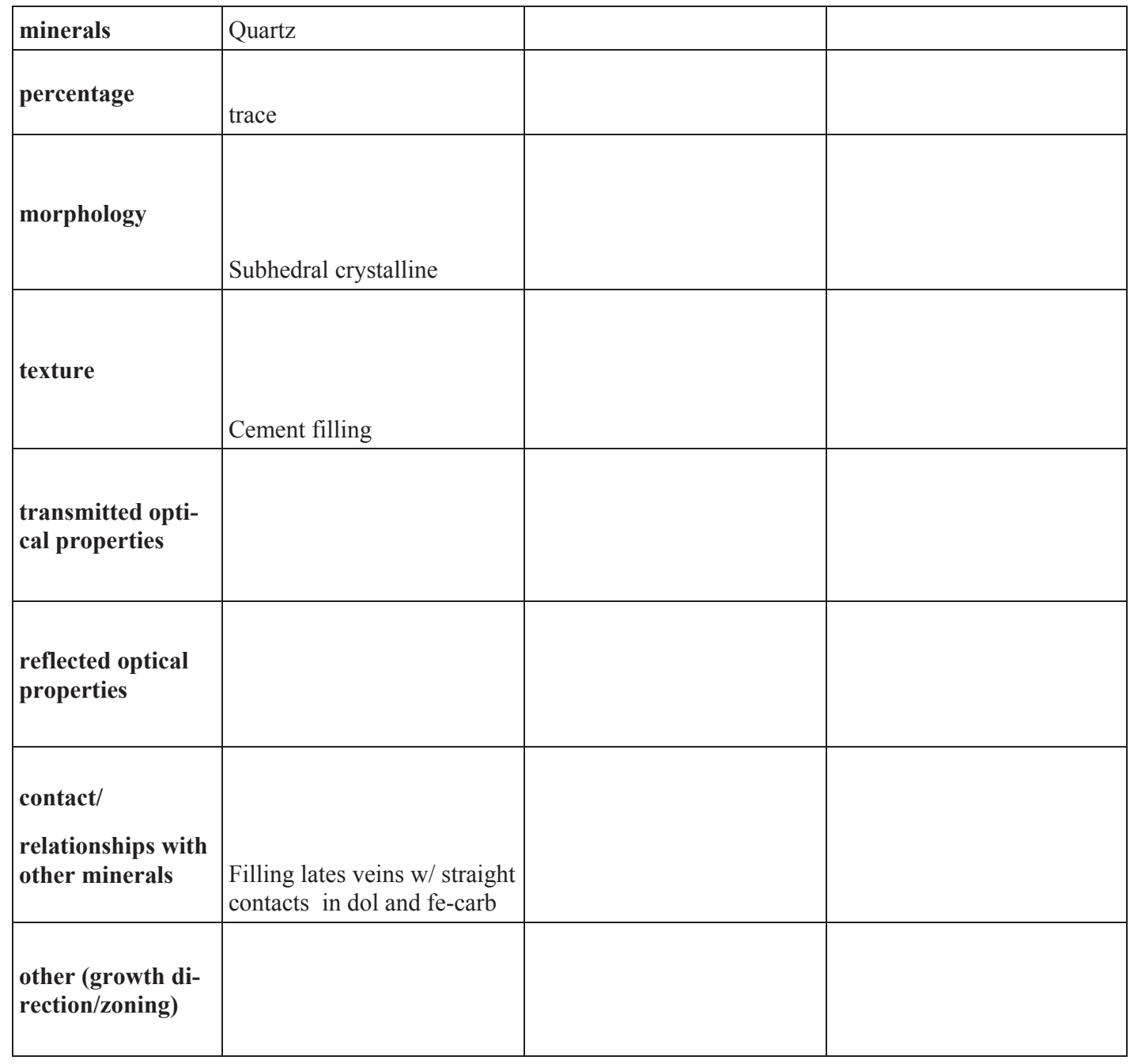

Paragenesis:

Time

Early

Late

Dol

Fe-carb

$\mathrm{Hm}$

Qtz 
Geologic Unit: Upper?/Lower Morro do Pinheiro?

Lithological Unit (Votorantim): Marl (MG)

Sample Photograph
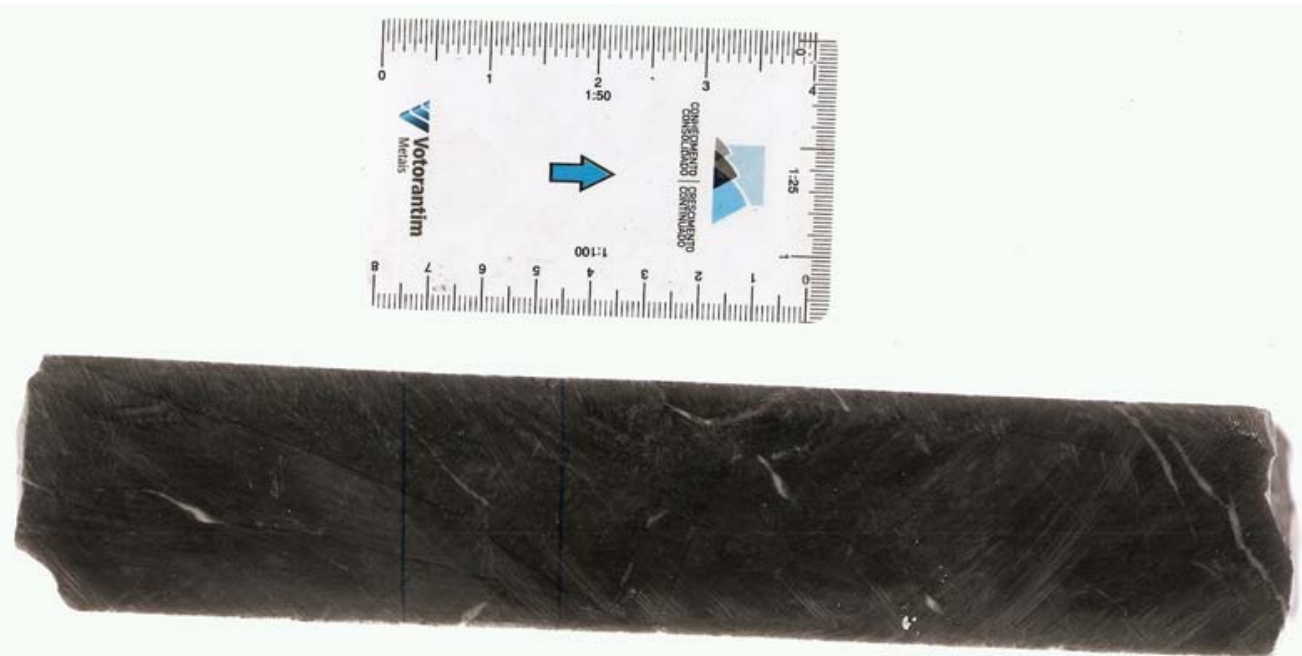

VEMIF
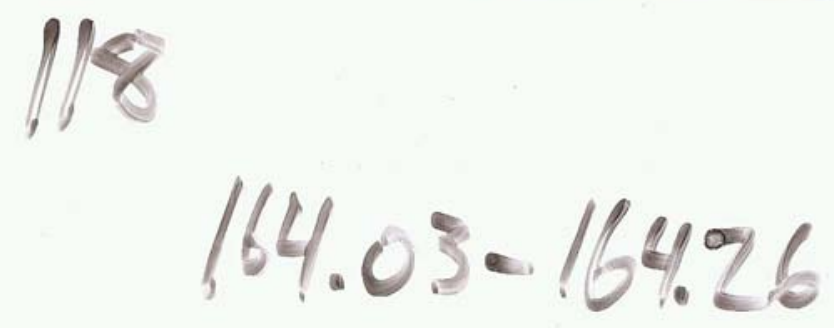

Other Sample Information: 


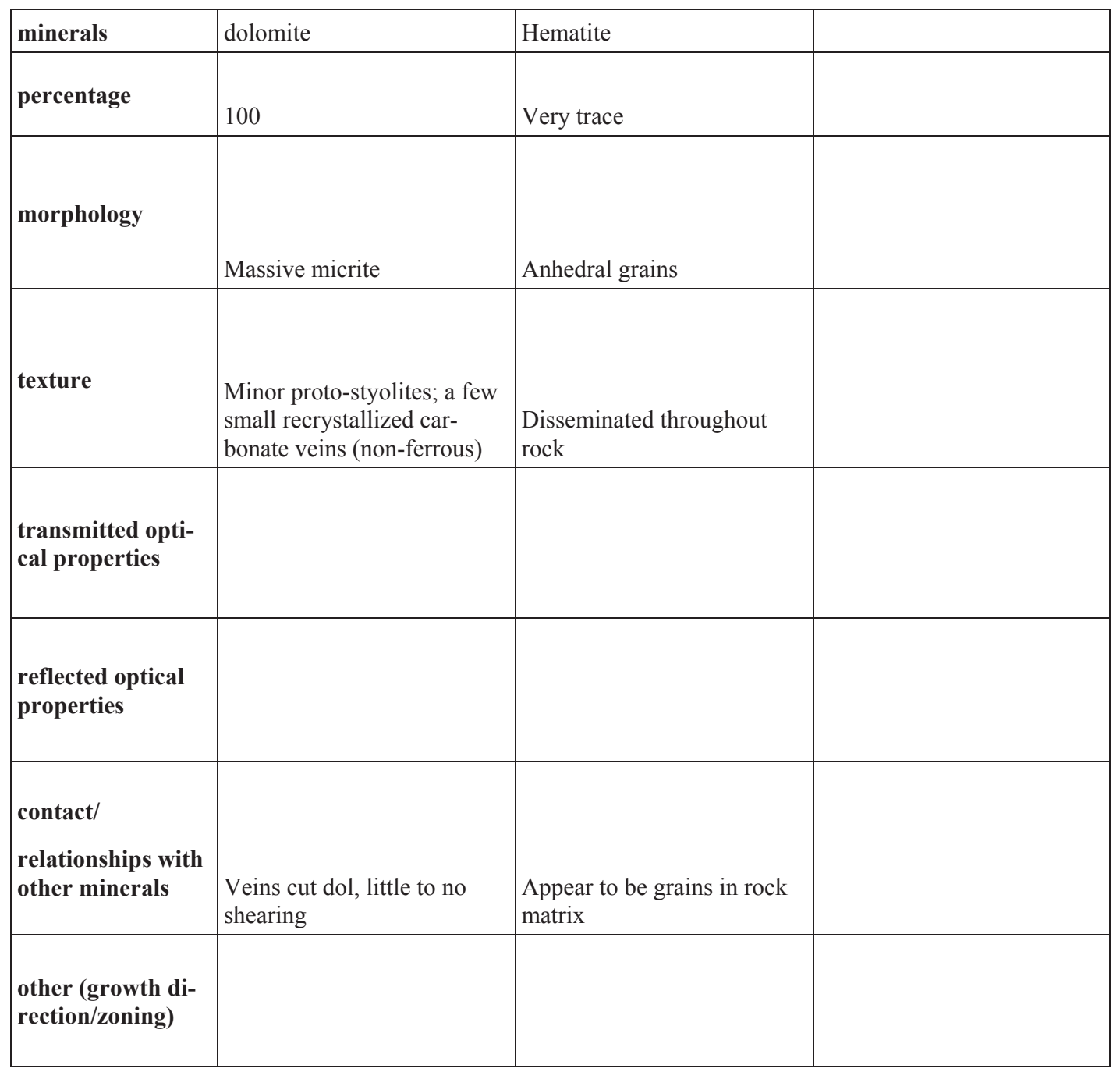


Geologic Unit: Hydrothermal Breccia

Lithological Unit (Votorantim): Massive Silicate (SM in BH)

Sample Photograph

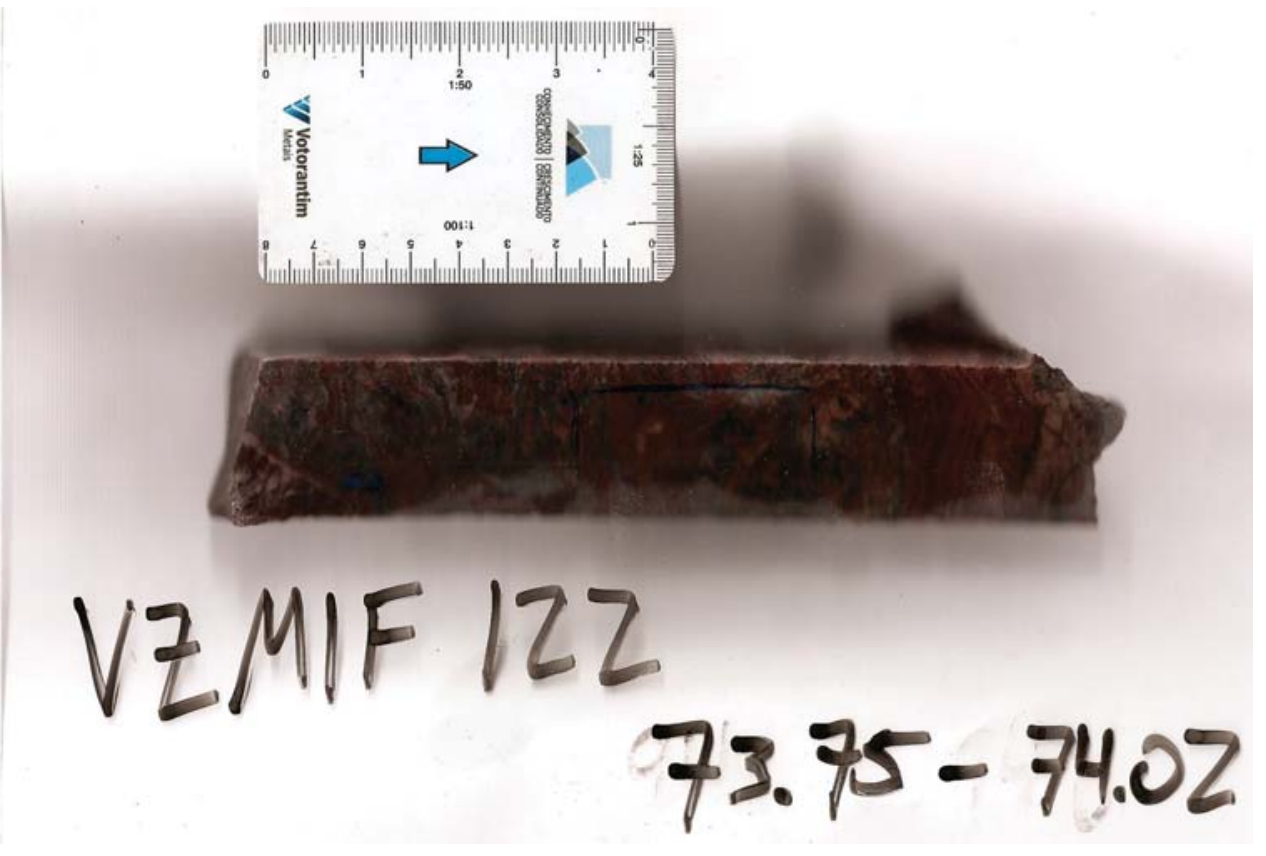

Other Sample Information: 


\begin{tabular}{|c|c|c|c|}
\hline minerals & Willemite & Hematite & Franklinte \\
\hline percentage & 45 & 12 & trace \\
\hline morphology & $\begin{array}{l}\text { 1st gen. granulare and } 2 \text { nd } \\
\text { gen. lath }\end{array}$ & $\begin{array}{l}\text { Small anhedral xtals includ- } \\
\text { ed in wl and large laths and } \\
\text { needles }\end{array}$ & Subhedral xtals \\
\hline texture & $\begin{array}{l}\text { Semi-massive replacement } \\
1 \text { st gen replacing carb and } \\
\text { qtz, } 2 \text { nd gen replacing } 1 \text { st } \\
\text { gen wl, carb and qtz }\end{array}$ & $\begin{array}{l}\text { Replacement net-like texture } \\
\text { and inclusions }\end{array}$ & disseminated \\
\hline $\begin{array}{l}\text { transmitted opti- } \\
\text { cal properties }\end{array}$ & & & \\
\hline $\begin{array}{l}\text { reflected optical } \\
\text { properties }\end{array}$ & & & \\
\hline $\begin{array}{l}\text { contact/ } \\
\text { relationships with } \\
\text { other minerals }\end{array}$ & $\begin{array}{l}\text { Shreddy contacts with wl } \\
\text { and carb, more jagged con- } \\
\text { tacts w/ qtz and hm, being } \\
\text { replaced by hm, replacing } \\
\text { qtz and carb }\end{array}$ & $\begin{array}{l}\text { Replacing wl, carb, and qtz, } \\
\text { jagged contacts w/ these } \\
\text { mins }\end{array}$ & Replacing wl, included in wl \\
\hline $\begin{array}{l}\text { other (growth di- } \\
\text { rection/zoning) }\end{array}$ & & $\begin{array}{l}\text { Preferential replacement of } \\
\text { some carbonate overgrowths }\end{array}$ & \\
\hline
\end{tabular}




\begin{tabular}{|c|c|c|}
\hline minerals & Quartz & Carbonate \\
\hline percentage & 3 & 40 \\
\hline morphology & anhedral & $\begin{array}{l}\text { Anhedral hydrothermal spar- } \\
\text { rite } \\
\text { Fine-grained carb in veinlets }\end{array}$ \\
\hline texture & dissminated & $\begin{array}{l}\text { Hydrothermal rock-forming, } \\
\text { being replaced } \\
\text { Small, late cross-cutting } \\
\text { veinlets }\end{array}$ \\
\hline $\begin{array}{l}\text { transmitted opti- } \\
\text { cal properties }\end{array}$ & & \\
\hline $\begin{array}{l}\text { reflected optical } \\
\text { properties }\end{array}$ & & \\
\hline $\begin{array}{l}\text { contact/ } \\
\text { relationships with } \\
\text { other minerals }\end{array}$ & $\begin{array}{l}\text { Irregular and jagged contacts } \\
\text { with all mins, replacing carb, } \\
\text { but being replaced by wl and } \\
\text { hm }\end{array}$ & $\begin{array}{l}\text { Early stage being replaced } \\
\text { by all mins, late stage vein- } \\
\text { lets cutting all mins }\end{array}$ \\
\hline $\begin{array}{l}\text { other (growth di- } \\
\text { rection/zoning) }\end{array}$ & & \\
\hline
\end{tabular}

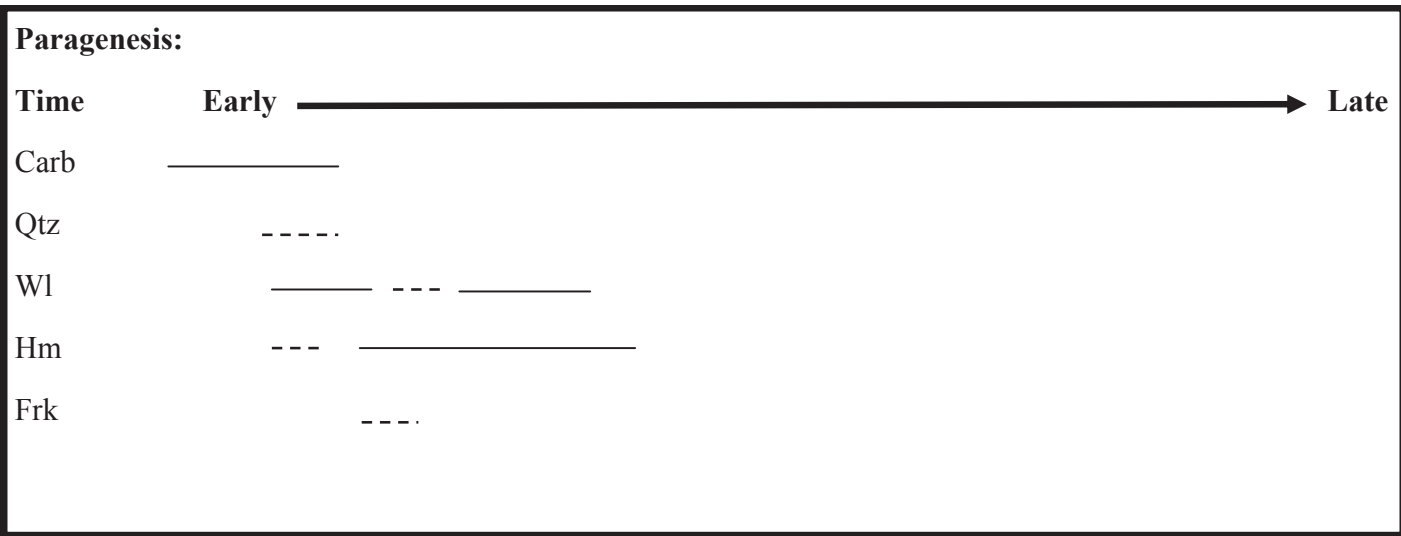


Geologic Unit: Hydrothermal Breccia

Lithological Unit (Votorantim): Massive Silicate (in BH)

Sample Photograph

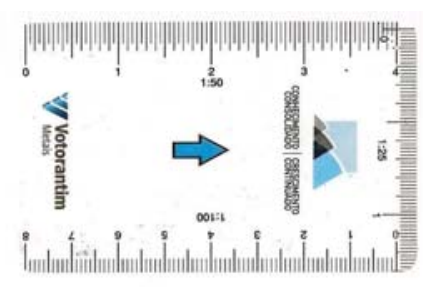

VZMIF IZZ

$75.8=76.45$

Other Sample Information: 


\begin{tabular}{|c|c|c|c|}
\hline minerals & Willemite & Hematite & Franklinte \\
\hline percentage & 70 & 10 & trace \\
\hline morphology & $\begin{array}{l}\text { 1st gen. granular } \\
\text { 2nd gen. lath-like and splays }\end{array}$ & $\begin{array}{l}\text { Needle and lath-shaped } \\
\text { xtals, oriented along veinlets }\end{array}$ & $\begin{array}{l}\text { Subhedral, proximal to } \mathrm{hm} \\
\text { but in wl }\end{array}$ \\
\hline texture & Massive & $\begin{array}{l}\text { Filling veinlets in a net- } \\
\text { texture }\end{array}$ & disseminated \\
\hline $\begin{array}{l}\text { transmitted opti- } \\
\text { cal properties }\end{array}$ & & & \\
\hline $\begin{array}{l}\text { reflected optical } \\
\text { properties }\end{array}$ & & & \\
\hline $\begin{array}{l}\text { contact/ } \\
\text { relationships with } \\
\text { other minerals }\end{array}$ & $\begin{array}{l}\text { Replacing qtz and carb and } \\
\text { itself, jagged contacts with } \\
\text { these mins } \\
\text { Being replaced by hm and } \\
\text { frk, also jagged contacts }\end{array}$ & $\begin{array}{l}\text { Replacing wl, qtz, and carb, } \\
\text { jagged and irregular contacts } \\
\text { with these mins }\end{array}$ & $\begin{array}{l}\text { Straight to embayed contacts } \\
\text { with wl }\end{array}$ \\
\hline $\begin{array}{l}\text { other (growth di- } \\
\text { rection/zoning) }\end{array}$ & $\begin{array}{l}\text { Some } 1 \text { st gen. wl xtals have } \\
\text { been recrystallized and man- } \\
\text { tled with more wl }\end{array}$ & & \\
\hline
\end{tabular}




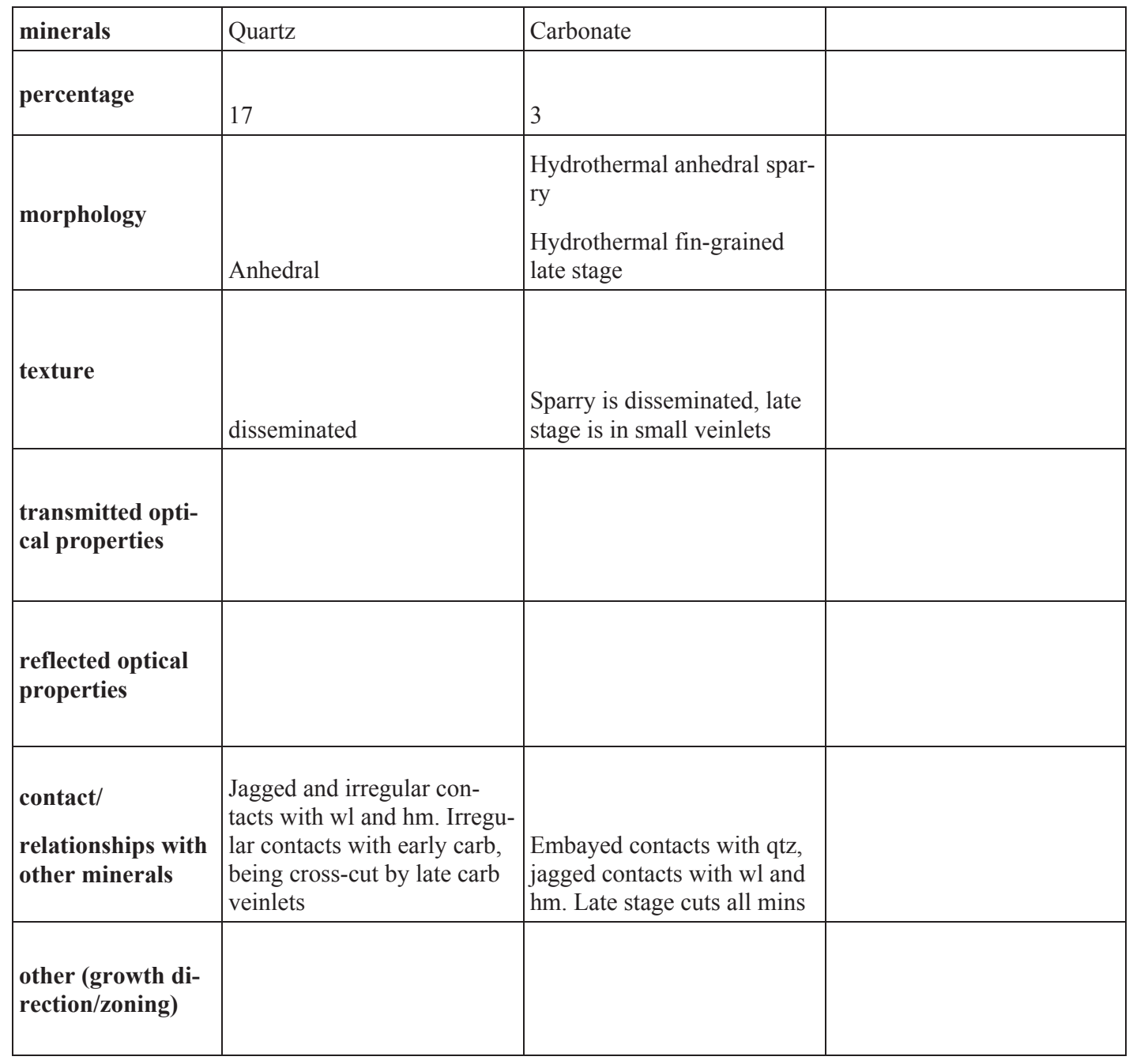

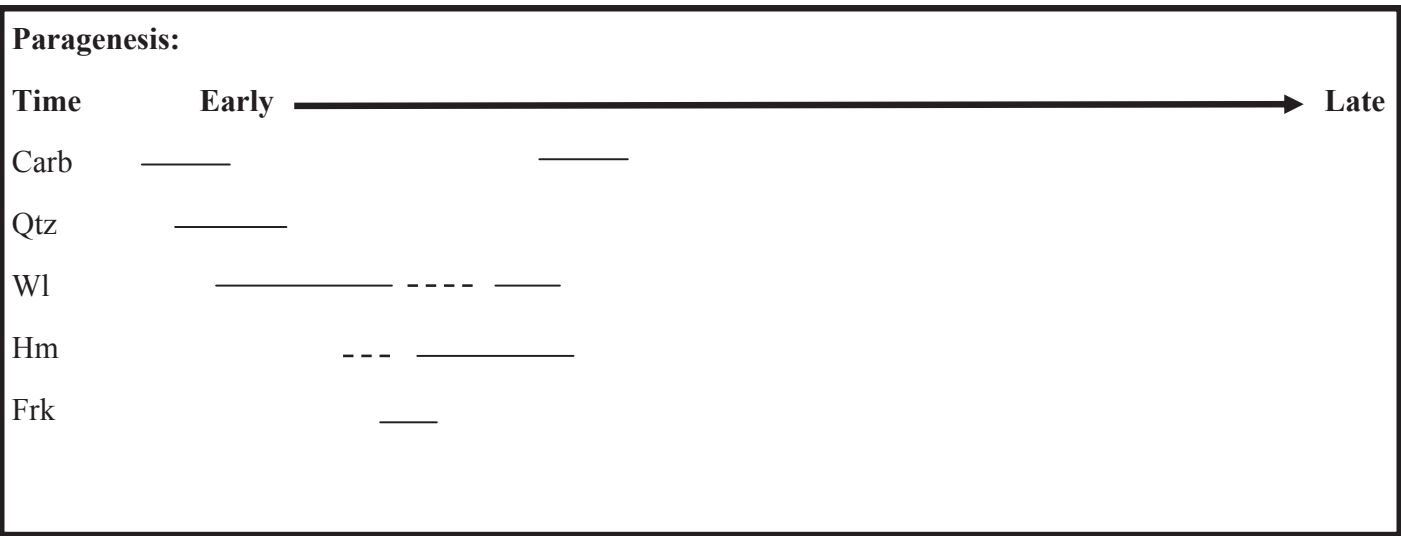


Structures: Qtz and other mins cut by brittle faults. Hm seems to be more ductile and flows around minerals as it replaces them, but it's still brittle deformation 
Geologic Unit: Lower Pamplona

Lithological Unit (Votorantim): Pink dolomite (DG)

Sample Photograph
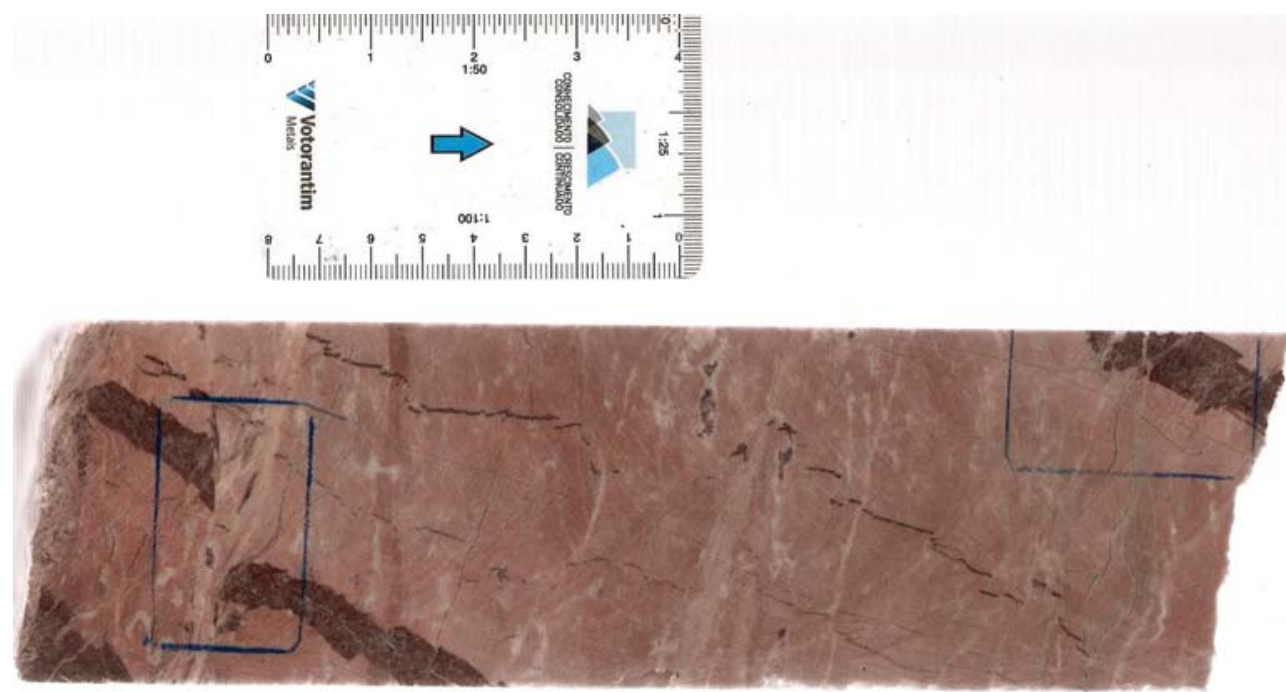

\section{VZMIF 122}

$$
94.25 \cdot 94.6
$$

Other Sample Information: 


\begin{tabular}{|c|c|c|c|}
\hline minerals & Dolomite & Fe-carbonate & Quartz \\
\hline percentage & 35 & 25 & 20 \\
\hline morphology & Micrite forming rock & Sparry veinlets & Anhedral, veinlets \\
\hline texture & Fine xtals of micrite & Veinlets & veinlets \\
\hline $\begin{array}{l}\text { transmitted opti- } \\
\text { cal properties }\end{array}$ & & & \\
\hline $\begin{array}{l}\text { reflected optical } \\
\text { properties }\end{array}$ & & & \\
\hline $\begin{array}{l}\text { contact/ } \\
\text { relationships with } \\
\text { other minerals }\end{array}$ & $\begin{array}{l}\text { Being cut by qtz and carb } \\
\text { veins, minor qtz replacement }\end{array}$ & $\begin{array}{l}\text { Cutting host rock and being } \\
\text { cut by qtz and fine-grained } \\
\text { carb }\end{array}$ & $\begin{array}{l}\text { Cutting Fe-carb and subpar- } \\
\text { allel to fine-grained car- } \\
\text { bonates }\end{array}$ \\
\hline $\begin{array}{l}\text { other (growth di- } \\
\text { rection/zoning) }\end{array}$ & & $\begin{array}{l}\text { Red color and hm included } \\
\text { in xtals }\end{array}$ & \\
\hline
\end{tabular}




\begin{tabular}{|c|c|c|}
\hline minerals & Fine-grained sheared carb & Hematite \\
\hline percentage & 20 & trace \\
\hline morphology & Shear vein, sparry & anhedral \\
\hline texture & Shear vein & Disseminated in Fe-carb \\
\hline $\begin{array}{l}\text { transmitted opti- } \\
\text { cal properties }\end{array}$ & & \\
\hline $\begin{array}{l}\text { reflected optical } \\
\text { properties }\end{array}$ & & \\
\hline $\begin{array}{l}\text { contact/ } \\
\text { relationships with } \\
\text { other minerals }\end{array}$ & $\begin{array}{l}\text { Cutting Fe-carb and host } \\
\text { rock, includes some qtz }\end{array}$ & $\begin{array}{l}\text { Irregular contacts with Fe- } \\
\text { carb }\end{array}$ \\
\hline $\begin{array}{l}\text { other (growth di- } \\
\text { rection/zoning) }\end{array}$ & & \\
\hline
\end{tabular}

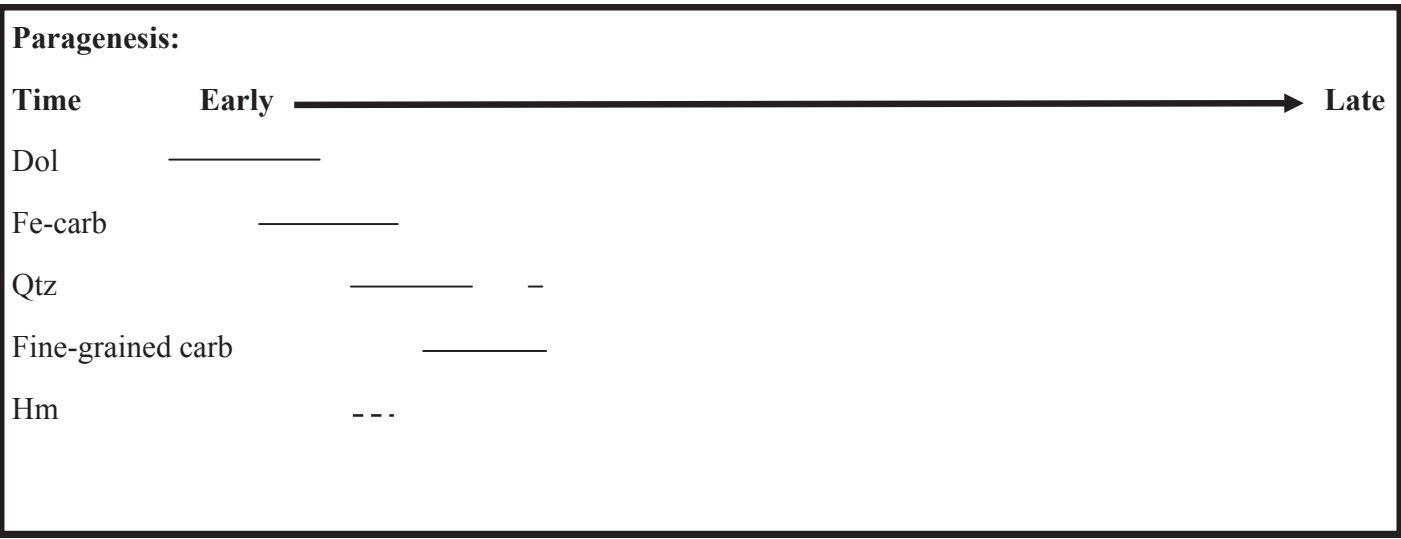


Geologic Unit: Lower Pamplona (phyllite)

Lithological Unit (Votorantim): Pink dolomite (DG)

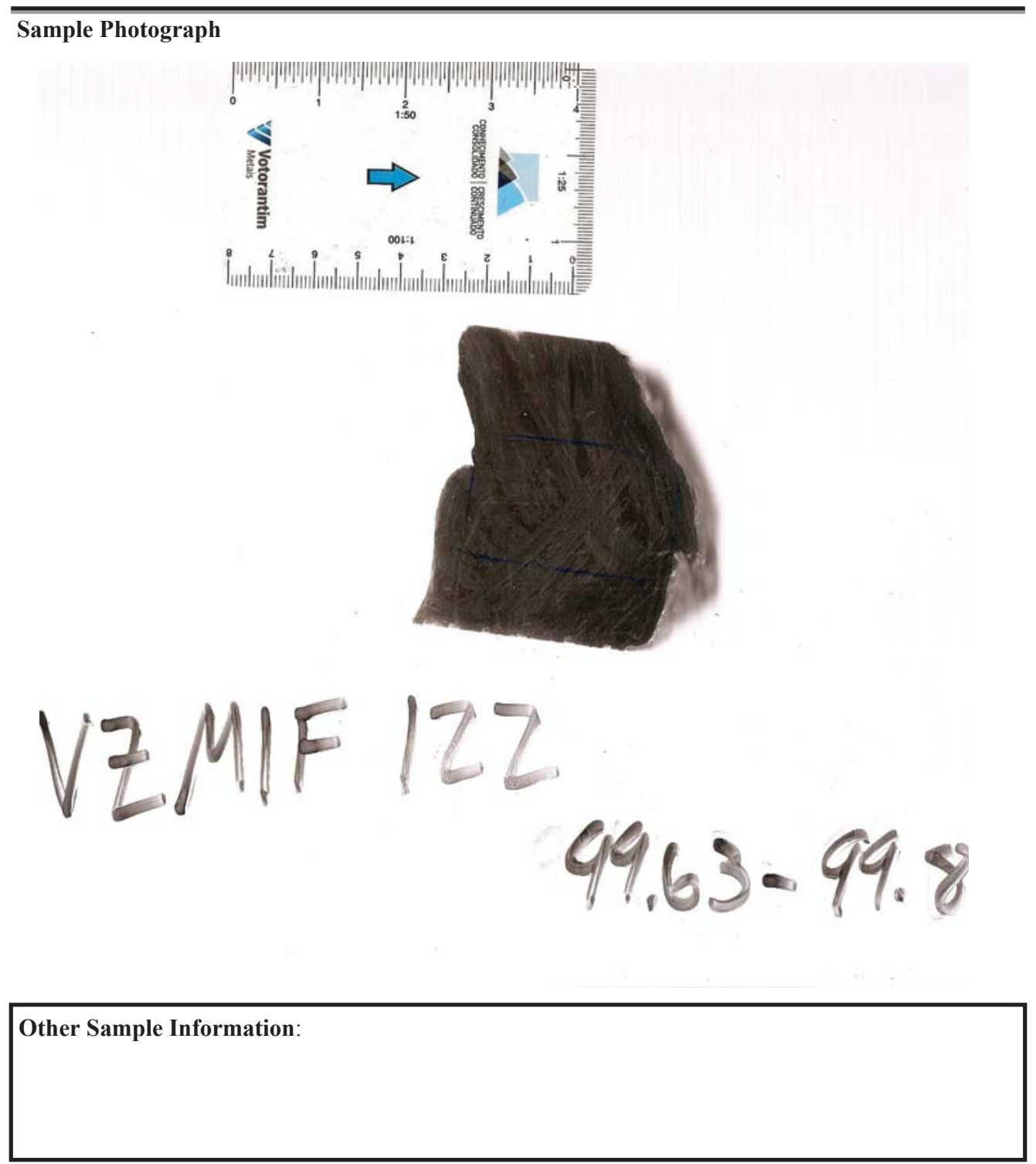




\begin{tabular}{|c|c|c|c|}
\hline minerals & Quartz & Muscovite & Carbonate? \\
\hline percentage & 60 & 10 & 30 \\
\hline morphology & $\begin{array}{l}\text { Angular to subrounded } \\
\text { grains }\end{array}$ & Euhedral laths & Fine grained micritic matrix \\
\hline texture & $\begin{array}{l}\text { Compose rock, oriented } \\
\text { along foliation (fold) }\end{array}$ & Oriented along foliation & $\begin{array}{l}\text { More cement in between } \\
\text { grains }\end{array}$ \\
\hline $\begin{array}{l}\text { transmitted opti- } \\
\text { cal properties }\end{array}$ & & & \\
\hline $\begin{array}{l}\text { reflected optical } \\
\text { properties }\end{array}$ & & & \\
\hline $\begin{array}{l}\text { contact/ } \\
\text { relationships with } \\
\text { other minerals }\end{array}$ & $\begin{array}{l}\text { Oriented with muscovite in } \\
\text { foliatiom }\end{array}$ & $\begin{array}{l}\text { Oriented with quartz in foli- } \\
\text { ation }\end{array}$ & amorphous flow texture \\
\hline $\begin{array}{l}\text { other (growth di- } \\
\text { rection/zoning) }\end{array}$ & & & \\
\hline
\end{tabular}




\begin{tabular}{|c|c|c|}
\hline minerals & Hematite & Zircon \\
\hline percentage & trace & Trace \\
\hline morphology & Sub angular grains & $\begin{array}{l}\text { Anhedral to euhedral grains; } \\
20-50 \text { microns in size }\end{array}$ \\
\hline texture & disseminated & Verry disseminated \\
\hline $\begin{array}{l}\text { transmitted opti- } \\
\text { cal properties }\end{array}$ & & High birefringence \\
\hline $\begin{array}{l}\text { reflected optical } \\
\text { properties }\end{array}$ & & Gray iand zoned n ref ppl \\
\hline $\begin{array}{l}\text { contact/ } \\
\text { relationships with } \\
\text { other minerals }\end{array}$ & $\begin{array}{l}\text { Part of grains comprising } \\
\text { phyllite }\end{array}$ & $\begin{array}{l}\text { Rounded or straight contacts } \\
\text { with musc, qtz, and hm }\end{array}$ \\
\hline $\begin{array}{l}\text { other (growth di- } \\
\text { rection/zoning) }\end{array}$ & & Zoned, some cracked \\
\hline
\end{tabular}

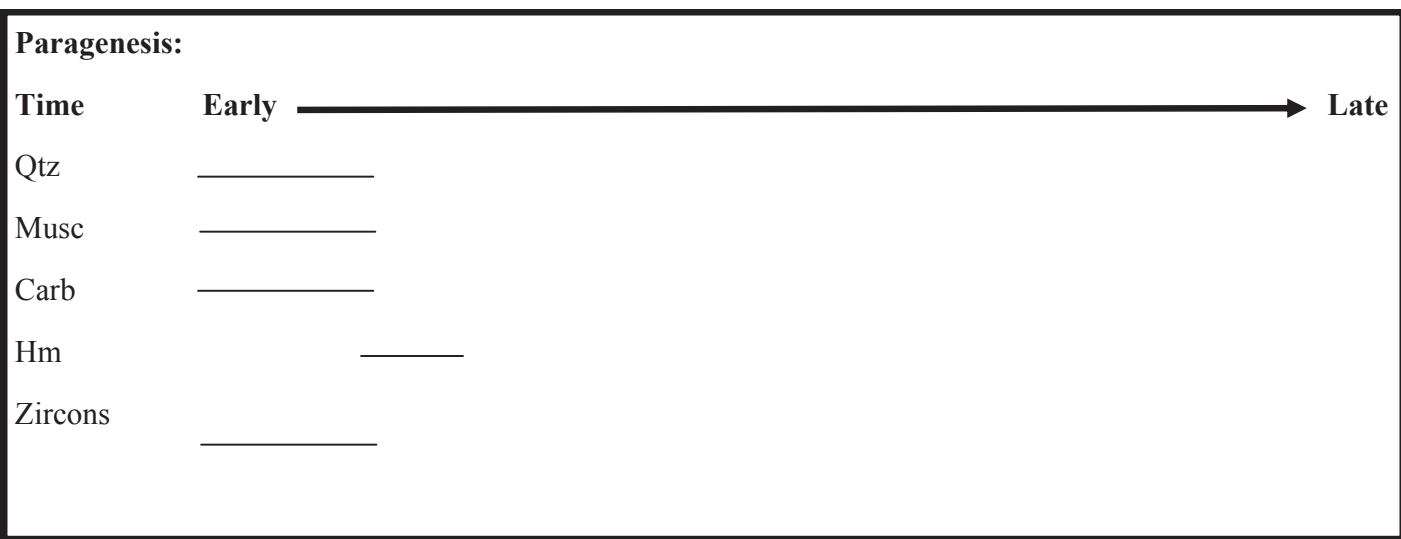


Geologic Unit: Lower Pamplona

Lithological Unit (Votorantim): Hydrothermal Breccia
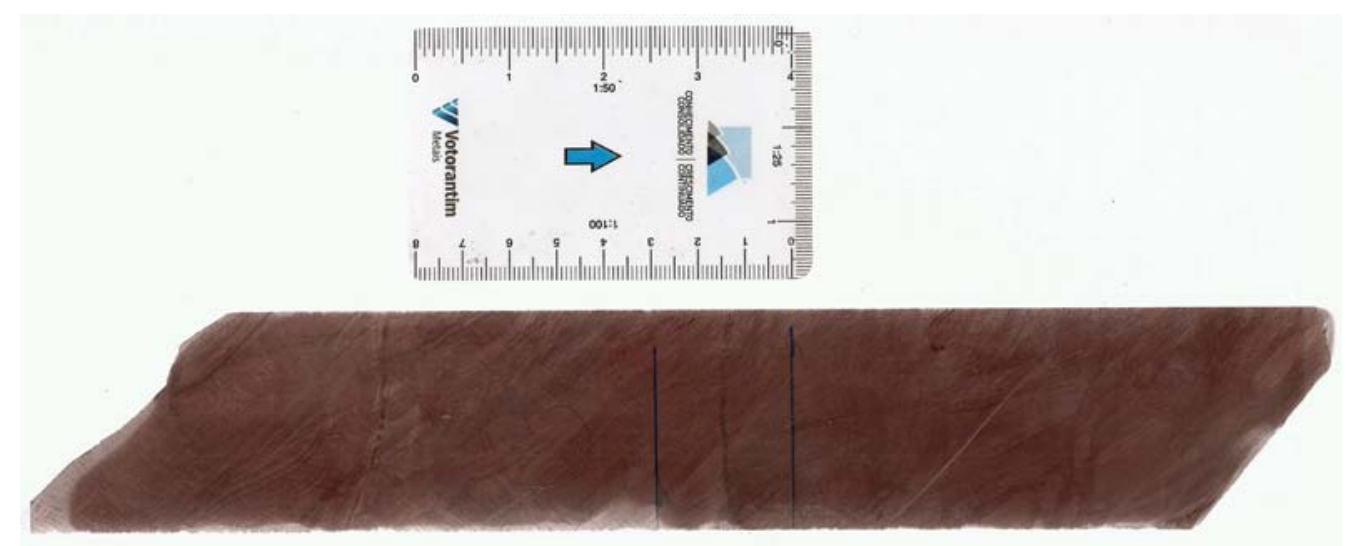

\section{VEMIF 123}

$46.62-46.9$

Other Sample Information: Altered rock, but not brecciated

**rock is a laminated quartz boundstone (Dunham, 1962) 


\begin{tabular}{|c|c|c|c|}
\hline minerals & Dolomite & Quartz & Muscovite \\
\hline percentage & 79 & 10 & 1 \\
\hline morphology & Small, sparry crystals & $\begin{array}{l}\text { Angular to subrounded } \\
\text { grains }\end{array}$ & Rectangular crystal laths \\
\hline texture & $\begin{array}{l}\text { Non planar dol; laminated } \\
\text { with qtz due to algal layer- } \\
\text { ing }\end{array}$ & Laminated with dolomite & disseminated \\
\hline $\begin{array}{l}\text { transmitted opti- } \\
\text { cal properties }\end{array}$ & & & \\
\hline $\begin{array}{l}\text { reflected optical } \\
\text { properties }\end{array}$ & & & \\
\hline $\begin{array}{l}\text { contact/ } \\
\text { relationships with } \\
\text { other minerals }\end{array}$ & $\begin{array}{l}\text { Straight contacts with qtz } \\
\text { and musc, sheared contact } \\
\text { with Fe-carb vein }\end{array}$ & $\begin{array}{l}\text { Straight contacts with musc } \\
\text { and dol }\end{array}$ & $\begin{array}{l}\text { Straight contacts with qtz } \\
\text { and dol }\end{array}$ \\
\hline $\begin{array}{l}\text { other (growth di- } \\
\text { rection/zoning) }\end{array}$ & & & \\
\hline
\end{tabular}




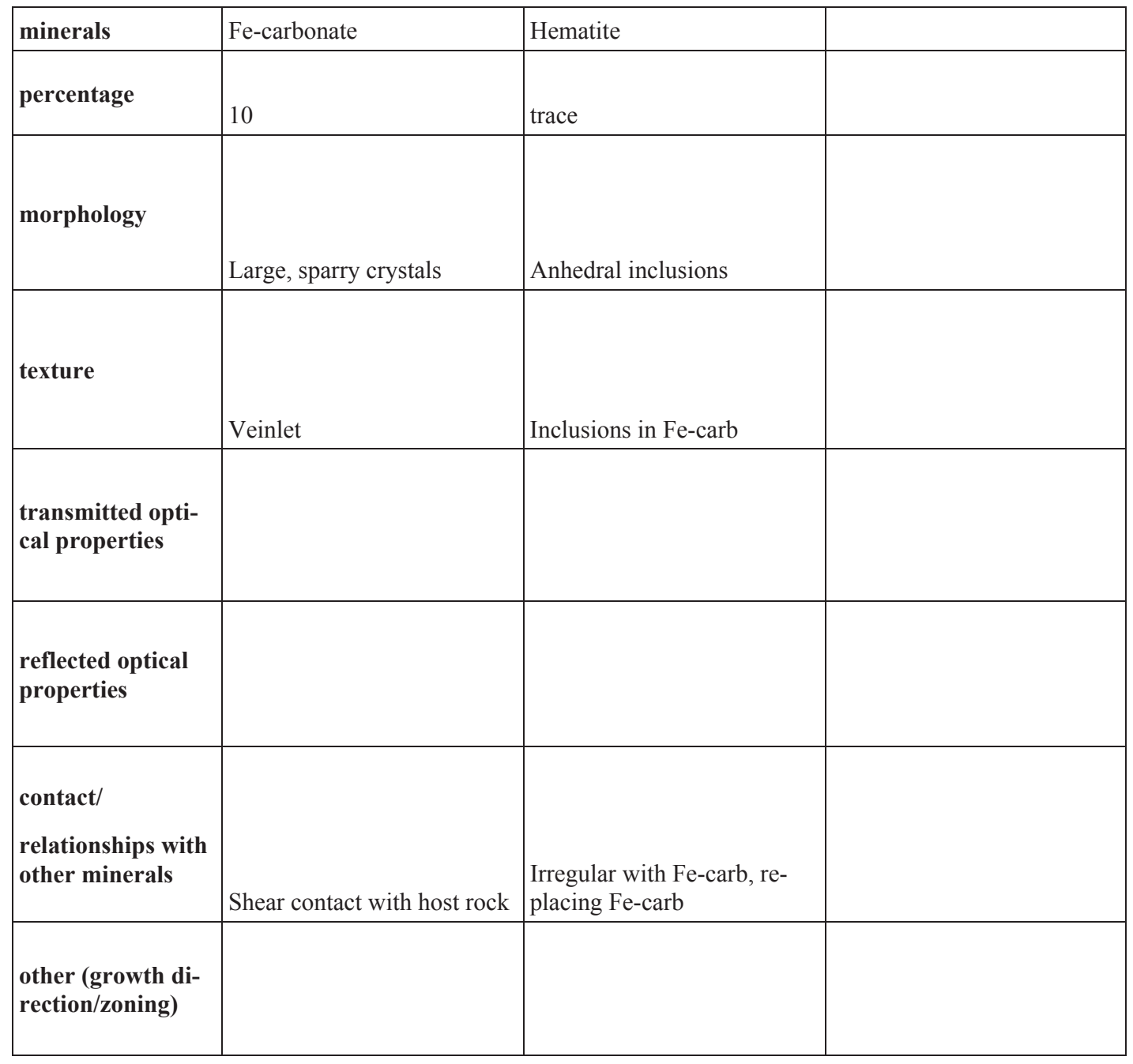

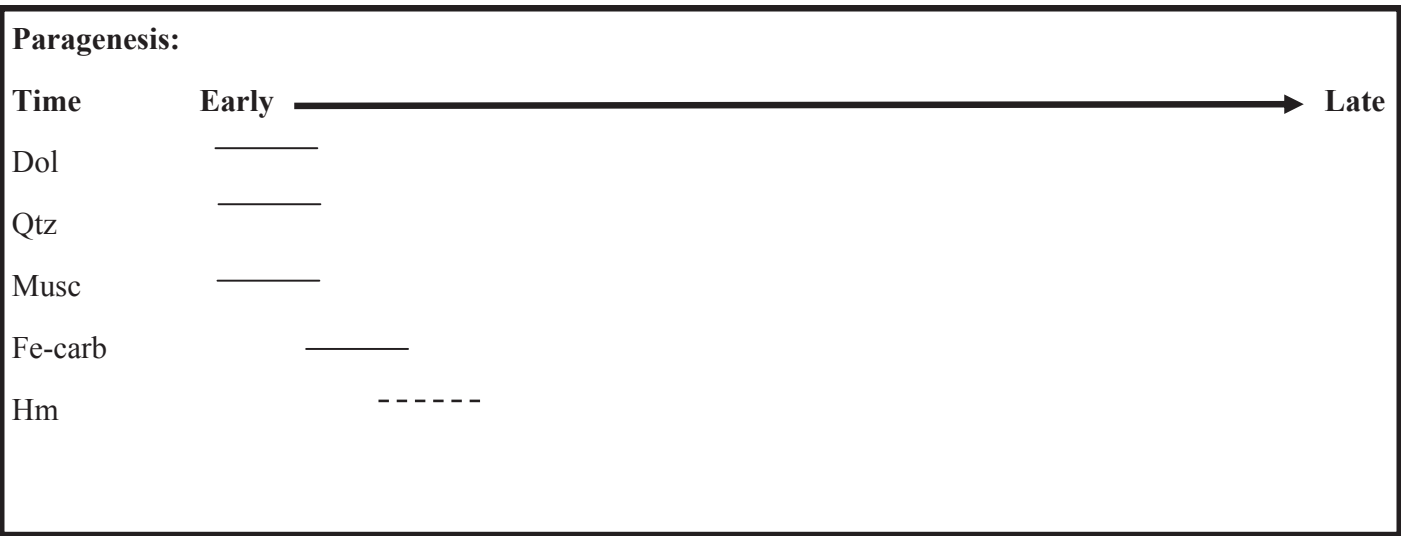


Geologic Unit: Hydrothermal Breccia

Lithological Unit (Votorantim): Massive Silicates (SM) in hydrothermal breccia Sample Photograph

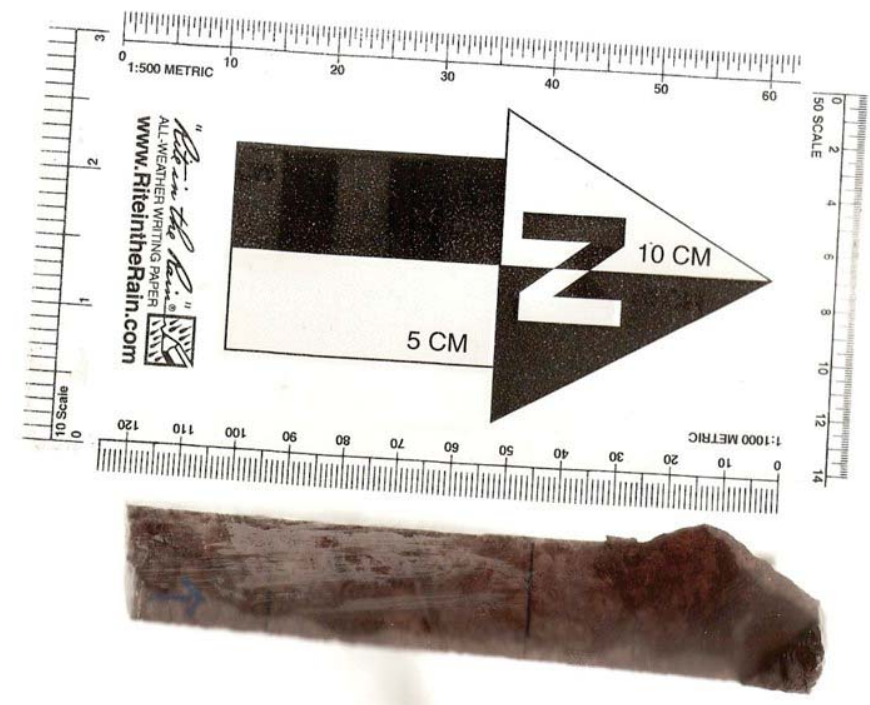

\section{V:M15 123}

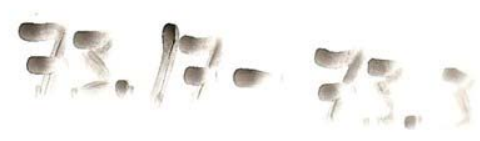

Other Sample Information: 


\begin{tabular}{|c|c|c|c|}
\hline minerals & Hematite & Specular Hm & Dolomite \\
\hline percentage & 25 & 10 & 3 \\
\hline morphology & $\begin{array}{l}\text { Blebs on and around grain } \\
\text { margins, related to specular } \\
\text { hematite }\end{array}$ & & $\begin{array}{l}\text { Being replaced by } \mathrm{Hm} \text { and } \\
\text { Wl, faded edges }\end{array}$ \\
\hline texture & Disseminated, blebby & & $\begin{array}{l}\text { Being replaced by } \mathrm{Hm} \text { and } \\
\text { W1 }\end{array}$ \\
\hline $\begin{array}{l}\text { transmitted opti- } \\
\text { cal properties }\end{array}$ & & opaque & \\
\hline $\begin{array}{r}\text { color } \\
\text { ppl } \\
\text { xpl }\end{array}$ & $\begin{array}{l}\text { Reddish-brown } \\
\text { Black }\end{array}$ & & $\begin{array}{l}\text { Clear } \\
\text { pastels }\end{array}$ \\
\hline pleochroism & $\mathrm{n} / \mathrm{a}$ & & $\mathrm{n} / \mathrm{a}$ \\
\hline birefringence & opaque & & 3 rd order \\
\hline $\begin{array}{r}\text { twinning/ } \\
\text { cleavage }\end{array}$ & $\mathrm{n} / \mathrm{a}$ & & Rhombohedral clvg \\
\hline other & & & Low relief \\
\hline $\begin{array}{l}\text { reflected optical } \\
\text { properties }\end{array}$ & $\mathrm{n} / \mathrm{a}$ & $\mathrm{N} / \mathrm{a}$ & $\mathrm{n} / \mathrm{a}$ \\
\hline color & & Whitish-grey & \\
\hline bireflectance & & Very weak, lighter to darker & \\
\hline anisotropy & & $\begin{array}{l}\text { Distinct, grey to greyish } \\
\text { blue }\end{array}$ & \\
\hline internal reflections & & Some, red & \\
\hline other & & & \\
\hline $\begin{array}{l}\text { contact/ } \\
\text { relationships with } \\
\text { other minerals }\end{array}$ & $\begin{array}{l}\text { On margins of specularite, } \\
\text { wl, and qtz, replacing dol }\end{array}$ & $\begin{array}{l}\text { In veins and in between } \\
\text { margins of wl and qtz, re- } \\
\text { placing dol }\end{array}$ & $\begin{array}{l}\text { Faded edges, in contact } \\
\text { with } \mathrm{Hm}\end{array}$ \\
\hline $\begin{array}{l}\text { other (growth di- } \\
\text { rection/zoning) }\end{array}$ & Oxidation? Of specularite & & \\
\hline
\end{tabular}




\begin{tabular}{|c|c|c|c|}
\hline minerals & Granular willemite & Bladed willemite & quartz \\
\hline percentage & 45 & 15 & 2 \\
\hline morphology & $\begin{array}{l}\text { Subhedral to euhedral granu- } \\
\text { lar, small to large }\end{array}$ & $\begin{array}{l}\text { Large, euhedral bladed/ } \\
\text { fibrous crystals }\end{array}$ & Small subhedra grains \\
\hline texture & $\begin{array}{l}\text { Massive, replacing car- } \\
\text { bonates }\end{array}$ & $\begin{array}{l}\text { Overgrowing existing W1 } \\
\text { and some Hm }\end{array}$ & Amongst willemite \\
\hline \multicolumn{4}{|l|}{$\begin{array}{l}\text { transmitted opti- } \\
\text { cal properties }\end{array}$} \\
\hline $\begin{array}{r}\text { color } \\
\text { ppl } \\
\mathrm{xpl}\end{array}$ & $\begin{array}{l}\text { Clear } \\
\text { *see birefringence }\end{array}$ & $\begin{array}{l}\text { Clear } \\
\text { *see birefringence }\end{array}$ & $\begin{array}{l}\text { Clear } \\
\text { White to black }\end{array}$ \\
\hline pleochroism & Weak greenish tan to white & Weak greenish tan to white & $\mathrm{n} / \mathrm{a}$ \\
\hline birefringence & 2nd to low 3 rd order & 2nd to low 3 rd order & White to black \\
\hline $\begin{array}{r}\text { twinning/ } \\
\text { cleavage }\end{array}$ & $\mathrm{N} / \mathrm{a}$ & Clvg seen on fibrous splays & Undulatory extinction \\
\hline other & Lower relief than qtz & Lower relief than qtz & High relief \\
\hline $\begin{array}{l}\text { reflected optical } \\
\text { properties }\end{array}$ & $\mathrm{n} / \mathrm{a}$ & $\mathrm{N} / \mathrm{a}$ & $\mathrm{n} / \mathrm{a}$ \\
\hline \multicolumn{4}{|l|}{ color } \\
\hline \multicolumn{4}{|l|}{ bireflectance } \\
\hline \multicolumn{4}{|l|}{ anisotropy } \\
\hline \multicolumn{4}{|l|}{ internal reflections } \\
\hline \multicolumn{4}{|l|}{ other } \\
\hline $\begin{array}{l}\text { contact/ } \\
\text { relationships with } \\
\text { other minerals }\end{array}$ & $\begin{array}{l}\text { Straight edges with qtz, } \\
\text { overgrowing Hm and replac- } \\
\text { ing dol }\end{array}$ & $\begin{array}{l}\text { Growing in splays on top of } \\
\text { granular wl and into specu- } \\
\text { larite, some ruddy hm on } \\
\text { edges }\end{array}$ & $\begin{array}{l}\text { Some } \mathrm{Hm} \text { on edges, straight } \\
\text { contact edges with } \mathrm{Wl}\end{array}$ \\
\hline $\begin{array}{l}\text { other (growth di- } \\
\text { rection/zoning) }\end{array}$ & $\begin{array}{l}\text { Zoned xtal growth inwards } \\
\text { of veins. (i.e. wl is growing } \\
\text { into vugs/veins) }\end{array}$ & $\begin{array}{l}\text { Growing into hm filled vugs } \\
\text { and fractures }\end{array}$ & $\begin{array}{l}\text { Occurs in between some } \\
\text { granular wl xtals }\end{array}$ \\
\hline
\end{tabular}


Structures: The sample has been brecciated, or at least fractured, creating open space in the dolomitic host rock. The wl crystals are replacing the carbonates and growing in the open space. The veins are filled with specular hematite as opposed to the ruddy hematite seen throughout the rest of the sample.

Paragenetic Sequence: dol $\rightarrow \mathrm{bx} \rightarrow \mathrm{Wl}$ (granular) $+\mathrm{Hm}$ specular $+\mathrm{qtz} \rightarrow \mathrm{Wl}$ (fibrous) $+\mathrm{Hm}$ (ruddy)

Paragenetic Reasoning: The dol is faded in the specular hm veins in which it appears, and its edges corroded by hm. The granular wl grows as small crystals in most areas along with qtz, except where there are large hematite veins and vugs, where it grows as large crystals towards the center of the open space. The specular hm is coeval with the granular wl as some wl occurs in the middle of the hm veins. The fibrous wl appears to be replacing some of the larger wl xtals on near the contacts of granular wl and specularite. The ruddy $\mathrm{hm}$ is later as it appears on the margins of all minerals, particularly the specular hematite.

Sample Highlights: -2 phases of wl

-open space filling of wl xtals

-coeval occurrence of $\mathrm{hm}$ and wl 
Geologic Unit: BH overprinting Lower Pamplona

Lithological Unit (Votorantim): Hydrothermal Breccia (BH)

Sample Photograph

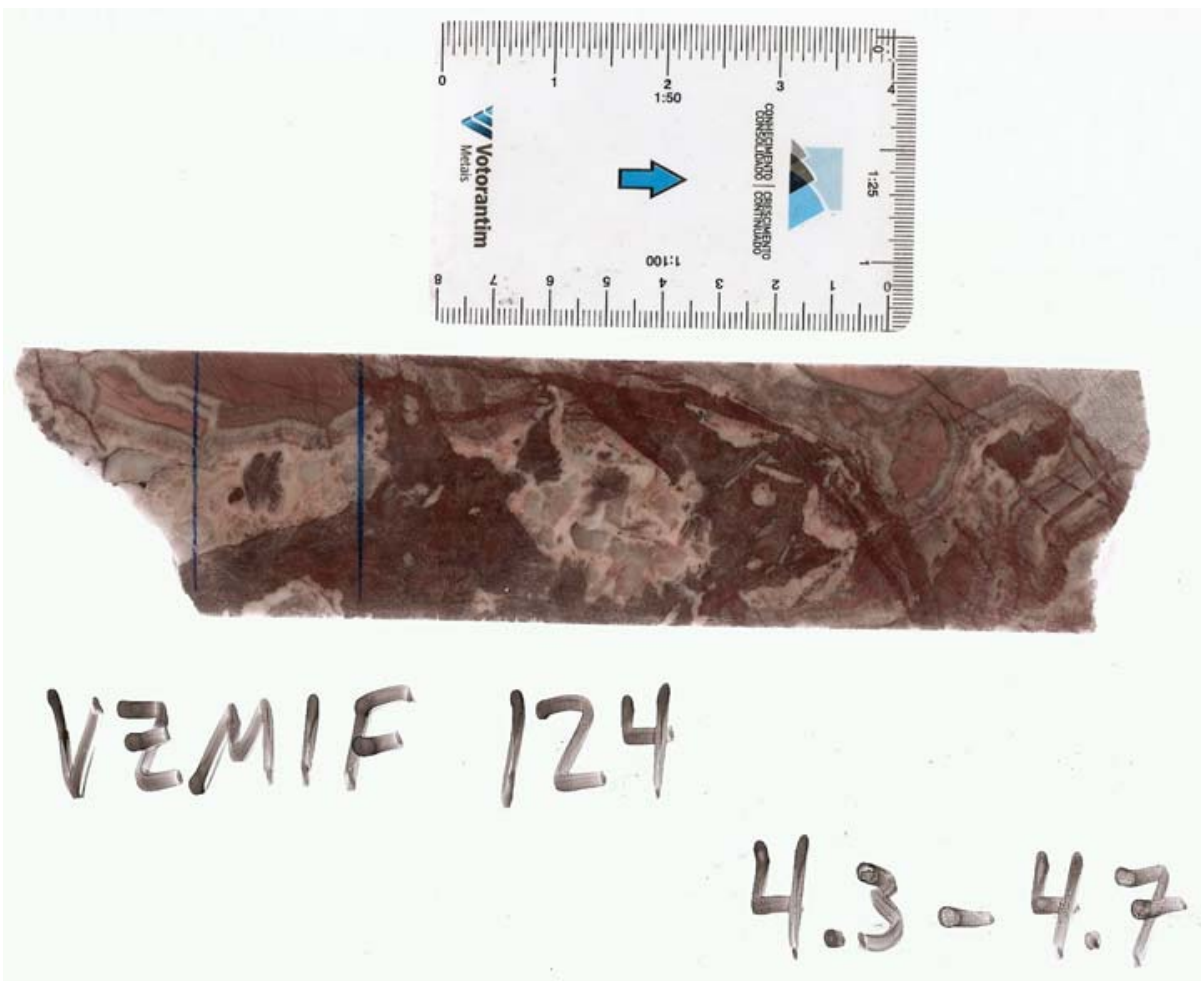

Other Sample Information: 


\begin{tabular}{|c|c|c|c|}
\hline minerals & Fe-carbonate & Hydrothermal carbonate & Quartz \\
\hline percentage & 45 & 45 & 10 \\
\hline morphology & Micritic breccia fragments & $\begin{array}{l}\text { Subhedral to euhedral spar- } \\
\text { rite }\end{array}$ & Large, anhedral crystals \\
\hline texture & Breccia fragments & $\begin{array}{l}\text { Cement b/w breccia frage- } \\
\text { ments }\end{array}$ & Associated with carb cement \\
\hline $\begin{array}{l}\text { transmitted opti- } \\
\text { cal properties }\end{array}$ & & & \\
\hline $\begin{array}{l}\text { reflected optical } \\
\text { properties }\end{array}$ & & & \\
\hline $\begin{array}{l}\text { contact/ } \\
\text { relationships with } \\
\text { other minerals }\end{array}$ & $\begin{array}{l}\text { Diffuse into hydrothermal } \\
\text { carbonate (nucleation site) }\end{array}$ & $\begin{array}{l}\text { Fe-carb micrite diffuses into } \\
\text { hydrothermal carbonate, this } \\
\text { carbonate grows out from } \\
\text { red Fe-carb fragments; em- } \\
\text { bayed with qtz (replacing } \\
\text { qtz) }\end{array}$ & $\begin{array}{l}\text { Irregular with carbonates, } \\
\text { being replaced by carb }\end{array}$ \\
\hline $\begin{array}{l}\text { other (growth di- } \\
\text { rection/zoning) }\end{array}$ & & & \\
\hline
\end{tabular}




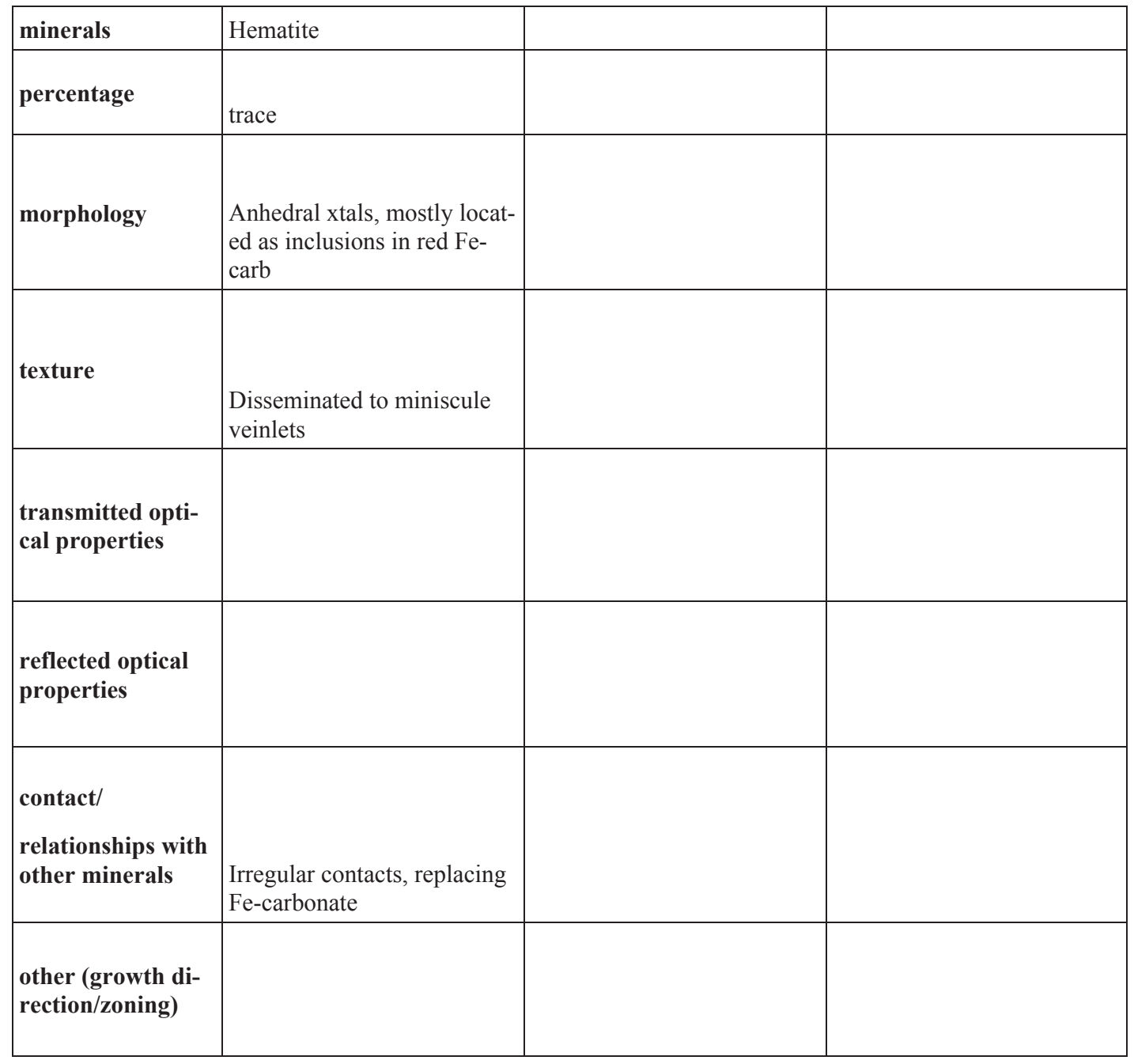
Paragenesis:
Time
Early
Late
Fe-carb
Carb Cement
Qtz
$\mathrm{Hm}$ 
Geologic Unit: BH overprinting Lower Pamplona

Lithological Unit (Votorantim): Hydrothermal Dolomite

Sample Photograph

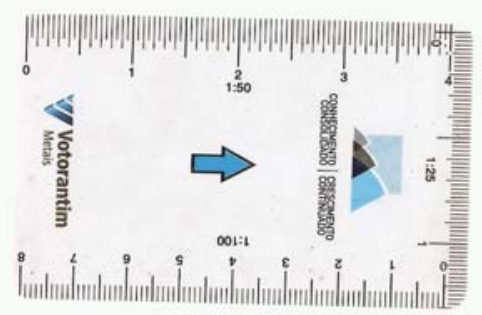

$\sqrt{a} / 1)=$

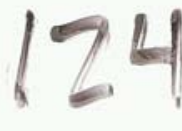

$72.55-72.76$

Other Sample Information: 


\begin{tabular}{|c|c|c|c|}
\hline minerals & Dolomomitic host rock & Fe-carb alteration & Hematite \\
\hline percentage & 80 & 17 & 3 \\
\hline morphology & $\begin{array}{l}\text { Massive micrite; sedimen- } \\
\text { tary breccia replaced by mi- } \\
\text { critic dolomite with car- } \\
\text { bonate cement (some neo- } \\
\text { morphic zones) }\end{array}$ & $\begin{array}{l}\text { Sparry carbonate with black } \\
\text { inclusions }\end{array}$ & Anhedral crystal inclusions \\
\hline texture & $\begin{array}{l}\text { Massive; sedimentary brec- } \\
\text { cia }\end{array}$ & $\begin{array}{l}\text { Veinlets cross-cutting the } \\
\text { host rock }\end{array}$ & Inclusions in Fe-carb \\
\hline $\begin{array}{l}\text { transmitted opti- } \\
\text { cal properties }\end{array}$ & & & \\
\hline $\begin{array}{l}\text { reflected optical } \\
\text { properties }\end{array}$ & & & \\
\hline $\begin{array}{l}\text { contact/ } \\
\text { relationships with } \\
\text { other minerals }\end{array}$ & $\begin{array}{l}\text { See Fe-carb; non planar do- } \\
\text { lomite }\end{array}$ & $\begin{array}{l}\text { Partially sheared and partial- } \\
\text { ly jagged contacts with host } \\
\text { roc }\end{array}$ & $\begin{array}{l}\text { Irregular contacts with Fe- } \\
\text { carb, replacing Fe-carb? } \\
\text { Some inclusions in host rock } \\
\text { as well, irregular contacts }\end{array}$ \\
\hline $\begin{array}{l}\text { other (growth di- } \\
\text { rection/zoning) }\end{array}$ & & & \\
\hline
\end{tabular}




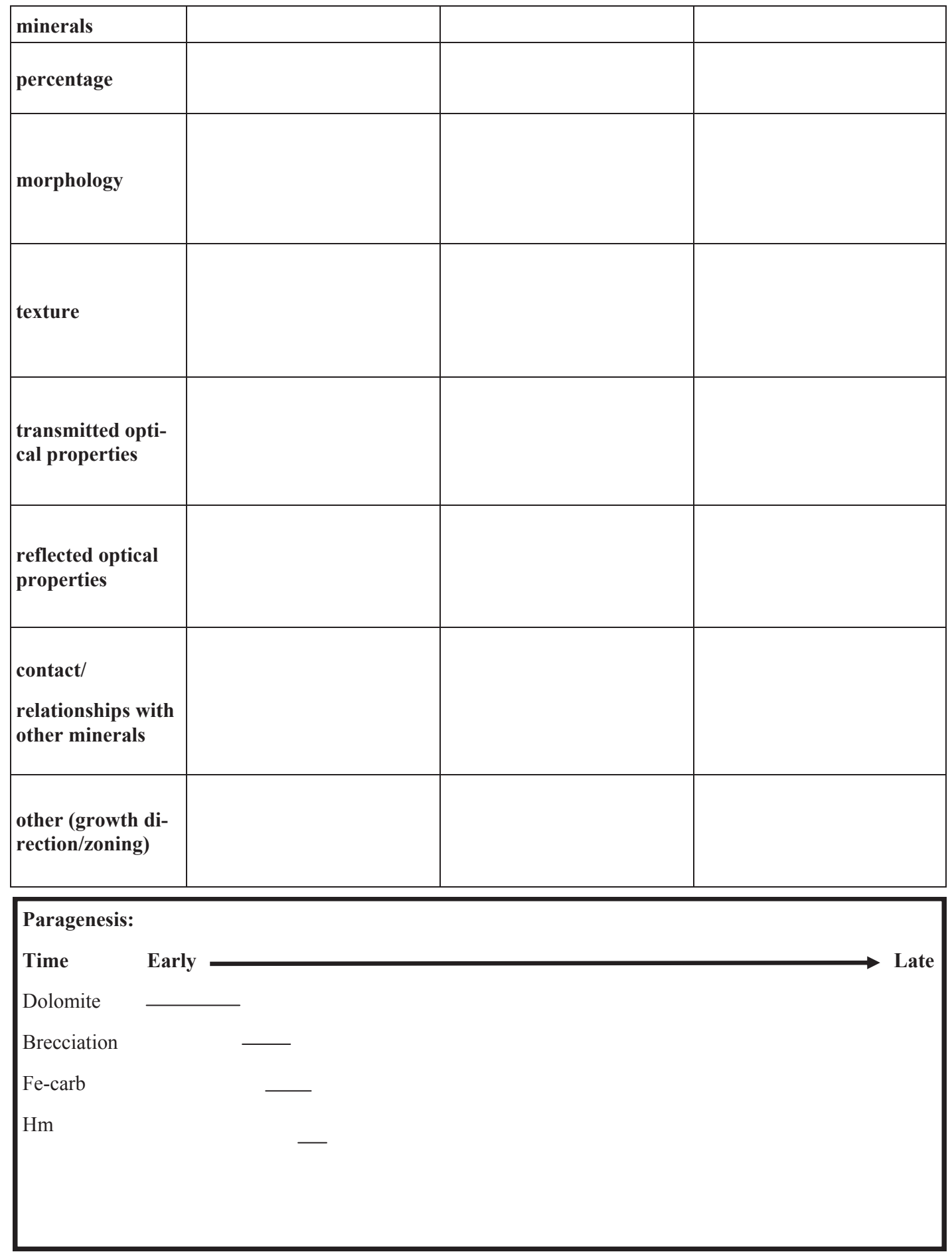


Geologic Unit: Hydrothermal Breccia

Lithological Unit (Votorantim): Massive Silicate in BH

Sample Photograph
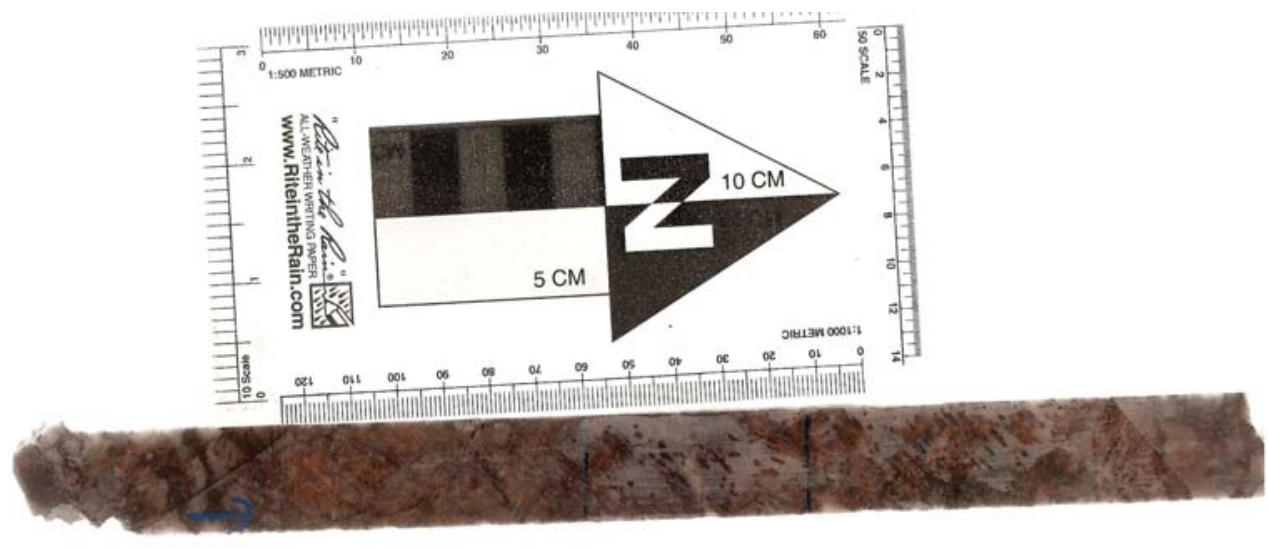

$\int_{-111}^{\infty}=121$

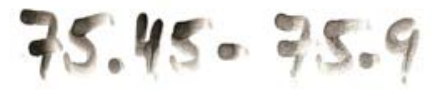

Other Sample Information: Interval runs $45 \% \mathrm{Zn}$ 


\begin{tabular}{|c|c|c|c|}
\hline minerals & Dolomite & Willemite & Quartz \\
\hline percentage & 5 & 79 & 5 \\
\hline morphology & $\begin{array}{l}\text { Anhedral micrite in matrix } \\
\text { and in veins }\end{array}$ & Euhedral granular xtals & Subhedral xtals \\
\hline texture & $\begin{array}{l}\text { Micritic, being replaced by } \\
\text { wl, replacing qtz }\end{array}$ & massive & $\begin{array}{l}\text { Veins, minor disseminated } \\
\text { xtals }\end{array}$ \\
\hline $\begin{array}{l}\text { transmitted opti- } \\
\text { cal properties }\end{array}$ & & & \\
\hline $\begin{array}{l}\text { reflected optical } \\
\text { properties }\end{array}$ & & & \\
\hline $\begin{array}{l}\text { contact/ } \\
\text { relationships with } \\
\text { other minerals }\end{array}$ & $\begin{array}{l}\text { Irregular, being replaced by } \\
\text { wl and hm }\end{array}$ & $\begin{array}{l}\text { Mostly straight with other } \\
\text { wl, irregular with hm and } \\
\text { dol (being replaced by hm) }\end{array}$ & $\begin{array}{l}\text { Irregular, being replaced by } \\
\text { dol in veins along contacts } \\
\text { with wl }\end{array}$ \\
\hline $\begin{array}{l}\text { other (growth di- } \\
\text { rection/zoning) }\end{array}$ & & & \\
\hline
\end{tabular}




\begin{tabular}{|c|c|c|}
\hline minerals & Hematite & Sphalerite \\
\hline percentage & 10 & 1 \\
\hline morphology & Xtal laths in shear zone & Anhedral to euhedral xtals \\
\hline texture & $\begin{array}{l}\text { Massive sheared vein, dis- } \\
\text { seminated in wl }\end{array}$ & disseminated \\
\hline $\begin{array}{l}\text { transmitted opti- } \\
\text { cal properties }\end{array}$ & & \\
\hline $\begin{array}{l}\text { reflected optical } \\
\text { properties }\end{array}$ & & \\
\hline $\begin{array}{l}\text { contact/ } \\
\text { relationships with } \\
\text { other minerals }\end{array}$ & $\begin{array}{l}\text { Replacing dol and wl, very } \\
\text { minor replacement of qtz } \\
\text { veins (most likely replacing } \\
\text { dol in qtz veins) }\end{array}$ & $\begin{array}{l}\text { Irregular and straight with } \\
\mathrm{hm} \text { and dol. Straight with wl. } \\
\text { Exosolution of hm in sl }\end{array}$ \\
\hline $\begin{array}{l}\text { other (growth di- } \\
\text { rection/zoning) }\end{array}$ & & $\begin{array}{l}\text { Associated with } \mathrm{hm} \text { in dol } \\
\text { xtals. Dol xtals are being } \\
\text { replaced by wl }\end{array}$ \\
\hline
\end{tabular}

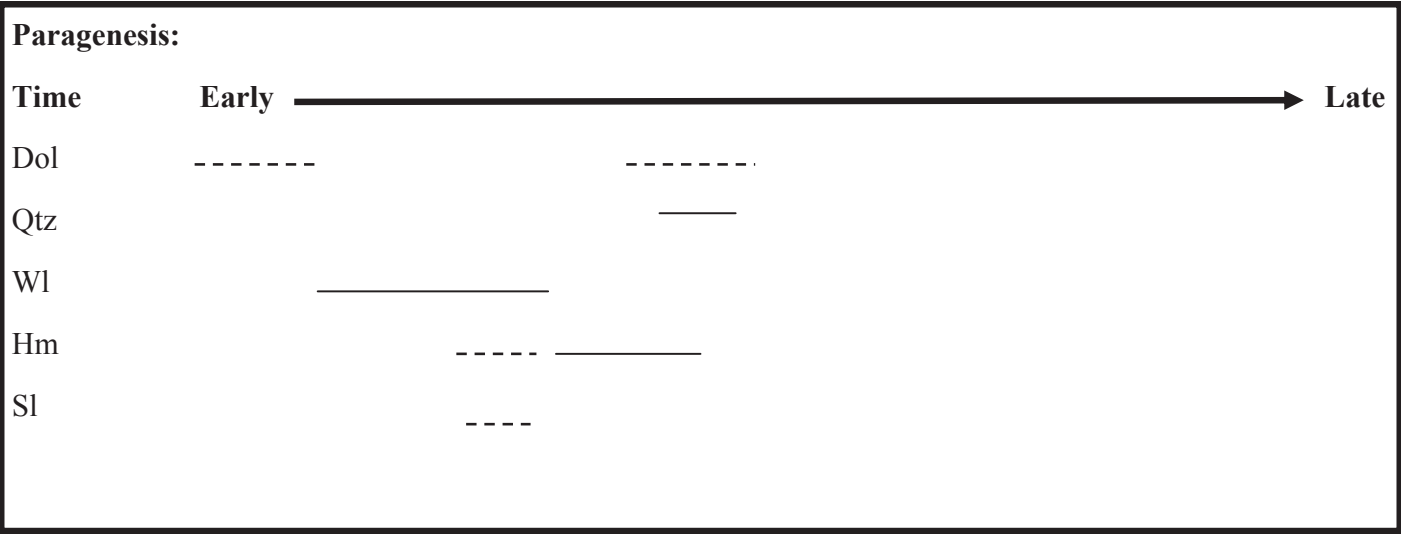


Structures: Sample is massive except for large shear vein/fault in middle of sample. Shearing caused some brecciation of wl. 3 vein styles present: 1) hm shear vein, 2) qtz and Fe-dol vein, and 3) qtz veins. Multiple veins see vein paragenesis below

Paragenetic Sequence: $\mathrm{hm}$ vein $\rightarrow \mathrm{qtz}+\mathrm{Fe}-\mathrm{dol} \rightarrow \mathrm{qtz}$

Paragenetic Reasoning: The qtz $+\mathrm{Fe}-\mathrm{dol}$ vein cross-cuts the hm shear vein, but is cut by later $\mathrm{qtz}$ veins that cut across entire sample. Qtz veins are being altered by carbonate. 
Geologic Unit: Hydrothermal Breccia

Lithological Unit (Votorantim): Massive Silicate in BH

Sample Photograph

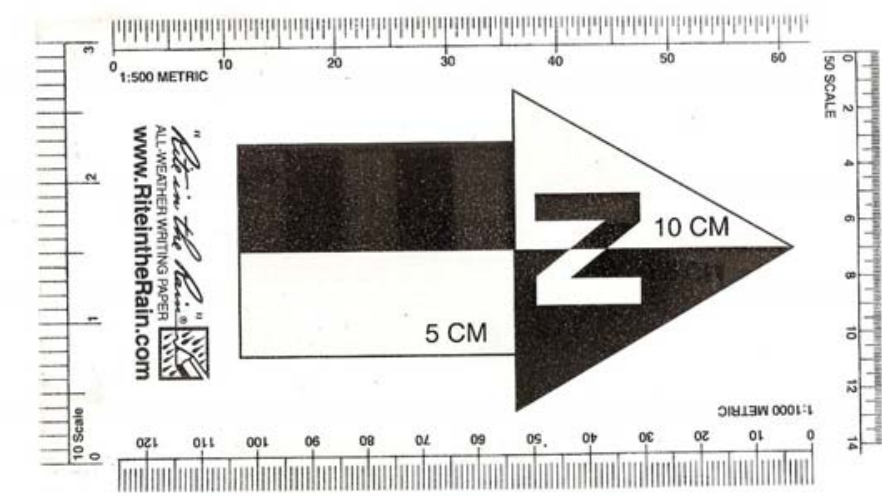

.

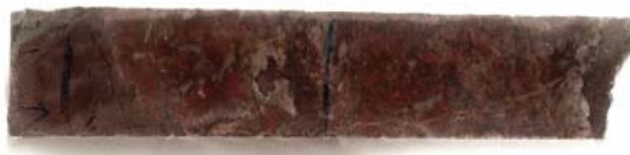

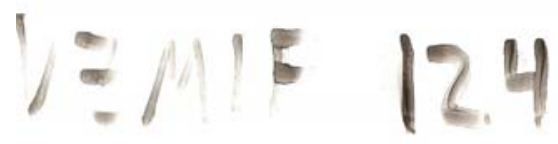

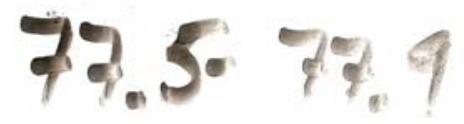

Other Sample Information: See sample 104-86 (mid grade 20\% Zn) 
Sample \#: 124-83

Geologic Unit: Hydrothermal Breccia

Lithological Unit (Votorantim): Hydrothermal Breccia

Sample Photograph
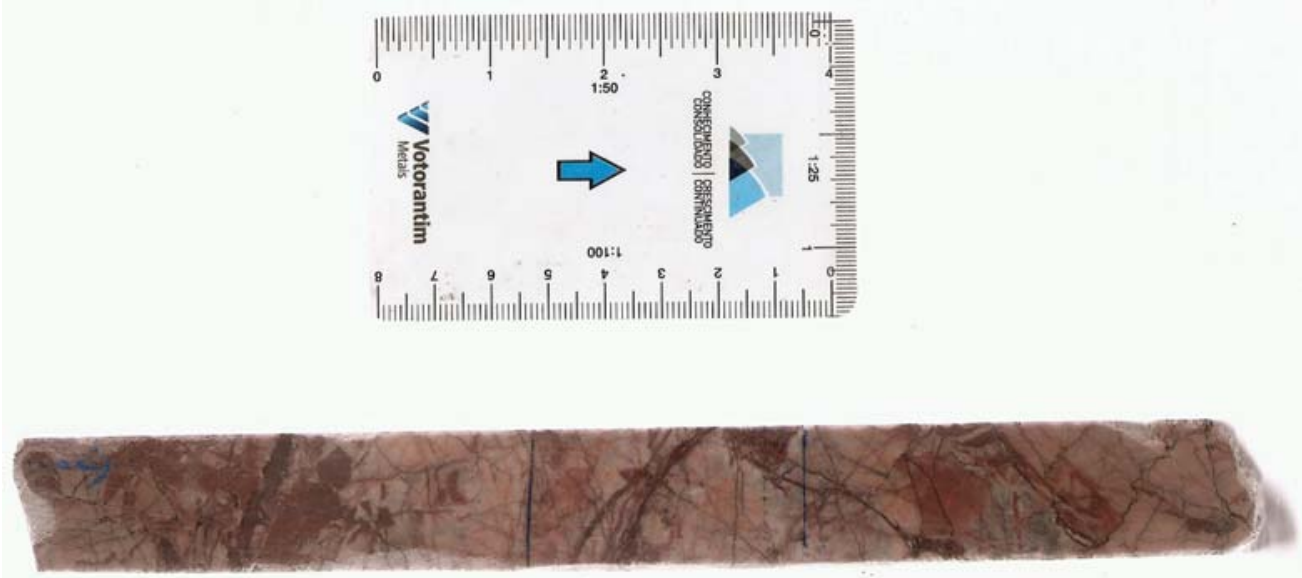

\section{VZMIF 124}

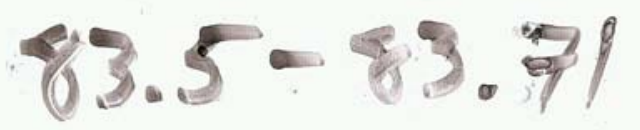

Other Sample Information: Same as $124-72 * *$ more hydrothermal breccia less sedimentary breccia framents 
Geologic Unit: Hydrothermal Breccia(?)

Lithological Unit (Votorantim): Disseminated Silicate (unknown bx)

Sample Photograph

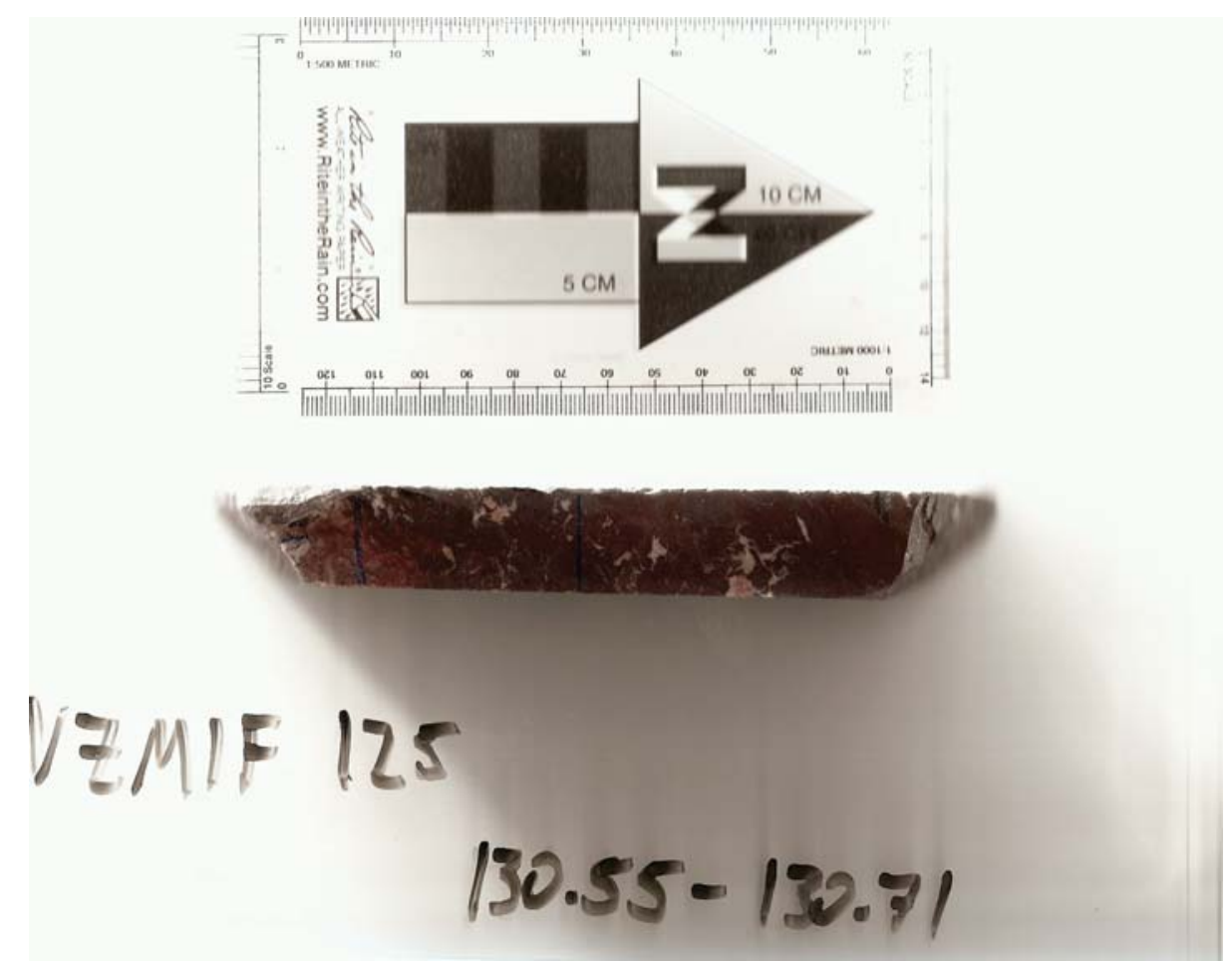

Other Sample Information: hematite shear zone, not in specific BH 


\begin{tabular}{|c|c|c|}
\hline minerals & Dolomite & Hematite (specular) \\
\hline percentage & 30 & 70 \\
\hline morphology & Large, anhedral dol crystals & Massive veins \\
\hline texture & $\begin{array}{l}\text { Primary dolostone being re- } \\
\text { placed by hm. }\end{array}$ & Migmatite,breccia veins \\
\hline $\begin{array}{l}\text { transmitted opti- } \\
\text { cal properties }\end{array}$ & & opaque \\
\hline reflected optical & $\mathrm{n} / \mathrm{a}$ & \\
\hline $\begin{array}{l}\text { contact/ } \\
\text { relationships with } \\
\text { other minerals }\end{array}$ & $\begin{array}{l}\text { Some secondary dol replac- } \\
\text { ing dol. All dol being re- } \\
\text { placed by hm }\end{array}$ & $\begin{array}{l}\mathrm{Hm} \text { is replacing dol. Hm } \\
\text { forms a shear vein with } \\
\text { proximal breccia. }\end{array}$ \\
\hline $\begin{array}{l}\text { other (growth di- } \\
\text { rection/zoning) }\end{array}$ & & \\
\hline
\end{tabular}

Paragenesis:

Time

Early

Late

Dol

$\mathrm{Hm}$ 
Structures: Hm forms a shear vein with surrounding breccia proximal to vein. Dol and hm veins are curved and migmatized.

Paragenetic Sequence: dol $\rightarrow$ 2ndary dol $\rightarrow \mathrm{hm}$

Paragenetic Reasoning: Dol xtals are being replaced by dolomite. All dol being replaced by hm laths. Dol xtal edges are irregular when in contact with hematite.

Sample Highlights: Migmatized dol 
Geologic Unit: Middle Pamplona

Lithological Unit (Votorantim): Grey Dolomite (DO)

Sample Photograph

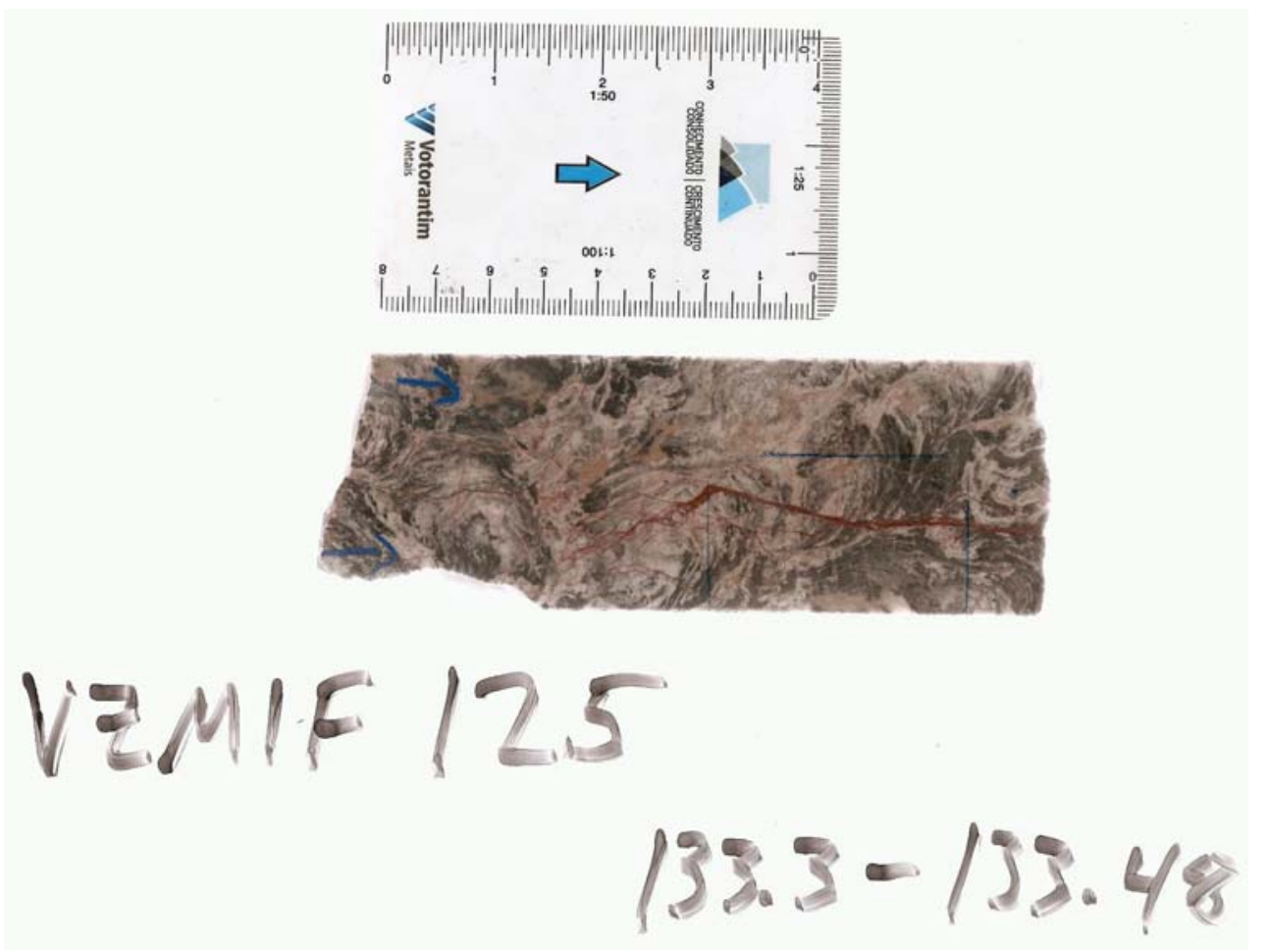

Other Sample Information: rock type the same as 118-132 (stromatolite boundstone)

Rest of sheet is alteration analysis only 


\begin{tabular}{|c|c|c|c|}
\hline minerals & Fe-carb & Quartz & Fine-grained carbonate \\
\hline percentage & 10 & 5 & 3 \\
\hline morphology & $\begin{array}{l}\text { Sparry carbonate with hm } \\
\text { inclusions }\end{array}$ & $\begin{array}{l}\text { Crystalline quartz } \\
\text { (subhedral) }\end{array}$ & $\begin{array}{l}\text { Very small carbonate crys- } \\
\text { tals }\end{array}$ \\
\hline texture & Veinlets & Veinlets & veinlets \\
\hline $\begin{array}{l}\text { transmitted opti- } \\
\text { cal properties }\end{array}$ & & & \\
\hline $\begin{array}{l}\text { reflected optical } \\
\text { properties }\end{array}$ & & & \\
\hline $\begin{array}{l}\text { contact/ } \\
\text { relationships with } \\
\text { other minerals }\end{array}$ & $\begin{array}{l}\text { Irregular contacts, jagged, } \\
\text { fine carb; being replaced by } \\
\text { fine carb. Straight and } \\
\text { curved edges with qtz }\end{array}$ & $\begin{array}{l}\text { Grew along same fracture as } \\
\text { Fe-carb, being replaced by } \\
\text { fine carb }\end{array}$ & $\begin{array}{l}\text { Irregular and spotty contacts } \\
\text { with Fe-carb and qtz vein, } \\
\text { replacing these mins }\end{array}$ \\
\hline $\begin{array}{l}\text { other (growth di- } \\
\text { rection/zoning) }\end{array}$ & & & \\
\hline
\end{tabular}

\section{Paragenesis:}

Time

Early

Fe-carb

Qtz

Fine carb 
Geologic Unit: Hydrothermal Breccia

Lithological Unit (Votorantim): Massive Silicate (SM) in BH

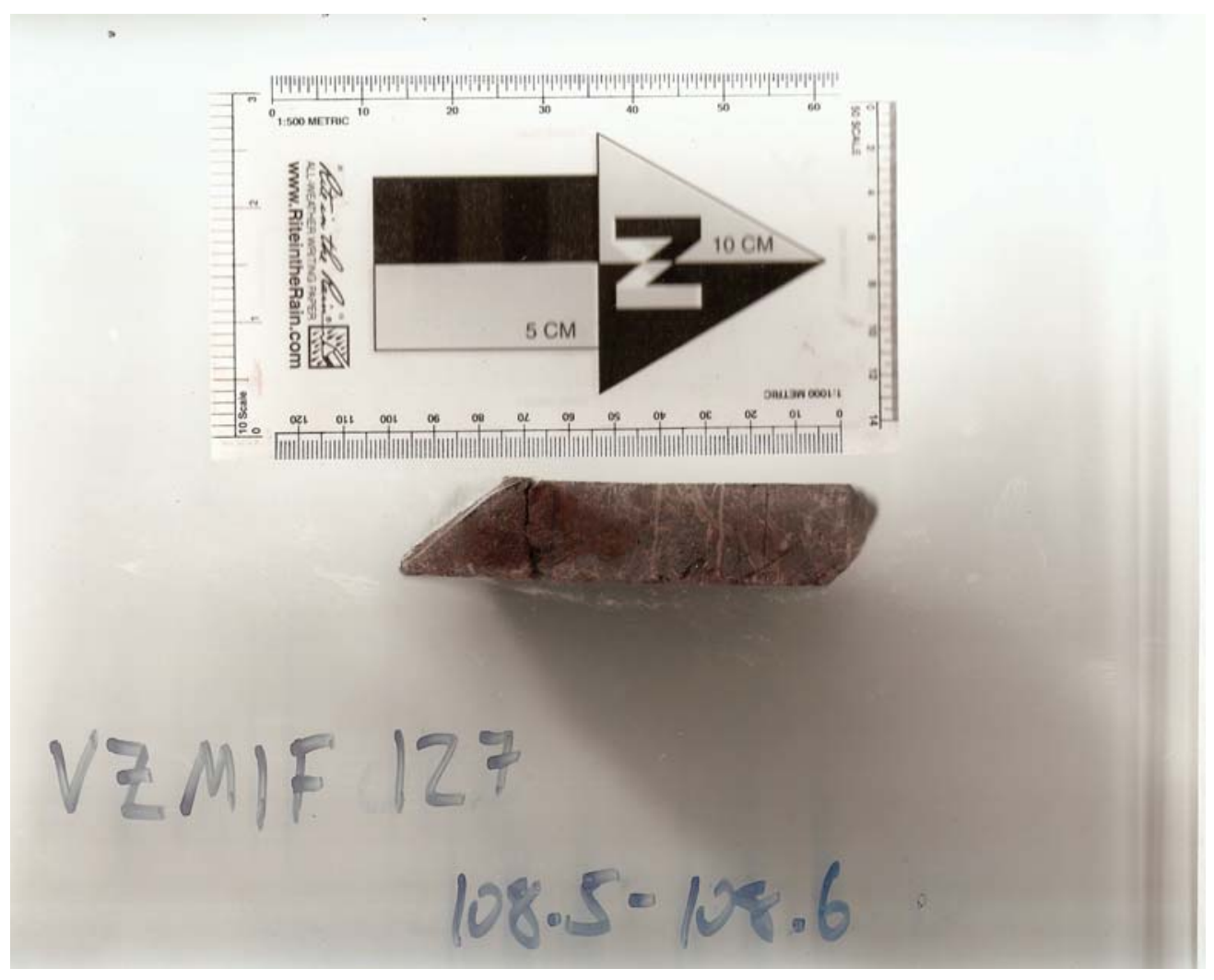

Other Sample Information: $16 \% \mathrm{Zn}$ 


\begin{tabular}{|c|c|c|c|}
\hline minerals & Dolomite & Quartz & willemite \\
\hline percentage & 20 & 6 & 8 \\
\hline morphology & $\begin{array}{l}2 \text { types: } \\
\text { 1) large, anhedral Fe-dol in } \\
\text { veins } \\
\text { 2) Small, subhedral dol }\end{array}$ & $\begin{array}{l}\text { A few small anhedrals xtals } \\
\text { with small dol. Mostly large } \\
\text { euhedral xtals in veins }\end{array}$ & $\begin{array}{l}\text { A few small anhedrals xtals } \\
\text { with small dol. Mostly large } \\
\text { euhedral xtals in veins }\end{array}$ \\
\hline texture & $\begin{array}{l}\text { Large dol is being replaced } \\
\text { by dol and hm, and replacing } \\
\text { qtz and wl } \\
\text { Small dol is being replaced } \\
\text { by hm }\end{array}$ & $\begin{array}{l}\text { Smaller xtals compose } \\
\text { groundmass. Large xtals } \\
\text { make up veins, being re- } \\
\text { placed by dol }\end{array}$ & $\begin{array}{l}\text { Smaller xtals compose } \\
\text { groundmass. Large xtals } \\
\text { make up veins, being re- } \\
\text { placed by dol }\end{array}$ \\
\hline $\begin{array}{l}\text { transmitted opti- } \\
\text { cal properties }\end{array}$ & & $\begin{array}{l}\text { Lower relief than wl. A few } \\
\text { xtals have qtz overgrowths } \\
\text { and rims }\end{array}$ & \\
\hline $\begin{array}{l}\text { reflected optical } \\
\text { properties }\end{array}$ & & & \\
\hline $\begin{array}{l}\text { contact/ } \\
\text { relationships with } \\
\text { other minerals }\end{array}$ & $\begin{array}{l}\text { Small dol is corroded by } \\
\text { hematite and being replaced } \\
\text { by small qtz and wl }\end{array}$ & $\begin{array}{l}\text { Large xtals in veins have } \\
\text { straight edges with wl and } \\
\text { other qtz, being slightly re- } \\
\text { placed by dol and hm }\end{array}$ & $\begin{array}{l}\text { Large xtals in veins have } \\
\text { straight edges with qtz and } \\
\text { other wl, being slightly re- } \\
\text { placed by dol and hm }\end{array}$ \\
\hline $\begin{array}{l}\text { other (growth di- } \\
\text { rection/zoning) }\end{array}$ & & Has euhedral growth rims & \\
\hline
\end{tabular}




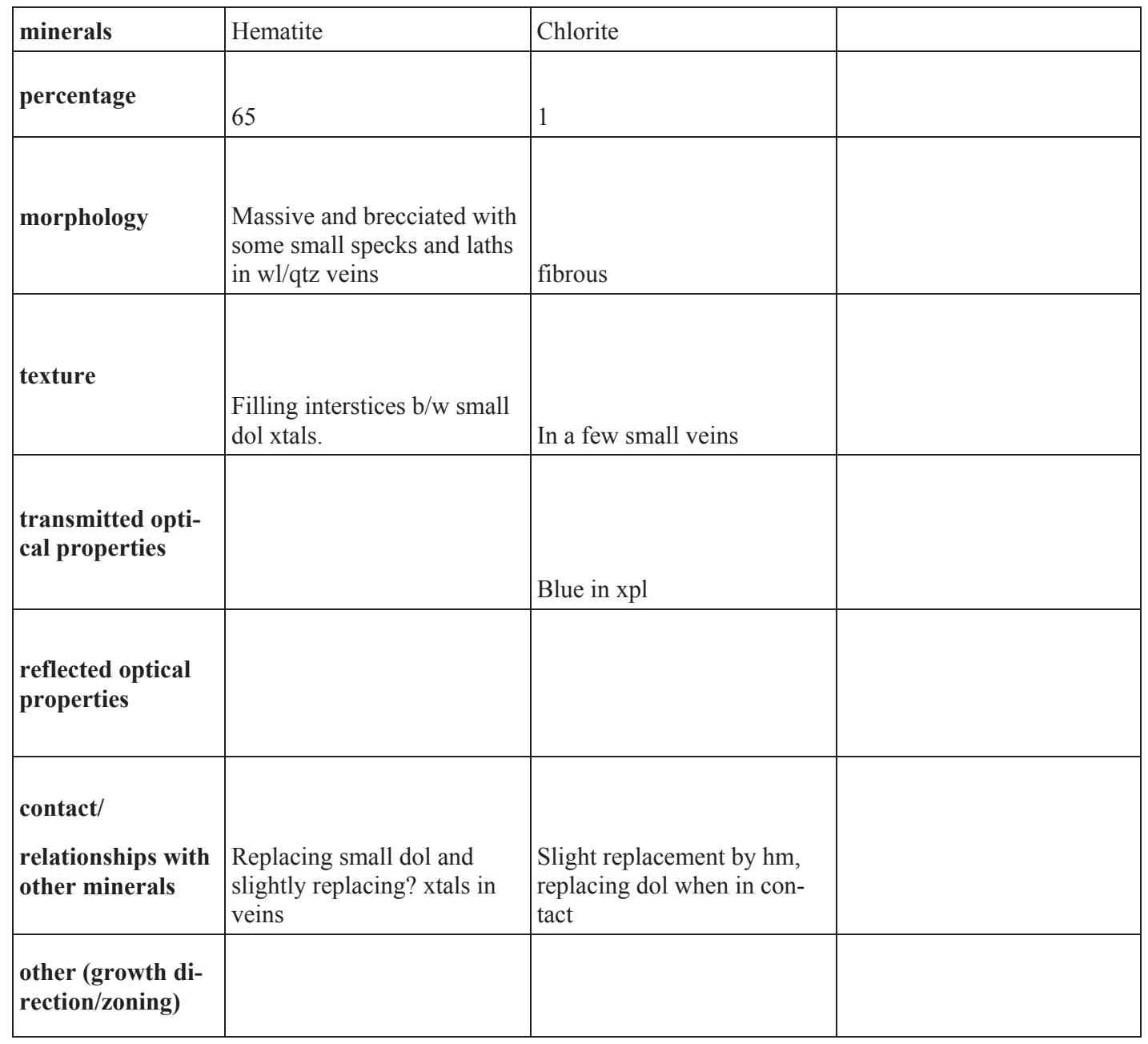

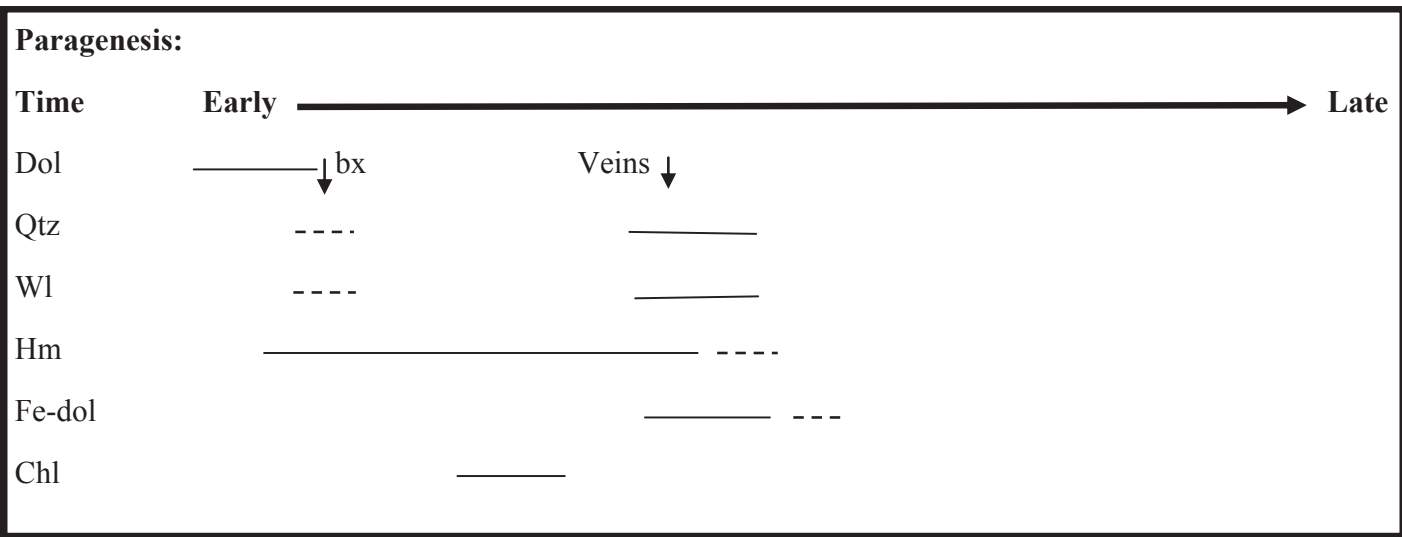


Structures: 2 large wl, qtz, and Fe-dol veins cross cut entire sample after main breccia event, as seen by there tendency to cut across breccia fragments

Paragenetic Sequence: $\mathrm{dol} \rightarrow \mathrm{wl}+\mathrm{qtz} \rightarrow \mathrm{hm}+\mathrm{chl} \rightarrow \mathrm{wl}+\mathrm{qtz}+\mathrm{Fe}-\mathrm{dol}$ in veins $\rightarrow$ shreddy Fe-carb

Paragenetic Reasoning: The rock-forming dol has small spots of qtz and wl replacement, but is thoroughly being replaced by $\mathrm{hm}$ found in between grains and in large bx fragments. The hm, in turn, is being cut by wl, qtz, and Fe-dol veins with some slight replacement of these veins by hm. Within the veins, Fe-dol is replacing qtz and wl (possible why high iron and no zinc...Fe-carb alteration). Some Fe-carb alteration on vein margins is replacing all minerals.

Sample Highlights: later wl and qtz veins with Fe-dol 
Geologic Unit: Hydrothermal Breccia

Lithological Unit (Votorantim): Massive Silicate (SM) in BH

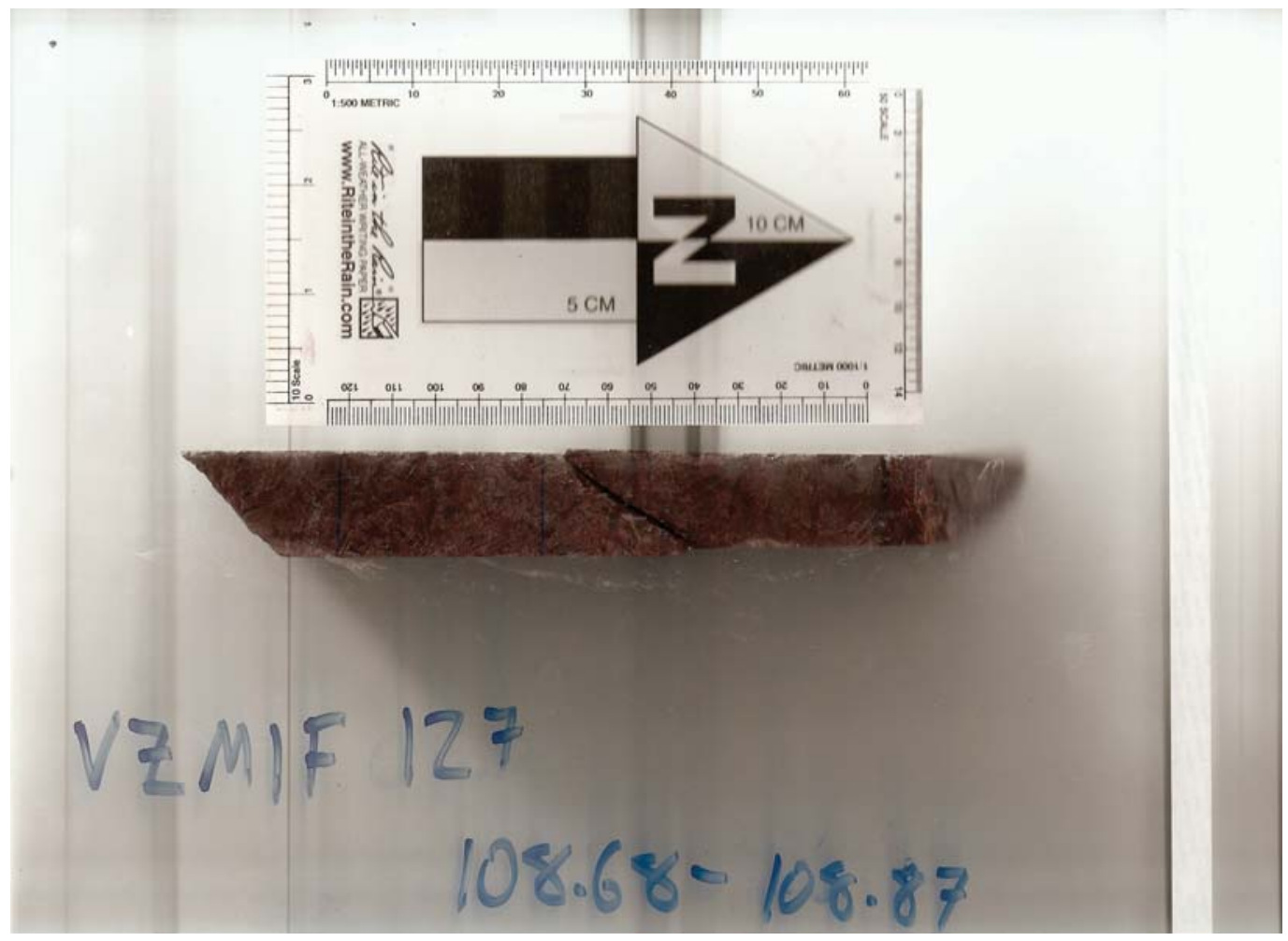

Other Sample Information: Similar to $127-108.5$ with faults instead of veins. Hm clasts in bx are sheared, faults orthogonal to shearing 


\begin{tabular}{|c|c|c|c|}
\hline minerals & Carbonate & Quartz & Willemite \\
\hline percentage & 15 & 20 & 15 \\
\hline morphology & $\begin{array}{l}\text { A few original xtals being } \\
\text { replaced by qtz and hm, } \\
\text { mostly small, fine Fe-rich } \\
\text { dol }\end{array}$ & $\begin{array}{l}\text { Anhedral to subhedral xtals, } \\
\text { makes up majority of non- } \\
\text { opaques }\end{array}$ & $\begin{array}{l}\text { Similar to qtz but with high- } \\
\text { er order interference colors }\end{array}$ \\
\hline texture & $\begin{array}{l}\text { Small Fe-dol is replacing qtz } \\
\text { and wl }\end{array}$ & $\begin{array}{l}\text { Cumulate replacement of } \\
\text { dol, being replaced by hm }\end{array}$ & $\begin{array}{l}\text { Similar to qtz but with high- } \\
\text { er order interference colors }\end{array}$ \\
\hline $\begin{array}{l}\text { transmitted opti- } \\
\text { cal properties }\end{array}$ & *sample cut thin & *sample cut thin & *sample cut thin \\
\hline $\begin{array}{l}\text { reflected optical } \\
\text { properties }\end{array}$ & & & \\
\hline $\begin{array}{l}\text { contact/ } \\
\text { relationships with } \\
\text { other minerals }\end{array}$ & $\begin{array}{l}\text { Irregular contacts with qtz } \\
\text { and wl as well as hm }\end{array}$ & $\begin{array}{l}\text { Irregular contacts with hm } \\
\text { and dol, but straight with } \\
\text { other qtz and wl }\end{array}$ & Same as qtz \\
\hline $\begin{array}{l}\text { other (growth di- } \\
\text { rection/zoning) }\end{array}$ & & & \\
\hline
\end{tabular}




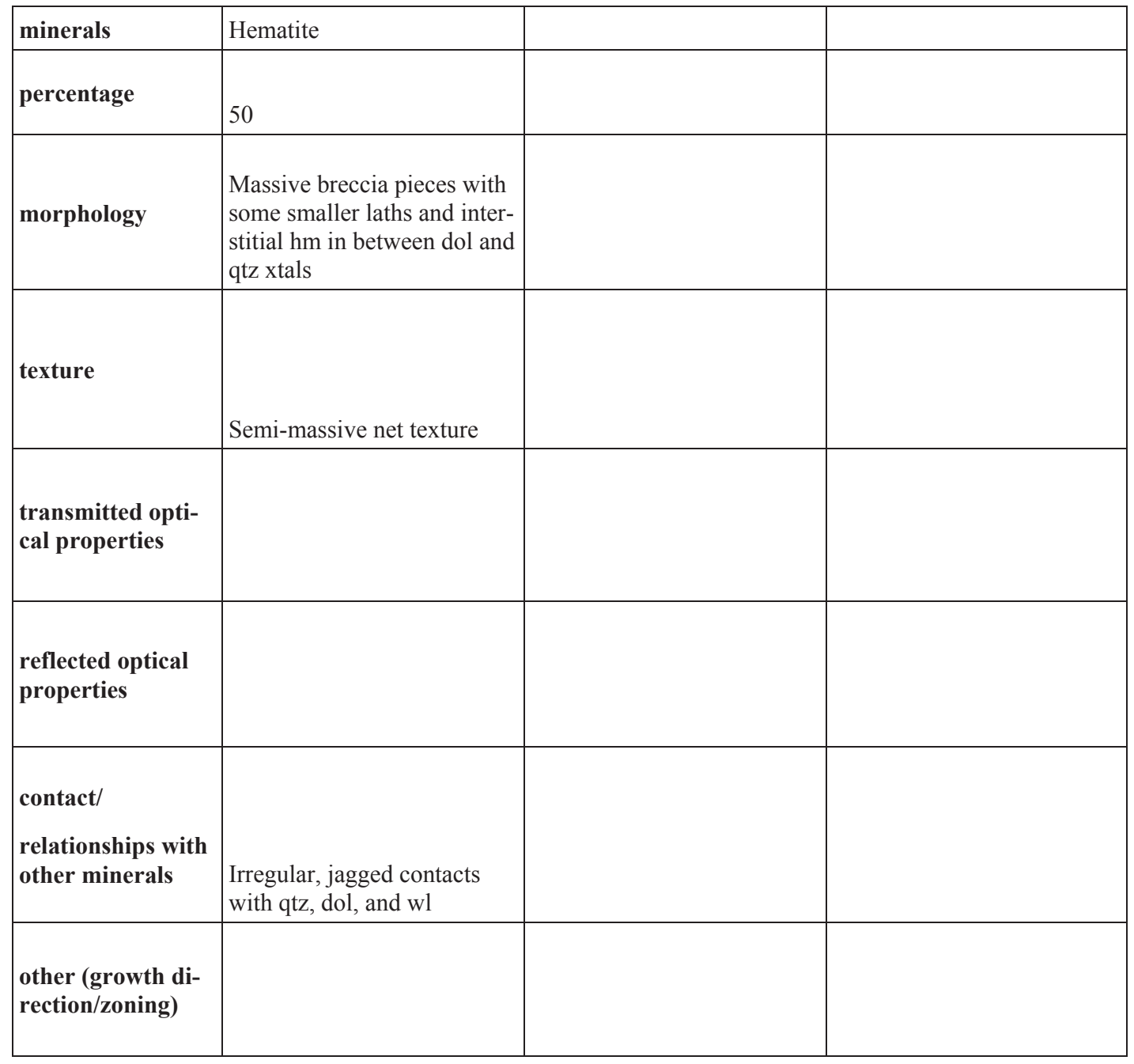

\begin{tabular}{l|l}
\hline Paragenesis: & \\
Time & Early $\longrightarrow$ Late \\
Dol & $\longrightarrow$ bx \\
Qtz & \\
Wl & \\
$\mathrm{Hm}$ &
\end{tabular}


Structures: A set of faults (and smaller fractures) orthogonal to the orientation of the sheared hematite bx fragments

Paragenetic Sequence: $\mathrm{dol} \rightarrow \mathrm{qtz}+\mathrm{wl} \rightarrow \mathrm{hm} \rightarrow \mathrm{Fe}-\mathrm{dol}$

Paragenetic Reasoning: The dolomite found is relict and being replaced by qtz and wl. The qtz and wl have irregular, wavy contacts with $\mathrm{hm}$, suggesting replacement by $\mathrm{hm}$. Lastly, the Fe-dol is fine and also replacing the qtz and wl regions, but it is being replaced or is interwoven with $\mathrm{hm}$.

Sample Highlights: fault orthogonal to shear 
Geologic Unit: Lower Pamplona

Lithological Unit (Votorantim): Hydrothermal Breccia

Sample Photograph
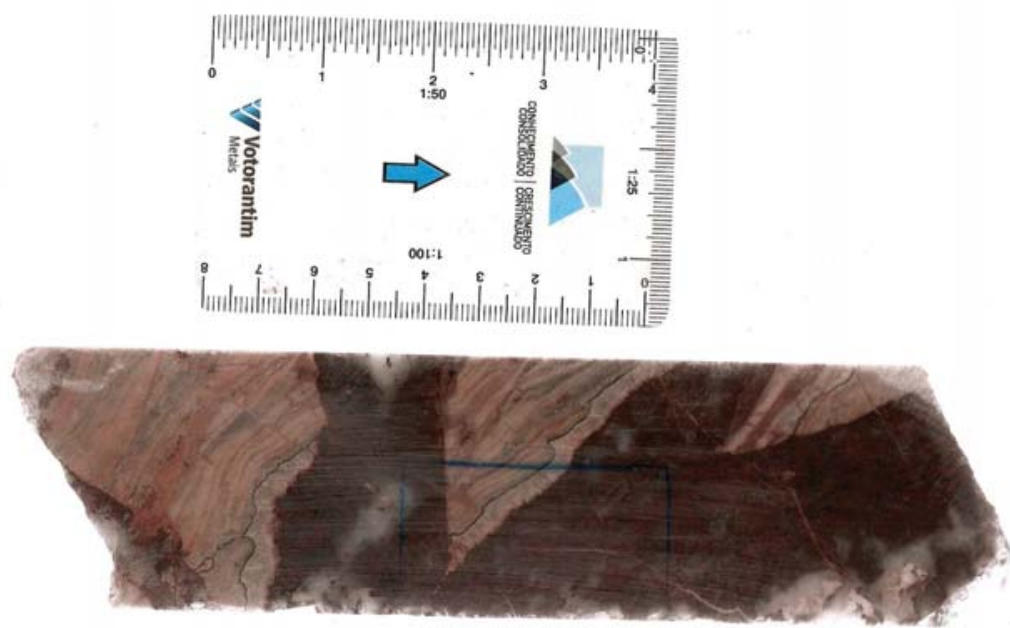

\section{VEMIF 129}

$$
138.0-138.18
$$

Other Sample Information: 


\begin{tabular}{|c|c|c|c|}
\hline minerals & Carbonate & Quartz & Hematite \\
\hline percentage & 25 & 72 & 3 \\
\hline morphology & Micritic to sparry & Mostly anhedral & anhedral \\
\hline texture & Breccia fragment & hydrothermal & disseminated \\
\hline $\begin{array}{l}\text { transmitted opti- } \\
\text { cal properties }\end{array}$ & & & \\
\hline $\begin{array}{l}\text { reflected optical } \\
\text { properties }\end{array}$ & & & \\
\hline $\begin{array}{l}\text { contact/ } \\
\text { relationships with } \\
\text { other minerals }\end{array}$ & $\begin{array}{l}\text { Somewhat irregular contact } \\
\text { with qtz, more likely b/c of } \\
\text { change in fluid content than } \\
\text { replacement }\end{array}$ & $\begin{array}{l}\text { See carb, irregular contacts } \\
\text { with } \mathrm{hm} \text {, replacing } \mathrm{hm} \text { ? }\end{array}$ & $\begin{array}{l}\text { Found included in qtz or be- } \\
\text { ing replaced by qtz }\end{array}$ \\
\hline $\begin{array}{l}\text { other (growth di- } \\
\text { rection/zoning) }\end{array}$ & & $\begin{array}{l}\text { Zoned with hm; lots of sec- } \\
\text { ondary fluid inclusion trails }\end{array}$ & \\
\hline
\end{tabular}




\begin{tabular}{|c|c|c|c|}
\hline minerals & barite & pyrite & unknown \\
\hline percentage & trace & Very trace & trace \\
\hline morphology & anhedral & anhedral & subhedral \\
\hline texture & Disseminated & Disseminated & Some triangular faces \\
\hline $\begin{array}{l}\text { transmitted opti- } \\
\text { cal properties }\end{array}$ & Low order birefringence & & \\
\hline $\begin{array}{l}\text { reflected optical } \\
\text { properties }\end{array}$ & & & $\begin{array}{l}\text { Very strong anisotropy, } \\
\text { higher reflectance than } \mathrm{hm}\end{array}$ \\
\hline $\begin{array}{l}\text { contact/ } \\
\text { relationships with } \\
\text { other minerals }\end{array}$ & $\begin{array}{l}\text { Irregular with qtz, being re- } \\
\text { placed by qtz? }\end{array}$ & & $\begin{array}{l}\text { Straighter contacts with qtz } \\
\text { than } \mathrm{hm}\end{array}$ \\
\hline $\begin{array}{l}\text { other (growth di- } \\
\text { rection/zoning) }\end{array}$ & & & \\
\hline
\end{tabular}

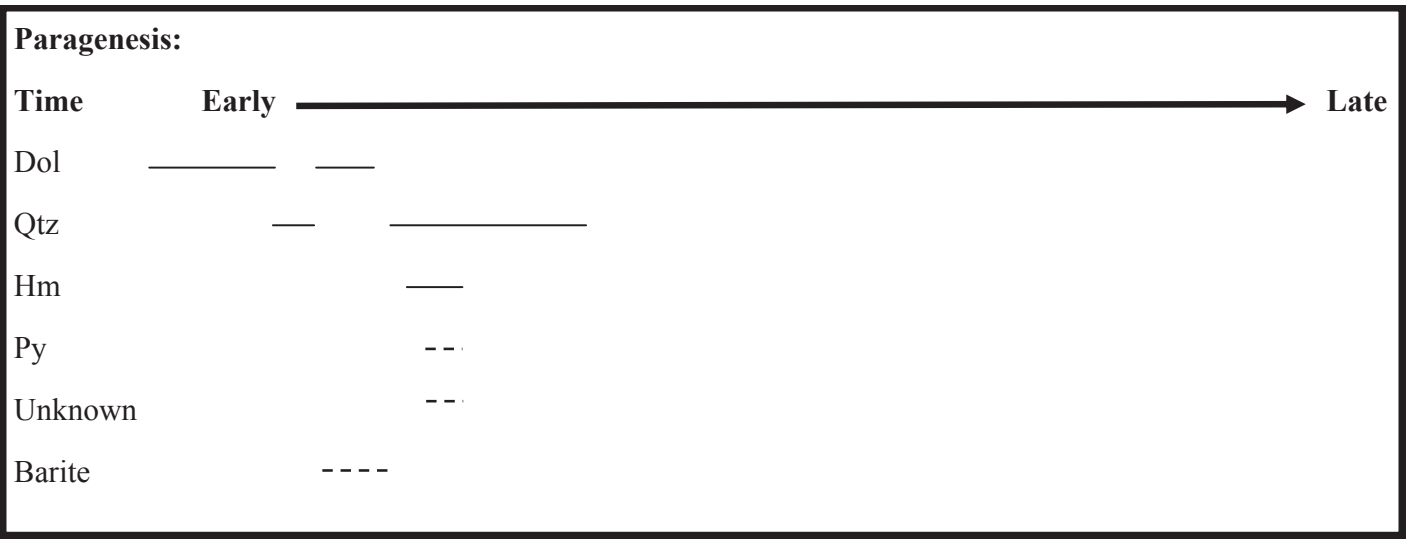


Geologic Unit: Hydrothermal Breccia

Lithological Unit (Votorantim): Massive Silicate (SM) in BH

Sample Photograph

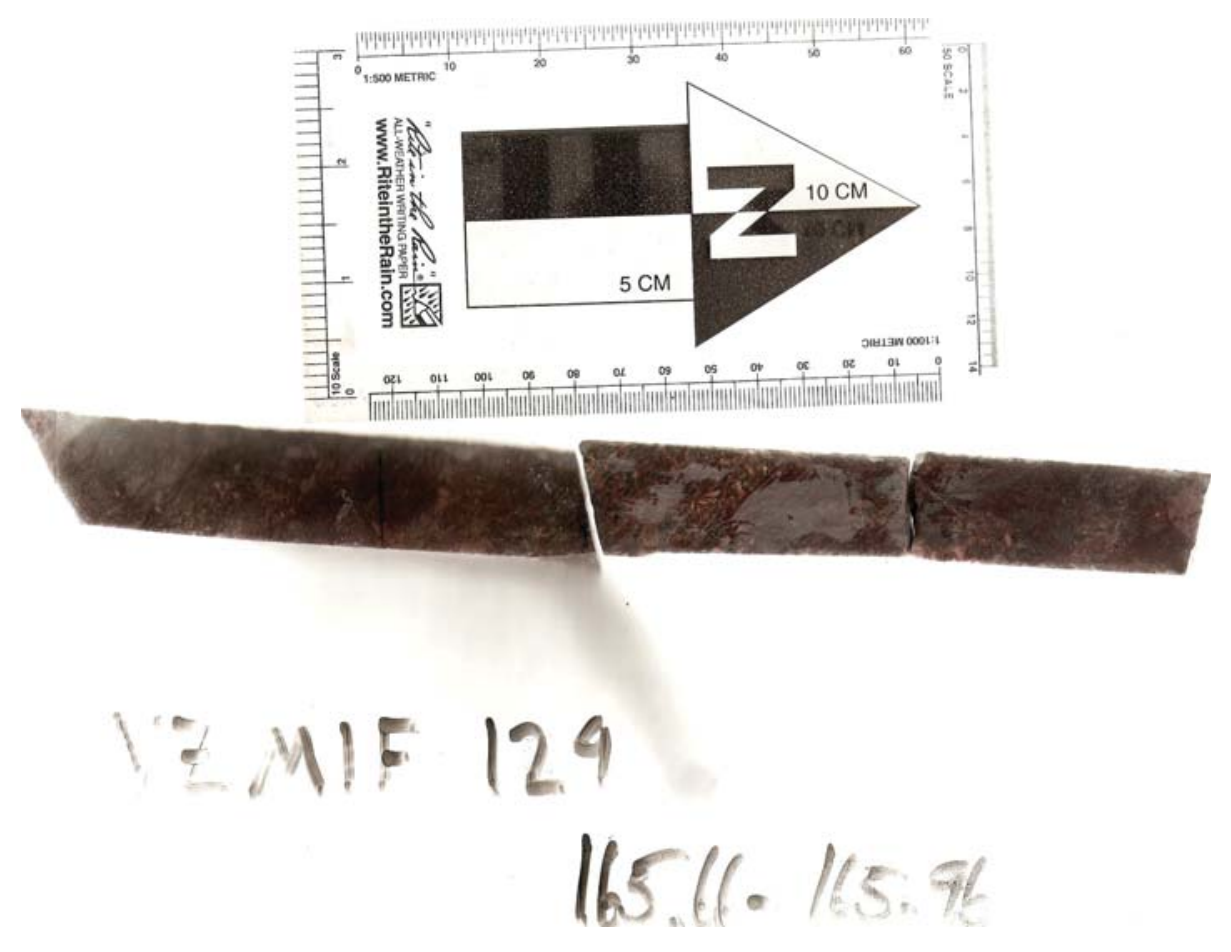

Other Sample Information: Standard appearance of willimite and hematite shear veins 


\begin{tabular}{|c|c|c|c|}
\hline minerals & Willemite & Dolomite & Hematite \\
\hline percentage & 65 & 20 & 15 \\
\hline morphology & $\begin{array}{l}\text { Small subhedral crystals to } \\
\text { large euhedral crystals fill- } \\
\text { ing in void spaces all replac- } \\
\text { ing dol }\end{array}$ & $\begin{array}{l}\text { 1) large anhedral dol } \\
\text { (primary) being replaced } \\
\text { by wl } \\
\text { 2)smaller secondary dol fill- } \\
\text { ing in veins }\end{array}$ & $\begin{array}{l}\text { Subhedral, associated with } \\
\text { wl and replacing dol. Also } \\
\text { filling in leftover space b/w } \\
\text { Wl xtals }\end{array}$ \\
\hline texture & $\begin{array}{l}\text { Filling in open space from } \\
\text { bx, replacing dol }\end{array}$ & $\begin{array}{l}\text { Veins and being replaced by } \\
\text { wl \& hm }\end{array}$ & $\begin{array}{l}\text { Replacing Wl and dol, fill- } \\
\text { ing in space after W1 }\end{array}$ \\
\hline \multicolumn{4}{|l|}{$\begin{array}{l}\text { transmitted opti- } \\
\text { cal properties }\end{array}$} \\
\hline \multicolumn{4}{|l|}{$\begin{array}{l}\text { reflected optical } \\
\text { properties }\end{array}$} \\
\hline $\begin{array}{l}\text { contact/ } \\
\text { relationships with } \\
\text { other minerals }\end{array}$ & $\begin{array}{l}\text { Straight edges with other wl. } \\
\text { Small irregularities with dol } \\
\text { contacts (replacement). Ir- } \\
\text { regular/jagged contact with } \\
\text { hm (lots of embayments) }\end{array}$ & $\begin{array}{l}\text { Irregular contacts with wl } \\
\text { and hm (wl overgrowing } \\
\text { dol) }\end{array}$ & $\begin{array}{l}\text { Filling in spaces b/w Wl } \\
\text { xtals }\end{array}$ \\
\hline $\begin{array}{l}\text { other (growth di- } \\
\text { rection/zoning) }\end{array}$ & & & \\
\hline
\end{tabular}




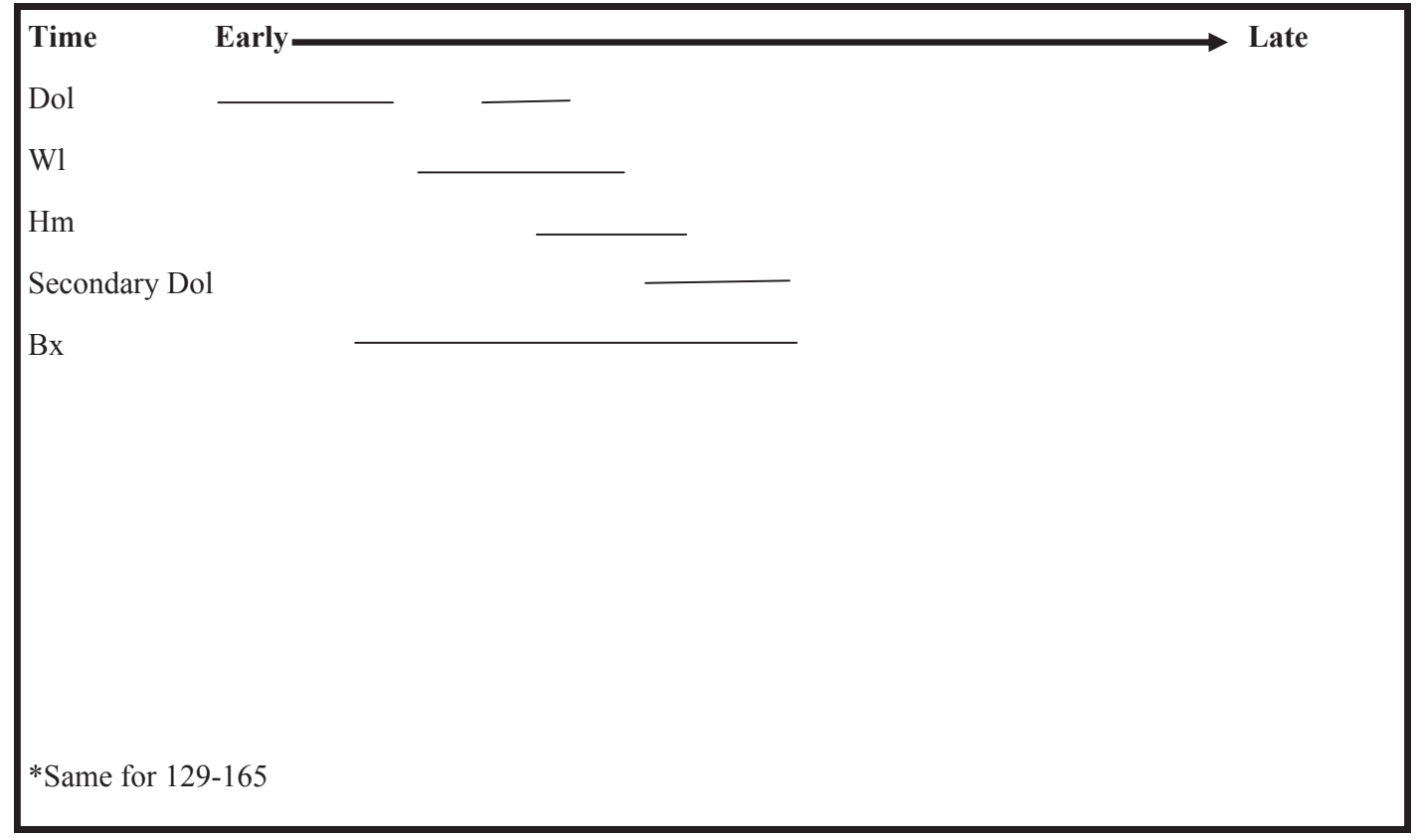


Structures: same as 129-165

Veins associated with bx

Paragenetic Sequence: $\mathrm{dol} \rightarrow \mathrm{Wl} \rightarrow \mathrm{Hm} \rightarrow$ late dol veins

Paragenetic Reasoning: same as $129-165$

Sample Highlights: similar to $129-165$, but less brecciated

Carbonate veins have larger xtals in faulted open space (See Fig) 
Geologic Unit: Hydrothermal Breccia

Lithological Unit (Votorantim): Massive Silicate (SM) in BH

\section{Sample Photograph}
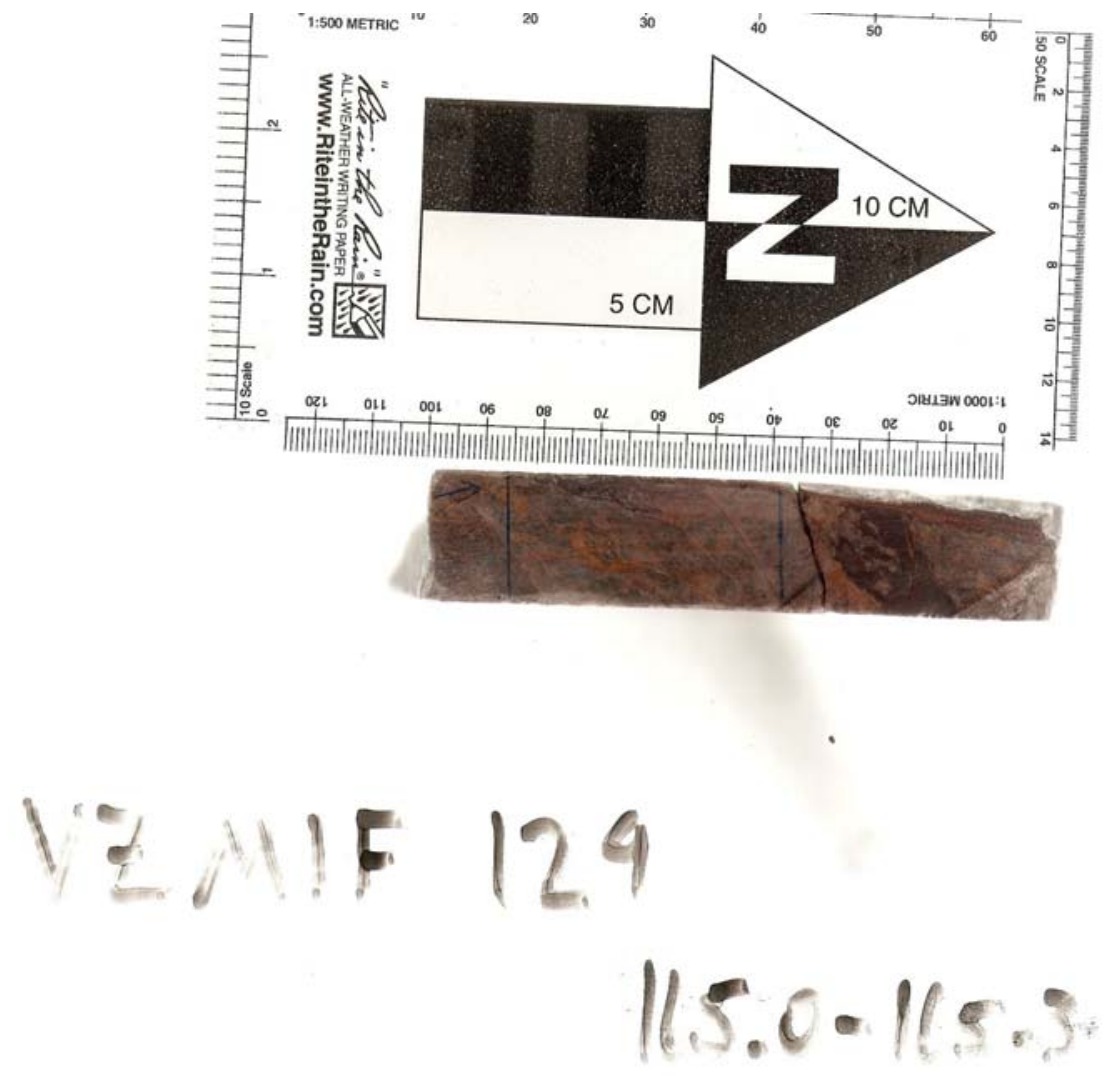

Other Sample Information: Willimite with non specular hematite veins. Alteration appears different than the usual $\mathrm{Wl}+\mathrm{Hm}$ shear vein (more orange and ruddy) 


\begin{tabular}{|c|c|c|c|}
\hline minerals & willimite & dolomite & Sphalerite \\
\hline percentage & 60 & 35 & 2 \\
\hline morphology & $\begin{array}{l}\text { Smaller crystals are more } \\
\text { anhedral. Larger crystals are } \\
\text { subhedral laths and grains } \\
\text { around voids }\end{array}$ & $\begin{array}{l}\text { Small, anhedral crystals } \\
\text { composing veins. A few } \\
\text { larger xtals }\end{array}$ & Disseminated, amorphous \\
\hline texture & $\begin{array}{l}\text { Brecciated, contains small } \\
\text { amounts of hematite }\end{array}$ & $\begin{array}{l}\text { Vein texture in between wl } \\
\text { breccia clasts, replacing wl }\end{array}$ & $\begin{array}{l}\text { Located as inclusions within } \\
\text { wl }\end{array}$ \\
\hline $\begin{array}{l}\text { transmitted opti- } \\
\text { cal properties }\end{array}$ & Cut thin & Cut thin & opaque \\
\hline reflected optical & $\mathrm{n} / \mathrm{a}$ & $\mathrm{n} / \mathrm{a}$ & \\
\hline $\begin{array}{l}\text { contact/ } \\
\text { relationships with } \\
\text { other minerals }\end{array}$ & $\begin{array}{l}\text { Shreddy, irregular contact } \\
\text { with Fe-carb, being replaced } \\
\text { by Fe-carb. Irregular contact } \\
\text { with Hm, replacing? Hm }\end{array}$ & $\begin{array}{l}\text { Replacing wl, hm, and pri- } \\
\text { mary dol }\end{array}$ & $\begin{array}{l}\text { Embayed and irregular con- } \\
\text { tact with all mins, }\end{array}$ \\
\hline $\begin{array}{l}\text { other (growth di- } \\
\text { rection/zoning) }\end{array}$ & Some minor circular zoning & & Internal reflections \\
\hline
\end{tabular}




\begin{tabular}{|l|l|l|l|}
\hline minerals & dolomite & & \\
\hline percentage & 3 & & \\
\hline morphology & Large, anhedral & & \\
\hline texture & $\begin{array}{l}\text { In voids, being replaced by } \\
\text { wl }\end{array}$ & & \\
\hline $\begin{array}{l}\text { transmitted opti- } \\
\text { cal properties }\end{array}$ & & & \\
\hline $\begin{array}{l}\text { reflected optical } \\
\text { properties }\end{array}$ & & & \\
\hline $\begin{array}{l}\text { contact/ } \\
\text { relationships with } \\
\text { other minerals }\end{array}$ & $\begin{array}{l}\text { Irregular contact with wil- } \\
\text { lemite, being replaced by wl }\end{array}$ & & \\
\hline $\begin{array}{l}\text { other (growth di- } \\
\text { rection/zoning) }\end{array}$ & & & \\
\hline
\end{tabular}

Paragenesis:

Time

Early

Dol

SI

W1

$\mathrm{Hm}$ ?

Carbonate Alt 
Structures: Sample is brecciated throughout and the fractures are filled with tiny, secondary carbonates, including dol and Fe-rich carbonates.

Paragenetic Sequence: dol $\rightarrow \mathrm{sl} \rightarrow \mathrm{wl} \rightarrow \mathrm{bx} \rightarrow 2$ nd carbonates

Paragenetic Reasoning: The primary dolomite is being replaced by the wl in the sample. The $\mathrm{Wl}$ is large and subhedral, indicating growth into possible open spaces (bx?). The Sl is associated with Wl and is in the middle of many Wl samples and becoming replaced by Wl. The bx has crushed and cut the $\mathrm{Wl}$ and the spaces are filled in with fine carbonates.

Sample Highlights: carbonate alteration and sphalerite 
Geologic Unit: Hydrothermal Breccia

Lithological Unit (Votorantim): Massive Silicate (SM) in BH

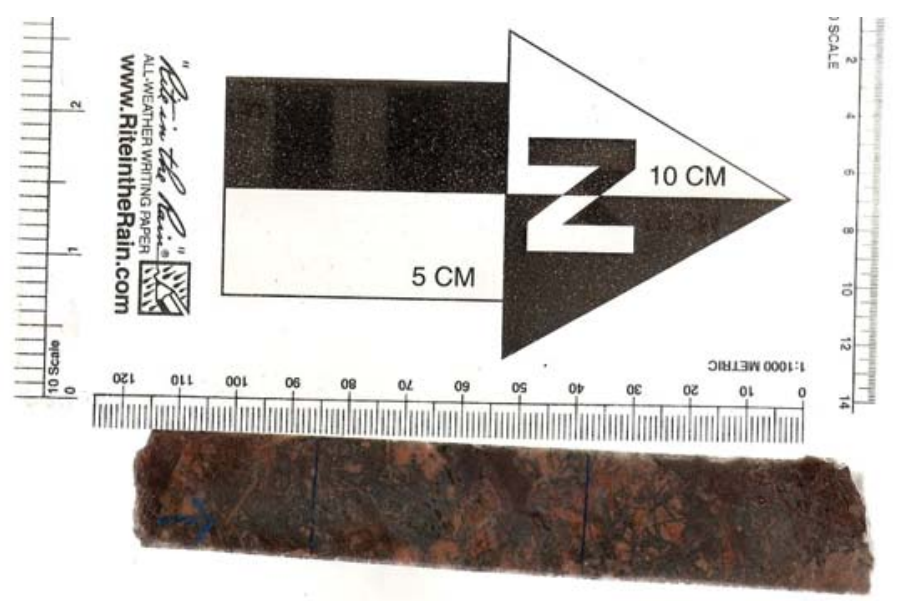

12 . 129

$$
167.11 \cdot 119.999
$$

Other Sample Information: Fe-carbonate altered dolomite clasts in mineralized breccia 


\begin{tabular}{|l|l|l|l|}
\hline minerals & Willemite & Quartz & Hematite \\
\hline percentage & 30 & 20 & 30 \\
\hline morphology & $\begin{array}{l}\text { Mostly fibrous xtals with } \\
\text { some smaller anhedral xtals }\end{array}$ & $\begin{array}{l}\text { Subhedrals xtals (silica alt?), } \\
\text { ing open space } \\
\text { ing places of later qtz fill- }\end{array}$ & $\begin{array}{l}\text { Massive in veins. Dissemi- } \\
\text { nated euhedral laths }\end{array}$ \\
\hline $\begin{array}{l}\text { texture } \\
\text { Filling open space, xtals } \\
\text { grow towards center of veins } \\
\text { and vugs. Replacing dol }\end{array}$ & Filling open space, & Veins and replacing wl and \\
qtz
\end{tabular}




\begin{tabular}{|c|c|c|c|}
\hline minerals & Dolomite & Sphalerite & Fe-carb \\
\hline percentage & 15 & trace & 5 \\
\hline morphology & anhedral & subhedral & Small, shreddy \\
\hline texture & $\begin{array}{l}\text { Being replaced by wl, hm, } \\
\text { qtz }\end{array}$ & $\begin{array}{l}\text { Dissemated, but associated } \\
\text { w/ Hm }\end{array}$ & In veins that cut everything \\
\hline $\begin{array}{l}\text { transmitted opti- } \\
\text { cal properties }\end{array}$ & & & \\
\hline $\begin{array}{l}\text { reflected optical } \\
\text { properties }\end{array}$ & & & \\
\hline $\begin{array}{l}\text { contact/ } \\
\text { relationships with } \\
\text { other minerals }\end{array}$ & $\begin{array}{l}\text { Irregular contacts with other } \\
\text { minerals. Lot of embay- } \\
\text { ments and jagged edges. W1 } \\
\text { tends to overgrow dol }\end{array}$ & $\begin{array}{l}\text { Somewhat irregular contacts } \\
\text { with wl, mostly straight edg- } \\
\text { es with Hm. }\end{array}$ & $\begin{array}{l}\text { Cut everything, contacts are } \\
\text { mottled, splotches of Fe- } \\
\text { carb on hm, wl, and qtz. Cut } \\
\text { through hm }\end{array}$ \\
\hline $\begin{array}{l}\text { other (growth di- } \\
\text { rection/zoning) }\end{array}$ & & & Late \\
\hline
\end{tabular}

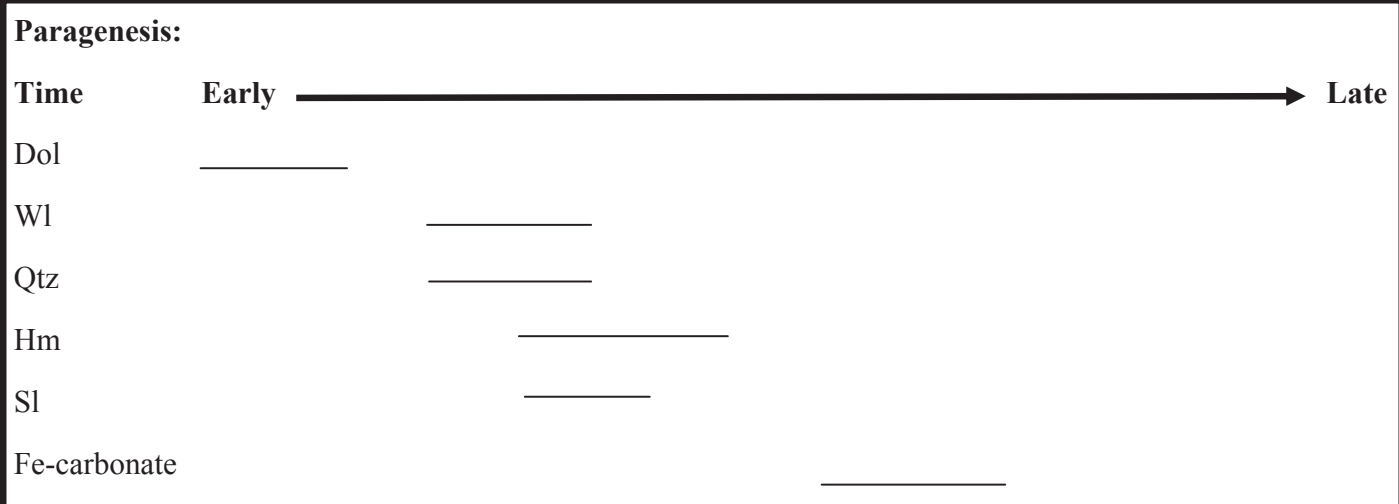


Structures: Sample has been brecciated and cut by hm shear veins. Silicates are replacing dol and filling in open space. Hm and Wl have intercalated growths next to hm shear vein, indicating "fluid pulses". Fe-carbonates create a stockwork of veins that cut through all minerals.

Paragenetic Sequence: dol $\rightarrow \mathrm{wl}+\mathrm{qtz} \rightarrow \mathrm{hm}+\mathrm{sl} \rightarrow \mathrm{Fe}$-carb

*hm overlaps with wl and is intergrown $\mathrm{w} / \mathrm{wl}$ in some instances

Paragenetic Reasoning: See wl and qtz overgrowing and replacing dol. Hm is replacing wl in main shear vein and has laths growing on qtz. Sl is associated? with $\mathrm{hm}$ next to some wl. Fe-carb veins cut all mins and have no specific order (i.e. V1, V2, ...)

Sample Highlights: Sphalerite, Fibrous wl + qtz 
Geologic Unit: Upper Morro do Pinheiro

Lithological Unit (Votorantim): Grey Dolomite (DO)

Sample Photograph

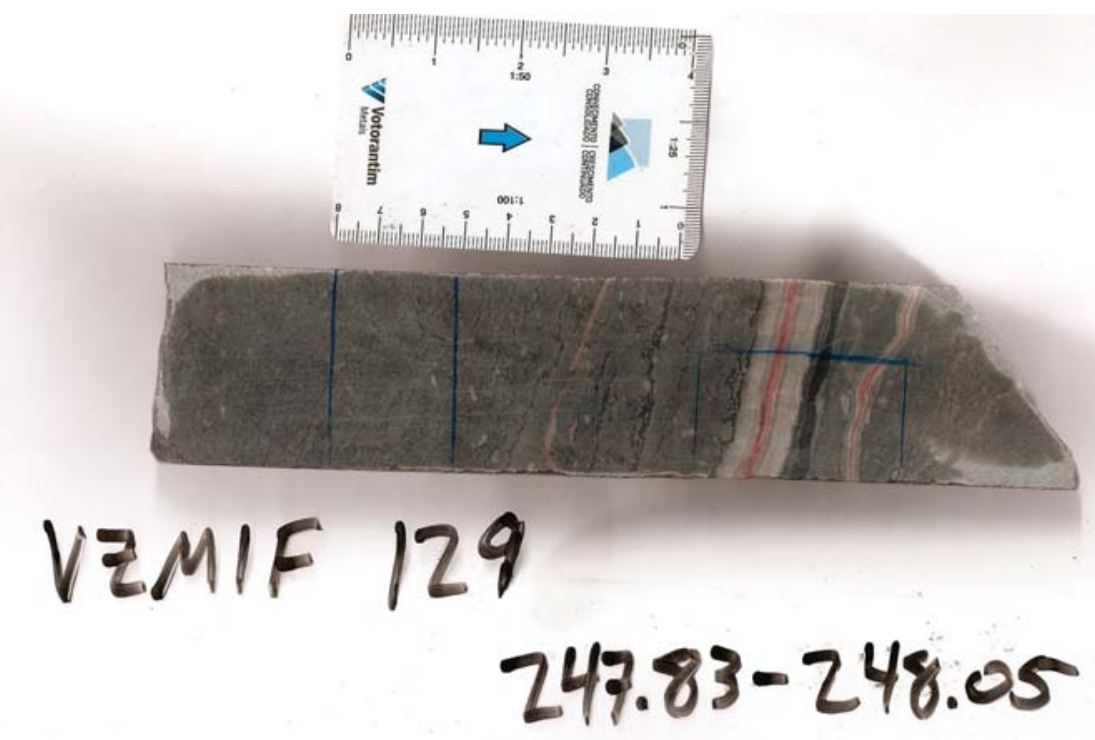

Other Sample Information: 


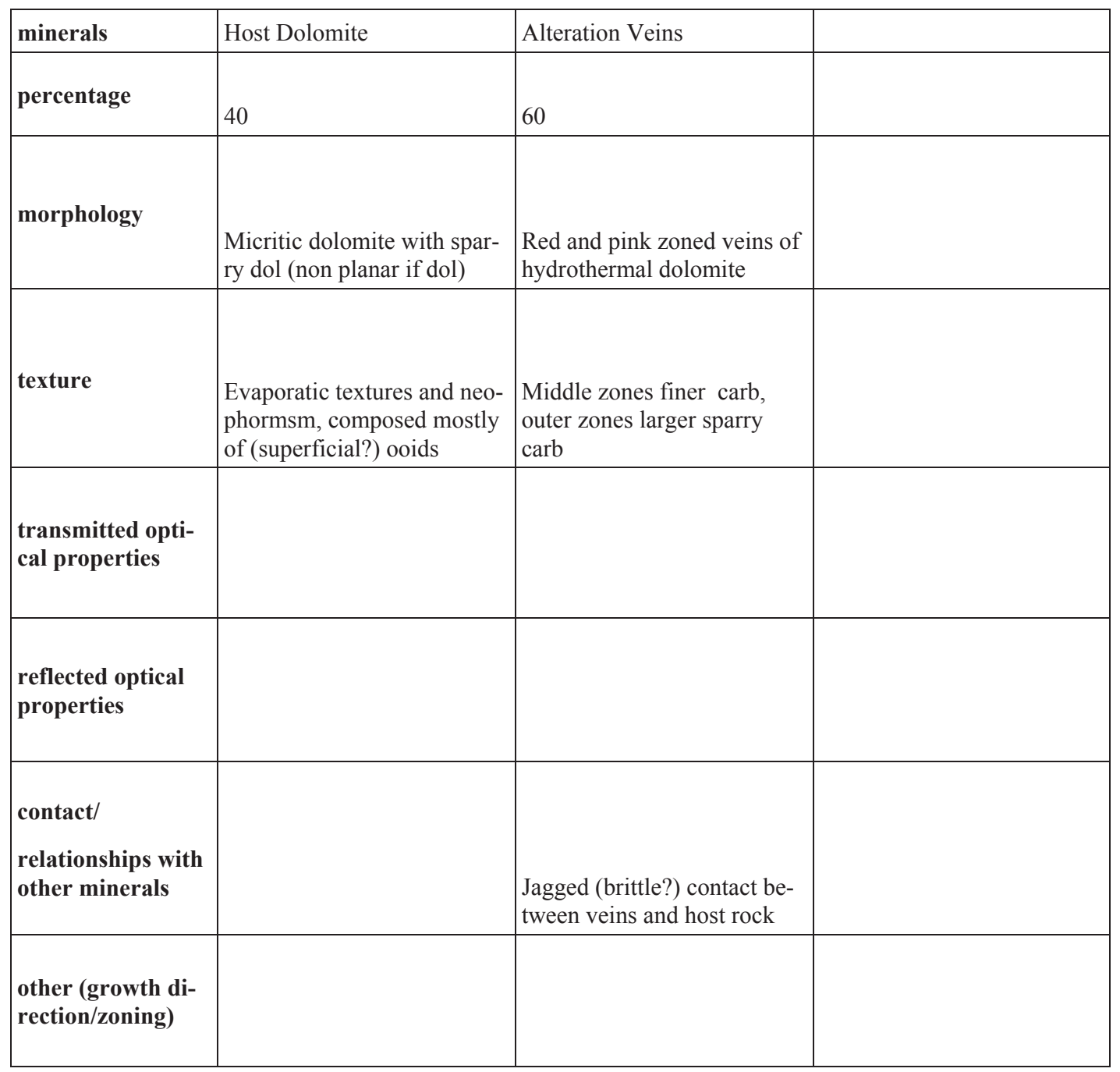


Geologic Unit: Middle Pamplona

Lithological Unit (Votorantim): Pink Dolomite (DO)

\section{Sample Photograph}

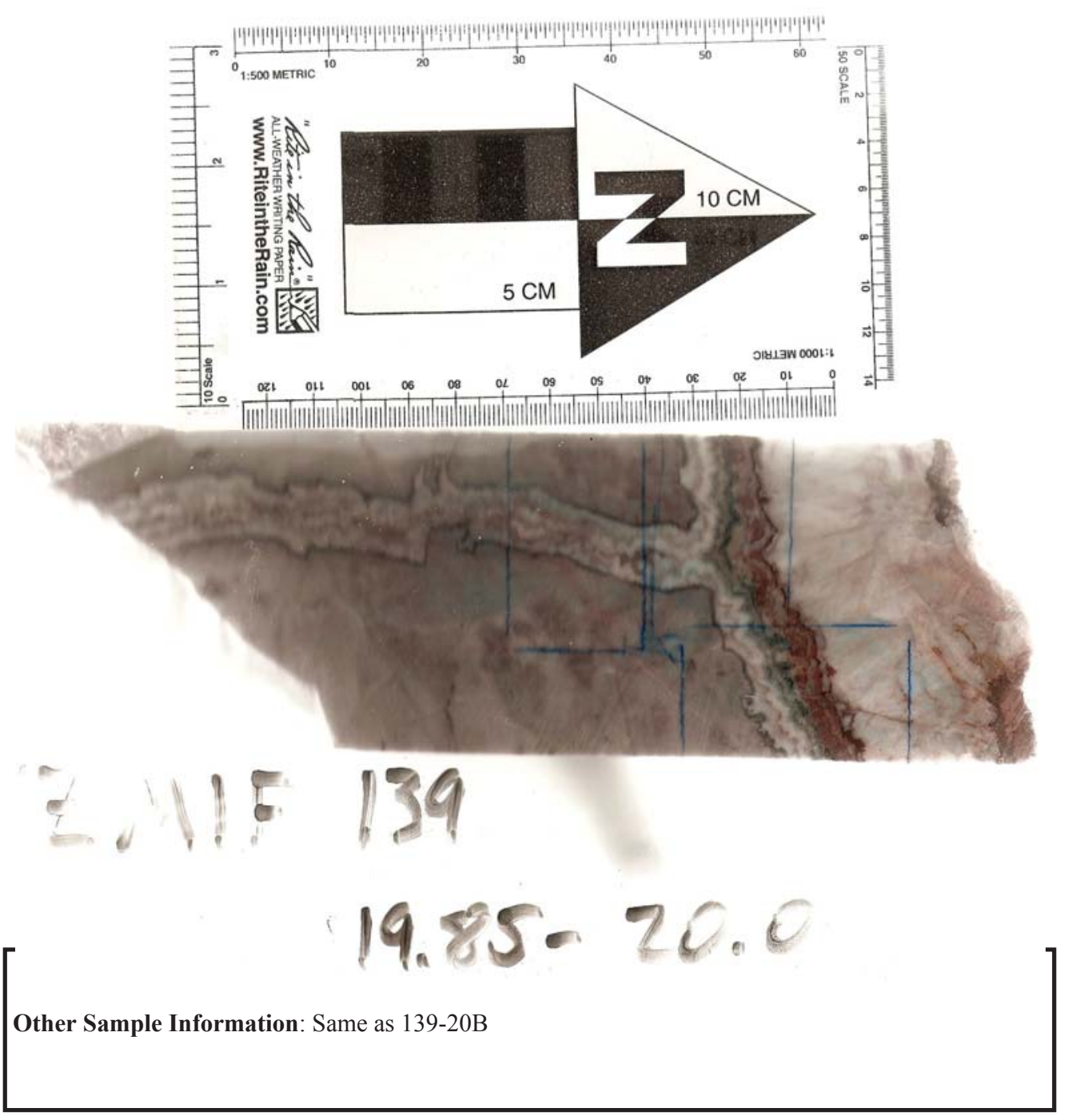


Geologic Unit: Middle Pamplona

Lithological Unit (Votorantim): Pink Dolomite (DO)

Sample Photograph

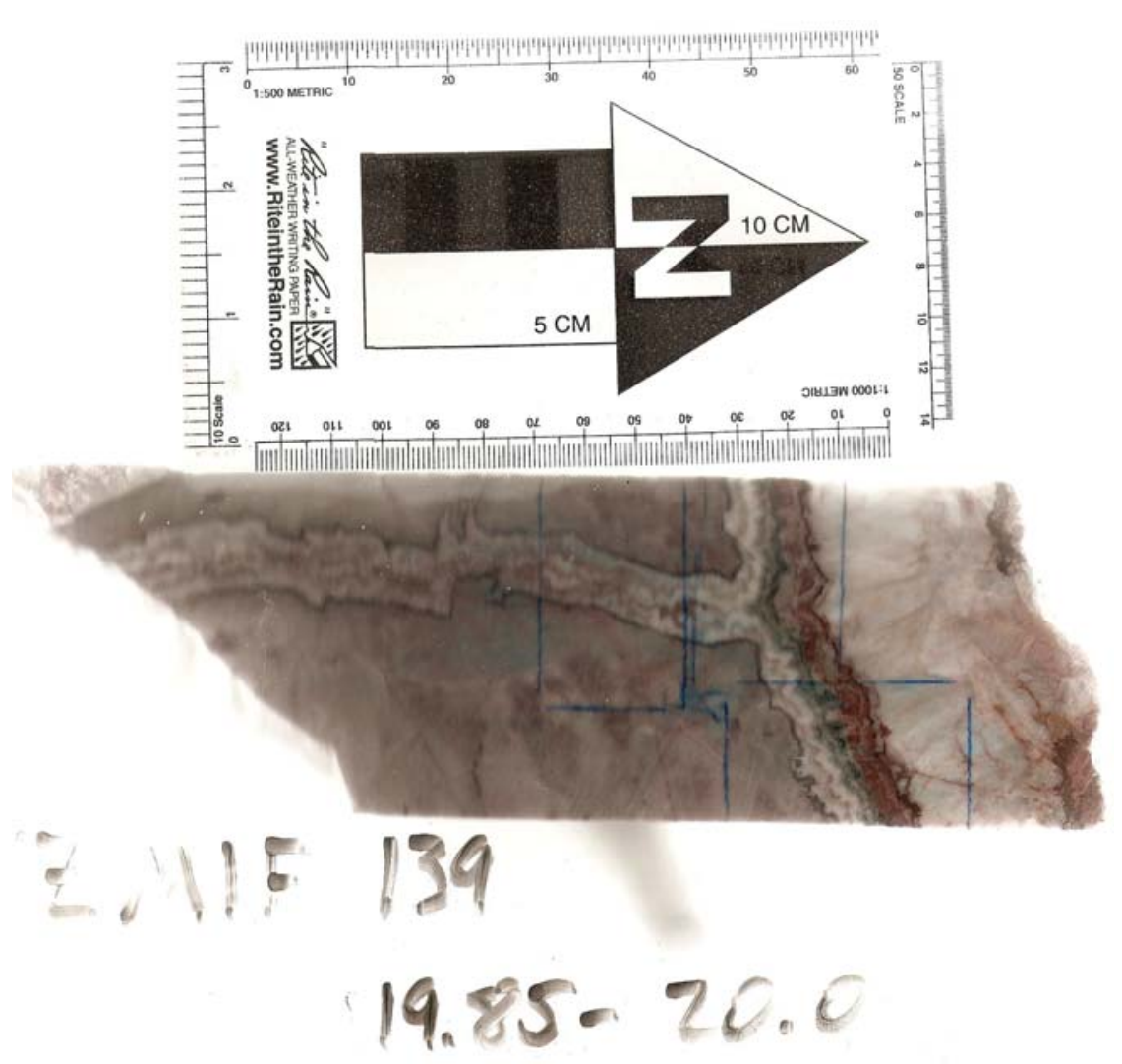

Other Sample Information: 


\begin{tabular}{|l|l|l|l|}
\hline minerals & Dolomite & Hydrothermal Dolomite & Quartz \\
\hline percentage & 20 & 72 & 5 \\
\hline morphology & anhedral & euhedral & euhedral \\
\hline texture & & & \\
\hline $\begin{array}{l}\text { transmitted opti- } \\
\text { cal properties }\end{array}$ & Host rock & Hydrothermal vein filling & Hydrothermal vein filling \\
\hline $\begin{array}{l}\text { reflected optical } \\
\text { properties }\end{array}$ & quartz & $\begin{array}{l}\text { Slightly irregular edges with } \\
\text { quartz. Irregular and em- } \\
\text { bayed edges with sp, py, and } \\
\text { fe-oxides, these minerals last } \\
\text { stage formation }\end{array}$ & Slightly irregular edges with \\
dol (rxn front?)
\end{tabular}




\begin{tabular}{|c|c|c|c|}
\hline minerals & Sphalerite & Pyrite & Fe-oxides/hydroxides \\
\hline percentage & 1 & 1 & 1 \\
\hline morphology & Anhedral xtal & Anhedral xtal & Anhedral xtal \\
\hline texture & $\begin{array}{l}\text { Disseminated last stage min- } \\
\text { eralization }\end{array}$ & $\begin{array}{l}\text { Disseminated last stage min- } \\
\text { eralization, some 'py dis- } \\
\text { ease' }\end{array}$ & Alteration from py \\
\hline $\begin{array}{l}\text { transmitted opti- } \\
\text { cal properties }\end{array}$ & & & \\
\hline $\begin{array}{l}\text { reflected optical } \\
\text { properties }\end{array}$ & & & \\
\hline $\begin{array}{l}\text { contact/ } \\
\text { relationships with } \\
\text { other minerals }\end{array}$ & $\begin{array}{l}\text { Irregular with dol, filling in } \\
\text { space }\end{array}$ & $\begin{array}{l}\text { Irregular with dol, filling in } \\
\text { space, minor 'py disease' in } \\
\text { sp-replacing sp }\end{array}$ & Altering from py \\
\hline $\begin{array}{l}\text { other (growth di- } \\
\text { rection/zoning) }\end{array}$ & & & \\
\hline
\end{tabular}

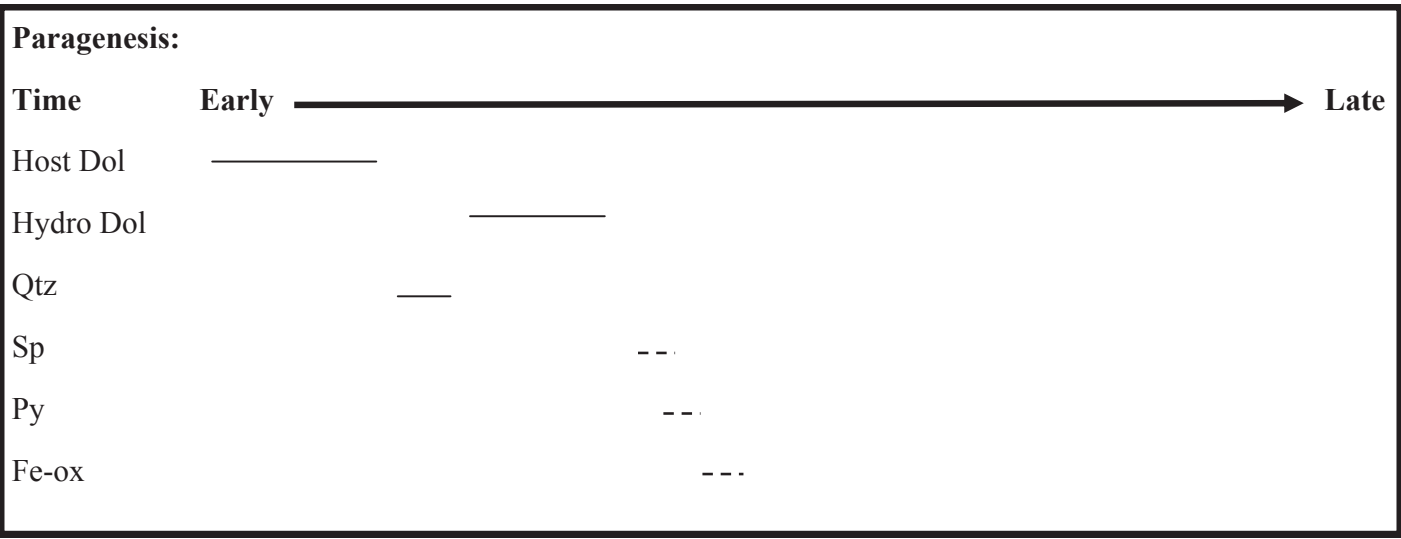


Structures: hydrothermal carbonate vein cutting host dolomite in Middle Pamplona. Late stage sulfides present. The sulfides are associated with the Fe-rich sections (red) of the hydrothermal dolomite 
Geologic Unit: Middle Pamplona

Lithological Unit (Votorantim): Pink Dolomite (DO)

\section{Sample Photograph}

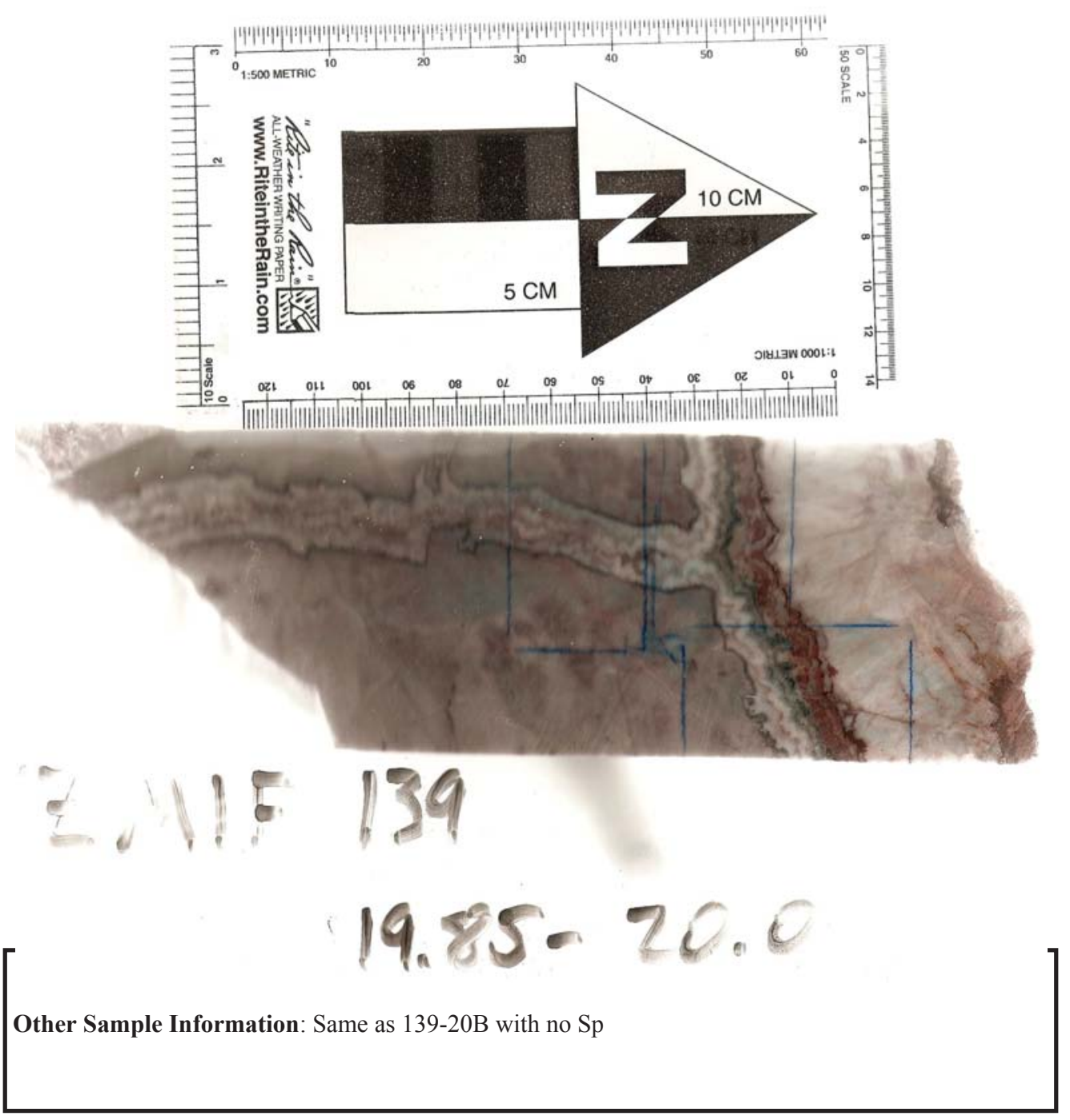


Geologic Unit: Middle Pamplona

Lithological Unit (Votorantim): Pink Dolomite (DO)

Sample Photograph

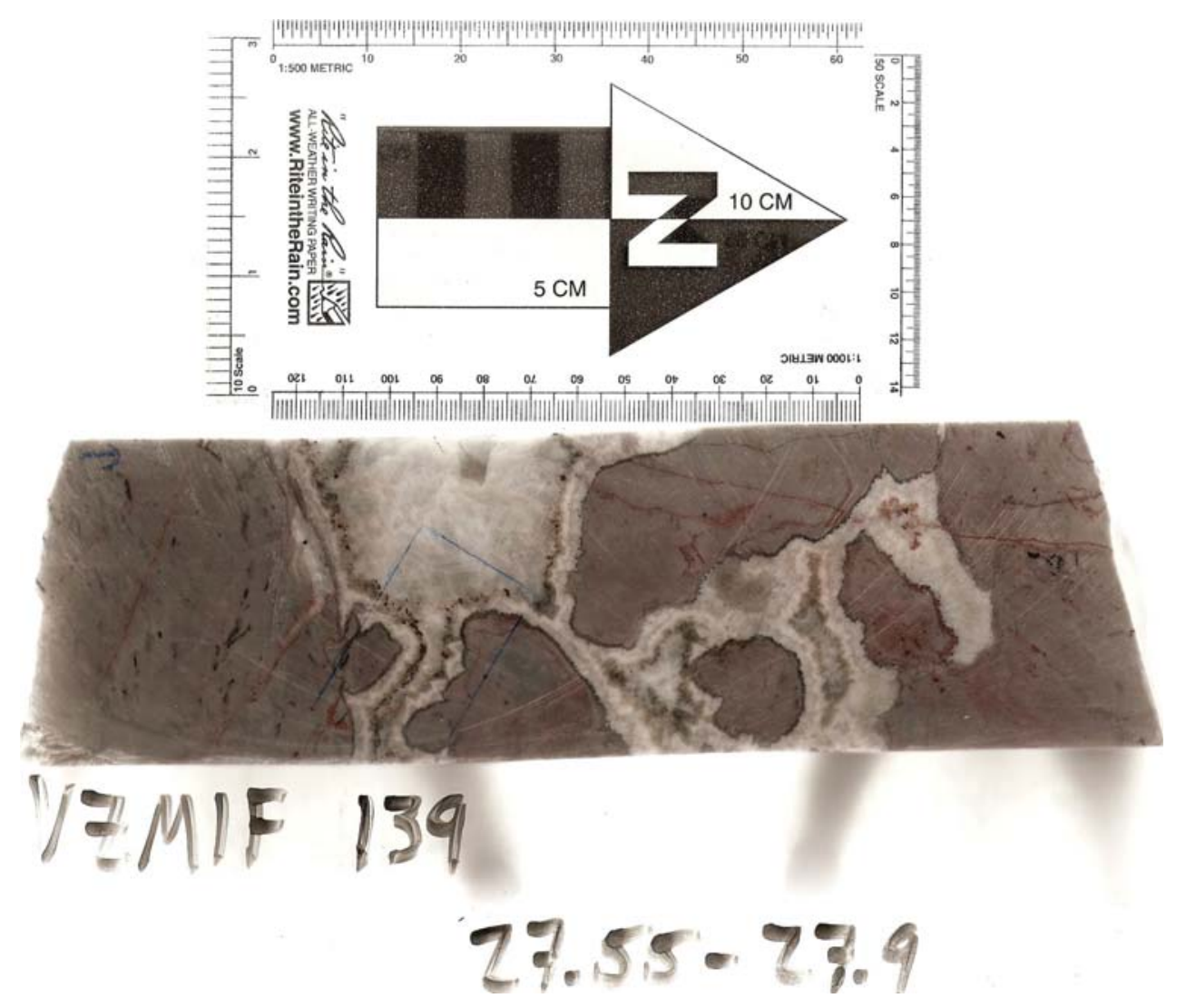

Other Sample Information: Same as 139-20B with no Sp 
Geologic Unit: breccia in Lower Morro do Pinheiro

Lithological Unit (Votorantim): Pink Dolomite (DO)

\section{Sample Photograph}

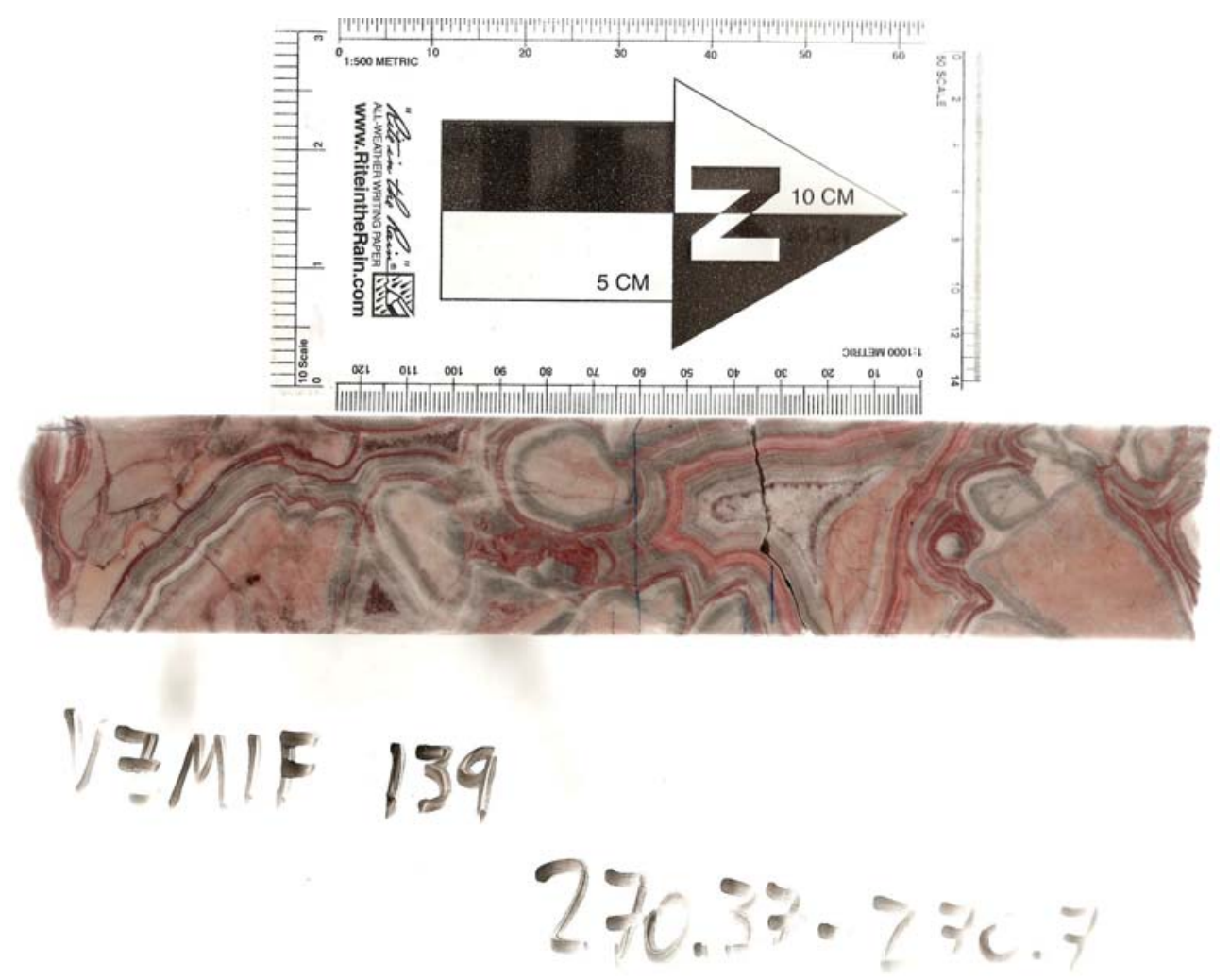

Other Sample Information: 


\begin{tabular}{|c|c|c|c|}
\hline minerals & Dolomite & Quartz & Hematite \\
\hline percentage & 99 & 1 & trace \\
\hline morphology & $\begin{array}{l}\text { Hydrothermal dolomite } \\
\text { overgrowths }\end{array}$ & Subhedral crystals & Very small euhedral xtals \\
\hline texture & Open space filling & Open space filling & Last mineral to form \\
\hline $\begin{array}{l}\text { transmitted opti- } \\
\text { cal properties }\end{array}$ & & & \\
\hline $\begin{array}{l}\text { reflected optical } \\
\text { properties }\end{array}$ & & & \\
\hline $\begin{array}{l}\text { contact/ } \\
\text { relationships with } \\
\text { other minerals }\end{array}$ & $\begin{array}{l}\text { Irregular with } \mathrm{hm} \text { and qtz, } \\
\text { replacing qtz. Hm is last to } \\
\text { form hence irregularity }\end{array}$ & Replaced by dol & \\
\hline $\begin{array}{l}\text { other (growth di- } \\
\text { rection/zoning) }\end{array}$ & & & \\
\hline
\end{tabular}


Geologic Unit: Middle Pamplona

Lithological Unit (Votorantim): DO - Gray dolomite

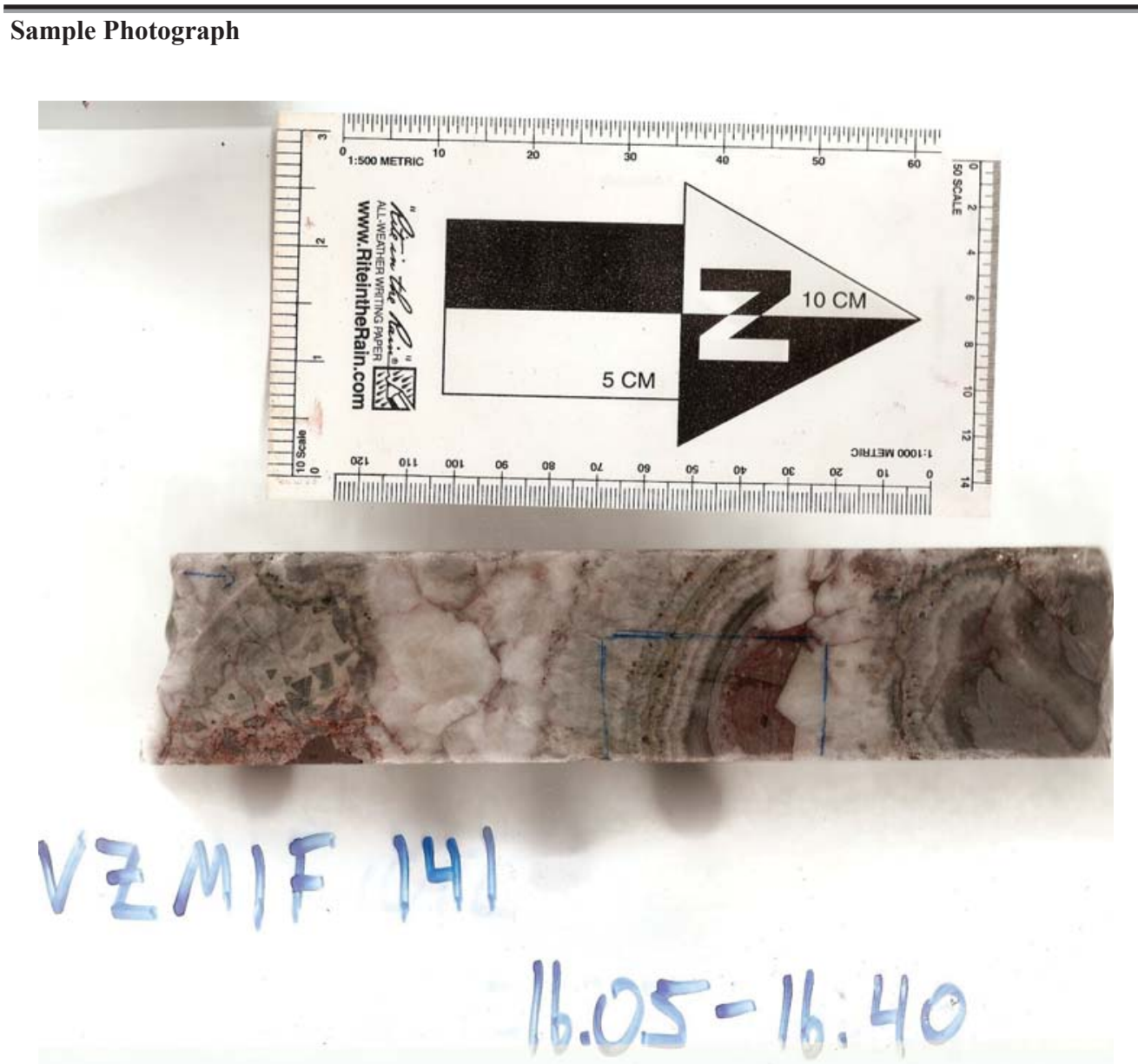

Other Sample Information: Hydrothermal bx vein in Middle Pamplona...with sphalerite 


\begin{tabular}{|c|c|c|c|}
\hline minerals & Dolomite & Quartz & Ferroan dolomite \\
\hline percentage & 40 & 20 & 30 \\
\hline morphology & Hydrothermal spar & Large, euhedral xtals & $\begin{array}{l}\text { Smaller anhedral to subhe- } \\
\text { dral xtal (sparry micrite?) }\end{array}$ \\
\hline texture & Large, crystalline & $\begin{array}{l}\text { Growing out from } \mathrm{Fe} \text {-dol } \\
\text { vein in center of slide }\end{array}$ & $\begin{array}{l}\text { One vein in center of sam- } \\
\text { ple, massive }\end{array}$ \\
\hline $\begin{array}{l}\text { transmitted opti- } \\
\text { cal properties }\end{array}$ & & Fluid inclusion trails & \\
\hline $\begin{array}{l}\text { reflected optical } \\
\text { properties }\end{array}$ & & & \\
\hline $\begin{array}{l}\text { contact/ } \\
\text { relationships with } \\
\text { other minerals }\end{array}$ & $\begin{array}{l}\text { Straight contacts with qtz. } \\
\text { Irregular edges with sp and } \\
\text { Fe-dol (replacing dol) }\end{array}$ & $\begin{array}{l}\text { Irregular edges with Fe-dol } \\
\text { and sp, replaced by sp }\end{array}$ & Replacing qtz and sparry dol \\
\hline $\begin{array}{l}\text { other (growth di- } \\
\text { rection/zoning) }\end{array}$ & & $\begin{array}{l}\text { Xtal overgrowths w/ fluid } \\
\text { inclusions }\end{array}$ & \\
\hline
\end{tabular}




\begin{tabular}{|c|c|c|c|}
\hline minerals & Sphalerite & Pyrite/Chalcopyrite & Hematite \\
\hline percentage & 10 & trace & trace \\
\hline morphology & amorphous & Minute blebs in sp, anhedral & Found in Fe-dol \\
\hline texture & Vein, minor disseminations & $\begin{array}{l}\text { Cpy-disease, disseminated } \\
\text { throughout sample }\end{array}$ & disseminated \\
\hline $\begin{array}{l}\text { transmitted opti- } \\
\text { cal properties }\end{array}$ & Yellow-brown in ppl & & \\
\hline $\begin{array}{l}\text { reflected optical } \\
\text { properties }\end{array}$ & Isotropic, yellow IR & & \\
\hline $\begin{array}{l}\text { contact/ } \\
\text { relationships with } \\
\text { other minerals }\end{array}$ & $\begin{array}{l}\text { Irregular with dol and qtz } \\
\text { (replacing these mins) }\end{array}$ & Smooth with sp & $\begin{array}{l}\text { Irregular, shreddy contacts } \\
\text { with Fe-dol, replacing Fe- } \\
\text { dol? }\end{array}$ \\
\hline $\begin{array}{l}\text { other (growth di- } \\
\text { rection/zoning) }\end{array}$ & & Exosolution from $\mathrm{sp}$ & $\begin{array}{l}\text { Forms reaction front b/w Fe- } \\
\text { dol and other mins (i.e. qtz) }\end{array}$ \\
\hline
\end{tabular}

Paragenesis: ???????????????????????????????????????????????

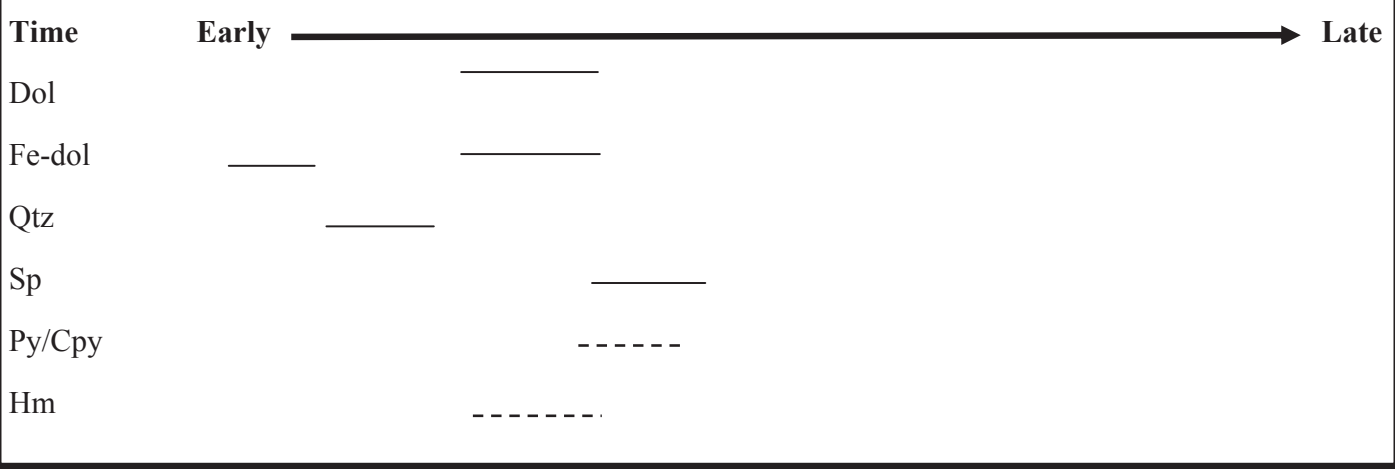


Structures: Fe-carbonate vein cuts sample in half. Quartz grows out from this vein. Sample is taken from a hydrothermal bx (not $\mathrm{BH})$.

Paragenetic Sequence: dol vein $\rightarrow \mathrm{qtz} \pm \mathrm{py} \rightarrow \mathrm{dol} \rightarrow \mathrm{sph} \rightarrow \mathrm{py}+\mathrm{sp}$

Paragenetic Reasoning: Vein order

Sample Highlights: Reaction front between dol and Fe-dol.

Rxn front b/w qtz and Fe-dol 
Geologic Unit: Hydrothermal Breccia

Lithological Unit (Votorantim): Hydrothermal Breccia

Sample Photograph

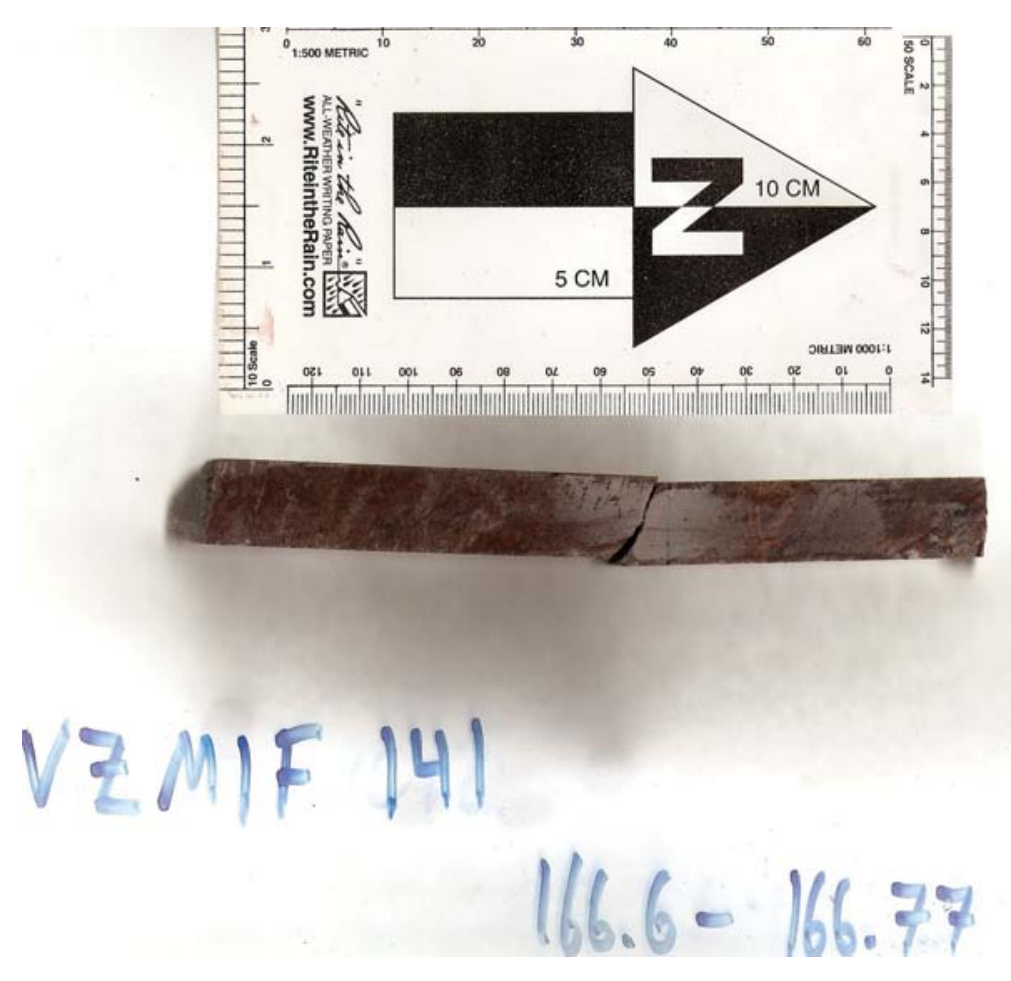

Other Sample Information: $\sim 32 \% \mathrm{Zn}$ in 166.5-166.9 $\mathrm{m}$ interval

*See Sample 129-167* 
Geologic Unit: Middle Pamplona

Lithological Unit (Votorantim): DO- gray dolomite

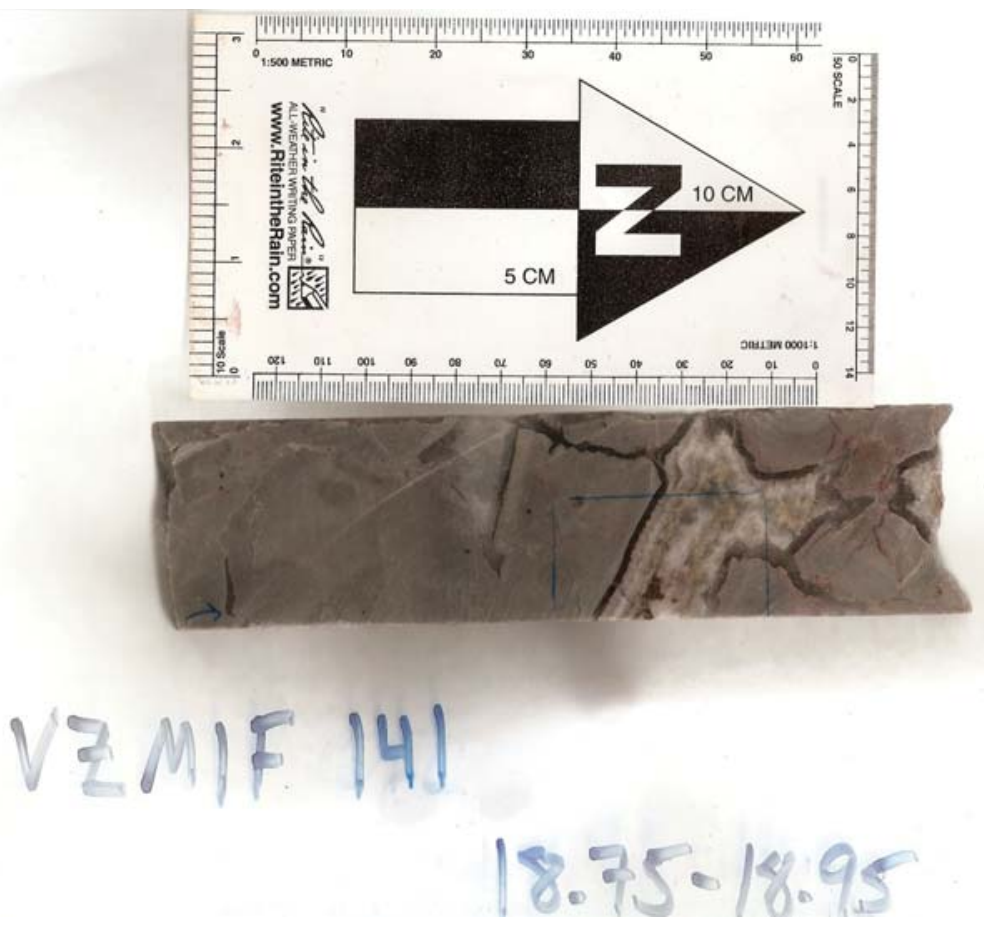

Other Sample Information: 


\begin{tabular}{|c|c|c|c|}
\hline minerals & Dolomite & Quartz & Pyrite \\
\hline percentage & 87 & 10 & 2 \\
\hline morphology & $\begin{array}{l}\text { Large sparry dol and regular } \\
\text { rock-forming dol (minor ne- } \\
\text { omorphism) }\end{array}$ & Euhedral xtals & blebs \\
\hline texture & $\begin{array}{l}\text { Rock-forming and secondary } \\
\text { vein-filling }\end{array}$ & Rimming dolostone clasts & disseminated \\
\hline $\begin{array}{l}\text { transmitted opti- } \\
\text { cal properties }\end{array}$ & & & \\
\hline $\begin{array}{l}\text { reflected optical } \\
\text { properties }\end{array}$ & & & \\
\hline $\begin{array}{l}\text { contact/ } \\
\text { relationships with } \\
\text { other minerals }\end{array}$ & $\begin{array}{l}\text { Straight with other dol. } \\
\text { Some minor irregularities } \\
\text { with qtz }\end{array}$ & $\begin{array}{l}\text { Straight with most dol and } \\
\text { other qtz }\end{array}$ & $\begin{array}{l}\text { Irregular with qtz, dol, and } \\
\text { Fe-oxides. Late stage in hy- } \\
\text { drothermal dol and qtz fluid. } \\
\text { Altering to Fe-hydroxides } \\
\text { and oxides }\end{array}$ \\
\hline $\begin{array}{l}\text { other (growth di- } \\
\text { rection/zoning) }\end{array}$ & & Overgrowths and zones & \\
\hline
\end{tabular}




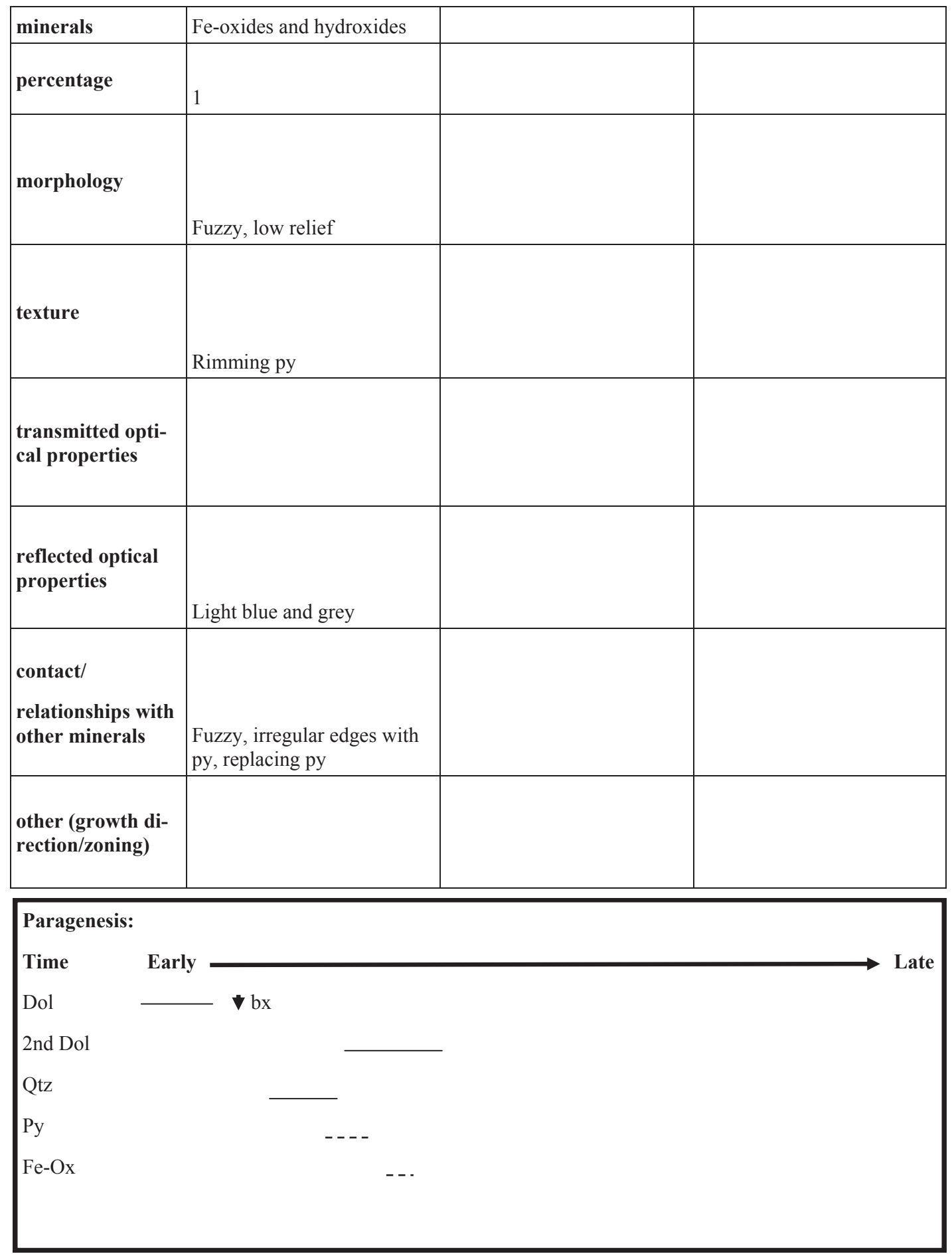


Structures: Breccia filled in with hydrothermal qtz and dol

Paragenetic Sequence: dolostone $\rightarrow \mathrm{qtz} \rightarrow$ hydrothermal dol $+\mathrm{py} \rightarrow \mathrm{Fe}-\mathrm{ox}$

Paragenetic Reasoning: Order of events in vein 
Geologic Unit: Middle Pamplona

Lithological Unit (Votorantim): Grey Dolomite (DO)

Sample Photograph
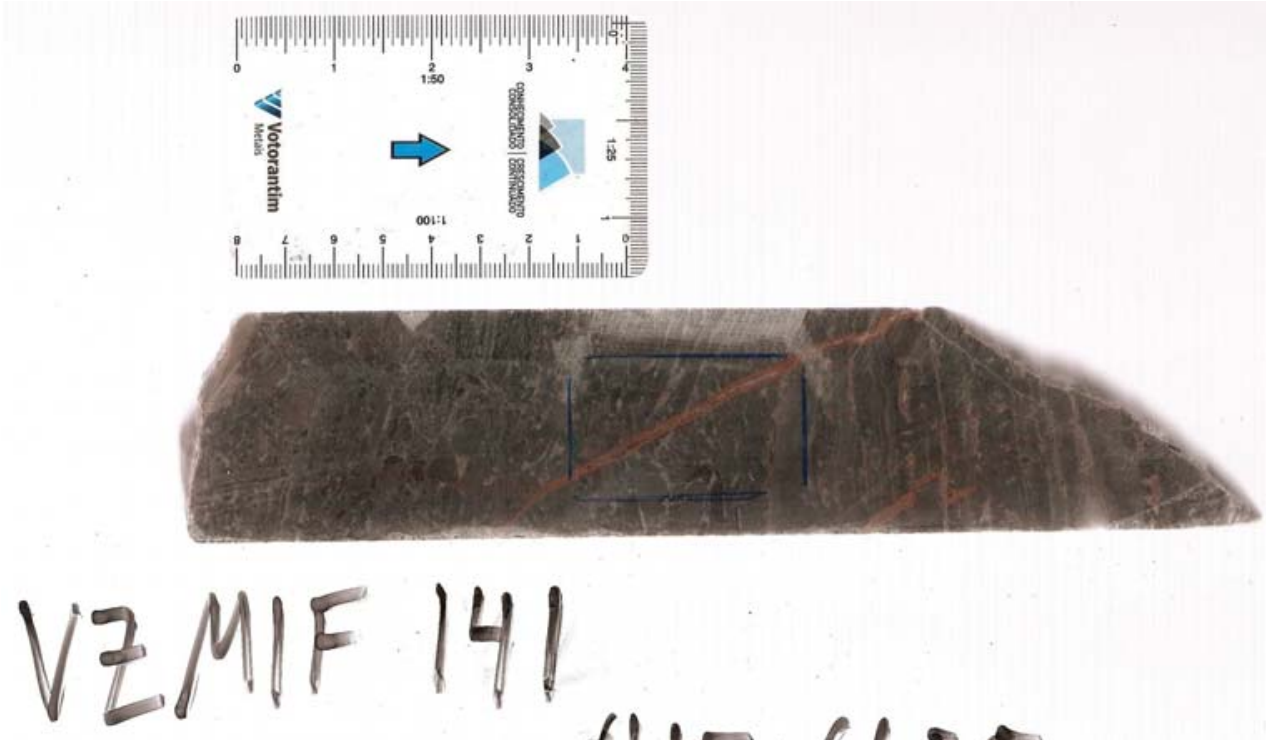

$4.15=61.35$

Other Sample Information: Using Wright (1992) and Embry and Klovan (1971) this is a clast supported rudstone with a sparry carbonate matrix 


\begin{tabular}{|l|l|l|l|}
\hline minerals & dolomite & Calcite? & quartz \\
\hline percentage & 65 & 24 & 1 \\
\hline morphology & Micritic dol clasts & Sparry calcite cement & Veinlets and alteration \\
\hline $\begin{array}{l}\text { texture } \\
\text { cal properties } \\
\text { transmitted opti- } \\
\text { cal }\end{array}$ & Rock forming intraclasts & matrix & \\
\hline $\begin{array}{l}\text { reflected optical } \\
\text { properties }\end{array}$ & & & $\begin{array}{l}\text { Veinlets and some minor } \\
\text { replacement of dol }\end{array}$ \\
\hline $\begin{array}{l}\text { contact/ } \\
\text { relationships with } \\
\text { other minerals } \\
\text { rection/zoning) }\end{array}$ & & & \\
\hline (growth di- & & & $\begin{array}{l}\text { Replacing dol in rudstone } \\
\text { clasts }\end{array}$ \\
\hline
\end{tabular}




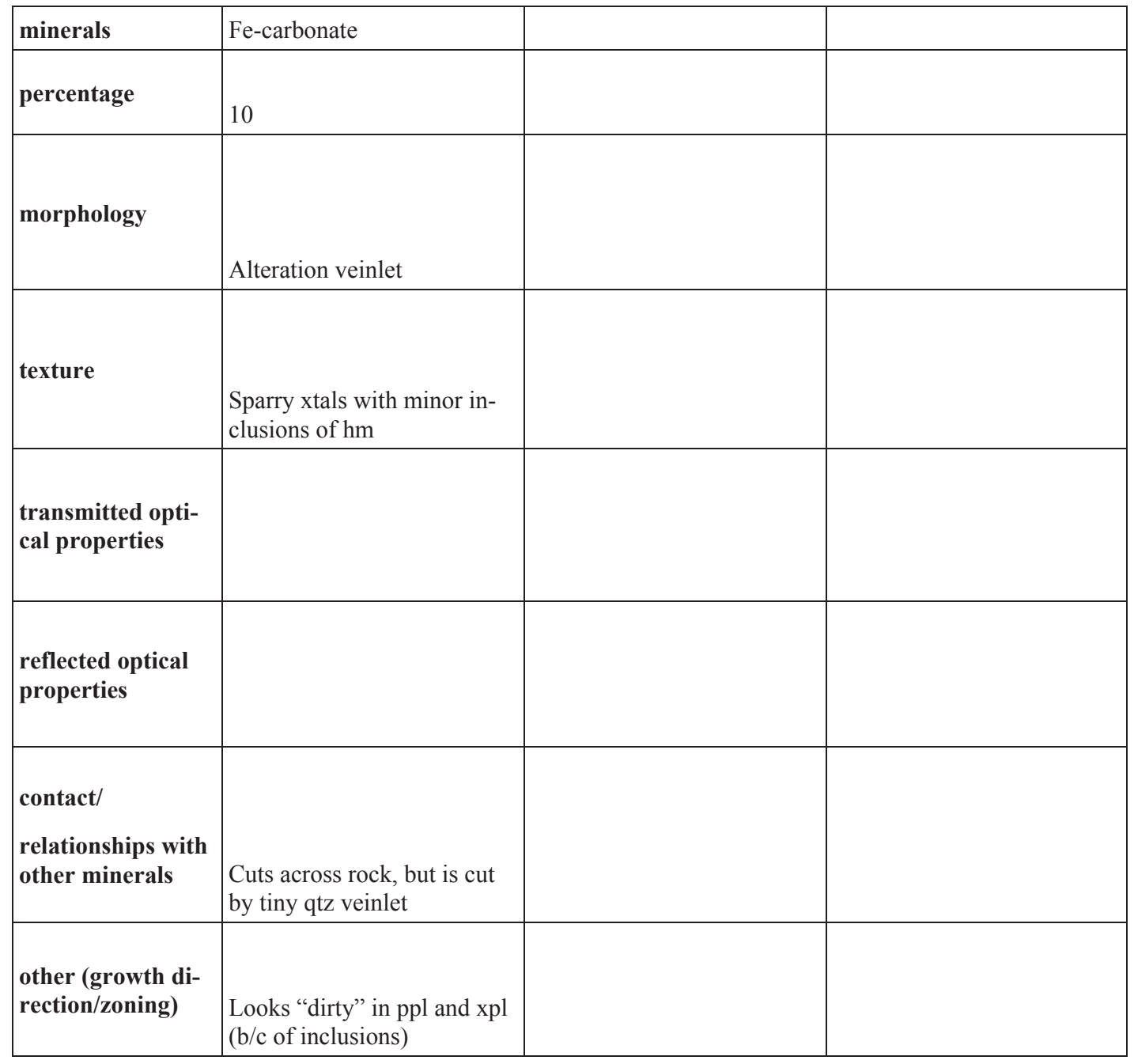

Paragenesis:

Time

Early 
Structures: Rock is a clast supported rudstone with sparry calcite matrix. An Fe-carbonate vein cuts across sample and looks "dirty" under microscope (possibly ankerite or siderite) 
Geologic Unit: Lower Pamplona

Lithological Unit (Votorantim): Grey Dolomite (DO)

Sample Photograph

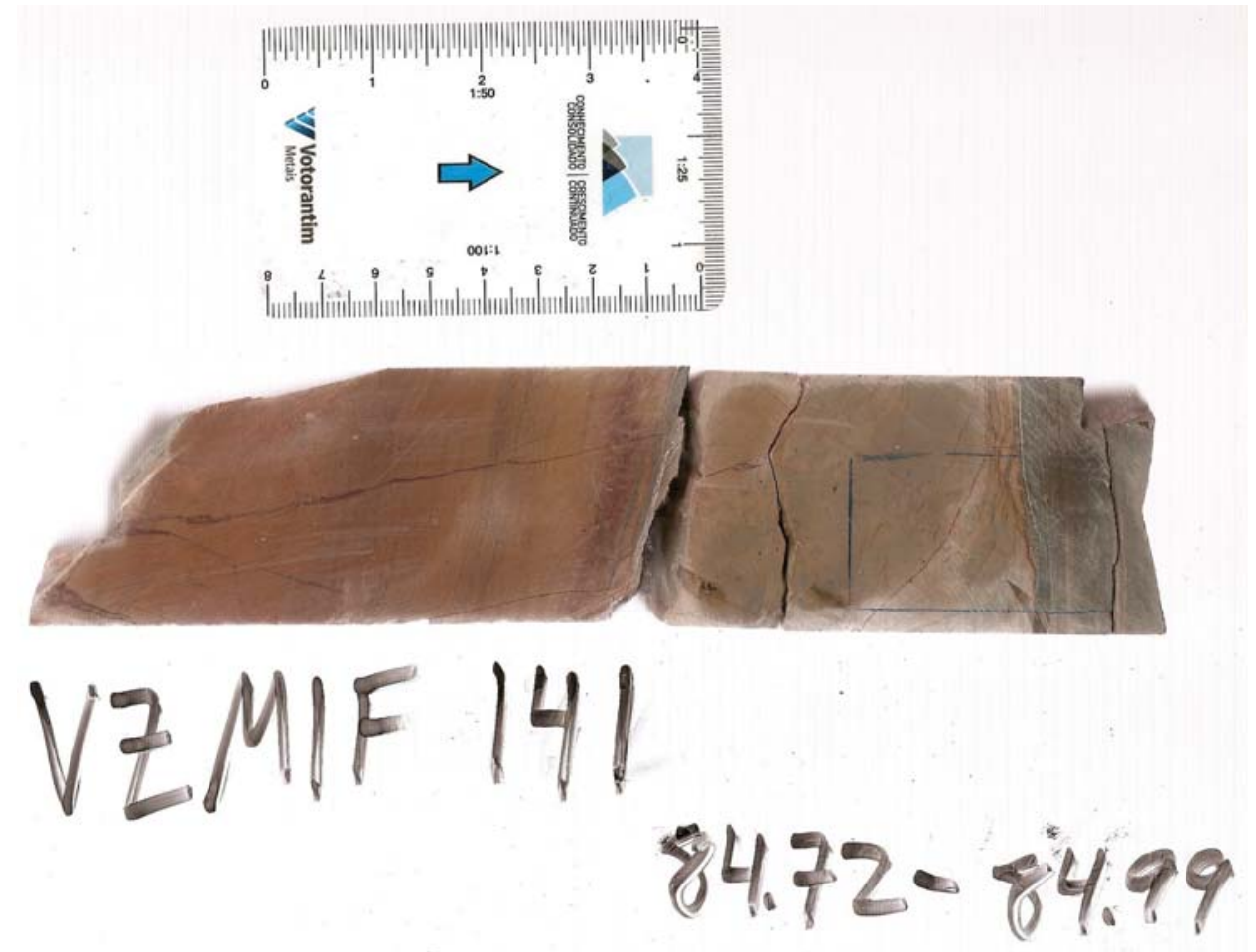

Other Sample Information: bottome part is a massive micrite with some disseminated angular to subrounded quartz grains

The top part of the section is a red micrite with laminattions of peloids and angular to subrounded qts grains. The carbonate has been recrystallized to form one crystal in the micrite 


\begin{tabular}{|c|c|c|c|}
\hline minerals & Red carbonate (phyllite) & Carbonate & quartz \\
\hline percentage & 20 & 73 & 5 \\
\hline morphology & micrite & Micrite & Angular to subrounded \\
\hline texture & $\begin{array}{l}\text { Contains intercalated lami- } \\
\text { nations of peloids and qtz } \\
\text { grains }\end{array}$ & Some styolites & $\begin{array}{l}\text { Disseminated in carbonate, } \\
\text { in red carbonate they are } \\
\text { laminated }\end{array}$ \\
\hline $\begin{array}{l}\text { transmitted opti- } \\
\text { cal properties }\end{array}$ & & & \\
\hline $\begin{array}{l}\text { reflected optical } \\
\text { properties }\end{array}$ & & & \\
\hline $\begin{array}{l}\text { contact/ } \\
\text { relationships with } \\
\text { other minerals }\end{array}$ & $\begin{array}{l}\text { A hummocky contact w/ car- } \\
\text { bonate }\end{array}$ & & \\
\hline $\begin{array}{l}\text { other (growth di- } \\
\text { rection/zoning) }\end{array}$ & $\begin{array}{l}\text { One crystal, was formerly a } \\
\text { "phyllite" }\end{array}$ & & \\
\hline
\end{tabular}




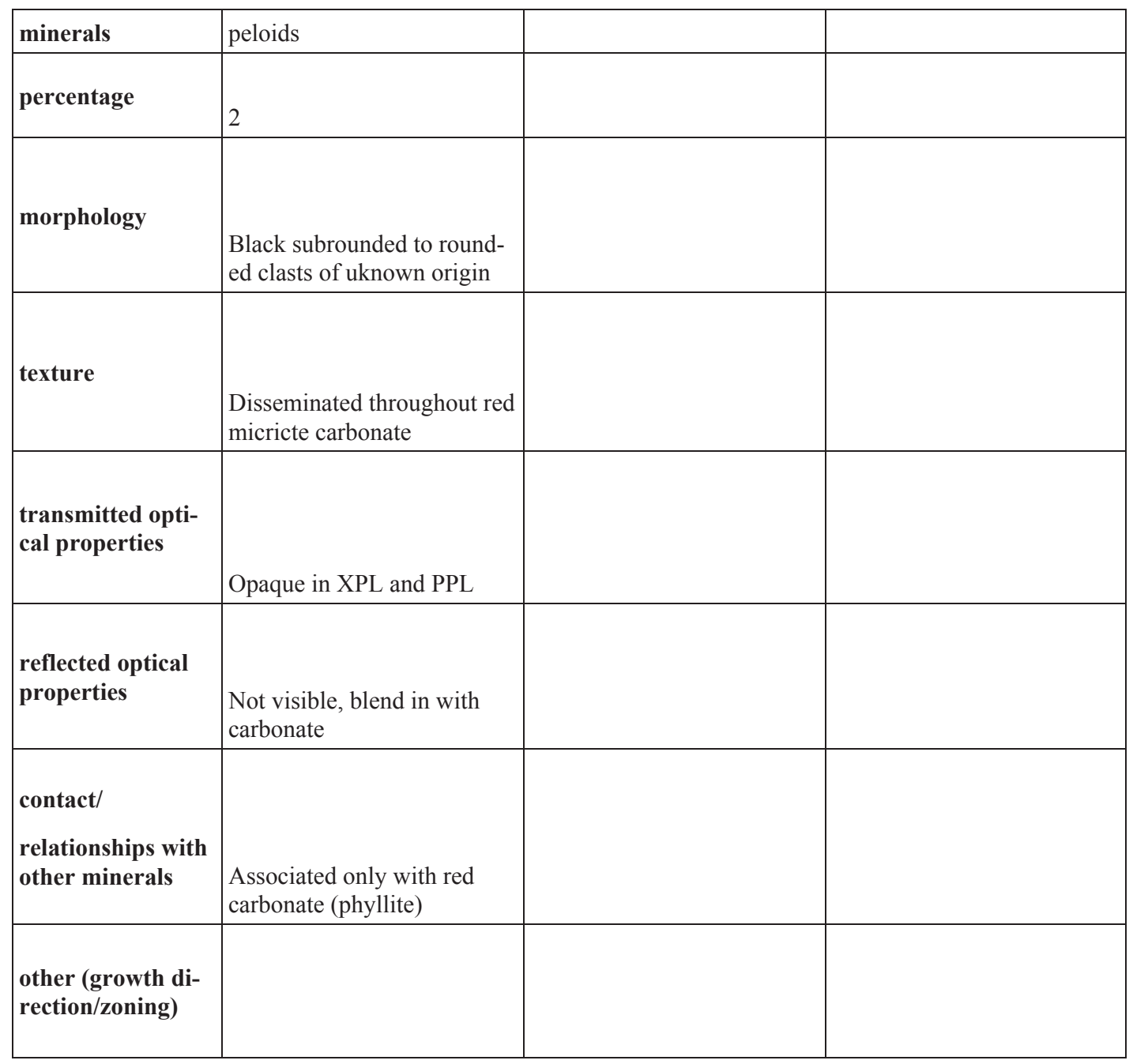

Paragenesis:

Time

Early 
Geologic Unit: Lower Pamplona

\section{Lithological Unit (Votorantim):}

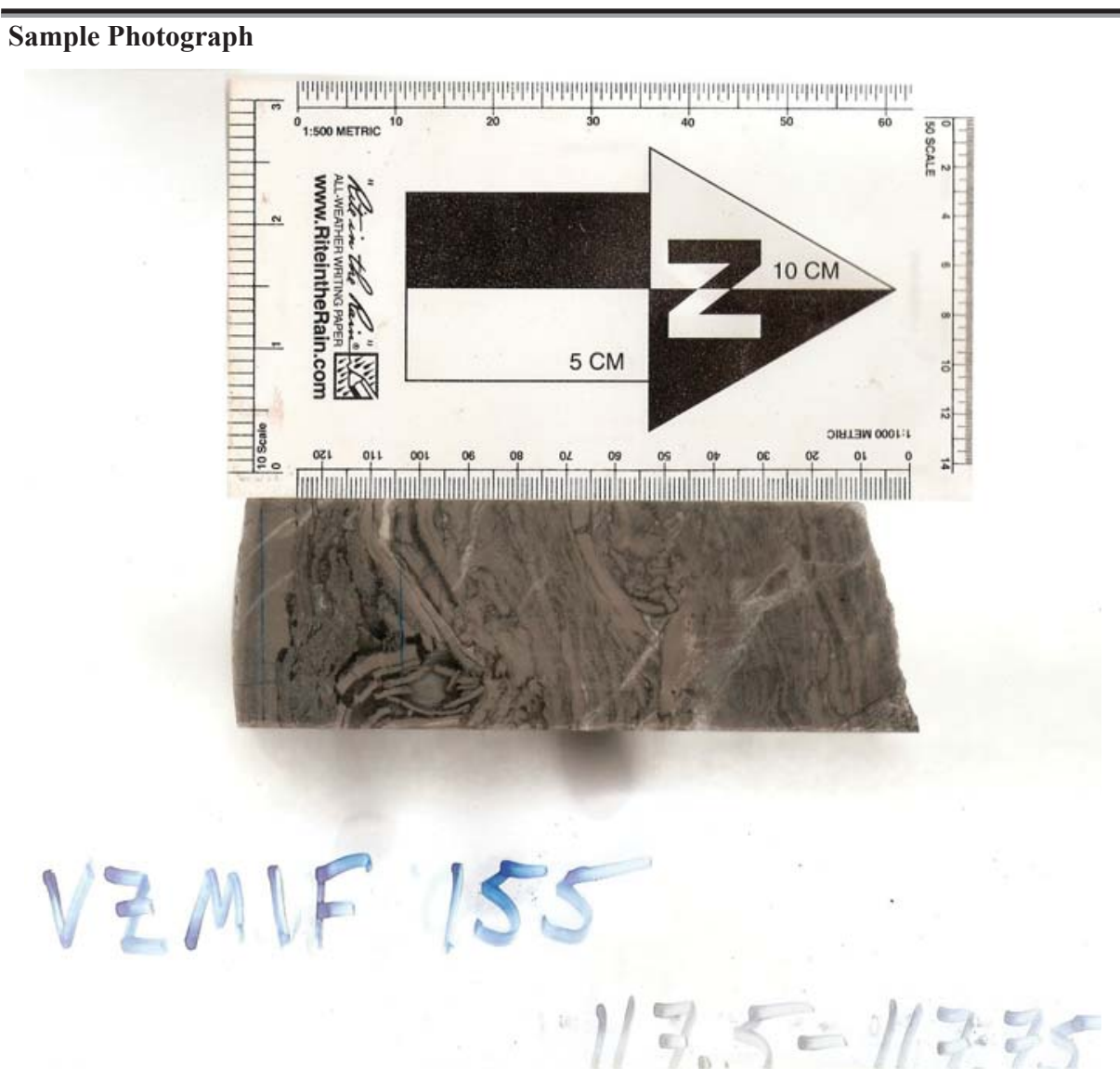

Other Sample Information: Sedimentary dolomite breccia with silica alteration 


\begin{tabular}{|c|c|c|c|}
\hline minerals & Dolomite & Quartz & Pyrite \& Hematite \\
\hline percentage & 70 & 30 & trace \\
\hline morphology & $\begin{array}{l}\text { Euhedral dolomite matrix } \\
\text { with larger dolomite xtals in } \\
\text { open space }\end{array}$ & $\begin{array}{l}\text { Very fine crystals to large } \\
\text { euhedral crystals in centers } \\
\text { of open space }\end{array}$ & subhedral \\
\hline texture & $\begin{array}{l}\text { Rock matrix, some neomor- } \\
\text { phosed areas }\end{array}$ & Filling open space & Singular grain in open space \\
\hline $\begin{array}{l}\text { transmitted opti- } \\
\text { cal properties }\end{array}$ & & & \\
\hline $\begin{array}{r}\text { colo } \\
\mathrm{pp} \\
\mathrm{xp}\end{array}$ & & & \\
\hline pleochroisn & & & \\
\hline birefringenc & & & \\
\hline $\begin{array}{r}\text { twinning } \\
\text { cleavag }\end{array}$ & & & \\
\hline othe & & & \\
\hline $\begin{array}{l}\text { reflected optical } \\
\text { properties }\end{array}$ & & & \\
\hline colo & & & \\
\hline bireflectanc & & & \\
\hline anisotrop & & & \\
\hline internal reflection & & & \\
\hline othe & & & \\
\hline $\begin{array}{l}\text { contact/ } \\
\text { relationships with } \\
\text { other minerals }\end{array}$ & & & \\
\hline $\begin{array}{l}\text { other (growth di- } \\
\text { rection/zoning) }\end{array}$ & $\begin{array}{l}\text { Filling open space, zoned } \\
\text { xtals }\end{array}$ & Filling open space & Zoned with hm \\
\hline
\end{tabular}


Structures: Sample is part of a sedimentary breccia. Dolomite clasts are surrounded by quartz and recrystallized dolomite. Some neomorphism of dolomite has occurred. There are also tension gashes across the quartz filled vugs.

Paragenetic Sequence: dol $\rightarrow$ fracturing $\rightarrow$ neomorphism + dolomite fracture fill $\rightarrow$ qtz fracture fill $\rightarrow$ tension gashes $+\mathrm{Py}$

Paragenetic Reasoning: Fracturing occurred allowing for dolomite to being to fill the void space with large, euhedral, sparry dolomite. Quartz began to fill in the rest of the fractures, filling the voids with large crystals in the center of voids along with some sparry dolomite. Pyrite is late?????

Sample Highlights: Tension Gashes, dolomite overgrowths 
Geologic Unit: Lower Pamplona

Lithological Unit (Votorantim): Hydrothermal Breccia

Sample Photograph
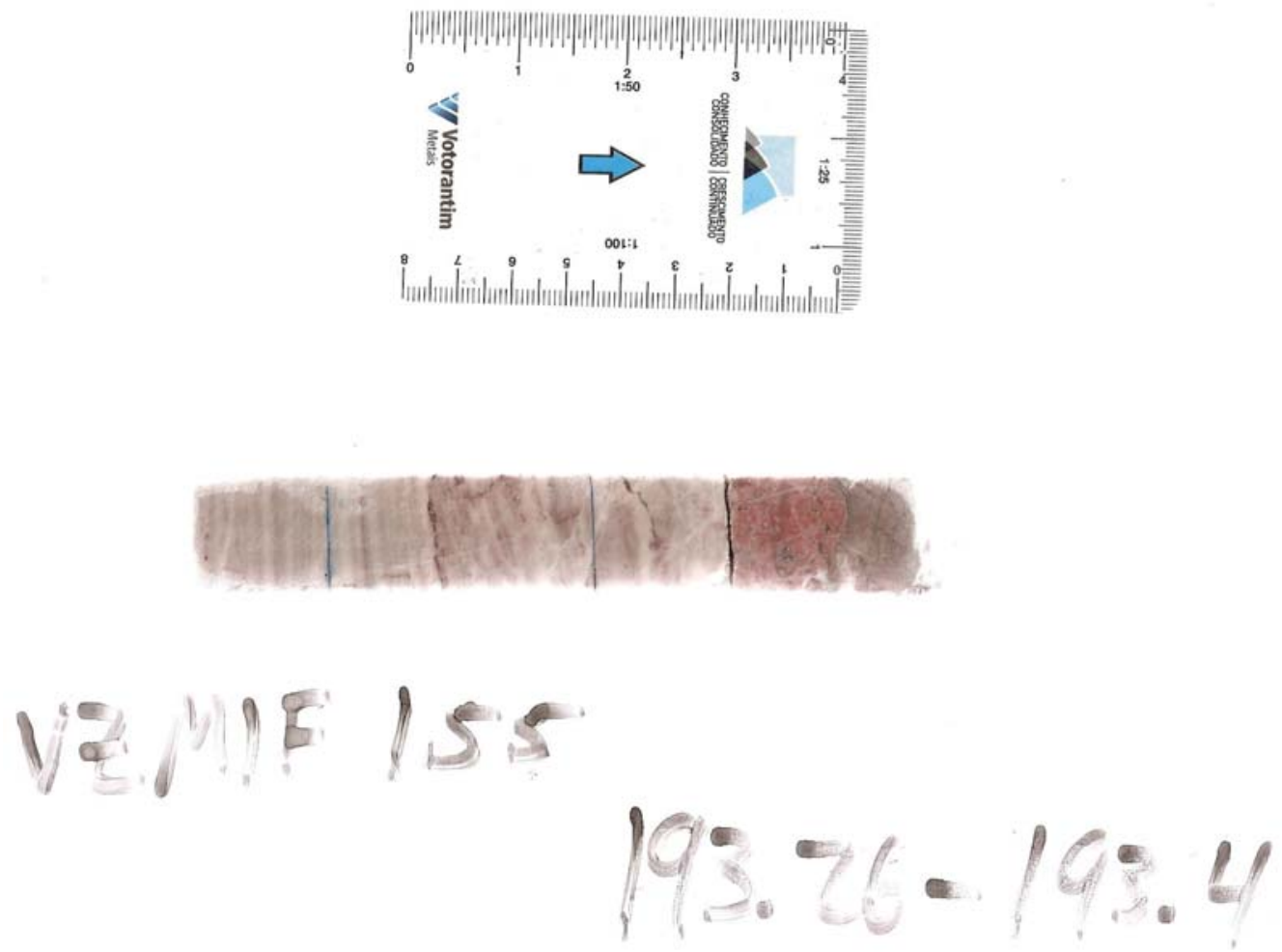

Other Sample Information: 


\begin{tabular}{|l|l|l|l|}
\hline minerals & Dolomite & quartz & Muscovite \\
\hline percentage & 95 & 5 & trace \\
\hline morphology & Micritic algal boundstone & Angular to subangular grains & Platy laths \\
\hline $\begin{array}{l}\text { texture } \\
\text { transmitted opti- } \\
\text { cal properties }\end{array}$ & $\begin{array}{l}\text { Algal laminations with qtz } \\
\text { (+ musc) intercalations }\end{array}$ & $\begin{array}{l}\text { Algal laminations with qtz } \\
\text { (+ musc) intercalations }\end{array}$ & \\
\hline $\begin{array}{l}\text { reflected optical } \\
\text { properties }\end{array}$ & & & \\
\hline $\begin{array}{l}\text { contact/ } \\
\text { relationships with } \\
\text { other minerals }\end{array}$ & $\begin{array}{l}\text { Forms rock; some unminer- } \\
\text { alized fractures }\end{array}$ & Forms rock & \\
\hline rection/zoning) & & & \\
\hline
\end{tabular}




\begin{tabular}{|c|c|c|c|}
\hline minerals & Fe-alt & & \\
\hline percentage & trace & & \\
\hline morphology & $\begin{array}{l}\text { Discontinuous veinlets with } \\
\text { very trace hematite }\end{array}$ & & \\
\hline texture & veinlets & & \\
\hline $\begin{array}{l}\text { transmitted opti- } \\
\text { cal properties }\end{array}$ & & & \\
\hline $\begin{array}{l}\text { reflected optical } \\
\text { properties }\end{array}$ & & & \\
\hline $\begin{array}{l}\text { contact/ } \\
\text { relationships with } \\
\text { other minerals }\end{array}$ & Overprints boundstone & & \\
\hline $\begin{array}{l}\text { other (growth di- } \\
\text { rection/zoning) }\end{array}$ & & & \\
\hline
\end{tabular}

Paragenesis:

Time

Early

Late

$\mathrm{Fe}$-alt is late compared to rock-forming mins 
Geologic Unit: Lower Pamplona

Lithological Unit (Votorantim): Pink Dolomite

Sample Photograph

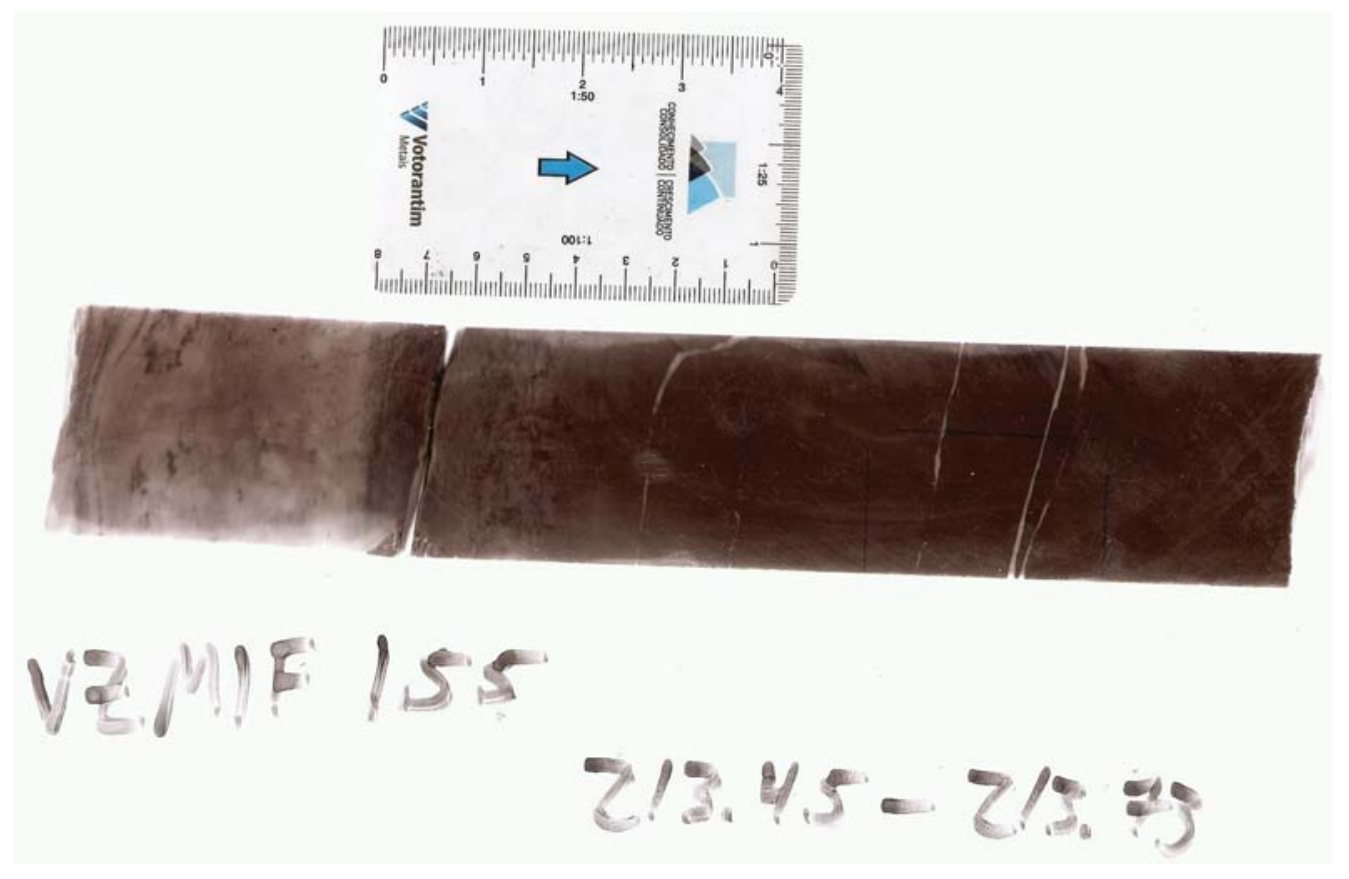

Other Sample Information: Same as 123-46. Not originally a phyllite as previously thought, just more altered carbonate 
Sample \#: GMN1-527 A

Geologic Unit: Hydrothermal Breccia

Lithological Unit (Votorantim): Hydrothermal Breccia

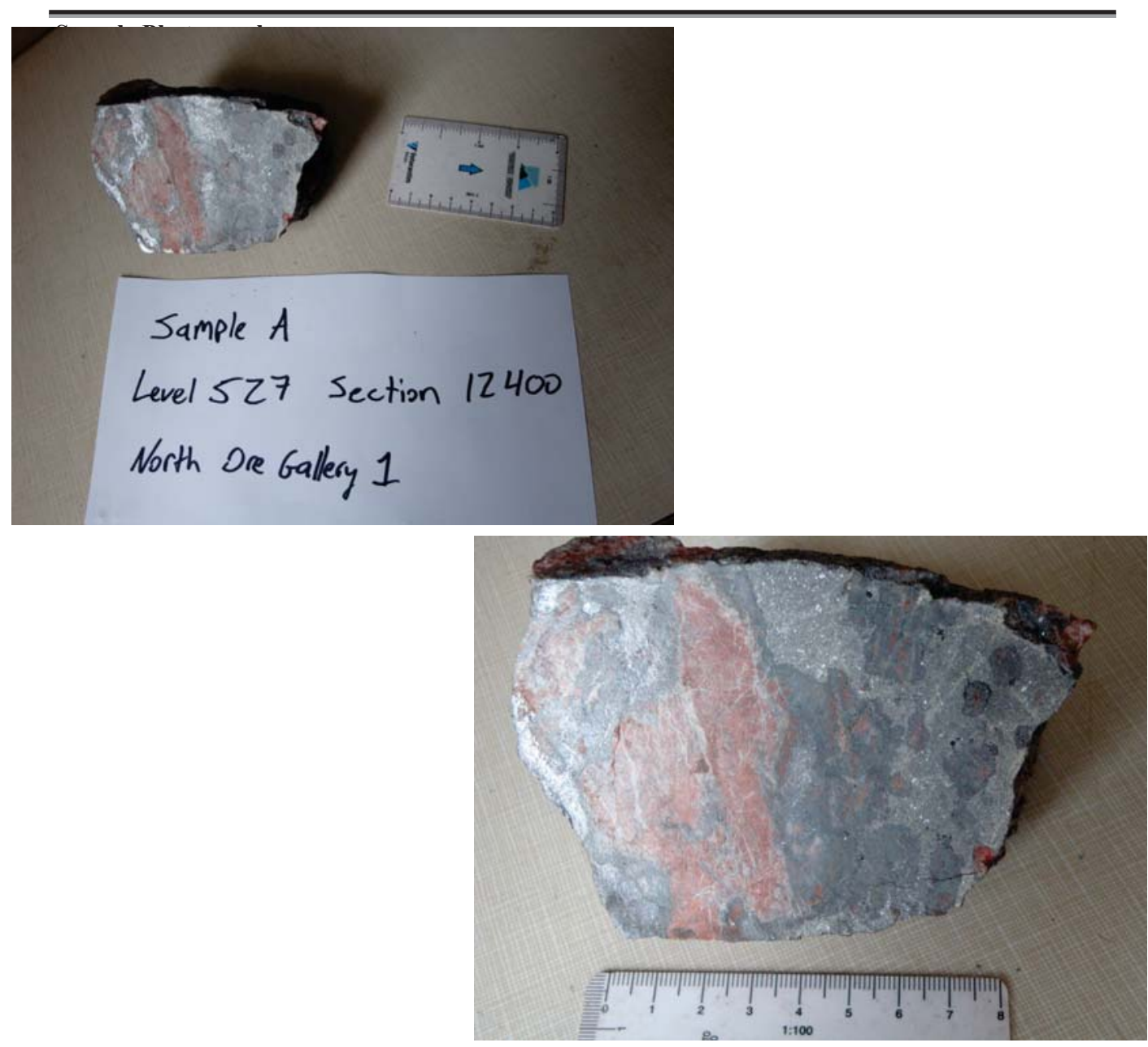

Other Sample Information: 


\begin{tabular}{|l|l|l|l|}
\hline minerals & Fe-carbonate & Willemite & Sphalerite \\
\hline percentage & 25 & 35 & 20 \\
\hline morphology & Anhedral, large, crystalline & $\begin{array}{l}\text { Euhedral laths and granular } \\
\text { crystals }\end{array}$ & Irregular crystals \\
\hline $\begin{array}{l}\text { texture } \\
\text { transmitted opti- } \\
\text { cal properties }\end{array}$ & "dirty" looking & Massive & \\
\hline $\begin{array}{l}\text { reflected optical } \\
\text { properties }\end{array}$ & & & Vein-forming \\
\hline $\begin{array}{l}\text { contact/ } \\
\text { relationships with } \\
\text { other minerals } \\
\text { rection/zoning) }\end{array}$ & $\begin{array}{l}\text { Being replaced by wl, sul- } \\
\text { fides }\end{array}$ & $\begin{array}{l}\text { Cross-cut and fractured by } \\
\text { sulfides, being replaced by } \\
\text { sp, gn, frk, and hm }\end{array}$ & $\begin{array}{l}\text { Intergrown with galena, co- } \\
\text { cveal with gn, hm, and frk, } \\
\text { cross-cutting wl and car- } \\
\text { bonate }\end{array}$ \\
\hline & & & \\
\hline
\end{tabular}




\begin{tabular}{|c|c|c|c|}
\hline minerals & Galena & Franklinite & hematite \\
\hline percentage & 10 & 7 & 3 \\
\hline morphology & Irregular crystalline & Euhedral & Euhedral \\
\hline texture & Vein-forming w/sp & $\begin{array}{l}\text { Disseminated in } \mathrm{sp}+\mathrm{gn} \text { vein, } \\
\text { close to contacts with W1 }\end{array}$ & Disseminated in $\mathrm{sp}+\mathrm{gn}$ vein \\
\hline $\begin{array}{l}\text { transmitted opti- } \\
\text { cal properties }\end{array}$ & & & \\
\hline $\begin{array}{l}\text { reflected optical } \\
\text { properties }\end{array}$ & & & \\
\hline $\begin{array}{l}\text { contact/ } \\
\text { relationships with } \\
\text { other minerals }\end{array}$ & $\begin{array}{l}\text { Intergrown with sp, being } \\
\text { replaced by srm and cv }\end{array}$ & $\begin{array}{l}\text { Straight contacts with gn and } \\
\text { sp }\end{array}$ & $\begin{array}{l}\text { Straight contacts with gn } \\
\text { and sp }\end{array}$ \\
\hline $\begin{array}{l}\text { other (growth di- } \\
\text { rection/zoning) }\end{array}$ & & & \\
\hline
\end{tabular}

Paragenesis:

Time

Early 


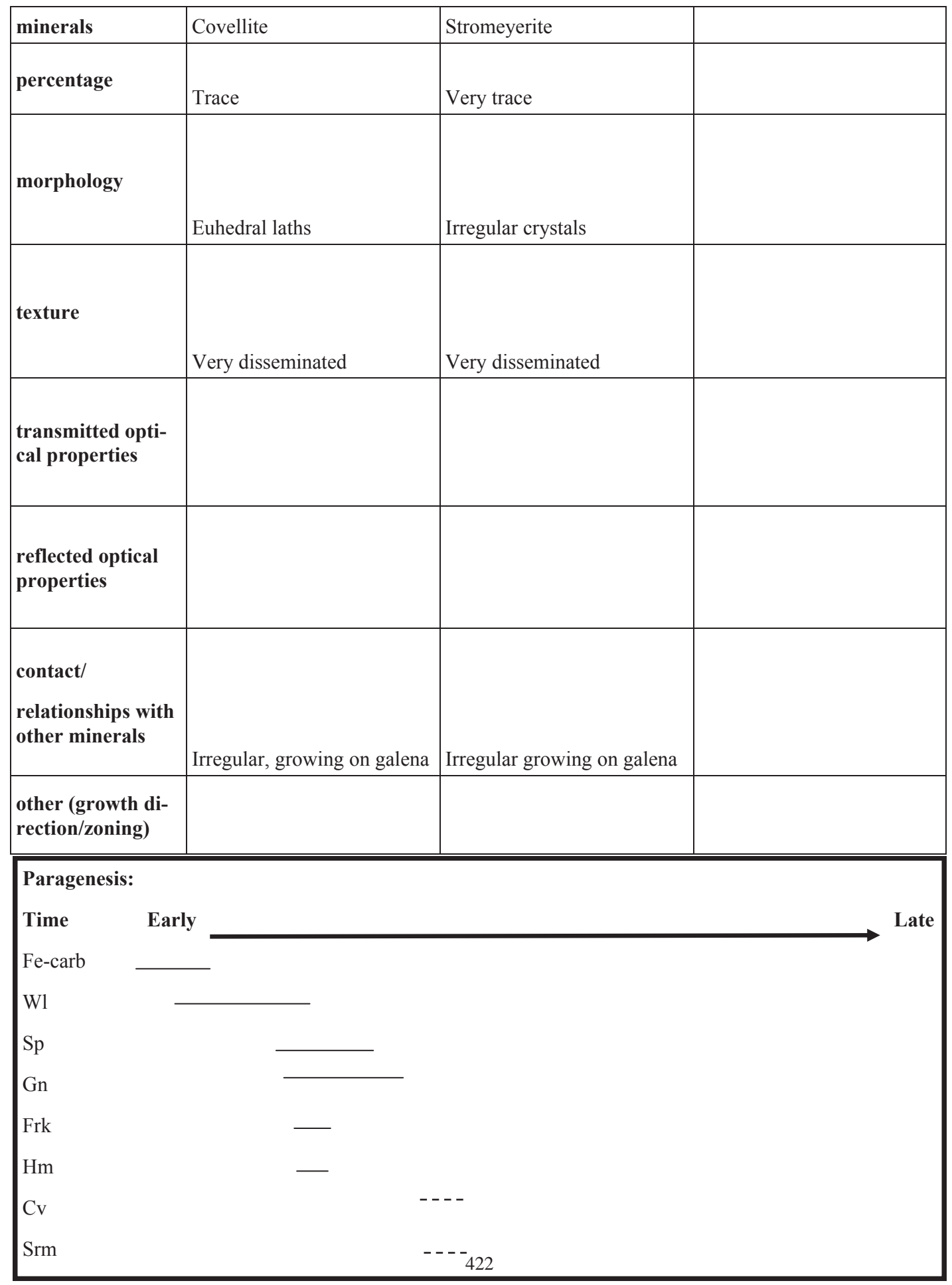


Sample \#: GMN1-527 D

Hole \#: Vazante

Sample Interval: Level 527; Section Mine 12,400; Northern Ore Gallery 1

Geologic Unit: Hydrothermal Breccia

Lithological Unit (Votorantim): Hydrothermal Breccia

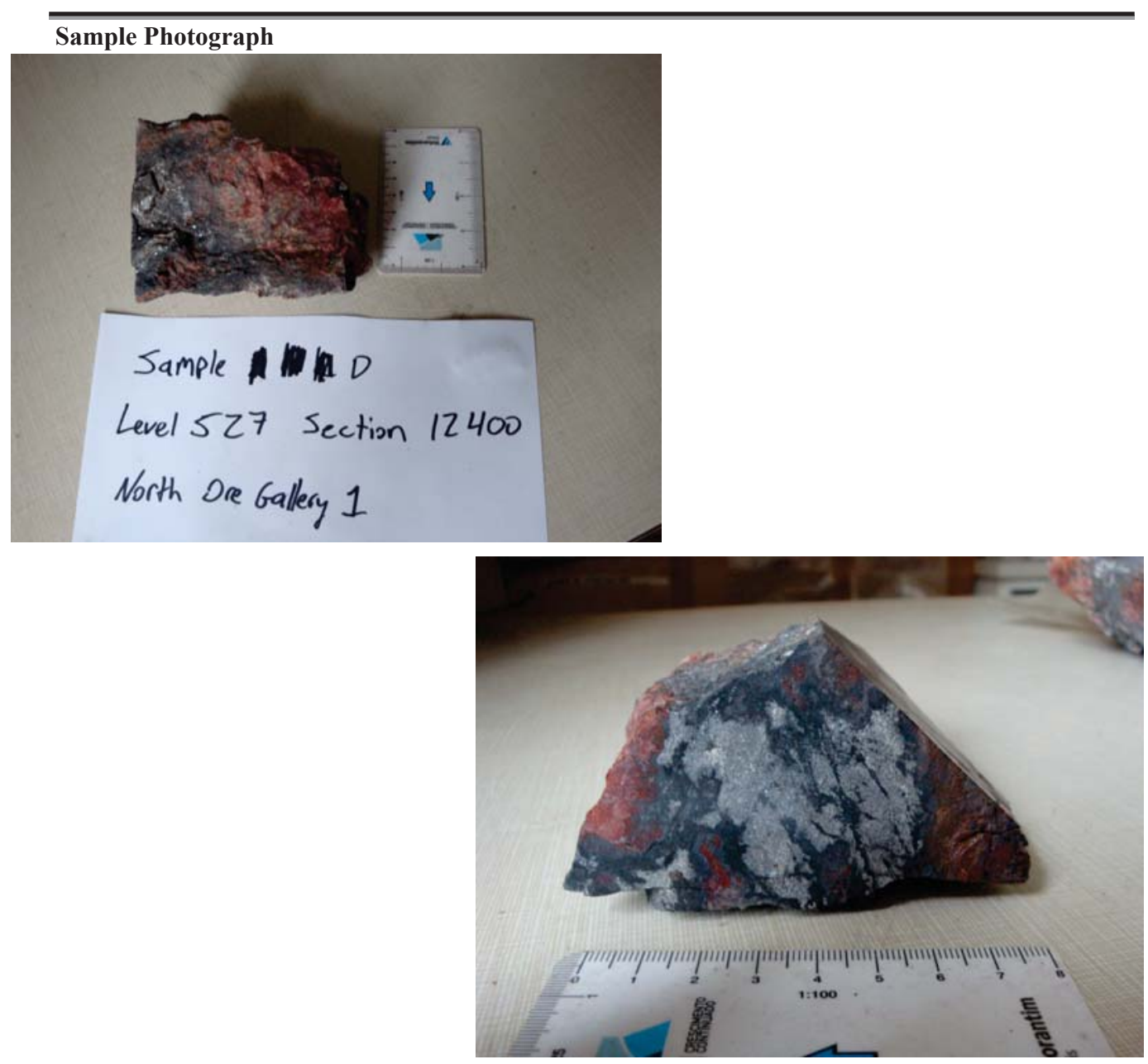

Other Sample Information: $527 \mathrm{~A}$ and B are the same with less carbonate and more willemite 


\begin{tabular}{|l|l|l|l|}
\hline minerals & Fe-carbonate & Willemite & Sphalerite \\
\hline percentage & 25 & 35 & 20 \\
\hline morphology & Anhedral, large, crystalline & $\begin{array}{l}\text { Euhedral laths and granular } \\
\text { crystals }\end{array}$ & Irregular crystals \\
\hline $\begin{array}{l}\text { texture } \\
\text { transmitted opti- } \\
\text { cal properties }\end{array}$ & "dirty" looking & Massive & \\
\hline $\begin{array}{l}\text { reflected optical } \\
\text { properties }\end{array}$ & & & Vein-forming \\
\hline $\begin{array}{l}\text { contact/ } \\
\text { relationships with } \\
\text { other minerals } \\
\text { rection/zoning) }\end{array}$ & $\begin{array}{l}\text { Being replaced by wl, sul- } \\
\text { fides }\end{array}$ & $\begin{array}{l}\text { Cross-cut and fractured by } \\
\text { sulfides, being replaced by } \\
\text { sp, gn, frk, and hm }\end{array}$ & $\begin{array}{l}\text { Intergrown with galena, co- } \\
\text { cveal with gn, hm, and frk, } \\
\text { cross-cutting wl and car- } \\
\text { bonate }\end{array}$ \\
\hline & & & \\
\hline
\end{tabular}




\begin{tabular}{|c|c|c|c|}
\hline minerals & Galena & Franklinite & hematite \\
\hline percentage & 10 & 7 & 3 \\
\hline morphology & Irregular crystalline & Euhedral & Euhedral \\
\hline texture & Vein-forming w/sp & $\begin{array}{l}\text { Disseminated in } \mathrm{sp}+\mathrm{gn} \text { vein, } \\
\text { close to contacts with W1 }\end{array}$ & Disseminated in $\mathrm{sp}+\mathrm{gn}$ vein \\
\hline $\begin{array}{l}\text { transmitted opti- } \\
\text { cal properties }\end{array}$ & & & \\
\hline $\begin{array}{l}\text { reflected optical } \\
\text { properties }\end{array}$ & & & \\
\hline $\begin{array}{l}\text { contact/ } \\
\text { relationships with } \\
\text { other minerals }\end{array}$ & $\begin{array}{l}\text { Intergrown with sp, being } \\
\text { replaced by srm and cv }\end{array}$ & $\begin{array}{l}\text { Straight contacts with gn and } \\
\text { sp }\end{array}$ & $\begin{array}{l}\text { Straight contacts with gn } \\
\text { and sp }\end{array}$ \\
\hline $\begin{array}{l}\text { other (growth di- } \\
\text { rection/zoning) }\end{array}$ & & & \\
\hline
\end{tabular}

Paragenesis:

Time

Early 


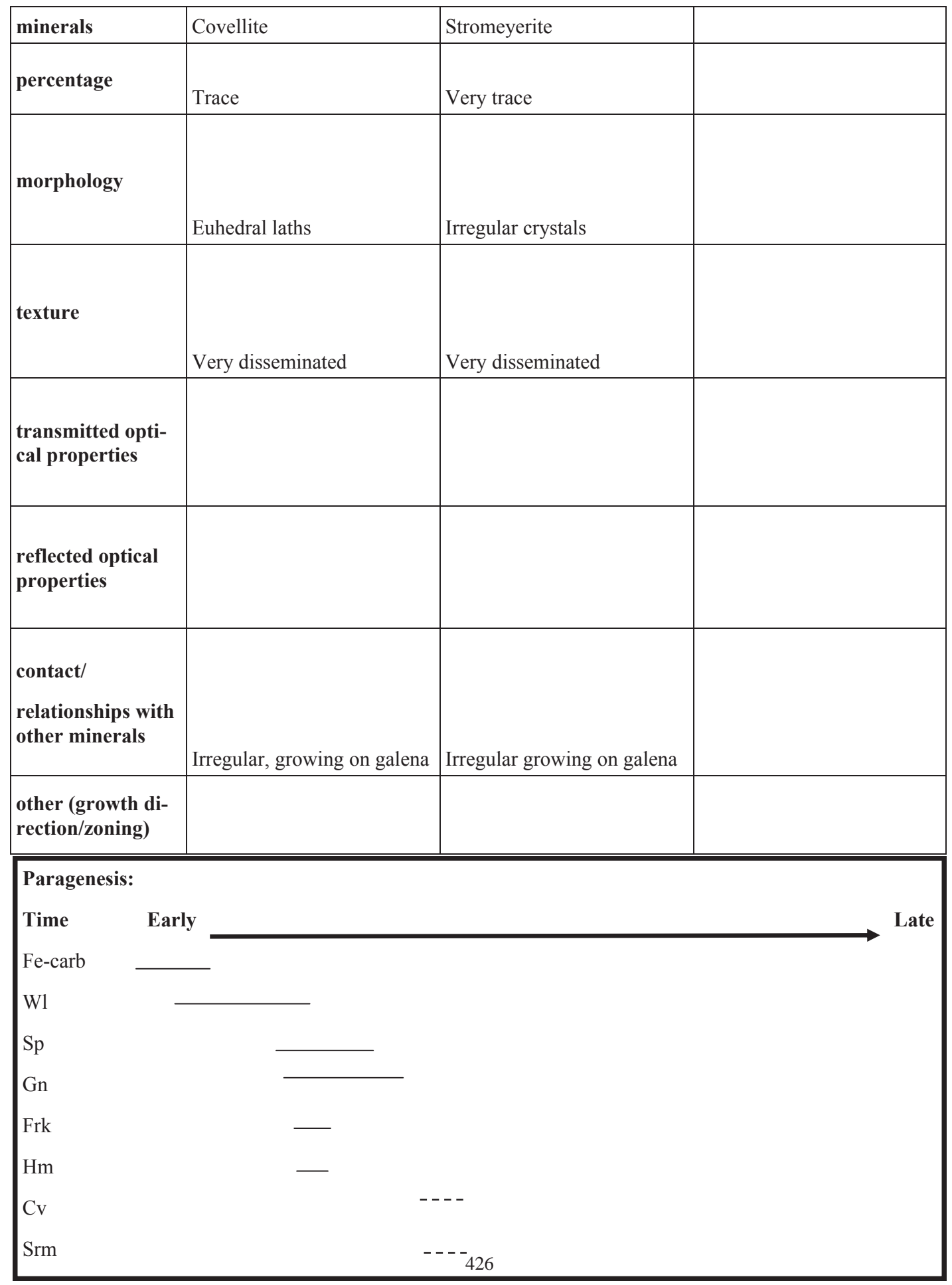


Geologic Unit: Upper Pamplona (silty marker bed)

Lithological Unit (Votorantim): Marl (MG)

Sample Photograph

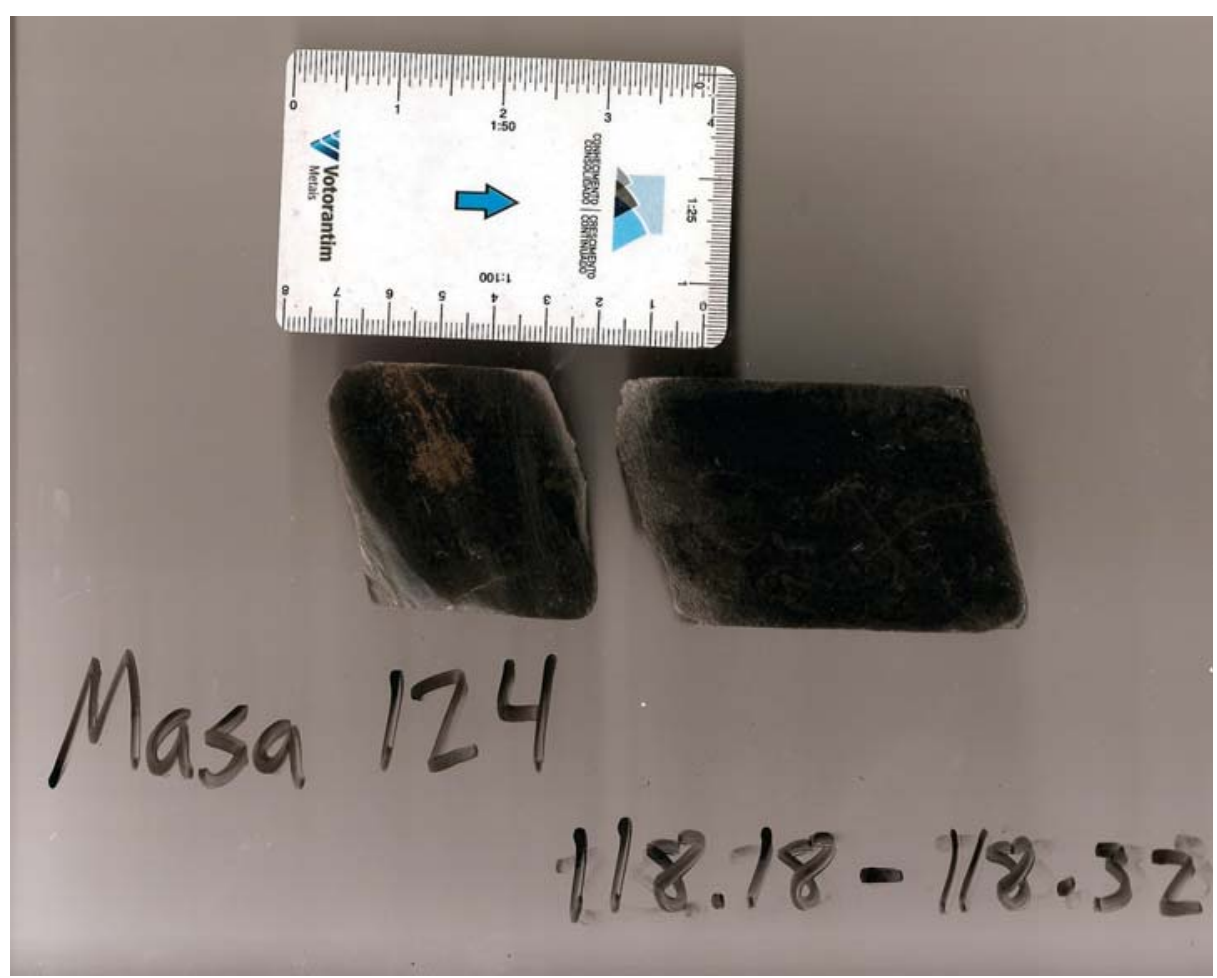

Other Sample Information: contact between the Upper and Middle Pamplona members 


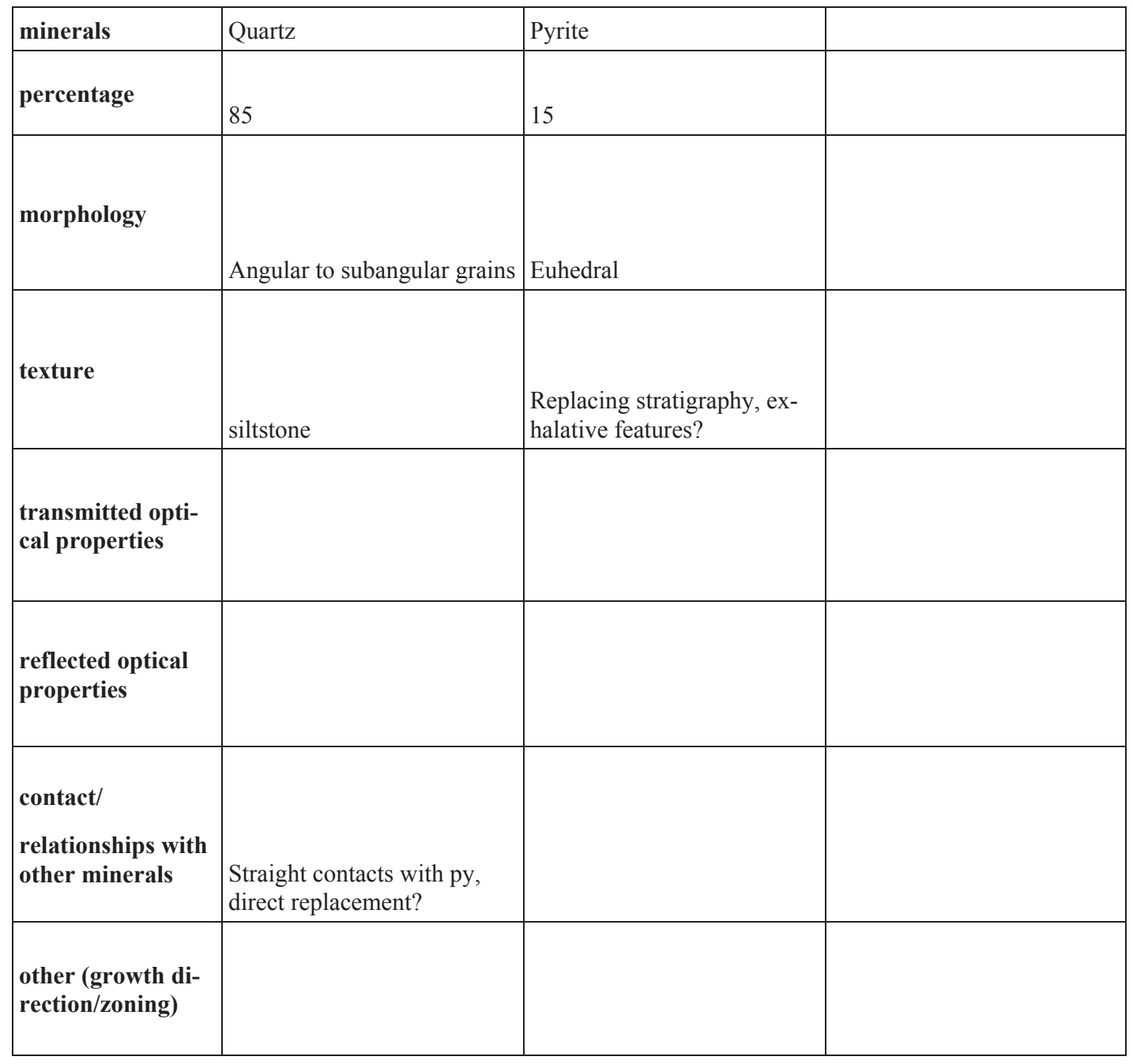

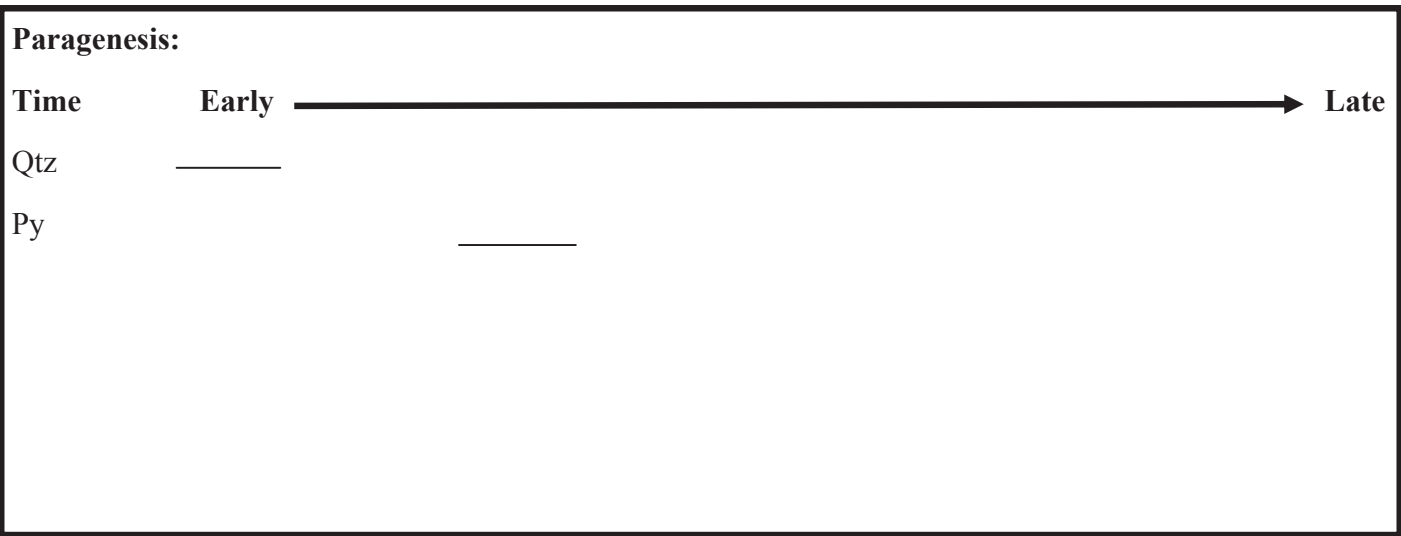


Paragenetic Reasoning: Possible that Py formed much later as a result of reducing sulfates or salts

being present in unit. Py forming around voids, possible exhalative escape structures. 
Geologic Unit: Lower Pamplona

Lithological Unit (Votorantim): Intercalated phyllites (DG)

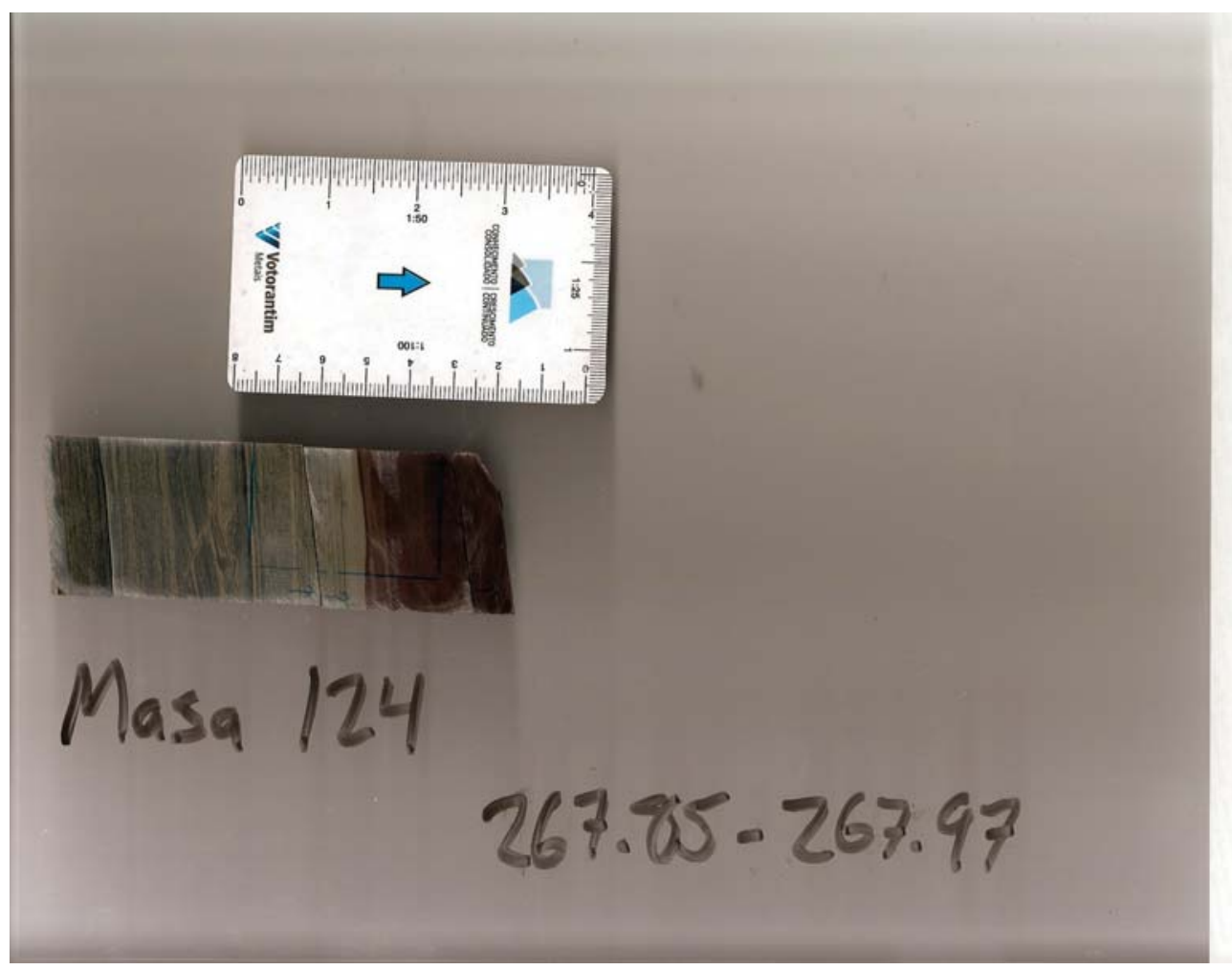

Other Sample Information: see $043-191$ * contains trace chalcocite and covellite in green zone 


\section{Geologic Unit: Upper Pamplona}

Lithological Unit (Votorantim): Grey dolomite (DO)

Sample Photograph

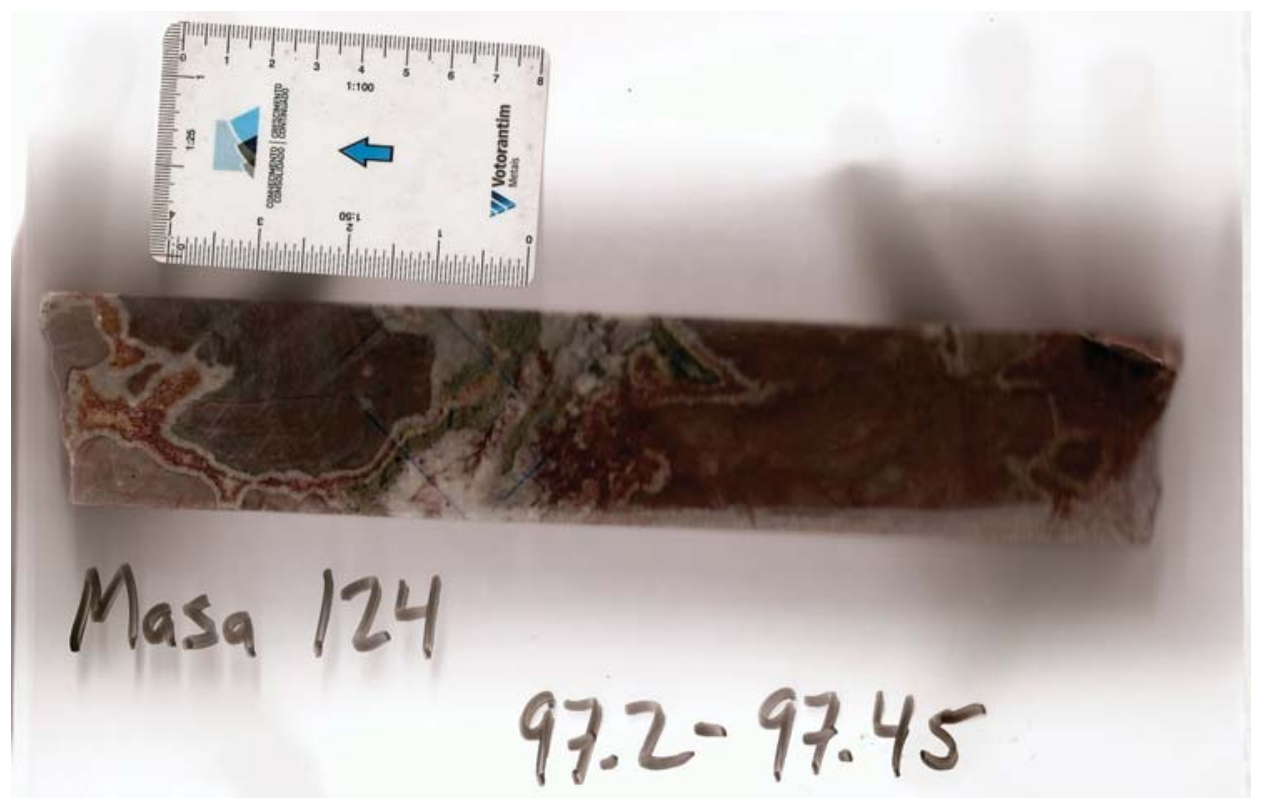

Other Sample Information: 


\begin{tabular}{|l|l|l|l|}
\hline minerals & Dolomite & Recrystallized dolomite & Quartz \\
\hline percentage & 30 & 47 & 1 \\
\hline morphology & Subhedral crystalline & Large euhedral crystalline & subangular \\
\hline $\begin{array}{l}\text { texture } \\
\text { transmitted opti- } \\
\text { cal properties }\end{array}$ & Pseudo-neomorphic & Vein-filling & \\
\hline $\begin{array}{l}\text { reflected optical } \\
\text { properties }\end{array}$ & & & disseminated \\
\hline $\begin{array}{l}\text { contact/ } \\
\text { relationships with } \\
\text { other minerals }\end{array}$ & $\begin{array}{l}\text { Host rock, being cut by re- } \\
\text { crystallized dol }\end{array}$ & $\begin{array}{l}\text { Being replaced by sp, irregu- } \\
\text { lar contacts }\end{array}$ & Coeval with dolomite \\
\hline $\begin{array}{l}\text { other (growth di- } \\
\text { rection/zoning) }\end{array}$ & & & \\
\hline
\end{tabular}




\begin{tabular}{|c|c|c|c|}
\hline minerals & Sphalerite & Pyrite & Fe-oxides (Goethite?) \\
\hline percentage & 15 & 2 & 5 \\
\hline morphology & Irregular crystalline & Irregular crystalline & Irregular crystalline \\
\hline texture & Vein-filling & $\begin{array}{l}\text { Disseminated and vein fill- } \\
\text { ing }\end{array}$ & Replacing Py \\
\hline $\begin{array}{l}\text { transmitted opti- } \\
\text { cal properties }\end{array}$ & Translucent in ppl & & \\
\hline $\begin{array}{l}\text { reflected optical } \\
\text { properties }\end{array}$ & & & \\
\hline $\begin{array}{l}\text { contact/ } \\
\text { relationships with } \\
\text { other minerals }\end{array}$ & $\begin{array}{l}\text { Replacing dol in vein and } \\
\text { contains disseminated py } \\
\text { (coeval) }\end{array}$ & Replacing dol in vein & Replacing Py and dol in vein \\
\hline $\begin{array}{l}\text { other (growth di- } \\
\text { rection/zoning) }\end{array}$ & & & \\
\hline
\end{tabular}

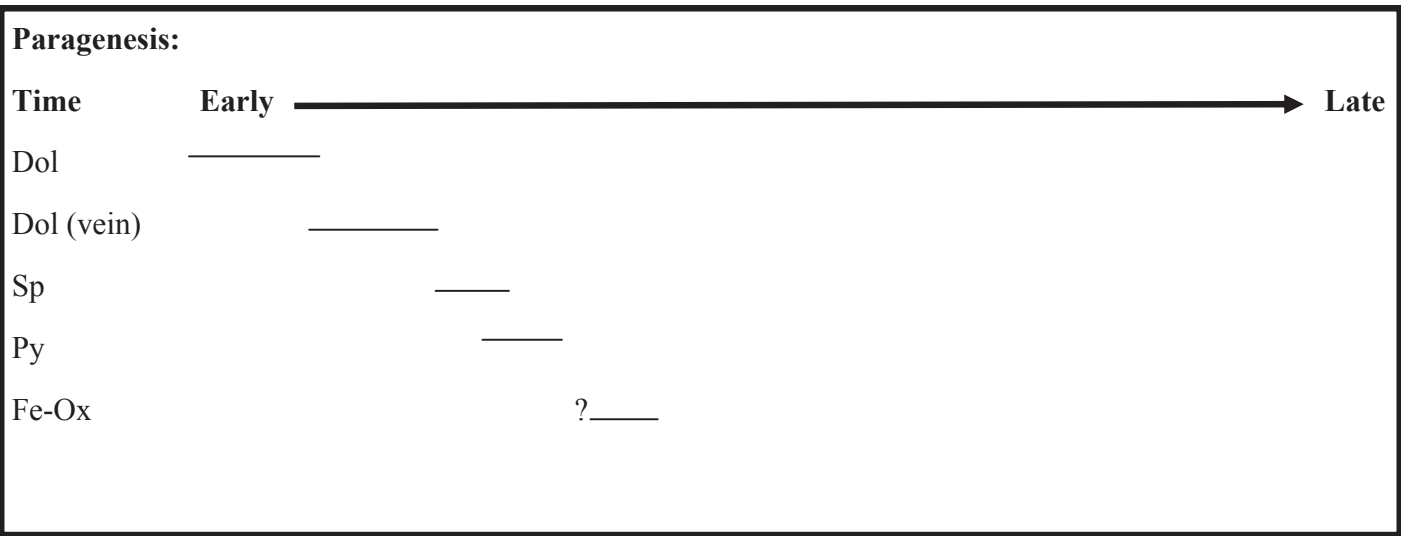




\section{Appendix D Whole Rock Geochemistry}

\section{D.1 ICP-MS Data}

\begin{tabular}{|c|c|c|c|c|c|c|c|}
\hline SAMPLE & & $12-347$ & $12-377$ & $136-112$ & $122-75$ & $115-150$ & $115-220$ \\
\hline Geologic & & $\mathrm{BH}$ & $\mathrm{BH}$ & $\mathrm{BH}$ & $\mathrm{BH}$ & $\mathrm{BH}$ & $\mathrm{BH}$ \\
\hline Unit & & & & & & & \\
\hline Description & & type 4 & type 2 & type 2 & type 4 & type 1 & type 4 \\
\hline Ag & ppm & 231 & 0.84 & 2.91 & 43.1 & 3 & 394 \\
\hline Al & $\%$ & 0.08 & 0.19 & 0.13 & 0.04 & 0.34 & 0.02 \\
\hline As & ppm & 42.4 & 5 & 4.4 & 42.5 & 5 & 96.6 \\
\hline $\mathbf{B a}$ & ppm & 1510 & 1980 & 1480 & 820 & 50 & 640 \\
\hline Be & ppm & 0.76 & 0.11 & 0.16 & 0.98 & 0.05 & 0.48 \\
\hline $\mathbf{B i}$ & ppm & 0.03 & 0.02 & 0.01 & 0.02 & 0.08 & 0.06 \\
\hline $\mathbf{C a}$ & $\%$ & 3.04 & 17.65 & 7.44 & 1.19 & 19.05 & 0.17 \\
\hline Cd & ppm & 178 & 7.75 & 214 & 59.1 & 278 & 89.2 \\
\hline $\mathrm{Ce}$ & ppm & 1.56 & 3.59 & 2.54 & 4.21 & 2.64 & 0.74 \\
\hline Co & ppm & 7.8 & 9 & 53.2 & 38.3 & 31.3 & 1.7 \\
\hline $\mathrm{Cr}$ & ppm & 11 & 4 & 19 & 9 & 6 & 7 \\
\hline Cs & ppm & 0.08 & 0.19 & 0.07 & 0.05 & 0.1 & 0.05 \\
\hline $\mathrm{Cu}$ & ppm & 338 & 6.9 & 7.2 & 74.9 & 18 & 2650 \\
\hline $\mathbf{F e}$ & $\%$ & 3.91 & 2.03 & 3.37 & 3.8 & 1.31 & 1.85 \\
\hline $\mathbf{G a}$ & ppm & 5.46 & 0.68 & 1.54 & 1.09 & 1.3 & 1.38 \\
\hline Ge & ppm & 0.06 & 0.05 & 0.05 & 0.06 & 0.05 & 0.07 \\
\hline Hf & ppm & 0.1 & 0.1 & 0.1 & 0.1 & 0.2 & 0.1 \\
\hline Hg & ppm & 8.3 & 0.22 & 0.1 & 1.05 & 0.31 & 8.2 \\
\hline In & ppm & 0.082 & 0.01 & 0.079 & 0.828 & 0.01 & 0.061 \\
\hline $\mathbf{K}$ & $\%$ & 0.03 & 0.08 & 0.01 & 0.01 & 0.02 & 0.01 \\
\hline $\mathbf{L a}$ & ppm & 0.8 & 1.9 & 2 & 2.5 & 1.7 & 0.5 \\
\hline $\mathbf{L i}$ & ppm & 4.4 & 4.3 & 2.8 & 5.2 & 3.3 & 10.9 \\
\hline Mg & $\%$ & 1.67 & 10.95 & 4.06 & 2.59 & 11.8 & 0.11 \\
\hline Mn & ppm & 185 & 720 & 1040 & 403 & 529 & 22 \\
\hline
\end{tabular}




\begin{tabular}{|c|c|c|c|c|c|c|c|}
\hline SAMPLE & & $12-347$ & $12-377$ & $136-112$ & $122-75$ & $115-150$ & $115-220$ \\
\hline Mo & ppm & 0.6 & 0.22 & 0.48 & 0.78 & 0.11 & 0.73 \\
\hline $\mathbf{N a}$ & $\%$ & 0.03 & 0.05 & 0.03 & 0.01 & 0.01 & 0.01 \\
\hline Nb & ppm & 0.3 & 0.6 & 0.3 & 0.2 & 0.8 & 0.1 \\
\hline $\mathbf{N i}$ & ppm & 3 & 2.9 & 13.9 & 7.1 & 9.1 & 1.2 \\
\hline $\mathbf{P}$ & ppm & 110 & 80 & 250 & 140 & 110 & 240 \\
\hline $\mathbf{P b}$ & ppm & 1295 & 130.5 & 917 & 1750 & 4730 & 87000 \\
\hline $\mathbf{R b}$ & ppm & 0.9 & 3.4 & 0.2 & 0.2 & 0.7 & 0.2 \\
\hline $\mathbf{R e}$ & ppm & 0.002 & 0.002 & 0.002 & 0.002 & 0.002 & 0.002 \\
\hline $\mathbf{S}$ & $\%$ & 0.05 & 0.06 & 0.04 & 0.04 & 0.03 & 0.72 \\
\hline $\mathbf{S b}$ & ppm & 2.2 & 0.32 & 0.99 & 5.63 & 0.25 & 1.02 \\
\hline Sc & ppm & 0.2 & 0.3 & 0.3 & 0.5 & 0.7 & 0.1 \\
\hline Se & ppm & 41 & 1 & 1 & 38 & 4 & 39 \\
\hline Sn & ppm & 0.2 & 0.2 & 0.2 & 0.2 & 0.2 & 0.2 \\
\hline $\mathrm{Sr}$ & ppm & 22.3 & 56.3 & 37 & 34.2 & 63.7 & 85.8 \\
\hline Ta & ppm & 0.05 & 0.05 & 0.05 & 0.05 & 0.05 & 0.05 \\
\hline Te & ppm & 0.05 & 0.05 & 0.05 & 0.05 & 0.05 & 0.05 \\
\hline Th & ppm & 0.2 & 0.2 & 0.2 & 0.2 & 0.3 & 0.2 \\
\hline $\mathbf{T i}$ & $\%$ & 0.005 & 0.009 & 0.006 & 0.005 & 0.018 & 0.005 \\
\hline Tl & ppm & 0.02 & 0.02 & 0.02 & 0.02 & 0.02 & 0.02 \\
\hline $\mathbf{U}$ & ppm & 1.5 & 0.6 & 0.4 & 0.7 & 0.6 & 0.7 \\
\hline V & ppm & 21 & 18 & 97 & 8 & 33 & 27 \\
\hline $\mathbf{W}$ & ppm & 0.2 & 0.3 & 0.3 & 0.2 & 0.1 & 0.2 \\
\hline $\mathbf{Y}$ & ppm & 0.5 & 1.5 & 2 & 1 & 2.1 & 0.3 \\
\hline $\mathbf{Z n}$ & ppm & 460000 & 4030 & 5440 & 378700 & 19000 & 489000 \\
\hline $\mathbf{Z r}$ & ppm & 1.3 & 4.2 & 2.9 & 0.6 & 6.3 & 0.7 \\
\hline
\end{tabular}




\begin{tabular}{|c|c|c|c|c|c|c|c|}
\hline SAMPLE & & $129-160$ & $129-167$ & $60-120$ & $60-136$ & $101-137$ & $15-382$ \\
\hline Geologic & & $\mathrm{BH}$ & $\mathrm{BH}$ & $\mathrm{BH}$ & $\mathrm{BH}$ & $\mathrm{BH}$ & $\mathrm{BH}$ \\
\hline Unit & & & & & & & \\
\hline Description & & type 1 & type 4 & type 4 & type 4 & type 4 & type 1 \\
\hline Ag & ppm & 2.31 & 24.1 & 19.55 & 257 & 3.58 & 0.13 \\
\hline Al & $\%$ & 1.21 & 0.65 & 0.1 & 0.06 & 0.03 & 0.62 \\
\hline As & ppm & 8 & 68.5 & 54.7 & 49.3 & 68.1 & 5 \\
\hline $\mathbf{B a}$ & ppm & 200 & 150 & 1940 & 1960 & 470 & 120 \\
\hline $\mathbf{B e}$ & ppm & 0.55 & 1.65 & 0.53 & 0.71 & 0.47 & 0.2 \\
\hline $\mathbf{B i}$ & ppm & 0.07 & 0.09 & 0.02 & 0.07 & 0.02 & 0.03 \\
\hline $\mathbf{C a}$ & $\%$ & 16.35 & 2.15 & 0.6 & 4.53 & 7.01 & 19.05 \\
\hline Cd & ppm & 11.05 & 222 & 14.55 & 900 & 812 & 5.53 \\
\hline $\mathrm{Ce}$ & ppm & 17.1 & 11.8 & 1.44 & 4.55 & 2.17 & 6.29 \\
\hline Co & ppm & 18.4 & 18.5 & 6.4 & 12.1 & 16.8 & 3.5 \\
\hline $\mathrm{Cr}$ & ppm & 8 & 30 & 3 & 8 & 11 & 4 \\
\hline Cs & ppm & 0.74 & 0.15 & 0.14 & 0.05 & 0.05 & 0.5 \\
\hline $\mathrm{Cu}$ & ppm & 15.7 & 132.5 & 77.6 & 191.5 & 16.6 & 4.6 \\
\hline $\mathbf{F e}$ & $\%$ & 1.34 & 12.4 & 13.3 & 7.21 & 1.91 & 0.69 \\
\hline Ga & ppm & 3.39 & 6.25 & 1.77 & 3.69 & 4.08 & 1.71 \\
\hline Ge & ppm & 0.05 & 0.25 & 0.21 & 0.17 & 0.05 & 0.07 \\
\hline Hf & ppm & 0.5 & 0.4 & 0.1 & 0.1 & 0.1 & 0.3 \\
\hline $\mathrm{Hg}$ & ppm & 0.07 & 0.71 & 2.12 & 0.8 & 0.23 & 0.01 \\
\hline In & ppm & 0.033 & 0.285 & 0.677 & 0.086 & 0.008 & 0.006 \\
\hline $\mathbf{K}$ & $\%$ & 0.54 & 0.01 & 0.01 & 0.01 & 0.01 & 0.35 \\
\hline La & ppm & 8 & 5.2 & 0.9 & 2.6 & 1.7 & 3.1 \\
\hline $\mathbf{L i}$ & ppm & 22.7 & 16.7 & 4 & 8.3 & 4.6 & 8.4 \\
\hline Mg & $\%$ & 10.55 & 1.5 & 0.36 & 2.5 & 3.83 & 11.9 \\
\hline Mn & ppm & 564 & 158 & 73 & 438 & 491 & 519 \\
\hline Mo & ppm & 0.18 & 1.99 & 1.91 & 1.12 & 0.37 & 0.05 \\
\hline $\mathbf{N a}$ & $\%$ & 0.03 & 0.01 & 0.03 & 0.04 & 0.01 & 0.02 \\
\hline Nb & ppm & 2.1 & 1.4 & 0.4 & 0.3 & 0.2 & 1.2 \\
\hline $\mathbf{N i}$ & ppm & 7.1 & 21.3 & 8.3 & 6.6 & 4.3 & 4.7 \\
\hline
\end{tabular}




\begin{tabular}{|c|c|c|c|c|c|c|c|}
\hline SAMPLE & & $129-160$ & $129-167$ & $60-120$ & $60-136$ & 101-137 & $15-382$ \\
\hline $\mathbf{P}$ & ppm & 1330 & 2150 & 120 & 150 & 290 & 170 \\
\hline $\mathbf{P b}$ & ppm & 1225 & 2960 & 1295 & 34700 & 2920 & 72.5 \\
\hline $\mathbf{R b}$ & ppm & 21.5 & 0.3 & 0.3 & 0.2 & 0.2 & 11.6 \\
\hline $\mathbf{R e}$ & ppm & 0.002 & 0.002 & 0.002 & 0.002 & 0.002 & 0.002 \\
\hline $\mathbf{S}$ & $\%$ & 0.01 & 0.02 & 0.2 & 0.24 & 0.02 & 0.01 \\
\hline $\mathbf{S b}$ & ppm & 1.13 & 12.75 & 2.99 & 2.95 & 1.36 & 0.12 \\
\hline Sc & ppm & 2.1 & 1.2 & 0.2 & 0.2 & 0.4 & 1 \\
\hline Se & ppm & 1 & 37 & 15 & 41 & 35 & 1 \\
\hline Sn & ppm & 0.4 & 0.4 & 0.2 & 0.2 & 0.2 & 0.2 \\
\hline $\mathbf{S r}$ & ppm & 49.7 & 12.1 & 1895 & 86.8 & 17.6 & 56 \\
\hline Ta & ppm & 0.13 & 0.07 & 0.05 & 0.05 & 0.05 & 0.06 \\
\hline Te & ppm & 0.05 & 0.07 & 0.05 & 0.05 & 0.05 & 0.05 \\
\hline Th & ppm & 1.7 & 0.9 & 0.2 & 0.2 & 0.2 & 0.7 \\
\hline $\mathbf{T i}$ & $\%$ & 0.056 & 0.03 & 0.005 & 0.005 & 0.005 & 0.027 \\
\hline Tl & ppm & 0.15 & 0.02 & 0.02 & 0.02 & 0.02 & 0.04 \\
\hline $\mathbf{U}$ & ppm & 1.3 & 7.3 & 3.8 & 3.5 & 0.5 & 0.4 \\
\hline $\mathbf{V}$ & ppm & 18 & 47 & 33 & 76 & 20 & 8 \\
\hline $\mathbf{W}$ & ppm & 0.3 & 0.5 & 0.2 & 0.3 & 0.1 & 0.1 \\
\hline $\mathbf{Y}$ & ppm & 9.4 & 2.3 & 0.6 & 1.2 & 1.1 & 1.9 \\
\hline Zn & ppm & 3160 & 401200 & 170500 & 373800 & 382900 & 1470 \\
\hline $\mathbf{Z r}$ & ppm & 19.9 & 12.9 & 1 & 0.8 & 0.5 & 9.3 \\
\hline
\end{tabular}




\begin{tabular}{|c|c|c|c|c|c|c|c|}
\hline SAMPLE & & $125-55$ & $123-73$ & $139-270$ & $28-47$ & $124-62$ & $124-72$ \\
\hline Geologic & & $\mathrm{BH}$ & $\mathrm{BH}$ & $\mathrm{BH}$ & $\mathrm{BH}$ & $\mathrm{BH}$ & $\mathrm{BH}$ \\
\hline Unit & & & & & & & \\
\hline Description & & type 3 & type 4 & Other & type 2 & type 3 & type 1 \\
\hline Ag & ppm & 0.99 & 30 & 0.03 & 0.1 & 1.01 & 0.46 \\
\hline Al & $\%$ & 0.78 & 0.07 & 0.33 & 0.67 & 0.61 & 0.92 \\
\hline As & ppm & 104 & 90.2 & 5 & 5 & 115.5 & 13 \\
\hline $\mathbf{B a}$ & ppm & 100 & 150 & 600 & 170 & 50 & 30 \\
\hline $\mathbf{B e}$ & ppm & 1.63 & 0.74 & 0.17 & 0.36 & 2.09 & 0.26 \\
\hline $\mathbf{B i}$ & ppm & 0.15 & 0.02 & 0.02 & 0.06 & 0.08 & 0.02 \\
\hline $\mathbf{C a}$ & $\%$ & 6.72 & 1.56 & 20 & 19.7 & 7.04 & 18.05 \\
\hline Cd & ppm & 108 & 55.3 & 1.86 & 8.69 & 189 & 553 \\
\hline $\mathrm{Ce}$ & ppm & 9.78 & 6.23 & 4.22 & 7.59 & 4.96 & 14.7 \\
\hline Co & ppm & 61.9 & 9.5 & 2.7 & 5.8 & 60.3 & 57.4 \\
\hline $\mathrm{Cr}$ & ppm & 5 & 12 & 4 & 6 & 5 & 8 \\
\hline Cs & ppm & 1 & 0.05 & 0.26 & 0.58 & 0.75 & 0.16 \\
\hline $\mathrm{Cu}$ & ppm & 9 & 62.5 & 2.1 & 3.3 & 16.2 & 15.9 \\
\hline $\mathbf{F e}$ & $\%$ & 43.1 & 15.85 & 0.45 & 0.46 & 41.2 & 2.26 \\
\hline Ga & ppm & 3.17 & 1.72 & 0.85 & 1.79 & 3.61 & 3.46 \\
\hline Ge & ppm & 0.95 & 0.34 & 0.12 & 0.08 & 0.21 & 0.12 \\
\hline Hf & ppm & 0.1 & 0.1 & 0.2 & 0.3 & 0.1 & 0.4 \\
\hline $\mathrm{Hg}$ & ppm & 0.2 & 1.08 & 0.01 & 0.07 & 0.05 & 0.02 \\
\hline In & ppm & 2.94 & 0.231 & 0.014 & 0.011 & 2.89 & 0.064 \\
\hline $\mathbf{K}$ & $\%$ & 0.04 & 0.01 & 0.16 & 0.39 & 0.1 & 0.01 \\
\hline La & ppm & 3.7 & 3 & 1.9 & 3.7 & 2.8 & 9.2 \\
\hline $\mathbf{L i}$ & ppm & 10.1 & 12.6 & 5 & 10.8 & 13.2 & 17.1 \\
\hline Mg & $\%$ & 3.97 & 0.88 & 12.65 & 11.8 & 4.28 & 11.45 \\
\hline Mn & ppm & 451 & 115 & 131 & 207 & 385 & 637 \\
\hline Mo & ppm & 3.87 & 1.8 & 0.05 & 0.13 & 5.25 & 0.3 \\
\hline $\mathbf{N a}$ & $\%$ & 0.01 & 0.01 & 0.02 & 0.03 & 0.01 & 0.01 \\
\hline Nb & ppm & 1.4 & 0.5 & 0.9 & 1.4 & 0.2 & 1.4 \\
\hline $\mathbf{N i}$ & ppm & 48.5 & 6.1 & 3.5 & 5.4 & 31.5 & 32.3 \\
\hline
\end{tabular}




\begin{tabular}{|c|c|c|c|c|c|c|c|}
\hline SAMPL & & $\mathbf{1 2 5 - 5 5}$ & $\mathbf{1 2 3 - 7 3}$ & $\mathbf{1 3 9 - 2 7 0}$ & $\mathbf{2 8 - 4 7}$ & $\mathbf{1 2 4 - 6 2}$ & $\mathbf{1 2 4 - 7 2}$ \\
$\mathbf{P}$ & $\mathrm{ppm}$ & 260 & 280 & 110 & 2700 & 210 & 380 \\
$\mathbf{P b}$ & $\mathrm{ppm}$ & 1850 & 2030 & 99.8 & 187 & 2390 & 2060 \\
$\mathbf{R b}$ & $\mathrm{ppm}$ & 2.7 & 0.3 & 6.8 & 16.7 & 3.6 & 0.6 \\
$\mathbf{R e}$ & $\mathrm{ppm}$ & 0.002 & 0.002 & 0.002 & 0.002 & 0.004 & 0.003 \\
$\mathbf{S}$ & $\%$ & 0.12 & 0.07 & 0.02 & 0.01 & 0.01 & 0.01 \\
$\mathbf{S b}$ & $\mathrm{ppm}$ & 47.6 & 12.95 & 0.19 & 0.18 & 31 & 1.36 \\
$\mathbf{S c}$ & $\mathrm{ppm}$ & 0.5 & 0.2 & 0.7 & 1 & 0.6 & 1.8 \\
$\mathbf{S e}$ & $\mathrm{ppm}$ & 3 & 29 & 1 & 1 & 1 & 1 \\
$\mathbf{S n}$ & $\mathrm{ppm}$ & 0.2 & 0.2 & 0.2 & 0.3 & 0.3 & 0.3 \\
$\mathbf{S r}$ & $\mathrm{ppm}$ & 16.8 & 39 & 58 & 70.8 & 20.3 & 45.1 \\
$\mathbf{T a}$ & $\mathrm{ppm}$ & 0.05 & 0.05 & 0.05 & 0.09 & 0.05 & 0.1 \\
$\mathbf{T e}$ & $\mathrm{ppm}$ & 0.05 & 0.05 & 0.05 & 0.05 & 0.05 & 0.05 \\
$\mathbf{T h}$ & $\mathrm{ppm}$ & 0.2 & 0.2 & 0.5 & 1.3 & 0.2 & 1.4 \\
$\mathbf{T i}$ & $\%$ & 0.005 & 0.005 & 0.017 & 0.026 & 0.005 & 0.046 \\
$\mathbf{T l}$ & $\mathrm{ppm}$ & 0.02 & 0.02 & 0.03 & 0.07 & 0.04 & 0.02 \\
$\mathbf{U}$ & $\mathrm{ppm}$ & 7.2 & 3.8 & 0.4 & 0.7 & 15.3 & 1.3 \\
$\mathbf{V}$ & $\mathrm{ppm}$ & 153 & 138 & 4 & 6 & 146 & 23 \\
$\mathbf{W}$ & $\mathrm{ppm}$ & 1.2 & 0.4 & 0.1 & 0.1 & 1.1 & 0.1 \\
$\mathbf{Y}$ & $\mathrm{ppm}$ & 3.4 & 0.7 & 1.6 & 3.1 & 2 & 3.9 \\
$\mathbf{Z n}$ & $\mathrm{ppm}$ & 24800 & 324000 & 325 & 1180 & 12200 & 25700 \\
$\mathbf{Z r}$ & $\mathrm{ppm}$ & 4.2 & 1.1 & 8.2 & 11.3 & 4.2 & 12.7 \\
\hline
\end{tabular}




\begin{tabular}{|c|c|c|c|c|c|c|c|}
\hline SAMPLE & & $124-75$ & $124-78$ & $124-83$ & $139-314$ & $123-127$ & $101-128$ \\
\hline Geologic & & $\mathrm{BH}$ & $\mathrm{BH}$ & $\mathrm{BH}$ & $\mathrm{BH}$ & $\mathrm{BH}$ & $\mathrm{BH}$ \\
\hline Unit & & & & & & & \\
\hline Description & & type 4 & type 4 & type 1 & type 1 & type 1 & type 3 \\
\hline Ag & ppm & 5.7 & 37 & 0.25 & 0.07 & 0.1 & 5.59 \\
\hline Al & $\%$ & 0.03 & 0.07 & 0.3 & 0.53 & 0.41 & 0.43 \\
\hline As & ppm & 57.3 & 63.9 & 5 & 8 & 5 & 87.9 \\
\hline $\mathbf{B a}$ & ppm & 70 & 280 & 10 & 20 & 40 & 4570 \\
\hline $\mathbf{B e}$ & ppm & 0.73 & 1.42 & 0.15 & 0.3 & 0.3 & 0.73 \\
\hline $\mathbf{B i}$ & ppm & 0.02 & 0.01 & 0.02 & 0.03 & 0.03 & 0.08 \\
\hline Ca & $\%$ & 1.84 & 1.27 & 20.3 & 19.25 & 19.7 & 6.06 \\
\hline Cd & ppm & 32.5 & 68.5 & 145.5 & 289 & 4.84 & 335 \\
\hline $\mathrm{Ce}$ & $\mathrm{ppm}$ & 3.6 & 1.54 & 3.29 & 5.73 & 5.25 & 6.32 \\
\hline Co & ppm & 12.8 & 9.7 & 19.6 & 65.6 & 1.5 & 14.5 \\
\hline $\mathrm{Cr}$ & ppm & 5 & 12 & 3 & 3 & 6 & 1 \\
\hline Cs & ppm & 0.05 & 0.05 & 0.12 & 0.31 & 0.43 & 0.41 \\
\hline $\mathrm{Cu}$ & $\mathrm{ppm}$ & 18 & 62.5 & 5.6 & 2.5 & 3.8 & 10.4 \\
\hline $\mathbf{F e}$ & $\%$ & 6.02 & 12.1 & 1.04 & 1.2 & 0.35 & 25.1 \\
\hline Ga & ppm & 1.63 & 3.21 & 1.2 & 1.84 & 1.01 & 2.8 \\
\hline Ge & ppm & 0.12 & 0.12 & 0.1 & 0.12 & 0.12 & 0.12 \\
\hline Hf & ppm & 0.1 & 0.1 & 0.1 & 0.4 & 0.3 & 0.1 \\
\hline Hg & ppm & 0.14 & 1.27 & 0.07 & 0.16 & 0.04 & 0.32 \\
\hline In & ppm & 0.532 & 1.935 & 0.012 & 0.139 & 0.006 & 0.077 \\
\hline $\mathbf{K}$ & $\%$ & 0.01 & 0.01 & 0.01 & 0.02 & 0.21 & 0.01 \\
\hline La & ppm & 1.7 & 0.9 & 2 & 3.6 & 2.4 & 3.2 \\
\hline $\mathbf{L i}$ & ppm & 14.5 & 8.9 & 7.2 & 16.9 & 9.5 & 3.8 \\
\hline Mg & $\%$ & 0.89 & 0.63 & 12.35 & 11.9 & 11.65 & 3.54 \\
\hline Mn & ppm & 94 & 71 & 409 & 627 & 144 & 671 \\
\hline Mo & ppm & 1.14 & 2.22 & 0.14 & 0.16 & 0.08 & 3.81 \\
\hline $\mathbf{N a}$ & $\%$ & 0.01 & 0.01 & 0.02 & 0.02 & 0.02 & 0.07 \\
\hline Nb & ppm & 0.1 & 0.1 & 0.6 & 1.2 & 1 & 0.2 \\
\hline $\mathbf{N i}$ & $\mathrm{ppm}$ & 9.3 & 12.8 & 12.3 & 41 & 2.5 & 10.1 \\
\hline
\end{tabular}




\begin{tabular}{|c|c|c|c|c|c|c|c|}
\hline SAMPLE & & $\mathbf{1 2 4 - 7 5}$ & $\mathbf{1 2 4 - 7 8}$ & $\mathbf{1 2 4 - 8 3}$ & $\mathbf{1 3 9 - 3 1 4}$ & $\mathbf{1 2 3 - 1 2 7}$ & $\mathbf{1 0 1 - 1 2 8}$ \\
$\mathbf{P}$ & $\mathrm{ppm}$ & 1030 & 190 & 80 & 110 & 110 & 320 \\
$\mathbf{P b}$ & $\mathrm{ppm}$ & 1180 & 1290 & 1480 & 1410 & 314 & 2850 \\
$\mathbf{R b}$ & $\mathrm{ppm}$ & 0.1 & 0.2 & 0.6 & 1.1 & 7.9 & 0.7 \\
$\mathbf{R e}$ & $\mathrm{ppm}$ & 0.003 & 0.003 & 0.003 & 0.004 & 0.003 & 0.003 \\
$\mathbf{S}$ & $\%$ & 0.01 & 0.01 & 0.01 & 0.01 & 0.01 & 0.2 \\
$\mathbf{S b}$ & $\mathrm{ppm}$ & 3.26 & 11.05 & 0.42 & 0.33 & 0.09 & 13.5 \\
$\mathbf{S c}$ & $\mathrm{ppm}$ & 0.1 & 0.1 & 0.6 & 1.2 & 0.8 & 0.7 \\
$\mathbf{S e}$ & $\mathrm{ppm}$ & 6 & 8 & 1 & 1 & 1 & 1 \\
$\mathbf{S n}$ & $\mathrm{ppm}$ & 0.2 & 0.2 & 0.2 & 0.3 & 0.2 & 0.2 \\
$\mathbf{S r}$ & $\mathrm{ppm}$ & 23.7 & 12.5 & 58.8 & 81.5 & 83.8 & 444 \\
$\mathbf{T a}$ & $\mathrm{ppm}$ & 0.05 & 0.05 & 0.05 & 0.07 & 0.06 & 0.05 \\
$\mathbf{T e}$ & $\mathrm{ppm}$ & 0.05 & 0.05 & 0.05 & 0.05 & 0.05 & 0.05 \\
$\mathbf{T h}$ & $\mathrm{ppm}$ & 0.2 & 0.2 & 0.5 & 1 & 0.7 & 0.2 \\
$\mathbf{T i}$ & $\%$ & 0.005 & 0.005 & 0.015 & 0.033 & 0.023 & 0.006 \\
$\mathbf{T l}$ & $\mathrm{ppm}$ & 0.02 & 0.02 & 0.02 & 0.02 & 0.05 & 0.02 \\
$\mathbf{U}$ & $\mathrm{ppm}$ & 1.9 & 8.4 & 0.7 & 1.6 & 4.1 & 4.2 \\
$\mathbf{V}$ & $\mathrm{ppm}$ & 33 & 94 & 6 & 9 & 27 & 52 \\
$\mathbf{W}$ & $\mathrm{ppm}$ & 0.1 & 0.4 & 0.1 & 0.1 & 0.1 & 0.8 \\
$\mathbf{Y}$ & $\mathrm{ppm}$ & 1.6 & 0.8 & 2.1 & 3.7 & 2.4 & 1.9 \\
$\mathbf{Z n}$ & $\mathrm{ppm}$ & 486400 & 350000 & 12250 & 9280 & 542 & 45600 \\
$\mathbf{Z r}$ & $\mathrm{ppm}$ & 0.5 & 0.7 & 5.3 & 12.7 & 10.1 & 2.6 \\
\hline
\end{tabular}




\begin{tabular}{|c|c|c|c|c|c|c|c|}
\hline SAMPLE & & $101-148$ & $43-213$ & 43-232 & M124-284 & $\begin{array}{c}M 124- \\
289\end{array}$ & $\begin{array}{r}M 124 \\
300\end{array}$ \\
\hline Geologic & & $\mathrm{BH}$ & $\mathrm{BH}$ & $\mathrm{BH}$ & $\mathrm{BH}$ & $\mathrm{BH}$ & $\mathrm{BH}$ \\
\hline Unit & & & & & & & \\
\hline Description & & type 2 & type 1 & type 2 & type 1 & type 1 & type 1 \\
\hline Ag & ppm & 0.41 & 0.06 & 0.03 & 0.1 & 0.18 & 0.18 \\
\hline Al & $\%$ & 0.18 & 0.49 & 0.09 & 0.41 & 0.41 & 0.33 \\
\hline As & ppm & 10 & 5 & 6 & 5 & 7 & 5 \\
\hline $\mathbf{B a}$ & ppm & 320 & 350 & 390 & 30 & 70 & 20 \\
\hline Be & ppm & 0.32 & 0.3 & 0.08 & 0.15 & 0.26 & 0.09 \\
\hline $\mathbf{B i}$ & ppm & 0.02 & 0.02 & 0.01 & 0.02 & 0.04 & 0.01 \\
\hline $\mathrm{Ca}$ & $\%$ & 16.6 & 19.45 & 19.65 & 19.1 & 17.25 & 19.3 \\
\hline Cd & ppm & 1110 & 36.8 & 167.5 & 158.5 & 4.94 & 22.2 \\
\hline $\mathrm{Ce}$ & ppm & 4.71 & 6.4 & 5.76 & 3.48 & 6.29 & 3.42 \\
\hline Co & ppm & 36.5 & 17.5 & 27.2 & 15.6 & 6 & 6.6 \\
\hline $\mathrm{Cr}$ & ppm & 1 & 2 & 1 & 4 & 4 & 3 \\
\hline Cs & ppm & 0.07 & 0.51 & 0.08 & 0.16 & 0.32 & 0.17 \\
\hline $\mathrm{Cu}$ & ppm & 7.3 & 1.8 & 2.6 & 6.2 & 9.1 & 6.7 \\
\hline $\mathbf{F e}$ & $\%$ & 1.37 & 0.71 & 1.49 & 0.95 & 2.76 & 0.57 \\
\hline Ga & ppm & 2.31 & 1.35 & 0.34 & 1.29 & 1.1 & 0.68 \\
\hline Ge & ppm & 0.08 & 0.12 & 0.1 & 0.12 & 0.13 & 0.11 \\
\hline Hf & ppm & 0.1 & 0.2 & 0.1 & 0.1 & 0.1 & 0.2 \\
\hline Hg & ppm & 0.07 & 0.03 & 0.02 & 0.04 & 0.1 & 0.14 \\
\hline In & ppm & 0.017 & 0.006 & 0.006 & 0.005 & 0.006 & 0.005 \\
\hline $\mathbf{K}$ & $\%$ & 0.01 & 0.23 & 0.01 & 0.08 & 0.2 & 0.04 \\
\hline La & ppm & 2.6 & 3.2 & 3.3 & 1.9 & 2.9 & 1.9 \\
\hline $\mathbf{L i}$ & ppm & 4 & 9.5 & 3.5 & 7.6 & 5.8 & 6.6 \\
\hline Mg & $\%$ & 10.1 & 11.6 & 11.65 & 12 & 10.7 & 12.15 \\
\hline Mn & ppm & 825 & 613 & 1080 & 541 & 693 & 578 \\
\hline Mo & ppm & 0.14 & 0.05 & 0.1 & 0.11 & 0.32 & 0.11 \\
\hline $\mathbf{N a}$ & $\%$ & 0.01 & 0.02 & 0.02 & 0.02 & 0.01 & 0.02 \\
\hline $\mathrm{Nb}$ & $\mathrm{ppm}$ & 0.3 & 0.9 & 0.2 & 0.5 & 0.5 & 0.4 \\
\hline
\end{tabular}




\begin{tabular}{|c|c|c|c|c|c|c|c|}
\hline SAMPLE & & $101-148$ & $43-213$ & $43-232$ & M124-284 & $\begin{array}{c}M 124- \\
289\end{array}$ & $\begin{array}{c}M 124- \\
300\end{array}$ \\
\hline $\mathbf{N i}$ & ppm & 6.4 & 7.5 & 11.8 & 9.4 & 5.9 & 3.6 \\
\hline $\mathbf{P}$ & ppm & 80 & 120 & 160 & 110 & 230 & 270 \\
\hline $\mathbf{P b}$ & ppm & 2010 & 656 & 1410 & 1400 & 1445 & 740 \\
\hline $\mathbf{R b}$ & ppm & 0.5 & 8.7 & 0.4 & 2.5 & 6.2 & 1.3 \\
\hline $\mathbf{R e}$ & ppm & 0.003 & 0.003 & 0.003 & 0.002 & 0.002 & 0.002 \\
\hline $\mathbf{S}$ & $\%$ & 0.01 & 0.01 & 0.01 & 0.01 & 0.01 & 0.01 \\
\hline $\mathbf{S b}$ & ppm & 1.42 & 0.23 & 0.36 & 0.1 & 0.78 & 0.05 \\
\hline Sc & ppm & 0.3 & 0.8 & 0.1 & 0.7 & 0.6 & 0.5 \\
\hline $\mathbf{S e}$ & ppm & 1 & 1 & 1 & 1 & 1 & 1 \\
\hline Sn & ppm & 0.2 & 0.2 & 0.2 & 0.2 & 0.2 & 0.2 \\
\hline $\mathrm{Sr}$ & ppm & 28.1 & 75.9 & 75.3 & 57.6 & 46.1 & 47.3 \\
\hline Ta & ppm & 0.05 & 0.05 & 0.05 & 0.05 & 0.05 & 0.05 \\
\hline Te & ppm & 0.05 & 0.05 & 0.05 & 0.05 & 0.05 & 0.05 \\
\hline Th & ppm & 0.3 & 0.7 & 0.2 & 0.3 & 0.6 & 0.4 \\
\hline $\mathbf{T i}$ & $\%$ & 0.008 & 0.021 & 0.005 & 0.016 & 0.015 & 0.016 \\
\hline Tl & ppm & 0.02 & 0.03 & 0.02 & 0.02 & 0.03 & 0.02 \\
\hline $\mathbf{U}$ & ppm & 0.7 & 0.4 & 0.2 & 0.4 & 1 & 0.5 \\
\hline $\mathbf{V}$ & ppm & 17 & 6 & 13 & 12 & 28 & 5 \\
\hline $\mathbf{W}$ & ppm & 0.1 & 0.1 & 0.1 & 0.1 & 0.3 & 0.1 \\
\hline $\mathbf{Y}$ & ppm & 2.2 & 2 & 3.1 & 1.6 & 3.4 & 1.2 \\
\hline $\mathbf{Z n}$ & ppm & 107000 & 3660 & 8940 & 5350 & 1120 & 6170 \\
\hline $\mathbf{Z r}$ & ppm & 3.3 & 8.4 & 1.5 & 3.7 & 4.7 & 5.4 \\
\hline
\end{tabular}




\begin{tabular}{|c|c|c|c|c|c|}
\hline SAMPLE & & $\begin{array}{c}\text { M124- } \\
305\end{array}$ & M124-321 & $\begin{array}{c}M 124- \\
329\end{array}$ & $\begin{array}{r}\text { M124 } \\
347\end{array}$ \\
\hline Geologic & & $\mathrm{BH}$ & $\mathrm{BH}$ & $\mathrm{BH}$ & $\mathrm{BH}$ \\
\hline Unit & & & & & \\
\hline Description & & type 3 & Other-brecciated & type 4 & type 2 \\
\hline & & & phyllite & & \\
\hline Ag & ppm & 8.82 & 5.35 & 26.1 & 0.31 \\
\hline Al & $\%$ & 0.35 & 6.71 & 0.12 & 0.22 \\
\hline As & $\mathrm{ppm}$ & 147 & 3.5 & 82 & 8 \\
\hline Ba & ppm & 50 & 430 & 20 & 60 \\
\hline Be & ppm & 0.87 & 1.92 & 2.38 & 0.1 \\
\hline $\mathbf{B i}$ & ppm & 0.08 & 0.1 & 0.01 & 0.02 \\
\hline $\mathbf{C a}$ & $\%$ & 1.55 & 1.33 & 0.43 & 17.65 \\
\hline Cd & ppm & 50.7 & 11.65 & 10.55 & 628 \\
\hline $\mathrm{Ce}$ & $\mathrm{ppm}$ & 3.78 & 57.7 & 2.27 & 7.41 \\
\hline Co & ppm & 8.2 & 31.9 & 14.5 & 44 \\
\hline $\mathrm{Cr}$ & ppm & 17 & 46 & 10 & 2 \\
\hline Cs & ppm & 0.75 & 11.1 & 0.13 & 0.25 \\
\hline $\mathrm{Cu}$ & $\mathrm{ppm}$ & 48.9 & 26.8 & 22.1 & 2.7 \\
\hline $\mathbf{F e}$ & $\%$ & 54.31 & 2.12 & 13.4 & 4.81 \\
\hline Ga & ppm & 3.25 & 14 & 2.13 & 1.09 \\
\hline Ge & ppm & 0.18 & 0.22 & 0.21 & 0.14 \\
\hline Hf & ppm & 0.1 & 3.1 & 0.1 & 0.1 \\
\hline Hg & ppm & 0.34 & 0.15 & 0.01 & 0.03 \\
\hline In & ppm & 0.017 & 0.039 & 2.1 & 0.074 \\
\hline $\mathbf{K}$ & $\%$ & 0.03 & 2.99 & 0.01 & 0.04 \\
\hline $\mathbf{L a}$ & ppm & 1.9 & 29.3 & 1 & 3.9 \\
\hline $\mathbf{L i}$ & ppm & 3.3 & 78.3 & 5 & 5.7 \\
\hline Mg & $\%$ & 0.88 & 3.78 & 0.25 & 10.9 \\
\hline Mn & ppm & 357 & 200 & 80 & 899 \\
\hline Mo & ppm & 9.29 & 0.18 & 2.43 & 0.49 \\
\hline $\mathbf{N a}$ & $\%$ & 0.01 & 0.05 & 0.01 & 0.01 \\
\hline
\end{tabular}




\begin{tabular}{|c|c|c|c|c|c|}
\hline SAMPLE & & $\begin{array}{c}\text { M124- } \\
305\end{array}$ & M124-321 & $\begin{array}{c}M 124- \\
329\end{array}$ & $\begin{array}{r}M 124 \\
347\end{array}$ \\
\hline Nb & ppm & 0.3 & 10 & 0.1 & 0.4 \\
\hline $\mathbf{N i}$ & $\mathrm{ppm}$ & 11.4 & 25.4 & 10.1 & 14.4 \\
\hline $\mathbf{P}$ & $\mathrm{ppm}$ & 150 & 240 & 400 & 330 \\
\hline $\mathbf{P b}$ & ppm & 2680 & 702 & 1310 & 2230 \\
\hline $\mathbf{R b}$ & ppm & 1.5 & 107.5 & 0.4 & 1.4 \\
\hline $\operatorname{Re}$ & $\mathrm{ppm}$ & 0.002 & 0.002 & 0.002 & 0.002 \\
\hline $\mathbf{S}$ & $\%$ & 0.01 & 0.01 & 0.01 & 0.01 \\
\hline Sb & $\mathrm{ppm}$ & 9.11 & 1.51 & 4.71 & 1.15 \\
\hline Sc & ppm & 1.3 & 9.5 & 0.1 & 0.4 \\
\hline Se & $\mathrm{ppm}$ & 1 & 1 & 4 & 1 \\
\hline Sn & $\mathrm{ppm}$ & 0.2 & 1.5 & 0.2 & 0.2 \\
\hline $\mathrm{Sr}$ & $\mathrm{ppm}$ & 10.4 & 9 & 5.7 & 75.4 \\
\hline Ta & ppm & 0.05 & 0.73 & 0.05 & 0.05 \\
\hline Te & $\mathrm{ppm}$ & 0.05 & 0.05 & 0.05 & 0.05 \\
\hline Th & ppm & 0.2 & 8.1 & 0.2 & 0.3 \\
\hline $\mathbf{T i}$ & $\%$ & 0.007 & 0.331 & 0.005 & 0.011 \\
\hline Tl & ppm & 0.02 & 0.8 & 0.02 & 0.02 \\
\hline $\mathbf{U}$ & $\mathrm{ppm}$ & 4.7 & 1.7 & 2.9 & 1.5 \\
\hline $\mathbf{V}$ & ppm & 49 & 60 & 34 & 10 \\
\hline W & ppm & 0.4 & 0.8 & 0.2 & 0.2 \\
\hline $\mathbf{Y}$ & ppm & 1.1 & 10.1 & 0.8 & 3.6 \\
\hline $\mathbf{Z n}$ & $\mathrm{ppm}$ & 46600 & 8840 & 454200 & 7050 \\
\hline $\mathbf{Z r}$ & $\mathrm{ppm}$ & 2.1 & 102 & 0.6 & 3.6 \\
\hline
\end{tabular}




\begin{tabular}{|c|c|c|c|c|c|}
\hline SAMPLE & & $\begin{array}{c}\text { M124- } \\
353\end{array}$ & $\begin{array}{c}M 124- \\
356\end{array}$ & $124-4$ & $32-157$ \\
\hline $\begin{array}{c}\text { Geologic } \\
\text { Unit }\end{array}$ & & $\mathrm{BH}$ & $\mathrm{BH}$ & BH overprint on Lp & $\mathrm{BH} ? / \mathrm{SdG}$ \\
\hline Description & & type 3 & type 1 & type 2 & mineralized fault/vein \\
\hline Ag & ppm & 4.92 & 0.15 & 0.07 & 0.38 \\
\hline Al & $\%$ & 0.17 & 0.18 & 0.05 & 0.37 \\
\hline As & ppm & 54.7 & 5 & 5 & 5 \\
\hline $\mathbf{B a}$ & ppm & 30 & 20 & 50 & 40 \\
\hline Be & ppm & 0.82 & 0.09 & 0.08 & 0.54 \\
\hline $\mathbf{B i}$ & ppm & 0.04 & 0.01 & 0.02 & 0.08 \\
\hline $\mathbf{C a}$ & $\%$ & 2.09 & 20.1 & 20.3 & 16.05 \\
\hline Cd & ppm & 66.5 & 236 & 167.5 & 49.7 \\
\hline $\mathrm{Ce}$ & ppm & 1.56 & 3.16 & 2.3 & 12.95 \\
\hline Co & ppm & 25.4 & 28.6 & 56.7 & 34.5 \\
\hline $\mathrm{Cr}$ & ppm & 13 & 2 & 4 & 33 \\
\hline Cs & ppm & 0.3 & 0.09 & 0.06 & 2.81 \\
\hline $\mathbf{C u}$ & ppm & 21.9 & 3.1 & 10.7 & 3.1 \\
\hline $\mathrm{Fe}$ & $\%$ & 59.87 & 0.84 & 1.57 & 13.3 \\
\hline Ga & ppm & 5.11 & 0.66 & 0.26 & 1.5 \\
\hline Ge & ppm & 0.19 & 0.11 & 0.12 & 0.27 \\
\hline Hf & ppm & 0.1 & 0.1 & 0.1 & 0.1 \\
\hline Hg & ppm & 0.05 & 0.02 & 0.06 & 0.14 \\
\hline In & ppm & 0.613 & 0.047 & 0.014 & 0.151 \\
\hline $\mathbf{K}$ & $\%$ & 0.01 & 0.02 & 0.02 & 0.03 \\
\hline $\mathbf{L a}$ & ppm & 0.7 & 2.7 & 1.2 & 6.3 \\
\hline $\mathbf{L i}$ & ppm & 3.8 & 5.2 & 2 & 12 \\
\hline Mg & $\%$ & 1.33 & 12.45 & 11.9 & 9.03 \\
\hline Mn & ppm & 165 & 495 & 1140 & 778 \\
\hline Mo & ppm & 12.7 & 0.07 & 0.45 & 0.54 \\
\hline $\mathbf{N a}$ & $\%$ & 0.01 & 0.02 & 0.01 & 0.01 \\
\hline $\mathbf{N b}$ & ppm & 0.1 & 0.3 & 0.1 & 0.9 \\
\hline
\end{tabular}




\begin{tabular}{|c|c|c|c|c|c|}
\hline SAMPLE & & $\begin{array}{c}\text { M124- } \\
353\end{array}$ & $\begin{array}{c}\text { M124- } \\
356\end{array}$ & $124-4$ & $32-157$ \\
\hline $\mathrm{Ni}$ & ppm & 30 & 10.9 & 8.1 & 23 \\
\hline $\mathbf{P}$ & $\mathrm{ppm}$ & 60 & 380 & 90 & 110 \\
\hline $\mathbf{P b}$ & ppm & 1160 & 2080 & 2040 & 2580 \\
\hline $\mathbf{R b}$ & $\mathrm{ppm}$ & 0.3 & 0.5 & 0.6 & 3.6 \\
\hline $\mathbf{R e}$ & ppm & 0.002 & 0.002 & 0.003 & 0.002 \\
\hline $\mathbf{S}$ & $\%$ & 0.01 & 0.01 & 0.01 & 0.02 \\
\hline Sb & $\mathrm{ppm}$ & 32.7 & 0.08 & 0.9 & 0.73 \\
\hline Sc & $\mathrm{ppm}$ & 0.1 & 0.4 & 0.2 & 0.6 \\
\hline Se & ppm & 1 & 1 & 1 & 1 \\
\hline Sn & ppm & 1.1 & 0.2 & 0.2 & 0.2 \\
\hline $\mathrm{Sr}$ & $\mathrm{ppm}$ & 11.3 & 56 & 41.8 & 212 \\
\hline Ta & ppm & 0.05 & 0.05 & 0.05 & 0.05 \\
\hline Te & ppm & 0.05 & 0.05 & 0.05 & 0.05 \\
\hline Th & $\mathrm{ppm}$ & 0.2 & 0.3 & 0.2 & 0.2 \\
\hline $\mathbf{T i}$ & $\%$ & 0.005 & 0.009 & 0.005 & 0.009 \\
\hline Tl & ppm & 0.02 & 0.02 & 0.02 & 0.02 \\
\hline $\mathbf{U}$ & ppm & 6.8 & 0.8 & 0.2 & 3.7 \\
\hline $\mathbf{V}$ & $\mathrm{ppm}$ & 60 & 8 & 15 & 508 \\
\hline W & ppm & 0.5 & 0.1 & 0.2 & 0.2 \\
\hline $\mathbf{Y}$ & ppm & 0.7 & 1.9 & 1.7 & 8.3 \\
\hline Zn & ppm & 5390 & 6740 & 9800 & 3190 \\
\hline $\mathbf{Z r}$ & $\mathrm{ppm}$ & 0.5 & 3.4 & 0.9 & 4.2 \\
\hline
\end{tabular}




\begin{tabular}{|c|c|c|c|c|}
\hline SAMPLE & & $88-68$ & $139-231$ & $139-245$ \\
\hline Geologic & & $\mathrm{BH} ?$ & LMdP & LMdP? \\
\hline Unit & & & & \\
\hline Description & & Other-sulfide bx & least alt dolomite & selective Fe-carb \\
\hline & & & & alt selvages \\
\hline Ag & ppm & 44.9 & 0.03 & 0.22 \\
\hline Al & $\%$ & 0.28 & 0.12 & 0.98 \\
\hline As & ppm & 287 & 5 & 8 \\
\hline $\mathbf{B a}$ & ppm & 160 & 20 & 40 \\
\hline $\mathbf{B e}$ & ppm & 0.09 & 0.15 & 0.41 \\
\hline $\mathbf{B i}$ & ppm & 0.02 & 0.01 & 0.04 \\
\hline $\mathbf{C a}$ & $\%$ & 7.89 & 20.6 & 18.85 \\
\hline Cd & ppm & 508 & 10.55 & 10.2 \\
\hline $\mathrm{Ce}$ & ppm & 3.41 & 1.84 & 11.25 \\
\hline Co & ppm & 61.6 & 3.3 & 2.9 \\
\hline $\mathrm{Cr}$ & ppm & 1 & 2 & 7 \\
\hline Cs & ppm & 0.14 & 0.1 & 0.99 \\
\hline $\mathrm{Cu}$ & ppm & 37.6 & 3.3 & 5.1 \\
\hline $\mathbf{F e}$ & $\%$ & 14.25 & 0.27 & 0.49 \\
\hline Ga & ppm & 2.68 & 0.27 & 2.58 \\
\hline Ge & ppm & 0.3 & 0.06 & 0.13 \\
\hline Hf & ppm & 0.1 & 0.1 & 0.9 \\
\hline Hg & ppm & 15.7 & 0.1 & 0.02 \\
\hline In & ppm & 0.137 & 0.005 & 0.012 \\
\hline $\mathbf{K}$ & $\%$ & 0.09 & 0.06 & 0.59 \\
\hline $\mathbf{L a}$ & ppm & 1.4 & 0.9 & 5.1 \\
\hline $\mathbf{L i}$ & ppm & 3.1 & 4.3 & 18.5 \\
\hline Mg & $\%$ & 4.49 & 13.15 & 11.05 \\
\hline Mn & ppm & 769 & 194 & 180 \\
\hline Mo & ppm & 1.04 & 0.09 & 0.13 \\
\hline $\mathbf{N a}$ & $\%$ & 0.01 & 0.02 & 0.04 \\
\hline $\mathbf{N b}$ & $\mathrm{ppm}$ & 0.8 & 0.2 & 1.8 \\
\hline
\end{tabular}




\begin{tabular}{|c|c|c|c|c|}
\hline SAMPLE & & $88-68$ & $139-231$ & $139-245$ \\
\hline $\mathbf{N i}$ & ppm & 38 & 1.6 & 16.9 \\
\hline $\mathbf{P}$ & ppm & 50 & 570 & 3090 \\
\hline $\mathbf{P b}$ & ppm & 2960 & 119 & 176 \\
\hline $\mathbf{R b}$ & ppm & 2.5 & 2.2 & 21.5 \\
\hline $\mathbf{R e}$ & ppm & 0.002 & 0.002 & 0.004 \\
\hline $\mathbf{S}$ & $\%$ & 10 & 0.01 & 0.01 \\
\hline Sb & ppm & 1.56 & 0.05 & 0.22 \\
\hline Sc & ppm & 0.5 & 0.3 & 1.7 \\
\hline Se & ppm & 15 & 1 & 1 \\
\hline Sn & ppm & 0.2 & 0.2 & 0.4 \\
\hline $\mathrm{Sr}$ & ppm & 71.5 & 65.6 & 79.6 \\
\hline Ta & ppm & 0.05 & 0.05 & 0.12 \\
\hline Te & ppm & 0.05 & 0.05 & 0.05 \\
\hline Th & ppm & 0.3 & 0.2 & 1.5 \\
\hline $\mathbf{T i}$ & $\%$ & 0.011 & 0.005 & 0.048 \\
\hline Tl & ppm & 0.1 & 0.02 & 0.2 \\
\hline $\mathbf{U}$ & ppm & 0.5 & 0.3 & 1.8 \\
\hline $\mathbf{V}$ & ppm & 3 & 2 & 9 \\
\hline $\mathbf{W}$ & ppm & 0.1 & 0.1 & 0.2 \\
\hline $\mathbf{Y}$ & ppm & 2.2 & 0.7 & 4.7 \\
\hline $\mathbf{Z n}$ & ppm & 174300 & 539 & 754 \\
\hline $\mathbf{Z r}$ & ppm & 4.8 & 3.8 & 33.3 \\
\hline
\end{tabular}




\begin{tabular}{|c|c|c|c|c|c|}
\hline SAMPLE & & $143-124$ & $155-185$ & $\begin{array}{l}155- \\
214\end{array}$ & $43-97$ \\
\hline Geologic & & LP & LP & LP & LP \\
\hline Unit & & & & & \\
\hline Description & & least alt dolomite & dolomite & phyllite & red phyllite \\
\hline Ag & ppm & 0.02 & 0.06 & 0.28 & 0.06 \\
\hline Al & $\%$ & 0.93 & 2.48 & 7.75 & 8.77 \\
\hline As & ppm & 5 & 5 & 3.5 & 2.9 \\
\hline $\mathbf{B a}$ & ppm & 90 & 430 & 650 & 360 \\
\hline $\mathbf{B e}$ & ppm & 0.2 & 0.34 & 1.79 & 2.59 \\
\hline $\mathbf{B i}$ & ppm & 0.02 & 0.04 & 0.05 & 0.21 \\
\hline $\mathbf{C a}$ & $\%$ & 18.7 & 13.45 & 0.23 & 1.24 \\
\hline Cd & ppm & 0.16 & 0.29 & 0.08 & 0.03 \\
\hline $\mathrm{Ce}$ & ppm & 9.81 & 15 & 87.3 & 82.9 \\
\hline Co & ppm & 2.1 & 2.4 & 8.4 & 14.3 \\
\hline $\mathrm{Cr}$ & ppm & 9 & 9 & 59 & 72 \\
\hline Cs & ppm & 0.61 & 1.93 & 19.3 & 10.45 \\
\hline $\mathrm{Cu}$ & ppm & 2.4 & 3.3 & 7.8 & 1.1 \\
\hline $\mathbf{F e}$ & $\%$ & 0.73 & 0.66 & 3.84 & 6.15 \\
\hline Ga & ppm & 2.32 & 5.08 & 21.6 & 27.3 \\
\hline Ge & ppm & 0.05 & 0.14 & 0.21 & 0.27 \\
\hline Hf & ppm & 0.4 & 0.7 & 2.4 & 3.3 \\
\hline Hg & ppm & 0.1 & 0.1 & 0.1 & 0.01 \\
\hline In & ppm & 0.008 & 0.011 & 0.055 & 0.067 \\
\hline $\mathbf{K}$ & $\%$ & 0.78 & 2.7 & 5.4 & 4.68 \\
\hline La & ppm & 5 & 7.1 & 40.2 & 39.1 \\
\hline $\mathbf{L i}$ & ppm & 6.3 & 9.1 & 27.4 & 37.8 \\
\hline Mg & $\%$ & 11.35 & 8.39 & 1.44 & 2.16 \\
\hline Mn & ppm & 312 & 262 & 18 & 119 \\
\hline Mo & ppm & 0.05 & 0.1 & 0.1 & 0.14 \\
\hline $\mathbf{N a}$ & $\%$ & 0.03 & 0.03 & 0.04 & 0.06 \\
\hline $\mathrm{Nb}$ & $\mathrm{ppm}$ & 1.3 & 2.7 & 12.5 & 12.9 \\
\hline
\end{tabular}




\begin{tabular}{|c|c|c|c|c|c|}
\hline SAMPLE & & $143-124$ & $155-185$ & $\begin{array}{l}155- \\
214\end{array}$ & $43-97$ \\
\hline $\mathbf{N i}$ & ppm & 4.8 & 4.8 & 18.2 & 34.7 \\
\hline $\mathbf{P}$ & ppm & 90 & 190 & 250 & 430 \\
\hline $\mathbf{P b}$ & $\mathrm{ppm}$ & 3.2 & 9.4 & 177.5 & 11.4 \\
\hline $\mathbf{R b}$ & $\mathrm{ppm}$ & 17.2 & 46.2 & 145 & 112 \\
\hline $\mathbf{R e}$ & ppm & 0.002 & 0.002 & 0.002 & 0.002 \\
\hline $\mathbf{S}$ & $\%$ & 0.01 & 0.01 & 0.01 & 0.01 \\
\hline Sb & ppm & 0.16 & 0.2 & 0.8 & 0.59 \\
\hline Sc & ppm & 1.5 & 2.4 & 13.5 & 17.2 \\
\hline Se & ppm & 1 & 1 & 2 & 1 \\
\hline Sn & ppm & 0.2 & 0.4 & 2.6 & 2.7 \\
\hline $\mathrm{Sr}$ & ppm & 55.8 & 69.8 & 25.3 & 39.3 \\
\hline Ta & ppm & 0.09 & 0.17 & 0.82 & 0.91 \\
\hline $\mathbf{T e}$ & ppm & 0.05 & 0.05 & 0.05 & 0.05 \\
\hline Th & ppm & 1.1 & 1.6 & 10.7 & 11 \\
\hline $\mathbf{T i}$ & $\%$ & 0.043 & 0.062 & 0.33 & 0.385 \\
\hline Tl & ppm & 0.06 & 0.16 & 0.7 & 0.66 \\
\hline $\mathbf{U}$ & ppm & 0.9 & 0.5 & 1.7 & 1.1 \\
\hline $\mathbf{V}$ & ppm & 13 & 8 & 75 & 78 \\
\hline W & ppm & 0.2 & 0.2 & 1.2 & 1.2 \\
\hline $\mathbf{Y}$ & ppm & 3.8 & 6.5 & 20.8 & 23.7 \\
\hline $\mathbf{Z n}$ & ppm & 36 & 24 & 1370 & 78 \\
\hline $\mathbf{Z r}$ & ppm & 14.8 & 22.8 & 84.3 & 105.5 \\
\hline
\end{tabular}




\begin{tabular}{|c|c|c|c|c|}
\hline SAMPLE & & $105-113$ & $141-117$ & $139-194$ \\
\hline Geologic & & LP & LP & LP \\
\hline Unit & & & & \\
\hline \multirow[t]{3}{*}{ Description } & & dol-specularite & $\mathrm{Fe}-\mathrm{carb} / \mathrm{carb}$ and $\mathrm{hm}$ & selective Fe- \\
\hline & & vein & /py vein & carb \\
\hline & & & & alt selvages \\
\hline Ag & ppm & 0.15 & 0.06 & 0.07 \\
\hline Al & $\%$ & 0.84 & 0.54 & 0.51 \\
\hline As & ppm & 5 & 6 & 5 \\
\hline $\mathbf{B a}$ & ppm & 1350 & 50 & 130 \\
\hline $\mathbf{B e}$ & $\mathrm{ppm}$ & 0.23 & 0.16 & 0.23 \\
\hline $\mathbf{B i}$ & ppm & 0.21 & 0.03 & 0.02 \\
\hline $\mathbf{C a}$ & $\%$ & 18.55 & 20 & 19.4 \\
\hline Cd & ppm & 7.26 & 0.63 & 4.59 \\
\hline $\mathrm{Ce}$ & ppm & 7.9 & 5.2 & 5.48 \\
\hline Co & ppm & 3.7 & 2.1 & 2.7 \\
\hline $\mathrm{Cr}$ & ppm & 5 & 5 & 3 \\
\hline Cs & ppm & 0.66 & 0.32 & 0.62 \\
\hline $\mathbf{C u}$ & ppm & 2.1 & 2.6 & 2.3 \\
\hline $\mathbf{F e}$ & $\%$ & 0.66 & 0.83 & 0.74 \\
\hline Ga & ppm & 1.73 & 1.45 & 1.3 \\
\hline Ge & ppm & 0.09 & 0.14 & 0.1 \\
\hline Hf & ppm & 0.5 & 0.2 & 0.3 \\
\hline Hg & ppm & 0.2 & 0.03 & 0.04 \\
\hline In & $\mathrm{ppm}$ & 0.007 & 0.006 & 0.007 \\
\hline $\mathbf{K}$ & $\%$ & 0.74 & 0.38 & 0.25 \\
\hline La & ppm & 3.8 & 2.4 & 2.7 \\
\hline $\mathbf{L i}$ & ppm & 7.5 & 4.2 & 10 \\
\hline Mg & $\%$ & 11.55 & 11.95 & 11.65 \\
\hline Mn & ppm & 334 & 371 & 397 \\
\hline Mo & ppm & 0.05 & 0.48 & 0.11 \\
\hline $\mathbf{N a}$ & $\%$ & 0.03 & 0.03 & 0.02 \\
\hline
\end{tabular}




\begin{tabular}{|c|c|c|c|c|}
\hline SAMPLE & & $105-113$ & $141-117$ & 139-194 \\
\hline $\mathbf{N b}$ & ppm & 1.1 & 0.8 & 0.8 \\
\hline $\mathbf{N i}$ & ppm & 5.8 & 3.1 & 3.3 \\
\hline $\mathbf{P}$ & ppm & 170 & 60 & 100 \\
\hline $\mathbf{P b}$ & $\mathrm{ppm}$ & 469 & 19.1 & 195 \\
\hline $\mathbf{R b}$ & $\mathrm{ppm}$ & 18.2 & 8.5 & 8.4 \\
\hline $\mathbf{R e}$ & ppm & 0.002 & 0.003 & 0.003 \\
\hline $\mathbf{S}$ & $\%$ & 0.05 & 0.01 & 0.01 \\
\hline Sb & ppm & 0.05 & 0.13 & 0.38 \\
\hline Sc & ppm & 1.5 & 0.8 & 0.8 \\
\hline Se & ppm & 1 & 1 & 1 \\
\hline Sn & ppm & 0.2 & 0.2 & 0.2 \\
\hline $\mathrm{Sr}$ & ppm & 88.7 & 51.3 & 75.6 \\
\hline Ta & ppm & 0.07 & 0.05 & 0.05 \\
\hline Te & ppm & 0.05 & 0.05 & 0.05 \\
\hline Th & ppm & 1 & 0.7 & 0.7 \\
\hline $\mathbf{T i}$ & $\%$ & 0.038 & 0.023 & 0.023 \\
\hline Tl & ppm & 0.07 & 0.03 & 0.04 \\
\hline $\mathbf{U}$ & ppm & 0.5 & 0.7 & 0.5 \\
\hline $\mathbf{V}$ & ppm & 8 & 14 & 7 \\
\hline W & ppm & 0.1 & 0.1 & 0.1 \\
\hline $\mathbf{Y}$ & ppm & 2.7 & 1.7 & 2 \\
\hline $\mathbf{Z n}$ & ppm & 523 & 163 & 1170 \\
\hline $\mathbf{Z r}$ & ppm & 18.8 & 6.2 & 10.3 \\
\hline
\end{tabular}




\begin{tabular}{|c|c|c|c|c|}
\hline SAMPLE & & $123-46$ & $123-51$ & $101-222$ \\
\hline Geologic & & LP & LP & LP \\
\hline Unit & & & & \\
\hline Description & & Fe-carb selvage alt & Hm alt vein & mottled Fe-carb \\
\hline & & w/ no texture & & atl and veinlets \\
\hline Ag & $\mathrm{ppm}$ & 0.13 & 0.15 & 0.07 \\
\hline Al & $\%$ & 2.22 & 1.43 & 0.4 \\
\hline As & ppm & 9 & 17 & 5 \\
\hline $\mathbf{B a}$ & ppm & 50 & 40 & 750 \\
\hline $\mathbf{B e}$ & ppm & 0.77 & 0.75 & 0.22 \\
\hline $\mathbf{B i}$ & ppm & 0.05 & 0.08 & 0.02 \\
\hline $\mathbf{C a}$ & $\%$ & 15.45 & 16.8 & 19.45 \\
\hline Cd & ppm & 16.45 & 104 & 3.82 \\
\hline $\mathrm{Ce}$ & ppm & 21.4 & 13.8 & 5.99 \\
\hline Co & ppm & 14.3 & 42.6 & 16.5 \\
\hline $\mathrm{Cr}$ & ppm & 10 & 7 & 2 \\
\hline Cs & ppm & 1.07 & 0.8 & 0.25 \\
\hline $\mathbf{C u}$ & ppm & 5.4 & 10.6 & 9.7 \\
\hline $\mathbf{F e}$ & $\%$ & 1.14 & 5.11 & 0.95 \\
\hline Ga & ppm & 4.75 & 3.72 & 1.25 \\
\hline Ge & ppm & 0.13 & 0.11 & 0.06 \\
\hline Hf & ppm & 1.2 & 0.7 & 0.2 \\
\hline Hg & ppm & 0.69 & 0.07 & 0.06 \\
\hline In & $\mathrm{ppm}$ & 0.018 & 0.054 & 0.015 \\
\hline $\mathbf{K}$ & $\%$ & 0.86 & 0.55 & 0.29 \\
\hline La & ppm & 10.1 & 7 & 3 \\
\hline $\mathbf{L i}$ & ppm & 20.9 & 16.4 & 5.2 \\
\hline Mg & $\%$ & 9.83 & 10.55 & 12.05 \\
\hline Mn & ppm & 530 & 696 & 464 \\
\hline Mo & ppm & 0.07 & 0.6 & 0.21 \\
\hline $\mathrm{Na}$ & $\%$ & 0.06 & 0.02 & 0.01 \\
\hline $\mathbf{N b}$ & ppm & 2.9 & 2.1 & 1.1 \\
\hline
\end{tabular}




\begin{tabular}{|c|c|c|c|c|}
\hline SAMPLE & & $123-46$ & $123-51$ & $101-222$ \\
\hline $\mathbf{N i}$ & ppm & 9.4 & 24 & 6.6 \\
\hline $\mathbf{P}$ & ppm & 280 & 90 & 140 \\
\hline $\mathbf{P b}$ & $\mathrm{ppm}$ & 763 & 2870 & 59.1 \\
\hline $\mathbf{R b}$ & ppm & 27.7 & 18.5 & 7.4 \\
\hline $\mathbf{R e}$ & ppm & 0.003 & 0.004 & 0.002 \\
\hline $\mathbf{S}$ & $\%$ & 0.01 & 0.01 & 0.02 \\
\hline Sb & ppm & 0.62 & 3.17 & 0.19 \\
\hline Sc & ppm & 3.9 & 2.8 & 0.8 \\
\hline Se & ppm & 1 & 1 & 1 \\
\hline Sn & ppm & 0.6 & 0.4 & 0.2 \\
\hline $\mathbf{S r}$ & ppm & 44.9 & 28.1 & 72.3 \\
\hline $\mathbf{T a}$ & ppm & 0.2 & 0.13 & 0.06 \\
\hline $\mathbf{T e}$ & ppm & 0.05 & 0.05 & 0.05 \\
\hline Th & ppm & 2.9 & 1.9 & 0.5 \\
\hline $\mathbf{T i}$ & $\%$ & 0.112 & 0.067 & 0.019 \\
\hline Tl & ppm & 0.19 & 0.11 & 0.03 \\
\hline $\mathbf{U}$ & ppm & 0.6 & 1.6 & 0.4 \\
\hline $\mathbf{V}$ & ppm & 14 & 32 & 11 \\
\hline $\mathbf{W}$ & ppm & 0.2 & 0.4 & 0.1 \\
\hline $\mathbf{Y}$ & ppm & 7.7 & 4.7 & 1.8 \\
\hline Zn & ppm & 4070 & 9650 & 581 \\
\hline $\mathbf{Z r}$ & ppm & 40.7 & 21.3 & 10.7 \\
\hline
\end{tabular}




\begin{tabular}{|c|c|c|c|c|c|}
\hline SAMPLE & & $43-191$ & $88-93$ & $88-124$ & M124-199 \\
\hline Geologic & & LP & LP & LP & LP \\
\hline Unit & & & & & \\
\hline Description & & green phyllite & maroon phyllite & black phyllite & pink carb w/ \\
\hline & & & & & sillca alt \\
\hline Ag & ppm & 0.07 & 0.25 & 0.02 & 0.4 \\
\hline Al & $\%$ & 5.51 & 7.79 & 8.05 & 0.5 \\
\hline As & ppm & 1.7 & 8.9 & 3.1 & 5 \\
\hline $\mathbf{B a}$ & ppm & 370 & 1050 & 390 & 40 \\
\hline Be & ppm & 2.49 & 2.08 & 2.05 & 0.26 \\
\hline $\mathbf{B i}$ & ppm & 0.13 & 0.11 & 0.13 & 0.03 \\
\hline $\mathbf{C a}$ & $\%$ & 4.18 & 0.41 & 0.31 & 16.95 \\
\hline Cd & ppm & 11.4 & 0.41 & 1.5 & 1.65 \\
\hline $\mathrm{Ce}$ & ppm & 75.7 & 62.6 & 72.8 & 3.28 \\
\hline Co & ppm & 20.9 & 10.1 & 8.2 & 2.5 \\
\hline $\mathrm{Cr}$ & ppm & 29 & 71 & 74 & 8 \\
\hline Cs & ppm & 5.26 & 11.8 & 7.69 & 0.24 \\
\hline $\mathrm{Cu}$ & ppm & 3.6 & 23.7 & 3.8 & 6 \\
\hline $\mathbf{F e}$ & $\%$ & 2.35 & 5.5 & 4.9 & 0.83 \\
\hline Ga & ppm & 15.2 & 21.7 & 24.2 & 1.38 \\
\hline Ge & ppm & 0.21 & 0.19 & 0.2 & 0.11 \\
\hline Hf & ppm & 4 & 3.8 & 3.6 & 0.2 \\
\hline Hg & ppm & 0.01 & 0.02 & 0.01 & 0.14 \\
\hline In & ppm & 0.045 & 0.056 & 0.054 & 0.005 \\
\hline $\mathbf{K}$ & $\%$ & 3.86 & 5.56 & 5.75 & 0.39 \\
\hline La & ppm & 39.4 & 28.7 & 33.7 & 1.6 \\
\hline $\mathbf{L i}$ & ppm & 21.2 & 31.2 & 33 & 5.4 \\
\hline Mg & $\%$ & 3.2 & 1.58 & 1.38 & 10.45 \\
\hline Mn & ppm & 225 & 22 & 46 & 368 \\
\hline Mo & ppm & 0.17 & 0.57 & 0.12 & 0.76 \\
\hline $\mathbf{N a}$ & $\%$ & 0.03 & 0.05 & 0.07 & 0.02 \\
\hline $\mathrm{Nb}$ & ppm & 25.9 & 12.1 & 13.9 & 0.6 \\
\hline
\end{tabular}




\begin{tabular}{|c|c|c|c|c|c|}
\hline SAMPLE & & $43-191$ & $88-93$ & $88-124$ & M124-199 \\
\hline $\mathbf{N i}$ & ppm & 16.2 & 25.5 & 24 & 2.8 \\
\hline $\mathbf{P}$ & ppm & 290 & 400 & 230 & 60 \\
\hline $\mathbf{P b}$ & ppm & 41.3 & 53.3 & 16.4 & 67.1 \\
\hline $\mathbf{R b}$ & ppm & 120 & 118 & 116 & 8.1 \\
\hline $\mathbf{R e}$ & ppm & 0.004 & 0.003 & 0.004 & 0.002 \\
\hline $\mathbf{S}$ & $\%$ & 0.01 & 0.01 & 0.01 & 0.01 \\
\hline Sb & ppm & 0.54 & 0.99 & 0.26 & 0.08 \\
\hline Sc & ppm & 8.2 & 12.2 & 13.8 & 0.6 \\
\hline Se & ppm & 2 & 1 & 1 & 1 \\
\hline Sn & ppm & 1.8 & 2.3 & 2.4 & 0.2 \\
\hline $\mathrm{Sr}$ & ppm & 30.1 & 12 & 17.5 & 46.3 \\
\hline Ta & ppm & 0.77 & 0.87 & 0.96 & 0.05 \\
\hline Te & ppm & 0.05 & 0.05 & 0.05 & 0.05 \\
\hline Th & ppm & 10 & 9.4 & 10.3 & 0.5 \\
\hline $\mathbf{T i}$ & $\%$ & 0.27 & 0.38 & 0.405 & 0.017 \\
\hline Tl & $\mathrm{ppm}$ & 0.58 & 0.67 & 0.59 & 0.04 \\
\hline $\mathbf{U}$ & ppm & 1.1 & 2.9 & 1.7 & 0.8 \\
\hline $\mathbf{V}$ & ppm & 41 & 101 & 115 & 19 \\
\hline $\mathbf{W}$ & ppm & 1 & 0.8 & 0.9 & 0.1 \\
\hline $\mathbf{Y}$ & ppm & 20.9 & 10.6 & 12.4 & 1.1 \\
\hline $\mathbf{Z n}$ & ppm & 579 & 468 & 160 & 315 \\
\hline $\mathbf{Z r}$ & ppm & 154 & 117 & 118.5 & 5.7 \\
\hline
\end{tabular}




\begin{tabular}{|c|c|c|c|c|}
\hline SAMPLE & & M124-250 & $139-135$ & $141-16$ \\
\hline Geologic & & LP & LP & MP \\
\hline Unit & & & & \\
\hline Description & & chalcedonic silica alt & Fe-carb selvage & carb-alt veinlet \\
\hline & & & alt w/ qtz alt veinlets & \\
\hline Ag & $\mathrm{ppm}$ & 0.08 & 0.7 & 0.39 \\
\hline Al & $\%$ & 0.58 & 0.7 & 0.09 \\
\hline As & ppm & 5 & 8 & 9 \\
\hline $\mathbf{B a}$ & ppm & 160 & 110 & 20 \\
\hline $\mathbf{B e}$ & ppm & 0.21 & 0.22 & 0.1 \\
\hline $\mathbf{B i}$ & ppm & 0.02 & 0.02 & 0.01 \\
\hline $\mathbf{C a}$ & $\%$ & 15.4 & 15.3 & 17.25 \\
\hline Cd & ppm & 2.65 & 3.63 & 58 \\
\hline $\mathrm{Ce}$ & ppm & 5.18 & 4.86 & 2.52 \\
\hline Co & ppm & 0.9 & 2.7 & 1.6 \\
\hline $\mathrm{Cr}$ & ppm & 11 & 23 & 3 \\
\hline Cs & ppm & 0.4 & 0.22 & 0.08 \\
\hline $\mathbf{C u}$ & ppm & 4.5 & 17.6 & 6.7 \\
\hline $\mathbf{F e}$ & $\%$ & 0.5 & 0.99 & 1.33 \\
\hline Ga & ppm & 1.4 & 1.59 & 0.56 \\
\hline Ge & ppm & 0.11 & 0.13 & 0.09 \\
\hline Hf & ppm & 0.3 & 0.2 & 0.1 \\
\hline Hg & ppm & 0.04 & 0.13 & 1.7 \\
\hline In & ppm & 0.005 & 0.012 & 0.007 \\
\hline $\mathbf{K}$ & $\%$ & 0.41 & 0.72 & 0.04 \\
\hline $\mathbf{L a}$ & ppm & 2.6 & 2.1 & 1.6 \\
\hline $\mathbf{L i}$ & ppm & 5.1 & 3.6 & 1.4 \\
\hline Mg & $\%$ & 9.39 & 9.61 & 11.05 \\
\hline Mn & ppm & 228 & 249 & 825 \\
\hline Mo & ppm & 0.55 & 2.18 & 0.5 \\
\hline $\mathbf{N a}$ & $\%$ & 0.01 & 0.02 & 0.02 \\
\hline $\mathbf{N b}$ & ppm & 0.8 & 0.7 & 0.2 \\
\hline
\end{tabular}




\begin{tabular}{|c|c|c|c|c|}
\hline SAMPLE & & M124-250 & $139-135$ & 141-16 \\
\hline $\mathbf{N i}$ & ppm & 1.4 & 4.5 & 1.5 \\
\hline $\mathbf{P}$ & ppm & 60 & 80 & 90 \\
\hline $\mathbf{P b}$ & ppm & 80.6 & 118.5 & 82.2 \\
\hline $\mathbf{R b}$ & $\mathrm{ppm}$ & 8 & 9.6 & 1.2 \\
\hline $\mathbf{R e}$ & ppm & 0.002 & 0.004 & 0.002 \\
\hline $\mathbf{S}$ & $\%$ & 0.02 & 0.01 & 0.76 \\
\hline Sb & ppm & 0.1 & 0.32 & 0.37 \\
\hline Sc & ppm & 0.7 & 0.6 & 0.3 \\
\hline Se & ppm & 1 & 1 & 3 \\
\hline Sn & ppm & 0.2 & 0.3 & 0.2 \\
\hline $\mathrm{Sr}$ & ppm & 55 & 46.9 & 51.1 \\
\hline Ta & ppm & 0.06 & 0.05 & 0.05 \\
\hline Te & ppm & 0.05 & 0.05 & 0.05 \\
\hline Th & ppm & 0.6 & 0.6 & 0.2 \\
\hline $\mathbf{T i}$ & $\%$ & 0.022 & 0.017 & 0.006 \\
\hline Tl & ppm & 0.03 & 0.05 & 0.02 \\
\hline $\mathbf{U}$ & ppm & 0.7 & 1 & 1.4 \\
\hline $\mathbf{V}$ & ppm & 28 & 27 & 15 \\
\hline $\mathbf{W}$ & ppm & 0.1 & 0.2 & 0.1 \\
\hline $\mathbf{Y}$ & ppm & 1.9 & 2.3 & 1.7 \\
\hline $\mathbf{Z n}$ & ppm & 390 & 2140 & 12850 \\
\hline $\mathbf{Z r}$ & ppm & 8.6 & 7.4 & 1.3 \\
\hline
\end{tabular}




\begin{tabular}{|c|c|c|c|c|}
\hline SAMPLE & & $141-18$ & $110-150$ & $139-20$ \\
\hline Geologic & & MP & MP & MP \\
\hline Unit & & & & \\
\hline Description & & carb-alt veinlet & least alt dolomite & carb-alt veinlet \\
\hline Ag & ppm & 0.03 & 0.01 & 0.09 \\
\hline Al & $\%$ & 0.47 & 0.11 & 0.11 \\
\hline As & ppm & 5 & 5 & 5 \\
\hline Ba & ppm & 50 & 10 & 20 \\
\hline $\mathbf{B e}$ & ppm & 0.21 & 0.08 & 0.11 \\
\hline $\mathbf{B i}$ & ppm & 0.01 & 0.01 & 0.01 \\
\hline $\mathbf{C a}$ & $\%$ & 21.1 & 20.1 & 20 \\
\hline Cd & ppm & 0.32 & 0.13 & 2.77 \\
\hline $\mathrm{Ce}$ & ppm & 6.05 & 1.54 & 2.44 \\
\hline Co & ppm & 1.1 & 0.9 & 1.8 \\
\hline $\mathrm{Cr}$ & ppm & 8 & 2 & 3 \\
\hline Cs & ppm & 0.3 & 0.07 & 0.08 \\
\hline $\mathrm{Cu}$ & ppm & 1 & 2.2 & 3.5 \\
\hline $\mathbf{F e}$ & $\%$ & 0.37 & 0.27 & 0.74 \\
\hline Ga & ppm & 1.09 & 0.24 & 0.29 \\
\hline Ge & ppm & 0.07 & 0.07 & 0.08 \\
\hline Hf & ppm & 0.3 & 0.1 & 0.1 \\
\hline Hg & ppm & 0.1 & 0.1 & 0.2 \\
\hline In & ppm & 0.005 & 0.005 & 0.005 \\
\hline $\mathbf{K}$ & $\%$ & 0.26 & 0.05 & 0.05 \\
\hline La & ppm & 3.1 & 0.7 & 1.1 \\
\hline $\mathbf{L i}$ & ppm & 4.4 & 3.7 & 1.9 \\
\hline Mg & $\%$ & 12.55 & 12.9 & 13.15 \\
\hline Mn & ppm & 207 & 324 & 314 \\
\hline Mo & ppm & 0.06 & 0.09 & 0.48 \\
\hline $\mathrm{Na}$ & $\%$ & 0.01 & 0.02 & 0.01 \\
\hline $\mathrm{Nb}$ & ppm & 0.9 & 0.2 & 0.2 \\
\hline $\mathbf{N i}$ & ppm & 1.3 & 0.2 & 1.1 \\
\hline
\end{tabular}




\begin{tabular}{|c|c|c|c|c|}
\hline SAMPLE & & $141-18$ & $110-150$ & $139-20$ \\
\hline $\mathbf{P}$ & ppm & 1130 & 200 & 70 \\
\hline $\mathbf{P b}$ & ppm & 12.1 & 2.9 & 18.8 \\
\hline $\mathbf{R b}$ & ppm & 8.7 & 1.7 & 1.8 \\
\hline $\mathbf{R e}$ & ppm & 0.002 & 0.002 & 0.002 \\
\hline $\mathbf{S}$ & $\%$ & 0.01 & 0.01 & 0.01 \\
\hline $\mathbf{S b}$ & $\mathrm{ppm}$ & 0.13 & 0.11 & 0.2 \\
\hline Sc & ppm & 0.9 & 0.3 & 0.3 \\
\hline Se & $\mathrm{ppm}$ & 1 & 1 & 2 \\
\hline Sn & ppm & 0.2 & 0.2 & 0.2 \\
\hline $\mathrm{Sr}$ & ppm & 60.4 & 46.7 & 102 \\
\hline Ta & ppm & 0.06 & 0.05 & 0.05 \\
\hline Te & $\mathrm{ppm}$ & 0.05 & 0.05 & 0.05 \\
\hline Th & ppm & 0.9 & 0.2 & 0.2 \\
\hline $\mathbf{T i}$ & $\%$ & 0.028 & 0.006 & 0.005 \\
\hline $\mathbf{T l}$ & $\mathrm{ppm}$ & 0.03 & 0.02 & 0.02 \\
\hline $\mathbf{U}$ & ppm & 1.9 & 1.5 & 0.5 \\
\hline $\mathbf{V}$ & $\mathrm{ppm}$ & 19 & 9 & 16 \\
\hline $\mathbf{W}$ & $\mathrm{ppm}$ & 0.2 & 0.1 & 0.1 \\
\hline $\mathbf{Y}$ & $\mathrm{ppm}$ & 3.8 & 0.7 & 1.6 \\
\hline $\mathbf{Z n}$ & ppm & 133 & 17 & 980 \\
\hline $\mathbf{Z r}$ & ppm & 10.2 & 1.7 & 2 \\
\hline
\end{tabular}




\begin{tabular}{|c|c|c|c|c|c|}
\hline SAMPLE & & $115-67$ & $129-20$ & $139-68$ & $32-199$ \\
\hline Geologic & & MP & MP & MP & $\mathrm{SdG}$ \\
\hline Unit & & & & & \\
\hline Description & & dolomite & fe-carb veinlets & dolomite sed bx & phyllite \\
\hline & & w/carb- & & & \\
\hline & & qtz-py alt & & & \\
\hline Ag & ppm & 0.02 & 0.02 & 0.14 & 1.94 \\
\hline Al & $\%$ & 0.77 & 0.49 & 0.09 & 6.09 \\
\hline As & ppm & 5 & 5 & 6 & 234 \\
\hline $\mathbf{B a}$ & ppm & 90 & 60 & 20 & 100 \\
\hline $\mathbf{B e}$ & ppm & 0.31 & 0.14 & 0.08 & 1.61 \\
\hline $\mathbf{B i}$ & ppm & 0.08 & 0.02 & 0.01 & 0.39 \\
\hline $\mathrm{Ca}$ & $\%$ & 17.35 & 20.1 & 14.6 & 0.17 \\
\hline Cd & ppm & 0.3 & 0.67 & 1.67 & 1.34 \\
\hline $\mathrm{Ce}$ & $\mathrm{ppm}$ & 7.49 & 3.34 & 1.49 & 46.1 \\
\hline Co & ppm & 1.3 & 0.9 & 9.5 & 16.5 \\
\hline $\mathrm{Cr}$ & $\mathrm{ppm}$ & 9 & 4 & 8 & 56 \\
\hline Cs & ppm & 0.44 & 0.17 & 0.05 & 9.02 \\
\hline $\mathrm{Cu}$ & ppm & 4.2 & 1.9 & 4.7 & 219 \\
\hline $\mathbf{F e}$ & $\%$ & 0.71 & 0.36 & 0.6 & 11.2 \\
\hline Ga & ppm & 1.88 & 1.03 & 0.33 & 15.4 \\
\hline Ge & ppm & 0.13 & 0.16 & 0.05 & 0.26 \\
\hline Hf & ppm & 0.3 & 0.1 & 0.1 & 3.1 \\
\hline Hg & ppm & 0.01 & 0.01 & 0.01 & 0.5 \\
\hline In & ppm & 0.005 & 0.005 & 0.011 & 0.051 \\
\hline $\mathbf{K}$ & $\%$ & 0.52 & 0.47 & 0.03 & 2.55 \\
\hline La & ppm & 3.6 & 1.5 & 0.7 & 23 \\
\hline $\mathbf{L i}$ & ppm & 5.6 & 4.6 & 1.8 & 26.4 \\
\hline Mg & $\%$ & 10.5 & 12 & 9.14 & 0.84 \\
\hline Mn & ppm & 289 & 228 & 188 & 143 \\
\hline Mo & ppm & 0.57 & 0.42 & 0.77 & 14.65 \\
\hline $\mathbf{N a}$ & $\%$ & 0.03 & 0.03 & 0.01 & 0.24 \\
\hline
\end{tabular}




\begin{tabular}{|c|c|c|c|c|c|}
\hline SAMPLE & & $115-67$ & $129-20$ & $139-68$ & $32-199$ \\
\hline $\mathbf{N b}$ & ppm & 0.9 & 0.5 & 0.2 & 7.6 \\
\hline $\mathbf{N i}$ & $\mathrm{ppm}$ & 3.2 & 1.3 & 5.7 & 219 \\
\hline $\mathbf{P}$ & ppm & 90 & 60 & 120 & 510 \\
\hline $\mathbf{P b}$ & ppm & 7.3 & 33.9 & 23 & 71.7 \\
\hline $\mathbf{R b}$ & ppm & 11 & 7.2 & 1.1 & 123.5 \\
\hline $\mathbf{R e}$ & ppm & 0.003 & 0.003 & 0.002 & 0.019 \\
\hline $\mathbf{S}$ & $\%$ & 0.01 & 0.01 & 0.01 & 10 \\
\hline $\mathbf{S b}$ & ppm & 0.14 & 0.07 & 0.28 & 18.5 \\
\hline Sc & ppm & 0.8 & 0.4 & 0.3 & 9.7 \\
\hline $\mathbf{S e}$ & ppm & 1 & 1 & 1 & 15 \\
\hline Sn & $\mathrm{ppm}$ & 0.2 & 0.2 & 0.2 & 1.9 \\
\hline $\mathbf{S r}$ & ppm & 51.4 & 60.5 & 38.7 & 30.9 \\
\hline $\mathbf{T a}$ & ppm & 0.06 & 0.05 & 0.05 & 0.64 \\
\hline $\mathbf{T e}$ & ppm & 0.05 & 0.05 & 0.05 & 0.19 \\
\hline Th & $\mathrm{ppm}$ & 0.7 & 0.3 & 0.2 & 9.5 \\
\hline $\mathbf{T i}$ & $\%$ & 0.024 & 0.013 & 0.005 & 0.283 \\
\hline Tl & ppm & 0.06 & 0.03 & 0.03 & 3.45 \\
\hline $\mathbf{U}$ & ppm & 0.8 & 0.4 & 1.4 & 8.2 \\
\hline $\mathbf{V}$ & ppm & 26 & 3 & 19 & 187 \\
\hline $\mathbf{W}$ & ppm & 0.1 & 0.1 & 0.1 & 0.9 \\
\hline $\mathbf{Y}$ & ppm & 3 & 1.1 & 1 & 25.7 \\
\hline Zn & ppm & 50 & 75 & 1090 & 241 \\
\hline $\mathbf{Z r}$ & ppm & 8.9 & 4.6 & 1.6 & 115.5 \\
\hline
\end{tabular}




\begin{tabular}{|c|c|c|c|c|}
\hline SAMPLE & & $118-163$ & $4-407$ & $101-245$ \\
\hline Geologic & & UMdP & UMdP & UMdP \\
\hline Unit & & & & \\
\hline Description & & blk shale & least alt dolomite & grey dol w/ \\
\hline & & & & white carb alt vein \\
\hline Ag & ppm & 0.7 & 0.05 & 0.05 \\
\hline Al & $\%$ & 9.08 & 0.33 & 0.12 \\
\hline As & ppm & 7.1 & 7 & 5 \\
\hline $\mathbf{B a}$ & ppm & 3330 & 60 & 340 \\
\hline $\mathbf{B e}$ & ppm & 3.91 & 0.19 & 0.17 \\
\hline $\mathbf{B i}$ & ppm & 0.4 & 0.02 & 0.01 \\
\hline $\mathbf{C a}$ & $\%$ & 0.16 & 20.1 & 16.75 \\
\hline Cd & ppm & 1.06 & 0.6 & 10.1 \\
\hline $\mathrm{Ce}$ & ppm & 72.3 & 3.32 & 2.05 \\
\hline Co & ppm & 8.2 & 2.3 & 6.8 \\
\hline $\mathrm{Cr}$ & ppm & 79 & 4 & 5 \\
\hline Cs & ppm & 21.3 & 0.51 & 0.11 \\
\hline $\mathrm{Cu}$ & ppm & 22.2 & 1.5 & 6 \\
\hline $\mathbf{F e}$ & $\%$ & 2.1 & 0.23 & 0.5 \\
\hline Ga & ppm & 24.5 & 0.94 & 0.41 \\
\hline Ge & ppm & 0.16 & 0.15 & 0.06 \\
\hline Hf & ppm & 3.9 & 0.2 & 0.1 \\
\hline Hg & ppm & 0.1 & 0.02 & 0.01 \\
\hline In & ppm & 0.053 & 0.005 & 0.012 \\
\hline $\mathbf{K}$ & $\%$ & 5.13 & 0.19 & 0.08 \\
\hline La & ppm & 37.6 & 1.6 & 1 \\
\hline $\mathbf{L i}$ & ppm & 34 & 10.8 & 3.2 \\
\hline Mg & $\%$ & 1.29 & 12.7 & 10.35 \\
\hline Mn & ppm & 33 & 498 & 512 \\
\hline Mo & ppm & 0.44 & 0.05 & 0.42 \\
\hline $\mathbf{N a}$ & $\%$ & 0.05 & 0.02 & 0.01 \\
\hline $\mathbf{N b}$ & ppm & 14.8 & 0.8 & 0.3 \\
\hline
\end{tabular}




\begin{tabular}{|c|c|c|c|c|}
\hline SAMPLE & & $118-163$ & $4-407$ & $101-245$ \\
\hline $\mathbf{N i}$ & ppm & 32.8 & 4 & 2.6 \\
\hline $\mathbf{P}$ & ppm & 180 & 170 & 160 \\
\hline $\mathbf{P b}$ & ppm & 174 & 14.3 & 27.6 \\
\hline $\mathbf{R b}$ & ppm & 211 & 8.8 & 2 \\
\hline $\mathbf{R e}$ & ppm & 0.002 & 0.002 & 0.002 \\
\hline $\mathbf{S}$ & $\%$ & 0.48 & 0.01 & 0.01 \\
\hline Sb & ppm & 0.63 & 0.06 & 0.08 \\
\hline Sc & ppm & 15.7 & 0.7 & 0.3 \\
\hline Se & ppm & 2 & 1 & 1 \\
\hline Sn & ppm & 3.2 & 0.2 & 0.2 \\
\hline $\mathbf{S r}$ & ppm & 23.5 & 99.5 & 72.1 \\
\hline Ta & ppm & 1.16 & 0.05 & 0.05 \\
\hline Te & ppm & 0.05 & 0.05 & 0.05 \\
\hline Th & ppm & 17.6 & 0.5 & 0.2 \\
\hline $\mathbf{T i}$ & $\%$ & 0.361 & 0.017 & 0.005 \\
\hline Tl & ppm & 1.08 & 0.03 & 0.02 \\
\hline $\mathbf{U}$ & ppm & 9 & 0.9 & 0.7 \\
\hline $\mathbf{V}$ & ppm & 174 & 5 & 7 \\
\hline $\mathbf{W}$ & ppm & 1.6 & 0.1 & 0.1 \\
\hline $\mathbf{Y}$ & ppm & 21.1 & 1.3 & 0.8 \\
\hline $\mathbf{Z n}$ & ppm & 306 & 119 & 678 \\
\hline $\mathbf{Z r}$ & ppm & 134 & 7 & 2.8 \\
\hline
\end{tabular}




\begin{tabular}{|c|c|c|c|c|}
\hline SAMPLE & & $43-288$ & $88-174$ & M124-25 \\
\hline Geologic & & UMdP & UMdP & UP \\
\hline Unit & & & & \\
\hline Description & & grey dol with & grey sed bx with & least alt dolarenite \\
\hline & & Fe-carb veinlets & dol overgrowths & \\
\hline Ag & $\mathrm{ppm}$ & 0.17 & 0.1 & 0.06 \\
\hline Al & $\%$ & 0.36 & 0.27 & 0.5 \\
\hline As & ppm & 5 & 7 & 5 \\
\hline $\mathbf{B a}$ & $\mathrm{ppm}$ & 120 & 130 & 40 \\
\hline Be & ppm & 0.16 & 0.25 & 0.17 \\
\hline $\mathbf{B i}$ & ppm & 0.04 & 0.02 & 0.03 \\
\hline $\mathbf{C a}$ & $\%$ & 19.55 & 17.55 & 18.7 \\
\hline Cd & ppm & 2.23 & 2.05 & 0.49 \\
\hline $\mathrm{Ce}$ & ppm & 5.3 & 2.83 & 5.86 \\
\hline Co & ppm & 4.2 & 4.7 & 0.8 \\
\hline $\mathrm{Cr}$ & ppm & 2 & 7 & 4 \\
\hline Cs & $\mathrm{ppm}$ & 0.34 & 0.29 & 0.63 \\
\hline $\mathbf{C u}$ & ppm & 5.1 & 8.6 & 2.4 \\
\hline $\mathbf{F e}$ & $\%$ & 0.6 & 0.36 & 0.37 \\
\hline Ga & ppm & 1.05 & 0.65 & 1.21 \\
\hline Ge & ppm & 0.06 & 0.12 & 0.1 \\
\hline Hf & ppm & 0.2 & 0.2 & 0.3 \\
\hline Hg & ppm & 0.2 & 0.01 & 0.01 \\
\hline In & ppm & 0.007 & 0.005 & 0.006 \\
\hline $\mathbf{K}$ & $\%$ & 0.21 & 0.17 & 0.38 \\
\hline $\mathbf{L a}$ & ppm & 2.3 & 1.3 & 3 \\
\hline $\mathbf{L i}$ & ppm & 8.5 & 5.1 & 5.1 \\
\hline Mg & $\%$ & 12.25 & 10.55 & 11.3 \\
\hline Mn & ppm & 491 & 279 & 98 \\
\hline Mo & ppm & 0.13 & 0.64 & 0.11 \\
\hline $\mathbf{N a}$ & $\%$ & 0.01 & 0.02 & 0.02 \\
\hline $\mathbf{N b}$ & ppm & 0.8 & 0.4 & 0.8 \\
\hline
\end{tabular}




\begin{tabular}{|c|c|c|c|c|}
\hline SAMPLE & & $43-288$ & $88-174$ & M124-25 \\
\hline $\mathbf{N i}$ & ppm & 3.5 & 4 & 1.8 \\
\hline $\mathbf{P}$ & $\mathrm{ppm}$ & 430 & 110 & 120 \\
\hline $\mathbf{P b}$ & ppm & 30.7 & 20.6 & 6.6 \\
\hline $\mathbf{R b}$ & ppm & 8.1 & 4.6 & 10.8 \\
\hline $\mathbf{R e}$ & ppm & 0.002 & 0.002 & 0.002 \\
\hline $\mathbf{S}$ & $\%$ & 0.01 & 0.03 & 0.01 \\
\hline Sb & ppm & 0.09 & 0.08 & 0.05 \\
\hline Sc & ppm & 0.9 & 0.5 & 1 \\
\hline Se & ppm & 2 & 1 & 1 \\
\hline Sn & ppm & 0.2 & 0.2 & 0.2 \\
\hline $\mathrm{Sr}$ & ppm & 88.7 & 56.6 & 54.2 \\
\hline Ta & ppm & 0.05 & 0.05 & 0.07 \\
\hline Te & ppm & 0.05 & 0.05 & 0.05 \\
\hline Th & ppm & 0.7 & 0.3 & 1 \\
\hline $\mathbf{T i}$ & $\%$ & 0.017 & 0.013 & 0.028 \\
\hline Tl & ppm & 0.04 & 0.02 & 0.05 \\
\hline $\mathbf{U}$ & ppm & 0.5 & 0.6 & 1.6 \\
\hline $\mathbf{V}$ & ppm & 4 & 8 & 13 \\
\hline $\mathbf{W}$ & ppm & 0.1 & 0.1 & 0.1 \\
\hline $\mathbf{Y}$ & ppm & 2 & 1.3 & 2.5 \\
\hline $\mathbf{Z n}$ & ppm & 523 & 124 & 46 \\
\hline $\mathbf{Z r}$ & ppm & 9.7 & 5.6 & 8.7 \\
\hline
\end{tabular}




\begin{tabular}{|c|c|c|c|c|}
\hline SAMPLE & & M124-42 & M124-97 & M124-118 \\
\hline Geologic & & UP & UP & UP \\
\hline Unit & & & & \\
\hline Description & & sed bx w/ carb b/w clasts & MVT-alt w/sp & silty marker bed \\
\hline Ag & $\mathrm{ppm}$ & 0.04 & 1.38 & 0.11 \\
\hline Al & $\%$ & 0.18 & 0.14 & 4.92 \\
\hline As & ppm & 5 & 5 & 3.9 \\
\hline $\mathbf{B a}$ & ppm & 50 & 30 & 700 \\
\hline $\mathbf{B e}$ & ppm & 0.07 & 0.05 & 0.54 \\
\hline $\mathbf{B i}$ & ppm & 0.02 & 0.01 & 0.09 \\
\hline $\mathbf{C a}$ & $\%$ & 12.7 & 18.35 & 0.2 \\
\hline Cd & ppm & 0.54 & 124 & 0.58 \\
\hline $\mathrm{Ce}$ & ppm & 3.97 & 2.61 & 48.5 \\
\hline Co & ppm & 1.6 & 0.6 & 2.3 \\
\hline $\mathrm{Cr}$ & ppm & 9 & 7 & 21 \\
\hline Cs & ppm & 0.09 & 0.08 & 2.37 \\
\hline $\mathbf{C u}$ & ppm & 15.2 & 7.1 & 14.8 \\
\hline $\mathbf{F e}$ & $\%$ & 0.95 & 0.7 & 1.14 \\
\hline Ga & ppm & 0.42 & 1.2 & 8.47 \\
\hline Ge & ppm & 0.14 & 0.14 & 0.21 \\
\hline Hf & ppm & 0.1 & 0.1 & 3.1 \\
\hline Hg & ppm & 0.01 & 3.11 & 0.01 \\
\hline In & ppm & 0.005 & 0.03 & 0.014 \\
\hline $\mathbf{K}$ & $\%$ & 0.17 & 0.09 & 5.33 \\
\hline La & ppm & 1.9 & 1.4 & 20.9 \\
\hline $\mathbf{L i}$ & ppm & 1.5 & 2.4 & 8.1 \\
\hline Mg & $\%$ & 7.93 & 11.2 & 0.31 \\
\hline Mn & ppm & 281 & 397 & 34 \\
\hline Mo & ppm & 0.79 & 0.28 & 0.82 \\
\hline $\mathbf{N a}$ & $\%$ & 0.01 & 0.01 & 0.05 \\
\hline Nb & ppm & 0.3 & 0.2 & 4.3 \\
\hline $\mathbf{N i}$ & ppm & 3.3 & 2.6 & 6.6 \\
\hline
\end{tabular}




\begin{tabular}{|c|c|c|c|c|}
\hline SAMPLE & & M124-42 & M124-97 & M124-118 \\
\hline $\mathbf{P}$ & ppm & 150 & 280 & 90 \\
\hline $\mathbf{P b}$ & ppm & 4.7 & 43.7 & 26.7 \\
\hline $\mathbf{R b}$ & ppm & 2.9 & 2.4 & 78.7 \\
\hline $\mathbf{R e}$ & ppm & 0.002 & 0.002 & 0.004 \\
\hline $\mathbf{S}$ & $\%$ & 0.02 & 1.27 & 0.39 \\
\hline $\mathbf{S b}$ & ppm & 0.06 & 0.39 & 0.42 \\
\hline Sc & ppm & 0.4 & 0.3 & 3.6 \\
\hline Se & ppm & 1 & 6 & 1 \\
\hline Sn & ppm & 0.2 & 0.2 & 1.2 \\
\hline $\mathrm{Sr}$ & ppm & 44.3 & 45 & 43.6 \\
\hline Ta & ppm & 0.05 & 0.05 & 0.33 \\
\hline Te & ppm & 0.05 & 0.05 & 0.05 \\
\hline Th & ppm & 0.4 & 0.2 & 5.7 \\
\hline $\mathbf{T i}$ & $\%$ & 0.008 & 0.007 & 0.126 \\
\hline Tl & ppm & 0.02 & 0.03 & 0.59 \\
\hline $\mathbf{U}$ & ppm & 1.2 & 1.6 & 1.8 \\
\hline V & ppm & 10 & 35 & 19 \\
\hline $\mathbf{W}$ & ppm & 0.1 & 0.1 & 0.5 \\
\hline $\mathbf{Y}$ & ppm & 1.9 & 1.7 & 12.9 \\
\hline $\mathbf{Z n}$ & ppm & 50 & 25700 & 41 \\
\hline $\mathbf{Z r}$ & ppm & 3.4 & 2.5 & 105 \\
\hline
\end{tabular}




\section{D.2 XRF Analyses}

\begin{tabular}{|c|c|c|c|c|c|}
\hline SAMPLE & & $32-199$ & 141-16 & 141-18 & 143-124 \\
\hline Geologic & & $\mathrm{SdG}$ & MP & MP & LP \\
\hline \multicolumn{6}{|l|}{ Unit } \\
\hline Rock Type & & phyllite & carb-alt veinlet & carb-alt & least alt dolomite \\
\hline & & & & veinlet & \\
\hline $\mathrm{SiO2}$ & $\%$ & 53.99 & 14.52 & 2.25 & 7.91 \\
\hline $\mathrm{Al2O3}$ & $\%$ & 11.34 & 0.08 & 0.68 & 1.56 \\
\hline $\mathrm{Fe} 2 \mathrm{O3}$ & $\%$ & 15.69 & 1.62 & 0.43 & 0.91 \\
\hline $\mathrm{CaO}$ & $\%$ & 0.2 & 25.26 & 28.23 & 25.84 \\
\hline MgO & $\%$ & 1.49 & 16.63 & 20.96 & 19.61 \\
\hline $\mathrm{Na2O}$ & $\%$ & 0.29 & 0.03 & 0.05 & 0.05 \\
\hline K2O & $\%$ & 3.23 & 0.01 & 0.14 & 0.71 \\
\hline Cr2O3 & $\%$ & 0.01 & 0.01 & 0.01 & 0.01 \\
\hline TiO2 & $\%$ & 0.58 & 0.01 & 0.03 & 0.06 \\
\hline MnO & $\%$ & 0.02 & 0.09 & 0.02 & 0.03 \\
\hline P2O5 & $\%$ & 0.101 & 0.012 & 0.211 & 0.013 \\
\hline SrO & $\%$ & 0.01 & 0.01 & 0.01 & 0.01 \\
\hline $\mathrm{BaO}$ & $\%$ & 0.04 & 0.01 & 0.01 & 0.01 \\
\hline LOI & $\%$ & 11.3 & 38.6 & 45.3 & 42.1 \\
\hline Total & $\%$ & 98.27 & 96.86 & 98.31 & 98.8 \\
\hline
\end{tabular}




\begin{tabular}{|c|c|c|c|c|c|}
\hline SAMPLE & & 110-150 & 139-20 & 139-231 & 118-163 \\
\hline Geologic & & MP & MP & UMdP (LMdP?) & UMdP \\
\hline \multicolumn{6}{|l|}{ Unit } \\
\hline Rock Type & & least alt dolomite & carb-alt veinlet & least alt dolomite & blk shale \\
\hline $\mathrm{SiO} 2$ & $\%$ & 2.84 & 2.96 & 0.86 & 61.18 \\
\hline $\mathrm{Al2O3}$ & $\%$ & 0.12 & 0.12 & 0.14 & 17.88 \\
\hline $\mathrm{Fe} 2 \mathrm{O3}$ & $\%$ & 0.33 & 0.89 & 0.33 & 2.98 \\
\hline $\mathrm{CaO}$ & $\%$ & 28.33 & 27.99 & 29.32 & 0.21 \\
\hline MgO & $\%$ & 21.25 & 20.93 & 20.95 & 2.3 \\
\hline $\mathrm{Na2O}$ & $\%$ & 0.04 & 0.05 & 0.06 & 0.04 \\
\hline K2O & $\%$ & 0.03 & 0.01 & 0.03 & 6.29 \\
\hline $\mathrm{Cr} 203$ & $\%$ & 0.01 & 0.01 & 0.01 & 0.01 \\
\hline TiO2 & $\%$ & 0.01 & 0.01 & 0.01 & 0.85 \\
\hline MnO & $\%$ & 0.03 & 0.03 & 0.02 & 0.01 \\
\hline P2O5 & $\%$ & 0.033 & 0.008 & 0.105 & 0.031 \\
\hline SrO & $\%$ & 0.01 & 0.01 & 0.01 & 0.01 \\
\hline $\mathrm{BaO}$ & $\%$ & 0.01 & 0.01 & 0.01 & 0.38 \\
\hline LOI & $\%$ & 45.5 & 45.4 & 46.4 & 6.55 \\
\hline Total & $\%$ & 98.51 & 98.39 & 98.22 & 98.7 \\
\hline
\end{tabular}




\begin{tabular}{|c|c|c|c|c|c|c|}
\hline SAMPLE & & $155-185$ & $155-214$ & $4-407$ & 43-97 & $105-113$ \\
\hline Geologic & & LP & LP & UMdP & LP & LP \\
\hline \multicolumn{7}{|l|}{ Unit } \\
\hline Rock Type & & dolomite & phyllite & least alt dolomite & phyllite & dol-specularite vein \\
\hline $\mathrm{SiO} 2$ & $\%$ & 24.99 & 63.99 & 2.3 & 52.32 & 6.2 \\
\hline Al2O3 & $\%$ & 4.28 & 15.27 & 0.47 & 17.8 & 1.33 \\
\hline $\mathrm{Fe} 2 \mathrm{O3}$ & $\%$ & 0.87 & 5.38 & 0.28 & 8.87 & 0.82 \\
\hline $\mathrm{CaO}$ & $\%$ & 19.55 & 0.23 & 28.72 & 1.65 & 26.58 \\
\hline MgO & $\%$ & 13.93 & 2.49 & 20.79 & 3.91 & 19.66 \\
\hline $\mathrm{Na} 2 \mathrm{O}$ & $\%$ & 0.04 & 0.05 & 0.05 & 0.06 & 0.06 \\
\hline K2O & $\%$ & 2.84 & 6.68 & 0.12 & 7.91 & 0.63 \\
\hline Cr2O3 & $\%$ & 0.01 & 0.01 & 0.01 & 0.01 & 0.01 \\
\hline TiO2 & $\%$ & 0.12 & 0.6 & 0.01 & 0.74 & 0.13 \\
\hline MnO & $\%$ & 0.03 & 0.01 & 0.05 & 0.01 & 0.03 \\
\hline P2O5 & $\%$ & 0.034 & 0.048 & 0.029 & 0.087 & 0.028 \\
\hline SrO & $\%$ & 0.01 & 0.01 & 0.01 & 0.01 & 0.01 \\
\hline $\mathbf{B a O}$ & $\%$ & 0.05 & 0.07 & 0.01 & 0.04 & 0.15 \\
\hline LOI & $\%$ & 31.4 & 3.54 & 45.6 & 5.61 & 42.7 \\
\hline Total & $\%$ & 98.13 & 98.35 & 98.42 & 99.02 & 98.32 \\
\hline
\end{tabular}




\begin{tabular}{|c|c|c|c|c|c|c|}
\hline SAMPLE & & 124-62 & 124-75 & 124-78 & $123-46$ & $123-51$ \\
\hline Geologic & & $\mathrm{BH}$ & $\mathrm{BH}$ & $\mathrm{BH}$ & LP & LP \\
\hline \multicolumn{7}{|l|}{ Unit } \\
\hline Rock Type & & type 3 & type 4 & type 4 & Fe-carb selvage alt & Hm alt vein \\
\hline & & & & & w/ no texture & \\
\hline $\mathrm{SiO} 2$ & $\%$ & 2.1 & 21.54 & 33.85 & 18.09 & 4.76 \\
\hline $\mathrm{Al2O3}$ & $\%$ & 1.14 & 0.05 & 0.13 & 4.06 & 2.68 \\
\hline $\mathrm{Fe} 2 \mathrm{O3}$ & $\%$ & 59.75 & 8.36 & 17.76 & 1.66 & 7.61 \\
\hline $\mathrm{CaO}$ & $\%$ & 10.6 & 2.19 & 1.54 & 21.94 & 24.54 \\
\hline MgO & $\%$ & 7.45 & 1.2 & 0.9 & 15.89 & 17.39 \\
\hline $\mathrm{Na} 2 \mathrm{O}$ & $\%$ & 0.02 & 0.02 & 0.02 & 0.1 & 0.04 \\
\hline K2O & $\%$ & 0.08 & 0.01 & 0.01 & 0.99 & 0.6 \\
\hline $\mathrm{Cr} 2 \mathrm{O3}$ & $\%$ & 0.02 & 0.01 & 0.02 & 0.01 & 0.01 \\
\hline TiO2 & $\%$ & 0.01 & 0.01 & 0.01 & 0.19 & 0.11 \\
\hline MnO & $\%$ & 0.08 & 0.02 & 0.01 & 0.07 & 0.1 \\
\hline P2O5 & $\%$ & 0.056 & 0.194 & 0.042 & 0.062 & 0.025 \\
\hline SrO & $\%$ & 0.01 & 0.01 & 0.01 & 0.01 & 0.01 \\
\hline $\mathrm{BaO}$ & $\%$ & 0.01 & 0.01 & 0.03 & 0.01 & 0.01 \\
\hline LOI & $\%$ & 16.7 & 3.58 & 2.57 & 35.1 & 39.2 \\
\hline Total & $\%$ & 98.02 & 37.18 & 56.88 & 98.17 & 97.06 \\
\hline
\end{tabular}




\begin{tabular}{|c|c|c|c|c|c|}
\hline SAMPLE & & 101-128 & 101-245 & $43-191$ & $88-93$ \\
\hline Geologic & & $\mathrm{BH}$ & UMdP & LP & LP \\
\hline \multicolumn{6}{|l|}{ Unit } \\
\hline Rock Type & & type 3 & grey dol with & green phyllite & maroon phyllite \\
\hline & & & white carb alt vein & & \\
\hline $\mathrm{SiO2}$ & $\%$ & 12.38 & 19.92 & 58.17 & 58.98 \\
\hline Al2O3 & $\%$ & 0.96 & 0.2 & 10.34 & 16.53 \\
\hline $\mathrm{Fe} 2 \mathrm{O3}$ & $\%$ & 42.25 & 0.68 & 3.27 & 7.9 \\
\hline $\mathrm{CaO}$ & $\%$ & 9.37 & 24.07 & 5.65 & 0.47 \\
\hline MgO & $\%$ & 6.53 & 15.84 & 5.33 & 2.91 \\
\hline $\mathrm{Na} 2 \mathrm{O}$ & $\%$ & 0.11 & 0.01 & 0.06 & 0.11 \\
\hline K2O & $\%$ & 0.02 & 0.05 & 4.46 & 6.95 \\
\hline $\mathrm{Cr} 2 \mathrm{O3}$ & $\%$ & 0.01 & 0.01 & 0.01 & 0.01 \\
\hline TiO2 & $\%$ & 0.01 & 0.01 & 0.46 & 0.72 \\
\hline MnO & $\%$ & 0.12 & 0.06 & 0.02 & 0.01 \\
\hline P2O5 & $\%$ & 0.082 & 0.034 & 0.065 & 0.091 \\
\hline SrO & $\%$ & 0.04 & 0.01 & 0.02 & 0.01 \\
\hline $\mathbf{B a O}$ & $\%$ & 7.24 & 0.03 & 0.03 & 0.12 \\
\hline LOI & $\%$ & 14.45 & 37.4 & 10.7 & 3.86 \\
\hline Total & $\%$ & 93.56 & 98.3 & 98.57 & 98.66 \\
\hline
\end{tabular}




\begin{tabular}{|c|c|c|c|c|}
\hline SAMPLE & & 88-124 & 88-174 & M124-25 \\
\hline Geologic & & LP & UMdP & UP \\
\hline \multicolumn{5}{|l|}{ Unit } \\
\hline Rock Type & & black phyllite & grey sed bx with & least alt dolarenite \\
\hline & & & dol overgrowths & \\
\hline $\mathrm{SiO2}$ & $\%$ & 58.6 & 14.45 & 3.6 \\
\hline $\mathrm{Al2O3}$ & $\%$ & 17.63 & 0.48 & 0.88 \\
\hline $\mathrm{Fe} 2 \mathrm{O3}$ & $\%$ & 7.06 & 0.51 & 0.54 \\
\hline $\mathrm{CaO}$ & $\%$ & 0.43 & 25.81 & 29.61 \\
\hline MgO & $\%$ & 2.63 & 17.03 & 18.82 \\
\hline $\mathrm{Na} 2 \mathrm{O}$ & $\%$ & 0.13 & 0.01 & 0.01 \\
\hline K2O & $\%$ & 7.67 & 0.1 & 0.31 \\
\hline $\mathrm{Cr} 2 \mathrm{O3}$ & $\%$ & 0.01 & 0.01 & 0.01 \\
\hline TiO2 & $\%$ & 0.75 & 0.01 & 0.04 \\
\hline MnO & $\%$ & 0.01 & 0.04 & 0.01 \\
\hline P2O5 & $\%$ & 0.056 & 0.028 & 0.033 \\
\hline SrO & $\%$ & 0.02 & 0.01 & 0.01 \\
\hline $\mathbf{B a O}$ & $\%$ & 0.03 & 0.01 & 0.01 \\
\hline LOI & $\%$ & 3.75 & 40 & 44.6 \\
\hline Total & $\%$ & 98.77 & 98.48 & 98.46 \\
\hline
\end{tabular}




\begin{tabular}{|c|c|c|c|c|}
\hline SAMPLE & & M124-42 & M124-97 & M124-118 \\
\hline Geologic & & UP & UP & UP \\
\hline \multicolumn{5}{|l|}{ Unit } \\
\hline \multirow[t]{2}{*}{ Rock Type } & & sed bx w/ carb & MVT-alt w/ sp & silty marker bed \\
\hline & & between clasts & & \\
\hline $\mathrm{SiO2}$ & $\%$ & 32.63 & 3.32 & 76.68 \\
\hline $\mathrm{Al2O3}$ & $\%$ & 0.31 & 0.25 & 10.33 \\
\hline $\mathrm{Fe} 2 \mathrm{O3}$ & $\%$ & 1.36 & 1.09 & 1.58 \\
\hline $\mathrm{CaO}$ & $\%$ & 19.76 & 28.88 & 0.25 \\
\hline MgO & $\%$ & 13.12 & 20.8 & 0.65 \\
\hline $\mathrm{Na} 2 \mathrm{O}$ & $\%$ & 0.01 & 0.02 & 0.07 \\
\hline K2O & $\%$ & 0.15 & 0.03 & 7.08 \\
\hline $\mathrm{Cr} 2 \mathrm{O3}$ & $\%$ & 0.01 & 0.01 & 0.01 \\
\hline TiO2 & $\%$ & 0.01 & 0.01 & 0.27 \\
\hline MnO & $\%$ & 0.03 & 0.06 & 0.01 \\
\hline P2O5 & $\%$ & 0.035 & 0.074 & 0.025 \\
\hline SrO & $\%$ & 0.01 & 0.01 & 0.02 \\
\hline $\mathbf{B a O}$ & $\%$ & 0.01 & 0.01 & 0.07 \\
\hline LOI & $\%$ & 30.8 & 41.3 & 1.61 \\
\hline Total & $\%$ & 98.2 & 95.82 & 98.64 \\
\hline
\end{tabular}




\begin{tabular}{|c|c|c|c|c|c|}
\hline SAMPLE & & M124-199 & M124-250 & M124-284 & M124-289 \\
\hline Geologic & & LP & LP & $\mathrm{BH}$ & $\mathrm{BH}$ \\
\hline \multicolumn{6}{|l|}{ Unit } \\
\hline \multirow[t]{2}{*}{ Rock Type } & & pink carb w/ & chalcedonic & type 1 & type 1 \\
\hline & & silica alt & silica alt & & \\
\hline $\mathrm{SiO2}$ & $\%$ & 12.52 & 22.7 & 1.51 & 7.98 \\
\hline $\mathrm{Al2O3}$ & $\%$ & 0.9 & 1.06 & 0.72 & 0.74 \\
\hline $\mathrm{Fe} 2 \mathrm{O3}$ & $\%$ & 1.21 & 0.72 & 1.44 & 4.15 \\
\hline $\mathrm{CaO}$ & $\%$ & 25.75 & 22.77 & 29.15 & 26.98 \\
\hline MgO & $\%$ & 17.6 & 15.57 & 19.82 & 17.88 \\
\hline $\mathrm{Na} 2 \mathrm{O}$ & $\%$ & 0.01 & 0.01 & 0.04 & 0.02 \\
\hline K2O & $\%$ & 0.33 & 0.37 & 0.03 & 0.16 \\
\hline $\mathrm{Cr} 2 \mathrm{O3}$ & $\%$ & 0.01 & 0.01 & 0.01 & 0.01 \\
\hline TiO2 & $\%$ & 0.02 & 0.03 & 0.02 & 0.01 \\
\hline MnO & $\%$ & 0.05 & 0.03 & 0.08 & 0.1 \\
\hline P2O5 & $\%$ & 0.018 & 0.017 & 0.029 & 0.054 \\
\hline SrO & $\%$ & 0.01 & 0.01 & 0.01 & 0.01 \\
\hline $\mathbf{B a O}$ & $\%$ & 0.01 & 0.02 & 0.01 & 0.01 \\
\hline LOI & $\%$ & 40.3 & 35.4 & 45.3 & 40.9 \\
\hline Total & $\%$ & 98.72 & 98.71 & 98.14 & 98.99 \\
\hline
\end{tabular}




\begin{tabular}{|c|c|c|c|c|c|}
\hline SAMPLE & & M124-300 & M124-305 & M124-321 & M124-329 \\
\hline Geologic & & $\mathrm{BH}$ & $\mathrm{BH}$ & $\mathrm{BH}$ & $\mathrm{BH}$ \\
\hline \multicolumn{6}{|l|}{ Unit } \\
\hline Rock Type & & type 1 & type 3 & brecciatred phyllite & type 4 \\
\hline $\mathrm{SiO} 2$ & $\%$ & 1.71 & 7.49 & 62.83 & 20.95 \\
\hline $\mathrm{Al2O3}$ & $\%$ & 0.63 & 0.63 & 13.31 & 0.2 \\
\hline $\mathrm{Fe} 2 \mathrm{O3}$ & $\%$ & 0.84 & 77.65 & 2.92 & 20.92 \\
\hline $\mathrm{CaO}$ & $\%$ & 29.36 & 2.2 & 1.83 & 0.52 \\
\hline MgO & $\%$ & 20.28 & 1.6 & 6.3 & 0.3 \\
\hline $\mathrm{Na} 2 \mathrm{O}$ & $\%$ & 0.03 & 0.02 & 0.07 & 0.01 \\
\hline K2O & $\%$ & 0.02 & 0.04 & 3.49 & 0.01 \\
\hline Cr2O3 & $\%$ & 0.01 & 0.03 & 0.01 & 0.02 \\
\hline TiO2 & $\%$ & 0.02 & 0.01 & 0.57 & 0.01 \\
\hline MnO & $\%$ & 0.08 & 0.07 & 0.02 & 0.02 \\
\hline P2O5 & $\%$ & 0.063 & 0.039 & 0.056 & 0.083 \\
\hline SrO & $\%$ & 0.01 & 0.01 & 0.02 & 0.01 \\
\hline $\mathrm{BaO}$ & $\%$ & 0.01 & 0.01 & 0.04 & 0.01 \\
\hline LOI & $\%$ & 45.4 & 3.61 & 6.68 & 0.94 \\
\hline Total & $\%$ & 98.43 & 93.4 & 98.15 & 43.99 \\
\hline
\end{tabular}




\begin{tabular}{|c|c|c|c|c|}
\hline SAMPLE & & M124-347 & M124-353 & M124-356 \\
\hline Geologic Unit & & $\mathrm{BH}$ & $\mathrm{BH}$ & $\mathrm{BH}$ \\
\hline Rock Type & & type 2 & type 3 & type 1 \\
\hline $\mathrm{SiO} 2$ & $\%$ & 2.08 & 1.8 & 0.69 \\
\hline $\mathrm{Al} 203$ & $\%$ & 0.39 & 0.31 & 0.31 \\
\hline $\mathrm{Fe} 2 \mathrm{O3}$ & $\%$ & 7.37 & 85.6 & 1.16 \\
\hline $\mathrm{CaO}$ & $\%$ & 27.22 & 3.1 & 29.68 \\
\hline MgO & $\%$ & 18.74 & 2.45 & 19.9 \\
\hline $\mathrm{Na2O}$ & $\%$ & 0.02 & 0.01 & 0.04 \\
\hline K2O & $\%$ & 0.03 & 0.01 & 0.01 \\
\hline Cr2O3 & $\%$ & 0.01 & 0.03 & 0.01 \\
\hline TiO2 & $\%$ & 0.01 & 0.01 & 0.01 \\
\hline MnO & $\%$ & 0.13 & 0.05 & 0.04 \\
\hline P2O5 & $\%$ & 0.08 & 0.022 & 0.086 \\
\hline SrO & $\%$ & 0.01 & 0.01 & 0.01 \\
\hline $\mathrm{BaO}$ & $\%$ & 0.01 & 0.01 & 0.01 \\
\hline LOI & $\%$ & 42.5 & 5.08 & 46.2 \\
\hline Total & $\%$ & 98.59 & 98.48 & 98.12 \\
\hline
\end{tabular}




\section{D.3 ICP-MS Correlation Matrix}

The 48-elememt analysis ICP-MS data for each sample was placed in a correlation matrix in MS Excel 2010 using the Data Analysis function

\begin{tabular}{|c|c|c|c|c|c|c|c|c|c|c|}
\hline & Ag & Al & As & $\mathrm{Ba}$ & $\mathrm{Be}$ & $\mathrm{Bi}$ & $\mathrm{Ca}$ & Cd & $\mathrm{Ce}$ & Co \\
\hline Ag & 1 & & & & & & & & & \\
\hline Al & -0.12873 & 1 & & & & & & & & \\
\hline As & 0.251367 & -0.03983 & 1 & & & & & & & \\
\hline $\mathrm{Ba}$ & 0.22628 & 0.214831 & 0.061843 & 1 & & & & & & \\
\hline $\mathrm{Be}$ & 0.026392 & 0.751596 & 0.214511 & 0.287583 & 1 & & & & & \\
\hline $\mathbf{B i}$ & -0.01283 & 0.692163 & 0.271005 & 0.334459 & 0.680511 & 1 & & & & \\
\hline $\mathrm{Ca}$ & -0.33746 & -0.50112 & -0.49984 & -0.36118 & -0.72884 & -0.43087 & 1 & & & \\
\hline Cd & 0.2346 & -0.21269 & 0.215968 & 0.145021 & -0.11428 & -0.11728 & -0.03789 & 1 & & \\
\hline $\mathrm{Ce}$ & -0.1252 & 0.976522 & -0.07056 & 0.187849 & 0.745205 & 0.629547 & -0.50132 & -0.18283 & 1 & \\
\hline Co & -0.07366 & -0.05614 & 0.303785 & -0.0584 & 0.119717 & 0.036102 & -0.09343 & 0.474649 & -0.02424 & 1 \\
\hline $\mathrm{Cr}$ & -0.05784 & 0.919543 & 0.035384 & 0.214417 & 0.777761 & 0.690488 & -0.59341 & -0.18612 & 0.886668 & -0.0517 \\
\hline Cs & -0.09708 & 0.914235 & -0.0203 & 0.300092 & 0.746198 & 0.674468 & -0.47466 & -0.1836 & 0.889515 & -0.04537 \\
\hline $\mathrm{Cu}$ & 0.821524 & -0.05813 & 0.229538 & 0.074563 & 0.010688 & 0.053023 & -0.25973 & 0.021296 & -0.06711 & -0.09325 \\
\hline $\mathrm{Fe}$ & 0.008073 & -0.05579 & 0.556707 & 0.07007 & 0.311156 & 0.161255 & -0.46175 & 0.058103 & -0.05733 & 0.31899 \\
\hline Ga & -0.03816 & 0.967157 & 0.046699 & 0.254947 & 0.816453 & 0.685309 & -0.60508 & -0.11102 & 0.952336 & -0.01398 \\
\hline Ge & -0.0508 & 0.23489 & 0.407537 & -0.05331 & 0.419605 & 0.340094 & -0.38601 & -0.01944 & 0.250933 & 0.337077 \\
\hline $\mathrm{Hf}$ & -0.11695 & 0.965531 & -0.0374 & 0.196004 & 0.729502 & 0.686248 & -0.49663 & -0.21167 & 0.955108 & -0.06251 \\
\hline Hg & 0.551153 & -0.12314 & 0.595105 & 0.079253 & -0.07231 & -0.0707 & -0.2347 & 0.193127 & -0.12934 & 0.15554 \\
\hline In & 0.015506 & -0.10351 & 0.364488 & -0.05411 & 0.410277 & 0.05019 & -0.36044 & -0.00483 & -0.10314 & 0.379286 \\
\hline K & -0.12217 & 0.95946 & -0.11411 & 0.206959 & 0.661306 & 0.577798 & -0.48097 & -0.23735 & 0.946333 & -0.14431 \\
\hline La & -0.12394 & 0.972729 & -0.0712 & 0.199888 & 0.755377 & 0.643917 & -0.49461 & -0.16635 & 0.997124 & -0.00443 \\
\hline Li & -0.04421 & 0.780382 & -0.024 & 0.079127 & 0.658295 & 0.523563 & -0.421 & -0.14369 & 0.758067 & 0.11387 \\
\hline Mg & -0.36194 & -0.43454 & -0.53368 & -0.36299 & -0.69151 & -0.40045 & 0.992934 & -0.06764 & -0.4382 & -0.10025 \\
\hline$M n$ & -0.16529 & -0.40165 & -0.0588 & -0.01186 & -0.44167 & -0.27591 & 0.460364 & 0.43159 & -0.36707 & 0.548044 \\
\hline
\end{tabular}




\begin{tabular}{|c|c|c|c|c|c|c|c|c|c|c|}
\hline & Ag & Al & As & $\mathrm{Ba}$ & $\mathrm{Be}$ & $\mathrm{Bi}$ & $\mathrm{Ca}$ & $\mathrm{Cd}$ & $\mathrm{Ce}$ & Co \\
\hline Mo & -0.00087 & 0.058382 & 0.635117 & 0.00669 & 0.250396 & 0.390608 & -0.43215 & -0.03027 & 0.009456 & 0.125899 \\
\hline $\mathrm{Na}$ & -0.03527 & 0.549586 & 0.310289 & 0.246065 & 0.331717 & 0.676605 & -0.29805 & -0.12793 & 0.480986 & -0.1045 \\
\hline Nb & -0.10852 & 0.884804 & -0.07964 & 0.175062 & 0.734575 & 0.58883 & -0.44042 & -0.18489 & 0.930169 & -0.02221 \\
\hline $\mathbf{N i}$ & -0.07205 & 0.400333 & 0.567399 & -0.02174 & 0.369761 & 0.673075 & -0.33043 & 0.015917 & 0.34397 & 0.292145 \\
\hline $\mathbf{P}$ & -0.04845 & 0.012545 & 0.00552 & -0.09314 & 0.085302 & 0.0637 & 0.006218 & -0.06636 & 0.033927 & -0.09078 \\
\hline $\mathrm{Pb}$ & 0.869933 & -0.10838 & 0.20618 & 0.124047 & -0.01639 & 0.014692 & -0.23239 & 0.19965 & -0.10495 & -0.03414 \\
\hline $\mathbf{R b}$ & -0.1203 & 0.970803 & -0.03038 & 0.268483 & 0.753917 & 0.738819 & -0.47576 & -0.23252 & 0.947853 & -0.10305 \\
\hline $\mathbf{R e}$ & -0.07602 & 0.297546 & 0.427448 & -0.06684 & 0.191334 & 0.539654 & -0.18026 & -0.04779 & 0.245687 & 0.073584 \\
\hline $\mathbf{S}$ & 0.061321 & 0.142654 & & -0.03572 & 05027 & 0.366592 & -0.1971 & 4166 & 3341 & 0.195267 \\
\hline Sb & -0.00066 & -0.03808 & 0.513568 & 0.021118 & 0.344079 & 0.259043 & -0.40014 & 0.045483 & -0.04851 & 0.38626 \\
\hline Sc & -0.11983 & 0.981804 & -0.0372 & 0.216022 & 0.759671 & 0.692855 & -0.47846 & -0.19358 & 0.961022 & -0.0471 \\
\hline Se & 0.71822 & -0.14246 & 0.409487 & 0.200867 & 0.108758 & 0.025843 & -0.48335 & 0.348151 & -0.12721 & 0.014048 \\
\hline Sn & -0.10012 & 0.969162 & -0.00697 & 0.242221 & 0.784795 & 0.698892 & -0.54617 & -0.18558 & 0.952749 & -0.04329 \\
\hline $\mathrm{Sr}$ & 0.014102 & -0.10907 & 0.059112 & 0.350481 & -0.07906 & -0.06746 & -0.12052 & -0.02932 & -0.10912 & -0.07417 \\
\hline $\mathrm{Ta}$ & -0.09893 & 0.983663 & -0.03933 & 0.248309 & 0.785676 & 0.692846 & -0.51243 & -0.18833 & 0.966938 & -0.04859 \\
\hline Te & -0.02443 & 0.239817 & 0.488224 & -0.05372 & 0.169446 & 0.55716 & -0.20531 & -0.05051 & 0.180765 & 0.006142 \\
\hline Th & -0.11226 & 0.973466 & -0.03142 & 0.267006 & 0.780422 & 0.747257 & -0.48484 & -0.19787 & 0.955518 & -0.06193 \\
\hline Ti & -0.11775 & 0.98687 & -0.04421 & 0.182145 & 0.740942 & 0.662071 & -0.4828 & -0.20223 & 0.966846 & -0.04727 \\
\hline TI & -0.07377 & 0.676161 & 0.349203 & 0.095988 & 0.490796 & 0.771163 & -0.39621 & -0.14857 & 0.616163 & -0.02774 \\
\hline $\mathbf{U}$ & 0.013567 & 0.179938 & 0.470897 & 0.175887 & 0.589483 & 0.465026 & -0.50243 & 0.007829 & 0.14186 & 0.230339 \\
\hline v & 0.001128 & 0.290387 & 0.20792 & 0.121048 & 0.432142 & 0.4479 & -0.35506 & -0.03508 & 0.29748 & 0.229243 \\
\hline w & -0.04485 & 0.781313 & 0.222672 & 0.374142 & 0.877684 & 0.719525 & -0.65028 & -0.1056 & 0.782423 & 0.188608 \\
\hline $\mathbf{Y}$ & -0.15309 & 0.902076 & 0.048925 & 0.141616 & 0.67936 & 0.752839 & -0.41938 & -0.17742 & 0.919416 & 0.011399 \\
\hline Zn & 0.634092 & -0.22455 & 0.402831 & 0.137553 & 0.18477 & -0.11957 & -0.54802 & 0.30277 & -0.20256 & 0.019367 \\
\hline $\mathrm{Zr}$ & -0.12889 & 0.952672 & -0.02705 & 0.187715 & 0.719994 & 0.697469 & -0.47367 & -0.21593 & 0.951092 & -0.05924 \\
\hline
\end{tabular}




\begin{tabular}{|c|c|c|c|c|c|c|c|c|c|c|}
\hline & $\mathrm{Cr}$ & Cs & $\mathrm{Cu}$ & $\mathrm{Fe}$ & Ga & Ge & Hf & $\mathrm{Hg}$ & In & K \\
\hline $\mathrm{Cr}$ & 1 & & & & & & & & & \\
\hline Cs & 0.886469 & 1 & & & & & & & & \\
\hline $\mathrm{Cu}$ & -0.01298 & -0.03926 & 1 & & & & & & & \\
\hline $\mathrm{Fe}$ & 0.045646 & -0.02354 & -0.01524 & 1 & & & & & & \\
\hline Ga & 0.944134 & 0.894628 & -0.02574 & 0.059066 & 1 & & & & & \\
\hline Ge & 0.242223 & 0.209715 & -0.05917 & 0.529606 & 0.262185 & 1 & & & & \\
\hline Hf & 0.864917 & 0.831246 & -0.04654 & -0.0869 & 0.918726 & 0.203407 & 1 & & & \\
\hline $\mathrm{Hg}$ & -0.0997 & -0.09789 & 0.450022 & 0.059439 & -0.05044 & 0.078248 & -0.12018 & 1 & & \\
\hline In & -0.06614 & -0.07536 & -0.01704 & 0.57201 & -0.03272 & 0.569227 & -0.12986 & -0.00442 & 1 & \\
\hline K & 0.855924 & 0.843745 & -0.06151 & -0.10171 & 0.912943 & 0.187211 & 0.941922 & -0.12289 & -0.13267 & 1 \\
\hline La & 0.882156 & 0.892787 & -0.06622 & -0.06426 & 0.948548 & 0.234911 & 0.957026 & -0.13229 & -0.10918 & 0.931993 \\
\hline Li & 0.716017 & 0.723866 & 0.012793 & -0.03565 & 0.739191 & 0.23405 & 0.752469 & -0.10944 & -0.01827 & 0.649819 \\
\hline Mg & -0.54057 & -0.41245 & -0.2734 & -0.4929 & -0.54907 & -0.39505 & -0.43472 & -0.25924 & -0.3822 & -0.42398 \\
\hline$M n$ & -0.41457 & -0.36228 & -0.18971 & -0.06407 & -0.41409 & -0.13045 & -0.40334 & 0.023768 & -0.13255 & -0.43477 \\
\hline Mo & 0.163105 & 0.063546 & 0.044061 & 0.757809 & 0.124527 & 0.308234 & 0.065726 & -0.00211 & 0.306087 & -0.0226 \\
\hline $\mathrm{Na}$ & 0.51032 & 0.453473 & 0.002367 & -0.02194 & 0.506563 & 0.112125 & 0.565015 & -0.06894 & -0.14465 & 0.458603 \\
\hline $\mathrm{Nb}$ & 0.785763 & 0.78671 & -0.05626 & -0.07184 & 0.8708 & 0.211624 & 0.921481 & -0.10291 & -0.10331 & 0.853922 \\
\hline $\mathrm{Ni}$ & 0.429651 & 0.366585 & 0.0114 & 0.245306 & 0.401263 & 0.359516 & 0.408402 & 0.043409 & 0.144183 & 0.258763 \\
\hline $\mathbf{P}$ & 0.041488 & -0.00995 & -0.01041 & -0.04984 & 0.032084 & -0.00467 & 0.043447 & -0.08238 & -0.01559 & -0.01594 \\
\hline $\mathrm{Pb}$ & -0.06593 & -0.08222 & 0.937984 & 0.007023 & -0.06575 & -0.04187 & -0.10046 & 0.38884 & -0.01026 & -0.10727 \\
\hline $\mathbf{R b}$ & 0.88625 & 0.942488 & -0.04869 & -0.08802 & 0.926441 & 0.187526 & 0.950429 & -0.11697 & -0.12655 & 0.931544 \\
\hline $\operatorname{Re}$ & 0.287505 & 0.208659 & 0.024647 & 0.045536 & 0.259058 & 0.118591 & 0.356208 & -0.06279 & 0.002208 & 0.21457 \\
\hline $\mathbf{S}$ & 0.142725 & 0.129451 & 0.083839 & 0.093443 & 0.13973 & 0.202026 & 0.161544 & 0.584585 & -0.0355 & 0.068675 \\
\hline Sb & 0.019347 & -0.02152 & 0.002189 & 0.832643 & 0.04848 & 0.703141 & -0.06111 & -0.01987 & 0.745457 & -0.10593 \\
\hline Sc & 0.930827 & 0.921569 & -0.05436 & -0.05319 & 0.976214 & 0.202975 & 0.92479 & -0.11455 & -0.1189 & 0.911651 \\
\hline Se & 0.005667 & -0.10696 & 0.481809 & 0.054054 & -0.00768 & 0.045847 & -0.12024 & 0.473072 & 0.069505 & -0.15712 \\
\hline
\end{tabular}




\begin{tabular}{|c|c|c|c|c|c|c|c|c|c|c|}
\hline Sn & 0.929964 & 0.927637 & -0.03824 & 0.038112 & 0.974408 & 0.219161 & 0.92989 & -0.10494 & -0.07986 & 0.919506 \\
\hline & $\mathrm{Cr}$ & Cs & $\mathrm{Cu}$ & $\mathrm{Fe}$ & Ga & $\mathrm{Ge}$ & $\mathrm{Hf}$ & $\mathrm{Hg}$ & In & K \\
\hline $\mathrm{Sr}$ & -0.11876 & -0.08484 & 0.015975 & 0.071593 & -0.09779 & 0.038471 & -0.10746 & 0.075543 & 0.044689 & -0.10547 \\
\hline Ta & 0.926386 & 0.925341 & -0.03974 & -0.06024 & 0.96972 & 0.202895 & 0.958353 & -0.10274 & -0.10634 & 0.928037 \\
\hline Te & 0.286362 & 0.207008 & 0.064139 & 0.062817 & 0.222075 & 0.134244 & 0.270401 & -0.00919 & -0.02817 & 0.129312 \\
\hline Th & 0.903475 & 0.934088 & -0.04432 & -0.08082 & 0.946569 & 0.189223 & 0.954946 & -0.11225 & -0.12314 & 0.916506 \\
\hline $\mathrm{Ti}$ & 0.921834 & 0.896352 & -0.04941 & -0.07964 & 0.9646 & 0.199352 & 0.959019 & -0.11562 & -0.13019 & 0.926967 \\
\hline TI & 0.648654 & 0.628344 & 0.023204 & 0.001612 & 0.628231 & 0.192407 & 0.693165 & -0.04692 & -0.08167 & 0.574257 \\
\hline U & 0.287994 & 0.254029 & -0.00838 & 0.668678 & 0.262344 & 0.438729 & 0.161221 & -0.05132 & 0.664596 & 0.097749 \\
\hline v & 0.489647 & 0.371062 & 0.000168 & 0.34384 & 0.327926 & 0.439347 & 0.256726 & -0.06448 & 0.26928 & 0.221067 \\
\hline w & 0.759199 & 0.781451 & -0.01547 & 0.400782 & 0.819924 & 0.534141 & 0.730002 & -0.09887 & 0.34864 & 0.70082 \\
\hline$Y$ & 0.818006 & 0.830984 & -0.06579 & -0.0582 & 0.865171 & 0.246351 & 0.89086 & -0.12676 & -0.11549 & 0.838586 \\
\hline $\mathrm{Zn}$ & -0.07478 & -0.17769 & 0.438804 & 0.108422 & -0.07925 & 0.030888 & -0.20628 & 0.41495 & 0.29743 & -0.22351 \\
\hline $\mathrm{Zr}$ & 0.842448 & 0.820562 & -0.05297 & -0.09224 & 0.901024 & 0.204521 & 0.995855 & -0.12151 & -0.13415 & 0.927676 \\
\hline
\end{tabular}




\begin{tabular}{|c|c|c|c|c|c|c|c|c|c|c|}
\hline & La & Li & Mg & $M n$ & Mo & $\mathrm{Na}$ & $\mathrm{Nb}$ & $\mathrm{Ni}$ & $\mathbf{P}$ & $\mathrm{Pb}$ \\
\hline La & 1 & & & & & & & & & \\
\hline Li & 0.767795 & 1 & & & & & & & & \\
\hline Mg & -0.43052 & -0.34572 & 1 & & & & & & & \\
\hline Mn & -0.35032 & -0.30406 & 0.438956 & 1 & & & & & & \\
\hline Mo & 0.010319 & 0.005408 & -0.45066 & -0.16306 & 1 & & & & & \\
\hline $\mathrm{Na}$ & 0.481787 & 0.410585 & -0.27187 & -0.19551 & 0.474312 & 1 & & & & \\
\hline $\mathrm{Nb}$ & 0.943941 & 0.676551 & -0.38342 & -0.342 & -0.01576 & 0.400061 & 1 & & & \\
\hline $\mathrm{Ni}$ & 0.354258 & 0.349032 & -0.31769 & -0.07764 & 0.672583 & 0.810054 & 0.288536 & 1 & & \\
\hline $\mathbf{P}$ & 0.027645 & 0.17526 & -0.00461 & -0.18895 & -0.02521 & 0.07335 & 0.021646 & 0.059442 & 1 & \\
\hline $\mathrm{Pb}$ & -0.10235 & -0.01454 & -0.25201 & -0.09912 & -0.00086 & -0.06138 & -0.09417 & -0.0576 & -0.0326 & 1 \\
\hline $\mathbf{R b}$ & 0.953113 & 0.729038 & -0.41347 & -0.4094 & 0.0733 & 0.557886 & 0.89343 & 0.411938 & 0.018661 & -0.10491 \\
\hline $\operatorname{Re}$ & 0.252605 & 0.205395 & -0.17723 & -0.09986 & 0.594079 & 0.850526 & 0.220828 & 0.893308 & 0.056899 & -0.05763 \\
\hline $\mathbf{S}$ & 0.096515 & 0.053911 & -0.20369 & 0.038805 & 0.441788 & 0.560071 & 0.075939 & 0.715634 & -0.02409 & 0.03069 \\
\hline Sb & -0.05519 & 0.020534 & -0.4228 & -0.09106 & 0.686783 & 0.106121 & -0.06598 & 0.409344 & 0.018186 & -0.00122 \\
\hline Sc & 0.959865 & 0.770107 & -0.41133 & -0.37521 & 0.048077 & 0.528179 & 0.872633 & 0.395823 & 0.024136 & -0.1006 \\
\hline Se & -0.12437 & -0.04929 & -0.50429 & -0.17565 & 0.089982 & 0.016971 & -0.11895 & 0.064468 & 0.079293 & 0.512132 \\
\hline Sn & 0.951768 & 0.71077 & -0.48621 & -0.4107 & 0.153809 & 0.516623 & 0.876198 & 0.413263 & 0.016128 & -0.08695 \\
\hline Sr & -0.10816 & -0.11301 & -0.13728 & -0.06618 & 0.018571 & 0.032863 & -0.09421 & -0.04922 & -0.04649 & 0.010299 \\
\hline $\mathrm{Ta}$ & 0.971358 & 0.771434 & -0.44605 & -0.39107 & 0.044878 & 0.514451 & 0.918892 & 0.380583 & 0.013065 & -0.08666 \\
\hline Te & 0.187764 & 0.170274 & -0.19896 & -0.11902 & 0.632386 & 0.837382 & 0.140197 & 0.908554 & 0.096514 & -0.02608 \\
\hline Th & 0.96326 & 0.739391 & -0.4224 & -0.39154 & 0.063478 & 0.541524 & 0.904663 & 0.414029 & 0.026874 & -0.09606 \\
\hline $\mathrm{Ti}$ & 0.966014 & 0.81114 & -0.41242 & -0.38667 & 0.047048 & 0.554132 & 0.898072 & 0.40754 & 0.026053 & -0.09969 \\
\hline TI & 0.623732 & 0.520942 & -0.35742 & -0.28188 & 0.500116 & 0.907897 & 0.548214 & 0.876889 & 0.04891 & -0.06788 \\
\hline $\mathbf{U}$ & 0.148458 & 0.195612 & -0.51978 & -0.27021 & 0.61087 & 0.220281 & 0.110375 & 0.448141 & 0.082979 & -0.00746 \\
\hline
\end{tabular}




\begin{tabular}{|c|c|c|c|c|c|c|c|c|c|c|}
\hline & La & Li & Mg & Mn & Mo & $\mathrm{Na}$ & $\mathrm{Nb}$ & $\mathrm{Ni}$ & $\mathbf{P}$ & $\mathrm{Pb}$ \\
\hline v & 0.300029 & 0.280554 & -0.3687 & -0.03037 & 0.287424 & 0.241025 & 0.236656 & 0.396033 & -0.05485 & 0.018022 \\
\hline w & 0.784713 & 0.617311 & -0.61378 & -0.30556 & 0.324567 & 0.423271 & 0.726631 & 0.442246 & 0.018043 & -0.03325 \\
\hline $\mathbf{Y}$ & 0.923981 & 0.662979 & -0.36977 & -0.27948 & 0.165251 & 0.652266 & 0.8532 & 0.570077 & 0.071556 & -0.11967 \\
\hline $\mathrm{Zn}$ & -0.20226 & -0.07711 & -0.58285 & -0.23352 & 0.046756 & -0.16212 & -0.18973 & -0.07423 & 0.109333 & 0.454434 \\
\hline $\mathrm{Zr}$ & 0.955672 & 0.736546 & -0.41342 & -0.39142 & 0.077414 & 0.579969 & 0.934476 & 0.429846 & 0.053232 & -0.10953 \\
\hline
\end{tabular}

\begin{tabular}{|c|c|c|c|c|c|c|c|c|c|c|}
\hline & Th & $\mathrm{Ti}$ & $\mathrm{TI}$ & $\mathbf{U}$ & v & w & $\mathbf{Y}$ & $\mathrm{Zn}$ & $\mathrm{Zr}$ & \\
\hline Th & 1 & & & & & & & & & \\
\hline $\mathrm{Ti}$ & 0.960667 & 1 & & & & & & & & \\
\hline TI & 0.695212 & 0.673228 & 1 & & & & & & & \\
\hline $\mathbf{U}$ & 0.216054 & 0.144762 & 0.311176 & 1 & & & & & & \\
\hline V & 0.295613 & 0.276435 & 0.324732 & 0.517459 & 1 & & & & & \\
\hline w & 0.787572 & 0.747312 & 0.541921 & 0.610395 & 0.464279 & 1 & & & & \\
\hline $\mathbf{Y}$ & 0.912779 & 0.878854 & 0.783766 & 0.202234 & 0.380202 & 0.742074 & 1 & & & \\
\hline Zn & -0.19935 & -0.20836 & -0.14084 & 0.151795 & 0.014327 & -0.07581 & -0.249 & 1 & & \\
\hline $\mathrm{Zr}$ & 0.952015 & 0.945359 & 0.71122 & 0.162011 & 0.25551 & 0.728373 & 0.906297 & 0.22608 & & 1 \\
\hline
\end{tabular}

